OAK RIDGE

ORNL/TM-2001/285

NATIONAL LABORATORY

MANAGED BY UT-BATTELLE

FOR THE DEPARTMENT OF ENERGY

\title{
Caustic-Side Solvent Extraction Chemical And Physical Properties Progress in FY 2000 and FY 2001
}

Bruce A. Moyer, Spiro D. Alexandratos, Peter V. Bonnesen, Gilbert M. Brown, John E. Caton, Jr., Lætitia H. Delmau, Caroline R. Duchemin, Tamara J. Haverlock, Tatiana G. Levitskaia, Michael P. Maskarinec, Frederick V. Sloop, Jr., and Christy L. Stine

February 2002

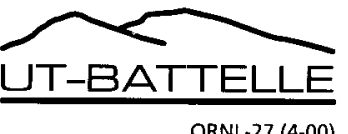




\section{DOCUMENT AVAILABILITY}

Reports produced after January 1, 1996, are generally available free via the U.S. Department of Energy (DOE) Information Bridge:

Web site: http://www.osti.gov/bridge

Reports produced before January 1, 1996, may be purchased by members of the public from the following source:

National Technical Information Service

5285 Port Royal Road

Springfield, VA 22161

Telephone: 703-605-6000 (1-800-553-6847)

TDD: 703-487-4639

Fax: 703-605-6900

E-mail: info@ntis.fedworld.gov

Web site: http://www.ntis.gov/support/ordernowabout.htm

Reports are available to DOE employees, DOE contractors, Energy Technology Data Exchange (ETDE) representatives, and International Nuclear Information System (INIS) representatives from the following source:

Office of Scientific and Technical Information

P.O. Box 62

Oak Ridge, TN 37831

Telephone: 865-576-8401

Fax: 865-576-5728

E-mail: reports@adonis.osti.gov

Web site: http://www.osti.gov/contact.html

This report was prepared as an account of work sponsored by an agency of the United States Government. Neither the United States government nor any agency thereof, nor any of their employees, makes any warranty, express or implied, or assumes any legal liability or responsibility for the accuracy, completeness, or usefulness of any information, apparatus, product, or process disclosed, or represents that its use would not infringe privately owned rights. Reference herein to any specific commercial product, process, or service by trade name, trademark, manufacturer, or otherwise, does not necessarily constitute or imply its endorsement, recommendation, or favoring by the United States Government or any agency thereof. The views and opinions of authors expressed herein do not necessarily state or reflect those of the United States Government or any agency thereof. 
ORNL/TM-2001/285

\author{
Chemical Sciences Division \\ Caustic-Side Solvent Extraction Chemical and Physical Properties \\ Progress in FY 2000 and FY 2001
}

Bruce A. Moyer, Spiro D. Alexandratos, ${ }^{\dagger}$ Peter V. Bonnesen, Gilbert M. Brown, John E. Caton, Jr., Lætitia H. Delmau, Caroline R. Duchemin, Tamara J. Haverlock, Tatiana G. Levitskaia, Michael P. Maskarinec, Frederick V. Sloop, Jr., and Christy L. Stine ${ }^{\dagger}$

Date Published-February 2002

\author{
Prepared by \\ OAK RIDGE NATIONAL LABORATORY \\ Oak Ridge, Tennessee 37831-6285 \\ managed by \\ UT-BATTELLE, LLC \\ for the \\ U.S. DEPARTMENT OF ENERGY \\ under contract DE-AC05-00OR22725
}

${ }^{\dagger}$ University of Tennessee, Knoxville 


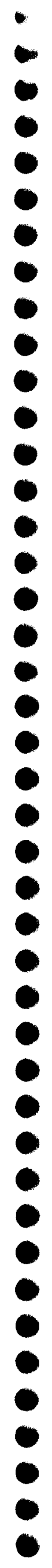




\section{CONTENTS}

LIST OF FIGURES. ix

LIST OF TABLES $\mathrm{xi}$

ABBREVIATIONS, ACRONYMS, AND SYMBOLS xvii

PREFACE.

xix

ACKNOWLEDGMENTS

$\mathrm{xxi}$

EXECUTIVE SUMMARY

PURPOSE

GENERAL CESIUM DISTRIBUTION BEHAVIOR.

CHEMICAL AND THERMAL STABILITY

RADIOLYTIC STABILITY.

SOLUBILITY PHENOMENA

PARTITIONING OF SOLVENT COMPONENTS

DISTRIBUTION OF MAJOR AND MINOR INORGANIC COMPONENTS

DISTRIBUTION OF ORGANIC COMPONENTS.

SOLVENT CLEANUP.

RISK EVALUATION

xxiii

xxiii

xxiii

xxiv

xxiv

$\mathrm{xxV}$

$\mathrm{xxv}$

xxvi

xxvi

xxviii

$\mathrm{xxix}$

ABSTRACT

$\mathrm{xxxi}$

1. INTRODUCTION

1.1 BACKGROUND.

1.2 TECHNICAL RISKS ADDRESSED.

1.3 WORK SCOPE.

1.4 CHAPTER ORGANIZATION

\section{1}

2. BASELINE SOLVENT, SIMULANTS, AND PROTOCOLS ......................................... 5

2.1 BASELINE SOLVENT..................................................................................

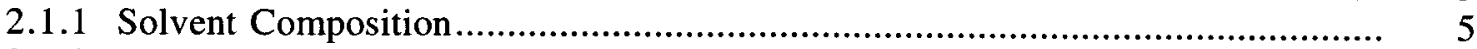

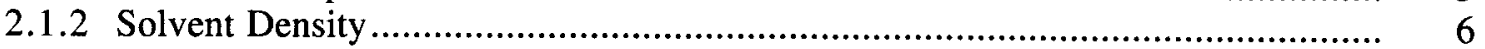

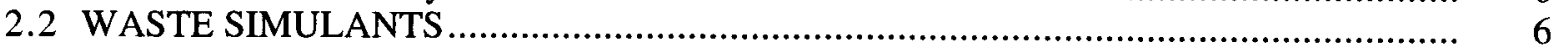

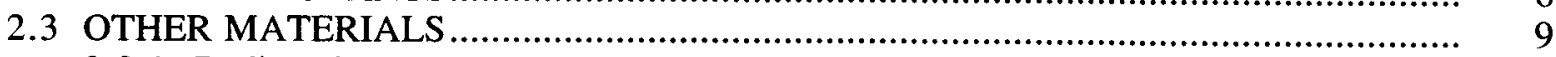

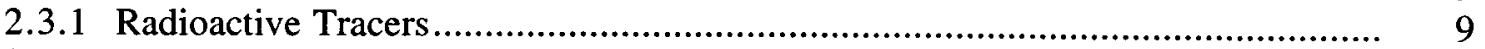

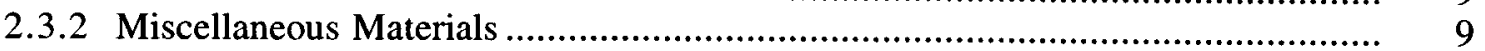

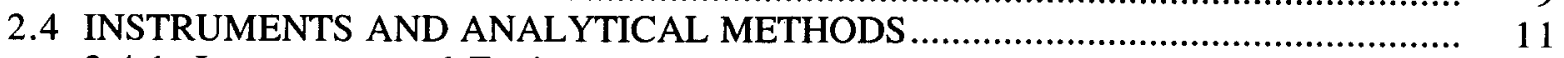

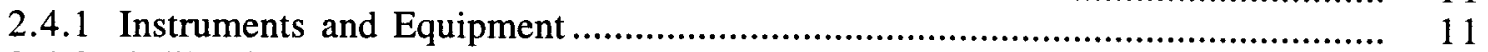

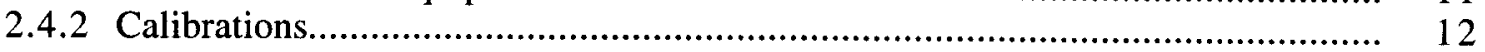

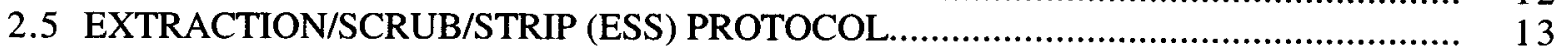

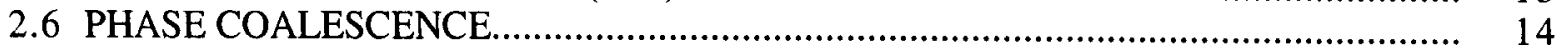

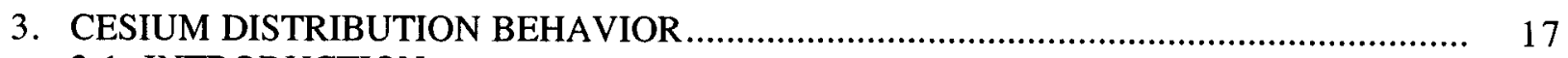

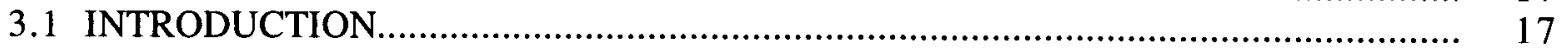


3.1.1 Purpose

3.1.2 Process Goals ................................................................................ 17

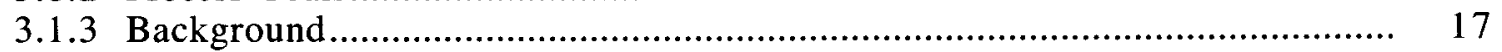

3.1.4 Experimental Design.............................................................................. 18

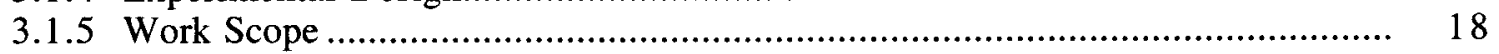

3.2 EXPERIMENTAL SECTION ..................................................................... 18

3.2.1 General ................................................................................... 18

3.2.2 Forward Scrubbing and Stripping ................................................................ 18

3.2.3 Temperature Variation .............................................................................. 19

3.2.4 Effect of Potassium..................................................................................... 19

3.2.5 Effect of 4-sec-Butylphenol................................................................ 19

3.2.6 Effects of Selected Organic Compounds ………............................................. 20

3.2.7 Trials of Different Simulant Recipes................................................................ 21

3.2.8 Effect of Multiple Solvent Contacts with Simulant ........................................... 21

3.2.9 Multi-Cycle Behavior ..................................................................................... 22

3.3 RESULTS AND DISCUSSION ........................................................................... 22

3.3.1 Extract/Scrub/Strip Performance.............................................................. 22

3.3.2 Simple Cesium Loading Model..................................................................... 24

3.3.3 Effect of Cesium Concentration (Loading Behavior) .......................................... 26

3.3.4 Effect of O/A Ratio .................................................................................. 27

3.3.5 Effect of Cs Concentration on Extract/Scrub/Strip Performance......................... 27

3.3.6 Effect of Forward Scrubbing and Stripping..................................................... 28

3.3.7 Extract/Scrub/Strip Performance with Two Extraction Steps............................. 29

3.3.8 Effect of Temperature....................................................................................... $\quad 30$

3.3.9 Effect of Potassium..................................................................................... 33

3.3.10 Effect of 4-sec-Butylphenol...................................................................... 34

3.3.11 Effect of Surfactant Anions................................................................... 36

3.3.12 Effect of Dibutylphosphate......................................................................... 37

3.3.13 Effect of Other Organic Species in the Full Simulant....................................... 38

3.3.14 Trials of Different Simulant Recipes................................................................... 39

3.3.15 Effect of Multiple Solvent Contacts with Simulant............................................ 43

3.3.16 Multi-Cycle Behavior............................................................................ 45

3.3.17 Effect of Modifier Concentration..................................................................... 47

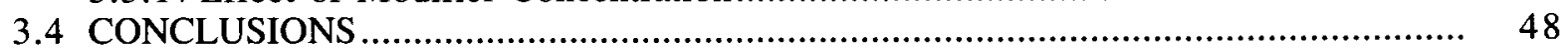

4. DISTRIBUTION OF SYSTEM COMPONENTS ………….............................................. 51

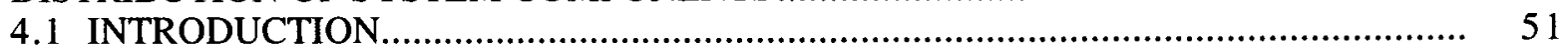

4.1.1 Purpose

4.1.2 Experimental Design................................................................................. 51

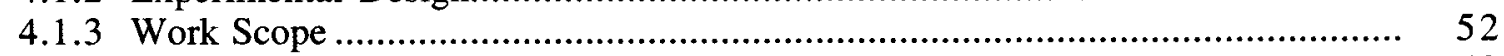

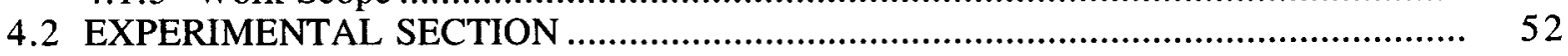

4.2.1 Chemicals and Materials............................................................................... 52

4.2.1.1 Distribution of Cations, Anions, and Solvent Components..................... 52

4.2.1.2 Distribution of Actinides, Strontium, and Technetium......................... 52

4.2.1.3 Anion-Exchange Resins......................................................................... 53

4.2.2 Phase-Contacting Protocols and Analytical Methods ………............................. 54

4.2.2.1 Partitioning of Solvent Components.................................................... 54

4.2.2.2 Distribution of Alkali Metal Cations........................................................ 55

4.2.2.3 Distribution of Other Metals and Selected Radionuclides ...................... 55

4.2.2.4 Distribution of Anions by Ion Chromatography ................................. 56

4.2.2.5 Distribution Behavior in Multi-Cycle Batch Tests................................. 57

4.2.2.6 Dibutylphosphate Distribution Measurement by NMR.......................... 58

4.2.2.7 Phase-Contacting Protocol for 4-sec-Butylphenol Partitioning ……….... 58

4.2.2.8 Partitioning of Surfactant Anions..................................................... $\quad 60$ 
4.2.2.9 Partitioning of Amines................................................................ 63

4.2.2.10Ion Exchange of Organic Anions ................................................ 64

4.2.2.11 Partitioning of Tributylphosphate and 1-Butanol............................. 64

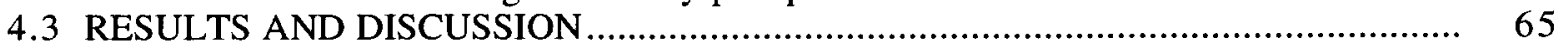

4.3.1 Partitioning of Solvent Components ...................................................... 65

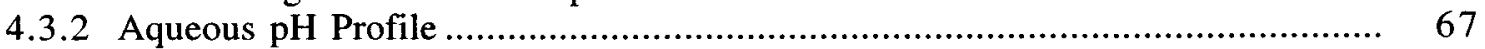

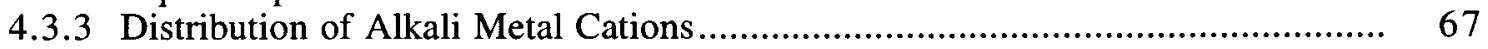

4.3.3.1 Generál Approach .................................................................. 67

4.3.3.2 Distribution of Alkali Metal Cations in the Absence of BOBCalixC6... 68

4.3.3.3 Extraction of Alkali Metal Cations From Simple Salt Matrices ............ 70

4.3.3.4 Extraction of Alkali Metal Cations From the Full Simulant Containing Variable Cesium or Potassium Concentration as a Function of Solvent Modifier Concentration

4.3.3.5 Effect of Anion on Alkali Metal Distribution Behavior Using Solvent With or Without BOBCalixC6 Extractant ............................................ 84

4.3.3.6 Effect of O/A Ratio........................................................................... $\quad 90$

4.3.3.7 Effect of Multiple Solvent Contacts With Simulant........................... 90

4.3.4 Distribution of Other Metals and Selected Radionuclides.............................. 94

4.3.5 Distribution of Anions by Ion Chromatography......................................... 95

4.3.6 Distribution Behavior in Multi-Cycle and Multiple-Contact Batch Tests............ 98

4.3.6.1 Five-Cycle Test Results .................................................................. 98

4.3.6.2 Electrospray Mass-Spectrometry Results on Multiple Contacts ............ 99

4.3.7 Dibutylphosphate Partitioning as Measured by NMR ................................. 101

4.3.7.1 Three-Contact NMR Experiment ................................................ 101

4.3.7.2 Partitioning of DBP Between the Solvent and the Simulant by NMR .... 101

4.3.7.3 Partitioning of DBP Between the Solvent and $\mathrm{NaOH}$ Solutions............ 101

4.3.8 Partitioning of 4-sec-Butylphenol ....................................................... 102

4.3.9 Partitioning of Surfactant Anions.......................................................... 104

4.3.10 Partitioning of Amines.................................................................. 107

4.3.11 Ion Exchange of Organic Anions.......................................................... 108

4.3.12 Partitioning of Tributylphosphate and 1-Butanol ...................................... 113

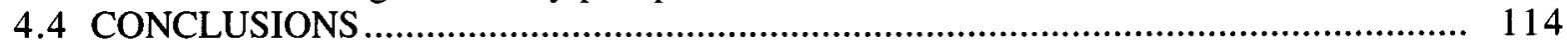

5. THERMAL STABILITY ............................................................................... 119

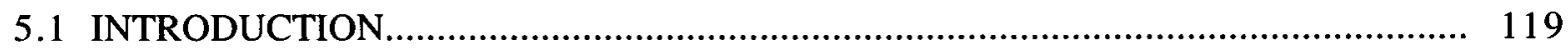

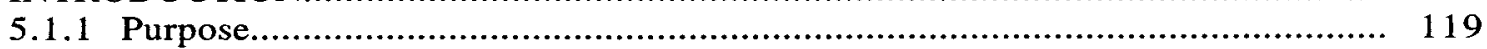

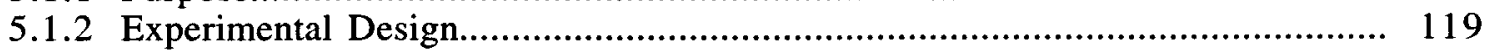

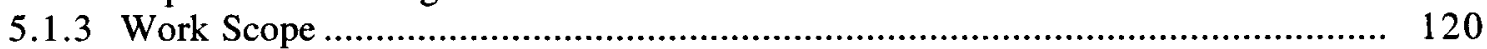

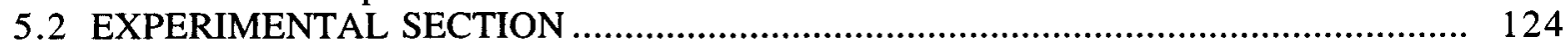

5.2.1 Chemicals, Materials, and Equipment .................................................. 124

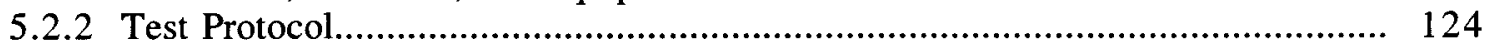

5.2.3 Extract/Scrub/Strip Protocol ............................................................... 125

5.2.4 Break Time (Coalescence) Measurements................................................ 126

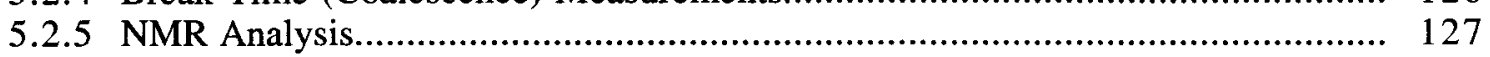

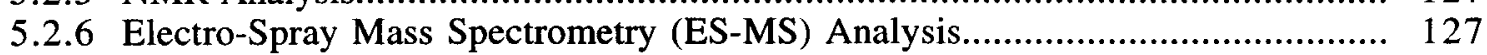

5.2.7 Chemical Analysis.......................................................................... 128

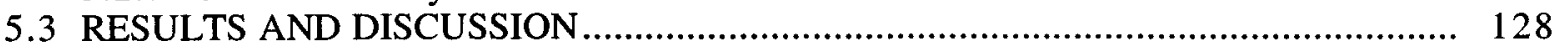

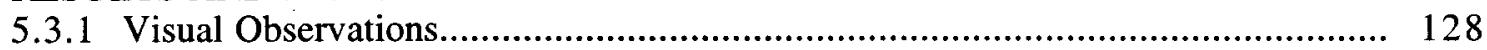

5.3.1.1 Thermal-Stability Test With Flowsheet Aqueous Phases...................... 128

5.3.1.2 Nitric Acid Thermal-Stability Test ............................................. 130

5.3.2 Break-Time Results and Observations for Thermal-Stability Samples .............. 130

5.3.3 Extract/Scrub/Strip Performance of Thermal-Stability Samples Over the

Course of 235 Days ....................................................................... 132

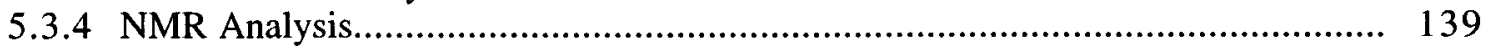


5.3.4.1 Thermal-Stability Test With Flowsheet Aqueous Phases..................... 139

5.3.4.2 Nitric Acid Thermal-Stability Test .................................................... 139

5.3.5 Electro-Spray Mass Spectrometry

5.3.5.1 Experimental Approach and General Observations ............................... 141

5.3.5.2 Anion-Mode Analysis.................................................................... 141

5.3.5.3 Cation-Mode Analysis .................................................................. 144

5.3.6 Chemical Analysis of Thermal-Stability Samples by GC and RP-HPLC............ 144

5.4 CONCLUSIONS.

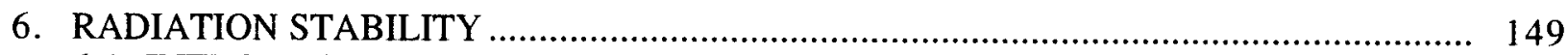

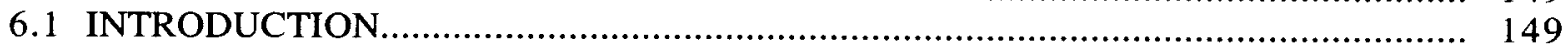

6.1 .1 Purpose

6.1.2 Experimental Design.................................................................................... 149

6.1.3 Work Scope …….................................................................................... 150

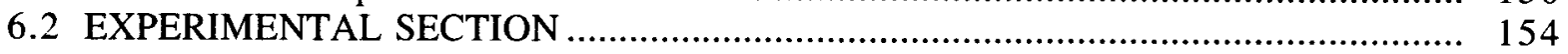

6.2.1 Irradiation Samples................................................................................. 154

6.2.2 Analytical Procedures ........................................................................... 154

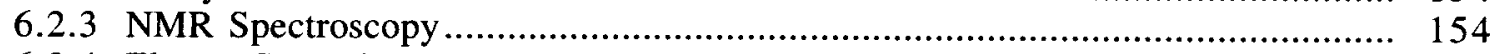

6.2.4 Electro-Spray Mass Spectrometry ……..................................................... 156

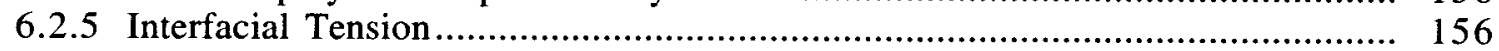

6.2.6 Sodium Extraction Capacity........................................................................ 156

6.2.7 Extract/Scrub/Strip Protocol .......................................................................... 156

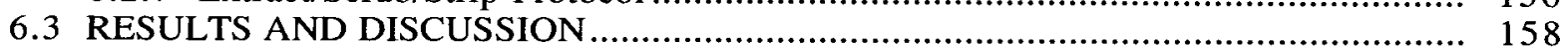

6.3.1 Visual Observations....................................................................................... 158

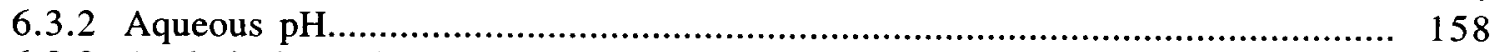

6.3.3 Analytical Results.................................................................................... 158

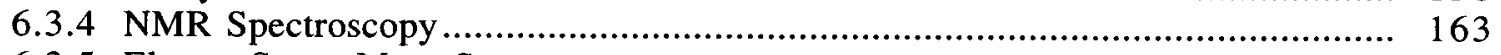

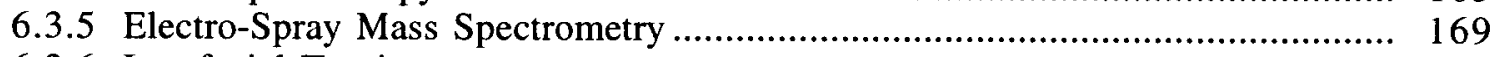

6.3.6 Interfacial Tension .................................................................................... 169

6.3.7 Sodium Extraction Capacity.......................................................................... 170

6.3.8 Extract/Scrub/Strip Performance................................................................. 170

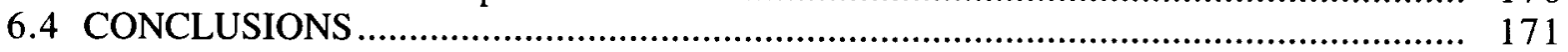

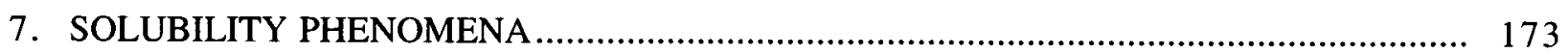

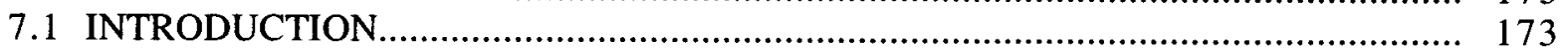

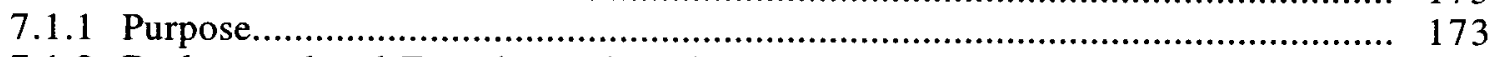

7.1.2 Background and Experimental Design ............................................................... 173

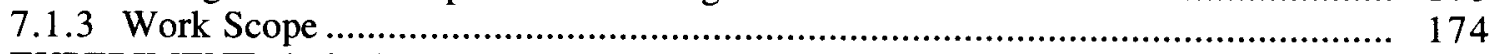

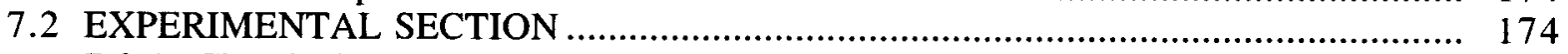

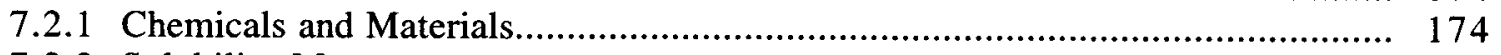

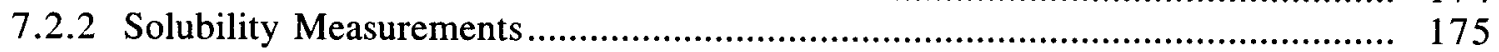

7.2.2.1 Preparation of the Solubility Samples .................................................... 175

7.2.2.2 Preparation of Solubility Samples for HPLC Analysis ........................... 176

7.2.2.3 Preparation of Standard Samples for HPLC Analysis ............................ 176

7.2.3 Solids-Formation Tests............................................................................. 177

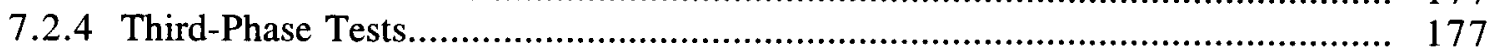

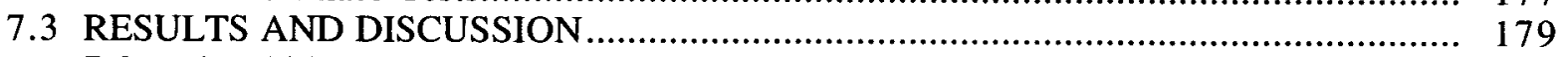

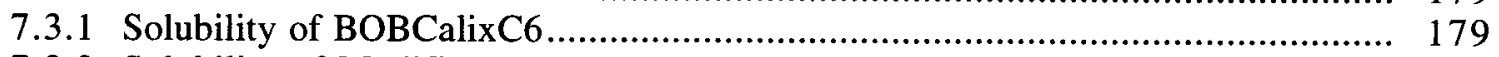

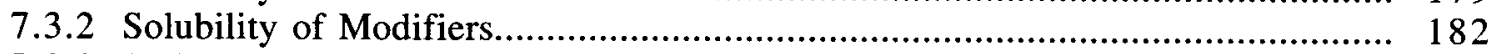

7.3.3 Solids-Formation Tests.................................................................................. 183

7.3.4 Third-Phase Formation .................................................................................. 184

7.3.4.1 Visual Observations ....................................................................... 184

7.3.4.2 Effect of BOBCalixC6 Loading ..................................................... 185

7.3.4.3 Effect of $\mathrm{Cs}^{+}$and $\mathrm{K}^{+}$in Full Simulant and Cs-7SB Modifier in Solvent 185 
7.3.4.4 Effect of Temperature ....................................................... 188

7.3.4.5 Effect of Scrub.................................................................... 189

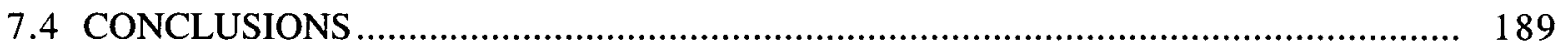

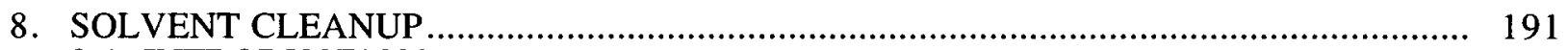

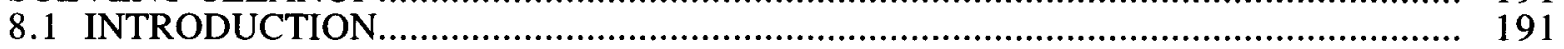

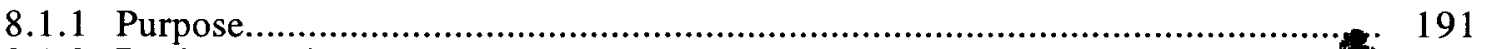

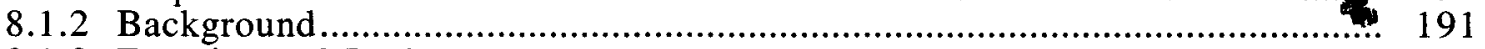

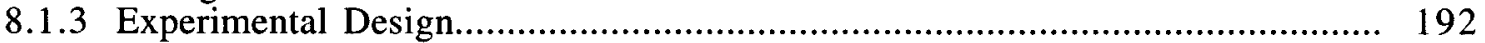

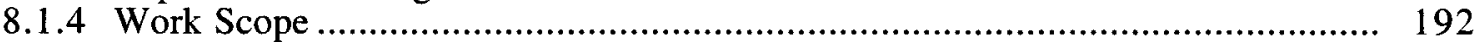

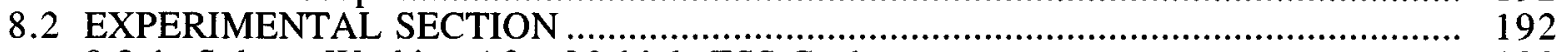

8.2.1 Solvent Washing After Multiple ESS Cycles ............................................... 192

8.2.2 Solvent Washing After Multiple Contacts With Full Simulant .............................. 193

8.2.3 Washing Internally Irradiated Solvent Samples........................................... 193

8.2.4 ESS Procedure for Washed Internally Irradiated Samples................................. 194

8.2.5 Washing Externally Irradiated or Thermally Treated Samples ........................ 194

8.2.6 ESS of Washed Solvents Used in Contactor Tests............................................. 194

8.3 RESULTS AND DISCUSSION ................................................................. 195

8.3.1 Solvent Washing after Multiple ESS Cycles ............................................... 195

8.3.2 Solvent Washing after Multiple Contacts with the Full Simulant .......................... 196

8.3.3 Solvent Washing of Internally Irradiated Solvent Samples ............................ 196

8.3.3.1 Genesis of Solvent Washing Procedure Due to Emulsion Formation.... 196

8.3.3.2 ESS Performance of Washed Internally Irradiated Samples................. 199

8.3.4 Solvent Washing of Externally Irradiated Samples............................................... 201

8.3.5 Solvent Washing of 235-Day Thermal-Stability Samples.............................. 202

8.3.6 ESS Performance of Solvents Used in Contactor Tests....................................... 202

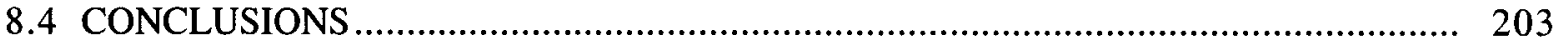

9. CONCLUSIONS AND PATH FORWARD ............................................................. 205

9.1 TECHNOLOGY RISKS ADDRESSED...................................................... 205

9.2 QUESTIONS REMAINING AND SUGGESTIONS FOR FURTHER WORK ............... 206

9.2.1 Summary Remarks................................................................................... 206

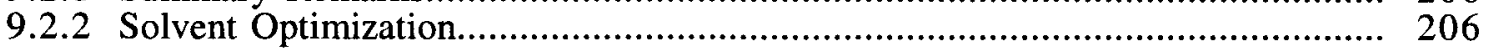

9.2.3 Actinide and Strontium Extraction ..................................................... 206

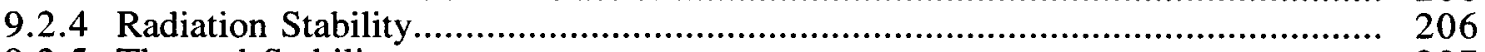

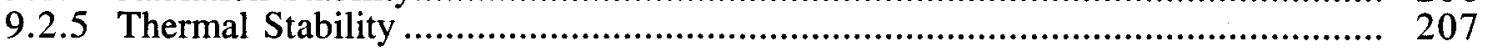

9.2.6 Solvent Cleanup ............................................................................................. 207

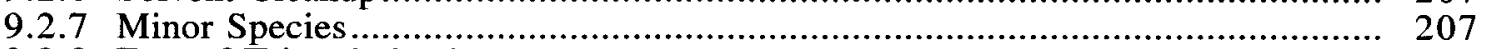

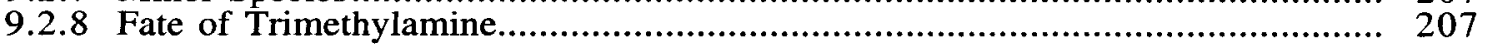

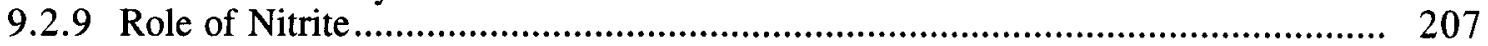

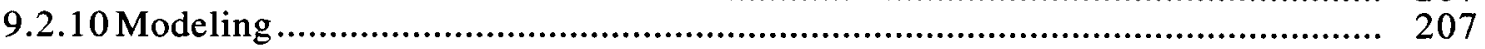

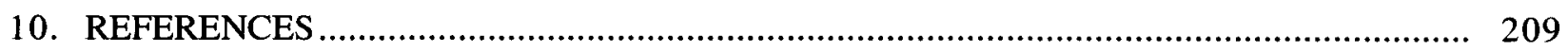




\section{LIST OF FIGURES}

Figure

Page

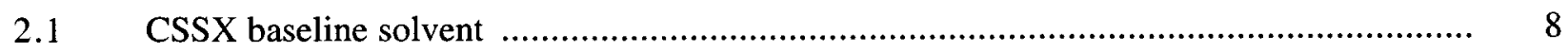

2.2 Extract/scrub/strip (ESS) sequential contacting protocol..................................... 13

2.3 Dispersion number vs. temperature on extraction phase of ESS ............................. 14

3.1 Temperature dependence of the cesium distribution ratio between full simulant and baseline solvent at $\mathrm{O} / \mathrm{A}=0.33$.

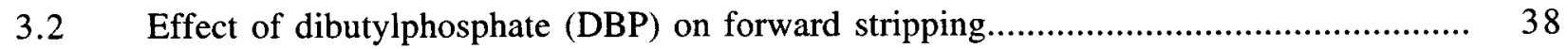

3.3 Cesium distribution in the fourth strip of the ESS protocol as a function of $\mathrm{NaNO}_{2}$ concentration in tank simulants....

3.4 Cesium distribution ratio in an $\mathrm{E}_{5} \mathrm{SS}_{6}$ test with different simulants............................ 44

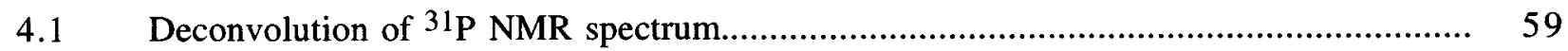

4.2 Effect of $\mathrm{Cs}^{+}$concentration on $\mathrm{Cs}^{+}$distribution ratio, loading, and third-phase formation 73

4.3 Effect of $\mathrm{K}^{+}$ion concentration on $\mathrm{Cs}^{+}$distribution ratio, loading, and third-phase formation.

4.4 Effect of $\mathrm{Na}^{+}$ion concentration in the aqueous phase on $\mathrm{Na}^{+}$distribution ratio............. 76

4.5 Effect of aqueous $\mathrm{Cs}^{+}$ion concentration on $D_{\mathrm{Cs}}, D_{\mathrm{K}}$, and BOBCalixC6 loading............ 79

4.6 Effect of Cs-7SB modifier concentration in the solvent on total BOBCalixC6 loading at different $\mathrm{Cs}^{+}$concentrations in the full simulant................................. 80

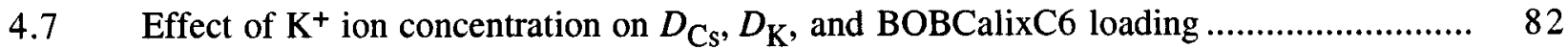

4.8 Effect of Cs-7SB modifier concentration in the baseline solvent on total BOBCalixC6 loading at different $\mathrm{K}^{+}$concentrations in the full simulant .................................... 83

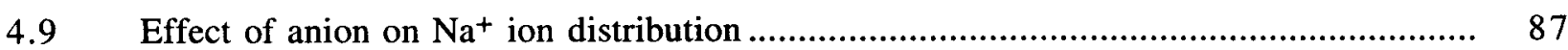

4.10 Distribution of $\mathrm{CsNO}_{3}$ from $0.5 \mathrm{mM} \mathrm{CsNO}_{3}, 60 \mathrm{mM} \mathrm{KNO}_{3}$ in variable $\mathrm{NaX} \ldots \ldots \ldots \ldots . . . . . . \quad 89$

4.11 Distribution of $\mathrm{KNO}_{3}$ from $0.5 \mathrm{mM} \mathrm{CsNO}_{3}, 60 \mathrm{mM} \mathrm{KNO}$ in variable $\mathrm{NaX}_{3} \ldots \ldots \ldots \ldots . . . . . . . \quad 89$

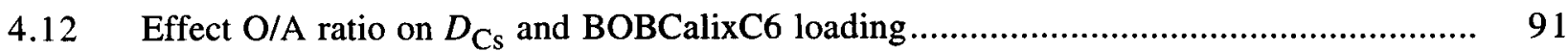

4.13 Effect of multiple contacts of the baseline solvent with the full simulant on (A) $D_{\mathrm{Cs}}$ and (B) BOBCalixC6 loading

4.14 Surfactant anion partitioning between baseline solvent and $\mathrm{NaOH}$ solutions at $25{ }^{\circ} \mathrm{C} \ldots . . \quad 106$ 
5.1 Contacting scheme for solvent samples thermally treated in contact with waste simulant. 121

5.2 Contacting scheme for solvent samples thermally treated in contact with scrub solution. 122

5.3 Contacting scheme for solvent samples thermally treated in contact with strip solution... 123

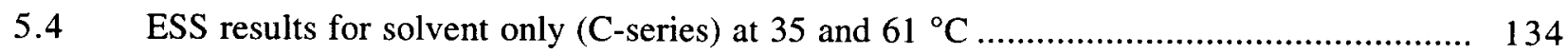

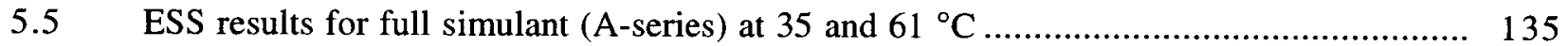

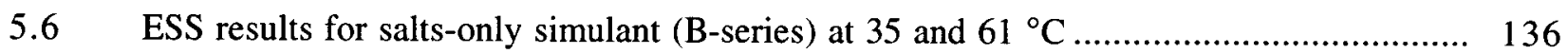

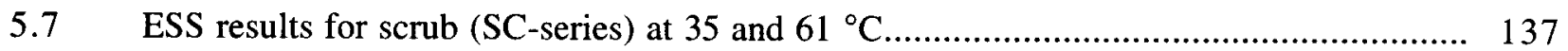

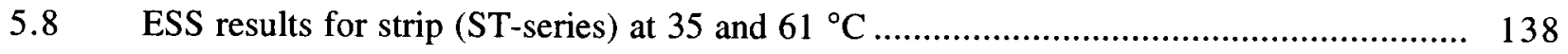

5.9 TOA and DOA concentrations in scrub (SC) and strip (ST) samples as a function

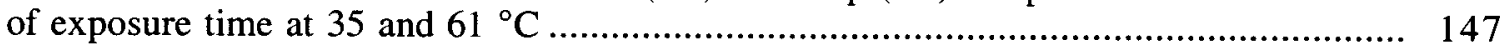

6.1 Contacting scheme for solvent samples treated by external gamma irradiation in contact with waste simulant

6.2 Contacting scheme for solvent samples treated by external gamma irradiation in contact with scrub solution

6.3 Contacting scheme for solvent samples treated by external gamma irradiation in contact with strip solution

6.4 Concentration of TOA in the baseline solvent irradiated over flowsheet solutions........... 161

7.1 Forward solubility of BOBCalixC6 in Isopar L as a function of Cs-7SB modifier concentration

7.2 Modifiers having different alkyl structures

8.1 Rejuvenation of the solvent upon washing with $10 \mathrm{mM} \mathrm{NaOH}$ following five cycles... 


\section{LIST OF TABLES}

Table

Page

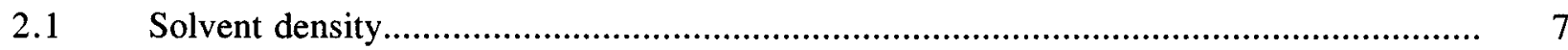

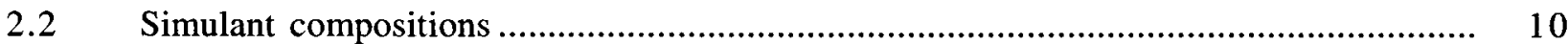

3.1 Experimental volumes in $\mathrm{mL}$ pertaining to the multi-contact experiment................... 21

3.2 Experimental volumes in $\mathrm{mL}$ pertaining to the multi-cycle experiment....................... 22

3.3 Cesium distribution ratios from extract/scrub/strip experiments (ESS) at $25^{\circ} \mathrm{C}$ with baseline solvent and full simulant ........................................................................... 23

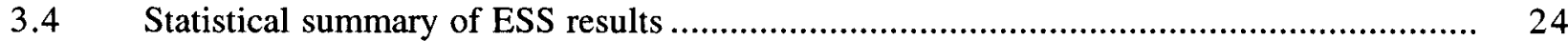

3.5 Cesium extraction from the full simulant by the baseline solvent at $25^{\circ} \mathrm{C}$ as a function of aqueous cesium concentration

3.6 Cesium extraction from the full simulant by the baseline solvent at $25^{\circ} \mathrm{C}$ as a function of $\mathrm{O} / \mathrm{A}$ ratio

3.7 Cesium distribution ratios from extract/scrub/strip experiments with increasing Cs concentration in the full simulant...

3.8 Cesium distribution ratios in forward scrubbing and stripping

3.9 Cesium distribution ratios from extract/scrub/strip experiments with two extraction steps $\left(\mathrm{E}_{2} \mathrm{SS}\right)$ at $25^{\circ} \mathrm{C}$ with baseline solvent and full simulant

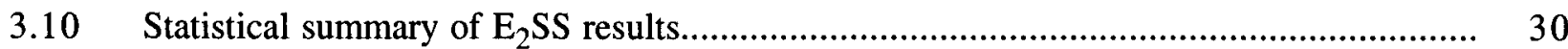

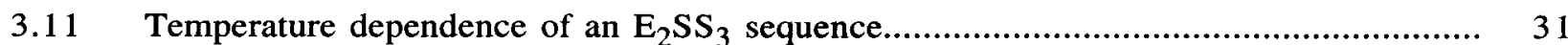

3.12 Temperature dependence of $D_{\mathrm{Cs}}$ between baseline solvent and full simulant............... 32

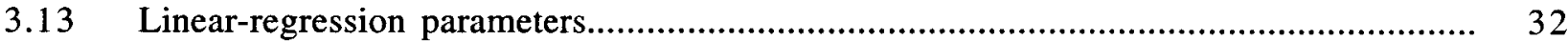

3.14 Results from batch internal-irradiation experiment ........................................ 32

3.15 Cesium extraction from the full simulant by the baseline solvent as a function of aqueous potassium concentration .............................................................................

3.16 Effect of added 4-sec-butylphenol on $D_{\mathrm{Cs}}$ in forward stripping............................... 35

3.17 Effect of 4-sec-butylphenol concentration on Cs distribution ratios in ESS tests ............ 36

3.18 Values of $D_{\mathrm{CS}}$ determined for one ESS cycle using pristine washed solvent containing $0.2 \mathrm{mM}$ SDS or SA at $25^{\circ} \mathrm{C}$ 
3.19 Values of $D_{\mathrm{Cs}}$ determined for one ESS cycle using pristine washed solvent containing dibutylphosphate at $25{ }^{\circ} \mathrm{C}$.

3.20 Summary of the effects of organic species on cesium distribution in ESS tests

3.21 Simulant recipes.

3.22 Effect of simulant recipe on the distribution of sodium, potassium, and cesium in the extraction step of an ESS with baseline solvent at $25^{\circ} \mathrm{C}$.

3.23 Detailed distribution results for effect of simulant recipe

3.24 Effect of substituting chloride for nitrite in simulant recipes.

3.25 Effect of adding nitrite in the scrub step.

3.26 Cesium distribution in an $\mathrm{E}_{5} \mathrm{SS}_{6}$ test with different simulants.

3.27 Cesium distribution ratios in the five-cycle experiment.

3.28 Cesium distribution ratios in three-cycle experiment.

3.29 Cesium distribution ratios for full simulant containing varying cesium ion concentrations and baseline solvent with three Cs-7SB modifier concentrations at $25{ }^{\circ} \mathrm{C}$

4.1 Preparation of $1000 \mathrm{mg} / \mathrm{L}$ anion standard.

4.2 Species analyzed in five-cycle experiment

4.3 Activity and quenching test results.

4.4 Batch test experimental matrix.

4.5 Partition ratios of trioctylamine, modifier, and extractant for various aqueous solutions.

4.6 Aqueous pH profile in batch ESS tests..

4.7 Cesium distribution ratios and organic-phase concentrations observed in distribution tests at $25^{\circ} \mathrm{C}$ using $0.5 \mathrm{M} \mathrm{Cs-7SB}$ modifier and $0.001 \mathrm{M}$ TOA in Isopar L

4.8 $\mathrm{K}^{+}$and $\mathrm{Na}^{+}$distribution ratios and organic-phase concentrations at $25^{\circ} \mathrm{C}$ using 0.5 M Cs-7SB modifier and 0.001 M TOA in Isopar L

4.9 $\mathrm{Cs}^{+}$ion distribution ratios, organic phase concentrations, and BOBCalicC6 loading observed in $\mathrm{Cs}^{+}$ion extraction tests using baseline solvent

4.10 $\mathrm{K}^{+}$ion distribution ratios, organic phase concentrations, and BOBCalixC6 loading observed in $\mathrm{K}^{+}$extraction tests using the baseline solvent

$4.11 \mathrm{Na}^{+}$ion distribution ratios and organic phase concentrations observed in $\mathrm{Na}^{+}$ extraction tests using full solvent.... 
4.12 Effect of $\mathrm{Cs}^{+}$concentration in the simulant on distribution behavior of alkali metal ions and total BOBCalixC6 loading at different Cs-7SB modifier

concentrations in the solvent.

4.13 Effect of $\mathrm{K}^{+}$ion concentration in the simulant on distribution behavior of alkali metal ions and total BOBCalixC6 loading at different Cs-7SB modifier concentrations in the solvent......

4.14 $\mathrm{Na}^{+}$ion distribution ratios and organic phase concentrations at $\mathrm{O} / \mathrm{A}=1$ and $25^{\circ} \mathrm{C}$

using solvent with or without BOBCalixC6 extractant

4.15 Distribution ratios of cesium and potassium from constant cesium and potassium concentration and variable $\mathrm{NaX}\left(\mathrm{X}=\mathrm{OH}^{-}, \mathrm{NO}_{3}^{-}, \mathrm{NO}_{2}^{-}, \mathrm{Cl}^{-}\right)$concentration

4.16 Effect of $\mathrm{O} / \mathrm{A}$ ratio on distribution behavior of alkali metal ions

4.17 Effect of multiple solvent contacts with simulant on distribution of alkali metal ions......

4.18 Cesium distribution in the presence of $\mathrm{U}, \mathrm{Np}, \mathrm{Pu}, \mathrm{Sr}$, and $\mathrm{Tc}$

4.19 Concentration of nitrate anion found in scrub solutions.

4.20 Concentration of anions found in the strip solution after subtraction of $\mathrm{HNO}_{3}$

4.21 Comparison of single- and multiple-contact strip solutions

4.22 Results of washing by $10 \mathrm{mM} \mathrm{NaOH}$.

4.23 Concentration of anions released in deionized water stripping of loaded solvents.

4.24 Cesium and total phosphorous content of the organic phases over five cycles.

4.25 Cation concentrations of the aqueous phases over five cycles.

4.26 Nitrate and nitrite concentrations of the aqueous phases over five cycles.

4.29 Partition ratios for ${ }^{14} \mathrm{C}$-labeled surfactants in batch tests

4.30 The partitioning of ${ }^{14} \mathrm{C}$-labeled anionic surfactants in the ESS protocol.

4.31 Surfactant anion partitioning between baseline solvent and $\mathrm{NaOH}$ solutions.

4.32 Partitioning of dioctylamine...... 
4.35 Distribution ratios and percentage of organic anion removed from the solvent using anion-exchange resins in the hydroxide form....

4.36 Distribution ratios and percentage of organic anion removed from the solvent using anion-exchange resins in the chloride form.

4.37 Tributylphosphate partitioning in batch systems

4.38 1-Butanol partitioning in batch systems

5.1 Scheme for thermal stability samples placed in $34.8^{\circ} \mathrm{C}$ incubator.

5.2 Appearance of organic and aqueous phases following 12 ( -2 series), 46 ( -7 series), 110 (-16 series), and 235 (-33 series) day incubation at $34.8^{\circ} \mathrm{C}$ and $61.2{ }^{\circ} \mathrm{C}$.

5.3 Appearance of organic and aqueous phases following 4 weeks incubation at $34.8^{\circ} \mathrm{C}$ and $61.2^{\circ} \mathrm{C}$ with aqueous phases of varying nitric acid concentration

5.4 Phase disengagement break times at $0,12,46,110$, and 235 days

5.5 Extraction, scrub, and strip protocol results for thermal-stability test samples

5.6 Amount of product formed by replacement of the modifier sec-butyl group with a nitro group as a function of time, temperature, and nitric acid concentration.

5.7 Possible species present in anion mode

5.8 Possible species present in cation mode.

5.9 GC and RP-HPLC analyses of thermal-stability test samples for TOA, DOA, and SBP....

6.1 Solvent systems planned for external-irradiation experiments

6.2 Externally irradiated samples received from SRTC

6.3 Volumes contacted in the second ESS cycle.

6.4 $\mathrm{pH}$ values for aqueous phases in irradiated samples

6.5 Effect of external gamma dose on baseline solvent composition

6.6 Estimates of solvent loss and makeup rates

6.7 Analysis of aqueous phase in external irradiation experiments.

6.8 NMR analyses of irradiated solvent sample organic phases received from SRS in June...

6.9 Fluorine-19 NMR analyses of irradiated solvent sample aqueous phases received from SRS in June.

6.10 Proton NMR analyses of irradiated solvent sample organic phases received from SRS in July and August. 
6.11 Fluorine-19 NMR analyses of selected aqueous phases of irradiated samples received from SRS in August

6.12 Interfacial tension of control and irradiated samples

6.13 Sodium extraction capacity of EXT series as a function of dose

6.14 ESS data on externally irradiated samples.

7.1 Preparation of samples for determination of BOBCalixC6 solubility.

7.2 Samples observed in the solids-formation test.

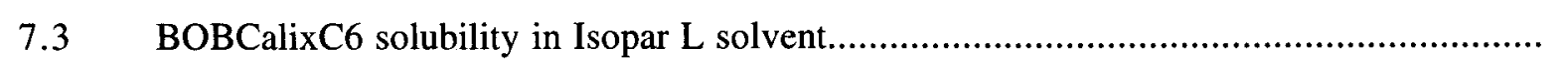

7.4 Effect of BOBCalixC6 loading on third-phase formation.

7.5 $\mathrm{CsNO}_{3}$ or $\mathrm{KNO}_{3}$ concentration in the aqueous phase and $\mathrm{O} / \mathrm{A}$ ratios corresponding to third-phase formation in extraction experiments using solvent containing 0.02 M BOBCalixC6

7.6 Formation of the third phase as a function of temperature, solvent, and simulant compositions

8.1 Internally irradiated solvents selected for establishing a wash-based cleanup process......

8.2 Trioctylamine (TOA) content of test samples and amount of TOA added to replenish that which was consumed in earlier testing

8.3 ESS performance after washing.

8.4 Performance of multiply contacted solvent following $\mathrm{NaOH}$ washing

8.5 Volume of persistent emulsion layers in internally irradiated samples following various centrifugations

8.6 Observations of internally irradiated samples following wash sequence.

8.7 Emulsion layer characteristics of internally irradiated solvent samples following final wash and centrifugation procedure.

8.8 Summary of the effectiveness of washing on cesium distribution ratios of selected solvent samples from ORNL internal-irradiation tests.

8.9 Extraction, scrub, and strip results for externally irradiated samples.

8.10 ESS performance of 235-day thermal-stability test samples before and after washing protocol

8.11 Summary of effects of solvent washing upon $D_{\mathrm{Cs}}$ values in solvents used in contactor tests. 


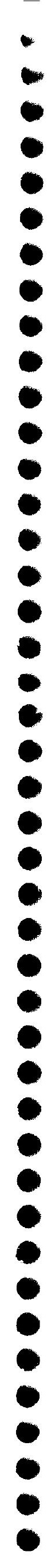




\section{ACRONYMS AND SYMBOLS}

amu
ANL
B
BDL
BOBCalixC6
BuOH
CSD
CF
Ci
Cs-3
Cs-6
Cs-7SB
Cs-7SBT
CSEX
CSSX
CTD
$D$

DCs or D(Cs)
$D$ M
DBP
DF
DI
DOA
ELD
ES-MS
ESS
EXT
E $S_{m} S_{p}$
FID
FY
FW
g
GC
GC-MS
GPC
HLW
HMB
HPLC
IC
ICP-AES
ICP-MS
ID
M
MBP
min
MW
$m / z$
$N_{\text {Di }}$

Atomic mass unit

Argonne National Laboratory

BOBCalix 6 in chemical equations

Below detection limits

Calix[4]arene-bis(tert-octylbenzo-crown-6)

1-Butanol

Chemical Sciences Division

Concentration Factor

Curie

1-(1,1,2,2-tetrafluoroethoxy)-3-(4-tert-octylphenoxy)-2-propanol

1-(2,2,3,3-tetrafluoropropoxy)-3-(4-tert-octylphenoxy)-2-propanol

1-(2,2,3,3-tetrafluoropropoxy)-3-(4-sec-butylphenoxy)-2-propanol

Compound Cs-7SB made from technical-grade 4-sec-butylphenol

Cesium Solvent Extraction

Caustic-Side Solvent Extraction

Chemical Technology Division

Distribution ratio, equal to the organic- to aqueous-phase molarity of a

given species, especially a metal ion or complex

Distribution ratio for cesium

Distribution ratio for any metal

Dibutylphosphate

Decontamination factor

Deionized

Dioctylamine

Evaporative light-scattering detector

Electro-spray mass spectrometry

Extract/scrub/strip batch test

Sample code for a sample treated under extraction conditions

An ESS batch test with $n$ extraction, $m$ scrub, and $p$ strip consecutive steps

Flame ionization detector

Fiscal year (Oct. 1 to Sept. 30)

Formula weight, g/mol

Gravitational acceleration constant, equal to $9.81 \mathrm{~m} / \mathrm{s}^{2}$

Gas chromatography

Gas chromatography - mass spectrometry

Gel permeation chromatography

High-level waste

Hexamethylbenzene

High-performance liquid chromatography

Ion chromatography

Inductively coupled plasma atomic emission spectrometry

Inductively coupled plasma mass spectrometry

Identification code for samples

Moles per liter

Monobutylphosphate

Minute

Molecular weight, $\mathrm{g} / \mathrm{mol}$

Mass-to-charge ratio

Dispersion number, characterizing coalescence rate 


$\begin{array}{ll}\text { NA } & \text { Not analyzed } \\ \text { ND } & \text { Not determined } \\ \text { NMR } & \text { Nuclear magnetic resonance } \\ \text { O/A } & \text { Organic-to-aqueous volume ratio } \\ \text { ORNL } & \text { Oak Ridge National Laboratory } \\ P & \text { Partition ratio, equal to the organic- to aqueous-phase molarity of an } \\ & \text { organic species, especially a neutral compound } \\ \text { ppm } & \text { Parts per million } \\ \text { ppt } & \text { Precipitate } \\ \text { PTFE } & \text { Polytetrafluoroethylene } \\ \text { QA } & \text { Quality assurance } \\ \text { RMAL } & \text { ORNL Radioanalytical Materials Laboratory } \\ \text { RP } & \text { Reverse phase } \\ \text { SA } & \text { Stearic acid } \\ \text { SBP } & \text { 4-sec-butylphenol } \\ \text { SC } & \text { Sample code for a sample treated under scrubbing conditions } \\ \text { SDS } & \text { Sodium dodecyl sulfate } \\ \text { SPE } & \text { Solid-phase extraction } \\ \text { SRS } & \text { Savannah River Site } \\ \text { SRTC } & \text { Savannah River Technology Center } \\ \text { ST } & \text { Sample code for a sample treated under stripping conditions } \\ T & \text { Temperature, usually in }{ }^{\circ} \text { C, unless otherwise noted as K } \\ t_{\text {B }} & \text { Break time of a dispersion, in seconds } \\ t_{1 / 2} & \text { Half life } \\ \text { TBP } & \text { Tributylphosphate } \\ \text { Teflon FEP } & \text { Fluorinated Ethylene Propylene } \\ \text { TIC } & \text { Total inorganic carbon } \\ \text { TMA } & \text { Trimethyl amine } \\ \text { TOA } & \text { Trioctyl amine } \\ \text { TOC } & \text { Total organic carbon } \\ \text { USDOE } & \text { United States Department of Energy } \\ \text { USEPA } & \text { United States Environmental Protection Agency } \\ \text { UV } & \text { Ultraviolet } \\ \text { WSRC } & \text { Westinghouse Savannah River Company } \\ \text { WSM } & \text { Work Scope Matrix } \\ \Delta Z & \text { Initial thickness of the dispersion band, in meters } \\ \phi & \text { Organic-to-aqueous volume ratio } \\ & \\ & \end{array}$




\section{PREFACE}

This report describes progress made in the period April 2000 to March 2001 on the chemical and physical properties of the solvent system employed in the Caustic Side Solvent Extraction (CSSX) process. The work reported was carried out in the Chemical and Analytical Sciences Division (now Chemical Sciences Division) of Oak Ridge National Laboratory (ORNL). As will be reflected by numerous references within this report, however, this work was closely linked with experimental tasks carried out in two other ORNL divisions (formerly Chemical Technology Division and Robotics and Process Systems Division), Savannah River Technology Center, and Argonne National Laboratory. The results of this work supported the down-selection from three candidate separation technologies to a single preferred technology for cesium removal from the Savannah River alkaline high-level waste. A preliminary version of the present report having the same authors and title (Document CERS/SR/SX/019, Oak Ridge National Laboratory, Oak Ridge, TN, Apr. 24, 2001) was issued for timely use in this decision. The down-selection took place in May 2001, culminating in the naming of CSSX as the preferred technology, as reported in the Federal Register on October 17, 2001 (Vol. 66, No. 201, pp. 52752-52756).

The present report is an edited version of the preliminary report, meeting the format requirements of the U.S. Department of Energy Office of Scientific and Technical Information. Except for addition of this preface and an abstract, no substantial material has been added or deleted from the earlier report. In addition to prescriptive format changes, editing included grammatical corrections, rephrasing to improve clarity, and reworking of some graphics. References were also collected into a single section to eliminate redundancy. Finally, citations of certain programmatic planning documents such as Technical Task Plans were removed. These references are not generally available to the public, and the information contained therein has since become available through resultant reports that were subsequently generated in the course of the planned work. In general, cited reports bearing Document Nos. beginning with "CERS/SR/SX" can be obtained through the Document Control Center, Nuclear Sciences \& Technology Division, Oak Ridge National Laboratory. These and other reports of the Savannah River Salt Processing Project can also be downloaded through the web site http://www.srs.gov/general/srtech/spp/index.html. The U.S. Department of Energy, Savannah River, Salt Processing Division should be contacted for access to this web site.

Significant solvent development has taken place subsequent to March 2001, primarily in response to the discovery herein that the extant baseline solvent was potentially susceptible to precipitation of the crystalline form of the extractant, calix[4]arene-bis(tert-octylbenzo-crown-6) (BOBCalixC6). New data regarding the properties of the solvent over a range of concentrations of the solvent components have resulted in the adoption of a new baseline solvent formulation. Only the component concentrations have changed; no changes in the identity of the solvent components have been made. The new baseline solvent is $0.007 \mathrm{M}$ BOBCalixC6, $0.75 \mathrm{M}$ Cs-7SB modifier, and 0.003 $M$ tri- $n$-octylamine in Isopar L diluent. For information regarding the selection of the new solvent composition, the reader is referred to the following report: L. N. Klatt, J. F. Birdwell, Jr., P. V. Bonnesen, L. H. Delmau, L. J. Foote, D. D. Lee, R. A. Leonard, T. G. Levitskaia, M. P. Maskarinec, and B. A. Moyer, "Caustic-Side Solvent Extraction Solvent-Composition Recommendation," Document No. CERS/SR/SX/026, Rev. 0, Nuclear Sciences \& Technology Division, Oak Ridge National Laboratory, Nov. 8, 2001, $29 \mathrm{pp}$. The new baseline solvent removes any potential problem with BOBCalixC6 solubility, increases solvent resistance to third-phase formation (thereby lowering the operating range to $15^{\circ} \mathrm{C}$ ), and increases the tolerance of the solvent to anionic impurities. Data pertinent to the recommendation included BOBCalixC6 solubility, third-phase formation temperature limits, batch cesium distribution ratios, calculated flowsheet robustness, dispersion numbers, and solvent density. It should be noted that certain questions raised in this report (e.g., an apparent effect of nitrite on cesium stripping) have now been resolved, and the reader is referred to reports of work carried out in FY 2002. 


\section{ACKNOWLEDGMENTS}

The work reported herein was sponsored by the Office of Project Completion and the Tanks Focus Area of the Office of Science and Technology, Office of Environmental Management, U.S. Department of Energy, under contract number DE-AC05-00OR22725 with Oak Ridge National Laboratory, managed and operated by UT-Battelle, LLC. The funding from the Office of Project Completion covered the work in FY 2000 and was effected by an Interoffice Work Order to Oak Ridge National Laboratory via the U.S. Department of Energy Savannah River Operations Office, Aiken, South Carolina.

Invention and initial development of the Caustic-Side Solvent Extraction Process (CSSX) occurred in the period FY 1996-1999 under primary funding from the EM-50 Efficient Separations and Processing (ESP) Crosscutting Program, Office of Science and Technology, Office of Environmental Management, U.S. Department of Energy. An understanding of the extraction properties of the CSSX extractant BOBCalixC6 and the synthetic chemistry of calix-crown compounds was achieved via support from the Environmental Management Science Program, Offices of Science and Environmental Management, U.S. Department of Energy. This understanding was critical in FY 1998 and FY 1999 in devising an efficient method for stripping cesium from the solvent and for improvements to the solvent system. The framework of principles of ion recognition upon which all of this work was based was provided under support from the Division of Chemical Sciences, Geosciences, and Biosciences, Office of Basic Energy Sciences, U.S. Department of Energy.

The participation of Prof. Spiro D. Alexandratos and Christy L. Stine was made possible via a subcontract to the Dept. of Chemistry, University of Tennessee, Knoxville, in FY 2000.

This research was also supported in part by appointments (LHD and TGL) to the Oak Ridge National Laboratory Postdoctoral Research Associates Program administered jointly by the Oak Ridge Institute for Science and Education and Oak Ridge National Laboratory.

The authors wish to thank Charles F. Coleman, ORNL ret., for helpful discussions and for reviewing the manuscript. Thanks are also extended to Leon N. Klatt of the ORNL Nuclear Science \& Technology Division (NSTD) for his friendship, dedication, and thoughtful leadership, and for providing excellent project management. The authors have also enjoyed stimulating interaction with Roger D. Spence and Joseph F. Birdwell, both of ORNL NSTD, on engineering tests related to solvent stability and contactor performance. Analytical services were provided by Joseph M. Giaquinto, Dale A. Caquelin, Todd Skeen, and Sara Harmon of the ORNL Chemical Sciences Division (CSD). The authors are grateful for access to, and assistance with, electrospray mass spectrometry instrumentation provided by Gary J. Van Berkel and Vilmos Kertesz of CSD.

The authors thank Ralph A. Leonard and his colleagues at Argonne National Laboratory for many fruitful discussions regarding engineering tests and flowsheet design. We also thank our many colleagues at the Savannah River Site for their generous support, guidance, and team spirit. Many helpful information exchanges involved Robert A. Pierce, Reid A. Peterson, Paul L. Rutland, Michael E. Hodges, Michael A. Norato, D. Douglas Walker, Thomas L. White, and William R. Wilmarth. Special thanks are reserved for Kenneth J. Rueter, former CSSX Team Lead, and Major C. Thompson, current CSSX Team Lead, for their strong and skillful leadership, dedication, optimism, and enthusiasm at all stages of this project. 


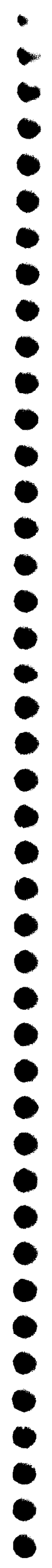




\section{EXECUTIVE SUMMARY}

\section{PURPOSE}

The purpose of this work was to provide chemical- and physical-property data addressing the technical risks of the Caustic-Side Solvent Extraction (CSSX) process as applied specifically to the removal of cesium from alkaline high-level salt waste stored at the U.S. Department of Energy Savannah River Site. As part of the overall Salt Processing Project, this effort supported decisionmaking in regards to selecting a preferred technology among three alternatives: (1) CSSX, (2) nonelutable ion-exchange with an inorganic silicotitanate material and (3) precipitation with tetraphenylborate. High risks, innate to CSSX, that needed specific attention included: (1) chemical stability of the solvent matrix, (2) radiolytic stability of the solvent matrix, (3) proof-of-concept performance of the proposed process flowsheet with simulated waste, and (4) performance of the CSSX flowsheet with actual SRS high-level waste. This body of work directly addressed the chemical-stability risk and additionally provided supporting information that served to plan, carry out, and evaluate experiments conducted by other CSSX investigators addressing the other high risks. Information on cesium distribution in extraction, scrubbing, and stripping served as input for flowsheet design, provided a baseline for evaluating solvent performance under numerous stresses, and contributed to a broad understanding of the effects of expected process variables. In parallel, other measurements were directed toward learning how other system components distribute in the flowsheet. Such components include the solvent components themselves, constituents of the waste, and solvent-degradation products. Upon understanding which components influence flowsheet performance, it was then possible to address in a rational fashion how to clean up the solvent and maintain its stable function.

\section{GENERAL CESIUM DISTRIBUTION BEHAVIOR}

Cesium distribution behavior under flowsheet conditions was characterized as a function of many variables and was shown to be reproducible by flexible batch methodology. Successful batch measurements require 1) that the temperature be constant and known, 2) that the phases be contacted with sufficient time and agitation to reach equilibrium, 3) that the phases be completely separated before sampling, and 4) that sampling be performed without contamination by the other phase. The particular type of analytical methodology used was not important as long as it was precise, reliable, and free of interferences. Within the expected operating temperature range $20-35{ }^{\circ} \mathrm{C}$, no issues were identified regarding cesium distribution or loading. However, a significant temperature dependence that causes the cesium distribution ratio $\left(D_{\mathrm{Cs}}\right)$ to decrease 6-10\% per degree with increasing temperature necessitates process-temperature management. It is likely that plant operators would need to systematically vary flowrates, especially the solvent flowrate, to maintain optimal performance as seasonal temperatures fluctuate. This property can be turned to advantage, by exploiting independent temperature control of extraction and stripping so as to significantly increase process performance, either to reduce footprint (number of stages) or to enhance process robustness in meeting process requirements (i.e., a decontamination factor of 40,000, concentration factor of 15 , and feed flow of $20.1 \mathrm{gal} / \mathrm{min}$ ). The chief competing species, $\mathrm{K}^{+}$ion, was found to reduce $\mathrm{Cs}^{+}$ion extraction and stripping performance, but within bounding conditions this competitive effect raises no issues regarding meeting process requirements. On the other hand, a survey of other minor components, focusing especially on organic species, confirmed that lipophilic anions such as dibutylphosphate can build up in the solvent on multiple cycles and impair stripping performance. Such anions, including dibutylphosphate, 4-sec-butylphenol, and surfactants having 12 or less carbons, can be removed readily by washing the solvent with dilute $\mathrm{NaOH}$ solutions. Including a $\mathrm{NaOH}$ wash step allows the solvent to be recycled multiple times without deterioration of 
performance, as demonstrated by extended engineering tests carried out in other tasks. Trials of various simulant recipes demonstrated the robustness of the solvent to handle widely varying waste compositions, and a computer model (reported elsewhere) was able to predict the value of $D_{\mathrm{Cs}}$. Not all chemical phenomena were completely understood, however. For example, the role of nitrite needs further investigation to understand why it impairs stripping when it is present at unexpectedly high concentrations (e.g., $2.6 \mathrm{M}$ ) in the simulant. Nevertheless, notwithstanding such questions outside bounding conditions, a good understanding of CSSX chemistry was obtained, and no major issues were identified with regards to meeting process requirements.

\section{CHEMICAL AND THERMAL STABILITY}

Results present a case for high solvent thermal stability. Batch extraction data show that acceptable extraction, scrubbing, and stripping performance is sustained for 235 days of constant exposure of the solvent to the full simulant, scrub solution, or strip solution at $35{ }^{\circ} \mathrm{C}$. This result corresponds to approximately 15 years of operation at the maximum sustained operating temperature of $35^{\circ} \mathrm{C}$ without solvent washing. The only solvent component that displays any sign of chemical instability to prolonged contact to aqueous solutions of the flowsheet is the trioctylamine, and the degradation manifests itself only under more severe conditions involving 110 days or longer of continuous contact with the scrub solution at $61^{\circ} \mathrm{C}$. The resulting decrease in TOA concentration was associated with an increase in the cesium distribution ratios in the scrub and stripping steps. Normal performance was re-established upon replenishing the TOA and solvent washing. For purposes of estimating the rate of replacement of solvent components, the TOA loss is taken to be approximately $10 \%(0.1 \mathrm{mM})$ per year. The chief degradation product of TOA was shown to be dioctylamine, which is expected to be washed out in the aqueous strip effluent. It is noteworthy that comparison of solvent degradation in the presence or absence of metal catalysts showed that potential catalysts in the full simulant have no apparent effect. Finally, tests to determine the threshold of nitration of either the extractant or modifier revealed that significant nitration requires heating of the solvent for 28 days at $61{ }^{\circ} \mathrm{C}$ with $1 \mathrm{M}$ nitric acid, conditions that the solvent will not experience in the process. Under these conditions, $4 \%$ nitration of the modifier was observed, while the extractant was not detectably nitrated.

\section{RADIOLYTIC STABILITY}

Based on the data obtained from solvent samples externally irradiated at the SRTC, the stability of the solvent to external irradiation, both with regard to absolute breakdown rate and performance, will readily meet performance requirements. No significant degradative loss of the primary solvent components nor consequential impairment of extraction, scrubbing, or stripping performance was observed over dose ranges well in excess of annual levels. For BOBCalixC6 and Cs-7SB, loss due to radiolytic breakdown was estimated to require less than $1 \%$ annual makeup. Even for TOA, the annual loss is conservatively expected to be $10 \%$. The primary degradation product observed was 4-sec-butylphenol, due to slight breakdown of the modifier. Its rate of buildup, however, is low, less than $0.1 \mathrm{mM}$ per annual dose. It was shown from partition ratios that this material would be washed out by the waste raffinate or $\mathrm{NaOH}$ wash solutions. Data from total organic carbon, solid-phase extraction, and NMR indicate that traces of organic products, especially fluorinated products from the modifier, distribute to the aqueous phases. NMR analyses show that detectable signs of degradation are only observed under high ( $>6 \mathrm{Mrad})$ doses, and even then the amount is small. Further, no changes between the irradiated samples and pristine solvent contacted with the full simulant, scrub, and strip solutions were observed by electrospray mass spectrometry. Interfacial behavior was also acceptable in the irradiated samples. Essentially no change in break time was observed. Interfacial tensions were all above $5 \mathrm{dyne} / \mathrm{cm}$, and the response was either increasing with dose (scrub conditions) or decreasing with dose (extraction or stripping conditions). In batch tests, extraction and scrubbing behavior was not much affected by external irradiation and remained acceptable. 
Stripping was also acceptable for all but the highest dose (16 Mrad, equivalent to the dose acquired under stripping conditions over a time period of 160 years), where it was shown that poor performance was related to the loss of $79 \%$ of the TOA. Unfortunately, it was not possible to restore performance of the most deteriorated solvent samples by replenishing the degraded TOA and solvent washing. However, solvent samples that had been irradiated internally in batch tests performed at ORNL were restored by replenishing lost TOA and washing.

\section{SOLUBILITY PHENOMENA}

Solubility phenomena were examined more extensively than planned, as some limiting features were encountered. Long-term observation of the baseline solvent under various conditions revealed no tendency to form solid phases at $25^{\circ} \mathrm{C}$ or at $4-6{ }^{\circ} \mathrm{C}$. Crystallization of a hydrate of the alternative modifier, Cs-6, was observed, eliminating this modifier from future consideration. However, the same crystallization phenomena were not found to apply to the case of Cs-7SB. Modifier Cs-6 exists as a pair of enantiomers due to the presence of one asymmetric carbon atom, whereas modifier Cs-7SB exists as four stereoisomers as a consequence of having two asymmetric carbon atoms. The greater number of isomers present for Cs-7SB is believed to play a key role in its resistance to solids formation. Although no issues were identified for the solubility of modifier, close inspection of the solubility of BOBCalixC6 revealed that the baseline solvent is somewhat supersaturated in BOBCalixC6 at $25{ }^{\circ} \mathrm{C}$. Highly supersaturated solutions consisting of $0.50 \mathrm{M}$ modifier and $0.05 \mathrm{M}$ BOBCalixC 6 can be prepared by means of warming and sonication. However, after standing at $25{ }^{\circ} \mathrm{C}$, the BOBCalixC6 is observed to crystallize out, yielding solutions having approximately $8 \mathrm{mM}$ of the extractant. When the modifier concentration is raised to $0.75 \mathrm{M}$, however, extractant solubility of $12.7 \mathrm{mM}$ is obtained, and thus there is motivation to employ higher modifier concentrations in future solvent-optimization tasks. It should be noted that lower BOBCalixC6 solubility values are obtained when the equilibrium is approached by dissolution of solid BOBCalixC6 vs. precipitation from supersaturated solution, raising the need to further investigate solubility phenomena. The higher modifier concentration also offers the benefit of stronger cesium extraction strength (higher $D_{\mathrm{Cs}}$ values), allowing the BOBCalixC6 concentration to be decreased with concomitant cost savings. An additional benefit entails greater resistance to third-phase formation, permitting a decreased operating temperature to $15^{\circ} \mathrm{C}$ (see below). Further investigation is needed, both to arrive at a more acceptable solvent composition and to understand the factors controlling extractant solubility.

Third-phase formation was found to occur as a result of either $\mathrm{Cs}^{+}$or $\mathrm{K}^{+}$ion loading and is associated with the generally recognized limited solubility of ion-pair complexes in nonpolar organic solvents. It was found that high $\mathrm{Cs}^{+}$ion loading can be tolerated, however, although conditions leading to high $\mathrm{Cs}^{+}$ion loading will not be found in the flowsheet. On the other hand, $\mathrm{K}^{+}$ion loading is significant, and the solubility limit of its complexes can be exceeded at the bounding $\mathrm{K}^{+}$ aqueous concentration if the temperature falls below $20{ }^{\circ} \mathrm{C}$. Hence, this property sets the lower operating limit of the process at $20^{\circ} \mathrm{C}$. If it is desirable to operate at a lower temperature for seasonal reasons, then feed blending or other control of the potassium concentration is needed. Alternatively, a higher modifier concentration can be employed, in which case the process temperature could be lowered to $15^{\circ} \mathrm{C}$ with $0.75 \mathrm{M} \mathrm{Cs}-7 \mathrm{SB}$.

\section{PARTITIONING OF SOLVENT COMPONENTS}

The partitioning of the baseline components of the solvent to the aqueous phase was found to be negligible. In the absence of solvent recovery, minor solvent replacement will likely be required owing purely to solubility loss. Partition ratios $(P)$ for BOBCalixC6, Cs-7SB, and TOA in the baseline solvent in contact with the process aqueous phases at $25{ }^{\circ} \mathrm{C}$ are all essentially too high for accurate measurement. The partition ratios are highest for the full simulant: $P_{\mathrm{BOBCalixC6}}>12,500, P_{\mathrm{Cs}-7 \mathrm{SB}}>$ 50,000 , and $P_{\text {TOA }}>30,000$. For scrub and strip solutions, the bounds are: $P_{\mathrm{BOBCalix} 6}>12,500$, $P_{\mathrm{Cs}-7 \mathrm{SB}}>8000$, and $P_{\mathrm{TOA}}>6000$. Using $P_{\mathrm{BOBCalix} 6}=12,500$ as the most conservative basis for 
extractant loss to the waste raffinate, 2800 solvent cycles at $\mathrm{O} / \mathrm{A}=0.33$ implies a cumulative loss of $49 \%$ of the BOBCalixC6 annually. This replacement meets the original goal of one solvent replacement per year. However, estimates of the true lipophilicity of BOBCalixC6 imply a $P_{\mathrm{BOBCalixC6}}$ value many orders of magnitude higher than the experimental lower bound of 12,500 . Thus, any need for solvent recovery or replacement is expected to arise predominantly due to entrainment losses, which can be dealt with by other means.

\section{DISTRIBUTION OF MAJOR AND MINOR INORGANIC COMPONENTS}

A large set of data was collected describing the distribution of the alkali metal cations $\mathrm{Na}^{+}, \mathrm{K}^{+}$, and $\mathrm{Cs}^{+}$from the full simulant, simple simulants, and simple one- or two-component electrolyte solutions. Some of these data were used for computer modeling reported in a separate report. Experiments comparing the baseline solvent with and without $\mathrm{BOBCalixC6}$, showed that the calixarene has little ability to bind $\mathrm{Na}^{+}$ion, and the observed $\mathrm{Na}^{+}$extraction by the baseline solvent is largely due to ion exchange of the modifier's proton. On the other hand, the calixarene binds $\mathrm{K}^{+}$ ions appreciably, and the resulting loading depresses $\mathrm{Cs}^{+}$extraction somewhat and raises the susceptibility of the solvent to third-phase formation (see above); neither effect is sufficient to jeopardize meeting process requirements. Both potassium and sodium report almost completely to the scrub solution. Measurements of the $\mathrm{pH}$ of the scrub and strip solutions under various conditions characterized aspects of the acid-balance behavior of the system, which entails uptake of acid from the scrub solution by TOA and modifier and subsequent partial release to the strip solution.

Experiments with simple salt solutions showed that the process should be relatively tolerant to variations in the anion content among the three major anions, nitrate, nitrite, and hydroxide. Each of these anions contributes significantly to the driving force for cesium extraction, as shown by computer modeling described elsewhere, and thus they should be mutually compensating at constant sodium concentration. This desirable property was in fact observed in more complex simulant recipes tested. Ion chromatography showed that nitrate, nitrite, chloride, and dibutylphosphate are the primary anions extracted from the simulant by the baseline solvent. All the anions except for nitrate and dibutylphosphate report to the scrub solution, while the nitrate and dibutylphosphate are washed out by $\mathrm{NaOH}$ solutions.

Five metals not included in the full simulant recipe, namely $\mathrm{U}, \mathrm{Np}, \mathrm{Pu}, \mathrm{Sr}$, and $\mathrm{Tc}$, were not significantly extracted by the CSSX baseline solvent. The presence of these five metals also does not impair extraction or stripping of cesium. Although more tests should be conducted to gather definitive data on controlled oxidation states (except Sr) in varied matrices, including real waste, it appears likely that the five metals should pass through the extraction stages and exit with the waste raffinate. As a tentative conclusion, these metals are expected to have little, if any, impact on the CSSX process and on the character of its solvent and strip effluent streams. The remaining question regarding placing the monosodium titanate strike after CSSX, then, is the extent of solids removal that would be required prior to CSSX to prevent fouling the contactors with solids. The possibility of performing minimal solids removal prior to CSSX and removing actinides and Sr after cesium removal potentially represents a major savings in the footprint of the highly shielded facility.

A survey of the elements present in the simulant, including $\mathrm{Al}, \mathrm{Cr}, \mathrm{Cu}, \mathrm{Fe}, \mathrm{Mo}, \mathrm{Hg}, \mathrm{Ag}, \mathrm{Pb}, \mathrm{Pd}, \mathrm{Rh}$, $\mathrm{Ru}, \mathrm{Zn}, \mathrm{Sn}, \mathrm{Cl}$, and $\mathrm{F}$, could not detect any buildup in the solvent on limited cycles, nor do these elements appear in the scrub or strip stages in significant concentrations.

\section{DISTRIBUTION OF ORGANIC COMPONENTS}

Given the use of solvent cleanup based on $\mathrm{NaOH}$ washing, the presence of lipophilic anionic species in the solvent as extracted from the waste or as formed upon degradation of the solvent components represents no particular risk. However, ample evidence was gathered to show that lipophilic anions at sufficient concentration in the solvent overwhelm the tolerance provided by the TOA suppressor and impair stripping. The question then amounts to the relative rate of buildup of 
lipophilic anions in the solvent as governed by their concentration in the feed or their formation (in the case of degradation products) vs. how fast they will be removed from the system as governed by their partition ratios with respect to process aqueous solutions and wash solutions.

A systematic test of the individual organic components of the full simulant revealed dibutylphosphate (DBP) to be the only organic waste component having a deleterious effect on stripping. Its partition ratio $P_{\mathrm{DBP}}$ was found here to be 2.3 between the baseline solvent and full simulant at $25^{\circ} \mathrm{C}$, which at $\mathrm{O} / \mathrm{A}=0.33$ implies that only a modest buildup to $0.27 \mathrm{mM}$ in the solvent is possible. Although this is just enough to start to impair the function of the TOA, any $\mathrm{NaOH}$ wash solutions containing from $10 \mathrm{mM}$ to $1 \mathrm{M} \mathrm{NaOH}$ efficiently washes out the DBP $\left(P_{\mathrm{DBP}} \leq 0.01\right)$.

4-sec-Butylphenol (SBP) is one of the two major solvent degradation products, the other being the breakdown product of TOA, dioctylamine (DOA). At concentrations of greater than $3 \mathrm{mM}$, SBP in the solvent begins to interfere with stripping, but conservative estimates set the annual production of SBP in the solvent at less than $0.3 \mathrm{mM}$. Hence, solvent washing to remove SBP is not expected to be needed. Moreover, partitioning data show that the waste raffinate washes out the SBP weakly (est. $6 \%$ per solvent cycle, $P_{\mathrm{SBP}}=\sim 50$ ), sufficient to keep the SBP at trace levels (est. $0.002 \mathrm{mM}$ ) in the solvent.

Although surfactant anions have not been specifically identified as waste components, results showed that they have significant potential to impair stripping performance. TOA negates their effect at trace levels, but it is important to understand the partitioning of typical surfactant anions in order to predict their buildup in the solvent and to optimize washing methods should this problem be later encountered. It was found that the 12-carbon surfactant anions dodeconoate (laurate) and dodecylsulfate and the 18-carbon surfactant octadecanoate (stearate) partition strongly to the solvent in contact with the simulant, scrub, or strip solutions. Partitioning of the anionic forms of the surfactants is driven by the sodium concentration in the aqueous phase, making it desirable to minimize the aqueous sodium concentration. On the other hand, sufficient alkalinity is needed to deprotonate the acid forms of the surfactants. For the carboxylate surfactants, the optimum $\mathrm{NaOH}$ concentration for washing was found to be $3 \mathrm{mM}\left(P_{\text {laurate }}=0.072, P_{\text {stearate }}=96\right)$. For dodecylsulfate (and presumably related sulfonate surfactants), the optimum is less than $0.001 \mathrm{M}$. As coalescence problems increase with decreasing $\mathrm{NaOH}$ concentration, $0.01 \mathrm{M} \mathrm{NaOH}$ was accepted as the best compromise for washing and was therefore recommended for use in extended contactor tests at ANL and SRTC. At this concentration of $\mathrm{NaOH}$ at $25{ }^{\circ} \mathrm{C}, P_{\text {laurate }}=0.12, P_{\text {stearate }}=150$, and $P_{\text {dodecylsulfate }}=1.7$. Using a $0.01 \mathrm{M} \mathrm{NaOH}$ wash at $\mathrm{O} / \mathrm{A}=5$ and $25{ }^{\circ} \mathrm{C}$, these $P$ values correspond respectively to $62 \%, 0.13 \%$, and $10 \%$ removal of these surfactant anions. Assuming the feed has $1 \times$ $10^{-5} \mathrm{M}$ of any of these anions, estimated steady-state concentrations in the solvent correspond respectively to $4.8 \times 10^{-5} \mathrm{M}, 0.023 \mathrm{M}$, and $3.0 \times 10^{-4} \mathrm{M}$. Such a buildup could be tolerated in the case of the 12-carbon surfactants, but not the 18-carbon surfactant. For this reason, it was judged prudent to demonstrate an effective solvent-cleanup alternative, and resin anion-exchange was shown to offer the requisite capability. Resins in the hydroxide form contacted directly with solvent spiked with stearic acid and sodium dodecylsulfate were particularly effective, with $K_{\mathrm{d}}$ values greater than $1000 \mathrm{~mL} / \mathrm{g}$ for three resins, including two commercially available resins.

The other major solvent degradation product, DOA does not partition to either the simulant or to $0.01 \mathrm{M} \mathrm{NaOH}$ wash solution, but it does partition weakly to the strip solution, where $P_{\mathrm{DOA}}=4.7$. At $\mathrm{O} / \mathrm{A}=5$, loss of DOA to the strip effluent would be expected to be $4 \%$ per solvent cycle, and the estimated steady-state concentration is $9 \times 10^{-7} \mathrm{M}$ based on $0.1 \mathrm{mM}$ DOA per year as the rate of production due to thermal or radiolytic degradation of TOA.

The partitioning properties of three neutral organic components of the full simulant, trimethylamine (TMA), tributylphosphate (TBP), and 1-butanol (BuOH) were examined to estimate their fate in the flowsheet. Among the three compounds, possible implications for process upset are greatest for TMA. It was found that TMA mildly partitions to the solvent during extraction $\left(P_{\mathrm{TMA}}=\right.$ 4.3 for baseline solvent and full simulant at $25^{\circ} \mathrm{C}$ ). Accordingly, the TMA will move with the solvent into the scrub stages in the flowsheet. There it will be protonated, partition into the aqueous phase $\left(P_{\mathrm{TMA}}=1.9 \times 10^{-4}\right.$ for baseline solvent and $50 \mathrm{mM} \mathrm{HNO} \mathrm{H}_{3}$ at $\left.25^{\circ} \mathrm{C}\right)$, and return to the feed stage, since the aqueous scrub effluent is added to the waste at the waste feed stage. With such a feedback loop, the TMA could in principle build up to very high concentrations in the solvent. As it is very 
volatile, however, the TMA probably leaves the system as a vapor from the feed hold tank or from the purged contactor head space in the extraction section. At this moment, the fate of TMA is not clear and is in need of further investigation. Tests starting with $10 \mathrm{mM}$ TMA in the solvent revealed no effect on extraction, scrub, and strip performance, and successful extended contactor tests with simulant at ANL and with real waste at the SRTC suggested no difficulties. If needed, straightforward options to deal with the TMA if needed include, for example, sparging the aqueous feed or reducing the solvent flow rate so that the O/A ratio in the extraction section gives an extraction factor less than unity.

TBP is present at very low concentrations in the waste (max. $1.9 \times 10^{-6} \mathrm{M}$ ) but partitions strongly to the solvent, where $P_{\mathrm{TBP}}=1880$ for the baseline solvent equilibrated at $25^{\circ} \mathrm{C}$ with the full simulant. This partition ratio indicates that TBP could only build up in the solvent to $3.5 \mathrm{mM}$ at steady state, as the raffinate exiting stage \#1 will start to wash out the TBP when it reaches this level in the solvent. This level is too low to have any effect on extraction, scrubbing, or stripping, as effects are not noticeable until the TBP concentration in the solvent reaches $30 \mathrm{mM}$. Notably, TBP is the only organic waste component found to depress $D_{\mathrm{Cs}}$ values, its action undoubtedly being to interact with the modifier, reducing its ability to effectively solvate the cesium ion-pair complex.

1-Butanol is present in the waste to at most $2.7 \times 10^{-5} \mathrm{M}$ and partitions mildly to the solvent, where $P_{\mathrm{BuOH}}=7.5$ for the baseline solvent equilibrated at $25^{\circ} \mathrm{C}$ with the full simulant. However, the partition ratio is not high enough to allow it to build up to more than $0.2 \mathrm{mM}$, again too low to have any effect on solvent performance. No effect of 1-butanol on extraction, scrubbing, and stripping was observed at $10 \mathrm{mM}$ in the solvent.

\section{SOLVENT CLEANUP}

Solvent cleanup was approached from the combined knowledge of the partitioning behavior of minor species in the solvent and their effects on extraction, scrubbing, and stripping. Thus, most of the basis for solvent washing has been presented above. Accordingly, it should be stressed that the primary need for solvent washing arises from the necessity of removing extractable minor waste components such as dibutylphosphate. The solvent breakdown products 4-sec-butylphenol and dioctylamine both partition sufficiently into, respectively, the simulant and strip aqueous phases as to render the solvent effectively free of all but traces of these compounds without solvent washing. A recommendation for solvent washing was therefore made according to the following chemical logic. Lipophilic anions represent the primary poison for the solvent, impairing stripping of cesium. The effect increases with the strength of the conjugate acid of the lipophilic anion. Stronger acids, such as dibutylphosphoric acid, will convert easily to the sodium salt on contact with caustic solution, whence in this form the lipophilic anion is most readily washed out. Since maximum alkalinity is needed for removal of phenol degradation products, hydroxide was considered the preferred anion for the wash solution (vs. carbonate). Suitable cations for washing include $\mathrm{Li}^{+}$and $\mathrm{Na}^{+}$. Larger alkali cations such as $\mathrm{K}^{+}$are too extractable by the calixarene and would inhibit removal of anions. Sodium salts, being most economical, were employed here. Chemical mass-action implies that removal of sodium salts from the organic solvent becomes increasingly efficient as the sodium concentration in the aqueous phase decreases. Economics also places value on minimizing the $\mathrm{NaOH}$ concentration. On the other hand, sufficient alkalinity is needed to maintain the anions in their deprotonated state, and sufficient ionic strength is needed for acceptable coalescence. As a compromise, $10 \mathrm{mM} \mathrm{NaOH}$ was chosen as a suitable wash solution for the extended contactor tests and for further flowsheet evaluation. Dispersion-number tests at ANL showed that this concentration is acceptable, though the tendancy to form emulsions seemed to be greater in batch tests.

Tests of solvent washing with various concentrations of $\mathrm{NaOH}$, including $10 \mathrm{mM} \mathrm{NaOH}$, were generally successful. In batch tests, washing allowed restoration of solvent function after multiple contacts with the full simulant or upon multiple cycles. It also restored function to the thermalstability and internal-irradiation test solvents after replenishing the TOA that had been degraded. It did not, however, restore function on archived samples from the external-irradiation test at the SRTC. The extended contactor tests at ANL with the full simulant and at the SRTC with real waste employed 
a $10 \mathrm{mM} \mathrm{NaOH}$ wash stage at $\mathrm{O} / \mathrm{A}=5$ and did not exhibit signs of degrading extraction or stripping decontamination factors. A backup solvent-cleanup technique involving anion exchange was successfully demonstrated to remove lipophilic anions, including an 18-carbon carboxylic acid. It is possible that other cleanup methods or aqueous washing strategies could be used, but their development awaits further investigation.

\section{RISK EVALUATION}

The overall conclusion of these studies is that the CSSX solvent meets all of the performance requirements within the bounding conditions defined by the Savannah River Site waste. These studies also show that chemical and thermal degradation under the operating conditions of the process is slow relative to the goal of one annual solvent replacement. Moreover, the trace products that were detected are not harmful and will wash out of the solvent, either into the process effluents or added wash stages. Solvent integrity with regard to component solubility loss to the aqueous phase, resistance to precipitation of solids or third-phases, and susceptibility to impurity effects is good with implementation of temperature management and solvent washing. This work also supported other tasks in addressing the radiation-stability risk, providing analytical and performance data showing that the solvent can withstand the dose equivalent of several years without the need for solvent washing. Finally, this undertaking provided batch distribution data and solvent-washing data that were critical in designing the proof-of-concept and real-waste tests. Overall, the data imply that the chemical and thermal stability risk for implementation of the CSSX process is low. 


\begin{abstract}
This report provides chemical- and physical-property data addressing the technical risks of the Caustic-Side Solvent Extraction (CSSX) process as applied specifically to removal of cesium from the alkaline high-level salt waste stored at the U.S. Department of Energy Savannah River Site. The work was conducted as part of the overall Salt Processing Project, supporting the selection of a preferred technology among three technology alternatives. In a coordinated effort with researchers at Argonne National Laboratory and the Savannah River Technology Center, it was the general goal to evaluate four high risks relating to CSSX, including (1) chemical stability of the solvent matrix, (2) radiolytic stability of the solvent matrix, (3) proof-of-concept performance of the proposed process flowsheet with simulated waste, and (4) performance of the CSSX flowsheet with actual Savannah River highlevel waste. Information on cesium distribution in extraction, scrubbing, and stripping served as input for flowsheet design and for establishing a baseline for evaluating solvent performance under numerous thermal, radiolytic, and chemical stresses. These data contributed to a broad understanding of the effects of expected process variables. In parallel, other measurements were directed toward learning how major and minor system components distribute in the flowsheet. Such components include the solvent components themselves, constituents of the waste, and solvent-degradation products. Upon understanding how various components influence flowsheet performance, it was then possible to address in a rational fashion how to clean up the solvent and maintain its stable function. Based on the results, recommendations for future research are presented.
\end{abstract}




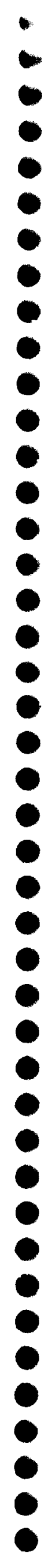




\section{INTRODUCTION}

\subsection{BACKGROUND}

Removal of cesium from high-level tank waste, such as that stored at the U.S. Department of Energy (USDOE) Savannah River Site (SRS) and Hanford site, continues to be a challenging problem, as judged by the large current investment being made under the auspices of the Office of Environmental Management and by current needs statements. ${ }^{1-2}$ Focusing in particular on the problems being addressed at the SRS, difficulties with benzene emissions owing to premature decomposition of tetraphenylborate precipitate in the In-Tank Precipitation process employed recently at the $S_{R S}{ }^{3}$ have led to an intensive phased program to identify and test alternative technologies and finally to select and implement a preferred technology to accomplish the cesium separation. ${ }^{4,5}$ Three technologies out of approximately 140 considered were selected for preconceptual design and further R\&D in Phase III. ${ }^{5}$ One of these alternatives, originally designated the alkaline-side cesium solvent-extraction (CSEX) process, was developed recently at the Oak Ridge National Laboratory (ORNL). ${ }^{6}$ An alternative acronym, CSSX (Caustic-Side Solvent Extraction), has been in use at the SRS and will be adopted here for consistency. The CSSX process makes use of an advanced type of extractant that is a hybrid of a crown ether and calixarene. ${ }^{7}$ The extraordinary selectivity and strength of this type of extractant, called a calix-crown, makes it possible for the first time to remove cesium from a concentrated alkaline sodium nitrate medium by solvent extraction. Based on the Phase III tests conducted in 1998,8-11 it was concluded that the CSSX process flowsheet ${ }^{12}$ meets the requirements for the removal of cesium from SRS high-level waste and is economically competitive with the alternative nonelutable ion exchange and precipitation technologies.5,13 However, owing to the relative immaturity of the process coupled with the demanding schedule for implementation, the process was not selected to proceed with higher-scale development, testing, and conceptual design in Phase IV. Under funding by the Efficient Separations and Crosscutting Program, the major issues of stability and susceptibility to impurity effects were successfully dealt with in FY 1999.11,14,15 A conceptual flowsheet meeting the SRS requirements in 22 stages was calculated based on the performance data for this second-generation solvent system. ${ }^{11,15}$ Based on these promising results and other considerations, an independent USDOE assessment ${ }^{16}$ and a review by the National Research Council of the National Academy of Sciences recently urged further testing of the CSSX process. ${ }^{17,18}$ The National Research Council in particular recommended that the CSSX process be brought quickly to a state of development comparable to the other alternatives. A comprehensive research and development plan was developed to accomplish this immediate objective, enabling a final selection of a technology in FY 2001 for design, pilot testing, and eventual implementation. ${ }^{4,19}$ This plan also provided a blueprint for work that will proceed in the post-downselect period.

\subsection{TECHNICAL RISKS ADDRESSED}

Process requirements include the ability to accept a feed solution at the rate of $20.1 \mathrm{gal} / \mathrm{min}$ that contains $5.6 \mathrm{M}$ sodium ion and an average ${ }^{137} \mathrm{Cs}$ activity of $0.375 \mathrm{Ci} / \mathrm{L}$ to yield a low-level waste stream containing $\leq 20 \mathrm{nCi} / \mathrm{g}$ and a strip effluent at $1.33 \mathrm{gal} / \mathrm{min}$ containing the separated cesium that is volumetrically concentrated by a factor of $15.1 .20-22$ High risks that must be addressed before further down-selection among the three alternative technologies include: ${ }^{4}$ (1) chemical stability of the solvent matrix, (2) radiolytic stability of the solvent matrix, (3) proof-of-concept performance of the proposed process flowsheet with simulated waste, and (4) performance of the CSSX flowsheet with actual SRS high-level waste. In FY 2000 and FY 2001, overall effort at ORNL, Argonne National Laboratory (ANL), and the Savannah River Technology Center (SRTC) focused on providing the data needed to evaluate these high risks as well as the risk associated with assuring adequate 
commercial availability of the solvent components. As reflected by the data presented in this report, the present work directly addresses the high risk due to solvent chemical stability and provides data and technical support needed by the tasks addressing the other high risks. The present report also addresses general risks associated with the CSSX solvent and flowsheet performance: predictability of performance, integrity of the solvent matrix over the range of expected operating conditions, tolerance of the solvent matrix to the effects of interfering minor waste components and solvent degradation products, effectiveness of solvent-cleanup methodology, and adequacy of analytical methodology.

The basic performance of the CSSX process derives from distribution behavior and hydraulics. Chemistry impacts these behaviors through the extraction equilibria and kinetics involving cesium, distribution of major and minor feed components to the solvent, solubility properties of all system components, and the stability of solvent components. Based on solvent-extraction plant practice in the hydrometallurgical and nuclear industries, ${ }^{23,24}$ we may realistically expect some degree of solvent degradation and interference from species that build up in the solvent. Risk arises in the effect of these eventualities, the rate at which problems develop, and whether an adequate method for solvent cleanup can be implemented.

Part of predicting performance entails understanding the distribution behavior of all of the minor and major system components between the solvent and the waste, scrub, strip, and wash solutions. Some information had been obtained on the distribution ratios of cesium, sodium, potassium, and several other metals under process conditions. ${ }^{15}$ Very limited information had been gathered on the dependence of these distributions on the system compositional variables, on minor-component distribution, and on solvent-component partitioning. $8,14,15$ A computer model had not yet been developed. Thus, at the outset of FY 2000 , there was a limited ability to predict how components distribute in a given flowsheet.

A critical question concerns the useful life of the solvent. Associated questions concern solventloss pathways, solvent recovery, cleanup methods, and process-suitable analytical methodology. Prior to this work, little has been learned about how minor metals and organic compounds distribute, including the fate of degradation products and the long-term perturbation of process performance as impurities build up in the system. It was previously found that solvent performance can be impaired by the presence of certain surfactant anions, such as dodecyl sulfonate. 8,14 It is particularly important, then, to investigate the distribution behavior and effect on performance of such anions as may be anticipated or as may be identified through analysis of degraded or used solvent. Such anions could include dibutylphosphate, surfactants, phenolates, and carboxylates. Possible effects could be poor phase coalescence, third-phase formation, crud formation, impaired stripping, loss of extraction capacity, and loss of selectivity. Although the amine component in the solvent nullifies the effect of lipophilic anions on stripping, its capacity can be exceeded. Thus, it is important to characterize the solvent tolerance for certain types of anions and understand how the anions partition to different aqueous solutions. Such knowledge can lead directly to solvent cleanup methods by washing procedures or possibly other methods, such as anion exchange.

\subsection{WORK SCOPE}

Within the scope outlined in the planning documents for the SRS Salt Processing Project, ${ }^{4,19}$ the present work was intended to begin to address the most pressing of the chemistry issues in FY 2000 and FY 2001. Solubility of solvent components and third-phase behavior were characterized as a function of temperature and aqueous conditions. Data on the distribution properties of the primary solvent components were collected to permit the estimation of the solvent loss rate by this pathway. The distribution properties of major and minor inorganic ions and organic impurities, including lipophilic anions, were measured. Cleanup methods were investigated, including solvent washing and anion exchange. A computer model to predict $\mathrm{Cs}$ distribution ratios was developed. Analytical methodology was developed to monitor solvent components and other species of interest. Samples received from contactor testing and from chemical and radiolytic degradation tests were subjected to analytical procedures, performance assessment, and cleanup tests. The receipt of samples created 
links to other tasks from Argonne National Laboratory (ANL), Savannah River Technology Corporation (SRTC), and the Chemical Technology Division (CTD) of ORNL.

\subsection{CHAPTER ORGANIZATION}

Topics covered by this report are presented in a logical order for the general reader. Chap. 2 lays out the major materials used in the experiments, together with major protocols. Chap. 3 provides experimental results on cesium distribution as a function of composition and temperature. A deeper understanding of system behavior is sought in Chap. 4, which deals with the distribution of the major solvent components, degradation products, and components of the waste feed. Chaps. 5 and 6 describe results on chemical (thermal) and radiolytic stability, respectively. The subject of solubility phenomena, including the solubility of the major solvent components and third-phase behavior, appears in Chap. 7. Finally, Chap. 8 details solvent washing strategies for the removal of interfering species that arise from the waste feed and solvent degradation. Conclusions and options for future work are presented in Chap. 9.

Results of subtasks to develop an equilibrium model for Cs distribution and to develop analytical methodology are covered under separate reports. 25,26 Given that the present report is long, it was taken as a matter of convenience that the progress reports on these subtasks could stand on their own without undue redundancy or loss of continuity. They are considered part of the final report for the task on CSSX chemical and physical properties. 


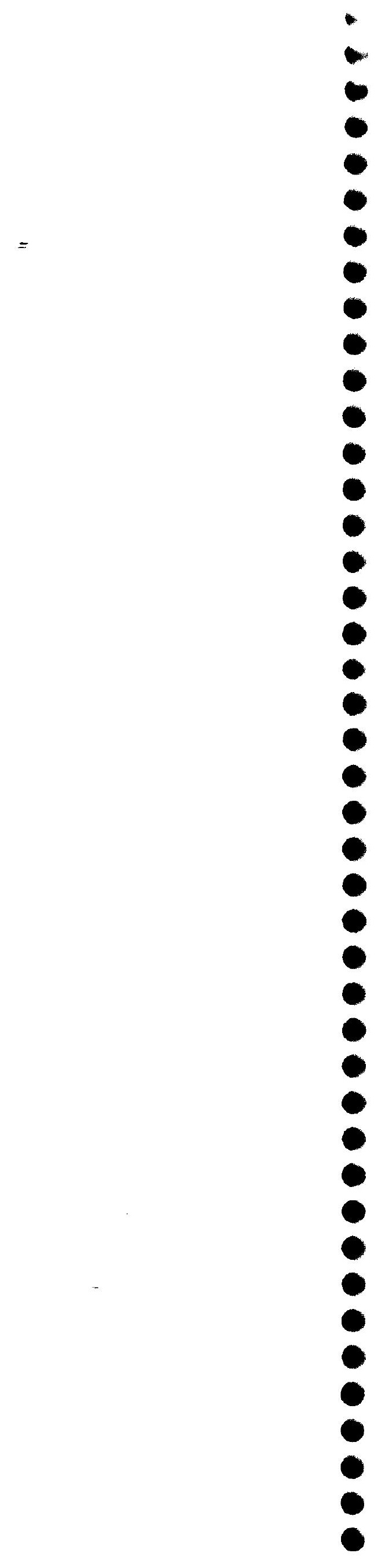




\section{BASELINE SOLVENT, SIMULANTS, AND PROTOCOLS}

\subsection{BASELINE SOLVENT}

\subsubsection{Solvent Composition}

The baseline solvent for all CSSX activities in FY 2000 and 2001 is shown in Fig. 2.1. It was essentially first formulated and tested in FY 1999.11,15 In that period, however, the modifier in use had been designated Cs-7SBT, the "T" indicating that a technical-grade of 4-sec-butylphenol (SBP) was employed in the synthesis. Otherwise, the structure is the same as the Cs-7SB modifier used in the baseline solvent, and there appears to be little or no difference in properties (see Chap. 6).

The baseline solvent consists of four components: $0.01 \mathrm{M}$ BOBCalixC6 extractant, $0.5 \mathrm{M}$ Cs-7SB modifier, and $0.001 \mathrm{M}$ trioctylamine, in Isopar $\mathrm{L}$ diluent. The extractant, calix[4]arene-bis(tertoctylbenzo-crown-6) (BOBCalixC6), possesses two cavities, only one of which is expected to be occupied by a $\mathrm{Cs}^{+}$cation at any time under normal CSSX operating conditions. ${ }^{7,27} \mathrm{~A}$ number of mono- and biscrown calixarenes have been introduced by Ungaro et al. and Vicens et al. and examined by Dozol et al. for potential in separations of cesium from nuclear waste. $7,28-30$ Calix [4]arene-crown-6 compounds in particular possess the appropriate crown bridging length (i.e., 6 oxygen atoms in the bridge) and calixarene ring size (i.e., 4 phenol units) for high selectivity toward $\mathrm{Cs}^{+}$ion. Representative calix[4]arene-crown-6 compounds have been surveyed at ORNL, and both mono- and biscrown calix[4]arene crown-6 compounds exhibit high extraction strength and selectivity. ${ }^{31}$

Mainly for synthetic accessibility and for supposed solubility reasons, BOBCalixC6 was chosen as the preferred extractant for the baseline solvent. It was obtained from IBC Advanced Technologies, Inc. at $97 \%$ nominal purity. The branched alkyl substituents are thought to increase the solubility of BOBCalixC6 in alkane diluents, though solubility data were previously lacking. ${ }^{8,27}$ They also boost the lipophilicity of BOBCalixC6, but it should be noted that the analog calix-crown without the two tert-octyl groups already possesses sufficient lipophilicity. ${ }^{27}$ Another effect of the two alkyl groups is to cause BOBCalixC6 to possess two enantiomers. A crystal structure of BOBCalixC6 has been determined, aided by the fact that the compound can be crystallized from dichloromethane. ${ }^{32}$ The two enantiomers crystallize together. Relative to the available literature on the structures of uncomplexed calix[4]arene-biscrown- 6 compounds, ${ }^{7,28,33}$ the structure of BOBCalixC6 is not particularly remarkable. As so often is the case for crown ethers, the flexible polyether chain twists in upon itself, and the cavity does not exist until a guest species such as $\mathrm{Cs}^{+}$ion is present.

A solvent modifier is needed especially for solvation of the ion-pair extraction complex formed when $\mathrm{CsNO}_{3}$, or competing $\mathrm{KNO}_{3}$, is extracted by BOBCalixC6.14,34 The solvating action of the modifier both improves solubility (prevents third-phase formation) of extraction complexes and increases the extraction power of the extractant. Certain fluorinated alkylphenoxyether alcohols have been shown to be especially effective, ${ }^{34}$ as linked to their effective hydrogen-bond-donation ability. 35 The modifier, 1-(2,2,3,3-tetrafluoropropoxy)-3-(4-sec-butylphenoxy)-2-propanol (Cs-7SB), was introduced to improve solvent stability, as the first-generation modifier, $1-(1,1,2,2-$ tetrafluoroethoxy)-3-(4-tert-octylphenoxy)-2-propanol (Cs-3), is thermally unstable. ${ }^{8}$ To promote the efficiency of caustic solutions to wash-out the parent alkyl phenol (an impurity left over from the modifier synthesis and also a degradation product) the tert-octyl group was replaced by the less lipophilic sec-butyl group. ${ }^{15}$ Among several choices of smaller alkyl groups, sec-butyl provided the best resistance to third-phase formation, likely owing to its producing four isomers (two asymmetric carbons) of Cs-7SB. Since Cs-7SB is a weaker modifier than Cs-3, it must be used at a higher concentration, and $0.5 \mathrm{M}$ was found to be suitable and subsequently adopted for the baseline solvent. ${ }^{15}$ 
The amine is present in the solvent to improve stripping performance. Without it, non-radioactive cesium nitrate must be added to the stripping solution. ${ }^{8}$ Even with the cesium nitrate added, the stripping efficiency is vulnerable to anionic impurities, especially surfactant anions. $8,14,36$ Since stripping is carried out under acidic conditions, the amine in the ammonium form provides an available concentration of cations in the solvent to act as counterions for traces of surfactant anions, dibutylphosphate, or other lipophilic anions that might be present. The effect of anions is therefore essentially suppressed, allowing cesium nitrate to be stripped. During extraction of cesium, the alkaline conditions will render the amine neutral, in which form its effect on extraction equilibria will be negligible. Trioctylamine (TOA) is a well-known commercial extractant and was an obvious choice of amine. Since its use was immediately successful, ${ }^{8,14}$ no survey of alternative commercial amine extractants was attempted, though triisooctylamine and Aliquat 336 were also found to suppress anion interference. ${ }^{8}$ Since $0.001 \mathrm{M}$ was the maximum concentration of TOA that was possible in the first-generation solvent system without noticeable loss of extraction performance, this concentration was adopted. With the use of higher modifier concentration, however, it is likely that TOA could be used at higher concentration without adverse effects, as loss of extraction performance is likely related to the modifier being effectively engaged in solvation of the ammonium nitrate salt.

An isoparaffinic diluent, Isopar L, available from Exxon Chemical Company, was chosen for the baseline solvent. ${ }^{15}$ Isopar $\mathrm{L}$ is a blend of $\mathrm{C}_{10}$ to $\mathrm{C}_{12}$ branched alkanes with a distillation range of $191-205{ }^{\circ} \mathrm{C}$, a viscosity of 1.6 centipoise at $25^{\circ} \mathrm{C}$, a specific gravity of $0.767 \mathrm{~g} / \mathrm{mL}$ at $60^{\circ} \mathrm{F}\left(15.5^{\circ} \mathrm{C}\right)$, and a Thermal Closed Cup flash point of $144{ }^{\circ} \mathrm{F}\left(62^{\circ} \mathrm{C}\right)$ (data courtesy of Exxon Chemical Company). Aliphatic diluents are desirable because they improve hydraulics (low density and viscosity) and have excellent stability. Straight-chain hydrocarbons would be preferred for maximum radiation stability, but it was found that use of the equivalent straight-chain diluent Norpar 12 , for example, entails a slightly greater tendency to third-phase formation. ${ }^{34}$

All solvent employed in the work reported herein was supplied by Bonnesen under a different task. Prior to delivery, pristine solvent had been washed in Teflon FEP separatory funnels with two equal volumes of $0.10 \mathrm{M} \mathrm{NaOH}$, two equal volumes of $0.05 \mathrm{M}$ nitric acid, and three equal volumes of water. The washes remove solvent impurities such as 4-sec-butylphenol, a residual starting material in the modifier synthesis. A quality-assurance (QA) procedure was also performed to verify uniform performance prior to delivery. 37 Referred to as "washed pristine," the solvent is water-saturated when it is received.

\subsubsection{Solvent Density}

Solvent densities are tabulated for several conditions in Table 2.1. All measurements were performed using volumetric flasks; an almost-negligible temperature correction for the volume of the flask was applied, ${ }^{38}$ affecting only the fourth decimal place. Uncertainties are propagated according to the tolerances for Class A volumetric flasks, ${ }^{39}$ or if precision was improved upon replication, a higher precision is indicated. Precision from lot to lot is excellent for pristine (dry) solvent. Washed pristine solvent appears to be slightly more dense than pristine solvent, though the value for $25.5{ }^{\circ} \mathrm{C}$ appears out of line.

\subsection{WASTE SIMULANTS}

Waste simulants were prepared under a different task using a staged recipe recommended by Westinghouse Savannah River Company (WSRC). ${ }^{21,22}$ Three simulants were prepared, designated salts only, salts + metals, and full simulant. Each simulant corresponded to successive completion of each of the three stages in the recipe. A large initial set of salts formed the primary salt matrix, which was then augmented by a smaller set of certain metals at low concentration and finally by a set of organic compounds known to be present in the waste. Although the salt matrix was designed to represent an average waste composition, the organic compounds in the full simulant were added at the highest concentration likely to be encountered in the SRS waste. Since organic compounds present 
Table 2.1. Solvent density

\begin{tabular}{ccccccc}
\hline $\begin{array}{c}\text { Temp }^{a} \\
\left({ }^{\circ} \mathrm{C}\right)\end{array}$ & $\begin{array}{c}\text { Density } \\
\left(\mathrm{g} \mathrm{cm}^{-3}\right)\end{array}$ & $\begin{array}{c}\text { Vol. } \\
(\mathrm{mL})\end{array}$ & Replicates & Wet or dry & Lot $^{c}$ & Reference \\
\hline 21.3 & $0.830 \pm 0.001$ & 1 & 3 & Wet & $156 \mathrm{~W}$ & This work \\
21.3 & $0.829 \pm 0.001$ & 5 & 5 & Wet & $156 \mathrm{~W}$ & This work \\
$25.5^{d}$ & $0.810 \pm 0.002$ & 10 & 1 & Wet & $124 \mathrm{~W}$ & $f$ \\
20.4 & $0.8262 \pm 0.0001$ & 2000 & 4 & Dry & $149 \mathrm{~W}$ & This work \\
20.5 & $0.8258 \pm 0.0001$ & 2000 & 9 & Dry & $31 \mathrm{~W}$ & This work \\
20.6 & $0.8262 \pm 0.0001$ & 2000 & 5 & Dry & $156 \mathrm{~W}$ & This work \\
20.8 & $0.8260 \pm 0.0001$ & 2000 & 9 & Dry & $148 \mathrm{~W}$ & This work \\
30.7 & $0.8185 \pm 0.0002$ & 2000 & 1 & Dry & $31 \mathrm{~W}$ & This work \\
33.6 & $0.8159 \pm 0.0002$ & 2000 & 1 & Dry & $31 \mathrm{~W}$ & This work \\
34.5 & $0.8154 \pm 0.0002$ & 2000 & 1 & Dry & $31 \mathrm{~W}$ & This work \\
35.0 & $0.8148 \pm 0.0002$ & 2000 & 1 & Dry & $31 \mathrm{~W}$ & This work \\
36.5 & $0.8135 \pm 0.0002$ & 2000 & 1 & Dry & $31 \mathrm{~W}$ & This work \\
19.4 & $0.8217 \pm 0.0002$ & 2000 & 1 & Dry & $0.5 \mathrm{M} \mathrm{Cs-7SB}{ }^{e}$ & This work \\
\hline
\end{tabular}

${ }^{a}$ Unless otherwise noted, solvent temperatures were measured directly by a thermometer.

${ }^{b}$ Dry is pristine solvent before it was washed; wet is washed pristine solvent.

${ }^{c}$ Full lot no. for $31 \mathrm{~W}$ is PVB B000894-31W. Full lot numbers for all others have the prefix PVB B000719-.

${ }^{d}$ Ambient temperature.

${ }^{e}$ The solvent was $0.5 \mathrm{M}$ Cs-7SB modifier in Isopar L (no BOBCalixC6 or TOA).

fJ.F. Birdwell, Jr.

the greatest likelihood of interference with the smooth operation of CSSX, the tests with the full simulant were expected to exhibit a worst case insofar as interference, especially on solvent recycle. The added metals in the salts + metals simulant provide potential catalysts for solvent-degradation reactions.

Table 2.2 summarizes the simulant compositions. The first column lists the species as added. The second column gives the composition of the simulant used in FY 1998 and FY 1999 studies designated SRS\#2.3,8,14,15 The third column gives the intended composition of the three simulants used in this work, divided into the three sets of components noted above. The composition of the full simulant as found by analysis (ORNL Radioactive Materials Analysis Laboratory) on three of four draws is given in the right-most three columns. Upon each draw, the simulant was pumped through a $0.5 \mu \mathrm{m}$ porous sintered stainless steel filter. It may be noted that cesium was left out of the simulants so that it could be added at desired concentrations before individual experiments. Typically, cesium as cesium nitrate was added at the prescribed concentration of $0.14 \mathrm{mM}$. A ${ }^{137} \mathrm{Cs}$ isotopic fraction is given as $22.56 \%, 12$ which implies an average ${ }^{137} \mathrm{Cs}$ activity of $0.375 \mathrm{Ci} / \mathrm{L}$.

The salts-only simulant differs from the SRS\#2 simulant employed in previous studies $3,8,14,15$ in having a $20 \%$ lower sodium content $(5.6 \mathrm{M}$ vs. $7.0 \mathrm{M})$, in accordance with the need to optimize performance of the preliminary strike with monosodium titanate. ${ }^{20}$ Since the lower sodium concentration is achieved by dilution with more dilute sodium hydroxide, the other major ions in the waste are lower in concentration in proportion with the decrease in sodium concentration, though with 


\section{Cesium Extractant}

@ $0.01 M$

- Synthetically attractive

- Rejects sodium

- Stable to alkaline simulant

\section{BOBCalixC6}

Calix [4]arene-bis(tert-octylbenzo-crown-6)

FW 1149.53

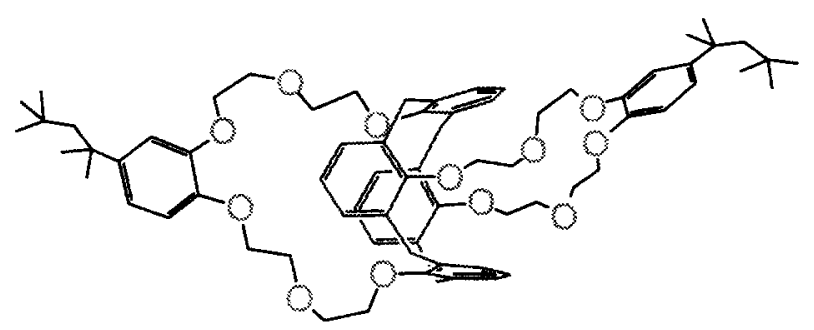

\section{Diluent Modifier}

@ $0.5 \mathrm{M}$

\section{Cs-7SB}

1-(2,2,3,3-Tetrafluoropropoxy)-3-(4-secbutylphenoxy)-2-propanol

FW 338.35

- Increases Cs extraction

- Increases extractant solubility

- Excellent stability characteristics

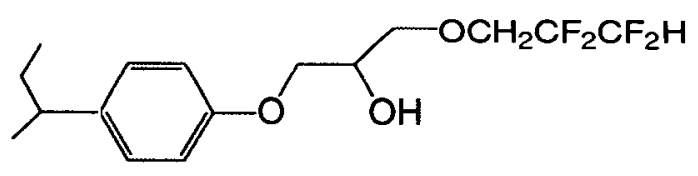

\section{Suppressor}

@ 0.001 M

\section{TOA}

Tri-n-octylamine

FW 353.69

- Suppresses impurity effects

- Suppresses ion-pair dissociation

- Improves and stabilizes stripping

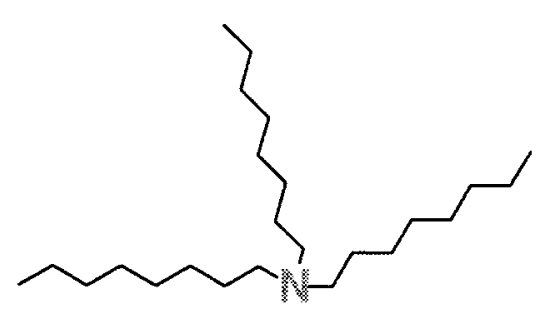

\section{Diluent}

- Isopar L

- Branched avg. 12-carbon aliphatic

- Promotes good hydraulics

Fig. 2.1. CSSX baseline solvent. 
some modifications. Free hydroxide concentration, however, increased from $1.9 \mathrm{M}$ in the SRS\#2 simulant to $2.06 \mathrm{M}$. Another significant difference is the substantially lower cesium concentration of $0.14 \mathrm{mM}$ vs. $0.7 \mathrm{mM}$ in the SRS\#2 simulant.

Table 2.2 shows that sodium was close to its target concentration, as assured in the preparation of the simulant. Potassium was higher than the intended $0.015 \mathrm{M}$, likely because of minor potassium impurities in the sodium salts used in simulant preparation. Owing to the competition between potassium and cesium in extraction, the simulant is thus expected to yield slightly lower extraction performance than the true average waste composition (other concentrations being equal). Most other salt constituents were not very different from the targeted concentrations. Interestingly, transition metals were not largely lost to precipitation, though copper, chromium, palladium, and tin were found at significantly lower than intended concentrations. Trends with time suggested partial redissolution of these four metals. A white precipitate is characteristically formed during simulant preparation. It continues to appear in filtered simulant and even in extended-duration extraction experiments.

A ${ }^{31} \mathrm{P}$ NMR analysis (see Section 2.4) of full simulant, $700 \mu \mathrm{L}$ diluted with $300 \mu \mathrm{L}$ of $\mathrm{D}_{2} \mathrm{O}$, shows the presence of free phosphate, what is believed to be MBP and DBP, and no TBP. Assuming the free phosphate concentration is the target $0.007 \mathrm{M}$, by integration against the phosphate peak the MBP concentration is $0.15 \pm 0.03 \mathrm{mM}$, and the DBP concentration is $0.19 \pm 0.04 \mathrm{mM}$. The target concentrations in the simulant are $25 \mathrm{mg} / \mathrm{L}(0.16 \mathrm{mM}), 25 \mathrm{mg} / \mathrm{L}(0.12 \mathrm{mM})$, and $0.5 \mathrm{mg} / \mathrm{L}(0.0019$ $\mathrm{mM}$ ) respectively for MBP, DBP, and TBP.

Despite initial expectations that cesium distribution ratios $\left(D_{\mathrm{Cs}}\right)$ would be lower than observed with the former SRS\#2 simulant because of the lower nitrate concentration, $D_{\mathrm{Cs}}$ values with the new simulant turned out to be higher (see below). In line with a more determined modeling effort, ${ }^{25}$ several reasonable hypotheses may be offered. First, the Cs concentration is lower and thus does not load the solvent as much. Second, the free hydroxide concentration is higher, favoring a hypothesized cation-exchange mechanism that augments the ion-pair extraction mechanism. Third, the activity of cesium nitrate drops as the ionic strength increases at high ionic strength.

\subsection{OTHER MATERIALS}

\subsubsection{Radioactive Tracers}

${ }^{137}$ Cesium was initially obtained as ${ }^{137} \mathrm{CsCl}$ in $0.1 \mathrm{M} \mathrm{HCl}$ from Isotope Products Laboratories, Burbank, CA. ${ }^{22}$ Sodium was obtained as ${ }^{22} \mathrm{NaCl}$ in water from Isotope Products Laboratories.

$1-{ }^{14} \mathrm{C}$-Lauric acid (specific activity of $57 \mathrm{mCi} / \mathrm{mmol}, 0.5 \mathrm{mCi} / \mathrm{mL}$ in hexane) was acquired from ICN Biomedicals, Costa Mesa, CA. Sodium $1{ }^{14} \mathrm{C}$-dodecyl sulfate (specific activity $55 \mathrm{mCi} / \mathrm{mmol}, 0.1$ $\mathrm{mCi} / \mathrm{mL}$ in sterile water) was obtained from American Radiolabeled Chemicals, Inc., St. Louis, MO. ${ }^{1-14} \mathrm{C}$-Stearic acid $\left(56 \mathrm{mCi} / \mathrm{mmol}, 0.1 \mathrm{mCi} / \mathrm{mL}\right.$ in toluene) and $1{ }^{14} \mathrm{C}$-lauric acid $(55 \mathrm{mCi} / \mathrm{mmol}, 100$ $\mu \mathrm{Ci}$ in $1 \mathrm{~mL}$ ethanol) were provided by Moravek Biochemicals, Brea, CA.

\subsubsection{Miscellaneous Materials}

Unless otherwise specified, all chemicals were reagent grade, and water was $18 \mathrm{M} \Omega$ distilled, deionized water obtained from a Barnstead Nanopure II system. When experiments required solvent compositions other than washed pristine solvent described above, BOBCalixC6 was received from IBC Advanced Technologies, Inc.; modifiers Cs-7SB or Cs-6 [1-(2,2,3,3-tetrafluoropropoxy)-3-(4-tertoctylphenoxy)-2-propanol] were received from P. V. Bonnesen, as prepared under a separate task; trioctylamine (Aldrich) was recrystallized as the $\mathrm{HCl}$ salt; and Isopar $\mathrm{L}$ and Norpar 12 was obtained from Exxon Chemicals. For some experiments as noted, BOBCalixC6 was recrystallized from dichloromethane. 4-sec-Butylphenol, $98+\%$ by GC, was purchased from TCI America.

Scrub $\left(0.05 \mathrm{M} \mathrm{HNO}_{3}\right)$ and strip $\left(0.001 \mathrm{M} \mathrm{HNO}_{3}\right)$ solutions were prepared by appropriately diluting $69.3 \% \mathrm{HNO}_{3}$ J.T. Baker Ultrex II Ultrapure Reagent. The initial dilution to $50 \mathrm{mM} \mathrm{HNO}_{3}$ was titrated in triplicate using a Mettler DL77 Titrator. Subsequent dilution provided the 1-mM $\mathrm{HNO}_{3}$ strip solution. Sodium hydroxide solutions were prepared from $50 \% \mathrm{NaOH}$ solution and stored under Ascarite. 
Table 2.2. Simulant compositions

\begin{tabular}{|c|c|c|c|c|c|c|c|c|}
\hline \multirow[t]{2}{*}{ Species $^{a}$} & \multicolumn{8}{|c|}{ Concentrations $(\mathbf{M})^{b}$} \\
\hline & SRS\#2 & This work & & & & Draw \#1 & Draw \#3 & Draw \#4 \\
\hline $\begin{array}{l}\mathrm{Na}^{+} \\
\mathrm{K}^{+} \\
\mathrm{Cs}^{+} \\
\mathrm{OH}^{-} \\
\mathrm{NO}_{3}^{-} \\
\mathrm{NO}_{2}^{-} \\
\mathrm{AlO}_{2}^{-} \\
\mathrm{CO}_{3}^{2-} \\
\mathrm{SO}_{4}^{2-} \\
\mathrm{Cl}^{-} \\
\mathrm{F}^{-} \\
\mathrm{PO}_{4}^{3-} \\
\mathrm{C}_{2} \mathrm{O}_{4}^{2-} \\
\mathrm{SiO}_{3}^{2-} \\
\mathrm{MoO}_{4}^{2-} \\
\mathrm{NH}_{3}\end{array}$ & $\begin{array}{c}7.00 \\
0.02 \\
7.0 \times 10^{-4} \\
1.90 \\
2.70 \\
1.00 \\
0.40 \\
0.20 \\
0.22 \\
0.10 \\
0.050\end{array}$ & $\begin{array}{c}5.6 \\
0.015 \\
1.4 \times 10^{-4} \\
2.06 \\
2.03 \\
0.5 \\
0.28 \\
0.15 \\
0.14 \\
0.024 \\
0.028 \\
0.007 \\
0.008 \\
0.03 \\
7.0 \times 10^{-5} \\
0.001\end{array}$ & $\begin{array}{l}\mathbf{I} \\
\mathbf{y}\end{array}$ & \begin{tabular}{l|}
$\mathbf{S}$ \\
$\mathbf{a}$ \\
$\mathbf{1}$ \\
$\mathbf{t}$ \\
$\mathbf{S}$ \\
\\
+
\end{tabular} & $\begin{array}{l}\mathbf{F} \\
\mathbf{u} \\
\mathbf{l} \\
\mathbf{l}\end{array}$ & $\begin{array}{c}5.52 \\
0.020 \\
\text { N/A } \\
\text { N/A } \\
1.98 \\
0.52 \\
0.25 \\
\text { N/A } \\
0.11 \\
0.018 \\
0.021 \\
0.005 \\
\text { N/A } \\
\text { N/A } \\
4 \times 10^{-5} \\
\text { N/A }\end{array}$ & $\begin{array}{c}5.65 \\
0.020 \\
\text { N/A } \\
\text { N/A } \\
1.90 \\
0.51 \\
0.24 \\
\text { N/A } \\
0.11 \\
0.017 \\
0.024 \\
0.006 \\
\text { N/A } \\
\text { N/A } \\
8 \times 10^{-5} \\
\text { N/A }\end{array}$ & $\begin{array}{c}5.35 \\
0.021 \\
\text { N/A } \\
\text { N/A } \\
2.23 \\
0.61 \\
0.25 \\
\text { N/A } \\
0.15 \\
0.019 \\
0.029 \\
0.008 \\
\text { N/A } \\
\text { N/A } \\
5 \times 10^{-5} \\
\text { N/A }\end{array}$ \\
\hline $\begin{array}{l}\mathrm{Cu}(\mathrm{II}) \\
\mathrm{Cr}(\mathrm{VI}) \\
\mathrm{Ru}(\mathrm{III}) \\
\mathrm{Pd}(\mathrm{II}) \\
\mathrm{Rh}(\mathrm{III}) \\
\mathrm{Fe}(\mathrm{III}) \\
\mathrm{Zn}(\mathrm{II}) \\
\mathrm{Sn}(\mathrm{II}) \\
\mathrm{Hg}(\mathrm{II}) \\
\mathrm{Pb}(\mathrm{II}) \\
\mathrm{Ag}(\mathrm{I})\end{array}$ & 0.015 & $\begin{array}{l}2.27 \times 10^{-5} \\
1.44 \times 10^{-3} \\
8.11 \times 10^{-6} \\
3.85 \times 10^{-6} \\
2.04 \times 10^{-6} \\
2.58 \times 10^{-5} \\
1.22 \times 10^{-4} \\
2.02 \times 10^{-5} \\
2.49 \times 10^{-7} \\
1.01 \times 10^{-5} \\
9.27 \times 10^{-8}\end{array}$ & & \begin{tabular}{c|}
$\mathbf{m}$ \\
$\mathbf{e}$ \\
$\mathbf{t}$ \\
$\mathbf{a}$ \\
$\mathbf{1}$ \\
$\mathbf{s}$
\end{tabular} & $\begin{array}{c}\mathbf{s} \\
\mathbf{i} \\
\mathbf{m} \\
\mathbf{u} \\
\mathbf{I} \\
\mathbf{a} \\
\mathbf{n} \\
\mathbf{t}\end{array}$ & $\begin{array}{r}1.57 \times 10^{-5} \\
4.25 \times 10^{-4} \\
9.73 \times 10^{-6} \\
1.01 \times 10^{-6} \\
2.68 \times 10^{-6} \\
3.29 \times 10^{-5} \\
9.28 \times 10^{-5} \\
4.55 \times 10^{-6} \\
<2.5 \times 10^{-6} \\
3.54 \times 10^{-5} \\
<9.3 \times 10^{-7}\end{array}$ & $\begin{array}{r}1.62 \times 10^{-5} \\
7.98 \times 10^{-4} \\
1.12 \times 10^{-5} \\
2.93 \times 10^{-6} \\
2.61 \times 10^{-6} \\
2.79 \times 10^{-5} \\
9.74 \times 10^{-5} \\
7.41 \times 10^{-6} \\
<2.5 \times 10^{-7} \\
4.03 \times 10^{-5} \\
<9.3 \times 10^{-7}\end{array}$ & $\begin{array}{l}1.89 \times 10^{-5} \\
8.48 \times 10^{-4} \\
1.01 \times 10^{-5} \\
2.23 \times 10^{-6} \\
2.50 \times 10^{-6} \\
4.30 \times 10^{-5} \\
1.05 \times 10^{-4} \\
8.73 \times 10^{-6} \\
<2.5 \times 10^{-7} \\
3.84 \times 10^{-5} \\
<9.3 \times 10^{-7}\end{array}$ \\
\hline $\begin{array}{l}\text { TBP } \\
\text { DBP } \\
\mathrm{MBP} \\
\mathrm{BuOH} \\
\mathrm{HCO}_{2}^{-} \\
\mathrm{TMA}^{-} \\
\end{array}$ & & $\begin{array}{l}1.88 \times 10^{-6} \\
1.19 \times 10^{-4} \\
1.62 \times 10^{-4} \\
2.70 \times 10^{-5} \\
3.33 \times 10^{-2} \\
1.69 \times 10^{-4}\end{array}$ & & 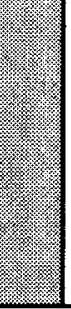 & & $\begin{array}{l}\text { NA } \\
\text { NA } \\
\text { NA } \\
\text { NA } \\
\text { NA } \\
\text { NA }\end{array}$ & $\begin{array}{l}\text { NA } \\
\text { NA } \\
\text { NA } \\
\text { NA } \\
\text { NA } \\
\text { NA }\end{array}$ & $\begin{array}{l}\text { NA } \\
\text { NA } \\
\text { NA } \\
\text { NA } \\
\text { NA } \\
\text { NA }\end{array}$ \\
\hline $\begin{array}{l}\text { TOC }^{c} \\
\text { TIC }^{c}\end{array}$ & & & & & & $\begin{array}{l}4.17 \times 10^{-2} \\
1.92 \times 10^{-1}\end{array}$ & $\begin{array}{l}1.26 \times 10^{-1} \\
1.86 \times 10^{-1}\end{array}$ & $\begin{array}{l}8.83 \times 10^{-2} \\
2.25 \times 10^{-1}\end{array}$ \\
\hline
\end{tabular}

${ }^{a}$ As added. Abbreviations: TBP, tri- $n$-butyl phosphate; DBP, di- $n$-butyl phosphate; MBP, monon-butyl phosphate; $\mathrm{BuOH}$, 1-butanol; TMA, trimethylamine.

$b$ NA refers to "Not Analyzed." Simulant preparation was completed on Jun. 9, 2000. Draws \#1, \#3, and \#4 were made on Jun. 13, Jul. 20, and Aug. 8, 2000, respectively, and analytical results shown in the three right columns were obtained within a month after each draw.

cTotal organic carbon (TOC) or total inorganic carbon (TIC), converted from $\mathrm{mg} / \mathrm{L}$ to $\mathrm{mol} / \mathrm{L}$. 


\subsection{INSTRUMENTS AND ANALYTICAL METHODS}

\subsubsection{Instruments and Equipment}

${ }^{137}$ Cesium and ${ }^{22} \mathrm{Na}$ activity in samples was counted using a Packard Cobra Quantum Model 5003 gamma counter equipped with a 3" $\mathrm{NaI}(\mathrm{Tl})$ crystal through-hole type detector. A counting window of 580 to $750 \mathrm{keV}\left({ }^{137 \mathrm{~m}} \mathrm{Ba}\right)$ was used to determine ${ }^{137} \mathrm{Cs}$ activity. The ${ }^{22} \mathrm{Na}$ activity in each phase was determined by monitoring the $1022 \mathrm{keV}$ peak. ${ }^{14}$ Carbon activity was counted using a Packard Tri-Carb 2500TR Liquid Scintillation Analyzer. Count times were routinely of 10-min duration using a counting window of 4.0 to $156 \mathrm{keV}$. Samples, typically 200-250 $\mu \mathrm{L}$ in volume, were placed in $20-\mathrm{mL}$ glass scintillation vials to which $10 \mathrm{~mL}$ of Packard UltraGold XR scintillation cocktail was added. Samples were dark-adapted for at least $30 \mathrm{~min}$ prior to counting.

Certain inorganic cation constituents (especially $\mathrm{Na}, \mathrm{K}$, and $\mathrm{Al}$ ) were analyzed by Inductively Coupled Argon Plasma (ICAP) emission spectrometer (Thermo Jarrell Ash, model IRIS, Franklin, MA). Ion chromatography was performed using a Dionex Model DX500 equipped with GP40 pump and ED40 conductivity detector. Anions were separated using an AS11 analytical column coupled with AG11 guard column and an anion trap column (ATC-1). Detection limits were determined by running standards at successively lower dilutions until the deviation from the expected result was greater than $10 \%$. Detection limits for each anion were as follows: $\mathrm{Cl}^{-}=7.05 \times 10^{-6} \mathrm{M}, \mathrm{NO}_{2}^{-}=2.2$ $\times 10^{-6} \mathrm{M}, \mathrm{NO}_{3}^{-}=8.06 \times 10^{-6} \mathrm{M}, \mathrm{SO}_{4}{ }^{2-}=2.0 \times 10^{-6} \mathrm{M}, \mathrm{PO}_{4}{ }^{3-}=1.05 \times 10^{-6} \mathrm{M}$. The cations, $\mathrm{Na}^{+}$, $\mathrm{K}^{+}$, and $\mathrm{Cs}^{+}$were separated using a CS12A analytical column coupled with a CG12A guard column. The analysis used $20 \mathrm{mN} \mathrm{H} \mathrm{SO}_{4}$ eluent at $1 \mathrm{~mL} / \mathrm{min}$ in an isocratic run of $20 \mathrm{~min}$. Background conductivity was $0.2 \mu \mathrm{S}$ using CSRS-Ultra suppressor in auto-regeneration mode set at $300 \mathrm{~mA}$.

Acid-base titrations were performed using a Mettler DL77 Titrator. Measurements of aqueous $\mathrm{pH}$ were carried out with an ORION Portable $\mathrm{pH}$ meter, model 230A, equipped with an ORION GLS microelectrode.

Electrospray Mass Spectrometry (ES-MS) was performed on a PE SCIEX API 165 single quadrupole mass spectrometer (Concord, Ontario, Canada) outfitted with a TurboIonSpray source. A $30-\mathrm{cm}$ long, Teflon encapsulated stainless steel transfer tube $(75 \mathrm{~mm}$-id. stainless steel encapsulated in 1/16 in. o.d. Teflon, CETAC Technologies, Inc.) connected a $3.5-\mathrm{cm}$-long stainless steel ES emitter (400 mm-o.d., 100-mm-i.d.) to the stainless steel $254-\mathrm{mm}$ i.d. bore-through bulkhead grounding port built into the source. The emitter held at ca. $4.5 \mathrm{kV}$ was placed $1.5-2.5 \mathrm{~cm}$ from the curtain gas plate aperture and angled to spray across the aperture. Nitrogen was used for sample nebulization. No "turbo gas" was used in these experiments. Sample was introduced to the instrument using a syringe pump to deliver solution loaded into $1.0-\mathrm{mL}$ plastic syringes (Becton Dickinson Co., Franklin Labs, NJ) at a flow rate of $10.0 \mu \mathrm{L} / \mathrm{min}$. Resulting spectra were the sum of ten individual scans from $\mathrm{m} / \mathrm{z}$ 50-3000 (in cation mode) and from $\mathrm{m} / \mathrm{z}$ 30-2000 (in anion mode) using a $0.1 \mathrm{~m} / \mathrm{z}$ step size, a $1.0-\mathrm{ms}$ dwell time, and a pause of $5 \mathrm{~ms}$. All the electrospray rinses and cleanup were done with a $50 / 50$ vol\% mixture of dichloromethane (EM, HPLC grade) and acetonitrile (anhydrous, EM). Tenfold dilutions of all the samples were also made with this mixture.

Nuclear Magnetic Resonance spectra were obtained on a Bruker Avance 400 wide-bore NMR spectrometer. Nuclei observed were proton $(400.13 \mathrm{MHz}),{ }^{13} \mathrm{C}(100.61 \mathrm{MHz}),{ }^{19} \mathrm{~F}(376.498 \mathrm{MHz})$, and ${ }^{31} \mathrm{P}(161.975 \mathrm{MHz})$. All proton, carbon, and fluorine NMR spectra on solvent samples were obtained by diluting typically $100 \mu \mathrm{L}$ aliquots with a fixed volume of deuterochloroform (99.8 atom\% D, obtained from Aldrich Chemical Company) in a 5-mm Wilmad NMR tube. The deuterochloroform also contained a known concentration of hexamethylbenzene (HMB, Aldrich, 99\%) as an internal integration standard. The precise procedure (volume of deuterochloroform, concentration of HMB) depended on the nature of the solvent samples being analyzed (irradiated or thermally degraded), and is provided in the experimental sections in Chaps. 5 and 6. Other sample preparations will be described in the experimental sections of the appropriate chapters. 


\subsubsection{Calibrations}

The calibration of Eppendorf Reference Pipetteman pipetters was checked monthly by weighing aliquots of distilled, deionized water. Standard volumetric flasks were employed in preparing stock solutions.

An H-B U.S.A. VWBrand thermometer was calibrated using an ice-water bath. The temperature controller on the water bath Linberg/Blue M, Model Number RSWB3222A-1 was found to agree with the calibrated thermometer within $0.2{ }^{\circ} \mathrm{C}$ over the $5-35{ }^{\circ} \mathrm{C}$ interval. This thermometer was used to check the temperature of incubators and to test the accuracy of the pipettes used. Two mercury-inglass thermometers were calibrated by the ORNL Instrument and Controls Division Metrology Laboratory and certified traceable to National Institute of Standards and Technology (NIST). Thermometer ID M093698, with resolution of $0.05{ }^{\circ} \mathrm{C}$ was used to check incubators and air box at 25,35 , and $60{ }^{\circ} \mathrm{C}$. The Labline Imperial III incubators used to conduct the thermal stability experiments were set to $36 \pm 0.5^{\circ} \mathrm{C}$ and $60 \pm 1{ }^{\circ} \mathrm{C}$, and the actual operating temperature ranges, when checked against the calibrated thermometer, were $34.8 \pm 0.5^{\circ} \mathrm{C}$ and $61.2 \pm 0.6{ }^{\circ} \mathrm{C}$, respectively.

The glass $\mathrm{pH}$ microelectrode was calibrated with two aqueous buffers at $\mathrm{pH} 4.01$ and 7.00 . Subsequent checks during sample tests confirmed the absence of drift.

For ${ }^{1} \mathrm{H}$ and ${ }^{13} \mathrm{C}$ NMR spectra obtained using deuterochloroform as the NMR solvent, chemical shifts were referenced against the residual chloroform peak $(7.25 \mathrm{ppm})$ for proton spectra, and against the carbon resonance for deuterochloroform $(77.0 \mathrm{ppm})$ for ${ }^{13} \mathrm{C}$ spectra. For ${ }^{19} \mathrm{~F}$ NMR, all chemical shifts were referenced against $\mathrm{CFCl}_{3}$ (external standard reference sample set at $0.0 \mathrm{ppm}$ ). For ${ }^{31} \mathrm{P}$ NMR, chemical shifts were referenced against phosphoric acid, set to $0.0 \mathrm{ppm}$ by way of an external standard reference sample.

A sodium hydroxide solution $(0.1 \mathrm{M} \mathrm{NaOH})$ was standardized using dried KHP (potassium hydrogen phthalate). The standardized $\mathrm{NaOH}$ solution was then used to standardize a stock $50 \mathrm{mM}$ nitric acid solution.

Internal calibration of the ICP spectrometer was done each day by using the internal $\mathrm{Hg}$ reference lamp (Hg Profile). External calibration of each metal was done prior to analysis with check standards run in between samples frequently. Standards were diluted volumetrically with $2 \%$ Ultrex II $\mathrm{HNO}_{3}$ from purchased ICP standards (JT Baker), typically $1000 \mathrm{mg} / \mathrm{L}$ to $10,000 \mathrm{mg} / \mathrm{L}$. Typically standards are made to bracket expected sample concentration by $\pm 10 \%$.

Anion analysis by ion chromatography (IC) used a two-level external standardization for each anion from a stock 5-anion standard prepared at $1000 \mathrm{~g} / \mathrm{L}$ from dried salts (Table 4.1). A QC standard (Combined Seven Anion Standard) was purchased from Dionex Corp. An external standardization using two levels of calibration was made for each anion. For anions $\mathrm{Cl}^{-}, \mathrm{NO}_{2}{ }^{-}, \mathrm{SO}_{4}{ }^{2-}$, and $\mathrm{PO}_{4}{ }^{3-}$, calibration was made using $0.1-$ and $1.0-\mathrm{ppm}$ standards. For $\mathrm{NO}_{3}{ }^{-}$anion, calibration was made using 1.0- and 10-ppm standards. An $r^{2}$ value of 0.9999 or better was achieved for each linear fit. To analyze dibutylphosphate-containing solutions, an external standardization of the anions was done with two levels of calibration using concentrations of $1 \mathrm{ppm}$ and $9.26 \mathrm{ppm}$. Dibutylphosphate (DBP) was calibrated at $5 \mathrm{ppm}$ and $92.6 \mathrm{ppm}$ in water. A separate response standard at $100 \mathrm{ppm}$ was run in $0.01 \mathrm{M} \mathrm{NaOH}$ to calibrate samples run in basic solutions. Typically the acid strip solutions were made basic to $\mathrm{pH}>10$ prior to analysis for DBP.

A five-level external standardization for each cation, $\mathrm{Na}^{+}, \mathrm{K}^{+}$, and $\mathrm{Cs}^{+}$, was used at $0.1,0.5,1.0$, 5.0 , and $10.0 \mathrm{ppm}$. Goodness of calibration $\left(r^{2}\right)$ fit was better than 0.9999 . Standards were prepared using dilutions of the dried nitrate salts prepared at 50 or $100 \mathrm{mM}$ and diluted in deionized water. Duplicates were run for each sample and were analyzed with $\pm 2 \%$ error.

The Packard Cobra II AutoGamma counter is calibrated and tested for background level on a daily basis before any samples are counted. The calibration procedure calls for the counting of an empty tube first, then of a sealed $0.25-\mu \mathrm{Ci}{ }^{137} \mathrm{Cs}$ source. This procedure corrects the counting efficiency and any potential window shift in the region of interest around the cesium peak.

The ES-MS spectrometer is calibrated before any series of analyses using a multi-ion standard inhouse prepared solution. The peaks corresponding to the ions (cation mode or anion mode) are referenced and adjustments are made automatically so that the spectrometer is tuned for best 
performance (match of the $m / z$ values and intensities). The ions in the standard solution covered and even exceeded the $\mathrm{m} / \mathrm{z}$ ranges of interest for our experiments.

\subsection{EXTRACTION/SCRUB/STRIP (ESS) PROTOCOL}

A simple test protocol was devised to provide useful information on flowsheet performance in batch mode. Since sequential contacting is labor intensive, a minimal test was desired. The chosen protocol entailed cross-current contacting of a given volume of solvent with simulant, then scrub solution (0.05 $\left.\mathrm{M} \mathrm{HNO}_{3}\right)$, and then three times with strip solution $\left(0.001 \mathrm{M} \mathrm{HNO}_{3}\right)$. Referred to as the "ESS" Protocol, the sequence is depicted in Fig. 2.2. In approximate accordance with the baseline flowsheet, ${ }^{20}$ the O/A ratios in the ESS Protocol are respectively $0.33,5$, and 5 . Throughout this report, reference to ESS implies the exact procedure just described. However, particular needs of certain experiments at times dictated some departures that will be pointed out as appropriate. Protocol departures involving different sequences are indicated by adding subscripts to indicate how many times a certain step was repeated. Thus, two extractions, one scrub, and five strips might be designated as $\mathrm{E}_{2} \mathrm{SS}_{5}$.

In a typical ESS experiment, the following steps are performed: An aliquot of simulant is spiked with ${ }^{137} \mathrm{Cs}$ (activity approximately $0.2 \mu \mathrm{Ci} / \mathrm{mL}$ ) in a Teflon FEP tube or polypropylene vial and put in contact with an aliquot of solvent at an O/A ratio of 0.33. The tube or vial is then capped and mounted by clips on a disk which is then rotated in a constant-temperature air box at $25 \pm 0.5^{\circ} \mathrm{C}$ for $30 \mathrm{~min}$ or longer. The gentle end-over-end tumbling produced by this technique is adequate to bring the samples to equilibrium in the contact interval used as long as sufficient head space is left in the tube or vial to allow good dispersion of the phases. It is recommended that head space be at least $25 \%$ of the container volume, as in one instance when the head space was $16 \%$ of container volume at an $\mathrm{O} / \mathrm{A}$ of 5 , the aqueous phase was not well dispersed and equilibrium was not reached. Since dispersion depends on disk rotation speed, phase ratio, and vial diameter, direct observation of phase

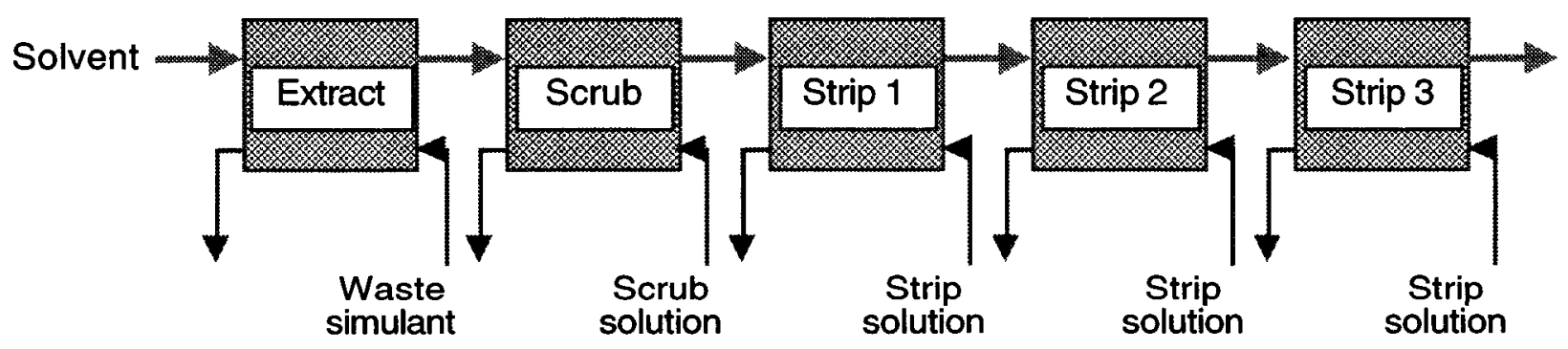

Fig. 2.2. Extract/Scrub/Strip (ESS) Sequential Contacting Protocol.

dispersion is prudent in any case. The contact time interval is also needed for the phases to reach thermal equilibrium. The fact that consistent distribution behavior from sample to sample (see Chap. 3 ) is observed even at longer equilibrium times (e.g., overnight) indicates that overall equilibrium is reached within the 30-min minimum contact time. Other contacting techniques are possible and were at times employed, such as vortexing and shaking. For any contacting technique, the same precautions regarding the attainment of chemical and thermal equilibrium are applicable. After equilibration, the tube or vial is centrifuged for $3 \mathrm{~min}$ at $25^{\circ} \mathrm{C}$ in a temperature-controlled centrifuge. An aliquot of each phase is subsampled to be counted. To avoid cross-contamination of phases, subsampling of the lower phase (aqueous in all cases here) can best be accomplished by completely withdrawing the aqueous phases via disposable pipette to a clean vial, from which accurate subsamples may be drawn. If solvent is in short supply, the solvent subsample can optionally be returned to the 
main volume of equilibrated solvent after counting. Proceeding to the next step in the ESS protocol, a known volume of solvent that had been equilibrated with simulant is then pipetted into a clean equilibration tube or vial with scrub solution at and O/A ratio of 5. The contacting, centrifugation, subsampling, and counting is then repeated. The solvent is then carried through as many strips as desired at an O/A ratio of 5 . On the second and subsequent strip steps, the aqueous phase must usually be spiked again with ${ }^{137} \mathrm{Cs}$ activity. Regarding gamma counting, since it is the ${ }^{137 \mathrm{~m}} \mathrm{Ba}$ daughter $\left(t_{1 / 2}=2.55 \mathrm{~min}\right)$ that is actually counted, sufficient time must be allowed for secular equilibrium to be reached. This depends on the magnitude of the distribution ratios that are involved and the accuracy needed, but for present purposes, 20-30 min is adequate.

\subsection{PHASE COALESCENCE}

The dispersion number $\left(N_{\mathrm{Di}}\right)^{40}$ for the extraction step of the ESS protocol predicted good contactor performance over a range of temperatures (Fig. 2.3). Break time $\left(t_{\mathrm{B}}\right)$ in seconds was measured for the baseline solvent agitated with the full simulant at $25,32,35,37$, and $40{ }^{\circ} \mathrm{C}$. The temperatures of the solutions were independently measured with a thermometer before each contact. The phases were contacted in $100-\mathrm{mL}$ glass, graduated cylinders with ground-glass stoppers using the baseline $\mathrm{O} / \mathrm{A}$ ratio of 0.33 . For each measurement, $75 \mathrm{~mL}$ of full simulant was added to the cylinder, and the baseline solvent was added with sufficient care not to disperse the phases. The distance from the bottom of the cylinder to the interface and also to the top of the liquid level ( $\Delta Z$, in meters) was measured using a ruler. After the solutions were put into contact, without mixing, the cylinder was

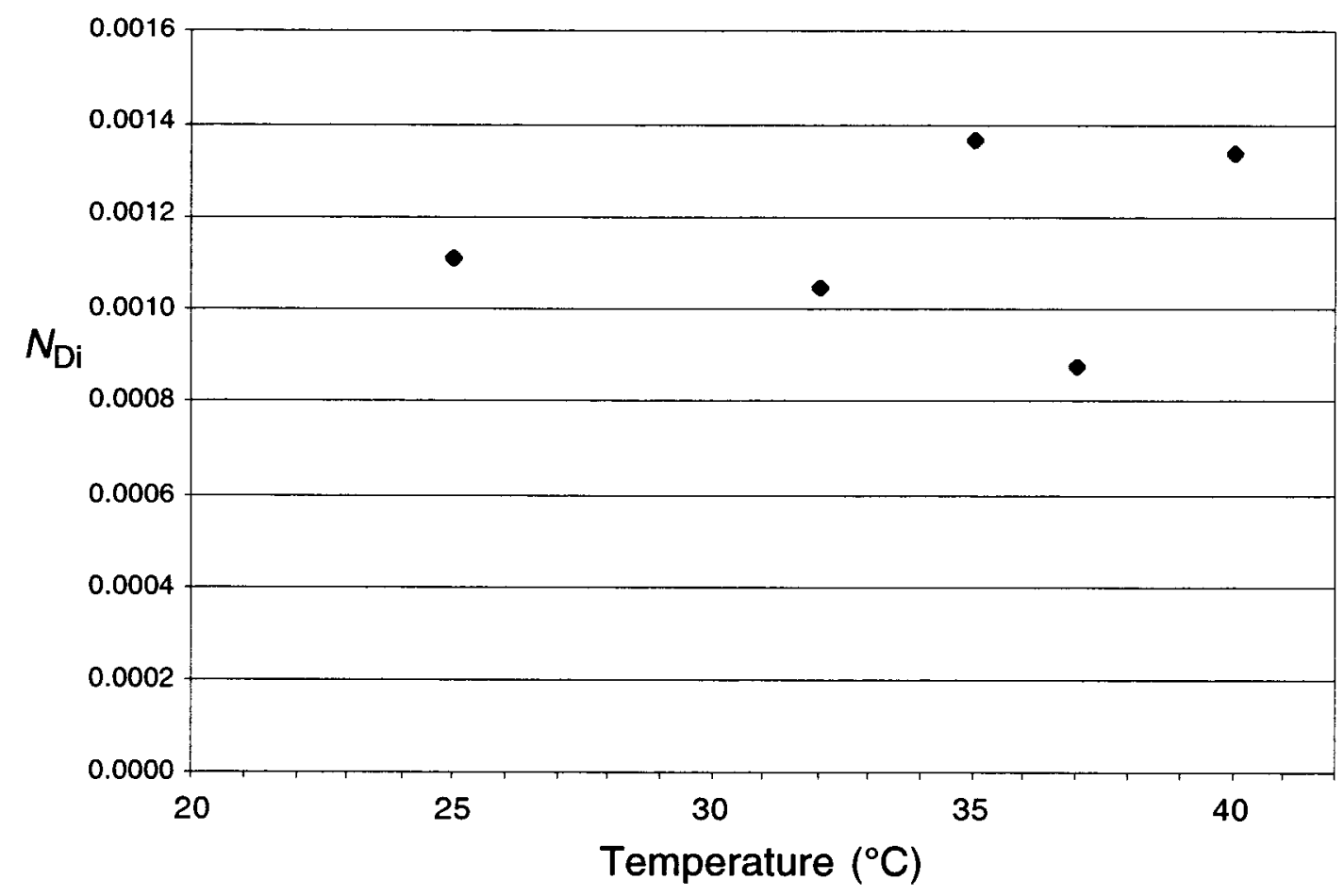

Fig. 2.3. Dispersion number vs. temperature on extraction phase of ESS. 
submerged in the temperature-controlled water bath or incubator box for another 30 min to ensure thermal equilibrium. The measurements were then repeated. The contacting was done by inverting the cylinder twice in a gentle, rolling motion, then by shaking with ten quick shakes. The break-time in seconds was recorded when all small drops had broken. The position of the interface was remeasured. After the phases were completely disengaged, the same solutions were placed back in the temperature device for $30-40 \mathrm{~min}$ and the process repeated. Each contact was repeated five times.

The average dispersion number $N_{\mathrm{Di}}$ was found to be $(1.15 \pm 0.21) \times 10^{-3}$ using the equation 40

$$
N_{D i}=\frac{1}{t_{\mathrm{B}}} \sqrt{\frac{\Delta Z}{\mathrm{~g}}}
$$

where $t_{\mathrm{B}}$ is measured in seconds, $\Delta Z$ refers to the initial thickness of the dispersion band in meters, and $\mathrm{g}$ is the gravitational acceleration $\left(9.81 \mathrm{~m} / \mathrm{s}^{2}\right)$. No trend with temperature can be seen in Fig. 2.3. 


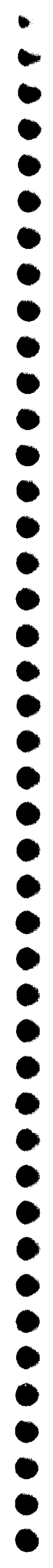




\section{CESIUM DISTRIBUTION BEHAVIOR}

\subsection{INTRODUCTION}

\subsubsection{Purpose}

In addition to the kinetic and hydraulic factors underlying contactor performance, cesium distribution behavior represents the primary predictor of whether the flowsheet will meet cesium decontamination and concentration goals. The purpose of the work presented in this chapter is to characterize how cesium distributes under conditions of extraction, scrubbing, and stripping as influenced by key chemical and operational variables. The dependence of $D_{\mathrm{Cs}}$ on temperature, aqueous-phase composition, and contacting parameters (e.g., O/A ratios, number of cycles, etc.) will be described, focusing on experiments in which waste simulants, as opposed to simple matrices, are used as the aqueous phase in extraction. The data provide a basis for prediction of flowsheet performance, for interpreting other test results, as well as for setting bounds within which successful operation can be guaranteed.

It should be noted that prediction of performance through modeling has been reported separately. ${ }^{25}$ In the modeling study, refinement of equilibrium parameters was carried out by regression of systematic distribution data taken first from simple systems and subsequently from more realistic waste-like matrices. Ultimately, the model provides a quantitative framework for understanding the distribution of all key system components in each stage of the process. Plans call for application of the model to optimization of the CSSX flowsheet. ${ }^{4}$ In complementary fashion, efforts described in the present chapter aim to identify key variables important to incorporate in the model.

\subsubsection{Process Goals}

To meet the process goals, $D_{\mathrm{Cs}}$ values are constrained by certain design criteria described elsewhere. ${ }^{20}$ The baseline flowsheet requires that the value of $D_{\mathrm{Cs}}$ on extraction be at least 8 . To avoid pinching at an O/A ratio of 5 , the $D_{\mathrm{Cs}}$ values on scrubbing and stripping must be no higher than 0.2 . In practice, it is considered desirable that $D_{\mathrm{Cs}}$ values fall to a steady value less than 0.10 in stripping. It should be noted that these target $D_{\mathrm{Cs}}$ values arise because of the particular flow rates chosen in the baseline flowsheet. Given that a concentration factor of 15 fixes the ratio of waste feed flow rate to strip effluent flow rate, the solvent flow rate remains as the flowsheet variable to be optimized for maximum robustness. ${ }^{15}$ Since robustness has a large margin, process designers have considerable latitude in varying solvent flow rate. For example, to lessen the possibility of pinching in the strip section, a slower solvent flow rate might be desirable, especially for low operating temperatures. In that case, the required minimum $D_{\mathrm{Cs}}$ on extraction would increase and the required maximum $D_{\mathrm{Cs}}$ for stripping would increase.

Sustained recyclability of the solvent is also required, as it will be cycled an estimated 2800 times in $80 \%$ of a full year's operation. ${ }^{41,42}$ Thus, the cesium distribution ratio must remain constant in extraction, scrubbing, and stripping as the solvent is reused, picking up minor impurities from the waste (Chap. 4) and from degradation process (Chaps. 5 and 6). As discussed in Chap. 7, the solvent must also retain its integrity with respect to absence of third-phase formation and precipitated solid phases.

\subsubsection{Background}

Cesium extraction, scrubbing, and stripping behavior in the first-generation solvent and flowsheet was described previously. 8,14 From that work, significant effects on flowsheet performance could be expected due to temperature, loading, and key interfering species such as potassium and lipophilic 
anions. Limited information regarding these effects on the performance of the second-generation system have been reported. 15 Owing to the more complex nature of the full simulant described in Chap. 2, new questions have arisen regarding flowsheet performance and its response to these and other variables.

It should be noted that kinetic and hydraulic aspects of CSSX solvent performance are being addressed in other tasks within the overall SRS Salt Processing Project. ${ }^{19}$ Both of these aspects of CSSX performance were found to be satisfactory in 1998 tests with the first-generation solvent. Kinetic tests using simple vortexing techniques showed that extraction and stripping were rapid. ${ }^{8}$ In addition, phase disengagement was good to excellent. ${ }^{10,11}$ However, stage efficiencies were found to be lower than desired, not because of kinetic issues, but because of inter-stage slug flow of the aqueous streams in the $2-\mathrm{cm}$ contactors. This peculiarity of $2-\mathrm{cm}$ contactor performance, ${ }^{43}$ not an issue for larger contactors, was successfully addressed in FY 2000 by certain modifications of the ANL 2-cm contactors, and good stage efficiencies of $(89.5 \pm 2.0) \%$ were obtained. ${ }^{44}$ Stage efficiencies in $5.5-\mathrm{cm}$ contactors are not yet available ${ }^{45}$ but on the basis of scale-up experience ${ }^{46}$ are expected to be significantly higher than that obtainable with $2-\mathrm{cm}$ contactors.

\subsubsection{Experimental Design}

Batch-contacting protocols described in Chap. 2 were considered to be adequate for most of the experiments to be conducted here. For greatest fidelity to flowsheet conditions, the baseline solvent was employed, except where noted, and the aqueous phase was usually the full simulant used directly or modified in a systematic manner.

\subsubsection{Work Scope}

Tasks of the Work Scope Matrix (WSM) ${ }^{19}$ examined in this chapter include those dealing with cesium distribution and the effects of temperature and system composition. Experiments include the standard batch extract/scrub/strip contacting protocol and systematic batch tests as a function of selected compositional variables. Cesium distribution is covered under WSM task 5.1.3, including the effect of the concentration of the major cations, especially potassium. WSM task 5.2 specifies efforts to understand the effect of major and minor components that are expected to be in the waste. In that certain organic compounds directly influence extraction and stripping and thereby introduce a degree of technical risk, WSM task 5.2.3 aimed to characterize the effect of lipophilic anions such as dibutylphosphate, surfactants, and 4-sec-butylphenoxide. Other organic compounds expected to be in the waste and included in the full simulant were also examined with regard to their perturbation on ESS performance. Selected minor inorganic components were also examined (WSM 5.2.4). Finally, the effects of temperature (WSM 5.4.1.1) and solvent-component concentrations (WSM 5.4.1.2) have been studied. As mentioned above, modeling activities under WSM 5.3 will be reported separately. ${ }^{25}$

\subsection{EXPERIMENTAL SECTION}

\subsubsection{General}

Unless otherwise noted, all materials, methods, and ESS protocol have been described in Chap. 2 .

\subsubsection{Forward Scrubbing and Stripping}

Regarding the forward scrubbing and stripping tests (Section 3.3.6), the procedure was the same as an ESS test, except that the pristine solvent was contacted respectively with the scrub solution or the strip solution containing a spike of ${ }^{133} \mathrm{Cs}$, and the initial contact with simulant was skipped. The aqueous cesium concentration was adjusted to what it would be in the solvent respectively after extraction or after scrub as if full simulant containing ${ }^{133} \mathrm{Cs}$ at a concentration of $1.4 \times 10^{-4} \mathrm{M}$ were used. To approximate this concentration, $D_{\text {ext }}=16$ and $D_{\text {scrub }}=1.6$ were assumed. The total Cs 
concentration present in the scrub stage if all the cesium were in the organic phase is calculated from $\left[\mathrm{Cs}^{+}\right]_{\text {org,ext,eq }}=\left[D_{\text {ext }} /\left(1+\phi D_{\text {ext }}\right)\right]\left[\mathrm{Cs}^{+}\right]_{\text {init }}=[16 /(1+16 / 3)] 1.4 \times 10^{-4} \mathrm{M}=3.54 \times 10^{-4} \mathrm{M}$. Since the spike was added to the aqueous phase, a concentration 5 times higher was added, namely $17.7 \times 10^{-4} \mathrm{M}$. Similarly, in a regular ESS, the concentration of Cs in the organic phase for the scrub stage at equilibrium is $3.15 \times 10^{-4} \mathrm{M}$. This is the total concentration present in the strip stage if all the cesium were in the organic phase. Since the spike was added to the aqueous phase, a concentration 5 times higher was added, namely $15.7 \times 10^{-4} \mathrm{M}$. In addition to the spike of ${ }^{133} \mathrm{Cs}$, a spike of ${ }^{137} \mathrm{Cs}$ was added to the aqueous phase prior to contact. The solution was then about 0.2 $\mu \mathrm{Ci} / \mathrm{mL}$.

\subsubsection{Temperature Variation}

The temperature dependence of $D_{\mathrm{Cs}}$ was determined in batch tests performed in the usual manner except that the phases were contacted by vortexing. In ESS tests at $32{ }^{\circ} \mathrm{C}$ tests, baseline solvent (Lot PVB000718-156W), full simulant (4th draw), $50 \mathrm{mM}$ scrub solution, and $1 \mathrm{mM}$ strip solution were employed. The aqueous and organic phases were thermally equilibrated at the prescribed temperatures prior to use, and volumes were delivered at the prescribed temperatures. A series of contacts between the solvent and simulant were performed at varying temperature with the aid of a water bath. The vortexing was performed as follows: $30 \mathrm{~s}$ on a GenieII Vortex mixer; $1 \mathrm{~min}$ in the thermostated bath. This sequence was repeated three times. The centrifuge was maintained at the temperature of extraction. Samples were centrifuged for $3 \mathrm{~min}$ at $3600 \mathrm{rpm}$. During subsampling for gamma counting, the tubes that were not handled were allowed to remain in the water bath. An ESS test was also performed using the same contacting procedure. A $5-\mu \mathrm{L}$ spike of ${ }^{137} \mathrm{Cs}$ was added to the third stripping solution.

Cesium distribution experiments at variable temperature were performed similarly. Baseline solvent (Lot PVB B000718-156W) and full simulant (draw \#4) solutions were thermally equilibrated at the desired temperature in the water bath for at least $30 \mathrm{~min}$, pipetted into the sample vial at $\mathrm{O} / \mathrm{A}=$ 0.33 , and kept in the water bath for an additional $10 \mathrm{~min}$ without agitation. These thermally equilibrated samples were then vortexed three times for $10 \mathrm{sec}$. Between vortexing steps, samples were placed in the water bath for $30 \mathrm{sec}$. Subsequently, samples were centrifuged at the contact temperature and subsampled for counting. Duplicate determinations of $D_{\mathrm{Cs}}$ were performed at each temperature. Density approximations were performed on subsamples of thermally equilibrated solvent and simulant solutions at the various temperatures by weighing pipetted volumes, with standard deviations determined from 5 to 11 replicate measurements.

\subsubsection{Effect of Potassium}

To determine $D_{C s}$, in the Cs-K loading experiment (Section 3.3.9), each phase was subsampled

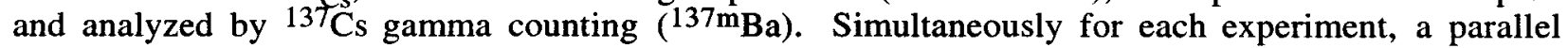
cold experiment was conducted to study $\mathrm{K}^{+}$distribution behavior under the same conditions. To determine potassium distribution ratio $D_{\mathrm{K}}$, subsamples of the solvent phase were mixed with 1,2-diisopropylbenzene in 1:1 ratio, stripped with deionized water, and analyzed for $\mathrm{K}^{+}$by ICP-AES. The value of $D_{\mathrm{K}}$ was calculated assuming that mass balance of $100 \%$ is sustained for each sample.

\subsubsection{Effect of 4-sec-Butylphenol}

In the investigation of the effect of 4-sec-butylphenol (SBP; see Section 3.3.10), the following chemicals, materials, and methods were used. Washed pristine baseline solvent (Lot PVB B000718$110 \mathrm{~W}$ ) was used in the forward stripping test, while Lot PVB B000718-124W was used for the full ESS procedure. 4-sec-Butylphenol was obtained from TCI America (Lot. FBQ01, 98\% by GC). Calibrated Eppendorf Reference Pipetteman pipetters were used for all liquid transfers less than $5 \mathrm{~mL}$. All solutions and dilutions were carried out in non-glass volumetric and graduated lab ware.

A forward-stripping contacting series was carried out as a quick survey to learn if 4-secbutylphenol (SBP) affects the distribution of cesium. A series of six reactions, plus a control, were 
carried out in duplicate. SBP was added to solvent at concentrations of $0.1,1.0,5.0,10.0,20.0$, and $50.0 \mathrm{mM}$. The control contained no SBP. The series was made by adding SBP to the baseline solvent at a concentration of $50 \mathrm{mM}$, then diluting with solvent in series down to $0.1 \mathrm{mM}$. The aqueous phase contained $1 \mathrm{mM} \mathrm{HNO}, 0.1 \mathrm{mM} \mathrm{CsNO}_{3}$, and $0.3 \mu \mathrm{Ci} / \mathrm{mL}^{137} \mathrm{Cs}$. The equilibrations were carried out in 5-mL sterile polypropylene tubes (Nalgene Cryoware Cryogenic vials) at an O/A ratio of 5. A $2.5-\mathrm{mL}$ volume of the solvent and $0.5 \mathrm{~mL}$ of the aqueous phase were contacted in duplicate by end-over-end rotation in a temperature-controlled air box at $25{ }^{\circ} \mathrm{C}$ for $30 \mathrm{~min}$. After contacting, the samples were centrifuged at 3,600 RPM for $3 \mathrm{~min}$ in a temperature-controlled Sanyo MSE Mistral 2000R centrifuge set at $25^{\circ} \mathrm{C}$. Following separation, both phases were subsampled ( 350 $\mu \mathrm{L}$ each) for gamma counting.

For the ESS test, full simulant containing $0.14 \mathrm{mM} \mathrm{CsNO}_{3}$ was spiked with ${ }^{137} \mathrm{Cs}$ to an activity of $0.2 \mu \mathrm{Ci} / \mathrm{mL}$ (by adding $8.5 \mu \mathrm{L}$ of $1.77 \mathrm{mCi} / \mathrm{mL}^{137} \mathrm{Cs}$ stock to each reaction bottle) and SBP added to a concentration of $0,0.3,3.0$, and $30.0 \mathrm{mM}$ (relative to the volume of solvent). All cold components for the extraction step were introduced into the extraction bottles prior to the addition of ${ }^{137} \mathrm{Cs}$ spike. The combined cold components were temperature-equilibrated in a $25{ }^{\circ} \mathrm{C}$ air box overnight. The phenol was seen to dissolve into the solvent phase upon phase contacting. Duplicates were run for each condition in $250-\mathrm{mL}$ heavy-walled Teflon bottles for the extraction step and 50-mL Teflon bottles for the scrub and strip steps. O/A ratios for each step were 0.33 (extraction) and 5 (scrubs and strips). Glass-Col rotators maintained in a $25{ }^{\circ} \mathrm{C}$ temperature-controlled air box were used for contacting the phases. Volumes employed were as follows: extraction - $25 \mathrm{~mL}$ solvent, $75 \mathrm{~mL}$ simulant; scrub - $24 \mathrm{~mL}$ solvent, $4.8 \mathrm{~mL} 50 \mathrm{mM} \mathrm{HNO}_{3}$; strip \#1 - $22.5 \mathrm{~mL}$ solvent, $4.5 \mathrm{~mL} 1 \mathrm{mM}$ $\mathrm{HNO}_{3}$; strip \#2 - $21 \mathrm{~mL}$ solvent, $4.2 \mathrm{~mL} 1 \mathrm{mM} \mathrm{HNO}$; and strip \#3 - $20 \mathrm{~mL}$ solvent, $4.0 \mathrm{~mL} 1 \mathrm{mM}$ $\mathrm{HNO}_{3}$. Following each 50-min contact, the phases were separated using a Beckman Coulter Allegra $6 \mathrm{KR}$ Centrifuge operated at $25 \mathrm{C}, 3040 \mathrm{rpm}(2111 \times \mathrm{g})$ for $4 \mathrm{~min}$. A $350-\mu \mathrm{L}$ aliquot was then removed from each of the respective organic and aqueous phases for gamma counting in a Packard Cobra Quantum gamma counter, Model 5003. All counting was done using a 580-750 keV window and a 10-min counting period. Background counts using simulant, solvent, and water were typically in the range of $24-25 \mathrm{cpm}$.

\subsubsection{Effects of Selected Organic Compounds}

A survey of possible effects on ESS performance by 5 of the 6 organic compounds found in full simulant was undertaken. The survey mimics the circumstance where organic constituents might build up in the solvent after repeated CSSX Process contacts with a waste stream or, as in this case, with simulant. Individual ESS tests were carried out for each of the organic compounds tested. This survey serves as a means to quickly check for any noticeable effects these selected organic compounds might have on the distribution of ${ }^{137} \mathrm{Cs}$ in the individual ESS steps.

Trimethylamine (TMA, Chem. Tech. Division Lot $13423 \mathrm{CU}$ ), tributylphosphate (TBP, Aldrich Chem. Co.), dibutylphosphate (DBP, Fluka Lot 360440/1), 1-butanol (Chem Tech Div Lot 6263306), and formate (sodium formate, Aldrich Chem. Co. Lot \# TQ-04022JQ) were investigated individually using salts and metals simulant (ORNL Chemical Technology Division, Lot B000747-p.35) containing $0.14 \mathrm{mM}$ cesium and a $0.2 \mu \mathrm{Ci} / \mathrm{mL}{ }^{137} \mathrm{Cs}$ spike, and washed solvent Cs-7SB/Isopar $\mathrm{L}$, ORNL Lot PVB B000718-156W. Monobutylphosphate was not surveyed, owing to its unavailability and its likely lack of effect (expected to partition to the solvent on the order of 100-fold weaker than dibutylphosphate). The concentrations of the individual organic compounds, relative to solvent, were $30 \mathrm{mM}$ TBP, $2 \mathrm{mM}$ DBP, $10 \mathrm{mM}$ TMA, $100 \mathrm{mM}$ formate, and $10 \mathrm{mM}$ 1-butanol. A salts and metals simulant blank served as a control. A typical ESS protocol was followed with O/A ratios of 0.33 for extraction and 5 for the scrub and three strip steps. Contacts were performed singly in $50-\mathrm{mL}$ Teflon tubes for extractions and $15-\mathrm{mL}$ polypropylene tubes for scrubs and strips. Contacts were made for $45 \mathrm{~min}$ in a temperature-controlled air box at $25{ }^{\circ} \mathrm{C}$ using end-over-end rotation. Phase separation was achieved by centrifugation at $2820 \mathrm{RPM}(1820 \times \mathrm{g})$ for $4 \mathrm{~min}$ in a Beckman Coulter Allegra $6 \mathrm{KR}$ centrifuge operated at $25^{\circ} \mathrm{C}$. A $300-\mu \mathrm{L}$ aliquot was removed from each post-contact organic and aqueous phase for gamma counting (10 min each, 580-750 keV window) in a Packard Cobra Quantum gamma counter, Model 5003. 


\subsubsection{Trials of Different Simulant Recipes}

An ESS protocol was performed on different simulants with and without ${ }^{137} \mathrm{Cs}$ tracer. Cesium was analyzed by gamma-counting techniques, while sodium, and potassium were analyzed in the aqueous phase of each scrub and strip contact by ICP-AES. This procedure used solvent Lot B000718-156W. Analysis by ICP-AES for sodium and potassium employed external calibration of the metals using ICP standard solutions (J.T. Baker) diluted to $\pm 10 \%$ of expected metals in the scrub and strip solutions. Typically, standards were used at 1 and $100 \mathrm{ppm}$.

\subsubsection{Effect of Multiple Solvent Contacts with Simulant}

An ESS protocol with six strips was performed after the solvent had been in contact four times with fresh full simulant, $\mathrm{O} / \mathrm{A}=0.33$ and $T=25^{\circ} \mathrm{C}$ (i.e., an $\mathrm{E}_{5} \mathrm{SS}_{6}$ sequence). The simulants that were used included (see Table 2.2): SRS\#2, salts only, salts + metals, and full simulant.

This experiment was conducted first with the full simulant only, then repeated with this simulant and compared with the three others. It was also run using only natural cesium $\left({ }^{133} \mathrm{Cs}\right)$ and the full simulant to perform a few electrospray analyses. In all cases, an aliquot of $7 \mathrm{~mL}$ of solvent (Lot PVB B000718-156W) was contacted with $21 \mathrm{~mL}$ of simulant four times. After each 30-min contact, wherein the sample was gently rocked end-over-end at $25^{\circ} \mathrm{C}$ and centrifuged at $3600 \mathrm{rpm}$ for $3 \mathrm{~min}$, the aqueous phase was removed and replaced by an equal volume of fresh simulant.

For the samples prepared for the electrospray experiment, the solvent was contacted one more time with the full simulant. An aliquot of $1 \mathrm{~mL}$ of solvent was removed. The remaining $6 \mathrm{~mL}$ were contacted with $1.2 \mathrm{~mL}$ of scrub solution. Again, $1 \mathrm{~mL}$ was removed and the remaining $5 \mathrm{~mL}$ were contacted with $1 \mathrm{~mL}$ of strip solution, then similarly subsampled for analysis. All the contacts were performed as described above.

For the experiments using cesium radiotracer to monitor the distribution ratios, the fifth contact with the simulant contained a spike of ${ }^{137} \mathrm{Cs}$. Also, the $1-\mathrm{mM}$ nitric acid strip solution was spiked to have an activity of about $0.08 \mu \mathrm{Ci}$. All the contacts were performed as described above with the volumes mentioned in the Table 3.1 below:

Table 3.1. Experimental volumes in $\mathrm{mL}$ pertaining to the multi-contact experiment

\begin{tabular}{lcc}
\hline Stage & $\begin{array}{c}\text { Organic phase } \\
(\mathrm{mL})\end{array}$ & $\begin{array}{c}\text { Aqueous phase } \\
(\mathrm{mL})\end{array}$ \\
\hline Extraction & 6.5 & 19.5 \\
Scrub & 5.0 & 1.0 \\
Strip \#1 & 4.5 & 0.9 \\
Strip \#2 & 4.0 & 0.8 \\
Strip \#3 & 3.75 & 0.75 \\
Strip \#4 & 3.4 & 0.68 \\
Strip \#5 & 3.0 & 0.6 \\
Strip \#6 & 2.6 & 0.52 \\
\hline
\end{tabular}

aThese two extra strips were not performed in the full simulant experiment. At each step, a $0.350-\mathrm{mL}$ subsample was counted from individual phases to determine cesium distribution ratios. 


\subsubsection{Multi-Cycle Behavior}

In the batch multi-cycle experiment, each cycle consisted of one extraction step, one scrub step and four strip steps. All contacts were performed following the general procedure described in Chap. 2 , and $\mathrm{Cs}$ distribution was monitored by ${ }^{137} \mathrm{Cs}$ tracer. In addition, the experiment was designed to allow for chemical analysis of the organic and aqueous phases at selected stages. A minimum of 10 $\mathrm{mL}$ of aqueous phase was necessary for those analyses, necessitating a minimum of $50 \mathrm{~mL}$ of organic phase to remain at the end of each cycle and a total initial volume of $90 \mathrm{~mL}$. This volume was split into 3 vials, which allowed triplicate determination of all distribution ratios. Table 3.2 gives the volumes (in $\mathrm{mL}$ ) used in each of the five cycles and at each step.

Table 3.2. Experimental volumes in $\mathrm{mL}$ pertaining to the multi-cycle experiment

\begin{tabular}{lllllllllll}
\hline \multirow{2}{*}{ Stage } & \multirow{2}{*}{ Type of container } & \multicolumn{2}{c}{ Cycle \#1 } & \multicolumn{3}{c}{ Cycle \#2 } & \multicolumn{3}{c}{ Cycle \#3 } & \multicolumn{3}{c}{ Cycle \#5 } \\
& & Org & Aq & Org & Aq & Org & Aq & Org & Aq \\
\hline Extraction & 250-mL PPE bottle & 30 & 90 & 27 & 81 & 24 & 72 & 21 & 63 \\
Scrub & 50-mL Teflon tube & 29 & 5.8 & 26 & 5.2 & 23 & 4.6 & 20 & 4 \\
Strip \#1 & 50-mL Teflon tube & 28.5 & 5.7 & 25.5 & 5.1 & 22.5 & 4.5 & 19.5 & 3.9 \\
Strip \#2 & 50-mL Teflon tube & 28 & 5.6 & 25 & 5 & 22 & 4.4 & 19 & 3.8 \\
Strip \#3 & 50-mL Teflon tube & 27.5 & 5.5 & 24.5 & 4.9 & 21.5 & 4.3 & 18.5 & 3.7 \\
Strip \#4 & 50-mL Teflon tube & 27 & 5.4 & 24 & 4.8 & 21 & 4.2 & 18 & 3.6 \\
\hline
\end{tabular}

\subsection{RESULTS AND DISCUSSION}

\subsubsection{Extract/Scrub/Strip Performance}

In the course of many experiments reported in later sections of this report and elsewhere, many control ESS experiments were performed on the baseline solvent with the full simulant at $25{ }^{\circ} \mathrm{C}$, enabling calculation of reliable averages and standard deviations for the cesium distribution ratio $\left(D_{\mathrm{Cs}}\right)$ in the ESS sequence. Results obtained by five individual ORNL researchers using varied methodology are listed in Table 3.3.

Most experiments were carried out as described in Section 2.5, but there were significant differences in techniques. Set \#2 used a simulant prepared at the SRTC. Set \#25 used overhead stirring at ambient temperature with correction for the temperature difference; analysis was by ICPMS. Sets \#32 and \#33 employed vortexing as the contacting method. Otherwise, protocols were similar except for minor differences, such as in vial size and subsampling technique.

The obvious consistent performance of the ESS tests indicates that the variations in techniques were inconsequential. In addition, the consistency of results also indicates no evolution of performance with time. In Table 3.3, entries are listed in the order in which the measurements were carried out over a 5-month period. Since the same simulant was used in each case, it may be concluded that the CSSX distribution performance is not affected by the aging of the simulant.

Table 3.4 summarizes simple statistics corresponding to the ESS data listed in Table 3.3. As shown, overall standard deviations (Stdev) lay in the range 5-9\%. The table also gives the minimum (Min) and maximum (Max) limits of the 95\% confidence interval together with the number of individual trials. Given that no attempt was made to exactly standardize the ESS protocol, the values in Table 3.4 exhibit remarkable precision. As counting precision is generally reduced to less than $\pm 1 \%$ by taking sufficient counts $(>10,000)$, the observed precision is thought to result from propagated volumetric errors coupled with the strong temperature sensitivity of $D_{\mathrm{Cs}}$. The value of 
Table 3.3. Cesium distribution ratios from extract/scrub/strip experiments (ESS) at $25^{\circ} \mathrm{C}$ with baseline solvent and full simulant

\begin{tabular}{|c|c|c|c|c|c|c|c|c|}
\hline Set \# & $\begin{array}{c}\text { Extraction } \\
D_{\mathrm{Cs}} \\
\end{array}$ & $\begin{array}{c}\text { Scrub } \\
D_{\mathrm{Cs}} \\
\end{array}$ & $\begin{array}{c}\text { Strip \#1 } \\
D_{\mathrm{Cs}} \\
\end{array}$ & $\begin{array}{c}\text { Strip \#2 } \\
D_{\mathrm{Cs}} \\
\end{array}$ & $\begin{array}{c}\text { Strip \#3 } \\
D_{\mathrm{Cs}} \\
\end{array}$ & $\begin{array}{c}\text { Strip \#4 } \\
D_{\mathrm{Cs}} \\
\end{array}$ & $\begin{array}{c}\text { Strip \#5 } \\
D_{\mathrm{Cs}}\end{array}$ & Source ${ }^{a}$ \\
\hline $1^{c}$ & $13.2^{b}$ & 1.61 & 0.149 & 0.085 & 0.065 & & & \\
\hline $2^{c, d}$ & 15.8 & 1.48 & $0.119^{b}$ & 0.077 & 0.054 & & & \\
\hline 3 & 14.6 & & & & & & & Ref. 44 \\
\hline $4^{e}$ & 17.3 & 1.60 & & 0.100 & 0.066 & & & \\
\hline $5^{e}$ & & 1.81 & 0.147 & & 0.067 & & & \\
\hline $6^{e}$ & & 1.72 & 0.145 & & 0.068 & & & \\
\hline $7 f$ & & & 0.162 & 0.084 & & & & \\
\hline $8^{f}$ & & & 0.169 & 0.089 & & & & \\
\hline 9 & & & 0.164 & 0.089 & 0.064 & & & \\
\hline 10 & 16.2 & 1.60 & 0.146 & 0.086 & 0.060 & & & \\
\hline 11 & & 1.55 & 0.145 & 0.093 & 0.060 & & & \\
\hline 12 & 15.3 & 1.55 & 0.150 & 0.086 & 0.063 & & & \\
\hline 13 & 16.9 & 1.63 & 0.151 & 0.088 & 0.067 & & & \\
\hline 14 & 16.0 & & & & & & & \\
\hline 15 & 16.0 & & & & & & & \\
\hline 16 & 18.2 & & & & & & & \\
\hline 17 & 17.6 & & & & & & & \\
\hline 18 & 17.2 & & & & & & & \\
\hline 19 & 16.8 & & & & & & & \\
\hline 20 & 18.7 & 1.63 & 0.155 & 0.094 & 0.071 & 0.061 & & \\
\hline 21 & 18.4 & 1.64 & 0.153 & 0.094 & 0.072 & 0.060 & & \\
\hline 22 & 18.8 & 1.63 & 0.154 & 0.091 & 0.070 & 0.061 & & \\
\hline 23 & 17.5 & 1.55 & 0.144 & 0.089 & 0.068 & 0.055 & & \\
\hline 24 & 17.9 & 1.57 & 0.147 & 0.089 & 0.069 & 0.056 & & \\
\hline $25 f$ & 18.0 & 1.57 & 0.143 & & & & & Ref. 47 \\
\hline 26 & 16.6 & 1.49 & 0.145 & 0.084 & 0.062 & 0.053 & 0.049 & \\
\hline 27 & 18.5 & & & & & & & \\
\hline 28 & 16.8 & 1.50 & 0.141 & 0.084 & 0.063 & 0.055 & & \\
\hline 29 & 17.5 & 1.59 & 0.152 & 0.094 & 0.077 & 0.062 & & \\
\hline 30 & 18.2 & 1.56 & 0.156 & 0.097 & 0.080 & 0.062 & & \\
\hline $31 g$ & 16.7 & 1.47 & 0.130 & 0.078 & & 0.057 & & \\
\hline $32^{h}$ & 15.1 & & & & & & & \\
\hline $33^{h}$ & 16.7 & & & & & & & \\
\hline
\end{tabular}

$a_{\text {Data }}$ are from this Chap., unless otherwise indicated.

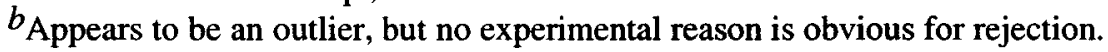

$c$ Very small sample volumes used. Organic solvent recovered after counting.

$d_{\text {Simulant was from the SRTC. }}$

${ }^{e}$ Stripping value rejected (left blank). System had not reached equilibrium, because of insufficient head space in sample vials. Subsequent strip values did not appear to be affected.

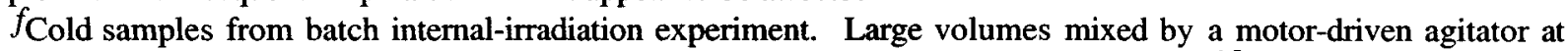
23.6-25.3 ${ }^{\circ} \mathrm{C}$. Values are corrected using previously reported temperature coefficients. ${ }^{15}$ Analysis of Cs was performed by ICP-MS.

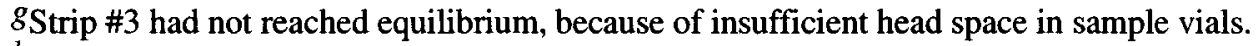

$h_{\text {Contacting by vortex. }}$ 
Table 3.4. Statistical summary of ESS results

\begin{tabular}{lccccccc}
\multicolumn{1}{c}{} & Extraction & Scrub & Strip \#1 & Strip \#2 & Strip \#3 & Strip \#4 & Strip \#5 \\
\cline { 2 - 8 } Avg. $D_{\mathrm{Cs}}$ & 16.9 & 1.59 & 0.148 & 0.089 & 0.067 & 0.058 & 0.049 \\
Stdev & $8 \%$ & $5 \%$ & $7 \%$ & $7 \%$ & $9 \%$ & $6 \%$ & $7 \%$ \\
Min & 14.2 & 1.42 & 0.127 & 0.077 & 0.054 & 0.052 & 0.043 \\
Max & 19.6 & 1.75 & 0.170 & 0.100 & 0.079 & 0.065 & 0.055 \\
No. trials & 27 & 20 & 22 & 20 & 19 & 10 & 1
\end{tabular}

$D_{\mathrm{Cs}}$ varies 6-10\% per degree. ${ }^{14}$ Temperature control in the constant-temperature air box is $\pm 0.5{ }^{\circ} \mathrm{C}$, and some re-equilibration of samples at ambient lab temperature (typically $23 \pm 2{ }^{\circ} \mathrm{C}$ ) may be expected during handling and subsampling.

From the data in Table 3.4, it is clear how the process behaves, from strong extraction of Cs, to retention of the $\mathrm{Cs}$ in the solvent during scrubbing, to efficient stripping. Data presented in later sections of this report show that certain competing inorganic (esp., $\mathrm{Na}^{+}$and $\mathrm{K}^{+}$) and organic ions (e.g., dibutylphosphate) are partially extracted from the simulant. The inorganic ions are scrubbed out in the scrub step, while DBP remains in the solvent to build up somewhat upon solvent cycling. As discussed further below, the DBP acts synergistically with the extractant BOBCalixC6 in the usual fashion 48,49 to increase $D_{C s}$ above what it would otherwise be in the absence of the DBP. The plateau value of $D_{\mathrm{Cs}}$ on successive stripping depends on the DBP content of the solvent.

It should be understood that the ESS protocol is at best a crude predictor of counter-current behavior in the baseline flowsheet. First, the mild "reflux" of cesium between the first extraction stage (stage 15) and first scrub stage (stage 16) in the baseline flowsheet raises the cesium concentration in the solvent somewhat above what is extracted in a single extraction step. Second, it takes two scrub steps to remove the bulk of the potassium and sodium from the solvent. In the ESS protocol, the single scrub allows a fraction of these metals to be carried into the first strip step, causing the aqueous nitrate concentration to be correspondingly higher. Hence, $D_{\mathrm{Cs}}$ will be higher in the first strip step than it would otherwise be.

The $D_{\mathrm{Cs}}$ values may be observed to decline gradually on successive strip steps. In addition to the effect of potassium and sodium just mentioned, two other effects contribute to this decline. The first is simply the gradual stripping out of the cesium, which in the same way contributes extra nitrate to the aqueous phase. Secondly, extracted acid is partially released at the lower acid concentration. In the scrub step, acid is expected to be extracted by TOA, as well as weakly by the solvent matrix, and this acid will re-equilibrate when the aqueous $\mathrm{pH}$ drops by 1.6 units when the scrubbed solvent is first stripped. Although these chemical effects have not yet been modeled, together they are thought to account for the observed decline in stripping $D_{\mathrm{Cs}}$ values. Noting that the stripping solution was set at an ambitiously low value of $0.001 \mathrm{M} \mathrm{HNO}_{3}$, the observed behavior seems not at all surprising.

\subsubsection{Simple Cesium Loading Model}

To assist in the interpretation of data, a simple loading model was employed. Sophisticated modeling of the extraction of $\mathrm{CsNO}_{3}$ by BOBCalixC6 in 1,2-dichloroethane has been performed previously using the program SXLSQI, including multiple equilibria and activity coefficients for all solute species in both phases. ${ }^{27}$ The same type of modeling has been carried out on the CSSX process system and reported separately. ${ }^{25}$ For purposes of the present report, however, it is instructive to use a simple analog model that can be applied in spreadsheet calculations to account for the effect of cesium loading on extraction from the simulant. As such, this model is not intended to account for any of the other effects known to play a role in determining the value of $D_{\mathrm{Cs}}$. The model is essentially the same as presented elsewhere ${ }^{11,15}$ and assumes the formation of only a 1:1 complex between the BOBCalixC6 molecule and $\mathrm{Cs}^{+}$ion. The organic-phase equilibrium concentration of $\mathrm{Cs}$ is given as 


$$
[\mathrm{Cs}]_{\mathrm{org}}=C_{\mathrm{Cs}}[\mathrm{Cs}]_{\mathrm{aq}}[\mathrm{B}]_{\mathrm{org}}
$$

where $C_{\mathrm{Cs}}$ is a fitting constant that depends on key aqueous compositional properties such as nitrate and free hydroxide concentration and $\mathrm{B}$ is the extractant BOBCalixC6. Mass-balance expressions corresponding to $\mathrm{Cs}$ and $\mathrm{B}$ may be written:

$$
\begin{aligned}
& {[\mathrm{B}]_{\mathrm{org}}=[\mathrm{B}]_{\mathrm{org}, \text { init }}-[\mathrm{Cs}]_{\mathrm{org}}} \\
& {[\mathrm{Cs}]_{\mathrm{aq}}=[\mathrm{Cs}]_{\mathrm{aq}, \text { init }}-\phi[\mathrm{Cs}]_{\mathrm{org}}}
\end{aligned}
$$

where $\phi$ is the $\mathrm{O} / \mathrm{A}$ volume ratio. Solution of eqs. 3.1-3.3 together yields a quadratic expression in $[\mathrm{B}]_{\text {org }}$ :

$$
\phi C_{\mathrm{Cs}}[\mathrm{B}]_{\mathrm{org}}^{2}+\left(1+C_{\mathrm{Cs}}[\mathrm{Cs}]_{\mathrm{aq}, \text { init }}-\phi C_{\mathrm{Cs}}[\mathrm{B}]_{\mathrm{org}, \text { init }}\right)[\mathrm{B}]_{\mathrm{org}}-[\mathrm{B}]_{\mathrm{org}, \text { init }}=0
$$

Solution of eq. 3.4 for its root $[\mathrm{B}]_{\mathrm{org}}$ between 0 and 0.010 then enables calculation of $D_{\mathrm{Cs}}$ :

$$
D_{\mathrm{Cs}}=C_{\mathrm{Cs}}[\mathrm{B}]_{\mathrm{org}}
$$

To find the fitting constant $C_{\mathrm{Cs}}$ for a given temperature, solvent, and aqueous matrix, one may iteratively fit eqs. 3.4 and 3.5 to a set of data in which the aqueous Cs concentration and O/A phase ratio may vary.

Regarding the extraction mechanism, it has been presumed that the primary process occurring corresponds to the simple ion-pair extraction equilibrium

$$
\mathrm{Cs}^{+}{ }_{\mathrm{aq}}+\mathrm{NO}_{3 \mathrm{aq}}^{-}+\mathrm{B}_{\mathrm{org}} \rightleftharpoons \mathrm{CsBNO}_{3 \mathrm{org}}
$$

where B again represents BOBCalixC6. Under the acidic conditions of scrubbing and stripping, the observed unit log-log dependence of $D_{\mathrm{Cs}}$ on aqueous nitrate and calixarene concentrations in both the first- and second-generation solvent systems clearly support eq. 3.6.8,15 Detailed computer modeling in simple systems using 1,2-dichloroethane and alcohol-modified dodecane leave little doubt as to the predominance of this equilibrium under neutral and weakly acidic conditions. 25,50 However, the weakly acidic nature of the class of fluorinated alcohols from which the modifier Cs-7SB is derived has been implicated as promoting ion-exchange under strongly alkaline conditions. 51,52 A process in which a macrocycle and fluorinated alcohol act in concert to extract $\mathrm{Na}^{+}$ion by a synergistic ion-exchange reaction has been hypothesized. ${ }^{53}$ Hence, the equilibrium shown below may be proposed:

$$
\mathrm{Cs}^{+}{ }_{\mathrm{aq}}+\mathrm{OH}^{-}{ }_{\mathrm{aq}}+\mathrm{HA}_{\text {org }}+\mathrm{B}_{\text {org }} \rightleftharpoons \mathrm{CsBA}_{\text {org }}+\mathrm{H}_{2} \mathrm{O}_{\mathrm{aq}}
$$

where HA corresponds to the modifier Cs-7SB. Indeed, computer modeling of the baseline solvent shows that this equilibrium is consistent with the $\mathrm{Cs}^{+}$ion extraction behavior from $\mathrm{NaOH}$ solutions. ${ }^{25}$ This same modeling implies that eqs. 3.6 and 3.7 are both operative as the most significant processes occurring in extraction of $\mathrm{Cs}^{+}$from the full simulant by the baseline solvent. Lesser contributions are made by equilibria analogous to eq. 3.6 wherein other anions such as $\mathrm{NO}_{2}^{-}$and $\mathrm{Cl}^{-}$play the same role as $\mathrm{NO}_{3}{ }^{-}$. It may be readily seen that eqs. 3.6 and 3.7, either alone or as simultaneous reactions, lead to the relation given in eq. 3.1. That is, the loading behavior follows as a consequence of a $1: 1$ complexation of the $\mathrm{Cs}^{+}$ion by BOBCalixC6 and does not depend on the particular anion that balances the charge, provided that the aqueous anion concentrations remain essentially independent of loading. 


\subsubsection{Effect of Cesium Concentration (Loading Behavior)}

Cesium loading behavior was studied using full simulant solutions containing $\mathrm{Cs}^{+}$ion (added as $\mathrm{CsNO}_{3}$ ) at concentrations in the range from $1.4 \times 10^{-4} \mathrm{M}$ (baseline $\mathrm{Cs}^{+}$concentration) to $1 \times 10^{-2} \mathrm{M}$. The high upper limit was set so as to explore boundaries for third-phase formation upon high loading, discussed in Chap. 7. Extraction experiments were performed at $25{ }^{\circ} \mathrm{C}$ and $\mathrm{O} / \mathrm{A}=0.33$. The solvent was the baseline solvent containing $0.01 \mathrm{M}$ BOBCalixC6, $0.5 \mathrm{M}$ Cs-7SB, and $0.001 \mathrm{M}$ TOA. The modifier concentration was also varied, and organic-phase $\mathrm{Na}^{+}$and $\mathrm{K}^{+}$concentrations were determined in the same experiment, as will be described later.

Table 3.5 shows the dependence of $D_{\mathrm{Cs}}$ on the initial $\mathrm{Cs}^{+}$concentration in the full simulant. Good reproducibility was obtained. The data together with $\mathrm{O} / \mathrm{A}$ data in Table 3.6 were smoothed with the simple cesium loading model derived in the previous section, with fitting constant $C_{\mathrm{Cs}}=1771$, and satisfactory agreement with the model was obtained overall, given by the standard error of fitting of $\pm 9.6 \%$. This good agreement supports the formation of a simple 1:1 BOBCalixC6-Cs ${ }^{+}$complex in the solvent, in agreement with more detailed equilibrium modeling. ${ }^{25}$

As the concentration of $\mathrm{Cs}^{+}$in the simulant increases, it may be seen that $D_{\mathrm{Cs}}$ gradually declines due to the progressive loading of the BOBCalixC6 extractant. Defining loading as a percent of the $0.01 \mathrm{M}$ BOBCalixC6 concentration, nearly complete loading of cesium was achieved, $92.5 \%$, and no third-phase formation was observed. At the average cesium concentration in the expected waste feed, $1.4 \times 10^{-4} \mathrm{M}$, the loading is inconsequential (3.6\%), and the average value of $D_{\mathrm{Cs}}$ was found to be 17.0, in good agreement with 16.9 given in Table 3.4. At trace $\mathrm{Cs}^{+}$loading, $D_{\mathrm{Cs}}$ would be expected from the model to rise slightly to 17.7. At the maximum aqueous $\mathrm{Cs}^{+}$concentration expected in the waste stream $(0.42 \mathrm{mM}$, corresponding to $4.3 \mathrm{Ci} / \mathrm{gal}),{ }^{54}$ the model implies a $D_{\mathrm{Cs}}$ value of 15.8 . Even at $1.0 \mathrm{mM}$ initial aqueous Cs concentration, the value of $D_{\mathrm{Cs}}(12.4)$ is well above the needed value of 8. Under baseline conditions, loading of the solvent by $\mathrm{Cs}^{+}$is, therefore, not an issue insofar as meeting process goals, provided that reflux from the scrubbing section is minor.

Table 3.5. Cesium extraction from the full simulant by the baseline solvent at $25^{\circ} \mathrm{C}$ as a function of aqueous cesium concentration

\begin{tabular}{cccc}
\hline$[\mathrm{Cs}]_{\text {init }}$ & $\begin{array}{c}D_{\mathrm{Cs}} \\
\text { Obs }\end{array}$ & $\begin{array}{c}D_{\mathrm{Cs}} \\
\text { Calc }\end{array}$ & $\begin{array}{c}{[\mathrm{Cs}]_{\text {org }}} \\
\text { Obs }\end{array}$ \\
\hline $1.4 \times 10^{-4}$ & 17.6 & 17.1 & $3.59 \times 10^{-4}$ \\
$1.4 \times 10^{-4}$ & 18.2 & 17.1 & $3.61 \times 10^{-4}$ \\
$1.4 \times 10^{-4}$ & 16.0 & 17.1 & $3.54 \times 10^{-4}$ \\
$1.4 \times 10^{-4}$ & 16.0 & 17.1 & $3.54 \times 10^{-4}$ \\
$3.0 \times 10^{-4}$ & 16.8 & 16.4 & $7.64 \times 10^{-4}$ \\
$3.0 \times 10^{-4}$ & 16.8 & 16.4 & $7.65 \times 10^{-4}$ \\
$7.0 \times 10^{-4}$ & 15.4 & 14.6 & $1.76 \times 10^{-3}$ \\
$7.0 \times 10^{-4}$ & 14.6 & 14.6 & $1.74 \times 10^{-3}$ \\
$1.0 \times 10^{-3}$ & 12.0 & 13.4 & $2.40 \times 10^{-3}$ \\
$1.0 \times 10^{-3}$ & 12.7 & 13.4 & $2.43 \times 10^{-3}$ \\
$3.0 \times 10^{-3}$ & 5.11 & 6.70 & $5.67 \times 10^{-3}$ \\
$3.0 \times 10^{-3}$ & 5.12 & 6.70 & $5.68 \times 10^{-3}$ \\
$1.0 \times 10^{-2}$ & 1.39 & 1.34 & $9.52 \times 10^{-3}$ \\
$1.0 \times 10^{-2}$ & 1.28 & 1.34 & $8.99 \times 10^{-3}$ \\
\hline
\end{tabular}




\subsubsection{Effect of O/A Ratio}

An experiment was conducted to examine the effect of extraction O/A ratio on cesium distribution. A low $\mathrm{O} / \mathrm{A}$ ratio on extraction will be used in the flowsheet to aid in attaining the needed concentration factor of 15.1. At the present, the baseline flowsheet specifies an extraction $\mathrm{O} / \mathrm{A}$ ratio of 0.31 . It is possible, perhaps likely, that a final plant design will specify a different value, or even allow for adjustment within a range to accommodate changes in temperature or other operating conditions.

As might be expected from the loading dependence described in the previous section, it was found that the solvent is able to sustain a fairly constant value of $D_{\mathrm{Cs}}$ over a wide range of O/A ratios. Table 3.6 shows the effect of extraction $\mathrm{O} / \mathrm{A}$ ratio on $D_{\mathrm{Cs}}$ for the baseline solvent and full simulant at $25{ }^{\circ} \mathrm{C}$. No third-phase formation was noted at any O/A ratio. It may be seen that the $D_{\mathrm{Cs}}$ values decrease as the O/A ratio decreases, owing to the increasing $\mathrm{Cs}^{+}$loading. Since the loadings involved are not high, the decrease in $D_{C s}$ is minor. Calculated values based on the simple cesium loading model (Section 3.3.2) applied simultaneously to the data in Tables 3.5 and 3.6 are shown in the third column. The model predicts a slightly faster decrease in $D_{\mathrm{Cs}}$ than is observed as O/A ratio decreases, and it also under-predicts the $D_{\mathrm{Cs}}$ values at high $\mathrm{O} / \mathrm{A}$ ratios. Although the discrepancies are within experimental error, other considerations suggest that they may be real. Namely, dibutylphosphate distributes to the solvent and may keep O/A ratio slightly high at low O/A ratios, and competition due to potassium extraction lessens at high $\mathrm{O} / \mathrm{A}$ ratios. Both of these phenomena are explored in later sections.

Table 3.6. Cesium extraction from the full simulant by the baseline solvent at $25^{\circ} \mathrm{C}$ as a function of $\mathrm{O} / \mathrm{A}$ ratio

\begin{tabular}{cccc}
\hline O/A & $\begin{array}{c}D_{\mathrm{Cs}} \\
\text { Obs }\end{array}$ & $\begin{array}{c}D_{\mathrm{Cs}} \\
\text { Calc }\end{array}$ & $\begin{array}{c}{[\mathrm{Cs}]_{\text {org }}} \\
\text { Obs }\end{array}$ \\
\hline 0.05 & 16.5 & 15.5 & $1.27 \times 10^{-3}$ \\
0.1 & 16.2 & 16.2 & $8.66 \times 10^{-4}$ \\
0.1 & 16.5 & 16.2 & $8.72 \times 10^{-4}$ \\
0.1 & 17.2 & 16.2 & $8.85 \times 10^{-4}$ \\
0.1 & 17.3 & 16.2 & $8.88 \times 10^{-4}$ \\
0.3 & 17.2 & 17.0 & $3.91 \times 10^{-4}$ \\
0.3 & 16.8 & 17.0 & $3.89 \times 10^{-4}$ \\
1 & 16.9 & 17.5 & $1.32 \times 10^{-4}$ \\
1 & 18.0 & 17.5 & $1.33 \times 10^{-4}$ \\
3 & 18.2 & 17.6 & $4.58 \times 10^{-5}$ \\
3 & 18.4 & 17.6 & $4.58 \times 10^{-5}$ \\
10 & 19.5 & 17.7 & $1.39 \times 10^{-5}$ \\
10 & 18.8 & 17.7 & $1.39 \times 10^{-5}$ \\
\hline
\end{tabular}

\subsubsection{Effect of Cs Concentration on Extract/Scrub/Strip Performance}

An ESS experiment was designed to address the question as to whether a potential pinch could occur in the strip section of the flowsheet if cesium concentrations in the organic phase build up sufficiently to give distribution ratios higher than 0.2 . The experiment entailed varying the 
concentration of ${ }^{133} \mathrm{Cs}$ ("cold" Cs) in the full simulant and subsequently performing an ESS sequence using the baseline solvent. It was found that if the initial cesium concentration in the waste does not exceed $0.5 \mathrm{mM}$ (over three times the baseline concentration) or, in fact, if the organic cesium concentration entering the stripping section in the solvent does not exceed $1 \mathrm{mM}$, no problem should be anticipated. Within those concentration limits, the stripping stages should yield low distribution ratios and an efficient release of the complexed cesium to the aqueous phase.

Table 3.7. Cesium distribution ratios from extract/scrub/strip experiments with increasing Cs concentration in the full simulant ${ }^{a}$

\begin{tabular}{ccccccccc}
\hline & {$[\mathrm{Cs}]_{\text {init }}(\mathrm{mM})$} & Extract & Scrub & Strip \#1 & Strip \#2 & Strip \#3 & Strip \#4 & Strip \#5 \\
\hline$D_{\mathrm{Cs}}$ & 0.14 & 16.6 & 1.49 & 0.145 & 0.084 & 0.062 & 0.053 & 0.049 \\
{$[\mathrm{Cs}]_{\mathrm{org}}(\mathrm{mM})$} & & $3.6 \times 10^{-1}$ & $3.1 \times 10^{-1}$ & $1.3 \times 10^{-1}$ & $3.9 \times 10^{-2}$ & $9.3 \times 10^{-3}$ & $1.9 \times 10^{-3}$ & $3.8 \times 10^{-4}$ \\
{$[\mathrm{Cs}]_{\mathrm{aq}}(\mathrm{mM})$} & & $2.1 \times 10^{-2}$ & $2.1 \times 10^{-1}$ & $9.1 \times 10^{-1}$ & $4.6 \times 10^{-1}$ & $1.5 \times 10^{-1}$ & $3.7 \times 10^{-2}$ & $7.8 \times 10^{-3}$ \\
$D_{\mathrm{Cs}}$ & 0.20 & 17.0 & 1.44 & 0.154 & 0.092 & 0.065 & 0.055 & 0.061 \\
{$[\mathrm{Cs}]_{\text {org }}(\mathrm{mM})$} & & $5.1 \times 10^{-1}$ & $4.5 \times 10^{-1}$ & $2.0 \times 10^{-1}$ & $6.1 \times 10^{-2}$ & $1.5 \times 10^{-2}$ & $3.2 \times 10^{-3}$ & $7.6 \times 10^{-4}$ \\
{$[\mathrm{Cs}]_{\mathrm{aq}}(\mathrm{mM})$} & & $3.0 \times 10^{-2}$ & $3.1 \times 10^{-1}$ & 1.3 & $6.7 \times 10^{-1}$ & $2.3 \times 10^{-1}$ & $5.9 \times 10^{-2}$ & $1.2 \times 10^{-2}$ \\
$D_{\mathrm{Cs}}$ & 0.30 & 16.4 & 1.48 & 0.174 & 0.108 & 0.076 & 0.060 & 0.053 \\
{$[\mathrm{Cs}]_{\mathrm{org}}(\mathrm{mM})$} & & $7.6 \times 10^{-1}$ & $6.7 \times 10^{-1}$ & $3.1 \times 10^{-1}$ & $1.1 \times 10^{-1}$ & $3.0 \times 10^{-2}$ & $7.0 \times 10^{-3}$ & $1.5 \times 10^{-3}$ \\
{$[\mathrm{Cs}]_{\mathrm{aq}}(\mathrm{mM})$} & & $4.6 \times 10^{-2}$ & $4.5 \times 10^{-1}$ & 1.8 & 1.0 & $4.0 \times 10^{-1}$ & $1.2 \times 10^{-1}$ & $2.8 \times 10^{-2}$ \\
$D_{\mathrm{Cs}}$ & 0.50 & 15.3 & 1.30 & 0.203 & 0.135 & 0.097 & 0.071 & 0.058 \\
{$[\mathrm{Cs}]_{\mathrm{org}}(\mathrm{mM})$} & & 1.3 & 1.1 & $5.5 \times 10^{-1}$ & $2.2 \times 10^{-1}$ & $7.2 \times 10^{-2}$ & $1.9 \times 10^{-2}$ & $4.2 \times 10^{-3}$ \\
{$[\mathrm{Cs}]_{\mathrm{aq}}(\mathrm{mM})$} & & $8.2 \times 10^{-2}$ & $8.4 \times 10^{-1}$ & 2.7 & 1.6 & $7.4 \times 10^{-1}$ & $2.7 \times 10^{-1}$ & $7.3 \times 10^{-2}$ \\
$D_{\mathrm{Cs}}$ & 0.70 & 14.5 & 1.19 & 0.225 & 0.157 & 0.111 & 0.081 & 0.061 \\
{$[\mathrm{Cs}]_{\mathrm{org}}(\mathrm{mM})$} & & 1.7 & 1.5 & $7.9 \times 10^{-1}$ & $3.5 \times 10^{-1}$ & $1.2 \times 10^{-1}$ & $3.6 \times 10^{-2}$ & $8.3 \times 10^{-3}$ \\
{$[\mathrm{Cs}]_{\mathrm{aq}}(\mathrm{mM})$} & & $1.2 \times 10^{-1}$ & 1.3 & 3.5 & 2.2 & 1.1 & $4.4 \times 10^{-1}$ & $1.4 \times 10^{-1}$ \\
\hline
\end{tabular}

${ }^{a} \mathrm{O} / \mathrm{A}$ ratios were 0.33 on extraction and 5.0 on scrubbing and stripping; $T=25^{\circ} \mathrm{C}$.

From the data in Table 3.7, the expected cesium-loading effect on extraction may be noted. The loading is also reflected in depressed $D_{\mathrm{Cs}}$ values on scrubbing. On stripping, the opposite effect occurs, as the added cesium nitrate in the aqueous phase indeed elevates the $D_{\mathrm{Cs}}$ values. For example, when the initial aqueous concentration of cesium is $0.70 \mathrm{mM}$, the cesium concentration in the aqueous phase is $3.5 \mathrm{mM}$, and thus, the aqueous-phase nitrate concentration is increased more than 4-fold above that of the initial strip solution. Correspondingly, the $D_{\mathrm{Cs}}$ value increases more than 4-fold. On successive strips, the $D_{C s}$ values converge, though the convergence is slower, taking more strip steps the greater the initial cesium concentration in the simulant.

\subsubsection{Effect of Forward Scrubbing and Stripping}

In the context of this report, "forward" scrubbing and stripping will refer to a particular type of batch experiment in which pristine solvent is contacted directly with aqueous scrub or strip solution to which is added various components of interest. The extraction step is omitted. In this section, $\mathrm{CsNO}_{3}$ (spiked with ${ }^{137} \mathrm{Cs}$ tracer) was added at the concentration that it would be as if a standard ESS test were conducted. This test therefore allows an examination of the behavior of $D_{\mathrm{Cs}}$ without the superimposed effect of the other components that are extracted from the simulant. 
In the test conducted (Table 3.8), five strips were performed. The control experiment employed an initial extraction from the full simulant, and the sequence of extraction, scrubbing, and stripping is accordingly indicated by the notation $\mathrm{ESS}_{5}$. In the same notation, $\mathrm{SS}_{5}$ denotes a forward scrub experiment in which the cesium is introduced to the aqueous scrub solution without an initial contact with simulant, and subsequently five strips are performed. Likewise, $S_{5}$ denotes a forward strip experiment in which the cesium is introduced to the aqueous strip solution without an initial contact with simulant and scrub, and subsequently four more strips are performed.

In the forward scrub experiment $\left(\mathrm{SS}_{5}\right)$, the $D_{\mathrm{Cs}}$ values are less than the corresponding values in the $\mathrm{ESS}_{5}$ experiment, because the effects of the components extracted from the simulant are absent, including $\mathrm{Na}^{+}, \mathrm{K}^{+}$, and dibutylphosphate. The values for the forward stripping experiment $\left(\mathrm{S}_{5}\right)$ are even less, because the acid normally extracted from the scrub by the TOA and modifier is not adding to the nitrate concentration of the aqueous phase. In this case, the solvent actually takes up some of the nitric acid from the strip solution, and the plateau value of $D_{\mathrm{Cs}}$ is approached from below.

Table 3.8. Cesium distribution ratios in forward scrubbing and stripping ${ }^{a}$

\begin{tabular}{lcll}
\hline & ESS $_{5}$ & $\mathrm{SS}_{5}$ & $\mathrm{~S}_{5}$ \\
\hline Extract & 16.6 & & \\
Scrub & 1.49 & 1.26 & \\
Strip \#1 & 0.145 & 0.106 & 0.060 \\
Strip \#2 & 0.084 & 0.069 & 0.028 \\
Strip \#3 & 0.062 & 0.053 & 0.025 \\
Strip \#4 & 0.053 & 0.046 & 0.027 \\
Strip \#5 & 0.049 & 0.044 & 0.029 \\
\hline
\end{tabular}

${ }^{a}$ The $\mathrm{ESS}_{5}$ is a control experiment for comparison, using the full simulant with the baseline value of $0.14 \mathrm{mM}$ Cs. The $\mathrm{SS}_{5}$ and $\mathrm{S}_{5}$ are forward scrubbing and stripping experiments, respectively (see text).

\subsubsection{Extract/Scrub/Strip Performance with Two Extraction Steps}

Since the mild "reflux" of cesium between the first extraction stage (stage 15) and first scrub stage (stage 16) in the baseline flowsheet raises the cesium concentration in the solvent somewhat, it is thought that two successive extraction steps mimic the conditions of stage 15 better than a single extraction contact. Hence, it is of interest to examine a modified ESS experiment, here designated $\mathrm{E}_{2} \mathrm{SS}$, that employs two contacts of the solvent with fresh simulant. Table 3.9 gives the results compiled from data supplied by ANL and SRTC collaborators, together with ORNL results. Table 3.10 gives the corresponding statistical summary.

It may be seen that, notwithstanding the greater variation expected from different laboratories using different simulants, contacting protocols, and analytical procedures, the extraction and scrubbing behavior in the $\mathrm{E}_{2} \mathrm{SS}$ tests is on average in fair agreement with the results of the ESS tests given in Table 3.4. Much greater variation may be seen in stripping, however. This variation is understandable, given the sensitivity of stripping to the solvent salt and acid content, which could well vary according to the differences in simulant makeup. For example, our own target potassium content was $0.015 \mathrm{M}$, but 0.020 was the obtained concentration (Table 2.2). If set \#2 in Table 3.9 is taken as the most direct comparison to the ESS results from ORNL (Table 3.4), it may be concluded that adding a second extraction step causes the stripping $D_{C s}$ values to be higher for a given stripping step. Since the values seem to decline normally, albeit delayed by one step, it seems reasonable to 
Table 3.9. Cesium distribution ratios from extract/scrub/strip experiments with two extraction steps ( $\mathrm{E}_{2} \mathrm{SS}$ ) at $25^{\circ} \mathrm{C}$ with baseline solvent and full simulant

\begin{tabular}{ccccccccc}
\hline Set \# & $\begin{array}{c}\text { Extraction } \\
D_{\mathrm{Cs}}\end{array}$ & $\begin{array}{c}\text { Extraction } \\
D_{\mathrm{Cs}}\end{array}$ & $\begin{array}{c}\text { Scrub } \\
D_{\mathrm{Cs}}\end{array}$ & $\begin{array}{c}\text { Strip \#1 } \\
D_{\mathrm{Cs}}\end{array}$ & $\begin{array}{c}\text { Strip \#2 } \\
D_{\mathrm{Cs}}\end{array}$ & $\begin{array}{c}\text { Strip \#3 } \\
D_{\mathrm{Cs}}\end{array}$ & $\begin{array}{c}\text { Strip \#4 } \\
D_{\mathrm{Cs}}\end{array}$ & Source $^{a}$ \\
\hline 1 & 14.6 & 15.1 & 1.08 & 0.125 & 0.085 & 0.053 & 0.054 & Ref. 44 \\
2 & 18.5 & 17.0 & 1.56 & 0.188 & 0.115 & 0.086 & 0.060 & \\
$3^{b}$ & & 15.4 & 1.8 & 0.32 & 0.20 & 0.16 & & Ref. 55 \\
$4^{b}$ & & & 1.5 & 0.22 & 0.28 & & & Ref. 55 \\
$5^{b}$ & & & & 0.38 & & & & Ref. 55 \\
$6^{c}$ & & 20.7 & 1.96 & 0.189 & 0.106 & 0.076 & & \\
$7^{c}$ & & & 1.53 & 0.121 & 0.047 & 0.028 & & \\
$8^{c}$ & & & & 0.292 & 0.121 & 0.083 & & \\
\hline
\end{tabular}

$a_{\text {Data }}$ are from this Chap., unless otherwise indicated.

$b$ Results from the SRTC external irradiation experiment at zero dose.

${ }^{c}$ These are zero-dose controls received from the SRTC and carried the rest of the way through the $\mathrm{E}_{2} \mathrm{SS}$ protocol at ORNL.

Table 3.10. Statistical summary of $E_{2} S S$ results

\begin{tabular}{l|ccccccc}
\multicolumn{1}{c}{} & Extraction & Extraction & Scrub & Strip \#1 & Strip \#2 & Strip \#3 & Strip \#4 \\
\cline { 2 - 8 } Avg. $D_{\mathrm{Cs}}$ & 16.5 & 17.1 & 1.57 & 0.229 & 0.136 & 0.081 & 0.057 \\
Stdev & $17 \%$ & $15 \%$ & $19 \%$ & $41 \%$ & $57 \%$ & $55 \%$ & $7 \%$ \\
Min & 10.9 & 11.9 & 0.97 & 0.043 & 0.000 & 0.000 & 0.049 \\
Max & 22.2 & 22.2 & 2.18 & 0.416 & 0.293 & 0.170 & 0.065 \\
No. Trials & 2 & 4 & 6 & 8 & 7 & 6 & 2
\end{tabular}

attribute the higher stripping $D_{\mathrm{Cs}}$ values to the added cesium that must be stripped. We shall see later that DBP may play a role here, too.

Samples that were zero-dose controls from the SRTC external-irradiation experiment exhibit the most variation. Sets \#3-\#5 reported by the SRTC 55 exhibit high stripping $D_{\mathrm{Cs}}$ values, which decline more slowly than the other sets. Sets \#6-\#8 were zero-dose samples prepared at the SRTC but run at ORNL. These samples seem to give erratic stripping behavior, but the $D_{\mathrm{Cs}}$ values are, with one exception, significantly lower than the SRTC results. Since the SRTC experiments exhibited some inadvertent carryover of aqueous simulant into the scrub step, 55 the variation in sets \#3-\#8 is possibly explainable. In fact, the aqueous $\mathrm{pH}$ of the zero-dose scrub sample (set \#7) was alkaline, as will be reported in Chap. 6. Since the TOA will tend to extract acid in the strip steps, thereby lowering the aqueous nitrate concentration, the low $D_{\mathrm{Cs}}$ values seen in set $\# 7$ follow.

\subsubsection{Effect of Temperature}

Cesium extraction in CSSX was earlier found to be strongly temperature-dependent. ${ }^{15}$ The reaction being exothermic, $D_{\mathrm{Cs}}$ values in all stages decrease with increasing temperature. Depending on the particular step in a batch sequence, the decrease in $D_{\mathrm{Cs}}$ is $6-10 \%$ per degree at $25{ }^{\circ} \mathrm{C}$. This strong dependence leads to particular challenges in conducting reproducible distribution experiments (see Chap. 2) as well as in managing a flowsheet. ${ }^{44}$ In support of a better understanding of the temperature dependence of CSSX, further experiments were conducted to examine the impact of the 
use of the present simulant recipe vs. the earlier SRS\#2 simulant. A sequence of $\mathrm{E}_{2} \mathrm{SS}_{3}$ batch contacts involving 2 extractions $(\mathrm{O} / \mathrm{A}=0.33), 1$ scrub $(\mathrm{O} / \mathrm{A}=5)$, and 3 strips $(\mathrm{O} / \mathrm{A}=5)$ was run at $32{ }^{\circ} \mathrm{C}$. Vortexing was used as the method of contact. Precautions to ensure constant temperature in all circumstances (contact, centrifuging, subsampling) were taken. Samples were run in duplicate.

As shown in Table 3.11, the observed $D_{\mathrm{Cs}}$ values in the $\mathrm{E}_{2} \mathrm{SS}_{3}$ experiment conducted at $32{ }^{\circ} \mathrm{C}$ are in good agreement with those expected based on the earlier results with a more concentrated simulant (SRS\#2)..$^{15}$ Scrubbing and stripping at $32{ }^{\circ} \mathrm{C}$ perform almost as predicted, whereas extraction is approximately $10 \%$ weaker than predicted. Note that the prediction employs the reported slope ${ }^{15}$ (second line in the heading of Table 3.11) and a new intercept calculated from the value obtained at $25^{\circ} \mathrm{C}$ (Table 3.11). Thus, the prediction assumes a constant slope (enthalpy-related). As shown earlier, this is approximately true, though the intercept strongly depends on the exact aqueous composition. ${ }^{15}$

Table 3.11. Temperature dependence of an $\mathrm{E}_{2} \mathrm{SS}_{3}$ sequence ${ }^{a}$

\begin{tabular}{lcccccccc}
\hline & $T$ & Extr. \#1 & Extr. \#2 & Scrub & Strip \#1 & Strip \#2 & Strip \#3 & Strip \#4 \\
& & $m=2.402$ & $m=2.470$ & $m=3.246$ & $m=4.018$ & $m=4.306$ & $m=4.048$ & $m=3.666$ \\
& ${ }^{\circ} \mathrm{C}$ & $D_{\mathrm{Cs}}$ & \multicolumn{1}{c}{$D_{\mathrm{Cs}}$} & $D_{\mathrm{Cs}}$ & $D_{\mathrm{Cs}}$ & $D_{\mathrm{Cs}}$ & $D_{\mathrm{Cs}}$ & $D_{\mathrm{Cs}}$ \\
\hline Average $^{b}$ & 25 & 16.5 & 17.1 & 1.57 & 0.229 & 0.136 & 0.081 & 0.057 \\
Sample A $^{2}$ & 32 & 9.9 & 10.0 & 0.90 & 0.102 & 0.062 & 0.045 & \\
Sample B $^{2}$ & 32 & 10.0 & 9.5 & 0.87 & 0.104 & 0.062 & 0.046 & \\
Averagec $^{c}$ & 32 & 10.0 & 9.8 & 0.89 & 0.103 & 0.062 & 0.045 & \\
Predicted $^{d}$ & 32 & 10.78 & 11.0 & 0.88 & 0.112 & 0.063 & 0.040 & 0.030 \\
\hline
\end{tabular}

${ }^{a}$ In the second row are given the slope $m$ reported earlier, ${ }^{15}$ corresponding to the relationship $\log D_{\mathrm{Cs}}=m(1000 / T)+b$, where $T$ is expressed in $\mathrm{K}, m$ is the slope, and $b$ is the intercept. The slope and intercept varied according to the particular step in the sequence.

${ }^{b}$ From Table 3.10 .

${ }^{c}$ Average of Samples A and B.

${ }^{d}$ Predicted from the slope $m$, using the value given in the first line for $25{ }^{\circ} \mathrm{C}$ as the reference value to calculate the intercept $b$ in the relationship $b=\log D_{\mathrm{Cs}, 25}-m(1000 /$ 298.15).

The temperature dependence of $D_{\mathrm{Cs}}$ for the baseline solvent equilibrated with the full simulant at $\mathrm{O} / \mathrm{A}=0.33$ is given in Table 3.12. Two linear regressions were performed corresponding to the temperature ranges $25-37{ }^{\circ} \mathrm{C}$ and $25-40{ }^{\circ} \mathrm{C}$. The plots are shown in Fig. 3.1, and the regression parameters are given in Table 3.13. It may be seen that the points in the range $25-37{ }^{\circ} \mathrm{C}$ are nearly linear, whereas the points at $40{ }^{\circ} \mathrm{C}$ are both less precise and in poor agreement with the linear regression of the lower temperatures. For purposes of prediction and correlation, the regression of the $25-37{ }^{\circ} \mathrm{C}$ data are preferred here. The densities shown were determined by weighing small volumes of solvent as delivered by a disposable-tip pipetter and should be considered approximate.

For comparison with the results described above, it may be seen from Table 3.14 that cesium distribution from the batch internal-irradiation experiment ${ }^{47}$ are in good agreement with the present batch results after taking into account the temperature correction. Calculated results shown in the table correspond to the values predicted from the earlier correlations ${ }^{15}$ or from the temperature dependence of the extraction from full simulant (Table 3.13). It may be remarked that the good agreement persists despite the fact that a) the controls were mixed by overhead stirring and used ICPMS for the analytical method for Cs determination, b) the high-activity samples entailed considerable activity relative to the levels used here and were handled in a hot cell, and c) different personnel carried out the experiment. 
Table 3.12. Temperature dependence of $D_{\mathrm{Cs}}$ between baseline solvent and full simulant ${ }^{a}$

\begin{tabular}{|c|c|c|c|c|c|}
\hline \multirow{2}{*}{$\begin{array}{l}\text { Temp. } \\
{ }^{\circ} \mathrm{C}\end{array}$} & \multirow{2}{*}{$\begin{array}{c}\text { Temp. } \\
\text { K }\end{array}$} & \multirow{2}{*}{$\begin{array}{l}1 / T \\
\mathrm{~K}^{-1}\end{array}$} & \multirow{2}{*}{$\begin{array}{c}D_{\mathrm{Cs}} \\
\text { duplicates }\end{array}$} & \multicolumn{2}{|c|}{ Density $(\mathrm{g} / \mathrm{mL})$} \\
\hline & & & & Solvent & Simulant \\
\hline 25 & 298.15 & 0.00335 & 15.1 & $0.827 \pm 0.004$ & $1.259 \pm 0.006$ \\
\hline$-\overline{32^{\mathrm{a}}}$ & 305.15 & 0.00328 & $\begin{array}{r}16.7 \\
9.9\end{array}$ & & \\
\hline$--\cdots$ & 308.15 & 0.00325 & $-\frac{10.0}{8.3}$ & $0.819 \pm 0.004$ & $1.265 \pm 0.003$ \\
\hline$-\frac{-}{37}$ & 310.15 & 0.00322 & $-\frac{7.6}{7.1}$ & $0.817 \pm 0.004$ & $1.271 \pm 0.003$ \\
\hline 40 & 313.15 & 0.00319 & $\begin{array}{r}6.8 \\
4.1 \\
4.8\end{array}$ & $0.810 \pm 0.002$ & $1.272 \pm 0.004$ \\
\hline
\end{tabular}

${ }^{a}$ The data points at $32{ }^{\circ} \mathrm{C}$ are taken from Table 3.11 .

Table 3.13. Linear-regression parameters ${ }^{a}$

\begin{tabular}{ccccc}
\hline Range $\left({ }^{\circ} \mathrm{C}\right)$ & $m$ & $b$ & $\sigma$ & $r^{2}$ \\
\hline $25-37$ & $2767 \pm 132$ & $-8.08 \pm 0.43$ & 0.018 & 0.986 \\
$25-40$ & $3253 \pm 268$ & $-9.68 \pm 0.87$ & 0.046 & 0.948 \\
\hline
\end{tabular}

${ }^{a}$ Parameters were obtained by linear regression of the points shown in Fig. 3.1 in the temperature ranges indicated in the first column. In the second and third columns are given the slope $m$ and intercept $b$ corresponding to the relationship $\log D_{\mathrm{Cs}}=m(1000 / T)+b$, where $T$ is expressed in $\mathrm{K}$. The fourth and fifth columns give the standard error of fitting and the correlation coefficient, respectively.

Table 3.14. Results from batch internal-irradiation experiment ${ }^{a}$

\begin{tabular}{lccccccc}
\hline & \multicolumn{3}{c}{ Controls (no $\left.{ }^{137} \mathrm{Cs}\right)^{b}$} & & \multicolumn{3}{c}{ High-activity samples ${ }^{b}$} \\
\cline { 2 - 4 } \cline { 6 - 7 } & $\begin{array}{c}\text { Temp. } \\
\left({ }^{\circ} \mathrm{C}\right)\end{array}$ & $\begin{array}{c}D_{\mathrm{Cs}} \\
(\mathrm{Obs})\end{array}$ & $\begin{array}{c}D_{\mathrm{Cs}} \\
(\mathrm{Calc})^{d}\end{array}$ & & $\begin{array}{c}\text { Temp. } \\
\left({ }^{\circ} \mathrm{C}\right)\end{array}$ & $\begin{array}{c}D_{\mathrm{Cs}} \\
(\mathrm{Obs})\end{array}$ & $\begin{array}{c}D_{\mathrm{Cs}} \\
(\mathrm{Calc})^{d}\end{array}$ \\
\hline Extraction & 23.5 & 0 & $18.6(17.8)$ & 34.0 & 9.34 & $9.81(8.54)$ \\
Scrub & 25.3 & 1.54 & 1.55 & 34.3 & 0.52 & 0.74 \\
Strip & 25.0 & 0.14 & 0.15 & 34.8 & 0.04 & 0.06 \\
\hline
\end{tabular}

${ }^{a}$ Data taken from the results of the batch internal-irradiation experiment using baseline solvent, full simulant, and baseline O/A ratios ( 0.33 for extraction and 5 for scrubbing and stripping).

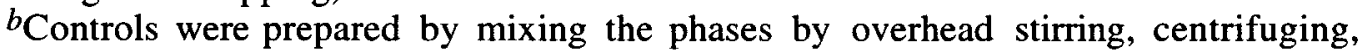
and analysis of the phases by ICP-MS.

${ }^{c}$ High-activity samples were prepared from a simulant containing $0.35 \mathrm{Ci} / \mathrm{L}{ }^{137} \mathrm{Cs}$ activity and were analyzed by gamma counting.

${ }^{d}$ An earlier correlation was used to calculate values for comparison, as described in Table 3.11.15 Values in parentheses for the extraction step used the correlation given in Table 3.13 for the temperature range $25-37{ }^{\circ} \mathrm{C}$. 


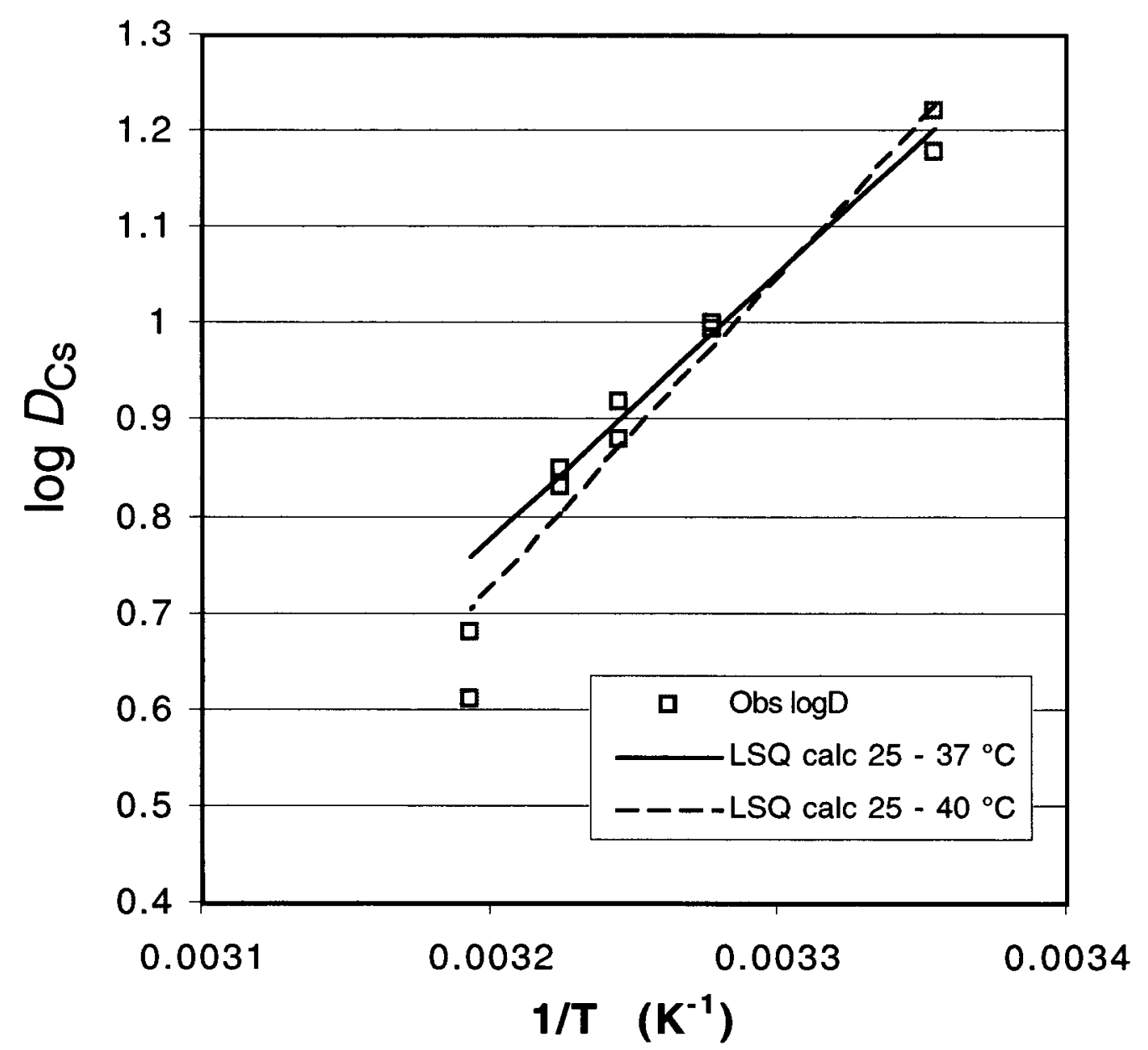

Fig. 3.1. Temperature dependence of the cesium distribution ratio between full simulant and baseline solvent at $\mathrm{O} / \mathrm{A}=0.33$. Two linear least-squares fits (LSQ calc in legend) are based on the temperature ranges and parameters given in Table 3.13.

\subsubsection{Effect of Potassium}

Potassium is known to be extracted by calix-crowns. Although it is extracted on the order of 100 -fold less strongly than $\mathrm{Cs}^{+}$ion, it competes significantly with $\mathrm{Cs}^{+}$extraction by virtue of the fact that it is normally present at much greater concentration (cf. $0.14 \mathrm{mM}$ for $\mathrm{Cs}^{+}$vs. $20 \mathrm{mM}$ for K $\mathrm{K}^{+}$). From Table 3.15, it may be seen that the presence of potassium indeed noticeably interferes with cesium extraction. In the next chapter, it will be shown that this is due to potassium loading, and at higher potassium concentrations, a third phase forms (Chap. 7). It should be noted that the bounding $\mathrm{K}^{+}$concentration in the feed is likely to be ca. $50 \mathrm{mM} .{ }^{54}$ At this level, the value of $D_{\mathrm{Cs}}$ is sufficiently high to meet flowsheet requirements. 
Table 3.15. Cesium extraction from the full simulant by the baseline solvent as a function of aqueous potassium concentration ${ }^{a}$

\begin{tabular}{cccc}
\hline $\begin{array}{c}{[\mathrm{Cs}]_{\text {init }}} \\
(\mathrm{mM})\end{array}$ & $\begin{array}{c}{[\mathrm{K}]_{\text {init }}} \\
(\mathrm{mM})\end{array}$ & $D_{\mathrm{Cs}}$ & $\begin{array}{c}{[\mathrm{Cs}]_{\text {org }}} \\
(\mathrm{mM})\end{array}$ \\
\hline 0.14 & 20 & 17.6 & 0.359 \\
0.14 & 20 & 18.2 & 0.361 \\
0.14 & 20 & 16.0 & 0.354 \\
0.14 & 20 & 16.0 & 0.354 \\
0.14 & 50 & 11.0 & 0.330 \\
0.14 & 50 & 11.9 & 0.336 \\
0.14 & 90 & 8.1 & 0.307 \\
0.14 & 90 & 7.8 & 0.304 \\
\hline
\end{tabular}

${ }^{a} \mathrm{O} / \mathrm{A}=0.33 ; 25^{\circ} \mathrm{C}$.

\subsubsection{Effect of 4-sec-Butylphenol}

4-sec-Butylphenol (SBP) is both an impurity in the synthesized Cs-7SB modifier and a degradation product. Studies of the effect of the related compound 4-tert-octylphenol in the firstgeneration solvent system found that stripping began to deteriorate at comparatively high levels of the phenol, ca. $10 \mathrm{mM}$ or greater. ${ }^{8}$ Given that the simulant has become more complex and the solvent has changed in composition, it was considered necessary to confirm that SBP has no important effect on Cs distribution within the expected concentration levels and to determine the level that effects on performance would become noticeable. It is estimated that the level of SBP in the modifier as synthesized is less than 0.1 mole\%. In the washing of the pristine solvent, this level of SBP decreases by an order of magnitude, judging by the data provided in Chap. 4 . Hence, the initial concentration of SBP in the solvent is estimated to be less than $0.1 \mathrm{mM}$. On external gamma irradiation, SBP is produced, but at negligible levels (Chap. 6). ${ }^{55}$ Its rate of buildup with dose was found to be at most $0.1 \mathrm{mM}$ per annual dose. Batch internal-irradiation studies found even less buildup of SBP. ${ }^{47}$ After a 10-year dose, less than $0.1 \mathrm{mM}$ of SBP was detected in the worst case. Thermal degradation of the solvent also did not implicate SBP as an important product either, its concentration being less than $0.02 \mathrm{mM}$ in all cases (Chap. 5). Overall, a conservative upper limit of $0.3 \mathrm{mM}$ may be established for SBP in the solvent.

Based on analytical results from both ORNL and SRTC, two experiments were carried out to test the effect of varying levels of 4-sec-butylphenol in the baseline solvent on $\mathrm{Cs}^{+}$distribution in forward stripping and batch ESS performance. The SBP concentration ranged from 0 to $50 \mathrm{mM}$ in the forward stripping experiment and 0 to $30 \mathrm{mM}$ in the ESS test. In forward stripping, the aqueous strip solution $\left(1 \mathrm{mM} \mathrm{HNO}_{3}\right)$ containing $0.1 \mathrm{mM} \mathrm{CsNO}_{3}$ was equilibrated at $\mathrm{O} / \mathrm{A}=5$ with baseline solvent at $25{ }^{\circ} \mathrm{C}$. A standard ESS test was also conducted with baseline solvent and full simulant.

Results from the forward-stripping experiment shown in Table 3.16 reveal a high tolerance for SBP. The value of $D_{C s}$ held constant at ca. 0.024 from $0 \mathrm{mM}$ through $5.0 \mathrm{mM}$ added SBP. A slight, but progressive increase in $D_{\mathrm{Cs}}$ was then seen at higher SBP concentrations through $50.0 \mathrm{mM}$. Presumably, with further increase in SBP concentration, $D_{\mathrm{Cs}}$ will continue to rise as dictated by the synergistic effect given in the proposed equilibrium

$$
\mathrm{Cs}^{+}{ }_{\mathrm{aq}}+\mathrm{HR}_{\mathrm{org}}+\mathrm{B}_{\mathrm{org}} \rightleftharpoons \mathrm{CsBR}_{\mathrm{org}}+\mathrm{H}^{+}{ }_{\mathrm{aq}}
$$

where HR represents the SBP (or indeed any organic acid) in the organic phase. Such synergistic 
Table 3.16. Effect of added 4-sec-butylphenol on $D_{\mathrm{Cs}}$ in forward stripping $a$

\begin{tabular}{cc}
$\begin{array}{c}\text { [4-sec-Butylphenol] } \\
(\mathrm{mM})\end{array}$ & $D_{\mathrm{Cs}}$ \\
\hline 0 & 0.023 \\
0 & 0.024 \\
0.1 & 0.025 \\
0.1 & 0.024 \\
1.0 & 0.025 \\
1.0 & 0.024 \\
5.0 & 0.024 \\
5.0 & 0.024 \\
10.0 & 0.026 \\
10.0 & 0.026 \\
20.0 & 0.027 \\
20.0 & 0.027 \\
50.0 & 0.031 \\
50.0 & 0.034 \\
\hline${ }^{a}$ Aqueous phase (1 mM $\mathrm{HNO}_{3}$ plus 0.1 \\
mM CsNO ${ }_{3}$ ) was contacted with baseline \\
solvent in the presence of variable \\
concentrations of 4-sec-butylphenol at 25 ${ }^{\circ} \mathrm{C}$.
\end{tabular}

effects are well known for the extraction of alkali metal cations by macrocycles in the presence of lipophilic organic acids, including carboxylic, sulfonic, and phosphoric acids. ${ }^{48,49}$ Given that the ion-pair extraction process given in eq. 3.6 governs the baseline extraction in the absence of SBP, eq. 3.8 provides further extraction that is unwanted in stripping. Fortunately, SBP is both low in concentration and acidity, such that eq. 3.8 is expected to be unimportant in the case of $\mathrm{HR}=\mathrm{SBP}$. In general, the synergism is expected to be driven to the right as the acidity of HR increases, and it will be seen below that more acidic species like dibutylphosphoric acid indeed exhibit greater ability to inhibit stripping.

One may note that increasing aqueous hydrogen ion concentration will be expected to promote stripping in the presence of an organic acid, as it reverses eq. 3.8. Obviously, the less basic anions such as organo sulfate, sulfonate, and phosphate will resist protonation. With regards to solvent cleanup, one can also see that even if one is able to strip the cesium by washing with aqueous acid, the regenerated organic acid HR would still remain in the solvent. As will be seen later (Chaps. 4 and 8), organic acids can be removed readily from the solvent by washing with $\mathrm{NaOH}$ solutions or by anion exchange.

More definitive results of the ESS batch test (see Table 3.17) confirm that the presence of SBP has little or no effect on $\mathrm{Cs}^{+}$extraction behavior at the maximum levels generated in the radiationstability tests (ca. $3 \mathrm{mM}$ ). ${ }^{55}$ The $D_{\mathrm{Cs}}$ values on extraction, scrub, and strip contacts are very close to each other for $0,0.3$, and $3.0 \mathrm{mM}$ added SBP. However, the $D_{\mathrm{Cs}}$ values for $30 \mathrm{mM}$ added SBP were considerably higher in all stages, and stripping was effectively prevented. The very high $D_{\mathrm{Cs}}$ values in the scrub and strip steps for the $30-\mathrm{mM}$ phenol case undoubtedly reflect the insufficient acid available in the scrub solution at $\mathrm{O} / \mathrm{A}=5$ to convert the sodium form of the phenol back to the neutral phenol. Hence, the weak extraction given by eq. 3.8 is made considerably stronger by conversion to the following process:

$$
\mathrm{Cs}^{+}{ }_{\mathrm{aq}}+\mathrm{Na}^{+} \mathrm{R}^{-}{ }_{\text {org }}+\mathrm{B}_{\text {org }} \rightleftharpoons \mathrm{CsBA}_{\text {org }}+\mathrm{Na}^{+}{ }_{\mathrm{aq}}
$$


Even if the calixarene is not present, the cation-exchange process favors $\mathrm{Cs}^{+}$ion vs. $\mathrm{Na}^{+}, 56$ but the strong binding of the $\mathrm{Cs}^{+}$cation by the calix-crown makes eq. 3.9 very favorable. As a result, the extraction step in the ESS is also enhanced by the SBP (Table 3.17).

It may be seen from the following metathesis reaction how the nitrate salt of TOA, namely $[\mathrm{TOAH}]_{\text {org }}^{+} \mathrm{NO}_{3}{ }^{-}$, allows the $\mathrm{Cs}^{+}$ion to be stripped even in the presence of limited concentrations of a lipophilic anion: 36

$$
[\mathrm{CsB}]^{+} \mathrm{A}_{\text {org }}^{-}+[\mathrm{TOAH}]^{+} \mathrm{NO}_{3}^{-}{ }_{\text {org }} \rightleftharpoons[\mathrm{CsB}]^{+} \mathrm{NO}_{3}^{-}{ }_{\text {org }}+[\mathrm{TOAH}]^{+}{ }_{\text {org }} \mathrm{A}^{-}
$$

In this equilibrium, the $\mathrm{Cs}^{+}$complex with BOBCalixC6 has been set off in brackets for clarity. The resulting $[\mathrm{CsB}]^{+} \mathrm{NO}_{3}{ }^{-}$org complex salt is free to be stripped according to the reverse of eq. 3.6. Naturally, the TOA at $1 \mathrm{mM}$ sets a limit on the tolerance of the solvent for lipophilic anions like SBP-

Table 3.17. Effect of 4-sec-butylphenol concentration on Cs distribution ratios in ESS tests ${ }^{a}$

\begin{tabular}{lcccc}
\hline \multirow{2}{*}{ Process step } & \multicolumn{4}{c}{$D_{\mathrm{Cs}}$} \\
& $0 \mathrm{mM}$ SBP & $0.3 \mathrm{mM} \mathrm{SBP}$ & $3.0 \mathrm{mM}$ SBP & $30.0 \mathrm{mM}$ SBP \\
\hline Extraction & 16.1 & 17.2 & 17.1 & 24.8 \\
Scrub & 1.59 & 1.57 & 1.57 & 49.4 \\
Strip \#1 & 0.151 & 0.151 & 0.154 & 21.1 \\
Strip \#2 & 0.087 & 0.088 & 0.090 & 16.6 \\
Strip \#3 & 0.065 & 0.066 & 0.068 & 9.8 \\
\hline
\end{tabular}

${ }^{a} \mathrm{O} / \mathrm{A}$ ratio was 0.33 on extraction and 5 on scrubbing and stripping; $T=25^{\circ} \mathrm{C}$.

At $30 \mathrm{mM}$ of SBP largely converted to its phenolate form, the TOA would clearly be overwhelmed. A nice feature of the TOA chemistry is that on contact with alkaline waste or $\mathrm{NaOH}$ wash solutions, the TOA is deprotonated and subsequently releases its anion to the aqueous phase (Chap. 4 ).

\subsubsection{Effect of Surfactant Anions}

Surfactant anions are commonly met impurities which potentially could be introduced into an organic solution by using, for instance, equipment washed with detergent but incompletely rinsed. It was seen above how the presence of such alkylphenolate anions in the solvent may affect $\mathrm{Cs}^{+}$ distribution behavior, and the same equilibrium relationships apply to surfactant anions. To investigate this effect further, two surfactants representing different classes of strong and weak organic acids were tested, namely sodium dodecyl sulfate (SDS) and stearic acid (SA), respectively. These surfactants were introduced into pristine washed solvent at $0.2 \mathrm{mM}$, just sufficient to start saturating the protection afforded by the TOA suppressor. Solvent solutions containing SDS or SA were carried through one ESS cycle using the full simulant at $25^{\circ} \mathrm{C}$. No emulsion formation was detected. Cesium distribution ratios for each ESS stage are collected in Table 3.18.

Comparison of the $D_{\mathrm{Cs}}$ values using solvent with and without surfactant at the $0.2 \mathrm{mM}$ level reveals little or no effect on extraction or scrubbing. However, stripping exhibits some adverse effect, especially on the first strip. Subsequent strips perform adequately, though with increased $D_{\mathrm{Cs}}$ values for SDS. The weaker acid, SA, clearly has minimal effect, as expected from the arguments made above regarding the effect of the acidity of the organic acid impurity. Based on experiments reported earlier, ${ }^{15}$ higher concentrations of SDS would quickly lead to poor stripping. Experiments reported in the next chapter, however, show how such surfactants can be removed from the solvent by $\mathrm{NaOH}$ washing or anion exchange. 
Table 3.18. Values of $D_{\mathrm{Cs}}$ determined for one ESS cycle using pristine washed solvent containing $0.2 \mathrm{mM}$ SDS or SA at $25^{\circ} \mathrm{C}$

\begin{tabular}{lccccc}
\hline \multirow{2}{*}{ Surfactant } & \multicolumn{5}{c}{$D_{\mathrm{Cs}}$} \\
\cline { 2 - 6 } & Extraction & Scrub & Strip \#1 & Strip \#2 & Strip \#3 \\
\hline SDS & 17.9 & 1.7 & 0.36 & 0.14 & 0.099 \\
SA & 16.9 & 1.5 & 0.23 & 0.087 & 0.070 \\
None $^{a}$ & 16.9 & 1.6 & 0.15 & 0.089 & 0.067 \\
\hline
\end{tabular}

${ }^{a}$ Average values from Table 3.4 .

\subsubsection{Effect of Dibutylphosphate}

Another lipophilic anion, dibutylphosphate (DBP), is present in the waste and is at its bounding concentration in the full simulant at $0.12 \mathrm{mM}(25 \mathrm{mg} / \mathrm{L})$. It readily partitions into the solvent phase, due to its moderate lipophilicity (Chap. 4). Thus, DBP potentially could build up in the solvent after several contacts with simulant. To examine the effect of DBP on cesium distribution behavior, it was deliberately introduced into pristine washed baseline solvent in the form of dibutylphosphoric acid. This solvent containing DBP was then taken through one ESS cycle at $25{ }^{\circ} \mathrm{C}$. Cesium distribution ratios obtained for each stage are listed in Table 3.19.

Table 3.19. Values of $D_{\text {Cs }}$ determined for an ESS cycle using pristine washed solvent containing dibutylphosphate at $25^{\circ} \mathrm{C}$

\begin{tabular}{ccccccc}
\hline $\begin{array}{c}{[\mathrm{DBP}]_{\text {org }}{ }^{a}} \\
(\mathrm{mM})\end{array}$ & Simulant & & \multicolumn{5}{c}{$D_{\mathrm{Cs}}$} \\
\cline { 3 - 6 } & & Extraction & Scrub & Strip \#1 & Strip \#2 & Strip \#3 \\
\hline 1.44 & Full $^{b}$ & 17.9 & 1.22 & 0.289 & 0.116 & 0.098 \\
2.00 & $\mathrm{~S}+\mathrm{M}^{c}$ & 17.0 & 1.58 & 0.221 & 0.124 & 0.110 \\
0.36 & Full $^{d}$ & 16.9 & 1.59 & 0.148 & 0.089 & 0.067 \\
None & $\mathrm{S}+\mathrm{M}^{e}$ & 16.8 & 1.46 & 0.121 & 0.072 & 0.055 \\
\hline
\end{tabular}

${ }^{a}$ Given as initial concentration relative to the solvent phase.

${ }^{b}$ Full simulant; extra DBP was added to the solvent.

${ }^{c}$ Salts + metals simulant (Chap. 2); DBP was added to the solvent.

${ }^{d}$ Full simulant; average values were taken from Table 3.4; no extra DBP added.

eSalts + metals simulant (Chap. 2); no DBP was added.

Comparison of the $D_{\mathrm{Cs}}$ values collected using solvent with and without extra DBP indicates that DBP does not significantly affect cesium distribution behavior at the extraction stage but does worsen stripping. Namely, $D_{\mathrm{Cs}}$ values are significantly higher at each stripping stage than the corresponding values obtained using the solvent without extra DBP (Table 3.4). Comparison of the results with full simulant and the salts + metals simulant reveals that the DBP content of the full simulant at $0.12 \mathrm{mM}$ noticeably worsens stripping. In accordance with discussions above, the synergistic effect of DBP could be explained considering the acidic nature of the dibutylphosphoric acid. During the contact with highly alkaline simulant solution, DBP anion becomes fully deprotonated, and the $\mathrm{Na}^{+}$ counterion is exchangeable (eq. 3.9). Like the other lipophilic anions studied above, it does not have a pronounced effect on the extraction step, which likely reflects a) the high concentration of $\mathrm{Na}^{+}$ion 
in the simulant, which competes with $\mathrm{Cs}^{+}$ion in eq. 3.9; and b) the fact that the total anion concentration in the solvent is high relative to DBP, owing to the significant extraction of $\mathrm{Na}^{+}$and $\mathrm{K}^{+}$ ions (Chap. 4). On scrubbing and stripping, the competition due to the aqueous $\mathrm{Na}^{+}$ions is minor or absent, and the other anions are washed out, intensifying the effect of the remaining DBP anions. Moreover, DBP has an aqueous $\mathrm{pKa}$ of $1.72^{57}$ and hence resists protonation under the mild, acidic conditions of scrubbing and stripping. Thus, at the scrub and strip stages, DBP plays the role of a lipophilic counteranion, which holds $\mathrm{Cs}^{+}$in the solvent phase.

To better understand the effect of DBP on cesium distribution behavior in stripping, a series of forward-stripping experiments was conducted at $25{ }^{\circ} \mathrm{C}$ (Fig. 3.2). In this study, the performance of the solvent was characterized by systematic variation of the DBP concentration $(0.3,1,3$, and $10 \mathrm{mM})$ in the solvent contacted with the stripping solution containing deliberately added $\mathrm{CsNO}_{3}$ at $0.1 \mathrm{mM}$. It is seen that increasing DBP concentration in the organic phase causes a steep increase in the value of $D_{\mathrm{Cs}}$ above $0.3 \mathrm{mM} \mathrm{DBP}$. That is, in this range the tolerance for anions afforded by the TOA is exceeded. At $3.5 \mathrm{mM} \mathrm{DBP}, D_{\mathrm{Cs}}$ value reaches unity, indicating that the stripping process becomes ineffective.

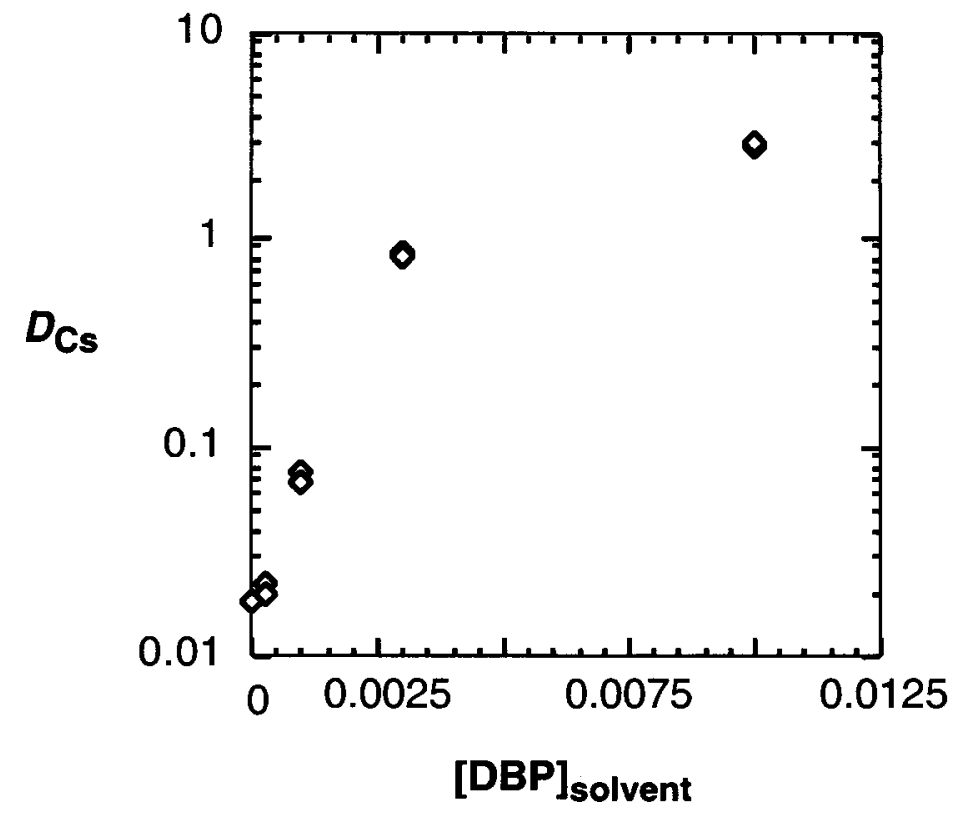

Fig. 3.2. Effect of dibutylphosphate (DBP) on forward stripping. Organic phase: washed pristine baseline solvent with variable DBP concentration. Aqueous phase: stripping solution containing $0.1 \mathrm{mM} \mathrm{CsNO}{ }_{3} . \mathrm{O} / \mathrm{A}=5 ; T=25^{\circ} \mathrm{C}$.

\subsubsection{Effect of Other Organic Species in the Full Simulant}

A survey of the behavior of $D_{\mathrm{Cs}}$ in ESS tests perturbed by the presence of various species present in the full simulant is given in Table 3.20. In this case, the salts + metals simulant was employed so that each species could be examined separately. Note that the data for the salts + metals simulant with and without DBP were also presented in Table 3.19 above, though the experiment shown in Table 3.20 was carried out as a single experiment. Except for formate, which was added at its nominal concentration in the full simulant, the organic species were added at considerably elevated concentrations: 1-butanol, 123-fold; TBP, 5320-fold; DBP, 17-fold; trimethylamine, 20-fold. 
From the data, it may be seen that, among the various organic species, only TBP causes any change in $D_{\mathrm{Cs}}$ in the extraction step. Given that the standard error of a single measurement is typically $\pm 1.4(8 \%)$, the observed $15 \%$ decrease may not be statistically significant, but it is considered real based on the chemical behavior of TBP, a base that can interact strongly by hydrogen bonding with the Cs-7SB modifier. This solvation interaction competes with the normal function of the modifier to solvate the cesium extraction complex, resulting in depressed extraction ability. Such an explanation gains further support from the fact that scrubbing and stripping performance is also depressed.

Among the other species tested, only DBP has any effect on scrubbing and stripping. Its effect was discussed at length in the previous section.

Table 3.20. Summary of the effects of organic species on cesium distribution in ESS tests ${ }^{a}$

\begin{tabular}{lcccccc}
\hline Step & $\begin{array}{c}1 \text {-Butanol } \\
(10 \mathrm{mM}) \\
D_{\mathrm{Cs}}\end{array}$ & $\begin{array}{c}\text { Trimethylamine } \\
(10 \mathrm{mM})\end{array}$ & $\begin{array}{c}\text { TBP } \\
(30 \mathrm{mM})\end{array}$ & $\begin{array}{c}\text { DBP } \\
(2 \mathrm{mM})\end{array}$ & $\begin{array}{c}\text { Formate } \\
(100 \mathrm{mM})\end{array}$ & Salts + metals \\
& $D_{\mathrm{Cs}}$ & $D_{\mathrm{Cs}}$ & $D_{\mathrm{Cs}}$ & $D_{\mathrm{Cs}}$ & $D_{\mathrm{Cs}}$ \\
\hline Extraction & 16.4 & 17.0 & 14.3 & 17.0 & 16.7 & 16.8 \\
Scrub & 1.39 & 1.41 & 1.16 & 1.58 & 1.44 & 1.46 \\
Strip \#1 & 0.118 & 0.120 & 0.096 & 0.221 & 0.120 & 0.121 \\
Strip \#2 & 0.068 & 0.070 & 0.056 & 0.124 & 0.071 & 0.072 \\
Strip \#3 & 0.057 & 0.056 & 0.044 & 0.110 & 0.055 & 0.055 \\
\hline
\end{tabular}

${ }^{a}$ Concentrations of organic species are given relative to solvent phase. Extraction $\mathrm{O} / \mathrm{A}=$ 0.33 , scrub and strip $\mathrm{O} / \mathrm{A}=5$; baseline solvent; salts + metals simulant; $T=25^{\circ} \mathrm{C}$.

\subsubsection{Trials of Different Simulant Recipes}

Based on input from the SRTC, ${ }^{58}$ five simple recipes corresponding to SRS tank supernatant wastes were prepared (see Table 3.21). It may be noted that the potassium concentration of one of them (Tank 13-H) exceeds the bounding concentration of 0.050 M. ${ }^{54}$ An ESS protocol using the baseline solvent with each simulant with and without ${ }^{137} \mathrm{Cs}$ tracer was performed to evaluate the effect of disparate salt concentrations on $D_{\mathrm{Cs}}$ at each stage. In addition, the selectivity of cesium over potassium and sodium was examined. For comparison, parallel measurements were performed on the full simulant.

Distribution results for $\mathrm{Na}^{+}, \mathrm{K}^{+}$, and $\mathrm{Cs}^{+}$ions in the extraction step of the ESS test $(\mathrm{O} / \mathrm{A}=0.33)$ are shown in Table 3.22. With one exception (tank $13-\mathrm{H})$, the $D_{\mathrm{Cs}}$ values are fairly constant. The single exception involves high nitrite concentration but low nitrate, needed for driving force. Its $D_{\mathrm{Cs}}$ value is low (tank 13-H), though it still exceeds the needed value of 8 . Extraction of competing potassium ion is significant overall, and its loading of the calixarene apparently reaches as high as $71 \%$ without loss of extraction strength. It may also be seen that sodium is weakly extracted relative to its large aqueous concentration. Nevertheless, the sodium concentration in the solvent reaches 4.2 $\mathrm{mM}$ (from full simulant), translating to over $20 \mathrm{mM}$ in the first aqueous scrub solution. Detailed treatment of these data by computer modeling is described elsewhere. 25

Complete ESS results, describing the pathway of $\mathrm{Na}^{+}, \mathrm{K}^{+}$, and $\mathrm{Cs}^{+}$ions through the contacting steps, are given in Table 3.23. Due to the low concentrations of the first two of these ions in the aqueous phase of the third strip step, only the first two strips were analyzed by ICP-AES. The effectiveness of the scrub step in preventing significant concentrations of $\mathrm{Na}^{+}$and $\mathrm{K}^{+}$ions from proceeding into stripping may be appreciated. It may be noted that the concentration of these ions in 
Table 3.21. Simulant recipes

\begin{tabular}{ccccccc}
\hline Tank no. & {$\left[\mathrm{Na}^{+}\right]$} & {$\left[\mathrm{K}^{+}\right]$} & {$\left[\mathrm{Cs}^{+}\right]$} & {$\left[\mathrm{OH}^{-}\right]$} & {$\left[\mathrm{NO}_{3}{ }^{-}\right]$} & {$\left[\mathrm{NO}_{2}^{-}\right]$} \\
\hline $13-\mathrm{H}$ & 5.6 & 0.067 & $5.12 \times 10^{-4}$ & 2.29 & 0.767 & 2.6 \\
$26-\mathrm{F}$ & 5.6 & 0.041 & $2.19 \times 10^{-4}$ & 4.71 & 0.956 & 0 \\
$33-\mathrm{F}$ & 3.3 & 0.005 & $8.03 \times 10^{-6}$ & 1.47 & 1.44 & 0.40 \\
$35-\mathrm{H}$ & 5.6 & 0.010 & $1.88 \times 10^{-4}$ & 2.93 & 1.40 & 1.3 \\
$46-\mathrm{F}$ & 5.6 & 0.032 & $3.78 \times 10^{-4}$ & 3.98 & 0.606 & 1.0 \\
Full simulant $^{a}$ & 5.6 & 0.020 & $1.40 \times 10^{-4}$ & 2.06 & 2.03 & 0.50 \\
\hline
\end{tabular}

${ }^{a}$ The full simulant described in Table 2.2 was used. Only the selected ions are shown here.

Table 3.22. Effect of simulant recipe on the distribution of sodium, potassium, and cesium in the extraction step of an ESS with baseline solvent at $25^{\circ} \mathrm{C}$

\begin{tabular}{ccccccc}
\hline Tank no. & $D_{\mathrm{Na}}$ & {$\left[\mathrm{Na}^{+}\right]_{\text {org }}$} & \multicolumn{1}{c}{$D_{\mathrm{K}}$} & {$\left[\mathrm{K}^{+}\right]_{\text {org }}$} & $D_{\mathrm{Cs}}$ & {$\left[\mathrm{Cs}^{+}\right]_{\text {org }}$} \\
\hline $13-\mathrm{H}$ & $6.08 \times 10^{-4}$ & $3.40 \times 10^{-3}$ & 0.080 & $5.22 \times 10^{-3}$ & 8.4 & $1.13 \times 10^{-3}$ \\
$26-\mathrm{F}$ & $1.27 \times 10^{-3}$ & $7.11 \times 10^{-3}$ & 0.183 & $7.07 \times 10^{-3}$ & 16.8 & $5.58 \times 10^{-4}$ \\
$33-\mathrm{F}$ & $4.23 \times 10^{-4}$ & $1.40 \times 10^{-3}$ & 0.111 & $5.35 \times 10^{-4}$ & 16.3 & $2.04 \times 10^{-5}$ \\
$35-\mathrm{H}$ & $9.01 \times 10^{-4}$ & $5.04 \times 10^{-3}$ & 0.232 & $2.15 \times 10^{-3}$ & 22.7 & $4.99 \times 10^{-4}$ \\
$46-\mathrm{F}$ & $1.02 \times 10^{-3}$ & $5.71 \times 10^{-3}$ & 0.185 & $5.58 \times 10^{-3}$ & 17.7 & $9.70 \times 10^{-4}$ \\
Full simulant & $7.56 \times 10^{-4}$ & $4.23 \times 10^{-3}$ & 0.123 & $2.36 \times 10^{-3}$ & 17.8 & $3.60 \times 10^{-4}$ \\
\hline
\end{tabular}

the aqueous phase in scrubbing is significant in relation to the initial nitrate concentration. Although only a minor concentration of these ions appears in the aqueous strip solutions, there is sufficient nitrate concentration associated with these components relative to the $1-\mathrm{mM}$ initial nitric acid concentration to increase $D_{C s}$ in stripping.

The ability of cesium to be removed in the fourth strip appeared to correlate with the initial amount of sodium nitrite in the tank recipe. This linear correlation is diagrammed in Fig. 3.3. Excluded from the plot were data from tank 13-H, which exhibited poor stripping. The reason for the poor stripping is not understood at this time. It appears to be real, as suggested by the correlation in Fig. 3.3.

That nitrite has an elevating effect on stripping is shown in Table 3.24. In the experiment, $\mathrm{Cl}^{-}$ion was substituted for $\mathrm{NO}_{2}^{-}$ion in the recipe without otherwise changing the composition. It may be seen that stripping has returned to near normal behavior. Apparently, nitrite also adds some driving force for extraction, as substitution of the less extractable chloride ion is seen to decrease $D_{\mathrm{Cs}}$ on extraction. This observation is borne out by further data shown in the next chapter. 
Table 3.23. Detailed distribution results for effect of simulant recipe ${ }^{a}$

\begin{tabular}{|c|c|c|c|c|c|c|c|c|}
\hline Tank & 13-H & $(\mathrm{M})$ & Stages & $D_{\mathrm{Cs}}$ & {$\left[\mathrm{K}^{+}\right]_{\text {org }}$} & {$\left[\mathrm{K}^{+}\right]_{\mathrm{aq}}$} & {$\left[\mathrm{Na}^{+}\right]_{\text {org }}$} & {$\left[\mathrm{Na}^{+}\right]_{\mathrm{ag}}$} \\
\hline$\left[\mathrm{Na}^{+}\right]$ & & 5.6 & Extraction & 8.38 & $5.2 \times 10^{-3}$ & $6.5 \times 10^{-2}$ & $3.4 \times 10^{-3}$ & 5.6 \\
\hline$\left[\mathrm{K}^{+}\right]$ & & 0.067 & Scrub & 2.09 & $3.1 \times 10^{-4}$ & $2.6 \times 10^{-2}$ & BDL & $\mathrm{BDL}$ \\
\hline$\left[\mathrm{Cs}^{+}\right]$ & & $5.12 \times 10^{-4}$ & Strip \#1 & 0.425 & $1.2 \times 10^{-6}$ & $1.5 \times 10^{-3}$ & $\mathrm{BDL}$ & $\mathrm{BDL}$ \\
\hline$\left[\mathrm{OH}^{-}\right]$ & & 2.29 & Strip \#2 & 0.348 & & & & \\
\hline$\left[\mathrm{NO}_{3}{ }^{-}\right]$ & & 0.767 & Strip \#3 & 0.389 & & & & \\
\hline$\left[\mathrm{NO}_{2}^{-}\right]$ & & 2.6 & Strip \#4 & 0.491 & & & & \\
\hline \multicolumn{9}{|c|}{ Tank 26-F } \\
\hline$\left[\mathrm{Na}^{+}\right]$ & & 5.6 & Extraction & 16.83 & $7.1 \times 10^{-3}$ & $3.9 \times 10^{-2}$ & $7.1 \times 10^{-3}$ & 5.6 \\
\hline$\left[\mathrm{K}^{+}\right]$ & & 0.041 & Scrub & 1.52 & $3.4 \times 10^{-5}$ & $3.5 \times 10^{-2}$ & $6.3 \times 10^{-7}$ & $3.5 \times 10^{-2}$ \\
\hline$\left[\mathrm{Cs}^{+}\right]$ & & $2.19 \times 10^{-4}$ & Strip \#1 & 0.143 & $1.7 \times 10^{-6}$ & $1.7 \times 10^{-4}$ & BDL & $\mathrm{BDL}$ \\
\hline$\left[\mathrm{OH}^{-}\right]$ & & 4.71 & Strip \#2 & 0.049 & & & & \\
\hline$\left[\mathrm{NO}_{3}^{-}\right]$ & & 0.956 & Strip \#3 & 0.027 & & & & \\
\hline$\left[\mathrm{NO}_{2}^{-}\right]$ & & 0.0 & Strip \#4 & 0.027 & & & & \\
\hline \multicolumn{9}{|c|}{ Tank 33-F } \\
\hline$\left[\mathrm{Na}^{+}\right]$ & & 3.3 & Extraction & 16.29 & $5.3 \times 10^{-4}$ & $4.8 \times 10^{-3}$ & $1.4 \times 10^{-3}$ & 3.3 \\
\hline$\left[\mathrm{K}^{+}\right]$ & & 0.005 & Scrub & 1.53 & $3.5 \times 10^{-6}$ & $2.7 \times 10^{-3}$ & $7.3 \times 10^{-7}$ & $7.0 \times 10^{-3}$ \\
\hline$\left[\mathrm{Cs}^{+}\right]$ & & $8.03 \times 10^{-6}$ & Strip \#1 & 0.090 & $4.8 \times 10^{-7}$ & $1.8 \times 10^{-5}$ & BDL & BDL \\
\hline$\left[\mathrm{OH}^{-}\right]$ & & 1.47 & Strip \#2 & 0.069 & & & & \\
\hline$\left[\mathrm{NO}_{3}^{-}\right]$ & & 1.440 & Strip \#3 & 0.060 & & & & \\
\hline$\left[\mathrm{NO}_{2}^{-}\right]$ & & 0.40 & Strip \#4 & 0.055 & & & & \\
\hline \multicolumn{9}{|c|}{ Tank 35-H } \\
\hline$\left[\mathrm{Na}^{+}\right]$ & & 5.6 & Extraction & 22.68 & $2.2 \times 10^{-3}$ & $9.3 \times 10^{-3}$ & $5.0 \times 10^{-3}$ & 5.6 \\
\hline$\left[\mathrm{K}^{+}\right]$ & & 0.010 & Scrub & 1.83 & $1.2 \times 10^{-5}$ & $1.1 \times 10^{-2}$ & $7.1 \times 10^{-7}$ & $2.5 \times 10^{-2}$ \\
\hline$\left[\mathrm{Cs}^{+}\right]$ & & $1.88 \times 10^{-4}$ & Strip \#1 & 0.204 & $1.1 \times 10^{-6}$ & $6.2 \times 10^{-5}$ & $\mathrm{BDL}$ & BDL \\
\hline$\left[\mathrm{OH}^{-}\right]$ & & 2.93 & Strip \#2 & 0.130 & & & & \\
\hline$\left[\mathrm{NO}_{3}{ }^{-}\right]$ & & 1.400 & Strip \#3 & 0.111 & & & & \\
\hline$\left[\mathrm{NO}_{2}^{-}\right]$ & & 1.3 & Strip \#4 & 0.094 & & & & \\
\hline \multicolumn{9}{|c|}{ Tank 46-F } \\
\hline$\left[\mathrm{Na}^{+}\right]$ & & 5.6 & Extraction & 17.68 & $5.6 \times 10^{-3}$ & $3.0 \times 10^{-2}$ & $5.7 \times 10^{-3}$ & 5.6 \\
\hline$\left[\mathrm{K}^{+}\right]$ & & 0.032 & Scrub & 2.07 & $2.0 \times 10^{-5}$ & $2.8 \times 10^{-2}$ & $4.7 \times 10^{-7}$ & $2.8 \times 10^{-2}$ \\
\hline$\left[\mathrm{Cs}^{+}\right]$ & & $3.78 \times 10^{-4}$ & Strip \#1 & 0.369 & $4.6 \times 10^{-6}$ & $1.0 \times 10^{-4}$ & $\mathrm{BDL}$ & $\mathrm{BDL}$ \\
\hline$\left[\mathrm{OH}^{-}\right]$ & & 3.98 & Strip \#2 & 0.201 & & & & \\
\hline$\left[\mathrm{NO}_{3}^{-}\right]$ & & 0.606 & Strip \#3 & 0.132 & & & & \\
\hline$\left[\mathrm{NO}_{2}^{-}\right]$ & & 1.0 & Strip \#4 & 0.083 & & & & \\
\hline \multicolumn{9}{|c|}{ Full simulant } \\
\hline$\left[\mathrm{Na}^{+}\right]$ & & 5.6 & Extraction & 17.83 & $2.3 \times 10^{-3}$ & $1.9 \times 10^{-2}$ & $4.2 \times 10^{-3}$ & 5.6 \\
\hline$\left[\mathrm{K}^{+}\right]$ & & 0.015 & Scrub & 1.58 & $6.8 \times 10^{-5}$ & $1.2 \times 10^{-2}$ & $\mathrm{BDL}$ & BDL \\
\hline$\left[\mathrm{Cs}^{+}\right]$ & & $1.40 \times 10^{-4}$ & Strip \#1 & 0.154 & $2.4 \times 10^{-6}$ & $3.4 \times 10^{-4}$ & BDL & BDL \\
\hline$\left[\mathrm{OH}^{-}\right]$ & & 2.06 & Strip \#2 & 0.096 & & & & \\
\hline$\left[\mathrm{NO}_{3}{ }^{-}\right]$ & & 2.030 & Strip \#3 & 0.079 & & & & \\
\hline$\left[\mathrm{NO}_{2}^{-}\right]$ & & 0.500 & Strip \#4 & 0.062 & & & & \\
\hline
\end{tabular}

${ }^{a}$ Detection limits for analysis of potassium and sodium were $4.6 \times 10^{-7} \mathrm{M}$ and $1.0 \times 10^{-7} \mathrm{M}$, respectively. Blanks in the table indicate that a measurement was not performed. 


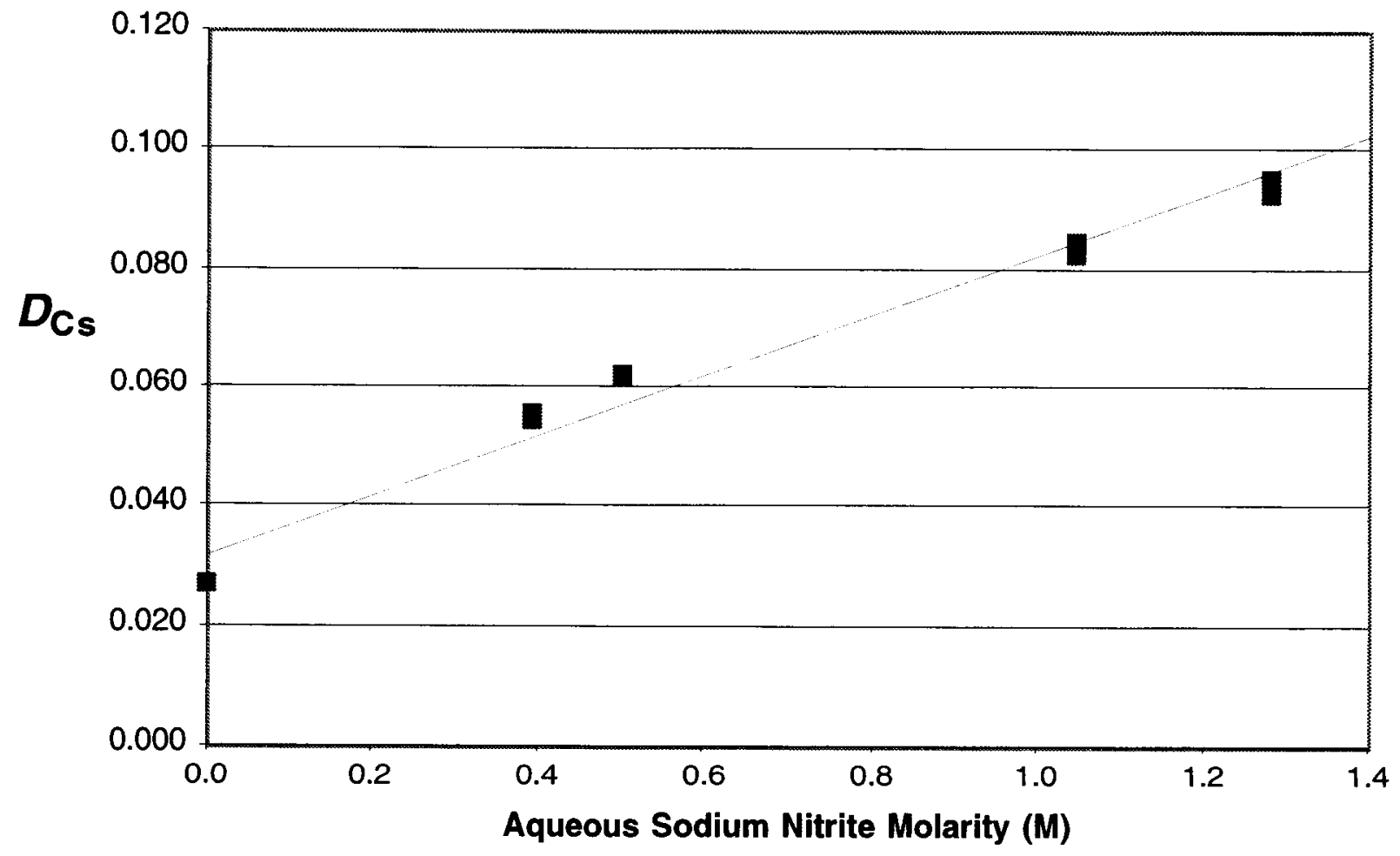

Fig. 3.3. Cesium distribution in the fourth strip of the ESS protocol as a function of $\mathrm{NaNO}_{2}$ concentration in tank simulants. The line shown is from linear regression $\left(r^{2}=0.981\right)$.

Table 3.24. Effect of substituting chloride for nitrite in simulant recipes

\begin{tabular}{lcll}
\hline \multicolumn{1}{c}{ Tank \#/Ion } & $(\mathrm{M})$ & \multicolumn{1}{c}{ Stages } & $D_{\mathrm{Cs}}$ \\
\hline Tank 13-H & & & \\
{$\left[\mathrm{Na}^{+}\right]$} & 5.6 & Extraction & 6.68 \\
{$\left[\mathrm{~K}^{+}\right]$} & 0.067 & Scrub & 1.20 \\
{$\left[\mathrm{Cs}^{+}\right]$} & $5.12 \times 10^{-4}$ & Strip \#1 & 0.197 \\
{$\left[\mathrm{OH}^{-}\right]$} & 2.29 & Strip \#2 & 0.115 \\
{$\left[\mathrm{NO}_{3}^{-}\right]$} & 0.767 & Strip \#3 & 0.093 \\
{$\left[\mathrm{Cl}^{-}\right]$} & 2.6 & Strip \#4 & 0.063 \\
Tank 46-F & & & \\
{$\left[\mathrm{Na}^{+}\right]$} & 5.6 & Extraction & 15.43 \\
{$\left[\mathrm{~K}^{+}\right]$} & 0.015 & Scrub & 1.29 \\
{$\left[\mathrm{Cs}^{+}\right]$} & $1.40 \times 10^{-4}$ & Strip \#1 & 0.162 \\
{$\left[\mathrm{OH}^{-}\right]$} & 2.06 & Strip \#2 & 0.084 \\
{$\left[\mathrm{NO}_{3}^{-}\right]$} & 2.030 & Strip \#3 & 0.067 \\
{$\left[\mathrm{Cl}^{-}\right]$} & 0.500 & Strip \#4 & 0.035 \\
\hline
\end{tabular}


The previous experiments showed a definite relation between the stripping performance and the initial concentration of nitrite in the simulant. To investigate this aspect further, spikes of sodium nitrite were added at the scrub stage to check if this addition impacted the subsequent steps in any way. Accordingly, a large volume of baseline solvent was contacted with the full simulant $(\mathrm{O} / \mathrm{A}=$ $0.33,25^{\circ} \mathrm{C}$ ). After centrifugation and subsampling, the contacted solvent was split into four different vials and contacted with the scrub solution in which a known amount of sodium nitrite had been added. The scrub stage was followed by four strip stages. Except for the addition of sodium nitrite, the entire ESS protocol was conducted as usual. The results summarized in Table 3.25 show that the addition of sodium nitrite at the scrub stage does not produce any stripping impairment and suggests that only the nitrite present in the initial alkaline media has an effect.

Table 3.25. Effect of adding nitrite in the scrub step

\begin{tabular}{ccccccc}
\hline $\mathrm{NaNO}_{2}$ conc. in scrub & Extraction & Scrub & Strip \#1 & Strip \#2 & Strip \#3 & Strip \#4 \\
\hline No addition & 17.6 & 1.60 & 0.156 & 0.085 & 0.072 & 0.063 \\
$1 \mathrm{mM}$ & 17.2 & 1.57 & 0.162 & 0.097 & 0.074 & 0.060 \\
$10 \mathrm{mM}$ & 17.6 & 1.65 & 0.157 & 0.090 & 0.071 & 0.062 \\
$100 \mathrm{mM}$ & 18.0 & 2.19 & 0.166 & 0.093 & 0.070 & 0.062 \\
\hline
\end{tabular}

\subsubsection{Effect of Multiple Solvent Contacts with Simulant}

In Section 3.3.7, it was observed that on two successive contacts of a volume of solvent with fresh full simulant at $\mathrm{O} / \mathrm{A}=0.33$, subsequent stripping was slightly less effective, suggesting the buildup of an anionic component in the solvent. Worsening stripping upon cycling the solvent was indeed confirmed by the test involving multiple cycles described in the next Section (3.3.16). It was found that the cesium distribution ratios tended to increase from one cycle to the next, particularly in the stripping stages. A test designed to reproduce this effect, but with much less experimental effort, was performed by contacting pristine solvent four times with fresh simulant, then running an ESS protocol with six strip contacts (Table 3.26 and Fig. 3.4). Hence, the sequence is designated $\mathrm{E}_{5} \mathrm{SS}_{6}$. This experiment was run with four different simulants (Table 2.2): salts only, salts + metals, full simulant, and SRS\#2. The simulant SRS\#2 was used in FY 1999 for most of the tests, including multiple cycles, and the solvent was shown to be recyclable over 10 cycles with inclusion of a $\mathrm{NaOH}$ wash each cycle. The use of the other three simulants was to detect the particular set of simulant components associated with the appearance of the loss of stripping performance, since the simulants were prepared successively. The results obtained after the $\mathrm{E}_{5} \mathrm{SS}_{6}$ protocol are presented in Table 3.26 and Fig. 3.4. The reader is also referred to Section 4.3.3.7, in which $\mathrm{Cs}^{+}, \mathrm{K}^{+}$, and $\mathrm{Na}^{+}$ distribution between the baseline solvent and the full simulant is measured at two O/A ratios with successive contacting up to 10 contacts. 


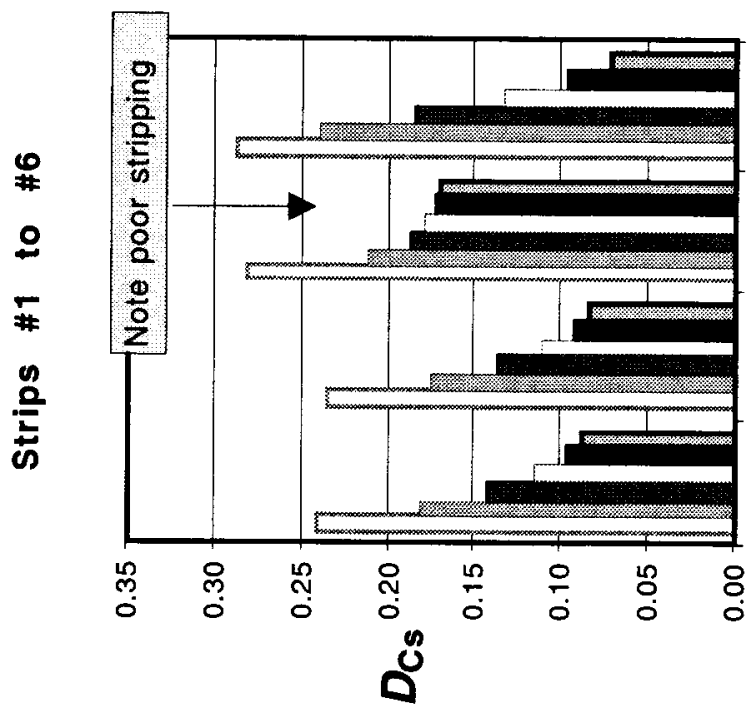

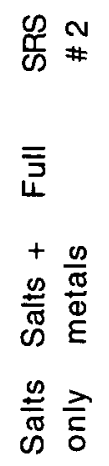

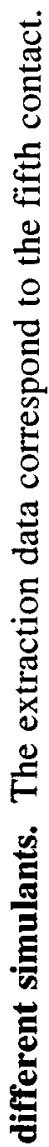
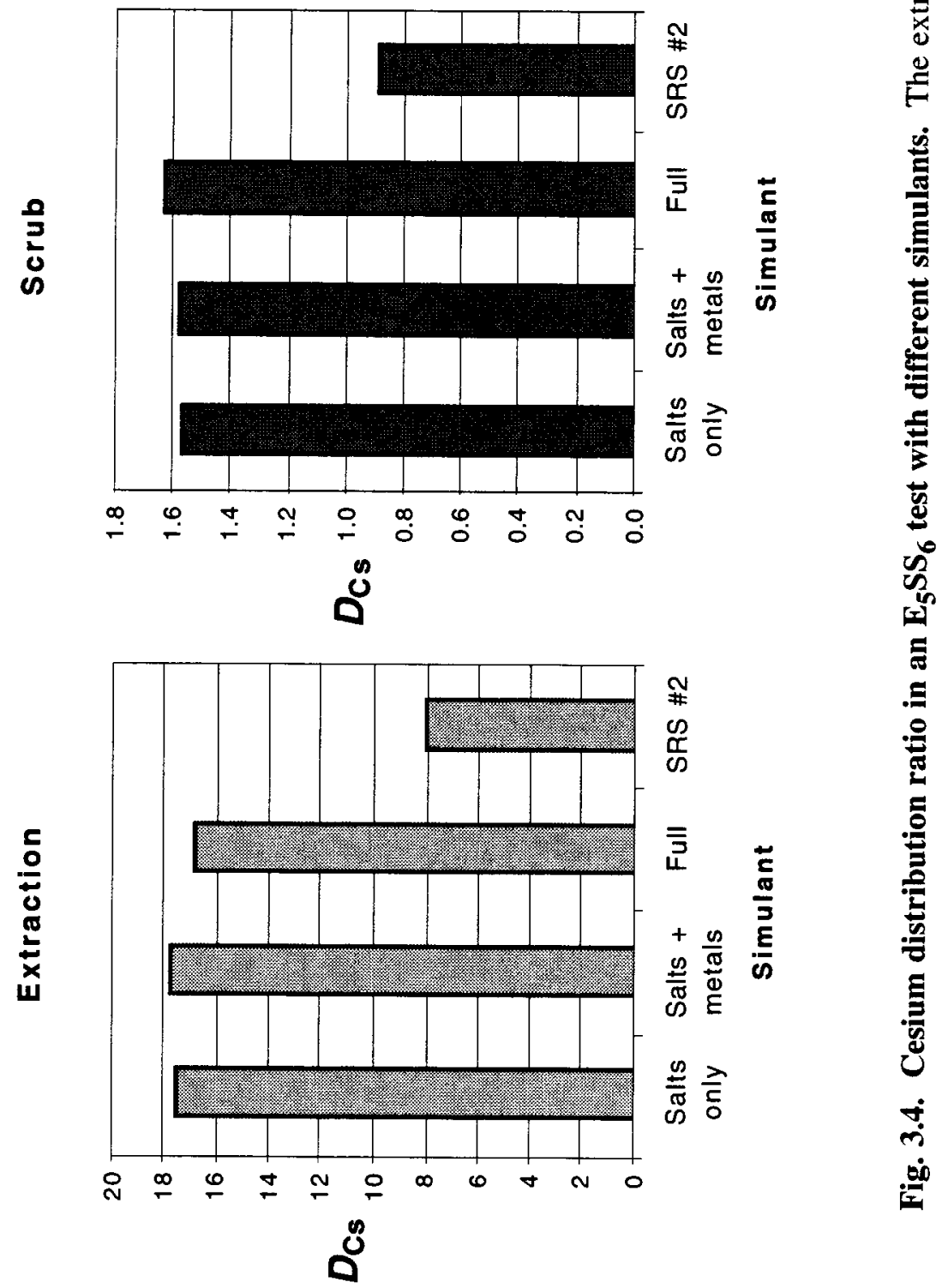
Table 3.26. Cesium distribution in an $\mathrm{E}_{5} \mathrm{SS}_{6}$ test with different simulants

\begin{tabular}{lccccccccc}
\hline & Extraction $^{a}$ & Scrub & Strip \#1 & Strip \#2 & Strip \#3 & Strip \#4 & Strip \#5 & Strip \#6 \\
\hline Salts on y & 17.5 & 1.57 & 0.241 & 0.181 & 0.143 & 0.115 & 0.097 & 0.088 \\
Salts + netals & 17.7 & 1.58 & 0.236 & 0.174 & 0.138 & 0.111 & 0.094 & 0.082 \\
Full & 16.9 & 1.63 & 0.282 & 0.212 & 0.189 & 0.179 & 0.173 & 0.169 \\
SRS\#2 & 8.12 & 0.89 & 0.288 & 0.240 & 0.186 & 0.132 & 0.097 & 0.072 \\
Full (avg. $\left.: \mathrm{SS}_{5}\right)^{b}$ & 16.9 & 1.59 & 0.148 & 0.089 & 0.067 & 0.058 & 0.049 & \\
Full, ESS & $0.7 \mathrm{mM} \mathrm{Cs}^{c}$ & 14.5 & 1.19 & 0.225 & 0.157 & 0.111 & 0.081 & 0.061 & \\
\hline
\end{tabular}

${ }^{a}$ Except as noted for the last line, these values correspond to the fifth contact..

${ }^{b}$ Averag: values from $\mathrm{ESS}_{5}$ sequence as taken from Table 3.4

$c$ Taken from Table 3.7 for the case of an $\mathrm{ESS}_{5}$ sequence performed using full simulant containing $(.70 \mathrm{mM}$ Cs.

It may he noted from the above data that the extraction and scrub $D_{\mathrm{Cs}}$ values for the SRS\#2 simulant are much lower than those observed for the more complete simulants studied in this work. The much lower $D_{\mathrm{Cs}}$ values are attributable to the high $\mathrm{Cs}^{+}$ion concentration $(0.7 \mathrm{mM})$ in the SRS\#2 simulant vs. $0.14 \mathrm{mM}$ in the other simulants. Obviously, five contacts gives rise to significant loading. However, even at such a high loading, the required $D_{\mathrm{Cs}}$ value of 8 is still obtained.

The results show very clearly that the only stripping behavior strongly impacted by the multiple contacts involve those stripping $D_{\mathrm{Cs}}$ values that were obtained starting with the full simulant. The values on the initial strip are high mainly due to the large concentration of nitrate extracted along with the alkiti cations, but in subsequent stripping, the $D_{\mathrm{Cs}}$ values decrease rapidly and level off to expected values for all cases except the full simulant. Therefore, the problem lies primarily in the presence of the lipophilic species present in the full simulant, most likely the dibutylphosphate that can build-uf and then retain cesium in the organic phase. It may be noted on close inspection, however, that stripping performance after five extraction contacts is not exactly equivalent to what is obtained after a single extraction contact with elevated cesium concentration. Even with the salts-only simulant, the stripping $D_{\mathrm{Cs}}$ values take longer to recover upon successive stripping. Overall, the experiment leads to the need to understand the partitioning of species in the system and possible means to wash these organic species from the solvent.

\subsubsection{Multi-Cycle Behavior}

Since the CSSX process is designed to reuse the solvent many times, it is essential to understand the behavior of the solvent upon repeated cycles. Previous data showed that up to 10 batch cycles of the second-generation solvent could be carried out with no apparent impact on cesium distribution. 14 In that experiment, a less complex waste simulant (SRS\#2, Table 2.2) was employed, and a $\mathrm{W}_{2} \mathrm{E}_{2} \mathrm{~S}_{2} \mathrm{~S}_{4}$ protocol was performed as follows (all contacts at $1: 1$ phase ratio at $25^{\circ} \mathrm{C}$ ): two washes with $0.5^{\mathrm{M}}$ $\mathrm{NaOH}$, two extractions from SRS\#2 simulant, two scrubs with $50 \mathrm{mM}$ nitric acid, and four strips with $1 \mathrm{mM}$ nitric acid. This protocol was highly favorable for recyclability, involving a solvent cleanup (at the time it was termed solvent conditioning) and use of a simpler simulant. For the present experiment, it was planned to withhold washing and to use the full simulant, where the presence of the additional minor metals and organic species would provide a more rigorous assessment of recyclability and need for solvent washing. Further information on this multi-cycle experiment is provided in Chaps. 4 (partitioning) and 8 (solvent cleanup). It should be noted that critical information on solvent cycling is also emerging from CSSX multistage centrifugal contactor tests being conducted as part of the SRS Salt Processing Project. ${ }^{19,42}$ In this regard, batch testing was 
fruitful as a prelude to the contactor tests and in fact suggested the benefit of the solvent-washing stage added in the extended simulant ${ }^{67}$ and real-waste tests. ${ }^{68}$

The data shown in Table 3.27 indicate that stripping becomes increasingly less effic ent with increasing number of cycles. By the third cycle, the value of $D_{\mathrm{Cs}}$ on the first strip is at or near the threshold value of 0.2 for pinching. The value of $D_{\mathrm{Cs}}$ for extraction shows a slight tendency to increase with cycling, and the value of $D_{\mathrm{Cs}}$ for scrubbing clearly increases. Based on the effect of organic anions observed above and on experience with impurity effects in the development of

Table 3.27. Cesium distribution ratios in the five-cycle experiment ${ }^{a}$

\begin{tabular}{|c|c|c|c|c|c|c|c|c|c|c|c|}
\hline Cycle & & Extract & Scrub & $\begin{array}{c}\text { Strip } \\
\# 1\end{array}$ & $\begin{array}{c}\text { Strip } \\
\# 2\end{array}$ & $\begin{array}{c}\text { Strip } \\
\# 3\end{array}$ & $\begin{array}{c}\text { Strip } \\
\# 4\end{array}$ & $\begin{array}{c}\text { Strip } \\
\# 5\end{array}$ & $\begin{array}{c}\text { Strip } \\
\# 6\end{array}$ & $\begin{array}{c}\text { Strip } \\
\# 7\end{array}$ & $\begin{array}{c}\text { Strip } \\
\# 8\end{array}$ \\
\hline \multirow[t]{4}{*}{$\# 1$} & A & 18.7 & 1.63 & 0.155 & 0.094 & 0.071 & 0.061 & & & & \\
\hline & B & 18.4 & 1.64 & 0.153 & 0.094 & 0.072 & 0.060 & & & & \\
\hline & $\mathrm{C}$ & 18.8 & 1.63 & 0.154 & 0.091 & 0.070 & 0.061 & & & & \\
\hline & Avg. & 18.6 & 1.63 & 0.154 & 0.093 & 0.071 & 0.061 & & & & \\
\hline \multirow{6}{*}{$=--$} & $\underline{\sigma}$. & 0.2 & _0.01 & $\underline{0.00} \underline{1}$ & -0.002 & $\underline{0.00} \underline{1}$ & 0.001 & & & & \\
\hline & A & 17.6 & 1.81 & 0.179 & 0.103 & 0.083 & 0.070 & & & & \\
\hline & B & 17.3 & 1.76 & 0.178 & 0.106 & 0.082 & 0.072 & & & & \\
\hline & $\mathrm{C}$ & 17.4 & 1.78 & 0.177 & 0.104 & 0.080 & 0.071 & & & & \\
\hline & Avg. & 17.4 & 1.78 & 0.178 & 0.104 & 0.082 & 0.071 & & & & \\
\hline & $\underline{\sigma}$ & 0.2 & -0.03 & $\underline{0.001}$ & -0.002 & $\underline{0.002}$ & 0.001 & & & & \\
\hline \multirow[t]{5}{*}{ \#3 } & A & 17.9 & 1.99 & 0.207 & 0.120 & 0.095 & 0.081 & & & & \\
\hline & B & 19.4 & 1.96 & 0.204 & 0.122 & 0.096 & 0.085 & & & & \\
\hline & $\mathrm{C}$ & 18.1 & 1.96 & 0.206 & 0.122 & 0.099 & 0.082 & & & & \\
\hline & Avg. & 18.4 & 1.97 & 0.206 & 0.121 & 0.097 & 0.083 & & & & \\
\hline & $\sigma_{-}$ & 0.8 & -0.02 & $\underline{0.002}$ & -0.001 & $\underline{0.002}$ & 0.002 & & & & \\
\hline \multirow[t]{5}{*}{ \#5 } & A & 20.1 & 2.26 & 0.273 & 0.168 & 0.151 & 0.138 & 0.126 & 0.120 & 0.120 & 0.122 \\
\hline & B & 20.2 & 2.21 & 0.271 & 0.165 & 0.150 & 0.133 & & & & \\
\hline & $\mathrm{C}$ & 19.6 & 2.24 & 0.273 & 0.165 & 0.148 & 0.135 & & & & \\
\hline & Avg. & 20.0 & 2.24 & 0.272 & 0.166 & 0.150 & 0.135 & & & & \\
\hline & $\sigma$ & 0.3 & 0.03 & 0.001 & 0.002 & 0.002 & 0.003 & & & & \\
\hline
\end{tabular}

${ }^{a}$ Data are shown in triplicate, as indicated by rows labeled $\mathrm{A}, \mathrm{B}$, and $\mathrm{C}$. The average and standard error are also shown. Baseline solvent was used with the full simulant at the baseline $\mathrm{O} / \mathrm{A}$ ratios at $25^{\circ} \mathrm{C}$.

CSSX, ${ }^{8,14}$ a reasonable hypothesis for the increased values of $D_{\mathrm{Cs}}$ in all stages of the flowsheet entails the buildup of lipophilic anions in the solvent. Indeed, this is shown to be the case in Chap. 4, as dibutylphosphate is demonstrated to partition to the solvent. It is proposed that the reason that extraction is only slightly affected is that the concentration of cesium, potassium, and sodium in the solvent is much greater than that of the lipophilic anion impurity. Once the cations are mostly scrubbed and stripped out, however, the reverse becomes true, and stripping is thus significantly affected. Note that for the anions to have an effect requires that they have concentrations in the solvent of a few tenths of a millimolar to overcome the suppressing effect of the TOA.14 At a concentration of $0.12 \mathrm{mM}$ in the full simulant, dibutylphosphate has a sufficient concentration to 
saturate the TOA, especially upon cycling. In Chap. 8 , it is shown that washing the solvent with dilute $\mathrm{NaOH}$ solutions restores the ESS performance, as dibutylphosphate and other anions readily wash out.

Table 3.28 shows the results of an independent multi-cycle experiment performed by another individual. Only three cycles were performed, and it may be seen that agreement with the previous experiment is very good. Mass-balance data are also provided and in most cases lend further confidence in the distribution results to within approximately $\pm 5 \%$.

Table 3.28. Cesium distribution ratios in three-cycle experiment ${ }^{a}$

\begin{tabular}{ccccccc}
\hline Sample & Cycle \#1 & Mass balance & Cycle \#2 & Mass balance & Cycle \#3 & Mass balance \\
\hline Extraction & 17.5 & $93.2 \%$ & 18.3 & $92.1 \%$ & 17.4 & $97.0 \%$ \\
& 17.9 & $94.4 \%$ & 18.2 & $90.5 \%$ & 18.4 & $97.1 \%$ \\
Scrub & 1.54 & $100.9 \%$ & 1.71 & $102.6 \%$ & 1.82 & $100.6 \%$ \\
& 1.56 & $99.9 \%$ & 1.65 & $102.0 \%$ & 1.75 & $99.2 \%$ \\
Strip \#1 & 0.144 & $99.3 \%$ & 0.166 & $98.1 \%$ & 0.205 & $101.5 \%$ \\
& 0.147 & $100.0 \%$ & 0.170 & $101.0 \%$ & 0.193 & $99.1 \%$ \\
Strip \#2 & 0.089 & $105.3 \%$ & 0.098 & $113.5 \%$ & 0.118 & $124.9 \%$ \\
& 0.089 & $105.5 \%$ & 0.099 & $112.72 \%$ & 0.117 & $124.8 \%$ \\
Strip \#3 & 0.068 & $98.4 \%$ & 0.076 & $95.9 \%$ & 0.093 & $96.9 \%$ \\
& 0.069 & $97.6 \%$ & 0.077 & $98.5 \%$ & 0.094 & $97.5 \%$ \\
Strip \#4 & 0.055 & $98.8 \%$ & 0.067 & $101.4 \%$ & 0.079 & $89.6 \%$ \\
& 0.056 & $98.6 \%$ & 0.063 & $98.7 \%$ & 0.088 & $91.3 \%$ \\
\hline
\end{tabular}

${ }^{a}$ Each experiment was run in duplicate as shown.

\subsubsection{Effect of Modifier Concentration}

As observed in solvent development, the nature of the modifier and its concentration govern the ability of the solvent to extract cesium and to avoid precipitation of a third phase. ${ }^{34}$ For reasons discussed at greater length in Chap. 7, it may be advantageous to increase the Cs-7SB concentration to $0.75 \mathrm{M}$, and indeed, optimization of the solvent composition is a part of the plan for future work. 4 A comprehensive experiment to investigate multiple aspects of metal ion distribution behavior and third-phase formation was conducted and will be described in detail in Chap. 4. One purpose of this experiment was to examine the effect of the Cs-7SB modifier concentration in the solvent on cesium ion distribution behavior, and this aspect of the experiment is described here. In the experiment, the aqueous phase was the full simulant solution containing $\mathrm{Cs}^{+}$ion (added as $\mathrm{CsNO}_{3}$ ) at concentrations in the range from $1.4 \times 10^{-4} \mathrm{M}$ (baseline $\mathrm{Cs}^{+}$concentration) to $1 \times 10^{-2} \mathrm{M}$. In addition to the baseline solvent $(0.5 \mathrm{M} \mathrm{Cs-7SB})$, two other solvent compositions were employed having the same composition as the baseline solvent except that they contained 0.25 and $0.75 \mathrm{M} \mathrm{Cs-7SB}$. Extraction experiments were performed in duplicate at $25{ }^{\circ} \mathrm{C}$ and $\mathrm{O} / \mathrm{A}=0.33$. Collected $D_{\mathrm{Cs}}$ values are listed in Table 3.29.

It was observed that $D_{\mathrm{Cs}}$ values increase with increasing modifier concentration in the solvent for the entire cesium concentration range. The most pronounced enhancement $(30-40 \%)$ of $D_{\mathrm{Cs}}$ values is observed when Cs-7SB concentration is raised from 0.25 to $0.5 \mathrm{M}$. Further increase in Cs-7SB concentration to $0.75 \mathrm{M}$ results in less significant increase $(2-15 \%)$ of $D_{\mathrm{Cs}}$ values, as the effect begins to plateau. The effect of modifier concentration upon $D_{\mathrm{Cs}}$ converges in accordance with the high cesium loading achieved. 
Table 3.29. Cesium distribution ratios for full simulant containing varying cesium ion concentrations and baseline solvent with three Cs-7SB modifier concentrations at $25{ }^{\circ} \mathrm{C}$.

\begin{tabular}{cccc}
\hline$[\mathrm{Cs}]_{\text {init }}$ & \multicolumn{3}{c}{$D_{\mathrm{Cs}}$} \\
& $0.25 \mathrm{M} \mathrm{Cs-7SB}$ & $0.5 \mathrm{M} \mathrm{Cs}$-7SB & $0.75 \mathrm{M} \mathrm{Cs}-7 \mathrm{SB}$ \\
\hline $1.4 \times 10^{-4}$ & 12.9 & 17.6 & 19.8 \\
$1.4 \times 10^{-4}$ & 13.6 & 18.2 & 20.2 \\
$3.0 \times 10^{-4}$ & 11.6 & 16.8 & 17.3 \\
$3.0 \times 10^{-4}$ & 12.2 & 16.8 & 18.4 \\
$7.0 \times 10^{-4}$ & 10.5 & 15.4 & 16.5 \\
$7.0 \times 10^{-4}$ & 11.3 & 14.6 & 16.7 \\
$1.0 \times 10^{-3}$ & 8.75 & 12.0 & 13.9 \\
$1.0 \times 10^{-3}$ & 9.02 & 12.7 & 13.2 \\
$3.0 \times 10^{-3}$ & Third phase & 5.11 & 5.22 \\
$3.0 \times 10^{-3}$ & Third phase & 5.12 & 5.16 \\
$1.0 \times 10^{-2}$ & Third phase & 1.39 & 1.41 \\
$1.0 \times 10^{-2}$ & Third phase & 1.28 & 1.39 \\
\hline
\end{tabular}

As described in Chap. 2 of this report, the modifier acts as a solvating agent in the solvent. Studies reported elsewhere ${ }^{35}$ show that its primary function is to solvate the ion-pair extraction complex composed of BOBCalixC6 and extracted cesium salt. Analysis of the thermodynamics of ion-pair extraction of metal salts by crown ethers suggests that the solvation mechanism largely lies in hydrogen-bond donation of the hydroxy group of the modifier to the nitrate anion. ${ }^{59}$ X-ray structural evidence for calix[4]arene-crown-6 complexes reveals that the $\mathrm{Cs}^{+}$ion is nearly encapsulated ${ }^{33}$ and therefore could not be strongly solvated by electron-pair donation by the modifier. The modifier also solvates the BOBCalixC6 molecule, increasing its solubility (Chap. 7). Although strong solvation of the extractant tends to weaken extraction, the net effect of the added solvation of the extractant and its ion-pair complex in the solvent is added driving force for extraction.

\subsection{CONCLUSIONS}

The obvious consistent performance of the ESS tests indicates that variations in batch contacting techniques, sampling, and analytical methods in different tests conducted by different individuals were inconsequential. In addition, the consistency of results also indicates little or no evolution of performance with time, suggesting that the CSSX distribution performance is not affected by the aging of the simulant or the solvent.

No particular issues regarding the ability of the CSSX flowsheet to meet process requirements within the envelope of expected operating conditions are identified. Cesium loading up to bounding conditions is not expected to be high enough to reduce $D_{\mathrm{Cs}}$ values below the value for onset of pinching in extraction or stripping at $25^{\circ} \mathrm{C}$, and wide latitude in setting $\mathrm{O} / \mathrm{A}$ ratios is possible. In addition, even at their bounding concentration in the waste, competing potassium ions are not expected to compromise process requirements. However, at the bounding temperature of $35{ }^{\circ} \mathrm{C}$, the falling $D_{\mathrm{Cs}}$ value with increasing temperature places the $D_{\mathrm{Cs}}$ value at the critical value for pinching for the full simulant at $\mathrm{O} / \mathrm{A}=0.33$. Thus, if feeds contain higher cesium concentrations or insufficient nitrate or hydroxide, it will be necessary to make an adjustment in solvent flow rate or to 
lower the temperature of the extraction section. Likewise, solvent flowrate adjustment or temperature management in the stripping section will likely be needed if the temperature of the stripping section is at the lower bound of $20^{\circ} \mathrm{C}$, especially if cesium loading is high.

A survey of the effect of various organic species on solvent performance confirmed that lipophilic organic anions remain the chief concern among minor feed components or solventdegradation products. A set of chemical equilibria was proposed as a model for anion effects, which primarily impact the effectiveness of stripping. Fortunately, it will be shown in later chapters that organic anions expected from the known composition of the wastes and from solvent-degradation processes can be readily removed from the solvent. Among the anions having an adverse impact on stripping were dibutylphosphate, 4-sec-butylphenol, and long-chain surfactants. The first of these is a constituent of the waste and by virtue of its acidity (as dibutylphosphoric acid) impacts stripping at fairly low concentrations. On the other hand, the modifier-degradation product 4-sec-butylphenol is weakly acidic and must be present at unexpectedly high concentrations to impact stripping. The surfactant anions are not specifically known as waste constituents. Small carboxylate anions like formate have no effect on solvent performance, presumably because they are too weakly extracted from the simulant. Neutral organic compounds present at low concentrations (e.g., tributylphosphate, 1-butanol, and trimethylamine) have little effect even at relatively high concentrations in the solvent. Their effect, if any, is primarily thought to involve interfering with the function of the modifier, thereby resulting in decreased $D_{\mathrm{Cs}}$ values on extraction.

Impurity buildup on multiple cycling necessitates solvent washing. It was observed that either multiple cycles or multiple extraction steps cause increased stripping $D_{\mathrm{Cs}}$ values. The resulting effectiveness of stripping is linked mainly to the set of organic constituents of the simulant. Among these constituents, dibutylphosphate is clearly the offending species.

Trials of various simulants with wide variations in the concentrations of major ionic constituents demonstrated that the solvent performance tolerates feed variations well. Computer modeling reported elsewhere ${ }^{25}$ was able to reasonably predict the value of $D_{\mathrm{Cs}}$. It was observed that nitrite concentration in the simulant correlates with stripping $D_{\mathrm{Cs}}$ values, and stripping becomes impaired when nitrite is present at very high concentration $(2.6 \mathrm{M})$ in the simulant. The reason for this correlation is not clear at present and needs to be investigated further.

Overall, the results point to a good understanding of the factors that influence cesium distribution behavior. No major issues were identified that cannot be dealt with outside normal engineering practice with regards to flow-rate adjustment, temperature management, and possibly feed blending. Not all chemical phenomena were completely understood, however. Some effects, such as that involving high nitrite concentrations, need to be explained, and other effects are understood only at a hypothetical or qualitative level. 


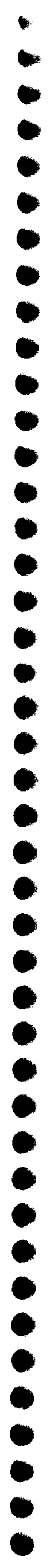




\section{DISTRIBUTION OF SYSTEM COMPONENTS}

\subsection{INTRODUCTION}

\subsubsection{Purpose}

Prior development of CSSX implicated certain metal ions such as $\mathrm{K}^{+}$, certain organic anions such as dodecylsulfonate, and solvent degradation products such as phenols as affecting flowsheet performance. ${ }^{8,14}$ Extensive characterization of the effects of such species on Cs distribution in the context of the present simulant and baseline solvent was seen in the previous chapter. Toward predicting flowsheet performance, identifying problems, and formulating solutions, the partitioning behavior of these species must be determined. Limited data were previously collected. In particular, it is desirable to quantify the distribution behavior of all waste components that have a significant tendency to be extracted, to build up in the solvent, or to interfere in some manner with the process. Similarly, the partitioning behavior of solvent degradation products must also be understood. Quantitative distribution data can then be correlated with key solvent properties, such as third-phase formation or $D_{\mathrm{Cs}}$ values on extraction, scrubbing, and stripping. Knowledge of the fate of various species can also provide understanding and control of effluent compositions. In addition, solvent cleanup methods can be devised in a rational manner. Thus a major objective of this chapter is to describe how various inorganic and organic solute species partition between the solvent and relevant aqueous solutions, migrate through the flowsheet, and possibly accumulate in the solvent.

Just as it is a concern that trace components of the waste partition into the solvent phase, it matters that traces of solvent components partition into the aqueous phase. Other than degradation or entrainment, partitioning represents a major pathway for loss of solvent components. In many hydrometallurgical solvent-extraction processes, solvent loss overall represents the chief operating cost. $^{23,60}$ In view of the high cost of $\mathrm{BOBCalixC6}$, loss pathways must be made manageable. Losses also represent a potential safety or environmental concern. ${ }^{60}$ Both cost and environmental concerns were addressed in the design of the reagents. In connection with testing the first-generation flowsheet, partition ratios were reported ${ }^{8}$ showing that $\mathrm{BOBCalixC6}$ and the earlier $\mathrm{Cs}-3$ modifier have negligible partitioning to the aqueous phase. Although $\mathrm{BOBCalixC} 6$ would also be expected to exhibit negligible partitioning to the aqueous phase in the current baseline flowsheet, the secondgeneration modifier Cs-7SB has a shorter alkyl chain and would likely suffer greater loss than Cs-3. As a standard extractant, TOA would not be expected to have undue partitioning losses, but its partitioning behavior has remained untested in this system.

\subsubsection{Experimental Design}

In general, measurement of partitioning phenomena required approximately the same batch contacting methods described in Chap. 2, unless otherwise noted. Experiments included the standard batch ESS contacting protocol and systematic batch tests as a function of selected compositional variables. The greatest challenge perhaps lay in devising sensitive analytical techniques for the very low concentrations of the various species of interest. Typically, each component tested required a unique analytical methodology. A portion of that methodology will be reported elsewhere. ${ }^{26}$ Selection of species for study was ongoing during the project. As information on solvent degradation products and interfering species was gathered, partitioning experiments were prioritized and scheduled. For example, at the outset of the project, the importance of dibutylphosphate in the waste and dioctylamine as a degradation product were not known. Surfactant species for study were mainly selected according to the availability of convenient ${ }^{14} \mathrm{C}$-labeled materials. 


\subsubsection{Work Scope}

Species whose partitioning behavior must be understood include primary solvent degradation products (WSM item 5.1.2), major cations in the waste and trace metals (WSM item 5.1.3), major and important minor inorganic anions (WSM item 5.1.4), lipophilic organic anions such as dibutylphosphate and dodecylsulfonate (WSM item 5.1.5). ${ }^{19}$ Solvent cleanup measures such as solvent washing will be examined in Chap. 8. WSM item 5.1.1 specified the determination of the distribution of the primary solvent components to flowsheet aqueous phases and wash solutions. Anion exchange was investigated as a possible solvent cleanup method (WSM 4.1.1.6).

\subsection{EXPERIMENTAL SECTION}

\subsubsection{Chemicals and Materials}

\subsubsection{Distribution of Cations, Anions, and Solvent Components}

Dioctylamine (98\%) was purchased and used as received from Aldrich. Dibutylphosphate was purchased from Fluka. The anion standards were prepared from dried salts at $1000 \mathrm{mg} / \mathrm{L}$ in nanopure deionized water (Table 4.1). $\mathrm{NaOH}$ pellets were used as received from J.T. Baker. The salts used to prepare the anion standards were $\mathrm{NaCl}, \mathrm{GR}$, EM Scientific; $\mathrm{NaNO}_{2}, 99.5 \%$, Aldrich; $\mathrm{NaNO}_{3}$, JT Baker, Reagent-grade crystal; $\mathrm{Na}_{2} \mathrm{SO}_{4}, \mathrm{GR}$, EM Scientific; $\mathrm{Na}_{2} \mathrm{HPO}_{4} \cdot 7 \mathrm{H}_{2} \mathrm{O}$, Baker Analyzed Reagent Grade. In addition, cesium nitrate was obtained from Alpha Aesar, $99.9 \%$ and dried prior to use. Potassium nitrate was obtained from EM Science.

Table 4.1. Preparation of $1000 \mathrm{mg} / \mathrm{L}$ anion standard

\begin{tabular}{ccc}
\hline Anion & Salt & Wt. salt in 1 Liter $(\mathrm{g})$ \\
\hline $\mathrm{Cl}^{-}$ & $\mathrm{NaCl}$ & 1.649 \\
$\mathrm{NO}_{2}{ }^{-}$ & $\mathrm{NaNO}_{2}$ & 1.500 \\
$\mathrm{NO}_{3}{ }^{-}$ & $\mathrm{NaNO}_{3}$ & 1.371 \\
$\mathrm{SO}_{4}{ }^{2-}$ & $\mathrm{Na}_{2} \mathrm{SO}_{4}$ & 1.480 \\
$\mathrm{PO}_{4}{ }^{3-}$ & $\mathrm{Na}_{2} \mathrm{HPO}_{4} \cdot 7 \mathrm{H}_{2} \mathrm{O}$ & 2.820 \\
\hline
\end{tabular}

In measurements of the partitioning of 4-sec-butylphenol, $\mathrm{NaOH}$ pellets were used as received to make 3- and 1-M stock solutions in distilled, deionized water. Other concentrations were prepared from dilutions to give $3.0,1.0,0.30,0.10,0.03,0.01,0.003$ and $0.001 \mathrm{M}$. Nitric acid solutions were prepared from J.T. Baker Ultrex II and deionized water. The full simulant was used from the fifth draw (ORNL, 9/22/00). The 4-sec-butylphenol was used as received from Aldrich $(96 \%)$ and added by volumetric preparation to make $1 \mathrm{mM}$ in the baseline solvent (Lot B000718-156W).

\subsubsection{Distribution of Actinides, Strontium, and Technetium}

A uniform concentration of $10^{-5} \mathrm{M}$ was desired for selected actinides, strontium, and technetium in the simulant. Four different simulant samples $(45 \mathrm{~mL})$ were prepared: one containing only uranium, one only neptunium, one only plutonium, and one containing all the actinides plus strontium and technetium. Solutions of ${ }^{233} \mathrm{U},{ }^{239} \mathrm{Pu},{ }^{237} \mathrm{~Np}$, and ${ }^{99} \mathrm{Tc}$ were obtained from stocks at ORNL. The purity of the ${ }^{233} \mathrm{U}$ and ${ }^{239} \mathrm{Pu}$ isotopes (absence of daughters) was checked by alpha spectrometry and determined to be satisfactory. The stock solution of ${ }^{237} \mathrm{~Np}$ was provided by 
Richard G. Haire of ORNL and used as received. A $100-\mu \mathrm{L}$ spike of each actinide stock solution was added to $900 \mu \mathrm{L}$ of nitric acid. In order to avoid precipitation, a pre-dilution step of the acidic actinide solutions into $2 \mathrm{~mL}$ of $1.75 \mathrm{M}$ sodium hydroxide was carried out followed by addition of the full $2 \mathrm{~mL}$ solution to the simulant. For $45 \mathrm{~mL}$ of simulant each acidic actinide solution was added as follows: $16.8 \mu \mathrm{L}$ of $\mathrm{U}, 76.8 \mu \mathrm{L}$ of $\mathrm{Np}$, and $161 \mu \mathrm{L}$ of $\mathrm{Pu}$. The color of the stock solutions of actinides $\left({ }^{233} \mathrm{U},{ }^{237} \mathrm{~Np}\right.$, and $\left.{ }^{239} \mathrm{Pu}\right)$ in nitric acid used for spiking was the only indication of the initial oxidation state of the actinides. Based on that observation, it can be reasonably assumed that uranium was $\mathrm{U}(\mathrm{VI})$, neptunium was $\mathrm{Np}(\mathrm{V})$, and plutonium was $\mathrm{Pu}(\mathrm{III})$. Strontium was added to the simulant as non-radioactive strontium. Strontium nitrate, 99.9\%, was obtained from Aldrich and used as received. A stock solution of strontium nitrate in water was prepared in a volumetric flask and used to spike the simulant. The ${ }^{99} \mathrm{Tc}$ stock was in the form of $3 \mathrm{mM}$ ammonium pertechnetate in water. In all cases, the cesium content was $1.4 \times 10^{-4} \mathrm{M}$, and the simulant was spiked with ${ }^{137} \mathrm{Cs}$ at ca. 0.1 $\mu \mathrm{Ci} / \mathrm{mL}$ for radioanalytical purposes.

\subsubsection{Anion-Exchange Resins}

General. The commercially available IRA-900 resin was purchased from Aldrich Chemical Co. Other resins were generously supplied by the respective manufacturer as follows: Sybron Ionac SR-6 from Sybron Chemicals, Inc., Birmingham, NJ; D3696-3 and A-850 from The Purolite Company, Bala Cynwyd, PA. Laboratory-prepared resins were synthesized at the University of Tennessee, Knoxville (see below). All resins were of the strong-base anion-exchange type with a macroporous morphology, except for Purolite A-850 and UTK $\mathrm{CS}_{3}-03-220$ which have gel-type morphology.

All resins were tested in the chloride and hydroxide forms except UTK $\mathrm{CS}_{3}-03-220$, which was used in the hydroxide form only. Prior to use, the resins were swollen in 1,4-dioxane for 2-12 hours, washed with dioxane/Isopar L and Isopar L, and kept in Isopar L over a weekend.

A percent-solids value was determined for each resin by the following procedure: Resin samples equilibrated with Isopar $\mathrm{L}$ were placed on a coarse glass filter and dried for 5-10 min (Isopar L no longer coming through the filter); resin subsamples were weighed and placed in the vacuum oven at $60{ }^{\circ} \mathrm{C}$ for $1-3$ days. Weight of subsamples was redetermined after air-equilibrating and cooling to room temperature. For the ion-exchange experiments, subsamples of the resins, $0.2-0.3 \mathrm{~g}$ each, were removed from the same glass filter-dried batch of resin used for the determination of percent-solids, weighed, and placed into washed pristine baseline solvent (Lot B000718-156W) for equilibration for at least 4 days.

Resin syntheses. In each case, $10 \mathrm{~g}$ of copolymer beads was contacted with the solution of amine in a 500-mL round-bottom flask equipped with an overhead stirrer, thermometer, and addition funnel. For resin $\mathrm{CS}_{2}-04-162,250 \mathrm{~mL}$ of $25 \%$ aqueous trimethylamine solution was added and the mixture refluxed for $17 \mathrm{~h}$. After cooling, the solution was removed, the beads washed with water and conditioned with $1 \mathrm{~L}$ of $1 \mathrm{~N} \mathrm{NaOH}, \mathrm{H}_{2} \mathrm{O}, 1 \mathrm{~N} \mathrm{HCl}$, and $\mathrm{H}_{2} \mathrm{O}$. For resins $\mathrm{CS}_{2}-04-187,-188,-198$, and $03-220,100 \mathrm{~mL}$ of dioxane was added to the copolymer beads and the beads allowed to swell in the solvent for $15 \mathrm{~h}$. Amine $(80 \mathrm{~mL}$ of trihexylamine, triethanolamine, tributylamine, and tributylamine for the resins as listed above, respectively) in $50 \mathrm{~mL}$ dioxane and $10 \mathrm{~mL}$ water were then added and the mixture refluxed for $17 \mathrm{~h}$. After cooling, the solution was removed, the beads washed with dioxane, dioxane/water (50/50), and water, and then conditioned as above.

Resins in the chloride form. The laboratory-made resins were synthesized in the chloride form, swollen in 1,4-dioxane, and used as received. The commercial resins were treated by successive washing ( $1 \mathrm{~L}$ per $10 \mathrm{~g}$ of resin) with: 


\author{
$1 \mathrm{M} \mathrm{NaOH}$, \\ Deionized $\mathrm{H}_{2} \mathrm{O}$ till $\mathrm{pH}$ of 7 , \\ $1 \mathrm{M} \mathrm{HCl}$, \\ Deionized $\mathrm{H}_{2} \mathrm{O}$ till $\mathrm{pH}$ of 7 , \\ Deionized $\mathrm{H}_{2} \mathrm{O}$ / ethanol, \\ Ethanol, \\ Ethanol / 1,4-dioxane, \\ 1,4-Dioxane.
}

Resins in the hydroxide form. All resins were converted to the hydroxide form by the successive washing ( $1 \mathrm{~L}$ per $10 \mathrm{~g}$ of resin) with:

Deionized $\mathrm{H}_{2} \mathrm{O}$,

$1 \mathrm{M} \mathrm{NaOH}$ prepared from $50 \% \mathrm{NaOH}$ aqueous solution and deionized $\mathrm{H}_{2} \mathrm{O}$ purged with argon for 20 min to remove carbon dioxide (negative $\mathrm{AgNO}_{3}$ test for $\mathrm{Cl}^{-}$ after acidification with $\mathrm{HNO}_{3}$ ),

Deionized $\mathrm{H}_{2} \mathrm{O}$ till $\mathrm{pH}$ of 7 ,

Deionized $\mathrm{H}_{2} \mathrm{O} /$ 1,4-dioxane,

1,4-Dioxane.

\title{
4.2.2 Phase-Contacting Protocols and Analytical Methods
}

\subsubsection{Partitioning of Solvent Components}

Three 2-mL aliquots of baseline solvent (Lot PVB B000718-156W) were contacted overnight at $25{ }^{\circ} \mathrm{C}$ respectively with $200 \mathrm{~mL}$ of the following solutions (directly vs. in ESS sequence): full simulant (third draw), scrub solution $(50 \mathrm{mM}$ nitric acid), and strip solution (1 $\mathrm{mM}$ nitric acid). These samples were centrifuged at $2500 \mathrm{rpm}$ for $5 \mathrm{~min}$ at $25{ }^{\circ} \mathrm{C}$ in a thermostated Beckman centrifuge. Both organic and aqueous phases were carefully removed, particularly to avoid any contamination of the aqueous phase, and placed in clean vials (rinsed with deionized water and $95 \%$ ethanol). It is of interest to note that a thin white film, likely uncoalesced fine dispersion, appeared at the interface (aqueous phase side) for the sample containing the strip solution. Note that for analytical purposes these volume ratios are quite different from those encountered in the flowsheet.

Seven solvent aliquots were equilibrated in the same manner as described in the previous paragraph except that 1) the aqueous phases were full simulant, $1 \mathrm{mM} \mathrm{HNO} 3,50 \mathrm{mM} \mathrm{HNO} 3,0.03 \mathrm{M}$ $\mathrm{NaOH}, 0.1 \mathrm{M} \mathrm{NaOH}, 0.3 \mathrm{M} \mathrm{NaOH}$, and $1 \mathrm{M} \mathrm{NaOH}$; and 2) the solvent samples were spiked with 0.5 ppm TBP, 25 ppm DBP, 25 ppm monobutylphosphoric acid, and $1 \mathrm{mM}$ SBP.

An experiment reproducing an ESS cycle was also performed. However, in this case, some values, such as the trace concentration of calixarene in the small volumes of aqueous phases, were expected to be below the detection limit. An 8-mL aliquot of solvent was first contacted with $24 \mathrm{~mL}$ of full simulant (third draw). The aqueous phase was saved along with a 1-mL subsample of solvent for analysis. The remaining $7 \mathrm{~mL}$ of solvent was contacted with $1.4 \mathrm{~mL}$ of scrub solution $(50 \mathrm{mM}$ nitric acid). Again, a small quantity of organic phase and all the aqueous phase were saved. The 5-mL solvent aliquot remaining was contacted with $1 \mathrm{~mL}$ of strip solution (1 mM nitric acid). All ESS contacts were performed by gentle end-over-end rocking at $60 \mathrm{rpm}$ for $1 \mathrm{~h}$ at $25{ }^{\circ} \mathrm{C}$, and samples were subsequently centrifuged at $3500 \mathrm{rpm}$ for $3 \mathrm{~min}$ at $25{ }^{\circ} \mathrm{C}$ in a thermostated Sanyo Mistral centrifuge. The white film observed in the previous experiment for the strip solution did not appear in this case.

Seven $1.2-\mathrm{mL}$ aliquots of the baseline solvent were contacted overnight at $25{ }^{\circ} \mathrm{C}$ with $30 \mathrm{~mL}$ of the nitric acid solutions at the following respective concentrations $(\mathrm{mM}): 0,1,2.5,10,25,50$, and 100. These samples were centrifuged at $3500 \mathrm{rpm}$ for $3 \mathrm{~min}$ at $25{ }^{\circ} \mathrm{C}$ in a thermostated Sanyo Mistral centrifuge. Both organic and aqueous phases were carefully removed, particularly to avoid any contamination of the aqueous phase, and placed in clean vials (rinsed with deionized water and 
95\% ethanol). The fine precipitate or emulsion observed previously was also noted here for the solvent in contact with deionized water, nitric acid $1 \mathrm{mM}$, and nitric acid $2.5 \mathrm{mM}$. Trioctylamine was determined by gas chromatography. Modifier Cs7-SB and calixarene concentrations were determined by reversed-phase HPLC.

\subsubsection{Distribution of Alkali Metal Cations}

Ion Chromatography (IC) was used to analyze cation extraction by simple salt mixtures that were the subject of computer modeling. Stock solutions of $\mathrm{NaNO}_{3}, \mathrm{NaNO}_{2}, \mathrm{NaCl}$, and $\mathrm{NaOH}$ were prepared and all other concentrations prepared as a dilution of the stock. Sodium concentrations prepared were $5.6,4.5,2.25,1.00,0.50,0.10$, and $0.01 \mathrm{M}$. $\mathrm{CsNO}_{3}$ and $\mathrm{KNO}_{3}$ were added directly to the sodium salts at $0.5 \mathrm{mM}$ and $60 \mathrm{mM}$, respectively, effecting a slight dilution of the initial sodium in solution. Binary salt solutions at anion ratios of $0,0.25,0.50,0.75,0.90$, and $1.0 \mathrm{M}$ and total sodium concentration of 4.5 or $5.6 \mathrm{M}$ were also prepared with $\mathrm{CsNO}_{3}$ added at $0.5 \mathrm{mM}$ as well, with and without $\mathrm{KNO}_{3}$ at $60 \mathrm{mM}$. Potassium extractions from $\mathrm{KNO}_{3}$ solutions at concentrations 1.0, $0.30,0.10,0.01 \mathrm{M}$ were also carried out. The baseline solvent (Lot B000718-156W) was contacted with equal volume salt solution in $2-\mathrm{mL}$ polypropylene vials for $1 \mathrm{~h}$ by rotation in a thermostated air box set at $25 \pm 0.1{ }^{\circ} \mathrm{C}$. All samples were centrifuged for $3 \mathrm{~min}$ at $3500 \mathrm{rpm}$ to ensure complete phase disengagement. The organic phase was then contacted with 5-10 times volume dilute $\mathrm{HNO}_{3}(1 \mathrm{mM})$ to strip. A second strip was done in the same manner, but results showed less than $2 \%$ metals recovery by the second strip. All results reported here are from the first strip only. The strip solutions were analyzed in duplicate by IC. The cations, sodium, potassium and cesium, were separated using a CS12A analytical column coupled with a CG12A guard column. The analysis used $20 \mathrm{mN} \mathrm{H}_{2} \mathrm{SO}_{4}$ eluent at $1 \mathrm{~mL} / \mathrm{min}$ in an isocratic run of $20 \mathrm{~min}$. Background conductivity was 0.2 $\mu S$ using CSRS-Ultra suppressor in auto-regeneration mode set at $300 \mathrm{~mA}$. Parallel experiments using ${ }^{137} \mathrm{Cs}$ radiotracer and ${ }^{22} \mathrm{Na}$ radiotracer were also performed.

Extraction behavior of the baseline solvent toward $\mathrm{Cs}^{+}, \mathrm{K}^{+}$, and $\mathrm{Na}^{+}$ions was characterized by systematic variation of the aqueous-phase metal ion concentration under acidic, neutral, or alkaline conditions at $25{ }^{\circ} \mathrm{C}$. Distribution measurements were carried out at $25{ }^{\circ} \mathrm{C}$ using an organic phase consisting of $0.5 \mathrm{M}$ Cs-7SB modifier and $0.001 \mathrm{M}$ TOA in Isopar L without BOBCalixC6 extractant. To investigate an effect of $\mathrm{pH}$, aqueous solutions containing the same cesium or potassium concentration were tested under neutral and alkaline conditions. Experiments using simulant solutions were conducted at $\mathrm{O} / \mathrm{A}$ of 0.33 and 0.1 . Cesium distribution ratios were measured by ${ }^{137} \mathrm{Cs}$ radiometric techniques, while $\mathrm{K}^{+}$and $\mathrm{Na}^{+}$distribution ratios were measured by ICP-AES.

\subsubsection{Distribution of Other Metals and Selected Radionuclides}

Sodium, potassium, and aluminum. During the investigation of an effect of $\mathrm{O} / \mathrm{A}$ ratio on alkali metal ion distribution into baseline solvent, the aqueous stripping phase was also examined for the presence of Al by ICP-AES. The experiment was performed in the following manner: A series of pristine washed solvent samples was contacted with simulant solution so that the O/A ratio was varied from 0.05 to 10 . To determine the cesium distribution ratio, each phase was subsampled and analyzed by ${ }^{137} \mathrm{Cs}$ gamma counting. A parallel cold experiment was conducted to study $\mathrm{K}^{+}$and $\mathrm{Na}^{+}$ distribution behavior under the same conditions. To determine $\mathrm{K}^{+}$and $\mathrm{Na}^{+}$distribution ratios, subsamples of the solvent phase were mixed with 1,3-diisopropylbenzene at a 1:1 ratio, stripped with deionized water, and analyzed by ICP-AES. Each distribution ratio was calculated assuming that mass balance of $100 \%$ is sustained for each sample. Each stripping sample was also analyzed for $\mathrm{Al}$ by ICP-AES. All samples showed an Al concentration at or below ICP detection limit. Thus, it is assumed based on an ICP-AES detection limit of $0.023 \mathrm{ppm}^{61}$ that the upper concentration limit of $\mathrm{Al}$ in the solvent is $3.5 \times 10^{-6} \mathrm{M}$. However, there is a possibility that experimental protocol adopted for the investigation of the transport behavior of alkali metal ions does not provide effective stripping of $\mathrm{Al}$ from the solvent phase and results in underestimation of the Al concentration in the solvent. This seems unlikely, as samples of organic phases submitted for direct analysis at the ORNL Radioanalytical Materials Analytical Laboratory (RMAL) failed to detect Al either. 
Actinides, strontium, and technetium. Samples were handled in a glove box. A $100-1000 \mu \mathrm{L}$ Eppendorf Pipetman and a 10-100 $\mu \mathrm{L}$ Eppendorf Pipetman were pre-calibrated with deionized water. A Genie II vortex mixer was used to contact the organic and aqueous phases. A VWR mini centrifuge able to hold six $2-\mathrm{mL}$ tubes was used to centrifuge all phases after contact.

Actinide concentrations were determined by alpha spectrometry or alpha counting. They were also checked by ICP-MS, along with strontium and technetium concentrations, using analytical methods developed by the ORNL staff of the RMAL. Spiked simulant solutions were counted four days after preparation and analyzed by ICP-MS six days after preparation.

The procedure adopted for the ESS sequence was as follows: A 5-mL volume of pristine solvent was contacted with $15 \mathrm{~mL}$ of simulant containing one or all elements in a $50-\mathrm{mL}$ Teflon tube. The tube was vortexed for $1 \mathrm{~min}$, then allowed to stand for $30 \mathrm{~s}$. This sequence was repeated three times. Because of the limited space in the glove box, only a small centrifuge could be accommodated. Small 2-mL Eppendorf tubes were used to centrifuge both the solvent from one contact to the next and the sample of the aqueous phase before analysis. These tubes had been washed and rinsed following the regular procedure for all other containers. The solvent was then placed back into clean $15-\mathrm{mL}$ tubes for scrubbing and stripping.

\subsubsection{Distribution of Anions by Ion Chromatography}

Baseline solvent (Lot $\mathrm{B} 000718-156 \mathrm{~W}$ ) was taken through an $\mathrm{ESS}_{3} \mathrm{~W}_{2}$ contacting protocol. As indicated, the sequence involved the addition of two washes of $10 \mathrm{mM} \mathrm{NaOH}$ after the third strip and employed a volume ratio of $\mathrm{O} / \mathrm{A}=1$ [labeled as solvent $(\mathrm{A})$ ]. Another aliquot of solvent was taken through an $\mathrm{E}_{5} \mathrm{SS}_{3} \mathrm{~W}_{2}$ sequence [labeled as solvent (B)]. Extractions employed the full simulant and baseline scrub and strip solutions. The contacting was carried out at $25{ }^{\circ} \mathrm{C}$ by gentle rotation in Teflon containers. All samples were centrifuged at $2500 \mathrm{rpm}$ for $5 \mathrm{~min}$ at $25{ }^{\circ} \mathrm{C}$ in a thermostated Beckman centrifuge. The aqueous phases were separated and diluted volumetrically as needed in Barnstead nanopure deionized water. All scrub and strip acid solutions were diluted, 50 times and 5 times respectively, and analyzed by $\mathrm{IC}$ for anions. The $\mathrm{NaOH}$ washes were analyzed directly. A small portion of the loaded solvent was also contacted with deionized water at ten times the organic volume and the aqueous phase analyzed. The washes and water strip solutions were analyzed for dibutylphosphate as well as for trace anions present in the solvent prior to the scrub and strips, as well as those retained by the solvent after the scrub and strips. Anions were separated using an AS 11 analytical column coupled with AG11 guard column and an anion trap column (ATC-1). Anions were separated using an IONPAC, AS11 analytical column coupled with an IONPAC, AG11 guard column. An Anion Trap Column (IONPAC, ATC-1) containing high capacity, low efficiency anion exchange resins was installed prior to the guard and analytical columns to remove trace anionic contaminants such as carbonate from the eluent. Detection limits were determined by running standards at subsequently lower dilutions until the deviation of the expected result was greater than 10\%. Detection limits for each anion were as follows: $\mathrm{Cl}^{-}, 7.0 \times 10^{-6} \mathrm{M} ; \mathrm{NO}_{2}^{-}, 2.2 \times 10^{-6} \mathrm{M}^{-} \mathrm{NO}_{3}^{-}$, $8.1 \times 10^{-6} \mathrm{M} ; \mathrm{SO}_{4}{ }^{2-}, 2.0 \times 10^{-6} \mathrm{M} ; \mathrm{PO}_{4}^{3-}, 1.0 \times 10^{-6} \mathrm{M}$.

Anion method by IC

IC Method: Dionex DX500 with GP40 pump and ED40 detector

Column type: AS11/AG11 with ATC-1

Eluent isocratic from $10.5 \mathrm{mM} \mathrm{NaOH}$ to $21 \mathrm{mM} \mathrm{NaOH}$

Column equilibrated for $7 \mathrm{~min}$ at $10.5 \mathrm{mM} \mathrm{NaOH}, 7 \mathrm{~min}$ to $15 \mathrm{~min}$ at $10.5 \mathrm{mM} \mathrm{NaOH}, 15$ to

$25 \mathrm{~min}$ at $21 \mathrm{mM} \mathrm{NaOH}$

Eluent pump flow rate: $1 \mathrm{~mL} / \mathrm{min}$

Injection loop: $200 \mu \mathrm{L}$

Pressure: 794 psi

Suppressor: ASRS-Ultra at $300 \mathrm{~mA}$ in autoregeneration mode

Background conductivity: $7.4 \mu \mathrm{S}$ 


\section{Anion method with dibutylphosphate separation}

IC method: Dionex Model DX500 with GP40 pump and ED40 detector

Column type: AS11/AG11 with ATC-1

Eluent gradient from $1.2 \mathrm{mM} \mathrm{NaOH}$ to $10.8 \mathrm{mM} \mathrm{NaOH}$ with $2 \% \mathrm{MeOH}$. Isocratic from $19 \mathrm{mM} \mathrm{NaOH} / 2 \% \mathrm{MeOH}$.

Column equilibrated for $7 \mathrm{~min}$ at $1.2 \mathrm{mM} \mathrm{NaOH} / 2 \% \mathrm{MeOH}, 7 \mathrm{~min}$ to $15 \mathrm{~min}$ at $1.2 \mathrm{mM}$ $\mathrm{NaOH} / 2 \% \mathrm{MeOH}$ gradient to $10.8 \mathrm{mM} \mathrm{NaOH} / 2 \% \mathrm{MeOH}, 15$ to $25 \mathrm{~min}$ at $19 \mathrm{mM}$ $\mathrm{NaOH} / 2 \% \mathrm{MeOH}$

Eluent pump flow rate: $1 \mathrm{~mL} / \mathrm{min}$

Injection loop: $200 \mu \mathrm{L}$

Pressure: 844 psi

Suppressor: ASRS-Ultra at $100 \mathrm{~mA}$ in External-Water mode

Background conductivity: $6.2-8.4 \mu \mathrm{S}$

\subsubsection{Distribution Behavior in Multi-Cycle Batch Tests}

Sample analyses were performed by the staff of the ORNL RMAL. Silver, cesium, molybdenum, lead, palladium, rhodium, ruthenium, and tin were analyzed by ICP-MS. Anions were analyzed by IC using the USEPA procedure SW846 9056. Mercury was analyzed using the USEPA procedure SW846 7471A. All other elements were analyzed using the USEPA procedure SW846 6010A. USEPA procedures were slightly modified at the RMAL to accommodate radioactive samples. Table 4.2 gives the limits of detection of the species analyzed for. In tabulating the analytical data from the test, certain anomalies were encountered, such as equal concentrations of $\mathrm{Cu}$ found in all samples and the presence of $U$ and $T h$ (which are not in the simulant).

Table 4.2. Species analyzed in five-cycle experiment

\begin{tabular}{cccc}
\hline $\begin{array}{c}\text { Element } \\
\text { or species }\end{array}$ & $\begin{array}{c}\text { Detection limit } \\
(\mathrm{ppm} \text { or } \mu \mathrm{g} / \mathrm{mL})\end{array}$ & $\begin{array}{c}\text { Detection limit } \\
(\mathrm{mol} / \mathrm{L})\end{array}$ & Method \\
\hline $\mathrm{Ag}$ & 0.01 & $9.3 \times 10^{-8}$ & ICP-MS \\
$\mathrm{Al}$ & 0.7 & $2.6 \times 10^{-5}$ & ICP-AES \\
$\mathrm{Br}$ & 0.5 & $6.3 \times 10^{-6}$ & IC \\
$\mathrm{Cl}$ & 0.5 & $1.4 \times 10^{-5}$ & IC \\
$\mathrm{Cr}$ & 0.45 & $8.6 \times 10^{-6}$ & ICP-AES \\
$\mathrm{Cu}$ & 1.3 & $2.0 \times 10^{-5}$ & ICP-AES \\
$\mathrm{F}$ & 0.50 & $2.6 \times 10^{-5}$ & ICP-AES \\
$\mathrm{Fe}$ & 3.0 & $5.4 \times 10^{-5}$ & ICP-AES \\
$\mathrm{Hg}$ & 0.025 & $1.2 \times 10^{-7}$ & Cold Vapor AA \\
$\mathrm{K}$ & 6.6 & $1.7 \times 10^{-4}$ & ICP-AES \\
$\mathrm{Mn}$ & 0.05 & $9.1 \times 10^{-7}$ & ICP-AES \\
$\mathrm{Mo}$ & 0.002 & $2.1 \times 10^{-8}$ & ICP-MS \\
$\mathrm{Na}$ & 2.6 & $1.1 \times 10^{-4}$ & ICP-AES \\
$\mathrm{NO}_{2}{ }^{-}$ & 0.5 & $1.1 \times 10^{-5}$ & IC \\
$\mathrm{Pb}$ & 0.01 & $4.8 \times 10^{-8}$ & ICP-MS \\
$\mathrm{Pd}$ & 0.002 & $1.9 \times 10^{-8}$ & ICP-MS \\
$\mathrm{PO}_{4}{ }^{3-}$ & 1.00 & $1.0 \times 10^{-5}$ & IC \\
$\mathrm{Rh}$ & 0.002 & $1.9 \times 10^{-8}$ & ICP-MS \\
$\mathrm{Ru}$ & 0.002 & $2.0 \times 10^{-8}$ & ICP-MS \\
$\mathrm{Sn}^{2-}$ & 0.01 & $8.4 \times 10^{-8}$ & ICP-MS \\
$\mathrm{SO}_{2}{ }^{2-}$ & 1 & $1.0 \times 10^{-5}$ & IC \\
$\mathrm{Zn}$ & 20 & $3.0 \times 10^{-4}$ & ICP-AES \\
\hline
\end{tabular}




\subsubsection{Dibutylphosphate Partitioning Measurement by NMR}

General. Phosphorus-31 Nuclear Magnetic Resonance spectra were obtained on a Bruker Avance 400 wide-bore spectrometer as described in Chap. 2. Chemical shifts were referenced against phosphoric acid, set to $0.0 \mathrm{ppm}$ by way of a separate standard sample (sealed tube from Bruker).

Three-contact experiment between solvent and simulant. Baseline solvent (Lot B000718-156W) was contacted thrice successively with simulant (third draw) at an $\mathrm{O} / \mathrm{A}$ ratio of 0.33 . Contacting was performed in 50-mL Teflon FEP centrifuge tubes by end-over-end rotation at $25{ }^{\circ} \mathrm{C}$ for $30 \mathrm{~min}$, centrifuging after each contact, as previously described. For the first contact, $9 \mathrm{~mL}$ of solvent was used; $8 \mathrm{~mL}$ of that solvent was used in the second contact with fresh simulant, and $7 \mathrm{~mL}$ of the solvent from the second contact was used in the third contact. A $700-\mu \mathrm{L}$ aliquot of solvent from the third contact was placed into a quartz 5-mm NMR tube along with $300 \mu \mathrm{L}$ of deuterochloroform containing tributylphosphate (TBP, Aldrich, 99.9\%) as an internal integration standard at $1.00 \mathrm{mM}$, giving a TBP concentration of $0.30 \mathrm{mM}$ in the NMR sample. The ${ }^{31} \mathrm{P}$ spectrum was then obtained, which revealed a DBP:TBP ratio of $0.95: 1.00$, indicating a DBP concentration in the sample of 0.285 $\mathrm{mM}$. No other phosphorus peaks were observed.

A 2-mL aliquot of solvent from the third contact was contacted in a $10-\mathrm{mL}$ Teflon FEP tube with an equal volume of $0.5 \mathrm{M} \mathrm{NaOH}$, in the same manner as described. After centrifugation, a $700-\mu \mathrm{L}$ aliquot of solvent was diluted with $300 \mu \mathrm{L}$ of deuterochloroform containing TBP. The ${ }^{31} \mathrm{P}$ spectrum showed only the presence of TBP, with no observable DBP being detected. A 700- $\mu \mathrm{L}$ sample of the $0.5 \mathrm{M} \mathrm{NaOH}$ aqueous phase was diluted with $300 \mu \mathrm{L}$ of $\mathrm{D}_{2} \mathrm{O}$ in a quartz tube, and the ${ }^{31} \mathrm{P}$ NMR clearly showed the presence of a peak with the correct chemical shift for DBP.

Partitioning of DBP between the solvent and the simulant or $\mathrm{NaOH}$ solutions. In these experiments, the simulant or solvent samples were analyzed directly with no dilution or addition of reagents for integration standards. Instead, aliquots of solvent or simulant were placed directly into $10-\mathrm{mm}$ quartz tubes. A quartz insert containing tributylphosphate (TBP) at $1 \mathrm{mM}$ in deuterochloroform was placed inside the $10-\mathrm{mm}$ tube, and this solution, external to the sample, was used as the deuterium lock and integration standard. The insert permitted solvent or simulant samples to be run neat without the need to dilute or mix with a standard solution. Before running sample unknowns, spectra of the empty external tube with the insert, and of the solvent containing 1 mM DBP (210 ppm) were acquired. For unknown samples, an overnight acquisition (10k-12k scans) was performed to ensure that a reasonable signal/noise ratio was achieved. Data-point files for each spectrum were converted to ASCII files and treated under MS Excel for deconvolution. Each peak was considered as a pure Lorentzian and approximated this way (Fig. 4.1). This manipulation allowed more precise determination of the peak areas.

\subsubsection{Phase-Contacting Protocol for 4-sec-Butylphenol Partitioning}

General. Contacts and back-extractions were carried out in Teflon FEP separatory funnels, centrifuge tubes, and polypropylene centrifuge tubes as appropriate. Acidity of aqueous phases prior to back-extractions were determined by $\mathrm{pH}$ paper. All contacts with the solvent were equilibrated at $25 \pm 0.5{ }^{\circ} \mathrm{C}$ by rotation on a Glas-Col laboratory rotator in a thermostated box. All samples were centrifuged for $25-30 \mathrm{~min}$.

Partitioning of 4-sec-butylphenol to the full simulant. Partition of 4-sec-butylphenol to the full simulant was contacted in 60 -mL Teflon FEP centrifuge tubes by handshaking at $20 \pm 1{ }^{\circ} \mathrm{C}$, followed by rotation on a Glas-Col laboratory rotator at $25 \pm 0.5^{\circ} \mathrm{C}$. All samples were run in duplicate with $\mathrm{O} / \mathrm{A}$ ratios of $1,0.33$, and 0.1 , corresponding to volumes $5 \mathrm{~mL} / 5 \mathrm{~mL}, 5 \mathrm{~mL} / 15 \mathrm{~mL}$, and $3 \mathrm{~mL} / 30 \mathrm{~mL}$. The samples were equilibrated for $2 \mathrm{~h}$ at $25{ }^{\circ} \mathrm{C}$ and were centrifuged for $25 \mathrm{~min}$ at the same temperature. A portion of the solvent phase was carefully removed and transferred to a clean, $\mathrm{dr}$ : glass vial for analysis. 

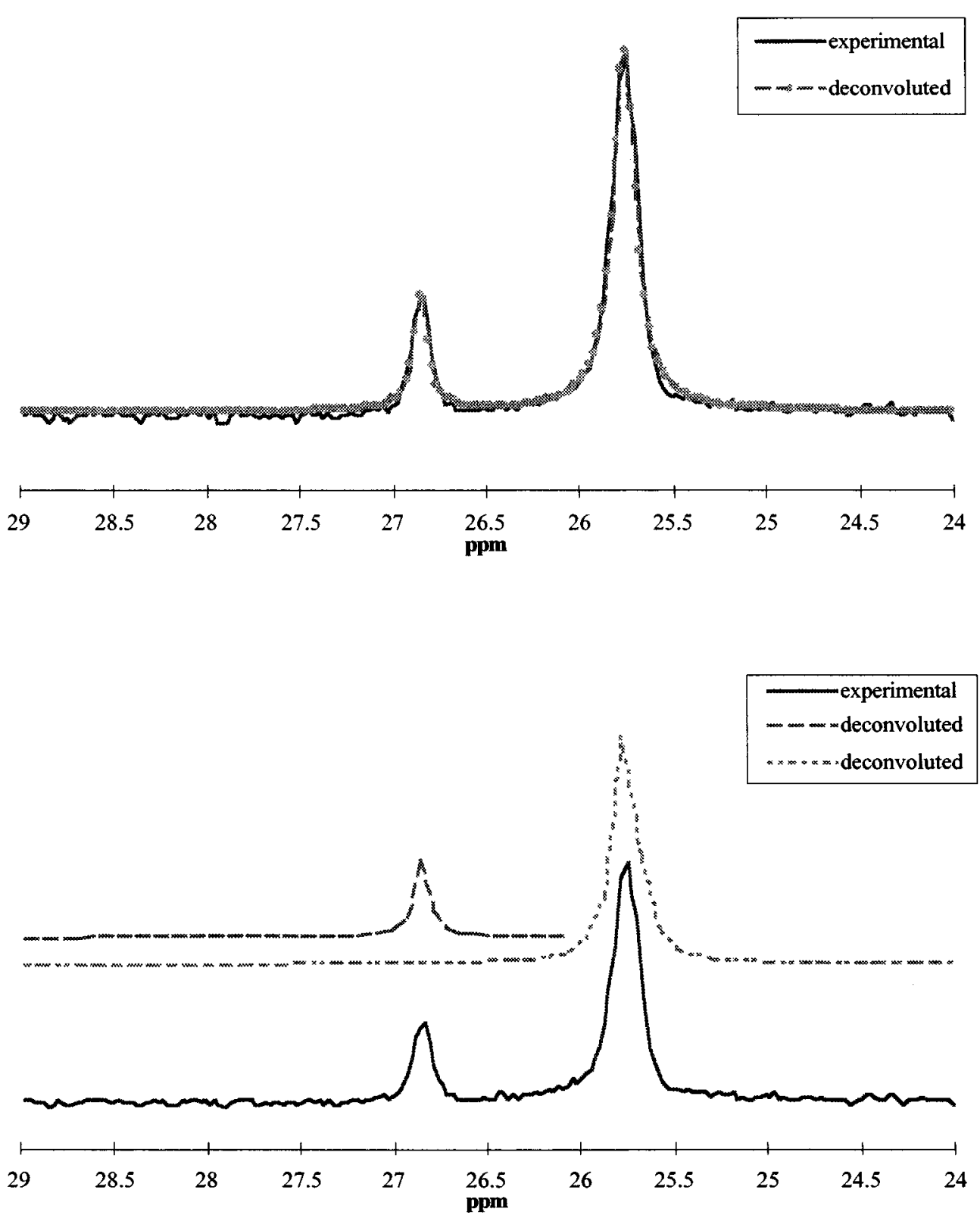

Fig. 4.1. Deconvolution of 31P NMR spectrum

Partitioning of 4-sec-butylphenol to the scrub and strip phases. The solvent with phenol was contacted with $50 \mathrm{mM} \mathrm{HNO}_{3}$ and $1 \mathrm{mM} \mathrm{HNO}_{3}$ using an O/A ratio of 0.10 . Volumes used were 10 $\mathrm{mL} / 100 \mathrm{~mL}$. The contact was done in $250-\mathrm{mL}$ Teflon FEP centrifuge bottles first by handshaking, followed by $2 \mathrm{~h}$ gentle contact at $25 \pm 0.5{ }^{\circ} \mathrm{C}$ as described above. The samples were again centrifuged for $25 \mathrm{~min}$ at $25 \pm 0.5{ }^{\circ} \mathrm{C}$. A portion of the solvent was removed for analysis, the remaining solvent plus some aqueous was removed from the contact sample. The remaining aqueous was carefully removed via pipette and transferred to a clean graduated cylinder. The volume of aqueous transferred was recorded and delivered to a clean 1-L Teflon FEP separatory funnel. Approximately $30 \mathrm{~mL}$ of methylene chloride was added to the aqueous sample in the funnel and vigorously hand-shaken for 1-2 min. The sample was allowed to separate completely and the 
methylene chloride carefully drained into a clean, tared brown glass bottle. The washing was repeated two more times with $\sim 30 \mathrm{~mL}$ fresh methylene chloride each washing.

Partitioning of 4-sec-butylphenol to various concentrations of $\mathrm{NaOH}$. The solvent with phenol was contacted with the following $\mathrm{NaOH}$ concentrations using an $\mathrm{O} / \mathrm{A}$ ratio of 5: 3.0, 1.0, 0.30, 0.10 , $0.03,0.01,0.003$, and $0.001 \mathrm{M}$. Volumes used were $30 \mathrm{~mL} / 6 \mathrm{~mL}$. The contacts were performed as described above in $60 \mathrm{~mL}$ Teflon FEP centrifuge tubes. A portion of the solvent after centrifuging was transferred to a clean vial for analysis. The remaining solvent was removed from the aqueous and the aqueous phase was carefully transferred to a $15 \mathrm{~mL}$ polypropylene tube. From this transfer, $5 \mathrm{~mL}$ of the aqueous phase (by Eppendorf pipette) was transferred to another clean 15-mL polypropylene tube. This $5-\mathrm{mL}$ volume was made acidic to $\mathrm{pH} 3$ by carefully adding Ultrex concentrated nitric acid. Back-extractions with equal volumes of methylene chloride were carried out in this $15-\mathrm{mL}$ tube, carefully removing with a glass disposable pipette most of the organic phase and transferring it to a clean, tared, $20-\mathrm{mL}$ glass vial.

Partitioning of 4-sec-butylphenol from $0.3 \mathrm{M} \mathrm{NaOH}$ to the solvent. A 5-mL sample of $0.3 \mathrm{M}$ $\mathrm{NaOH}$ was spiked with $1.36 \mathrm{mM}$ 4-sec-butylphenol. A portion of this sample was contacted with pristine solvent as outlined above, and then analyzed. Another portion of this sample was not contacted with solvent, but was acidified and immediately back-extracted into methylene chloride as a control. The back-extraction controls were analyzed along with the other samples, and the phenol was found to be $98 \%$ recoverable using this technique. All solvent and methylene chloride samples, along with pristine solvent and solvent spiked with the phenol, were submitted to the analytical team at ORNL RMAL for analysis.

Analytical method for 4-sec-butylphenol determination. The organic samples were diluted with isopropanol at a ratio of $1 / 10$. The methylene chloride layers from back-extractions were evaporated under a stream of nitrogen at $65-75^{\circ} \mathrm{C}$ to $<0.5 \mathrm{~mL}$. Isopropanol was added at a volume of $0.5 \mathrm{~mL}$ and the remaining methylene chloride evaporated. Isopropanol was again added to bring sample to final volume of 1.8 or $5 \mathrm{~mL}$. The analysis was carried out using a Hewlett-Packard Model 5890 Series 2 gas chromatograph coupled to an HP 5972 mass selective detector. The column used was a Restek-rtx-5, $30 \mathrm{M} \times 0.25 \mathrm{~mm} \times 0.25 \mu \mathrm{m}$ film thickness. The instrument was tuned using the autotune routine on each day prior to sample analysis. Calibration was performed prior to sample analysis using a $100 \mu \mathrm{g} / \mathrm{g}$ standard. Relative precision of replicate analyses was $\pm 3 \%$.

GC conditions were as follows:

Injector temperature: $250{ }^{\circ} \mathrm{C}$

Detector inlet temperature: $300{ }^{\circ} \mathrm{C}$

Initial column temperature: $40^{\circ} \mathrm{C}, 4$-min hold

Temperature control: $10^{\circ} \mathrm{C} / \mathrm{min}$. to $200^{\circ} \mathrm{C}, 50^{\circ} \mathrm{C} / \mathrm{min}$. to $300^{\circ} \mathrm{C}$, final hold $8 \mathrm{~min}$

Total run time: $30 \mathrm{~min}$

\subsubsection{Partitioning of Surfactant Anions}

General. For the studies on the distribution of lipophilic anions three ${ }^{14} \mathrm{C}$-labeled compounds were obtained: lauric acid (specific activity $57 \mathrm{mCi} / \mathrm{mmol}, 0.5 \mathrm{mCi} / \mathrm{mL}$ in hexane), sodium dodecylsulfate (specific activity $55 \mathrm{mCi} / \mathrm{mmol}, 0.1 \mathrm{mCi} / \mathrm{mL}$ in sterile water), and stearic acid $(56$ $\mathrm{mCi} / \mathrm{mmol}, 0.1 \mathrm{mCi} / \mathrm{mL}$ in toluene). The cold analogues for these anions were obtained as follows: lauric Acid, 99\%, Emery Uncommon Chemicals; sodium dodecylsulfate, 99+\%, Fluka; and stearic acid, 99\%, Fluka. Scintillation counting of samples was performed using $20-\mathrm{mL}$ glass vials, Packard UltraGold XR cocktail, and a Packard Tri-Carb 2500TR Liquid Scintillation Analyzer. Sample counting time was 10 min using a counting window of 4.0 to $156 \mathrm{keV}$.

Prior to performing batch tests, a simple test was performed to check the activity of the ${ }^{14} \mathrm{C}$ solutions as well as the quenching effects, if any, of the solvent, scrub, strip, and wash solutions on scintillation counting of ${ }^{14} \mathrm{C}$ stocks. Five reaction series were run (see Table 4.3 , below). In each 
case, the supporting matrix contained $10 \mathrm{~mL}$ of UltraGold XR scintillation cocktail. The series consisted of a blank, $200 \mu \mathrm{L}$ added solvent, $200 \mu \mathrm{L} 0.05 \mathrm{M} \mathrm{HNO}_{3}, 200 \mu \mathrm{L} 0.001 \mathrm{M} \mathrm{HNO}$, or $200 \mu \mathrm{L}$ $0.1 \mathrm{M} \mathrm{NaOH}$ added to the cocktail. The $200-\mu \mathrm{L}$ quantities were tested since this volume represents the sample size to be counted during batch testing. Each series component was then prepared without ${ }^{14} \mathrm{C}$ activity. The spiking solutions were made by adding $3 \mu \mathrm{L}(0.3 \mu \mathrm{Ci}, \sim 660 \mathrm{k} \mathrm{cpm})$ of either ${ }^{14} \mathrm{C}$-SDS, or ${ }^{14} \mathrm{C}$-stearic acid to $219 \mu \mathrm{L}$ solvent. Samples in the series were then spiked with $5 \mu \mathrm{L}$ of either ${ }^{14} \mathrm{C}$-SDS or ${ }^{14} \mathrm{C}$-stearic acid, as well as no activity. All tests were done in duplicate. The quenching results appear below in Table 4.3.

Table 4.3. Activity and Quenching Test Results

\begin{tabular}{|c|c|c|c|}
\hline & No added ${ }^{14} \mathrm{C}$ & $\begin{array}{c}\text { Spike } \\
{ }^{14} \text { C-SDS }\end{array}$ & $\begin{array}{c}\text { Spike } \\
{ }^{14} \mathrm{C}-\text { Stearic acid }\end{array}$ \\
\hline & \multicolumn{3}{|c|}{ CPM } \\
\hline Blank & 21.2 & 14717 & 32393 \\
\hline Blank & 20.6 & 14819 & 32804 \\
\hline Average & 20.9 & 14768 & 32599 \\
\hline Solvent & 20.8 & 15334 & 34665 \\
\hline Solvent & 19.3 & 15630 & 33836 \\
\hline Average & 20.1 & 15482 & 34251 \\
\hline $50 \mathrm{mM} \mathrm{HNO}_{3}$ & 19.7 & 15601 & 33763 \\
\hline $50 \mathrm{mM} \mathrm{HNO}_{3}$ & 19.6 & 15795 & 32044 \\
\hline Average & 19.6 & 15698 & 32904 \\
\hline $1 \mathrm{mM} \mathrm{HNO}_{3}$ & 23.4 & 16090 & 34738 \\
\hline $1 \mathrm{mM} \mathrm{HNO}_{3}$ & 21.7 & 15851 & 34872 \\
\hline Average & 22.6 & 15971 & 34805 \\
\hline $0.1 \mathrm{M} \mathrm{NaOH}$ & 20.4 & 14940 & 35329 \\
\hline $0.1 \mathrm{M} \mathrm{NaOH}$ & 19.0 & 14644 & 34240 \\
\hline Average & 19.7 & 14792 & 34785 \\
\hline Grand Average & 20.6 & 15342 & 33868 \\
\hline Standard Deviation & 1.2 & 542 & 1050 \\
\hline
\end{tabular}

Counting ${ }^{14} \mathrm{C}$-SDS and ${ }^{14} \mathrm{C}$-stearic acid appears to be unaffected by the presence of solvent, scrub, strip, or wash solutions. Backgrounds obtained under all conditions tested averaged $20.6 \pm 1.2$ $\mathrm{cpm}$. Counting of the ${ }^{14} \mathrm{C}$ labels under the various conditions produced deviations of total counts between all conditions tested of $\pm 3.0 \%$ to $\pm 3.5 \%$. Under these conditions, there was no indication of quenching by solvent, scrub, strip, or wash conditions. These results do indicate, however, that the specific activity of the ${ }^{14} \mathrm{C}$-stearic acid appears to be twice what was expected.

Lipophilic anions-batch test. The distribution of selected lipophilic anions (laurate, stearate, and dodecylsulfate) was investigated using the ${ }^{14} \mathrm{C}$-labeled surfactants. Two experimental approaches were taken. In the first simple series of batch tests $(\mathrm{O} / \mathrm{A}=1)$, the distribution of the anions as they were introduced into the solvent phase was investigated. The baseline solvent was employed as were the nominal scrub $\left(0.05 \mathrm{M} \mathrm{HNO}_{3}\right)$, strip $\left(0.001 \mathrm{M} \mathrm{HNO}_{3}\right)$, and aqueous phases. Additionally, a single wash solution $(0.1 \mathrm{M} \mathrm{NaOH})$ was also tested. The experimental matrix is shown in Table 4.4. 
Table 4.4. Batch test experimental matrix ${ }^{a}$

\begin{tabular}{|c|c|c|c|}
\hline & $\begin{array}{c}(\mathrm{A}) \\
50 \mathrm{mM} \mathrm{HNO} \\
\end{array}$ & $\begin{array}{c}\text { (B) } \\
1 \mathrm{mM} \mathrm{HNO}_{3}\end{array}$ & $\begin{array}{c}(\mathrm{C}) \\
0.1 \mathrm{M} \mathrm{NaOH} \\
\end{array}$ \\
\hline $\begin{array}{c}\text { 1S } \\
\text { Solvent only }\end{array}$ & Nos. $1-2$ & Nos. $3-4$ & Nos. $5-6$ \\
\hline $\begin{array}{c}2 \mathrm{~S} \\
\text { Solvent and } 2.0 \times 10^{-5} \mathrm{M} \\
14 \mathrm{C} \text {-Lauric acid } \\
3 \mathrm{~S}\end{array}$ & Nos. $7-8$ & Nos. $9-10$ & Nos. $11-12$ \\
\hline $\begin{array}{c}\text { Solvent and } 2.0 \times 10^{-5} \mathrm{M} \\
{ }^{14} \mathrm{C}-\mathrm{SDS} \\
4 \mathrm{~S}\end{array}$ & Nos. $13-14$ & Nos. $15-16$ & Nos. $17-18$ \\
\hline $\begin{array}{c}\text { Solvent and } 2.0 \times 10^{-5} \mathrm{M} \\
{ }^{14} \mathrm{C} \text {-Stearic acid }\end{array}$ & Nos. $19-20$ & Nos. $21-22$ & Nos. $23-24$ \\
\hline
\end{tabular}

${ }^{a} \mathrm{O} / \mathrm{A}=1, T=25^{\circ} \mathrm{C}$.

Contacts were performed in 15-mL polyethylene screw-capped tubes. Equal 1.5-mL volumes of the organic (baseline solvent) and aqueous phases were contacted. The surfactants, stearic acid, lauric acid, and SDS were added to the system at $2 \times 10^{-5} \mathrm{M}$. The ${ }^{14} \mathrm{C}$ activity for each contact was set at $0.2 \mu \mathrm{Ci} / \mathrm{mL}$ of solvent. Each contact, then, consisted of $1.5 \mathrm{~mL}$ solvent and $1.5 \mathrm{~mL}$ of the appropriate aqueous phase and $2 \times 10^{-5} \mathrm{M}$ of ${ }^{14} \mathrm{C}$-radiotracer-spiked surfactant. The labeled surfactant was introduced by adding $4.9 \mu \mathrm{L}$ of $5-\mathrm{mM}$ stock surfactant and $3 \mu \mathrm{L}$ of $0.1 \mu \mathrm{Ci} / \mu \mathrm{L}{ }^{14} \mathrm{C}$-labeled stearic acid, lauric acid, or SDS. All contacts were performed in duplicate. The contacts were performed using the standard rotating wheel for $50 \mathrm{~min}$ in the temperature-controlled air box set at $25{ }^{\circ} \mathrm{C}$. The phases were separated by centrifugation at 3000 RPM in a Sanyo MSE Mistral 2000R Centrifuge. A $200-\mu \mathrm{L}$ aliquot from each separated phase was removed for scintillation counting. These samples were transferred to $20-\mathrm{mL}$ scintillation vials containing $10 \mathrm{~mL}$ of Packard UltimaGold XR cocktail. The samples were counted for $10 \mathrm{~min}$ each in a Packard Tri-Carb 2500TR Liquid Scintillation Analyzer with the counting window set at 4.0 to $156 \mathrm{keV}$.

Lipophilic anions - ESS test. In the second, more detailed extraction process, the examination of the distribution of the lipophilic anions stearate (as stearic acid), laurate (as lauric acid), and dodecylsulfate (as SDS) was determined by performing the ESS Protocol using ${ }^{14} \mathrm{C}$-labeled anionic compounds. The surfactants were added at a concentration of $2 \times 10^{-5} \mathrm{M}$ with a specific activity of $0.1 \mu \mathrm{Ci} / \mathrm{mL}$ relative to the starting simulant phase. Aliquots of cold surfactant, 76.4-76.6 $\mu \mathrm{L}$ of 5 $\mathrm{mM}$ stock was added to the appropriate tubes along with $2.1 \mu \mathrm{Ci}$ of ${ }^{14} \mathrm{C}$-labeled surfactant. Extraction was performed using 50-mL Teflon screw-capped tubes while the scrub and three strip contacts were performed in $15-\mathrm{mL}$ conical-bottom polypropylene screw-capped tubes. Nominal O/A ratios were used, 0.33 for extraction $(7 \mathrm{~mL}$ of baseline solvent and $21 \mathrm{~mL}$ average full simulant, draw \#2) and 5 for the scrub and strips. The scrub operation used $6 \mathrm{~mL}$ of solvent and $1.2 \mathrm{~mL} 50 \mathrm{mM}$ $\mathrm{HNO}_{3}$; strip \#1 used $5 \mathrm{~mL}$ of solvent and $1.0 \mathrm{~mL}$ of $1 \mathrm{mM} \mathrm{HNO}$; strip \#2 used $4.0 \mathrm{~mL}$ of solvent, $0.8 \mathrm{~mL}$ of $1 \mathrm{mM} \mathrm{HNO}_{3}$; and strip \#3 used $3.0 \mathrm{~mL}$ of solvent and $0.6 \mathrm{~mL}$ of $1 \mathrm{mM} \mathrm{HNO}$. All ESS operations were performed in duplicate. The contacts were performed using the standard rotating wheel for $50 \mathrm{~min}$ in the temperature-controlled air box set at $25{ }^{\circ} \mathrm{C}$. The phases were separated by centrifugation at 3000 RPM in a Sanyo MSE Mistral 2000R Centrifuge. A $200 \mu \mathrm{L}$ aliquot from each separated phase was removed for scintillation counting. For better counting statistics, each sample involved four scintillation-counting operations. These operations were performed as checks on quenching by phase constituents as well as checks on background drift. Four 20-mL scintillation vials, each containing $10 \mathrm{~mL}$ of Packard UltimaGold XR cocktail, had the following additions made: 1) nothing, as this vial was a counting blank to determine background; 2) 200- $\mu \mathrm{L}$ phase sample; 3) 
$200-\mu \mathrm{L}$ phase sample plus a spike of $15-30 \mathrm{k} \mathrm{cpm}$ of the appropriate ${ }^{14} \mathrm{C}$-labeled surfactant; and 4 ) the spike alone. All samples were counted for $10 \mathrm{~min}$ each in a Packard Tri-Carb 2500TR Liquid Scintillation Analyzer with the counting window set at 4.0 to $156 \mathrm{keV}$.

Testing the effectiveness of a $\mathrm{NaOH}$ wash for removing surfactants from solvent. Should any of the various surfactants present in the SRS waste build up in the CSSX solvent, their removal will be necessary. To that end, a method for this treatment needed to be developed. One possible solution could be ion exchange. However, a simple wash protocol using base would more readily be integrated into the CSSX flowsheet.

To investigate this approach, a series of batch contacts were performed to test the ability of a $\mathrm{NaOH}$ wash to remove surfactants from solvent. ${ }^{14} \mathrm{C}$-labeled surfactants, SDS, stearic acid, and lauric acid, were added to solvent at $1 \times 10^{-5} \mathrm{M}$. Activity of each spike added was $0.2 \mu \mathrm{Ci} / \mathrm{mL}$. $\mathrm{NaOH}$ washes were contacted at an $\mathrm{O} / \mathrm{A}$ of 1 with solvent. Wash-solution concentrations tested included 1 , $0.3,0.1,0.03,0.01,0.003,0.001$, and zero $\mathrm{M} \mathrm{NaOH}$. Total test volume for each batch contact was $2 \mathrm{~mL}$.

\subsubsection{Partitioning of Amines}

Partitioning of dioctylamine. The partitioning of dioctylamine to several concentrations $\mathrm{HNO}_{3}$ was investigated. The partitioning of this compound to the process solutions was also examined. Dioctylamine was received from Aldrich $(98 \%)$ and was added at $50 \mathrm{mM}$ in the solvent (baseline solvent Lot B000718-156W). Methylene chloride was used as received from EM Science. Conditions for contacts remain the same as for 4-sec-butylphenol described in Section 4.2.2.7.

Partitioning of dioctylamine to the full simulant and to $0.01 \mathrm{M} \mathbf{N a O H}$. Contacting was performed by handshaking solvent containing dioctylamine and full simulant in $60 \mathrm{~mL}$ Teflon FEP centrifuge tubes at $20 \pm 1{ }^{\circ} \mathrm{C}$, followed by rotation on a Glas-Col laboratory rotator at $25 \pm 0.5{ }^{\circ} \mathrm{C}$ using an $\mathrm{O} / \mathrm{A}$ ratio of $0.33(5 \mathrm{~mL} / 15 \mathrm{~mL})$. The dioctylamine-containing solvent was also contacted with $0.01 \mathrm{M} \mathrm{NaOH}$ in the same manner using an $\mathrm{O} / \mathrm{A}$ ratio of $0.20(5 \mathrm{~mL} / 25 \mathrm{~mL})$. All samples were run in duplicate. The samples were equilibrated for $2 \mathrm{~h}$ at $25^{\circ} \mathrm{C}$ and were centrifuged for $25 \mathrm{~min}$ at the same temperature. A portion of the solvent phase was carefully removed and transferred to a clean, dry glass vial. The aqueous phase was removed via pipette and transferred to a clean graduated cylinder for measurement prior to transfer to a clean $60-\mathrm{mL}$ Teflon FEP centrifuge tube to be backextracted with methylene chloride. Each aqueous phase was back-extracted by handshaking three times successively with approximately $10 \mathrm{~mL}$ of fresh methylene chloride each time. The methylene chloride phase was carefully removed using glass, disposable pipettes and transferred to a clean, glass bottle.

Partitioning of dioctylamine to various concentrations of $\mathrm{HNO}_{3}$. The partitioning of dioctylamine to nitric acid was examined using $\mathrm{O} / \mathrm{A}$ ratios of 0.10 . The volumes used were $5 \mathrm{~mL}$ solvent spiked with $50 \mathrm{mM}$ dioctylamine to $50 \mathrm{~mL}$ acid in $250 \mathrm{~mL}$ Teflon FEP centrifuge bottles. These samples were contacted and separated in the same manner as described above. Nitric acid concentrations examined were $0.10,0.05,0.01$, and $0.001 \mathrm{M}$. The 0.01 - and $0.001-\mathrm{M}$ samples displayed fine, white precipitates, which were centrifuged to the interface several times while removing the aqueous phases for back-extraction. The solvent phases were transferred cleanly to glass bottles, while the aqueous phases were centrifuged repeatedly and carefully transferred and measured for back-extraction. These aqueous phases were adjusted to $\mathrm{pH} 12$ with $\mathrm{NaOH}$ and back-extracted with methylene chloride as described above. All solvent phases and methylene chloride phases from back-extractions were delivered to the analytical team at the ORNL RMAL for analysis.

Analytical method for dioctylamine determination. The organic samples were diluted at a ratio of $1 / 10$ with isopropanol containing $1 \%$ triethylamine (TEA). Triethylamine was added to all samples to ensure that the dioctylamine would exist in the neutral form. The methylene chloride phases from back-extractions were evaporated under a stream of nitrogen at $65-75{ }^{\circ} \mathrm{C}$ to $<0.5 \mathrm{~mL}$. 
Isopropanol was added at a volume of $0.5 \mathrm{~mL}$ and the remaining methylene chloride evaporated. Isopropanol was again added to bring sample to final volume of $2.2 \mathrm{~mL}$. The analysis was carried out using a Varian Model 3500 gas chromatograph equipped with a Varian Model 8100 autosampler and flame ionization detector. The column used was a J\&W Durabond DB-5, $30 \mathrm{M} \times 0.25 \mathrm{~mm} \times$ $0.25 \mu \mathrm{m}$ film thickness. Calibration was performed prior to sample analysis using a $100-\mu \mathrm{g} / \mathrm{g}$ standard. A control containing $50 \mathrm{mM}$ dioctylamine in the solvent was used for quantitation. Relative precision of replicate analyses was $\pm 2 \%$.

GC conditions were as follows:

Injector temperature: $250{ }^{\circ} \mathrm{C}$

Detector inlet temperature: $275^{\circ} \mathrm{C}$

Initial column temperature: $80^{\circ} \mathrm{C}, 8$-min hold

Temperature control: $10^{\circ} \mathrm{C} / \mathrm{min}$. to $275^{\circ} \mathrm{C}$.

Total run time: $35.5 \mathrm{~min}$

Partitioning of trimethylamine. The standard ESS protocol using baseline solvent and salts + metals simulant (Table 2.2) was employed at $25{ }^{\circ} \mathrm{C}$ using ${ }^{14} \mathrm{C}$-labeled trimethylamine (TMA) to facilitate analysis by beta liquid-scintillation counting. ${ }^{14} \mathrm{C}$-labeled TMA was added to the simulant at $0.2 \mu \mathrm{Ci} / \mathrm{mL}$. Phase-dependent quenching was corrected for using the internal-standard method.

\subsubsection{Ion exchange of Organic Anions}

Weighed samples of resins equilibrated with pristine washed solvent were separated from the liquid phase by careful decantation to ensure that no resin bits were carried over. A measured 2-3 $\mathrm{mL}$ volume of the stock solvent solution containing SDS or SA was added. Samples were equilibrated in the water bath at $25{ }^{\circ} \mathrm{C}$ with agitation. Small samples of the liquid phase $(0.01 \mathrm{~mL}$ aliquots) were withdrawn after $1 \mathrm{~h}$ and again after 18-22 $\mathrm{h}$ and added directly into $20-\mathrm{mL}$ standard scintillation vials containing $10 \mathrm{~mL}$ of Packard Ultima Gold XR scintillation cocktail. Following a dark-adaptation period for at least $30 \mathrm{~min}$, the ${ }^{14} \mathrm{C}$ activity was determined using a Packard Tricarb model 2700TR liquid-scintillation counter employing a standard counting protocol. The distribution ratio of SDS or SA (in $\mathrm{mL} / \mathrm{g}$ ) was calculated assuming that a mass balance of $100 \%$ is sustained for each sample as described previously: ${ }^{62}$

$$
K_{d}=\frac{\left[{ }^{14} \mathrm{C} c p m(\text { total })-{ }^{14} \mathrm{C} c p m(\text { solvent })\right] / g(\text { dry resin })}{{ }^{14} \mathrm{C} c p m(\text { solvent }) / m L(\text { solvent })}
$$

where ${ }^{14} \mathrm{Ccpm}$ (total) is the total number of counts per minute due to ${ }^{14} \mathrm{C}$ in the sample determined by the counting of the stock solvent solution with no resin added; ${ }^{14} \mathrm{Ccpm}$ (solvent) is the number of counts per min in the solvent contacted with the resin sample determined from the aliquot of the solvent of the known volume $(\mathrm{mL})$ in contact with the resin sample of the known weight. In the equation, the weight of dry resin ( $\mathrm{g}$ ) is used which was calculated from the known percent-solids determined for each resin as described above.

\subsubsection{Partitioning of Tributylphosphate and 1-Butanol}

Tributylphosphate (TBP) and 1-butanol partitioning experiments were carried out using standard batch contacting techniques in duplicate. Use of ${ }^{14} \mathrm{C}$-labeled TBP and 1-butanol facilitated analysis by beta liquid-scintillation counting. Phase-dependent quenching was corrected by use of the internal-standard method. ${ }^{14} \mathrm{C}$-labeled compounds were added at $0.1 \mu \mathrm{Ci} / \mathrm{mL}$ for $\mathrm{TBP}$ and 0.2 $\mathrm{mCi} / \mathrm{mL}$ for 1-butanol relative to the simulant volume for extraction and to the solvent volume for contacts involving scrub and strip solutions. 1-Butanol was added at its concentration in the full simulant, $2.7 \times 10^{-5} \mathrm{M}$, or equivalently, $8.1 \times 10^{-5} \mathrm{M}$ in the solvent (assuming quantitative extraction 
at $\mathrm{O} / \mathrm{A}=0.33$ ). However, the investigation of TBP was performed at $6.67 \times 10^{-6} \mathrm{M}$ in the simulant (or equivalently, $2.0 \times 10^{-5} \mathrm{M}$ in the solvent), since the specific activity of the ${ }^{14} \mathrm{C}$-labeled TBP (15 $\mathrm{mCi} / \mathrm{mmol}$ ) would not allow the test to be performed at the typical TBP simulant concentration of $1.88 \times 10^{-6} \mathrm{M}$.

\subsection{RESULTS AND DISCUSSION}

\subsubsection{Partitioning of Solvent Components}

Previous work involving the first-generation solvent showed that BOBCalixC6 and the Cs-3 modifier partition very weakly to the aqueous phases involved in the flowsheet, losses being low even when a very large aqueous volume is used. ${ }^{8}$ Hence, it was necessary to consider analytical detection limits so as to establish lower limits for partition ratios. The following criteria define the analytical detection limits applicable to the current baseline solvent components:

Key for minimum analytical requirements given below:

(1) the absolute weight that must be injected

(2) the concentration in the organic phase before any sample preparation

(3) the concentration in the aqueous phase before any sample preparation:
Calixarene by RP-HPLC:
(1) 9 ng injected
(2) $8 \times 10^{-5} \mathrm{M}$ in the original organic phase sample
(3) $8 \times 10^{-7} \mathrm{M}$ in the original aqueous phase sample

$\begin{array}{ll}\text { Modifier by RP-HPLC: } & \text { (1) } 40 \mathrm{ng} \text { injected } \\ & \text { (2) } 1 \times 10^{-3} \mathrm{M} \text { in the original organic phase sample } \\ \text { (3) } 1 \times 10^{-5} \mathrm{M} \text { in the original aqueous phase sample }\end{array}$

Trioctylamine by GC (Est.): (1) 0.8 ng injected

(2) $2 \times 10^{-5} \mathrm{M}$ in the original organic phase sample

(3) $2.5 \times 10^{-9} \mathrm{M}$ in the original aqueous phase sample

Note that partition ratios $P$ and distribution ratios $D$ have the same working definition, namely the organic-phase molarity divided by the aqueous-phase molarity of a species. Ideally, partitioning refers to a Nernstian process in which a species retains the same chemical form in the organic phase as it has in the aqueous phase. ${ }^{63}$ Thus, technically, the partition ratio $P$ is identical to the thermodynamic equilibrium constant (when $P$ is valid at infinite dilution), whereas the distribution ratio $D$ more generally corresponds to a chemical reaction that includes other species (e.g., hydrogen ion, extractant, etc.) and thereby is not necessarily identical to the associated equilibrium constant. Although there is widely differing terminology used in the literature, we have here chosen to associate partitioning with the distribution of an organic species, especially a neutral organic compound.

Table 4.5 defines the equilibrations that were performed and reports the results. All experiments were performed at $25^{\circ} \mathrm{C}$ at the indicated $\mathrm{O} / \mathrm{A}$ ratios. Very low $\mathrm{O} / \mathrm{A}$ ratios were usually employed so to both enhance loss to the aqueous phase that might be detected in the organic-phase analysis and increase the amount that could be detected upon concentrating the analyte from the aqueous phase by solid-phase extraction. Four sets of experiments were performed as separated by dotted lines in the table. The first set of three tests employed baseline solvent equilibrated directly with the aqueous flowsheet phases indicated. The second set involved the same aqueous phases but followed the ESS protocol with standard O/A ratios; that is, the solvent from the extraction was scrubbed and stripped, 


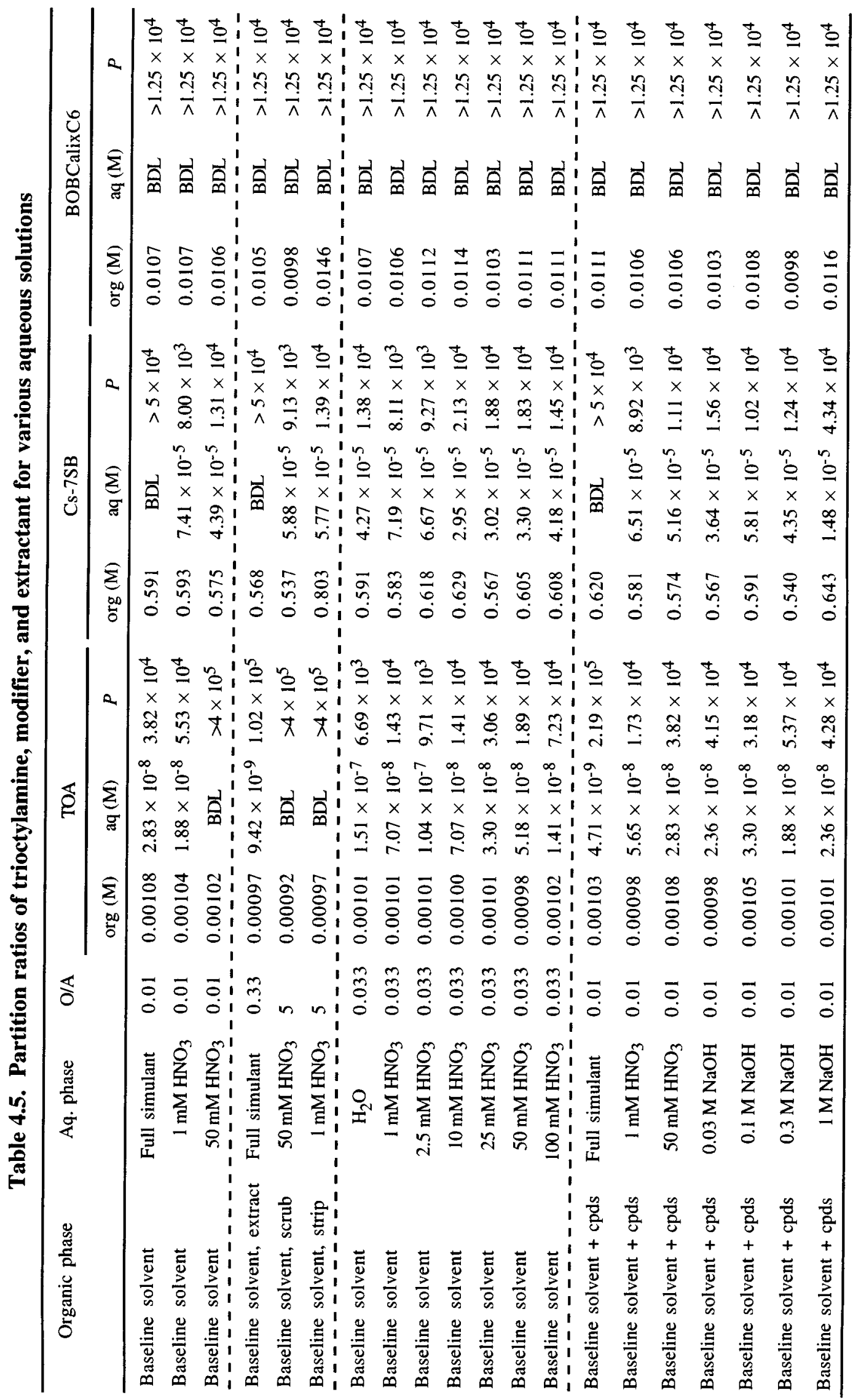


with analysis performed at each stage. The third set employed baseline solvent equilibrated directly with the aqueous phases indicated, with the intent to learn if an acid dependence could be discerned. The fourth set also employed baseline solvent equilibrated directly with the aqueous flowsheet phases indicated, except that the solvent contained the following additional compounds: $0.5 \mathrm{ppm}$ TBP, 25 ppm DBP, $25 \mathrm{ppm}$ monobutylphosphoric acid (MBP), and $1 \mathrm{mM} \mathrm{SBP.} \mathrm{It} \mathrm{was} \mathrm{the} \mathrm{intent} \mathrm{regarding}$ the fourth set to also determine the distribution of these additional compounds; however, it was found that further analytical methods development was required, and these determinations were carried out in separate experiments discussed later.

Overall, the organic-phase concentrations of TOA, Cs-7SB, and BOBCalixC6 were essentially unchanged relative to their nominal concentrations in the baseline solvent. Unfortunately, the Cs-7SB concentrations are on average $20 \%$ high, suggesting a calibration problem in the analyses. The aqueous-phase concentrations of three solvent components were all extremely low or below detection limits (BDL). Thus, all $P$ values are very high. Owing to the absence of trends or the lack of consistency among similar tests, it is doubtful that the reported $P$ values reflect real differences in partitioning. Rather, the values likely reflect the limitations of the analytical methods, minor levels of entrainment, or other artifacts. All concentrations of the calixarene in the aqueous phases were below detection limits, which indicates $P>12500$. Although measurable levels of TOA and Cs-7SB were usually obtained, the data clearly support the following lower bounds: $P_{\text {TOA }}>6000$ and $P_{\text {Cs-7SB }}>$ 8000. Likely, the actual values are much higher, and the data do suggest that the partition ratios are highest for the simulant: $P_{\mathrm{TOA}}>30,000$ and $P_{\mathrm{Cs}-7 \mathrm{SB}}>50,000$.

In terms of a process in which the solvent will be recycled an estimated 2800 times, ${ }^{41,42}$ the high partition ratios for the solvent components indicate that solubility losses and consequent need for solvent replacement or recovery on this account likely will be low. The highest aqueous flow is that of the waste, and the solvent partition ratios to this phase tend to be highest, presumably as a result of salting-out. Thus, the need for solvent recovery or replacement will arise predominantly due to entrainment losses, which can be dealt with by mechanical (e.g., centrifugation, sedimentation/decantation, etc.) or chemical (e.g., washing the aqueous phase with diluent).

\subsubsection{Aqueous pH Profile}

As discussed in Chap. 3, the $\mathrm{pH}$ of the aqueous phase reflects hydrogen-ion transfer in equilibrations of the solvent with various aqueous phases. It also can also serve as a convenient indicator of aqueous carry-over from extraction into scrubbing and stripping. To provide information on equilibrium acid balance, a series of measurements of aqueous $\mathrm{pH}$ was made in which a microelectrode was employed, as calibrated with aqueous buffer solutions. The aqueous phases were available from previous experiments described in Chap. 3.

As shown in Table 4.6, Series A-1, acid extracted from the scrub solution and partially released upon stripping makes the first strip solution slightly more acidic by ca. $0.35 \mathrm{mM} \mathrm{H} \mathrm{H}^{+}$ion concentration than the plateau value. Series C-1, which does not follow an extraction, shows almost the same behavior. However, forward-stripping Series C-2, which does not follow a contact with scrub solution, reveals uptake of acid and decreasing $\mathrm{pH}$ with successive strips.

\subsubsection{Distribution of Alkali Metal Cations}

\subsubsection{General Approach}

Analysis of the distribution behavior of alkali metal cations between aqueous and solvent phases is important for the understanding cesium extraction behavior from waste solutions. The nature of the waste/solvent extraction system makes this task especially hard because of the presence of multiple components in both phases; namely, their chemical and transport properties are mutually dependent and determine the state of the extraction system and thus cesium distribution. A thorough understanding of the behavior and functionality of the individual components of the system would allow quantitative prediction of the cesium extraction under different conditions, especially as aided 
Table 4.6. Aqueous pH profile in batch ESS tests

\begin{tabular}{|c|c|c|c|c|c|c|c|c|c|c|c|}
\hline Series $^{a}$ & $\begin{array}{l}\text { Contacting } \\
\text { sequence }\end{array}$ & Simulant & Scrub & $\begin{array}{c}\text { Strip } \\
\# 1\end{array}$ & $\begin{array}{c}\text { Strip } \\
\# 2\end{array}$ & $\begin{array}{c}\text { Strip } \\
\# 3\end{array}$ & $\begin{array}{c}\text { Strip } \\
\# 4\end{array}$ & $\begin{array}{l}\text { Strip } \\
\# 5\end{array}$ & $\begin{array}{c}\text { Strip } \\
\# 6\end{array}$ & $\begin{array}{c}\text { Strip } \\
\# 7\end{array}$ & $\begin{array}{c}\text { Strip } \\
\# 8\end{array}$ \\
\hline A-1 & $\mathrm{ESS}_{5}$ & Full & 1.78 & 2.84 & 2.90 & 2.94 & 2.96 & 2.95 & & & \\
\hline$A-2$ & $\mathrm{ESS}_{5}$ & Full @ $0.2 \mathrm{mM} \mathrm{Cs}$ & 1.80 & 2.83 & 2.92 & 2.94 & 2.95 & 3.07 & & & \\
\hline A-3 & $\mathrm{ESS}_{5}$ & Full @ $0.3 \mathrm{mM} \mathrm{Cs}$ & 1.81 & 2.83 & 2.93 & 2.94 & 2.96 & 2.99 & & & \\
\hline$A-4$ & $\mathrm{ESS}_{5}$ & Full @ $0.5 \mathrm{mM} \mathrm{Cs}$ & 1.84 & 2.83 & 2.95 & 2.95 & 2.95 & 2.98 & & & \\
\hline A-5 & $\mathrm{ESS}_{5}$ & Full @ $0.7 \mathrm{mM} \mathrm{Cs}$ & 1.84 & 2.87 & 2.96 & 2.95 & 2.93 & 2.95 & & & \\
\hline B-1 & $\mathrm{E}_{2} \mathrm{SS}_{5}$ & Full & 1.82 & 2.85 & 2.94 & $\begin{array}{r}2.96 \\
-\end{array}$ & 2.96 & $\begin{array}{r}2.99 \\
-\end{array}$ & & & \\
\hline$C-1$ & $\mathrm{SS}_{5}$ & None & 1.44 & 2.84 & 2.90 & 2.91 & 2.94 & 2.96 & & & \\
\hline $\begin{array}{ll}\mathrm{C}-2 \\
---\end{array}$ & $\mathrm{S}_{5}$ & None & & 4.02 & 3.46 & 3.24 & 3.17 & 3.15 & & & \\
\hline D-1 & $\mathrm{ESS}_{4}$ & Full & & & & 2.98 & 3.00 & & & & \\
\hline D-2 & $\left(\mathrm{ESS}_{4}\right)_{1}+\mathrm{ESS}_{4}$ & Full & 1.78 & 2.77 & 2.90 & 2.95 & 3.00 & & & & \\
\hline D-3 & $\left(\mathrm{ESS}_{4}\right)_{7}+\mathrm{ESS}_{4}$ & Full & 1.76 & 2.71 & 2.86 & 2.97 & 2.97 & & & & \\
\hline D-4 & $\left(\mathrm{ESS}_{4}\right)_{4}+\mathrm{ESS}_{8}$ & Full & 1.75 & 2.71 & 2.90 & 2.96 & 2.95 & 2.93 & 2.96 & 2.96 & 2.98 \\
\hline E-1 & $\mathrm{E}_{5} \mathrm{SS}_{6}$ & Full & 1.97 & 2.91 & 3.12 & 3.15 & 3.16 & 3.17 & 3.15 & & \\
\hline E-2 & $\mathrm{E}_{5} \mathrm{SS}_{6}$ & Salts + metals & 1.99 & 3.05 & 3.12 & 3.15 & 3.15 & 3.14 & 3.14 & & \\
\hline E-3 & $\mathrm{E}_{5} \mathrm{SS}_{6}$ & Salts only & 2.09 & 3.10 & 3.15 & 3.22 & 3.22 & 3.23 & 3.22 & & \\
\hline E-4 & $\mathrm{E}_{5} \mathrm{SS}_{6}$ & SRS\#2 & 1.79 & 2.92 & 2.99 & 3.00 & 3.02 & 3.05 & 2.99 & & \\
\hline
\end{tabular}

${ }^{a}$ Series A, B, C, D, and E correspond to the experiments described in Table 3.7, 3.9 (Set \#2), 3.8, 3.26, and Table 3.25.

by computer analysis reported under separate cover. 25 Sodium and potassium ions are the closest cesium analogs with many similar physical and chemical properties. Their presence in the waste solutions in large excess relative to cesium ion greatly affects cesium transport into the solvent phase.

Distribution experiments of the alkali metal cations were designed to provide data for the prediction of cesium distribution ratios and physical properties of the extraction system. The approach was to examine first the transport behavior of the alkali metal ions individually using simple aqueous phases containing single cesium, potassium, or sodium salts. Nitrate salts were selected for this purpose since nitrate is the most abundant inorganic anion in the waste. To elucidate the extraction mechanism, these experiments were carried out at varying acidity or alkalinity. Secondly, a systematic investigation was performed using waste-simulant solution. To this end, the distribution ratios of alkali metal ions were studied under conditions of variable cesium and potassium concentrations in the simulant, variable modifier concentration in the solvent, variable phase ratio, or variable number of contacts of solvent with simulant. Lastly, distribution experiments were performed to test transport properties of the solvent without BOBCalixC6 extractant so as to reflect upon that part of the extraction that is not due to complexation by the calixarene.

\subsubsection{Distribution of Alkali Metal Cations in the Absence of BOBCalixC6}

As may be seen in Table 4.7, the baseline solvent without the BOBCalixC6 extractant possesses a feeble ability to extract $\mathrm{Cs}^{+}$ion from the full simulant, and thus, almost all of the observed extraction by the baseline solvent may be attributable to complexation by the calixarene. The values of the cesium distribution ratio without $\mathrm{BOBCalixC} 6$ are over 6,000 times less than those obtained using the full solvent, containing $0.01 \mathrm{M}$ BOBCalixC6 $\left(D_{\mathrm{Cs}}=16.9\right.$, Table 3.4$)$. Only $0.4 \%$ of aqueous $\mathrm{Cs}^{+}$ion 
distributes into the organic phase in comparison with $85 \%$ in the presence of BOBCalixC6 (see below).

It may also be seen that values of $D_{\mathrm{Cs}}$ are very low for aqueous phases containing $\mathrm{CsNO}_{3}$ as a single salt at $\mathrm{pH}$ of 7 . These values increase over 50 -fold under increasing alkaline conditions. Such pronounced $\mathrm{pH}$ effects can be attributed to the two different distribution mechanisms taking place at neutral and at high $\mathrm{pH}$. Cs-7SB modifier is the major solvent component, and in the distribution experiments without extractant in the solvent, its ability to solvate ionic species determines the affinity of the $\mathrm{Cs}^{+}$ion for the organic phase. Under neutral conditions, $\mathrm{Cs}^{+}$ion along with the counter-anion is expected to be transported into the solvent phase by a simple ion-pair distribution mechanism, as reviewed in detail: 56

$$
\begin{gathered}
\mathrm{M}_{(\mathrm{aq})}^{+}+\mathrm{X}_{(\mathrm{aq})}^{-} \rightleftharpoons \mathrm{MX}_{(\mathrm{org})} \\
\mathrm{M}_{(\mathrm{aq})}^{+}+\mathrm{X}_{(\mathrm{aq})}^{-} \rightleftharpoons \mathrm{M}_{(\text {org })}^{+}+\mathrm{X}_{(\text {org })}^{-}
\end{gathered}
$$

The Cs-7SB modifier possesses an alcohol functionality and thus both hydrogen-bond donor and acceptor capabilities, allowing for more extensive solvation of both cation and anion than non-polar and aprotic diluents. Nevertheless, the ion-pair distribution mechanism shown is thermodynamically unfavorable because the solvation of the ions by the alcohol is weaker than the solvation by water molecules. This net hydrophilicity ultimately results in low cesium distribution values. At low concentration of ionic species in the solvent phase (Table 4.7), extraction of dissociated ion pairs is expected to dominate (Eq. 4.2). At high pH the weakly acidic modifier is partially deprotonated and is thought to function essentially as a cation exchanger. ${ }^{51,52}$ Thus, there is no need to transfer the counteranion to the solvent phase; instead, the modifier's proton is driven from the organic to the aqueous phase. Simultaneously, an aqueous cation is transported to the organic phase:

$$
\begin{gathered}
\mathrm{M}^{+}{ }_{(\mathrm{aq})}+\mathrm{OH}_{(\mathrm{aq})}^{-}+\mathrm{ROH}_{(\text {org })} \rightleftharpoons \mathrm{MOR}_{(\mathrm{org})}+\mathrm{H}_{2} \mathrm{O}_{(\mathrm{aq})} \\
\mathrm{M}_{(\mathrm{aq})}^{+}+\mathrm{OH}_{(\mathrm{aq})}^{-}+\mathrm{ROH}_{(\mathrm{org})} \rightleftharpoons \mathrm{RO}_{(\mathrm{org})}^{-}+\mathrm{M}^{+}{ }_{(\mathrm{org})}+\mathrm{H}_{2} \mathrm{O}_{(\mathrm{aq})}
\end{gathered}
$$

Cesium distribution by a cation-exchange mechanism at high $\mathrm{pH}$ is more thermodynamically favorable than simple ion-pair distribution and results in higher distribution values. In this case, total ionic strength of the solvent phase is increased and promotes ion pairing which additionally benefits the metal ion distribution by the ion-exchange mechanism in accord with Eq. 4.3.

Distribution results obtained for $\mathrm{K}^{+}$ion extraction in the absence of BOBCalixC6 closely resemble those observed for $\mathrm{Cs}^{+}$ion. Values of $D_{\mathrm{K}}$ were very low for aqueous phases containing single $\mathrm{KNO}_{3}$ salt at $\mathrm{pH} 7$ and increased nearly 30-fold under more alkaline conditions (Table 4.8). Distribution of $\mathrm{K}^{+}$ions is expected to be described by the mechanism proposed for $\mathrm{Cs}^{+}$ions (Eqs.

Table 4.7. Cesium distribution ratios and organic-phase concentrations observed in distribution tests at $25^{\circ} \mathrm{C}$ using $0.5 \mathrm{M}$ Cs-7SB modifier and $0.001 \mathrm{M}$ TOA in Isopar $L$

\begin{tabular}{cccc}
\hline Aqueous phase composition & $\mathrm{O} / \mathrm{A}$ & $D_{\mathrm{Cs}}$ & {$\left[\mathrm{Cs}^{+}\right]_{\text {org,eq }} \mathrm{M}$} \\
\hline $0.1 \mathrm{M} \mathrm{CsNO}_{3}$ & 1 & $(3.9 \pm 0.2) \times 10^{-5}$ & $3.9 \times 10^{-6}$ \\
$1 \mathrm{M} \mathrm{CsNO}_{3}$ & 1 & $(4.4 \pm 0.2) \times 10^{-5}$ & $4.4 \times 10^{-5}$ \\
$0.1 \mathrm{M} \mathrm{CsNO}_{3} / 2 \mathrm{M} \mathrm{NaOH}$ & 1 & $(2.84 \pm 0.02) \times 10^{-3}$ & $2.8 \times 10^{-4}$ \\
$1 \mathrm{M} \mathrm{CsNO}_{3} / 2 \mathrm{M} \mathrm{NaOH}$ & 1 & $(2.43 \pm 0.02) \times 10^{-3}$ & $2.4 \times 10^{-3}$ \\
Full simulant & 0.33 & $(2.78 \pm 0.02) \times 10^{-3}$ & $3.9 \times 10^{-7}$ \\
Full simulant & 0.1 & $(3.23 \pm 0.04) \times 10^{-3}$ & $4.5 \times 10^{-7}$ \\
\hline
\end{tabular}


4.1-4.4). Values of $D_{\mathrm{K}}$ observed with the full simulant and solvent containing no extractant are approximately 180 times less than those obtained using full solvent containing $0.01 \mathrm{M}$ BOBCalixC6 ( $D_{\mathrm{K}}=0.14$, see below). Less than $0.1 \%$ of the $\mathrm{K}^{+}$ions in the aqueous phase distribute into the solvent phase.

In contrast to the distribution results obtained with $\mathrm{Cs}^{+}$and $\mathrm{K}^{+}$ions, values of $D_{\mathrm{Na}}$ measured in the tests with full simulant (Table 4.8) are only about 2 times less than those obtained using full solvent containing $0.01 \mathrm{M}$ BOBCalixC6 $\left(D_{\mathrm{Na}}=6.4 \times 10^{-4}\right.$, see below). Such small differences can be attributed to several factors. Most importantly, this result confirms that BOBCalixC6 exhibits little ability to bind $\mathrm{Na}^{+}$ion, and the observed $\mathrm{Na}^{+}$extraction by the baseline solvent is largely due to ion exchange of the modifier's proton. Other factors that promote $\mathrm{Na}^{+}$ion distribution into organic phase include its high abundance in the simulant, which causes salting-out effects, and formation of the tight ion pair with alkoxide anion due to the high charge density on the $\mathrm{Na}^{+}$ion.

Table 4.8. $\mathrm{K}^{+}$and $\mathrm{Na}^{+}$distribution ratios and organic-phase concentrations at $25^{\circ} \mathrm{C}$ using $0.5 \mathrm{M}$ Cs-7SB modifier and 0.001 $M$ TOA in Isopar $L$

\begin{tabular}{cccccc}
\hline $\begin{array}{c}\text { Aqueous-phase } \\
\text { composition }\end{array}$ & $\mathrm{O} / \mathrm{A}$ & $D_{\mathrm{K}} \times 10^{4}$ & {$\left[\mathrm{~K}^{+}\right]_{\text {org,eq }}, \mathrm{M}$} & $D_{\mathrm{Na}} \times 10^{4}$ & {$\left[\mathrm{Na}^{+}\right]_{\text {org,eq }}, \mathrm{M}$} \\
\hline $0.3 \mathrm{M} \mathrm{KNO}_{3}$ & 0.33 & $0.33 \pm 0.02$ & $1.2 \times 10^{-5}$ & & \\
$1.5 \mathrm{M} \mathrm{KNO}_{3}$ & 0.33 & $0.40 \pm 0.04$ & $5.1 \times 10^{-5}$ & & \\
$0.3 \mathrm{M} \mathrm{KNO}_{3} / 2 \mathrm{M} \mathrm{NaOH}$ & 0.33 & $8.2 \pm 0.3$ & $2.4 \times 10^{-4}$ & $2.6 \pm 0.1$ & $5.3 \times 10^{-4}$ \\
$1.5 \mathrm{M} \mathrm{KNO}_{3} / 2 \mathrm{M} \mathrm{NaOH}$ & 0.33 & $9.2 \pm 0.1$ & $1.4 \times 10^{-3}$ & $3.3 \pm 0.1$ & $6.6 \times 10^{-4}$ \\
Full simulant & 0.33 & $8.4 \pm 0.5$ & $1.7 \times 10^{-5}$ & $3.9 \pm 0.1$ & $2.3 \times 10^{-3}$ \\
Full simulant & 0.10 & $9.9 \pm 0.6$ & $2.0 \times 10^{-5}$ & $4.5 \pm 0.1$ & $2.7 \times 10^{-3}$ \\
\hline
\end{tabular}

\subsubsection{Extraction of Alkali Metal Cations from Simple Salt Matrices}

Extraction behavior of the baseline solvent toward $\mathrm{Cs}^{+}, \mathrm{K}^{+}$, and $\mathrm{Na}^{+}$ions was characterized by systematic variation of the aqueous-phase metal ion concentration, varying the nitrate salt concentrations under acidic, neutral, or alkaline conditions (Figs. 4.2-4.4) at $25{ }^{\circ} \mathrm{C}$. A major reason for carrying out the experiment in this way was to correlate third-phase formation with loading. Qualitatively, $\mathrm{Cs}^{+}$ion yields stronger extraction than $\mathrm{K}^{+}$ion and much stronger than $\mathrm{Na}^{+}$ion. This bias could be rationalized in terms of the Gibbs energy of the overall extraction reaction that is governed by two principal processes, namely partitioning of the cation between the aqueous and organic phases and its subsequent complexation in the solvent by the macrocyclic extractant. The complexation chemistry of calix-crown compounds is now fairly well understood, and it is welldocumented that calix[4]arene-crown-6 receptors in the 1,3-alternate conformation preferentially bind $\mathrm{Cs}^{+}$ion among alkali metal ions. ${ }^{64}$ It was reported that in methanol or acetonitrile the binding constant of 1,3-alt-calix[4]arene-bis(benzocrown-6) with $\mathrm{Cs}^{+}$ion is about a half order of magnitude higher than that with $\mathrm{K}^{+}$ion and over three orders of magnitude higher than that with $\mathrm{Na}^{+}$ion. The same trend is expected for the structurally and conformationally similar BOBCalixC6. Also, the Gibbs energy of ion partitioning decreases in the sequence $\mathrm{Na}^{+}>\mathrm{K}^{+}>\mathrm{Cs}^{+}$as a reflection of the reduction of charge density on the alkali metal ion. Owing to these two factors, the strongest $\mathrm{Cs}^{+}$ extraction is thought to be the cumulative result of the favorable $\mathrm{Cs}^{+}$binding by BOBCalixC6 extractant and the least energy-consuming partitioning of this cation into the organic phase. Very weak $\mathrm{Na}^{+}$partitioning into the solvent phase is brought about by the inability of the extractant to bind this cation and by the high Gibbs energy of $\mathrm{Na}^{+}$ion distribution.

Tables 4.9-4.11 and Figs. 4.2-4.4 show the effect of aqueous-phase metal ion concentration on the $\mathrm{Cs}^{+}, \mathrm{K}^{+}$, and $\mathrm{Na}^{+}$distribution ratios. As the concentration of $\mathrm{Cs}^{+}$or $\mathrm{K}^{+}$ion in the aqueous phase increases, the distribution ratios increase and then gradually decline due to the progressive loading of 
the extractant. For $\mathrm{Cs}^{+}$ion, BOBCalixC6 loading values are very high, reaching $146 \%$. Loading higher than $100 \%$ indicates formation of $2: 1$, in addition to $1: 1$, metal:calixarene complex species. BOBCalixC6 has two identical oxygen-donor cavities and is able to accommodate two $\mathrm{Cs}^{+}$ions as was demonstrated previously. ${ }^{27}$ In the case of $\mathrm{K}^{+}$, loading does not exceed $84 \%$, and formation of a third phase was observed at a loading of $75 \%$ and higher (Fig. 4.3). In the experiments with $\mathrm{Cs}^{+}$ion, a third phase formed at loading near $100 \%$ under alkaline conditions and $140 \%$ under neutral and acidic conditions (Fig. 4.2). These results reveal that the solubility limit of the potassium-containing species in the solvent is much less than that of the cesium complex species. Detailed discussion of the third-phase question is given in Chap. 7 of this report.

Linear dependence on the aqueous sodium concentration was observed for $D_{\mathrm{Na}}$ under neutral and alkaline conditions (Fig. 4.4). The distribution of sodium gradually increased with slopes of 0.2 and 0.5 , respectively. This behavior indicates partial association of the ionic species in the solvent phase. ${ }^{56}$ The greater slope for the alkaline aqueous phase suggests more pronounced ion-pairing.

In Figs. 4.2-4.3, the distribution behavior of cesium and potassium observed under neutral and acidic conditions is very similar, suggesting an ion-pair extraction mechanism where the reaction of the cation complexation by the macrocyclic extractant is the driving force of the cationic transport from the aqueous phase into the organic phase. Taking into account that BOBCalixC6 loading with $\mathrm{Cs}^{+}$exceeds $100 \%$ at neutral or acidic $\mathrm{pH}$, formation of both $1: 1$ and $2: 1$ metal:calixarene species are implicated:

$$
\begin{gathered}
\mathrm{M}^{+}(\mathrm{aq})+\mathrm{X}^{-}(\mathrm{aq})+\mathrm{B}(\mathrm{org}) \rightleftharpoons \mathrm{MBX}_{(\text {org })} \\
\mathrm{M}^{+}(\mathrm{aq})+\mathrm{X}^{-}(\mathrm{aq})+\mathrm{B}(\mathrm{org}) \rightleftharpoons \mathrm{MB}^{+}(\text {org })+\mathrm{X}^{-}(\text {org }) \\
\mathrm{M}^{+}(\mathrm{aq})+\mathrm{X}^{-}(\mathrm{aq})+\mathrm{MBX}_{(\mathrm{org})} \rightleftharpoons \mathrm{M}_{2} \mathrm{BX}_{2}(\text { org })
\end{gathered}
$$

where $B$ represents the calixarene.

Application of an alkaline aqueous phase drastically changes the distribution performance of the solvent. Distribution values for all of the alkali metal ions obtained under the highly alkaline conditions are about one order of magnitude greater than the corresponding values observed at $\mathrm{pH} \leq$ 7 (Figs. 4.2-4.4). Despite the greater $D_{\mathrm{Cs}}$ values, $\mathrm{Cs}^{+}$ion loading is reduced and levels off at about 95\%. Formation of the third phase is observed for a wide range of $\mathrm{Cs}^{+}$or $\mathrm{K}^{+}$ion concentrations in the aqueous phase and indicates a change of the solvation environment in the organic phase. As demonstrated by the distribution experiments described above, under highly alkaline conditions, the weakly acidic Cs-7SB modifier is thought to act not only as an agent that solubilizes ionic species in the organic phase, but also as a cation exchanger. At high $\mathrm{pH}$ it becomes partially deprotonated and releases its proton into the aqueous phase. Simultaneously, to maintain electroneutrality, aqueous cations are transported into the solvent phase, where they may be incorporated into the BOBCalixC6 cavity. Thus, additional energetically favorable equilibrium reactions are involved:

$$
\begin{gathered}
\mathrm{M}^{+}(\mathrm{aq}) \\
+\mathrm{OH}_{(\mathrm{aq})}^{-}+\mathrm{ROH}_{(\mathrm{org})}+\mathrm{B}_{(\text {org })} \rightleftharpoons \mathrm{MBOR}_{(\text {org })}+\mathrm{H}_{2} \mathrm{O}_{(\mathrm{aq})} \\
\mathrm{M}_{(\mathrm{aq})}^{+}+\mathrm{OH}_{(\mathrm{aq})}^{-}+\mathrm{ROH}_{(\mathrm{org})}+\mathrm{B}_{(\mathrm{org})} \rightleftharpoons \mathrm{RO}_{(\mathrm{org})}^{-}+\mathrm{MB}_{(\text {org })}+\mathrm{H}_{2} \mathrm{O}_{(\mathrm{aq})}
\end{gathered}
$$

Accordingly, a mixed extraction mechanism is thought to be responsible for the boost of distribution values. High distribution values for $\mathrm{Na}^{+}$ion at high $\mathrm{pH}$ (Table 4.8) suggests that simple $\mathrm{Na}^{+}$ion exchange (Eqs. 4.10 and 4.11 ) contributes to the overall $\mathrm{Na}^{+}$ion extraction by the baseline solvent:

$$
\begin{gathered}
\mathrm{Na}_{(\mathrm{aq})}^{+}+\mathrm{OH}_{(\mathrm{aq})}^{-}+\mathrm{ROH}_{(\text {org })} \rightleftharpoons \mathrm{NaOR}_{(\text {org })}+\mathrm{H}_{2} \mathrm{O}_{(\mathrm{aq})} \\
\mathrm{Na}_{(\mathrm{aq})}+\mathrm{OH}_{(\mathrm{aq})}+\mathrm{ROH}_{(\text {org })} \rightleftharpoons \mathrm{RO}_{(\text {org })}^{-}+\mathrm{Na}^{+}{ }_{(\text {org })}+\mathrm{H}_{2} \mathrm{O}_{(\mathrm{aq})}
\end{gathered}
$$


Table 4.9. $\mathrm{Cs}^{+}$ion distribution ratios, organic-phase concentrations, and BOBCalixC6 loading observed in $\mathrm{Cs}^{+}$ion extraction tests using the baseline solvent ${ }^{a}$

\begin{tabular}{|c|c|c|c|}
\hline Aqueous-phase composition & $D_{\mathrm{Cs}}$ & {$\left[\mathrm{Cs}^{+}\right]_{\text {org,eq }}, \mathrm{M}$} & $\begin{array}{c}\text { BOBCalixC6 } \\
\text { loading, } \%\end{array}$ \\
\hline $1 \mathrm{M} \mathrm{CsNO}_{3}^{b}$ & $0.0139 \pm 0.0003$ & $1.37 \times 10^{-2}$ & 137 \\
\hline $1 \mathrm{M} \mathrm{CsNO}_{3}^{b}$ & $0.0134 \pm 0.0003$ & $1.32 \times 10^{-2}$ & 132 \\
\hline $0.3 \mathrm{M} \mathrm{CsNO}_{3}$ & $0.0503 \pm 0.0007$ & $1.44 \times 10^{-2}$ & 144 \\
\hline $0.3 \mathrm{M} \mathrm{CsNO}_{3}$ & $0.0503 \pm 0.0007$ & $1.44 \times 10^{-2}$ & 144 \\
\hline $0.1 \mathrm{M} \mathrm{CsNO}_{3}$ & $0.107 \pm 0.001$ & $9.69 \times 10^{-3}$ & 97 \\
\hline $0.1 \mathrm{M} \mathrm{CsNO}_{3}$ & $0.107 \pm 0.001$ & $9.66 \times 10^{-3}$ & 97 \\
\hline $0.03 \mathrm{M} \mathrm{CsNO}_{3}$ & $0.269 \pm 0.003$ & $6.36 \times 10^{-3}$ & 64 \\
\hline $0.03 \mathrm{M} \mathrm{CsNO}_{3}$ & $0.234 \pm 0.003$ & $5.69 \times 10^{-3}$ & 57 \\
\hline $0.01 \mathrm{M} \mathrm{CsNO}_{3}$ & $0.217 \pm 0.002$ & $1.78 \times 10^{-3}$ & 18 \\
\hline$\underline{0.01 \mathrm{M} \mathrm{CsNO}_{3}}$ & $0.196 \pm 0.002$ & $1.64 \times 10^{-3}$ & 16 \\
\hline $1 \mathrm{M} \mathrm{CsNO}_{3} / 0.05 \mathrm{M} \mathrm{HNO}_{3}^{b}$ & $0.0143 \pm 0.0003$ & $1.41 \times 10^{-2}$ & 142 \\
\hline $1 \mathrm{M} \mathrm{CsNO}_{3} / 0.05 \mathrm{M} \mathrm{HNO}_{3}^{b}$ & $0.0149 \pm 0.0002$ & $1.46 \times 10^{-2}$ & 146 \\
\hline $0.3 \mathrm{M} \mathrm{CsNO}_{3} / 0.05 \mathrm{M} \mathrm{HNO}_{3}$ & $0.0498 \pm 0.0007$ & $1.42 \times 10^{-2}$ & 142 \\
\hline $0.3 \mathrm{M} \mathrm{CsNO}_{3} / 0.05 \mathrm{M} \mathrm{HNO}_{3}$ & $0.0503 \pm 0.0006$ & $1.44 \times 10^{-2}$ & 144 \\
\hline $0.1 \mathrm{M} \mathrm{CsNO}_{3} / 0.05 \mathrm{M} \mathrm{HNO}_{3}$ & $0.130 \pm 0.001$ & $1.15 \times 10^{-2}$ & 115 \\
\hline $0.1 \mathrm{M} \mathrm{CsNO}_{3} / 0.05 \mathrm{M} \mathrm{HNO}_{3}$ & $0.132 \pm 0.001$ & $1.16 \times 10^{-2}$ & 116 \\
\hline $0.03 \mathrm{M} \mathrm{CsNO}_{3} / 0.05 \mathrm{M} \mathrm{HNO}_{3}$ & $0.386 \pm 0.004$ & $8.36 \times 10^{-3}$ & 84 \\
\hline $0.03 \mathrm{M} \mathrm{CsNO}_{3} / 0.05 \mathrm{M} \mathrm{HNO}_{3}$ & $0.371 \pm 0.004$ & $8.11 \times 10^{-3}$ & 81 \\
\hline $0.01 \mathrm{M} \mathrm{CsNO}_{3} / 0.05 \mathrm{M} \mathrm{HNO}_{3}$ & $0.847 \pm 0.009$ & $4.59 \times 10^{-3}$ & 46 \\
\hline $0.01 \mathrm{M} \mathrm{CsNO}_{3} / 0.05 \mathrm{M} \mathrm{HNO}_{3}$ & $0.852 \pm 0.009$ & $4.60 \times 10^{-3}$ & 46 \\
\hline $1 \mathrm{M} \mathrm{CsNO}_{3} / 2 \mathrm{M} \mathrm{NaOH}^{b}$ & $0.0081 \pm 0.0002$ & $8.00 \times 10^{-3}$ & 80 \\
\hline $0.3 \mathrm{M} \mathrm{CsNO}_{3} / 2 \mathrm{M} \mathrm{NaOH}^{b}$ & $0.0275 \pm 0.0004$ & $8.04 \times 10^{-3}$ & 80 \\
\hline $0.3 \mathrm{M} \mathrm{CsNO}_{3} / 2 \mathrm{M} \mathrm{NaOH}^{b}$ & $0.0336 \pm 0.0005$ & $9.75 \times 10^{-3}$ & 97 \\
\hline $0.1 \mathrm{M} \mathrm{CsNO}_{3} / 2 \mathrm{M} \mathrm{NaOH}^{b}$ & $0.0663 \pm 0.0008$ & $6.22 \times 10^{-3}$ & 62 \\
\hline $0.1 \mathrm{M} \mathrm{CsNO}_{3} / 2 \mathrm{M} \mathrm{NaOH}^{b}$ & $0.0770 \pm 0.0009$ & $7.15 \times 10^{-3}$ & 72 \\
\hline $0.03 \mathrm{M} \mathrm{CsNO}_{3} / 2 \mathrm{M} \mathrm{NaOH}^{b}$ & $0.344 \pm 0.004$ & $7.69 \times 10^{-3}$ & 77 \\
\hline $0.03 \mathrm{M} \mathrm{CsNO}_{3} / 2 \mathrm{M} \mathrm{NaOH}^{b}$ & $0.314 \pm 0.003$ & $7.17 \times 10^{-3}$ & 72 \\
\hline $0.01 \mathrm{M} \mathrm{CsNO}_{3} / 2 \mathrm{M} \mathrm{NaOH}^{b}$ & $3.35 \pm 0.04$ & $7.70 \times 10^{-3}$ & 77 \\
\hline $0.01 \mathrm{M} \mathrm{CsNO}_{3} / 2 \mathrm{M} \mathrm{NaOH}^{b}$ & $3.02 \pm 0.03$ & $7.51 \times 10^{-3}$ & 75 \\
\hline $0.003 \mathrm{M} \mathrm{CsNO}_{3} / 2 \mathrm{M} \mathrm{NaOH}$ & $5.07 \pm 0.06$ & $2.51 \times 10^{-3}$ & 25 \\
\hline $0.003 \mathrm{M} \mathrm{CsNO}_{3} / 2 \mathrm{M} \mathrm{NaOH}$ & $5.09 \pm 0.06$ & $2.51 \times 10^{-3}$ & 25 \\
\hline $0.001 \mathrm{M} \mathrm{CsNO}_{3} / 2 \mathrm{M} \mathrm{NaOH}$ & $13.6 \pm 0.3$ & $9.31 \times 10^{-4}$ & 9.3 \\
\hline $0.001 \mathrm{M} \mathrm{CsNO}_{3} / 2 \mathrm{M} \mathrm{NaOH}$ & $14.8 \pm 0.2$ & $9.37 \times 10^{-4}$ & 9.4 \\
\hline $0.0003 \mathrm{M} \mathrm{CsNO}_{3} / 2 \mathrm{M} \mathrm{NaOH}$ & $14.8 \pm 0.2$ & $2.81 \times 10^{-4}$ & 2.8 \\
\hline $0.0003 \mathrm{M} \mathrm{CsNO}_{3} / 2 \mathrm{M} \mathrm{NaOH}$ & $15.6 \pm 0.2$ & $2.82 \times 10^{-4}$ & 2.8 \\
\hline $0.0001 \mathrm{M} \mathrm{CsNO}_{3} / 2 \mathrm{M} \mathrm{NaOH}$ & $14.9 \pm 0.2$ & $9.37 \times 10^{-5}$ & 0.94 \\
\hline $0.0001 \mathrm{M} \mathrm{CsNO}_{3} / 2 \mathrm{M} \mathrm{NaOH}$ & $14.8 \pm 0.2$ & $9.37 \times 10^{-5}$ & 0.94 \\
\hline
\end{tabular}

$a^{a} \mathrm{O} / \mathrm{A}=1 ; \mathrm{T}=25^{\circ} \mathrm{C}$.

$b$ Third phase formed. 

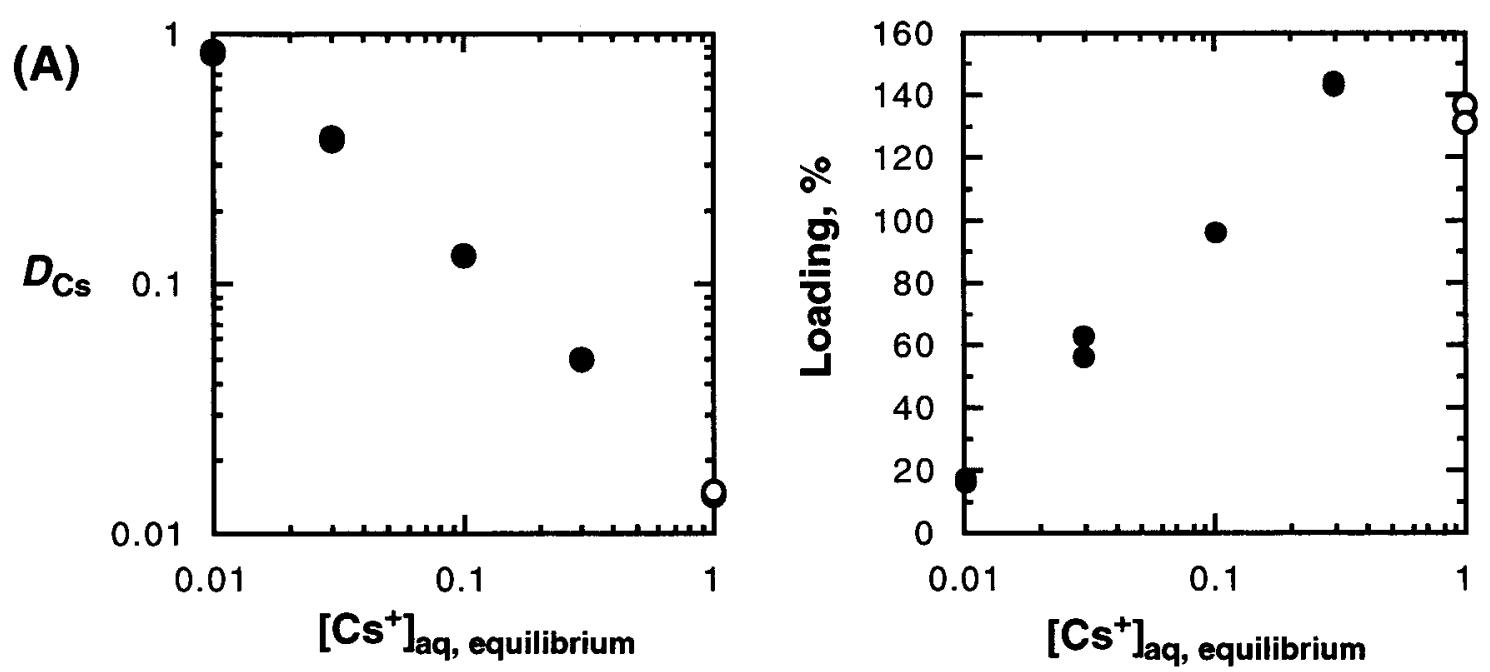

(B)
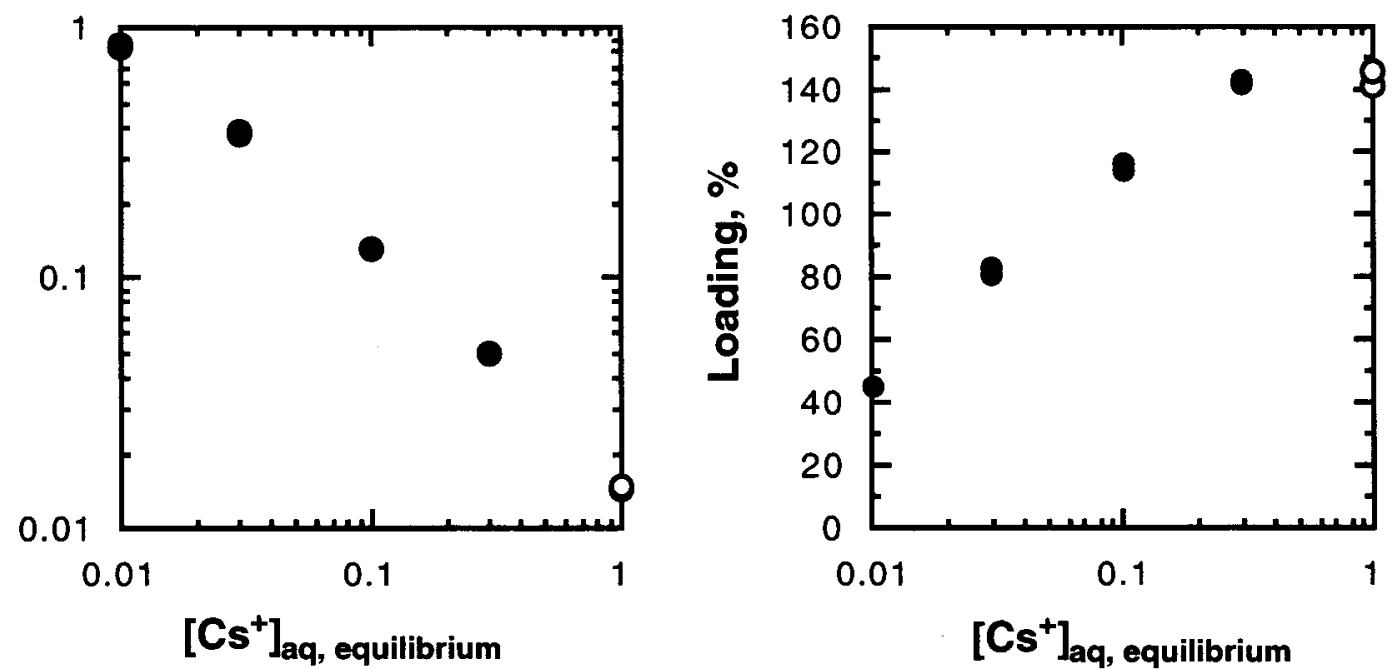

(C)
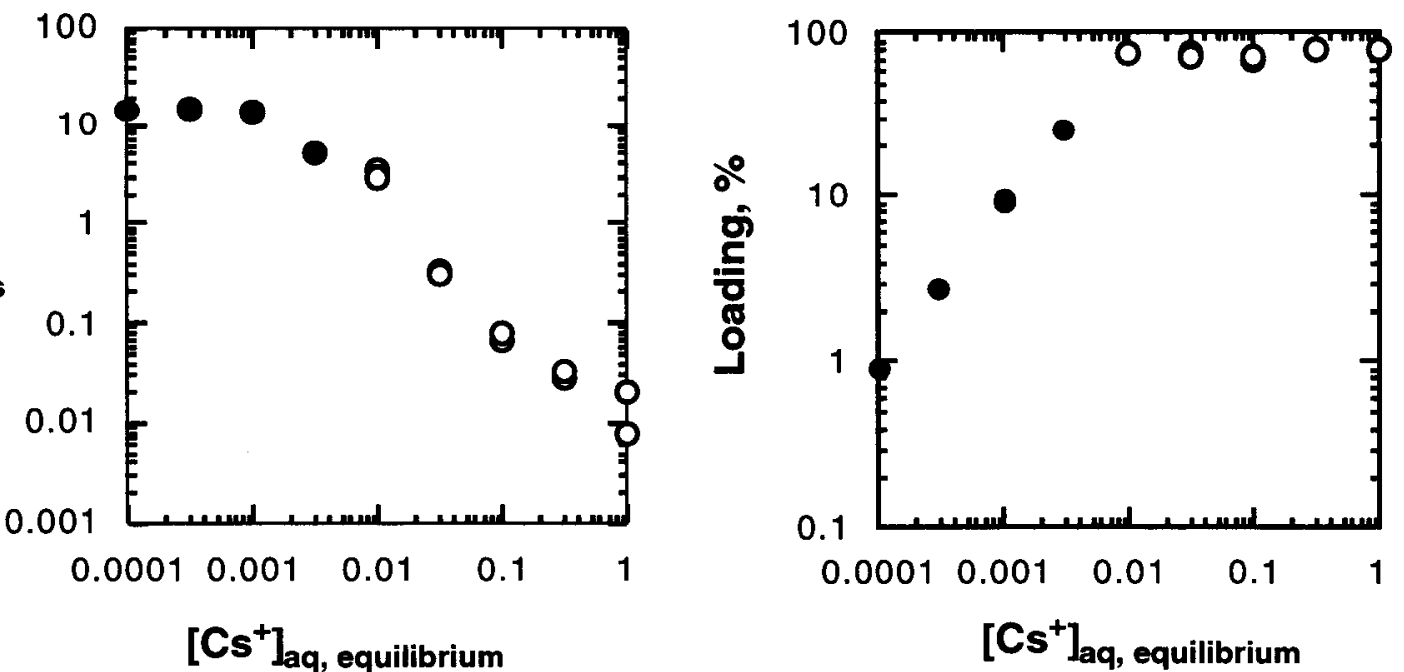

Fig. 4.2. Effect of $\mathrm{Cs}^{+}$concentration on $\mathrm{Cs}^{+}$distribution ratio, loading, and third-phase formation. Organic phase: Baseline solvent. Aqueous phase: (A) $\mathrm{CsNO}_{3}$; (B) $\mathrm{CsNO}_{3}, 0.05 \mathrm{M}$ $\mathrm{HNO}_{3} ;(\mathrm{C}) \mathrm{CsNO}_{3}, 2 \mathrm{M} \mathrm{NaOH}$. O/A $=1 ; T=25^{\circ} \mathrm{C}$. Open symbols denote third-phase formation. 
Table 4.10. $\mathrm{K}^{+}$ion distribution ratios, organic phase concentrations, and ВОВCalixC6 loading observed in $\mathrm{K}^{+}$extraction tests using the baseline solvent ${ }^{a}$

\begin{tabular}{|c|c|c|c|c|c|}
\hline Aqueous-phase composition & $D_{\mathrm{K}} \times 10^{3}$ & $\begin{array}{c}{\left[\mathrm{K}^{+}\right]_{\text {org }} \times 10^{3}} \\
(\mathrm{M})\end{array}$ & $\begin{array}{c}\text { BOBCalixC6 } \\
\text { loading, } \\
(\%)\end{array}$ & $D_{\mathrm{Na}} \times 10^{4}$ & $\begin{array}{c}{\left[\mathrm{Na}^{+}\right] \text {org } \times 10^{4}} \\
\text { (M) }\end{array}$ \\
\hline $1.5 \mathrm{M} \mathrm{KNO}_{3}{ }^{a}$ & $5.78 \pm 0.07$ & 8.7 & 87 & & \\
\hline $1.5 \mathrm{M} \mathrm{KNO}_{3}{ }^{a}$ & $5.66 \pm 0.06$ & 8.5 & 85 & & \\
\hline $0.75 \mathrm{M} \mathrm{KNO}_{3}$ & $9.92 \pm 0.07$ & 7.5 & 75 & & \\
\hline $0.75 \mathrm{M} \mathrm{KNO}_{3}$ & $9.95 \pm 0.07$ & 7.5 & 75 & & \\
\hline $0.3 \mathrm{M} \mathrm{KNO}_{3}$ & $13.0 \pm 0.4$ & 3.9 & 39 & & \\
\hline $0.3 \mathrm{M} \mathrm{KNO}_{3}$ & $13.3 \pm 0.3$ & 4.0 & 40 & & \\
\hline $0.1 \mathrm{M} \mathrm{KNO}_{3}$ & $8.15 \pm 0.01$ & 0.81 & 8.1 & & \\
\hline $0.1 \mathrm{M} \mathrm{KNO}_{3}$ & $8.36 \pm 0.07$ & 0.83 & 8.3 & & \\
\hline $2 \mathrm{M} \mathrm{KNO}_{3} / 0.05 \mathrm{M} \mathrm{HNO}_{3}^{b}$ & $3.7 \pm 0.1$ & 7.4 & 74 & & \\
\hline $2 \mathrm{M} \mathrm{KNO}_{3} / 0.05 \mathrm{M} \mathrm{HNO}_{3} b$ & $3.71 \pm 0.02$ & 7.4 & 74 & & \\
\hline $1 \mathrm{M} \mathrm{KNO}_{3} / 0.05 \mathrm{M} \mathrm{HNO}_{3}$ & $7.6 \pm 0.1$ & 7.6 & 76 & & \\
\hline $1 \mathrm{M} \mathrm{KNO}_{3} / 0.05 \mathrm{M} \mathrm{HNO}_{3}$ & $7.1 \pm 0.3$ & 7.1 & 71 & & \\
\hline $0.3 \mathrm{M} \mathrm{KNO}_{3} / 0.05 \mathrm{M} \mathrm{HNO}_{3}$ & $12.9 \pm 0.3$ & 3.9 & 39 & & \\
\hline $0.3 \mathrm{M} \mathrm{KNO}_{3} / 0.05 \mathrm{M} \mathrm{HNO}_{3}$ & $13.3 \pm 0.2$ & 4.0 & 40 & & \\
\hline $0.1 \mathrm{M} \mathrm{KNO}_{3} / 0.05 \mathrm{M} \mathrm{HNO}_{3}$ & $9.1 \pm 0.4$ & 0.91 & 9.1 & & \\
\hline $0.1 \mathrm{M} \mathrm{KNO}_{3} / 0.05 \mathrm{M} \mathrm{HNO}_{3}$ & $9.3 \pm 0.4$ & 0.93 & 9.3 & & \\
\hline $1.5 \mathrm{M} \mathrm{KNO}_{3} / 2 \mathrm{M} \mathrm{NaOH}^{b}$ & $5.16 \pm 0.05$ & 7.83 & 78 & $2.58 \pm 0.02$ & 5.15 \\
\hline $1.5 \mathrm{M} \mathrm{KNO}_{3} / 2 \mathrm{M} \mathrm{NaOH}^{b}$ & $4.8 \pm 0.1$ & 7.30 & 73 & $2.46 \pm 0.05$ & 4.90 \\
\hline $0.76 \mathrm{M} \mathrm{KNO}_{3} / 2 \mathrm{M} \mathrm{NaOH}^{b}$ & $10.2 \pm 0.1$ & 7.74 & 77 & $2.54 \pm 0.01$ & 5.08 \\
\hline $0.76 \mathrm{M} \mathrm{KNO}_{3} / 2 \mathrm{M} \mathrm{NaOH}^{b}$ & $9.6 \pm 0.2$ & 7.50 & 75 & $2.47 \pm 0.04$ & 4.94 \\
\hline $0.3 \mathrm{M} \mathrm{KNO}_{3} / 2 \mathrm{M} \mathrm{NaOH}^{b}$ & $25.6 \pm 0.7$ & 7.60 & 76 & $2.75 \pm 0.05$ & 5.50 \\
\hline $0.3 \mathrm{M} \mathrm{KNO}_{3} / 2 \mathrm{M} \mathrm{NaOH}^{b}$ & $24.9 \pm 0.2$ & 7.40 & 74 & $3.36 \pm 0.05$ & 6.72 \\
\hline $0.1 \mathrm{M} \mathrm{KNO}_{3} / 2 \mathrm{M} \mathrm{NaOH}$ & $68 \pm 1$ & 6.60 & 66 & $3.33 \pm 0.09$ & 6.70 \\
\hline $0.1 \mathrm{M} \mathrm{KNO}_{3} / 2 \mathrm{M} \mathrm{NaOH}$ & $68 \pm 2$ & 6.60 & 66 & $3.36 \pm 0.05$ & 6.72 \\
\hline
\end{tabular}

${ }^{a} \mathrm{O} / \mathrm{A}=0.33 ; \mathrm{T}=25^{\circ} \mathrm{C}$.

$b$ Third phase formed. 

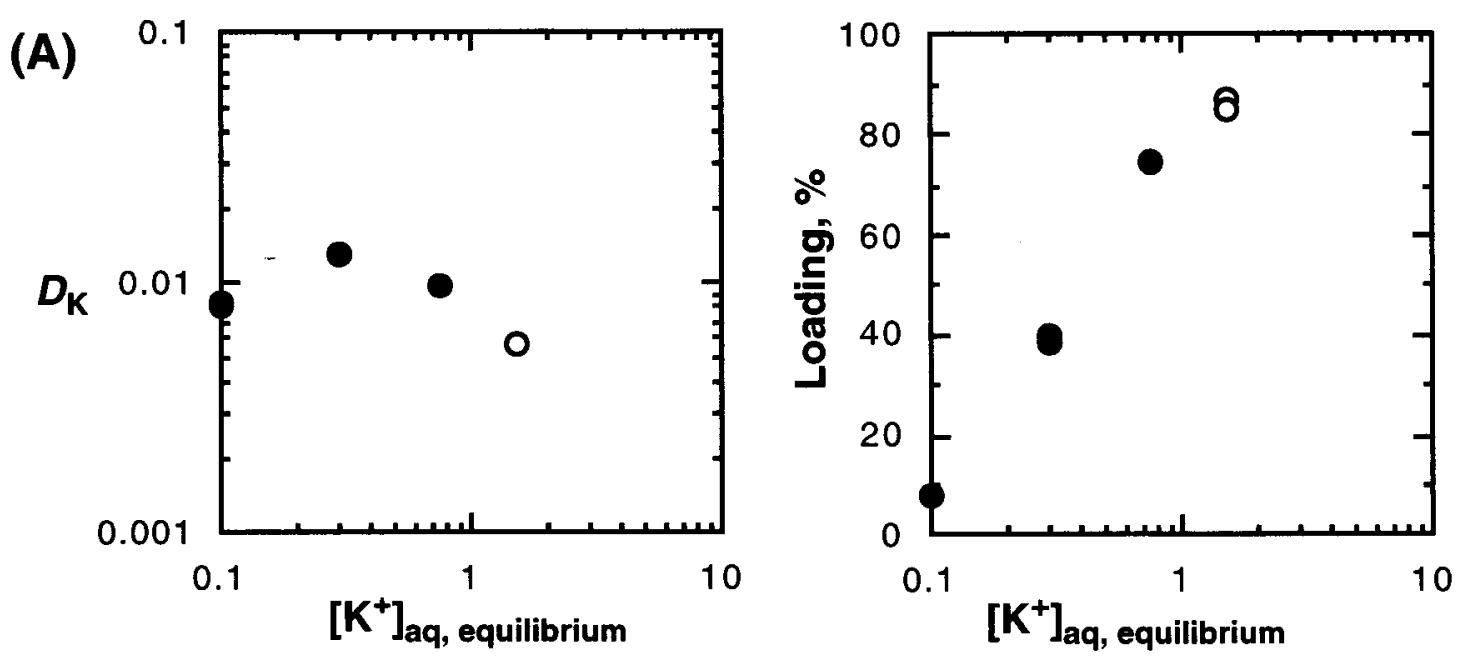

(B)
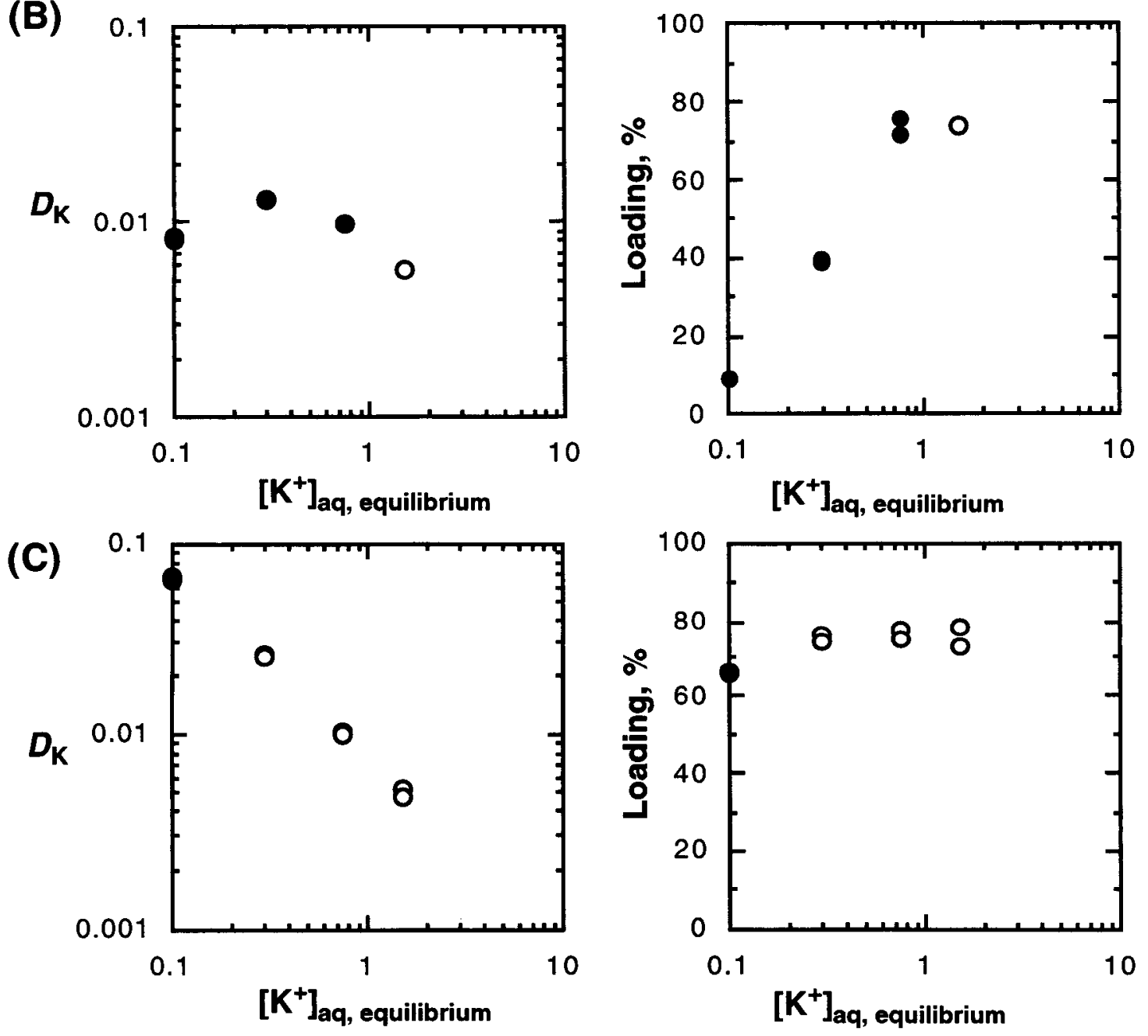

Fig. 4.3. Effect of $\mathrm{K}^{+}$ion concentration on $\mathrm{Cs}^{+}$distribution ratio, loading, and third-phase formation. Organic phase: Baseline solvent. Aqueous phase: (A) $\mathrm{KNO}_{3}$; (B) $\mathrm{KNO}_{3}, 0.05 \mathrm{M} \mathrm{HNO}_{3}$; (C) $\mathrm{KNO}_{3}, 2 \mathrm{M} \mathrm{NaOH}$. O/A $=0.33 ; T=25^{\circ} \mathrm{C}$. Open symbols denote third-phase formation. 
Table 4.11. $\mathrm{Na}^{+}$ion distribution ratios and organic-phase concentrations observed in $\mathrm{Na}^{+}$ extraction tests using full solvent

\begin{tabular}{|c|c|c|}
\hline Aqueous phase composition & $D_{\mathrm{Na}} \times 10^{4}$ & {$\left[\mathrm{Na}^{+}\right]_{\text {org }} \times 10^{4} \mathrm{M}$} \\
\hline $5 \mathrm{M} \mathrm{NaNO}_{3}$ & $4.9 \pm 0.4$ & 24.3 \\
\hline $3 \mathrm{M} \mathrm{NaNO}_{3}$ & $4.2 \pm 0.4$ & 12.6 \\
\hline $1 \mathrm{M} \mathrm{NaNO}_{3}$ & $2.4 \pm 0.4$ & 2.36 \\
\hline $1 \mathrm{M} \mathrm{NaNO}_{3}$ & $3.2 \pm 0.4$ & 3.21 \\
\hline $0.3 \mathrm{M} \mathrm{NaNO}_{3}$ & $2.6 \pm 0.4$ & 0.78 \\
\hline $0.3 \mathrm{M} \mathrm{NaNO}_{3}$ & $2.8 \pm 0.6$ & 0.84 \\
\hline $0.1 \mathrm{M} \mathrm{NaNO}_{3}$ & $2.0 \pm 0.6$ & 0.20 \\
\hline $0.1 \mathrm{M} \mathrm{NaNO}_{3}$ & $2.1 \pm 0.6$ & $\underline{0.21}$ \\
\hline $5 \mathrm{M} \mathrm{NaNO}_{3} / 2 \mathrm{M} \mathrm{NaOH}$ & $7.5 \pm 0.5$ & 52 \\
\hline $2.5 \mathrm{M} \mathrm{NaNO}_{3} / 2 \mathrm{M} \mathrm{NaOH}$ & $5.4 \pm 0.4$ & 24 \\
\hline $2.5 \mathrm{M} \mathrm{NaNO}_{3} / 2 \mathrm{M} \mathrm{NaOH}$ & $5.3 \pm 0.5$ & 24 \\
\hline $1.3 \mathrm{M} \mathrm{NaNO}_{3} / 2 \mathrm{M} \mathrm{NaOH}$ & $4.4 \pm 0.4$ & 14 \\
\hline $1.3 \mathrm{M} \mathrm{NaNO}_{3} / 2 \mathrm{M} \mathrm{NaOH}$ & $4.8 \pm 0.4$ & 15 \\
\hline $0.7 \mathrm{M} \mathrm{NaNO}_{3} / 2 \mathrm{M} \mathrm{NaOH}$ & $4.4 \pm 0.4$ & 11.9 \\
\hline $0.7 \mathrm{M} \mathrm{NaNO}_{3} / 2 \mathrm{M} \mathrm{NaOH}$ & $4.8 \pm 0.4$ & 12.9 \\
\hline $0.3 \mathrm{M} \mathrm{NaNO}_{3} / 2 \mathrm{M} \mathrm{NaOH}$ & $3.3 \pm 0.4$ & 7.6 \\
\hline $0.1 \mathrm{M} \mathrm{NaNO}_{3} / 2 \mathrm{M} \mathrm{NaOH}$ & $3.2 \pm 0.4$ & 6.7 \\
\hline $0.1 \mathrm{M} \mathrm{NaNO}_{3} / 2 \mathrm{M} \mathrm{NaOH}$ & $3.7 \pm 0.4$ & 7.7 \\
\hline $2 \mathrm{M} \mathrm{NaOH}$ & $4.0 \pm 0.4$ & 8.2 \\
\hline $2 \mathrm{M} \mathrm{NaOH}$ & $3.9 \pm 0.4$ & 7.6 \\
\hline
\end{tabular}

${ }^{a} \mathrm{O} / \mathrm{A}=0.33 ; \mathrm{T}=25^{\circ} \mathrm{C}$.

(A)

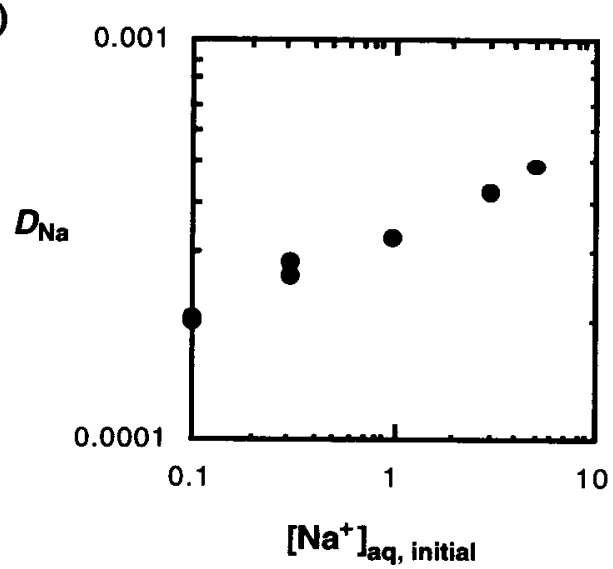

(B)

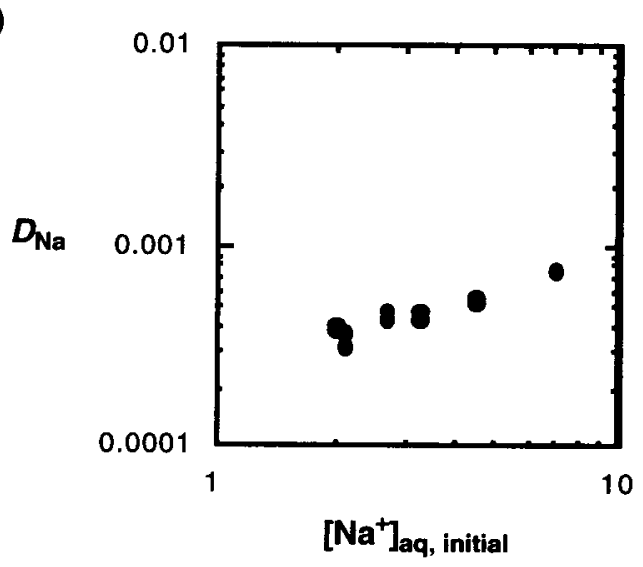

Fig. 4.4. Effect of $\mathrm{Na}^{+}$ion concentration in the aqueous phase on $\mathrm{Na}^{+}$distribution ratio. Organic phase: Baseline solvent. Aqueous phase: (A) $\mathrm{NaNO}_{3}$; (B) $\mathrm{NaNO}_{3}, 2 \mathrm{M} \mathrm{NaOH}$. $\mathrm{O} / \mathrm{A}=0.33$; $T=25^{\circ} \mathrm{C}$. 


\subsubsection{Extraction of Alkali Metal Cations from the Full Simulant Containing Variable Cesium or Potassium Concentration as a Function of Solvent Modifier Concentration}

As demonstrated in the previous section, BOBCalixC6 exhibits appreciably stronger extraction of $\mathrm{Cs}^{+}$ion over $\mathrm{K}^{+}$ion. However, the concentration of $\mathrm{K}^{+}$ion in the simulant solution is approximately 15 times greater than that of $\mathrm{Cs}^{+}$ion, promoting significant extraction of $\mathrm{K}^{+}$ion into solvent phase and significant overall loading of the BOBCalixC6 extractant. High loading of BOBCalixC6 with $\mathrm{K}^{+}$ ions decreases $\mathrm{Cs}^{+}$ion extraction and leads to formation of a third phase due to the limited solubility of the potassium species in the solvent, which is, in turn, highly dependent on the Cs-7SB modifier concentration in the solvent. For practical purposes, it is important to know the behavioral variation of the simulant/solvent extraction system under conditions both of different cesium and potassium concentrations in the simulant and of variable modifier concentrations in the solvent.

Distribution experiments to determine the effect of $\mathrm{Cs}^{+}$and $\mathrm{K}^{+}$concentrations in the simulant on solvent loading and third-phase formation were performed at $25{ }^{\circ} \mathrm{C}$ and $\mathrm{O} / \mathrm{A}=0.33$ using the baseline solvent containing three Cs-7SB concentrations:

\section{$0.25 \mathrm{M}$ Cs-7SB / $0.01 \mathrm{M}$ BOBCalixC6 / $0.001 \mathrm{M}$ TOA / Isopar L $0.50 \mathrm{M}$ Cs-7SB / 0.01 M BOBCalixC6 / $0.001 \mathrm{M}$ TOA / Isopar L 0.75 M Cs-7SB / 0.01 M BOBCalixC6 / 0.001 M TOA / Isopar L}

Two series of extraction experiments with each solvent were performed using simulant solutions containing:

1. $\mathrm{K}^{+}$ion concentration at the level in the simulant $(0.020 \mathrm{M})$ and variable $\mathrm{Cs}^{+}$ion concentration (Table 4.12 and Figs. 4.5 and 4.6);

2. $\mathrm{Cs}^{+}$ion concentration at the baseline level in the simulant $(0.00014 \mathrm{M})$ and variable $\mathrm{K}^{+}$ion concentration (Table 4.13 and Figs. 4.7 and 4.8).

It was observed that the increase of $\mathrm{Cs}^{+}$ion concentration in the simulant up to $0.001 \mathrm{M}$ with constant $0.020 \mathrm{M} \mathrm{K}^{+}$ion concentration caused only minor reduction of $D_{\mathrm{Cs}}$ and $D_{\mathrm{K}}$ values for all three solvent solutions (Table 4.12 and Fig. 4.5). Further increase of $\mathrm{Cs}^{+}$ion concentration in the simulant resulted in progressive decline of $D_{\mathrm{Cs}}$ and $D_{\mathrm{K}}$ values due to high loading of BOBCalixC6 (Table 4.12 and Figs. 4.5 and 4.6). It is shown in Fig. 4.6, that for the full simulant containing 0.14 $\mathrm{mM} \mathrm{Cs}^{+}$and $20 \mathrm{mM} \mathrm{K}^{+}$, the $\mathrm{K}^{+}$ion is the primary source of extractant loading. Note that, based on the results of the distribution experiments described above, all experiments with simulant solution result in minor contribution of sodium ions to the overall extractant loading and are thus excluded from calculation of loading. Under baseline conditions, the $\mathrm{K}^{+}$ion loading is $25.8 \%$. When the $\mathrm{Cs}^{+}$ ion concentration in the simulant is increased and becomes comparable with that of $\mathrm{K}^{+}$ion, the situation is reversed, and the majority of the extractant is loaded with the $\mathrm{Cs}^{+}$ion. The effect of modifier concentration on cesium extraction is discussed in Section 3.3.16.

A large effect of $\mathrm{K}^{+}$ion concentration was observed (Table 4.13 and Figs. 4.7 and 4.8). Values of $D_{\mathrm{K}}$ decrease as $\mathrm{K}^{+}$concentration in the simulant is increased (Fig. 4.7), attributed to the high loading of the extractant (Figs. 4.7 and 4.8). A further decline of the distribution values was demonstrated for the $\mathrm{Cs}^{+}$ion (Fig. 4.7) and explained by the dominant extraction of the $\mathrm{K}^{+}$ion and high extractant loading with the $\mathrm{K}^{+}$ion. Accordingly, the $\mathrm{Cs}^{+}$concentration in the solvent phase was greatly reduced as $\mathrm{K}^{+}$concentration in the simulant increased and the $\mathrm{Cs}^{+}$contribution to the total loading of the extractant became negligible (Fig. 4.8). These experiments demonstrated that $\mathrm{Cs}^{+}$ion distribution into the solvent phase is sensitive to potassium concentration in the simulant. Although within bounding conditions, this effect does not pose a serious problem in sustaining desired high $D_{\mathrm{Cs}}$ values, the potassium concentration in the feed solution should be closely monitored.

Effect of modifier concentration in the solvent on the $\mathrm{Cs}^{+}$and $\mathrm{K}^{+}$distribution behavior observed in the experiments with variable $\mathrm{K}^{+}$concentration in the simulant closely resembled that depicted in 
Table 4.12. Effect of $\mathrm{Cs}^{+}$concentration in the simulant on distribution behavior of alkali metal ions and total BOBCalixC6 loading at different Cs-7SB modifier concentrations in the solvent

\begin{tabular}{|c|c|c|c|c|c|}
\hline \multirow{2}{*}{$\begin{array}{l}{\left[\mathrm{Cs}^{+}\right]_{\text {simulant }}} \\
\quad(\mathrm{mM})\end{array}$} & \multicolumn{2}{|r|}{$\mathrm{Cs}^{+}$} & \multicolumn{2}{|c|}{$\mathrm{K}^{+}$} & \multirow[t]{2}{*}{ Total loading $a$} \\
\hline & $D_{\mathrm{Cs}}$ & {$\left[\mathrm{Cs}^{+}\right]_{\mathrm{org}}, \mathrm{mM}$} & $D_{\mathrm{K}}$ & {$\left[\mathrm{K}^{+}\right]_{\text {org }}, \mathrm{mM}$} & \\
\hline \multicolumn{6}{|c|}{$[\mathrm{Cs}-7 \mathrm{SB}]=0.25 \mathrm{M}$} \\
\hline 0.14 & 12.9 & 0.341 & 0.076 & 1.49 & 18.4 \\
\hline 0.14 & 13.6 & 0.344 & 0.078 & 1.53 & 18.8 \\
\hline 0.3 & 11.6 & 0.715 & 0.076 & 1.48 & 22.0 \\
\hline 0.3 & 12.2 & 0.723 & 0.076 & 1.48 & 22.1 \\
\hline 0.7 & 10.5 & 1.63 & 0.072 & 1.45 & 30.8 \\
\hline 0.7 & 11.3 & 1.66 & 0.066 & 1.32 & 29.8 \\
\hline 1 & 8.75 & 2.23 & 0.064 & 1.28 & 35.1 \\
\hline 1 & 9.02 & 2.25 & 0.066 & 1.33 & 35.8 \\
\hline $3^{b}$ & 2.41 & 4.01 & 0.022 & 0.45 & 44.6 \\
\hline $3^{b}$ & 2.44 & 4.04 & 0.023 & 0.47 & 45.1 \\
\hline $10^{b}$ & 0.65 & 5.35 & 0.0056 & 0.11 & 54.6 \\
\hline $10^{b}$ & 0.52 & 4.42 & 0.0048 & 0.099 & 45.2 \\
\hline \multicolumn{6}{|c|}{$[\mathrm{Cs}-7 \mathrm{SB}]=0.5 \mathrm{M}$} \\
\hline 0.14 & 17.6 & 0.359 & 0.13 & 2.44 & 28.0 \\
\hline 0.14 & 18.2 & 0.344 & 0.14 & 2.71 & 30.7 \\
\hline 0.3 & 16.8 & 0.764 & 0.12 & 2.25 & 30.1 \\
\hline 0.3 & 16.8 & 0.764 & 0.12 & 2.23 & 29.9 \\
\hline 0.7 & 15.4 & 1.76 & 0.099 & 1.98 & 37.4 \\
\hline 0.7 & 14.6 & 1.74 & 0.10 & 2.02 & 37.6 \\
\hline 1 & 12.0 & 2.40 & 0.10 & 2.07 & 44.7 \\
\hline 1 & 12.7 & 2.43 & 0.094 & 1.88 & 43.1 \\
\hline 3 & 5.11 & 5.67 & 0.057 & 1.15 & 68.2 \\
\hline 3 & 5.12 & 5.67 & 0.054 & 1.08 & 67.6 \\
\hline 10 & 1.39 & 9.52 & 0.015 & 0.30 & 98.2 \\
\hline 10 & 1.28 & 8.98 & 0.014 & 0.29 & 92.8 \\
\hline \multicolumn{6}{|c|}{$[\mathrm{Cs}-7 \mathrm{SB}]=0.75 \mathrm{M}$} \\
\hline 0.14 & 19.8 & 0.365 & 0.16 & 3.01 & 33.7 \\
\hline 0.14 & 20.2 & 0.366 & 0.14 & 2.75 & 31.2 \\
\hline 0.3 & 17.3 & 0.767 & 0.14 & 2.69 & 34.5 \\
\hline 0.3 & 18.4 & 0.774 & 0.13 & 2.57 & 33.4 \\
\hline 0.7 & 16.5 & 1.77 & 0.11 & 2.15 & 39.2 \\
\hline 0.7 & 16.7 & 1.78 & 0.11 & 2.24 & 40.2 \\
\hline 1 & 13.9 & 2.47 & 0.11 & 2.25 & 47.2 \\
\hline 1 & 13.2 & 2.44 & 0.11 & 2.25 & 46.9 \\
\hline 3 & 5.22 & 5.72 & 0.060 & 1.21 & 69.3 \\
\hline 3 & 5.16 & 5.69 & 0.054 & 1.07 & 67.6 \\
\hline 10 & 1.41 & 9.61 & 0.018 & 0.35 & 99.6 \\
\hline 10 & 1.39 & 9.51 & 0.016 & 0.34 & 98.5 \\
\hline
\end{tabular}

${ }^{a}$ Contribution of $\mathrm{Na}^{+}$ions to the total BOBCalixC6 loading is assumed to be negligible.

${ }^{b}$ Third phase formed. 

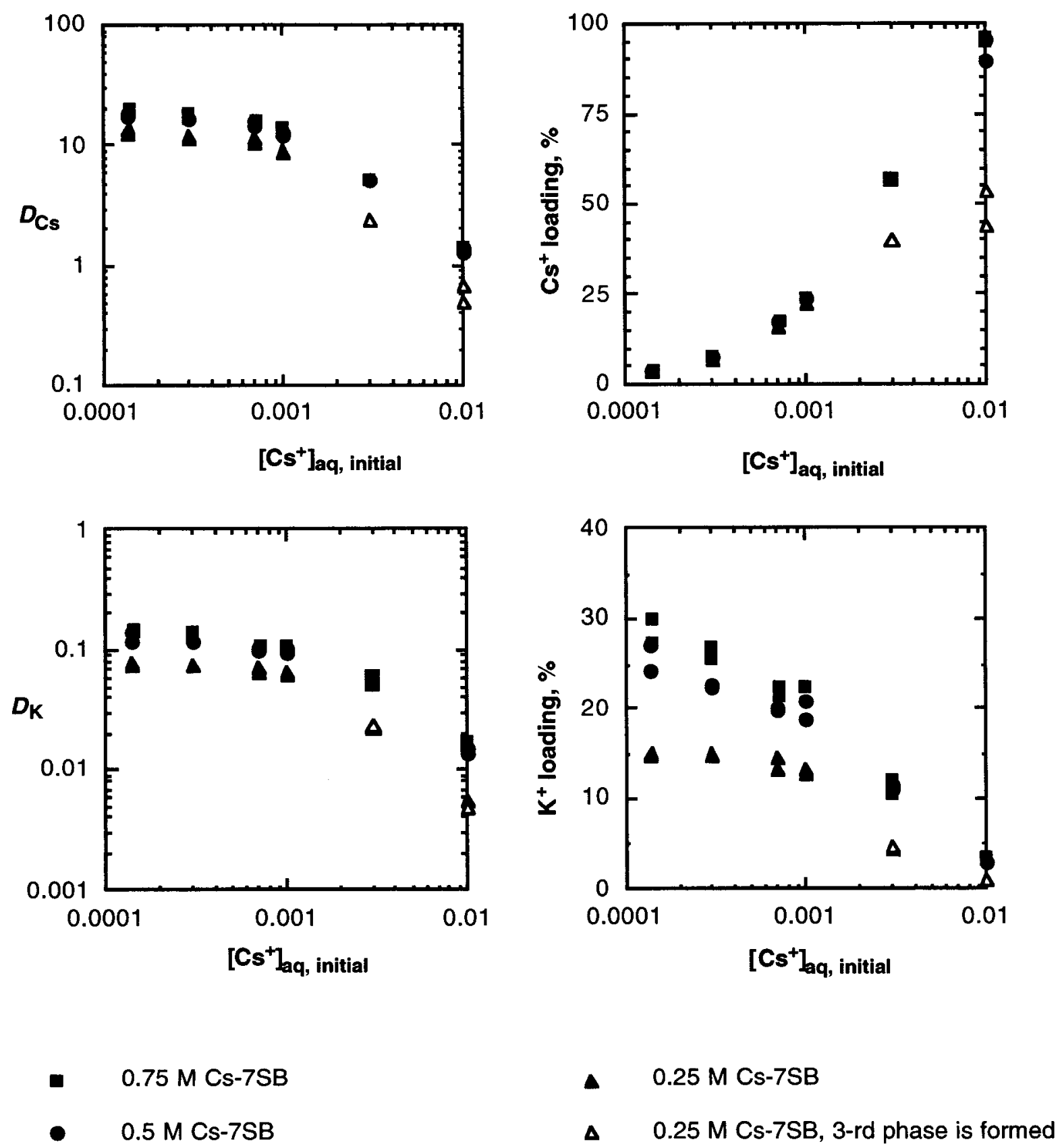

Fig. 4.5. Effect of aqueous $\mathrm{Cs}^{+}$ion concentration on $D_{\mathrm{Cs}}, D_{\mathrm{K}}$, and BOBCalixC6 loading. Organic phase: pristine washed solvent at $0.25,0.50$, or $0.75 \mathrm{M}$ Cs-7SB concentration. Aqueous phase: full simulant with variable $\mathrm{CsNO}_{3}$ concentration. $\mathrm{O} / \mathrm{A}=0.33 ; T=25^{\circ} \mathrm{C}$. Open symbols denote third-phase formation. 


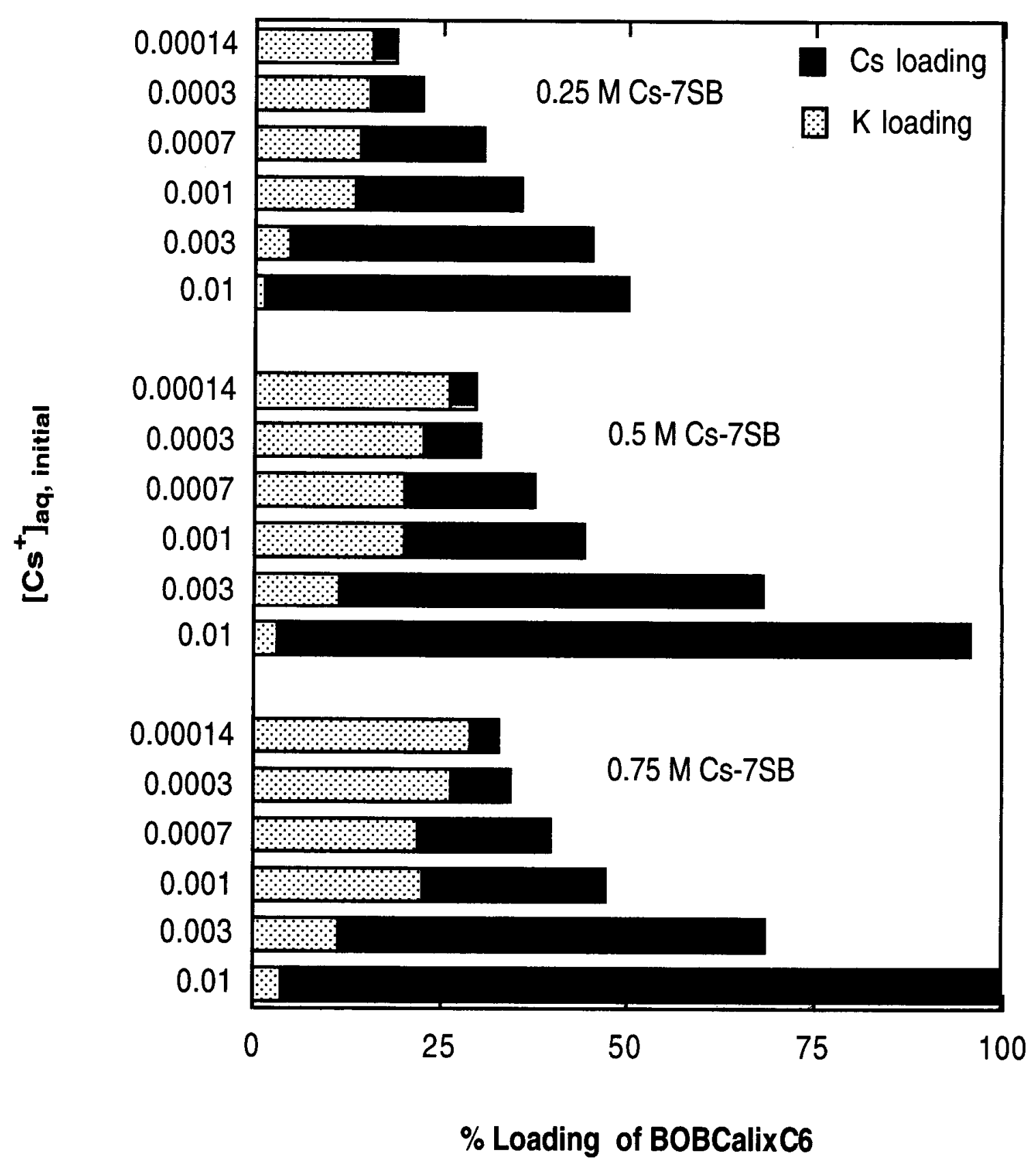

Fig. 4.6. Effect of Cs-7SB modifier concentration in the solvent on total BOBCalixC6 loading at different $\mathrm{Cs}^{+}$concentrations in the full simulant. Organic phase: pristine washed solvent with variable Cs-7SB concentration. Aqueous phase: full simulant with variable $\mathrm{Cs}^{+}$concentration. $\mathrm{O} / \mathrm{A}=$ $0.33 ; T=25^{\circ} \mathrm{C}$. 
Table 4.13. Effect of $\mathrm{K}^{+}$ion concentration in the simulant on distribution behavior of alkali metal ions and total BOBCalixC6 loading at different Cs-7SB modifier concentrations in the solvent

\begin{tabular}{|c|c|c|c|c|c|}
\hline \multirow{2}{*}{$\underset{(\mathrm{mM})}{\left[\mathrm{K}^{+}\right]_{\text {simulant }}}$} & \multicolumn{2}{|r|}{$\mathrm{Cs}^{+}$} & \multicolumn{2}{|r|}{$\mathrm{K}^{+}$} & \multirow[t]{2}{*}{ Total loading $a$} \\
\hline & $D_{\mathrm{Cs}}$ & {$\left[\mathrm{Cs}^{+}\right]_{\text {org }}, \mathrm{mM}$} & $D_{\mathrm{K}}$ & {$\left[\mathrm{K}^{+}\right]_{\text {org }}, \mathrm{mM}$} & \\
\hline \multicolumn{6}{|c|}{$[\mathrm{Cs}-7 \mathrm{SB}]=0.25 \mathrm{M}$} \\
\hline 20 & 12.9 & 0.341 & 0.077 & 1.50 & 18.4 \\
\hline 20 & 13.6 & 0.344 & 0.079 & 1.53 & 18.8 \\
\hline $50^{b}$ & 8.01 & 0.306 & 0.053 & 2.60 & 29.0 \\
\hline $50^{b}$ & & & 0.053 & 2.61 & 26.1 \\
\hline $90^{b}$ & 7.83 & 0.304 & 0.035 & 3.12 & 34.2 \\
\hline $90^{b}$ & 5.29 & 0.268 & 0.039 & 3.53 & 38.0 \\
\hline $120^{b}$ & 3.07 & 0.212 & 0.028 & 3.37 & 35.8 \\
\hline $120^{b}$ & 4.67 & 0.256 & 0.025 & 3.04 & 33.0 \\
\hline $320^{b}$ & 1.03 & 0.104 & 0.0078 & 2.51 & 26.1 \\
\hline $320^{b}$ & 1.04 & 0.108 & 0.0064 & 2.05 & 21.5 \\
\hline $520^{b}$ & 0.592 & 0.108 & 0.0044 & 2.31 & 23.8 \\
\hline $520^{b}$ & & 0.00692 & 0.0039 & 2.05 & 20.5 \\
\hline $860^{b}$ & 0.332 & 0.00419 & 0.0025 & 2.19 & 22.3 \\
\hline $860^{b}$ & 0.355 & 0.00444 & 0.0024 & 2.10 & 21.4 \\
\hline \multicolumn{6}{|c|}{$[\mathrm{Cs}-7 \mathrm{SB}]=0.5 \mathrm{M}$} \\
\hline 20 & 17.6 & 0.359 & 0.13 & 2.44 & 28.0 \\
\hline 20 & 18.2 & 0.344 & 0.14 & 2.71 & 30.7 \\
\hline 50 & 11.0 & 0.330 & 0.099 & 4.77 & 51.0 \\
\hline 50 & 11.9 & 0.335 & 0.094 & 4.57 & 49.0 \\
\hline 90 & 8.09 & 0.306 & 0.073 & 6.41 & 67.1 \\
\hline 90 & 7.85 & 0.304 & 0.074 & 6.47 & 67.7 \\
\hline 120 & 6.48 & 0.287 & 0.060 & 7.02 & 73.1 \\
\hline 120 & 6.53 & 0.288 & 0.061 & 7.16 & 74.5 \\
\hline $320^{b}$ & 2.70 & 0.199 & 0.026 & 8.12 & 83.2 \\
\hline $320^{b}$ & 2.73 & 0.200 & 0.026 & 8.24 & 84.4 \\
\hline $520^{b}$ & 1.52 & 0.141 & 0.016 & 8.48 & 86.2 \\
\hline $520^{b}$ & 1.69 & 0.152 & 0.016 & 8.50 & 86.6 \\
\hline $860^{b}$ & 0.895 & 0.0965 & & & \\
\hline $860^{b}$ & 0.921 & 0.0986 & 0.0097 & 8.31 & 84.1 \\
\hline \multicolumn{6}{|c|}{$[\mathrm{Cs}-7 \mathrm{SB}]=0.75 \mathrm{M}$} \\
\hline 20 & 19.8 & 0.365 & 0.16 & 3.01 & 33.7 \\
\hline 20 & 20.2 & 0.366 & 0.14 & 2.75 & 31.2 \\
\hline 50 & 13.2 & 0.342 & 0.12 & 5.60 & 59.3 \\
\hline 50 & 12.4 & 0.338 & 0.11 & 5.37 & 57.1 \\
\hline 90 & 7.80 & 0.303 & 0.078 & 7.00 & 73.0 \\
\hline 90 & 8.91 & 0.314 & 0.077 & 6.91 & 72.1 \\
\hline 120 & 6.77 & 0.291 & 0.064 & 7.62 & 79.1 \\
\hline 120 & 6.87 & 0.292 & 0.063 & 7.69 & 79.7 \\
\hline 320 & 2.89 & 0.206 & 0.039 & 10.5 & 106.8 \\
\hline 320 & 2.87 & 0.205 & 0.031 & 9.97 & 101.8 \\
\hline 520 & 1.88 & 0.162 & 0.021 & 11.1 & 112.7 \\
\hline 860 & 1.22 & 0.121 & 0.015 & 13.0 & 131.4 \\
\hline 860 & 1.20 & 0.120 & 0.016 & 13.6 & 136.5 \\
\hline
\end{tabular}

${ }^{a}$ Contribution of $\mathrm{Na}^{+}$ions to the total BOBCalixC6 loading is assumed to be negligible. $b$ Third phase formed. 


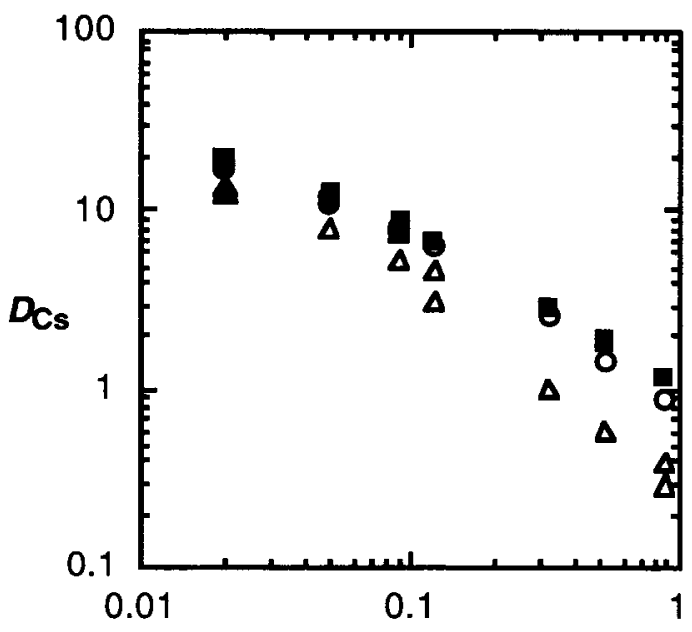

$\left[\mathrm{K}^{+}\right]_{\mathrm{aq} \text {, initial }}$

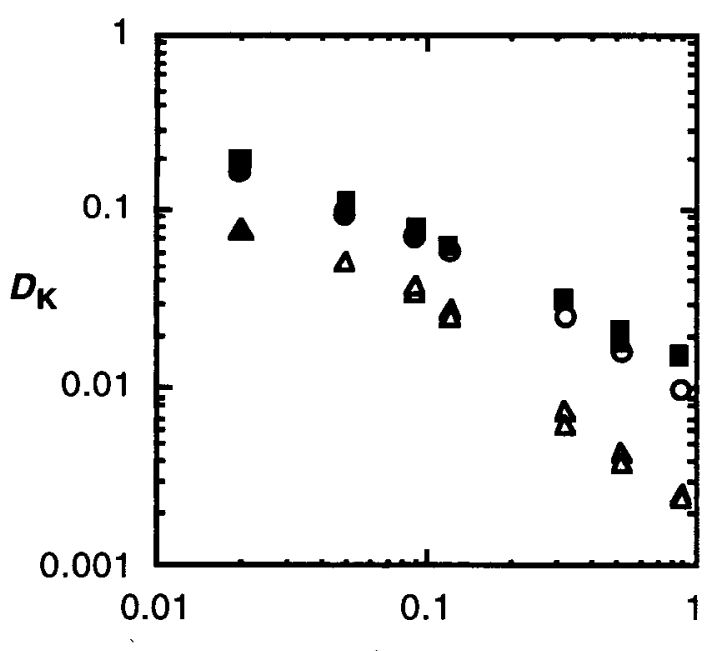

$\left[\mathbf{K}^{+}\right.$]aq, initial

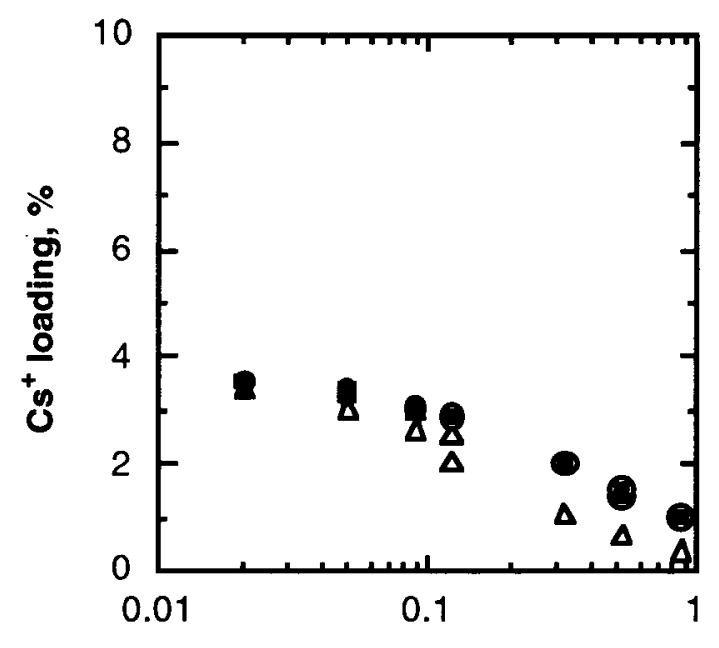

$\left[\mathbf{K}^{+}\right]$aq, initial

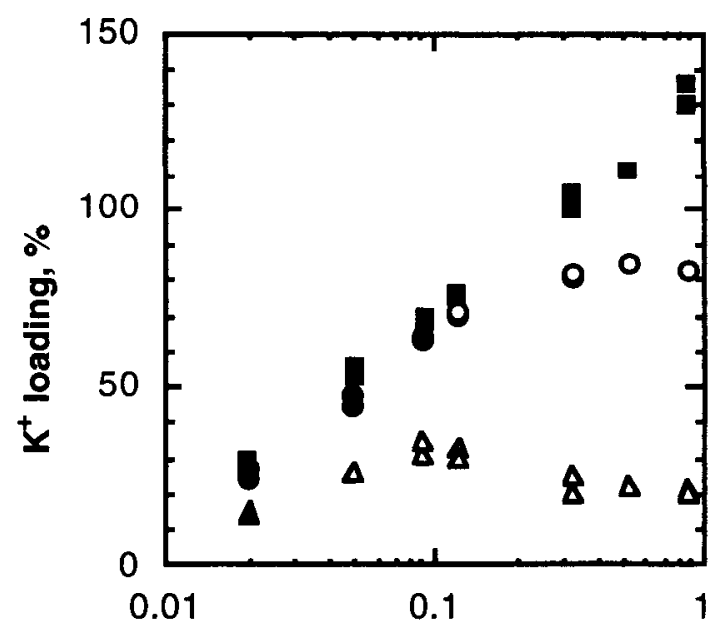

$\left[K^{+}\right]$aq, initial
- $\quad 0.75 \mathrm{M} \mathrm{Cs-7SB}$

- $\quad 0.5 \mathrm{M}$ Cs-7SB

$\Delta \quad 0.25 \mathrm{M} \mathrm{Cs}-7 \mathrm{SB}$
- $\quad 0.5 \mathrm{M} \mathrm{Cs-7SB}$, 3-rd phase is formed

$\Delta \quad 0.25 \mathrm{M} \mathrm{Cs}-7 \mathrm{SB}, 3-\mathrm{rd}$ phase is formed

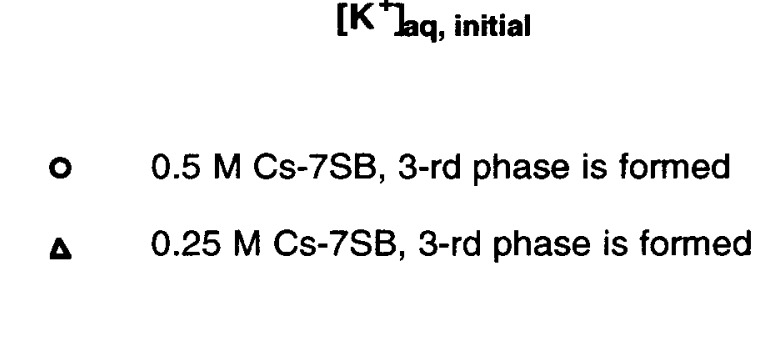

Fig. 4.7. Effect of $\mathrm{K}^{+}$ion concentration on $D_{\mathrm{Cs}}, D_{\mathrm{K}}$, and BOBCalixC6 loading. Organic phase: Baseline solvent with variable Cs-7SB concentration. Aqueous phase: full simulant with variable $\mathrm{K}^{+}$concentration. $\mathrm{O} / \mathrm{A}=0.33 ; T=25^{\circ} \mathrm{C}$. Open symbols denote third-phase formation. 
the analogous tests under the conditions of variable $\mathrm{Cs}^{+}$concentration in the simulant (Section 3.3.17). Distribution values were very similar for 0.5 and $0.75 \mathrm{M} \mathrm{Cs}-7 \mathrm{SB}$ and decreased for $0.25 \mathrm{M}$ Cs-7SB in the solvent. At 0.25 and $0.5 \mathrm{M}$ modifier in the solvent, the decrease of distribution values for both $\mathrm{Cs}^{+}$and $\mathrm{K}^{+}$ions was much steeper than those at $0.75 \mathrm{M}$. In this case of $0.25 \mathrm{M}$ modifier, formation of a third phase was observed for a wide $\mathrm{K}^{+}$concentration range, beginning slightly higher than the baseline $0.02 \mathrm{M}$ concentration. As a result, $\mathrm{Cs}^{+}$and $\mathrm{K}^{+}$ion distribution into the organic phase is greatly reduced, and total loading of the extractant levels off (Fig. 4.7 and 4.8). Interestingly, loading exceeding $100 \%$ without formation of a third phase is possible using solvent containing $0.75 \mathrm{M} \mathrm{Cs-7SB}$ (Table 4.13). This result suggests the desirability of employing a higher modifier concentration, as discussed at greater length in Chap. 7.

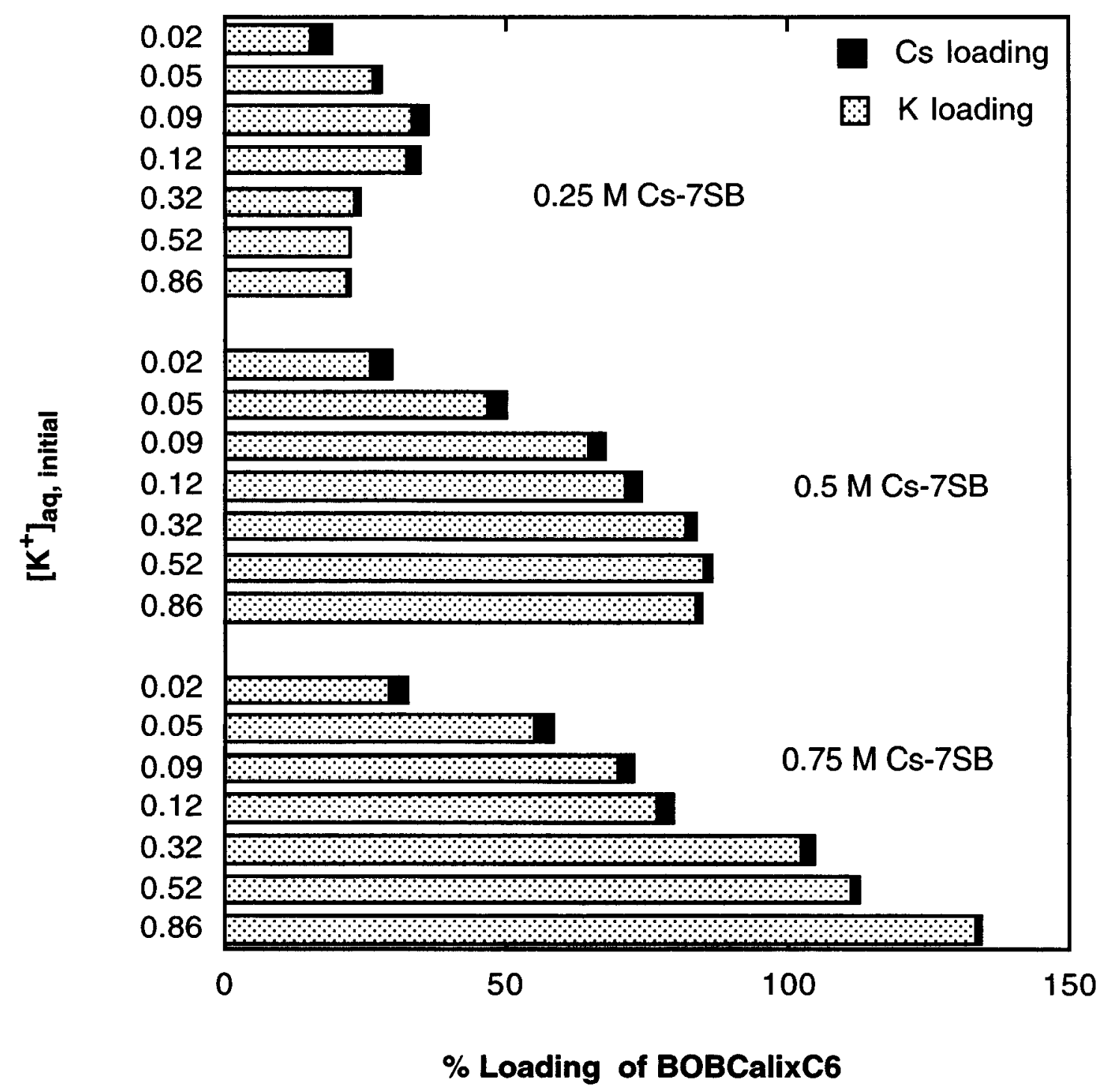

Fig. 4.8. Effect of Cs-7SB modifier concentration in the baseline solvent on total BOBCalixC6 loading at different $\mathrm{K}^{+}$concentrations in the full simulant. Organic phase: pristine washed solvent with variable $\mathrm{Cs}-7 \mathrm{SB}$ concentration. Aqueous phase: full simulant with variable $\mathrm{K}^{+}$concentration. $\mathrm{O} / \mathrm{A}=0.33 ; T=25^{\circ} \mathrm{C}$. 


\subsubsection{Effect of Anion on Alkali Metal Distribution Behavior Using Solvent With or Without BOBCalixC6 Extractant}

Effect of anion on sodium distribution with and without calixarene extractant. Distribution experiments performed using baseline simulant solution and solvent without BOBCalixC6 extractant (Section 4.3.3.1) indicated that BOBCalixC6 plays only a minor role in the distribution of $\mathrm{Na}^{+}$ion into the solvent phase. The sodium ion is extracted largely by an ion-exchange mechanism possible through partial deprotonation of the weakly acidic Cs-7SB modifier. ${ }^{25}$ Therefore, this result suggested the minor contribution of $\mathrm{Na}^{+}$ion to overall loading of BOBCalixC6. Extractant loading is one of the factors that determine the effectiveness of the cesium extraction into the solvent phase. In order to confirm our previous result that $\mathrm{Na}^{+}$ion adds little to the extractant loading, a study was performed to investigate the effect of several factors on the extraction and distribution of $\mathrm{Na}^{+}$ion using solvent both with or without BOBCalixC6 extractant. These factors include the nature of the anion and the sodium or hydroxide ion concentration in the aqueous phase.

Distribution experiments were performed with full solvent or BOBCalixC6-free solvent using aqueous phases containing 1) single $\mathrm{NaX}$ salt $\left(\mathrm{X}^{-}=\mathrm{NO}_{3}^{-}, \mathrm{NO}_{2}^{-}, \mathrm{Cl}^{-}, \mathrm{OH}^{-}\right)$in the concentration range from 0.1 to $5.6 \mathrm{M}$ or 2) $1 \mathrm{M} \mathrm{NaX}$ in $0.03-4.14 \mathrm{M} \mathrm{NaOH}$ solution. Obtained $D_{\mathrm{Na}}$ values and resulting $\mathrm{Na}^{+}$ion concentrations in the solvent phase are listed in Table 4.14. Distribution of $\mathrm{Na}^{+}$ion between the aqueous phase containing a single $\mathrm{NaX}$ salt and the organic phase of BOBCalixC6-free solvent was very low for $\mathrm{NaNO}_{2}$ salt and below the instrumental detection limit $\left(D_{\mathrm{Na}}<2 \times 10^{-5}\right)$ for $\mathrm{NaNO}_{3}$ and $\mathrm{NaCl}$ salt. Addition of BOBCalixC6 extractant resulted in an enhancement of sodium extraction; in the case of $\mathrm{NaNO}_{2}$, the enhancement was calculated to be about 4-fold. Linear dependence of $D_{\mathrm{Na}}$ values on aqueous $\mathrm{Na}^{+}$ion concentration was observed using full solvent. Slope values were calculated by linear-regression analysis to be $0.45 \pm 0.07,0.87 \pm 0.08$, and $1.54 \pm 0.09$ on the log-log scale for $\mathrm{NaNO}_{3}, \mathrm{NaNO}_{2}$, and $\mathrm{NaCl}$, respectively (Fig. 4.9-A). The value of the slope increased in the order $\mathrm{Cl}^{-}>\mathrm{NO}_{2}^{-}>\mathrm{NO}_{3}^{-}$and is explained by the anions' tendency for ion-pairing in the organic phase, which increases as anion size decreases. Thus, the least charge-dense nitrate anion exhibits the least electrostatic attraction to the sodium ion, which results in the smallest value of slope. Extraction equilibrium analysis indicates that, in case of complete dissociation of ions in the organic phase, no dependence of the $D_{\mathrm{Na}}$ values on aqueous $\mathrm{Na}^{+}$ion concentration is expected. ${ }^{56}$ The greater the slope, the more pronounced the ion-pairing in the solvent phase. Interestingly, it was observed that the value of the slopes obtained using aqueous phase containing single $\mathrm{NaNO}_{2}$ salt and solvent phase with and without BOBCalixC6 extractant are identical within observed standard deviations and equal to $0.87 \pm 0.08$ and $0.83 \pm 0.06$, respectively. This result demonstrates that $\mathrm{Na}^{+}$ ion exhibits the same ion-pairing independent of the presence of the BOBCalixC6 receptor in the solvent. Presumably, the BOBCalixC6 host molecule is not able to encapsulate $\mathrm{Na}^{+}$ion and saturate completely its inner coordination sphere, which leaves open a space for additional coordination of the anion.

Different $\mathrm{Na}^{+}$ion distribution behavior was observed under alkaline conditions (Table 4.14 and Fig. 4.9-B). The most important observation was that when the aqueous hydroxide concentration is greater than $1 \mathrm{M}, D_{\mathrm{Na}}$ values were very similar using solvents with and without BOBCalixC6 extractant. Addition of BOBCalixC6 into the extraction system resulted in no change or even slight reduction of $D_{\mathrm{Na}}$ values. The lack of dependence of $D_{\mathrm{Na}}$ values on the anion present in the aqueous phase with hydroxide concentration greater than $1 \mathrm{M}$ and correlation of $D_{\mathrm{Na}}$ values with aqueous hydroxide ion concentration strongly support an ion-exchange mechanism when $\mathrm{Na}^{+}$ion exchanges with the acidic $-\mathrm{OH}$ proton of the Cs-7SB modifier. The anion and BOBCalixC6 effects were observed only when aqueous hydroxide concentration was less than $1 \mathrm{M}$. These results directly confirm our previous conclusion that BOBCalixC6 does not participate in the $\mathrm{Na}^{+}$ion extraction mechanism when highly alkaline simulant solution (concentration of free-hydroxide ion is $2 \mathrm{M}$ ) is used and $\mathrm{Na}^{+}$ion does not contribute to overall extractant loading. 
Table 4.14. $\mathrm{Na}^{+}$ion distribution ratios and organic-phase concentrations at $\mathrm{O} / \mathrm{A}=1$ and $25^{\circ} \mathrm{C}$ using solvent with or without BOBCalixC6 extractant

\begin{tabular}{|c|c|c|}
\hline Aqueous-phase composition & $D_{\mathrm{Na}} \times 10^{4}$ & {$\left[\mathrm{Na}^{+}\right]_{\text {org }} \times 10^{4} \mathrm{M}$} \\
\hline \multicolumn{3}{|c|}{ Solvent without BOBCalixC6 extractant } \\
\hline $5.6 \mathrm{M} \mathrm{NaNO}_{3}$ & $\mathrm{BDL}^{a}$ & \\
\hline $2.25 \mathrm{M} \mathrm{NaNO}_{3}$ & $\mathrm{BDL}$ & \\
\hline $1 \mathrm{M} \mathrm{NaNO}_{3}$ & BDL & \\
\hline $0.1 \mathrm{M} \mathrm{NaNO}_{3}$ & BDL & \\
\hline $1 \mathrm{M} \mathrm{NaNO}_{3} / 4.14 \mathrm{M} \mathrm{NaOH}$ & $15.6 \pm 0.4$ & 80 \\
\hline $1 \mathrm{M} \mathrm{NaNO}_{3} / 1 \mathrm{M} \mathrm{NaOH}$ & $2.9 \pm 0.1$ & 5.8 \\
\hline $1 \mathrm{M} \mathrm{NaNO}_{3} / 0.3 \mathrm{M} \mathrm{NaOH}$ & $0.84 \pm 0.08$ & 1.1 \\
\hline $1 \mathrm{M} \mathrm{NaNO}_{3} / 0.1 \mathrm{M} \mathrm{NaOH}$ & $0.39 \pm 0.06$ & 0.48 \\
\hline $\begin{array}{c}1 \mathrm{M} \mathrm{NaNO} / 3 / 0.03 \mathrm{M} \mathrm{NaOH} \\
--\frac{\mathrm{M} \mathrm{NaNO}}{2}\end{array}$ & $\begin{array}{l}0.21 \pm 0.05 \\
--\overline{0.87} \pm 0.08\end{array}$ & $---\frac{0.22}{4.9}------$ \\
\hline $2.25 \mathrm{M} \mathrm{NaNO}_{2}$ & $0.41 \pm 0.06$ & 0.91 \\
\hline $1 \mathrm{M} \mathrm{NaNO}_{2}$ & $0.21 \pm 0.05$ & 0.2 \\
\hline $0.1 \mathrm{M} \mathrm{NaNO}_{2}$ & BDL & \\
\hline $1 \mathrm{M} \mathrm{NaNO}_{2} / 4.14 \mathrm{M} \mathrm{NaOH}$ & $14.2 \pm 0.4$ & 73 \\
\hline $1 \mathrm{M} \mathrm{NaNO}_{2} / 1 \mathrm{M} \mathrm{NaOH}$ & $2.9 \pm 0.1$ & 5.7 \\
\hline $1 \mathrm{M} \mathrm{NaNO}_{2} / 0.3 \mathrm{M} \mathrm{NaOH}$ & $1.33 \pm 0.08$ & 1.7 \\
\hline $1 \mathrm{M} \mathrm{NaNO}_{2} / 0.1 \mathrm{M} \mathrm{NaOH}$ & $0.29 \pm 0.06$ & 0.32 \\
\hline $\begin{array}{c}1 \mathrm{M} \mathrm{NaNO}_{2} / 0.03 \mathrm{M} \mathrm{NaOH} \\
4.5 \mathrm{M} \mathrm{NaCl}\end{array}$ & $\begin{array}{l}0.26 \pm 0.7 \\
-\mathrm{BDL}\end{array}$ & ------ \\
\hline $2.25 \mathrm{M} \mathrm{NaCl}$ & $\mathrm{BDL}$ & \\
\hline $1 \mathrm{M} \mathrm{NaCl}$ & BDL & \\
\hline $0.1 \mathrm{M} \mathrm{NaCl}$ & BDL & \\
\hline $1 \mathrm{M} \mathrm{NaCl} / 4.14 \mathrm{M} \mathrm{NaOH}$ & $12.6 \pm 0.3$ & 65 \\
\hline $1 \mathrm{M} \mathrm{NaCl} / 1 \mathrm{M} \mathrm{NaOH}$ & $2.7 \pm 0.1$ & 5.3 \\
\hline $1 \mathrm{M} \mathrm{NaCl} / 0.3 \mathrm{M} \mathrm{NaOH}$ & $1.47 \pm 0.07$ & 1.2 \\
\hline $1 \mathrm{M} \mathrm{NaCl} / 0.1 \mathrm{M} \mathrm{NaOH}$ & $1.29 \pm 0.05$ & 0.24 \\
\hline $1 \mathrm{M} \mathrm{NaCl} / 0.03 \mathrm{M} \mathrm{NaOH}$ & $\begin{array}{l}0.68 \pm 0.07 \\
21.1 \pm 0.5\end{array}$ & $-\frac{0.15}{94}-$ \\
\hline $3 \mathrm{M} \mathrm{NaOH}$ & $7.2 \pm 0.2$ & 16 \\
\hline $1 \mathrm{M} \mathrm{NaOH}$ & $2.2 \pm 0.1$ & 2.18 \\
\hline $0.1 \mathrm{M} \mathrm{NaOH}$ & $0.75 \pm 0.08$ & 0.08 \\
\hline
\end{tabular}

${ }^{a}$ Below detection limit. Detection limit of sodium corresponds to $D_{\mathrm{Na}}=2 \times 10^{-5}$. 
Table 4.14 cont.

\begin{tabular}{|c|c|c|}
\hline Aqueous-phase composition & $D_{\mathrm{Na}} \times 10^{4}$ & {$\left[\mathrm{Na}^{+}\right]_{\mathrm{org}} \times 10^{4} \mathrm{M}$} \\
\hline \multicolumn{3}{|l|}{$\begin{array}{c}\text { Solvent with BOBCalixC6 } \\
\text { extractant }\end{array}$} \\
\hline $5.6 \mathrm{M} \mathrm{NaNO}_{3}$ & $2.9 \pm 0.1$ & 16.2 \\
\hline $2.25 \mathrm{M} \mathrm{NaNO}_{3}$ & $2.1 \pm 0.1$ & 4.8 \\
\hline $1 \mathrm{M} \mathrm{NaNO}_{3}$ & $1.33 \pm 0.08$ & 1.3 \\
\hline $0.1 \mathrm{M} \mathrm{NaNO}_{3}$ & $0.39 \pm 0.06$ & 0.04 \\
\hline $1 \mathrm{M} \mathrm{NaNO}_{3} / 4.14 \mathrm{M} \mathrm{NaOH}$ & $13.5 \pm 0.4$ & 3.21 \\
\hline $1 \mathrm{M} \mathrm{NaNO}_{3} / 1 \mathrm{M} \mathrm{NaOH}$ & $4.8 \pm 0.2$ & 0.78 \\
\hline $1 \mathrm{M} \mathrm{NaNO}_{3} / 0.3 \mathrm{M} \mathrm{NaOH}$ & $9.7 \pm 0.3$ & 0.84 \\
\hline $\begin{array}{c}1 \mathrm{M} \mathrm{NaNO}_{3} / 0.1 \mathrm{M} \mathrm{NaOH} \\
-5.6 \mathrm{M} \mathrm{NaNO} \\
--1\end{array}$ & $\begin{array}{l}3.9 \pm 0.2 \\
-\overline{3.8 \pm 0.1}--\end{array}$ & $-\frac{0.20}{22}----$ \\
\hline $2.25 \mathrm{M} \mathrm{NaNO}_{2}$ & $1.75 \pm 0.09$ & 3.9 \\
\hline $1 \mathrm{M} \mathrm{NaNO}_{2}$ & $0.85 \pm 0.08$ & 0.85 \\
\hline $0.1 \mathrm{M} \mathrm{NaNO}_{2}$ & $\mathrm{BDL}^{a}$ & \\
\hline $1 \mathrm{M} \mathrm{NaNO}_{2} / 4.14 \mathrm{M} \mathrm{NaOH}$ & $13.5 \pm 0.4$ & 69 \\
\hline $1 \mathrm{M} \mathrm{NaNO}_{2} / 1 \mathrm{M} \mathrm{NaOH}$ & $3.6 \pm 0.1$ & 7.3 \\
\hline $1 \mathrm{M} \mathrm{NaNO}_{2} / 0.3 \mathrm{M} \mathrm{NaOH}$ & $2.7 \pm 0.1$ & 3.5 \\
\hline $\begin{array}{c}1 \mathrm{M} \mathrm{NaNO}_{2} / 0.03 \mathrm{M} \mathrm{NaOH} \\
4.5 \mathrm{M} \mathrm{NaCl}\end{array}$ & $\begin{array}{l}1.5 \pm 0.1 \\
--0 \pm--\overline{0} \\
1.03 \pm 0.08\end{array}$ & $-\frac{1.5}{4.6}-\cdots-$ \\
\hline $2.25 \mathrm{M} \mathrm{NaCl}$ & $0.36 \pm 0.06$ & 0.81 \\
\hline $1 \mathrm{M} \mathrm{NaCl}$ & $0.10 \pm 0.05$ & 0.1 \\
\hline $0.1 \mathrm{M} \mathrm{NaCl}$ & $\mathrm{BDL}$ & \\
\hline $1 \mathrm{M} \mathrm{NaCl} / 4.14 \mathrm{M} \mathrm{NaOH}$ & $12.3 \pm 0.3$ & 63 \\
\hline $1 \mathrm{M} \mathrm{NaCl} / 1 \mathrm{M} \mathrm{NaOH}$ & $2.6 \pm 0.2$ & 5.1 \\
\hline $1 \mathrm{M} \mathrm{NaCl} / 0.3 \mathrm{M} \mathrm{NaOH}$ & $1.5 \pm 0.1$ & 1.9 \\
\hline $1 \mathrm{M} \mathrm{NaCl} / 0.1 \mathrm{M} \mathrm{NaOH}$ & $1.3 \pm 0.1$ & 1.4 \\
\hline 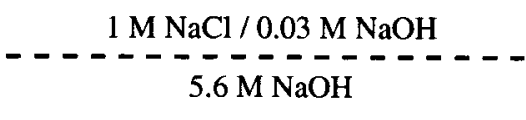 & $\begin{array}{l}0.7 \pm 0.07 \\
-17.2 \pm 0.5\end{array}$ & $-\frac{0.7}{77}----$ \\
\hline $3 \mathrm{M} \mathrm{NaOH}$ & $7.9 \pm 0.3$ & 17.7 \\
\hline $1 \mathrm{M} \mathrm{NaOH}$ & $2.7 \pm 0.1$ & 2.75 \\
\hline $0.1 \mathrm{M} \mathrm{NaOH}$ & $3.7 \pm 0.1$ & 0.36 \\
\hline
\end{tabular}

${ }^{a}$ Below detection limit. Detection limit of sodium corresponds to $D_{\mathrm{Na}}=2 \times 10^{-5}$. 

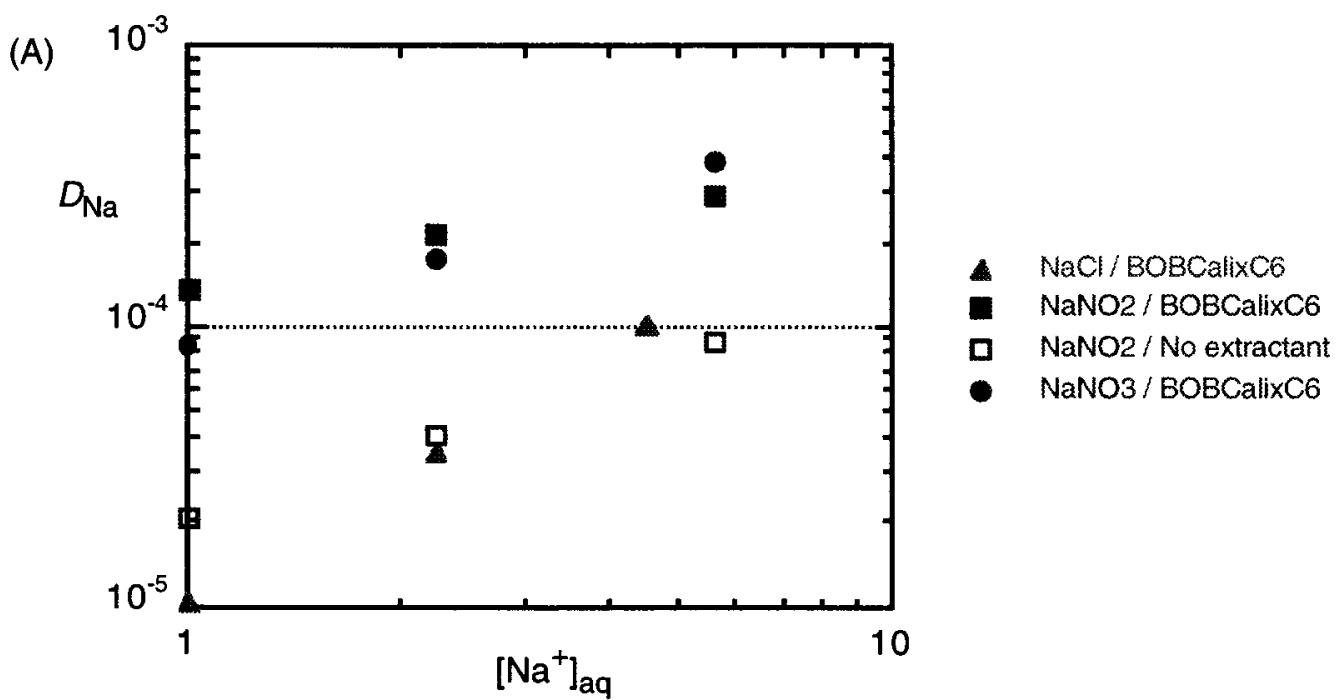

(B)

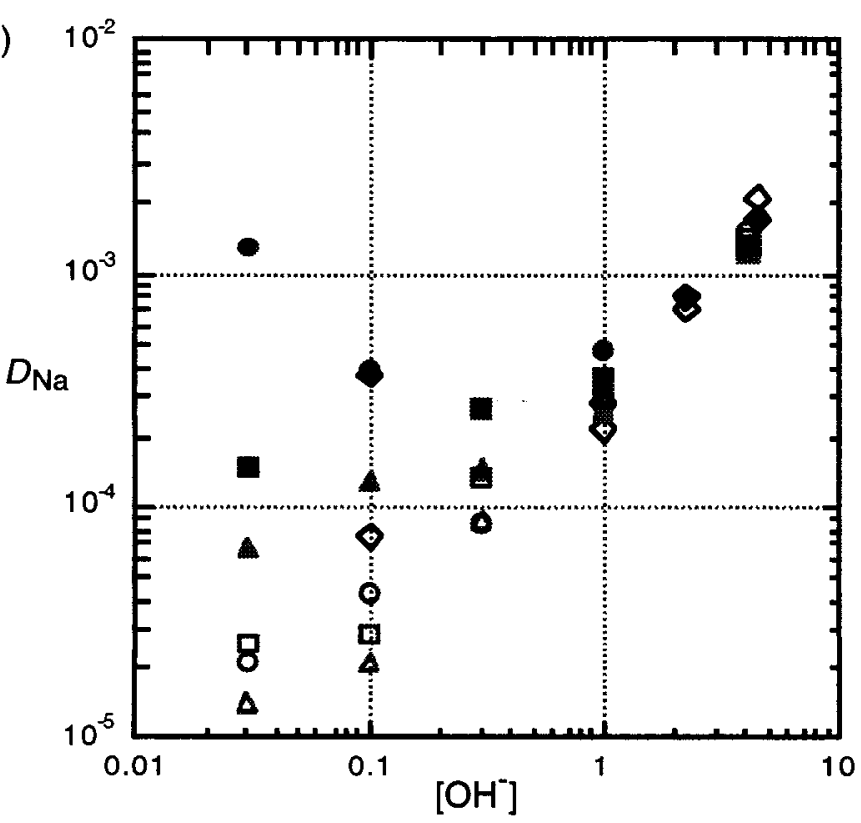

- NaOH/No extractant

- $\mathrm{NaOH} / \mathrm{BOBCalixC6}$

A $\mathrm{NaCl}+\mathrm{NaOH} / \mathrm{No}$ extractant

- $\mathrm{NaCl}+\mathrm{NaOH} / \mathrm{BOBCalixC6}$

- $\mathrm{NaNO} 3+\mathrm{NaOH} / \mathrm{No}$ extractant

- $\mathrm{NaNO} 3+\mathrm{NaOH} / \mathrm{BOBCalixC6}$

- $\mathrm{NaNO} 2+\mathrm{NaOH} / \mathrm{No}$ extractant

- $\mathrm{NaNO} 2+\mathrm{NaOH} / \mathrm{BOBCalixC6}$

Fig 4.9. Effect of anion on $\mathrm{Na}^{+}$ion distribution. Organic phase: pristine washed solvent with or without BOBCalixC6 extractant. Aqueous phase: (A) $\mathrm{NaX}\left(\mathrm{X}^{-}=\mathrm{NO}_{3}^{-}, \mathrm{NO}_{2}^{-}\right.$, and $\left.\mathrm{Cl}^{-}\right)$solution of variable concentration, (B) $1 \mathrm{M} \mathrm{NaX}\left(\mathrm{X}^{-}=\mathrm{NO}_{3}{ }^{-}, \mathrm{NO}_{2}{ }^{-}\right.$, and $\left.\mathrm{Cl}^{-}\right)$in $\mathrm{NaOH}$ solution of variable concentration. $\mathrm{O} / \mathrm{A}=1 ; T=25{ }^{\circ} \mathrm{C}$.

Effect of the anion on distribution of cesium and potassium from sodium salts. Sodium ion is the most abundant inorganic cation in the simulant solution, and for the prediction of the cesium distribution behavior it is necessary to know dependence of the extraction of $\mathrm{Cs}^{+}$and $\mathrm{K}^{+}$ions by the full solvent on the sodium ion concentration and the anion present in the aqueous phase. Accordingly, two series of distribution experiments at $25{ }^{\circ} \mathrm{C}$ and $\mathrm{O} / \mathrm{A}=1$ were performed using 
aqueous phases of constant $0.5 \mathrm{mM} \mathrm{CsNO}_{3}$ or $60 \mathrm{mM} \mathrm{KNO}{ }_{3}$ concentration and variable $\mathrm{NaX}\left(\mathrm{X}^{-}=\right.$ $\mathrm{NO}_{3}{ }^{-}, \mathrm{NO}_{2}{ }^{-}, \mathrm{Cl}^{-}$, and $\mathrm{OH}^{-}$) concentration in the range from $0.01 \mathrm{M}$ to $5.6 \mathrm{M}$. Alkali metal ions were analyzed using ${ }^{137} \mathrm{Cs}$ tracer analysis and ion chromatography outlined under analytical methods in this chapter.

Obtained $D_{\mathrm{Cs}}$ and $D_{\mathrm{K}}$ values are summarized in Table 4.15 and Figs. 4.10-4.11. Of direct relevance to process behavior, the distribution ratios for $\mathrm{Cs}^{+}$ion from sodium nitrate, nitrite, and hydroxide solutions are not grossly different at a given salt concentration. Thus, the process should be relatively tolerant to variations in the anion content among these three major anions, as they should be mutually compensating at constant sodium concentration. This desirable property was in fact observed in more complex simulant recipes tested in Chap. 3 (Section 3.3.14). Another interesting behavioral attribute is the stronger extraction from hydroxide than nitrate at salt concentrations greater than $1 \mathrm{M}$. The distribution results in Table 4.15 demonstrate a similar effect of anion and concentration of $\mathrm{NaX}$ in the aqueous phase on both $D_{\mathrm{Cs}}$ and $D_{\mathrm{K}}$ values. The extraction of both $\mathrm{Cs}^{+}$and $\mathrm{K}^{+}$ions was enhanced in the order $\mathrm{Cl}^{-}<\mathrm{NO}_{2}^{-}<\mathrm{NO}_{3}^{-}$and explained by the difference in the anion lipophilicity which is increased in the same sequence. Nearly linear log-log dependence of $D_{\mathrm{Cs}}$ and $D_{\mathrm{K}}$ values on aqueous concentrations of $\mathrm{NO}_{2}^{-}$and $\mathrm{Cl}^{-}$ions was observed, which suggests a simple ion-pair extraction mechanism. For $\mathrm{NaNO}_{3}$ aqueous matrix, a nearly linear dependence was observed when nitrate concentration in the aqueous phase was less $0.5 \mathrm{M}$. Further increase of nitrate concentration caused leveling off and then slight decrease of $D_{\mathrm{Cs}}$ and $D_{\mathrm{K}}$ values. No change in distribution values was found when nitric acid was used instead of $\mathrm{NaNO}_{3}$ in the aqueous phase (Table 4.15 and Figs. 4.10-4.11). This result supports our previous conclusion that $\mathrm{Na}^{+}$ion does not interfere with the extraction of $\mathrm{Cs}^{+}$and $\mathrm{K}^{+}$ions by BOBCalixC6 extractant. The flat region of the plot describing anion effect on $D_{\mathrm{K}}$ values which corresponds to the extraction of potassium ion from $\mathrm{HNO}_{3}$ solution is the reflection of complete dissociation of the ion pairs under conditions of low ionic strength in the solvent phase. The strongest extraction of $\mathrm{Cs}^{+}$and $\mathrm{K}^{+}$ions was observed from $\mathrm{NaOH}$ solutions and explained by mixed ion-pair and ion-exchange extraction mechanisms. As seen in Figs. 4.10-4.11, at low $\mathrm{NaOH}$ concentration in the aqueous phase $D_{\mathrm{Cs}}$ and $D_{\mathrm{K}}$ values are very similar to those collected using $\mathrm{NaNO}_{3}$ or $\mathrm{HNO}_{3}$ matrix and correspond to ionpair extraction of $\mathrm{Cs}^{+}$or $\mathrm{K}^{+}$ions with $\mathrm{NO}_{3}^{-}$counter-anion. As $\mathrm{NaOH}$ concentration in the aqueous phase increased, $D_{\mathrm{CS}}$ and $D_{\mathrm{K}}$ values are greatly enhanced due to an ion-exchange mechanism, which is turned on at elevated $\mathrm{pH}$. Similar results were demonstrated for $\mathrm{Na}^{+}$ion extraction and are described above in this section.

Table 4.15. Distribution ratios of cesium and potassium from constant cesium and potassium concentration and variable $\mathrm{NaX}\left(\mathrm{X}=\mathrm{OH}^{-}, \mathrm{NO}_{3}^{-}, \mathrm{NO}_{2}^{-}, \mathrm{Cl}^{-}\right)$concentration

\begin{tabular}{ccccccccc}
\hline$[\mathrm{NaX}]$ & \multicolumn{3}{c}{ Cesium Distribution Ratio } & \multicolumn{3}{c}{ Potassium Distribution Ratio } \\
\hline$(\mathrm{M})$ & $\mathrm{X}=\mathrm{OH}^{-}$ & $\mathrm{X}=\mathrm{NO}_{3}^{-}$ & $\mathrm{X}=\mathrm{NO}_{2}^{-}$ & $\mathrm{X}=\mathrm{Cl}^{-}$ & $\mathrm{X}=\mathrm{OH}^{-}$ & $\mathrm{X}=\mathrm{NO}_{3}^{-}$ & $\mathrm{X}=\mathrm{NO}_{2}^{-}$ & $\mathrm{X}=\mathrm{Cl}^{-}$ \\
\hline 0.01 & $2.5 \times 10^{-1}$ & $3.7 \times 10^{-1}$ & $1.2 \times 10^{-1}$ & $5.4 \times 10^{-2}$ & $1.1 \times 10^{-2}$ & $9.4 \times 10^{-3}$ & $9.2 \times 10^{-3}$ & $8.9 \times 10^{-3}$ \\
0.10 & $1.3 \times 10^{0}$ & $2.5 \times 10^{0}$ & $7.5 \times 10^{-1}$ & $2.5 \times 10^{-1}$ & $2.1 \times 10^{-2}$ & $1.7 \times 10^{-2}$ & $1.2 \times 10^{-2}$ & $9.5 \times 10^{-3}$ \\
0.50 & $5.1 \times 10^{0}$ & $7.2 \times 10^{0}$ & $2.8 \times 10^{0}$ & $7.9 \times 10^{-1}$ & $5.0 \times 10^{-2}$ & $3.2 \times 10^{-2}$ & $2.3 \times 10^{-2}$ & $1.3 \times 10^{-2}$ \\
1.0 & $9.4 \times 10^{0}$ & $9.9 \times 10^{0}$ & $5.0 \times 10^{0}$ & $1.3 \times 10^{0}$ & $7.6 \times 10^{-2}$ & $4.1 \times 10^{-2}$ & $3.3 \times 10^{-2}$ & $1.7 \times 10^{-2}$ \\
2.3 & $2.9 \times 10^{+1}$ & $1.1 \times 10^{+1}$ & $9.1 \times 10^{0}$ & $2.5 \times 10^{0}$ & $1.2 \times 10^{-1}$ & $4.7 \times 10^{-2}$ & $4.8 \times 10^{-2}$ & $2.7 \times 10^{-2}$ \\
4.5 & $6.3 \times 10^{+1}$ & $9.0 \times 10^{0}$ & $2.5 \times 10^{0}$ & $4.4 \times 10^{0}$ & $1.3 \times 10^{-1}$ & $4.3 \times 10^{-2}$ & $6.1 \times 10^{-2}$ & $4.4 \times 10^{-2}$ \\
5.6 & $1.0 \times 10^{+2}$ & $7.9 \times 10^{0}$ & $1.5 \times 10^{+1}$ & $a$ & $1.3 \times 10^{-1}$ & $4.0 \times 10^{-2}$ & $6.7 \times 10^{-2}$ & $a$ \\
\hline
\end{tabular}

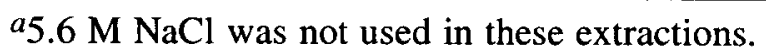




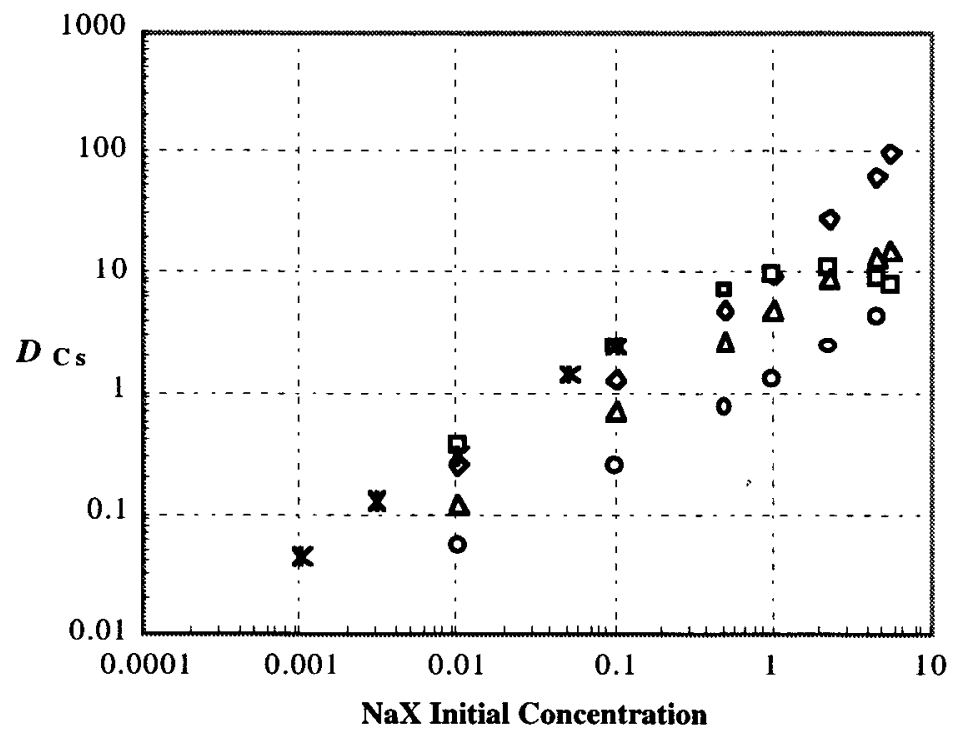

Fig. 4.10. Distribution of $\mathrm{CsNO}_{3}$ from $0.5 \mathrm{mM} \mathrm{CsNO}_{3}, 60 \mathrm{mM} \mathrm{KNO}_{3}$ in variable NaX. $(\diamond)$ designates $\mathrm{NaOH}$ extraction, ( ) designates $\mathrm{NaNO}_{3}$ extraction, $(\Delta)$ designates $\mathrm{NaNO}_{2}$ extraction, ( $\left.{ }^{\circ}\right)$ designates $\mathrm{NaCl}$ extraction, $(*)$ designates extraction from $\mathrm{HNO}_{3}$.

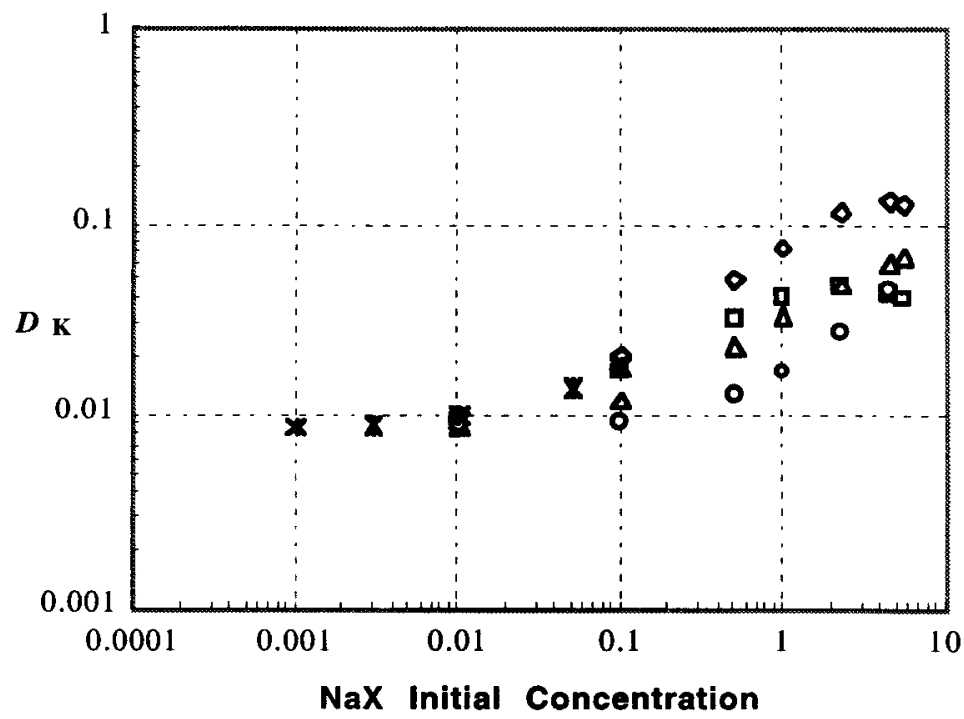

Fig. 4.11. Distribution of $\mathrm{KNO}_{3}$ from $0.5 \mathrm{mM} \mathrm{CsNO}_{3}, 60 \mathrm{mM} \mathrm{KNO}_{3}$ in variable NaX. ( () designates $\mathrm{NaOH}$ extraction, ( ) designates $\mathrm{NaNO}_{3}$ extraction, $(\Delta)$ designates $\mathrm{NaNO}_{2}$ extraction, (०) designates $\mathrm{NaCl}$ extraction, $(*)$ designates extraction from $\mathrm{HNO}_{3}$. 


\subsubsection{Effect of O/A Ratio}

The effect of O/A ratio on the distribution of alkali metal ions was examined. A series of pristine washed baseline solvent samples was contacted with full simulant solution at O/A in the range 0.05 to 10 (Table 4.16 and Fig. 4.12). The cesium data from this experiment were discussed in Section 3.3.4, where it was shown that changes in $\mathrm{O} / \mathrm{A}$ ratio caused relatively minor effects on $D_{\mathrm{Cs}}$ (Fig. 4.12 A). It may be observed that the distributions of $\mathrm{K}^{+}$and $\mathrm{Na}^{+}$ions are also not dependent upon changes in the $\mathrm{O} / \mathrm{A}$ ratio, except at the highest $\mathrm{O} / \mathrm{A}$ ratio in the case of $\mathrm{K}^{+}$(Fig. 4.12). Fig. 4.12-B shows the effect of O/A on the BOBCalixC6 loading. As O/A increases to high values, total loading gradually declines, as the aqueous-phase $\mathrm{K}^{+}$concentration is depleted. The main conclusion from this experiment is that the solvent is able to sustain a nearly constant value of $D_{M}$ for each alkali metal ion with variation in O/A ratio about the baseline value $(0.33)$ by as much as a factor of 3 .

Table 4.16. Effect of $\mathrm{O} / \mathrm{A}$ ratio on distribution behavior of alkali metal ions

\begin{tabular}{|c|c|c|c|c|c|c|c|}
\hline \multirow[t]{2}{*}{$\mathrm{O} / \mathrm{A}$} & \multicolumn{2}{|c|}{$\mathrm{Cs}^{+}$} & \multicolumn{2}{|c|}{$\mathrm{K}^{+}$} & \multicolumn{2}{|c|}{$\mathrm{Na}^{+}$} & \multirow{2}{*}{$\begin{array}{c}\text { Total } \\
\text { loading } a \\
\%\end{array}$} \\
\hline & $D_{\mathrm{Cs}}$ & $\begin{array}{c}{\left[\mathrm{Cs}^{+}\right]_{\mathrm{org}}} \\
(\mathrm{mM})\end{array}$ & $D_{\mathrm{K}}$ & $\begin{array}{c}{\left[\mathrm{K}^{+}\right]_{\mathrm{org}}} \\
(\mathrm{mM})\end{array}$ & $\begin{array}{c}D_{\mathrm{Na}} \\
\left(\times 10^{4}\right)\end{array}$ & $\begin{array}{l}{\left[\mathrm{Na}^{+}\right]_{\text {org }}} \\
(\mathrm{mM})\end{array}$ & \\
\hline 0.05 & 11.2 & 1.00 & 0.12 & 2.3 & 6.6 & 3.9 & 32.8 \\
\hline 0.05 & 16.5 & 1.27 & 0.11 & 2.2 & 7.1 & 4.2 & 34.8 \\
\hline 0.1 & 17.2 & 0.885 & 0.11 & 2.1 & & 6.8 & 29.9 \\
\hline 0.1 & 17.3 & 0.888 & 0.11 & 2.1 & 6.1 & 3.6 & 30.0 \\
\hline 0.3 & 17.2 & 0.391 & 0.12 & 2.3 & 6.5 & 3.8 & 27.3 \\
\hline 0.3 & 16.8 & 0.389 & 0.12 & 2.3 & 6.8 & 4.0 & 27.0 \\
\hline 1 & 16.9 & 0.132 & 0.12 & 2.2 & 6.4 & 3.8 & 22.9 \\
\hline 1 & 18.0 & 0.133 & 0.11 & 2.0 & 6.0 & 3.5 & 21.3 \\
\hline 3 & 18.2 & 0.0458 & 0.11 & 1.6 & 6.2 & 3.6 & 16.5 \\
\hline 3 & 18.4 & 0.0458 & 0.10 & 1.6 & 6.5 & 3.8 & 16.3 \\
\hline 10 & 19.4 & 0.0139 & 0.86 & 0.93 & 7.4 & 4.3 & 9.4 \\
\hline 10 & 18.8 & 0.0139 & 0.46 & 0.63 & 4.9 & 2.9 & 6.4 \\
\hline
\end{tabular}

${ }^{a}$ Contribution of $\mathrm{Na}^{+}$ions to the total $\mathrm{BOBCalixC} 6$ loading is assumed to be negligible.

\subsubsection{Effect of Multiple Solvent Contacts With Simulant}

An experiment was designed to investigate the alkali metal ion extraction behavior of the baseline solvent upon repeated contacts with the full simulant at $25{ }^{\circ} \mathrm{C}$ and at two different $\mathrm{O} / \mathrm{A}$ ratios, 0.33 and 0.1 (Table 4.17 and Fig. 4.13). Distribution ratios overall exhibited a marginal dependence on multiple solvent contacts with full simulant (Fig. 4.13-A). Only $D_{\mathrm{Cs}}$ decreases noticeably with increasing number of contacts, and the $\mathrm{Cs}^{+}$loading plateaus after 3 contacts. The values of $D_{\mathrm{K}}$ and $D_{\mathrm{Na}}$ are constant. No third phase was observed. The reader is also referred to the $\mathrm{E}_{5} \mathrm{SS}_{6}$ experiments described in Section 3.3.15. Although the results in Table 4.17 and Fig. 4.13 do not reveal appreciable change in distribution behavior in the extraction step, stripping worsens upon multiple contacts. 
(A)

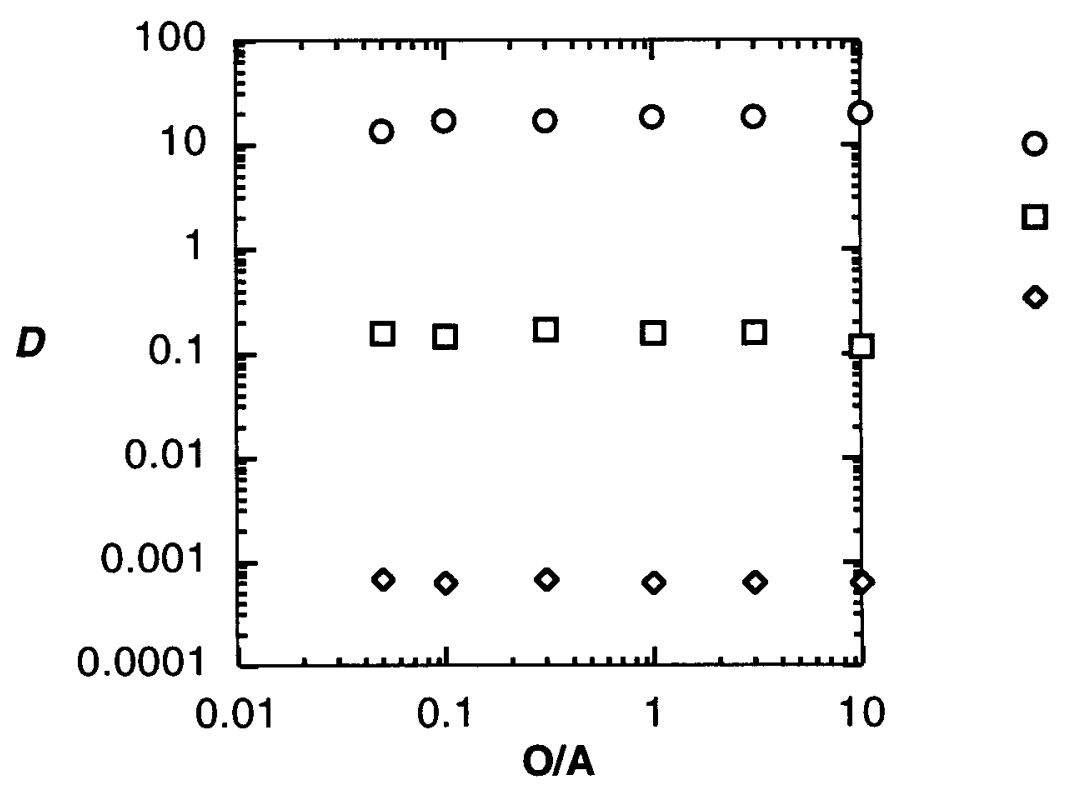

(B)

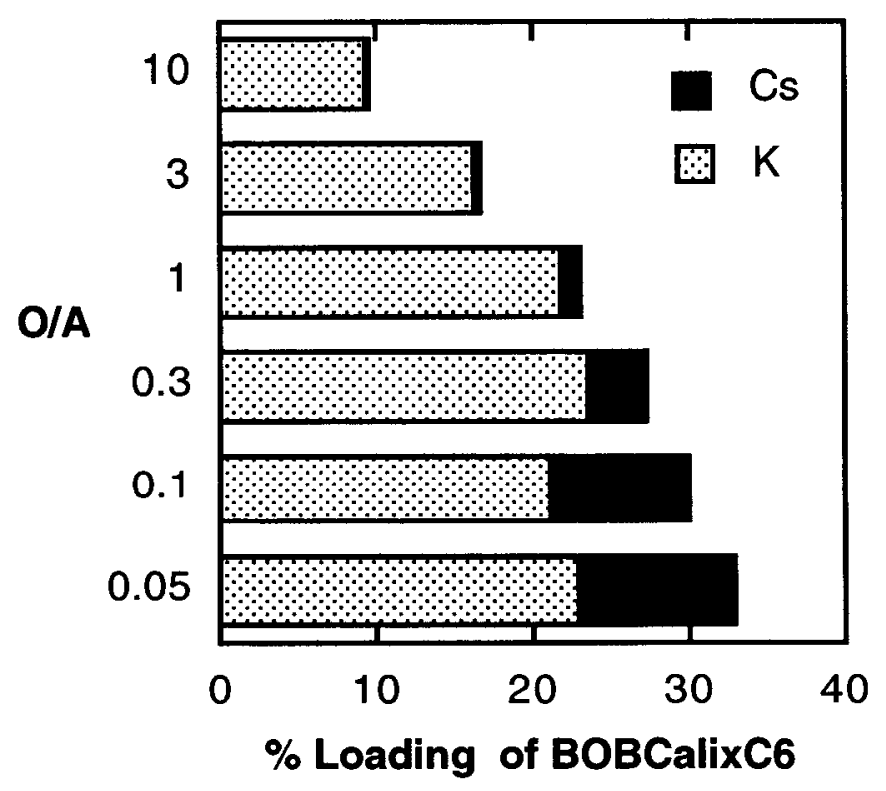

Fig. 4.12. Effect $O / A$ ratio on (A) $D_{\mathrm{Cs}}$ and (B) BOBCalixC6 loading. Organic phase: pristine washed solvent. Aqueous phase: full simulant. $\mathrm{O} / \mathrm{A}$ variable; $T=25^{\circ} \mathrm{C}$. 
Table 4.17. Effect of multiple solvent contacts with simulant on distribution of alkali metal ions

\begin{tabular}{|c|c|c|c|c|c|c|c|}
\hline \multirow{2}{*}{$\begin{array}{c}\text { No. of } \\
\text { contacts }\end{array}$} & \multicolumn{2}{|c|}{$\mathrm{Cs}^{+}$} & \multicolumn{2}{|c|}{$\mathrm{K}^{+}$} & \multicolumn{2}{|c|}{$\mathrm{Na}^{+}$} & \multirow{2}{*}{$\begin{array}{c}\text { Total } \\
\text { loading } a \\
\%\end{array}$} \\
\hline & $D_{\mathrm{Cs}}$ & $\begin{array}{c}{\left[\mathrm{Cs}^{+}\right]_{\text {org }}} \\
(\mathrm{mM})\end{array}$ & $D_{\mathrm{K}}$ & $\begin{array}{c}{\left[\mathrm{K}^{+}\right]_{\mathrm{org}}} \\
(\mathrm{mM})\end{array}$ & $\begin{array}{c}D_{\mathrm{Na}} \\
\left(\times 10^{4}\right)\end{array}$ & $\begin{array}{c}{\left[\mathrm{Na}^{+}\right]_{\mathrm{org}}} \\
(\mathrm{mM})\end{array}$ & \\
\hline \multicolumn{8}{|c|}{$\mathrm{O} / \mathrm{A}=0.33$} \\
\hline 1 & 16.0 & 0.354 & 0.12 & 2.3 & 6.7 & 4.0 & 26.5 \\
\hline 1 & 16.0 & 0.354 & 0.10 & 2.0 & 6.1 & 3.4 & 23.6 \\
\hline 2 & 15.3 & 0.647 & 0.12 & 2.3 & 6.3 & 3.7 & 29.4 \\
\hline 2 & 15.1 & 0.645 & 0.12 & 2.4 & 6.7 & 3.9 & 30.2 \\
\hline 3 & 16.1 & 0.899 & 0.13 & 2.4 & 6.7 & 3.9 & 33.3 \\
\hline 3 & 16.2 & 0.899 & 0.11 & 2.5 & 6.3 & 3.7 & 34.4 \\
\hline 5 & 16.0 & 1.26 & 0.11 & 2.4 & 6.6 & 3.9 & 36.9 \\
\hline 5 & 15.3 & 1.26 & 0.10 & & & & \\
\hline 10 & 15.5 & 1.76 & 0.10 & 2.3 & 6.8 & 4.0 & 40.3 \\
\hline 10 & 15.9 & 1.76 & 0.10 & 2.3 & 6.8 & 4.0 & 40.2 \\
\hline \multicolumn{8}{|c|}{$\mathrm{O} / \mathrm{A}=0.1$} \\
\hline 1 & 16.2 & 0.866 & 0.12 & 2.4 & 7.1 & 4.2 & 32.5 \\
\hline 1 & 16.5 & 0.872 & 0.12 & 2.3 & 5.8 & 3.4 & 31.9 \\
\hline 2 & 15.8 & 1.39 & 0.12 & 2.3 & 6.8 & 4.0 & 37.2 \\
\hline 2 & 14.4 & 1.34 & 0.12 & 2.2 & 6.6 & 3.9 & 35.8 \\
\hline 3 & 14.9 & 1.64 & 0.11 & 2.4 & 7.4 & 4.4 & 40.2 \\
\hline 3 & 13.6 & 1.58 & 0.11 & 2.3 & 7.2 & 4.2 & 38.5 \\
\hline 5 & 15.5 & 1.96 & 0.10 & 2.3 & & & 41.2 \\
\hline 5 & 14.2 & 1.96 & & & & & \\
\hline 10 & 17.1 & 2.11 & 0.10 & 2.2 & 7.2 & 4.2 & 43.2 \\
\hline 10 & 13.6 & 2.11 & 0.10 & 2.2 & 7.4 & 4.4 & 42.3 \\
\hline
\end{tabular}

${ }^{a}$ Contribution of $\mathrm{Na}^{+}$ions to the total BOBCalixC6 loading is assumed to be negligible. 
(A)

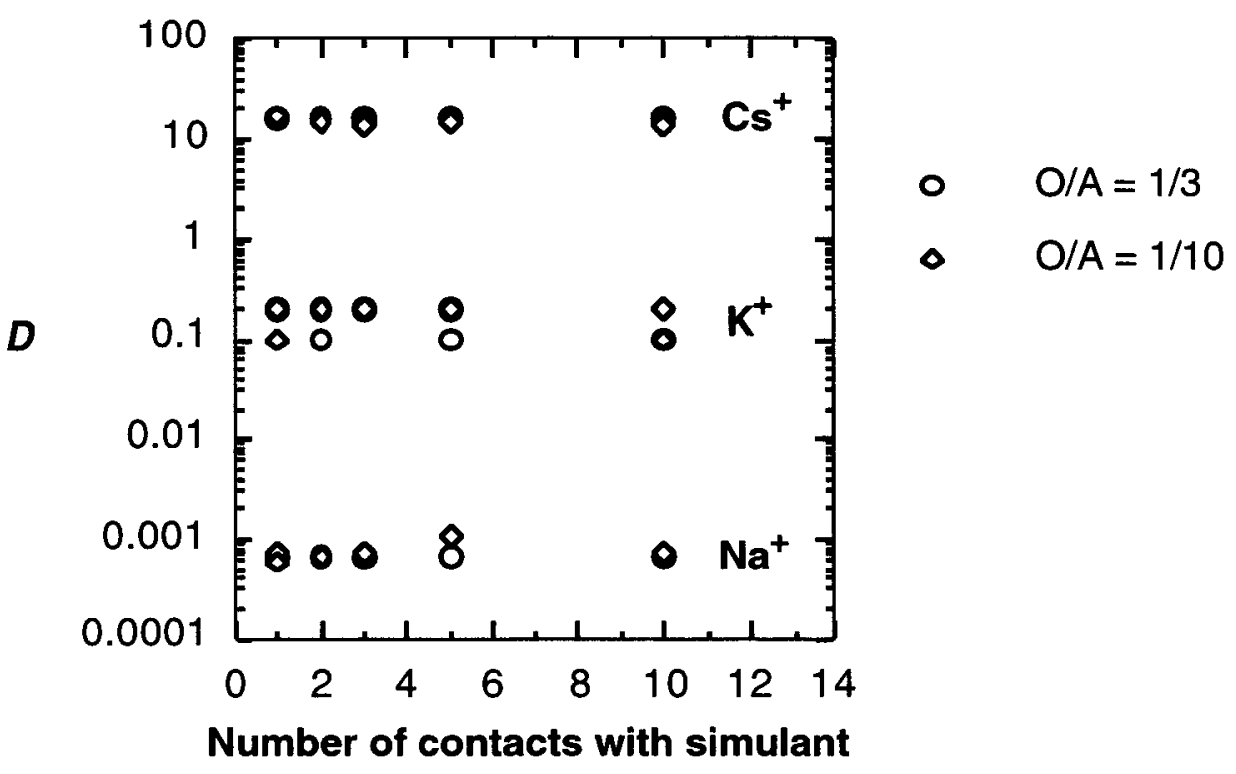

(B)

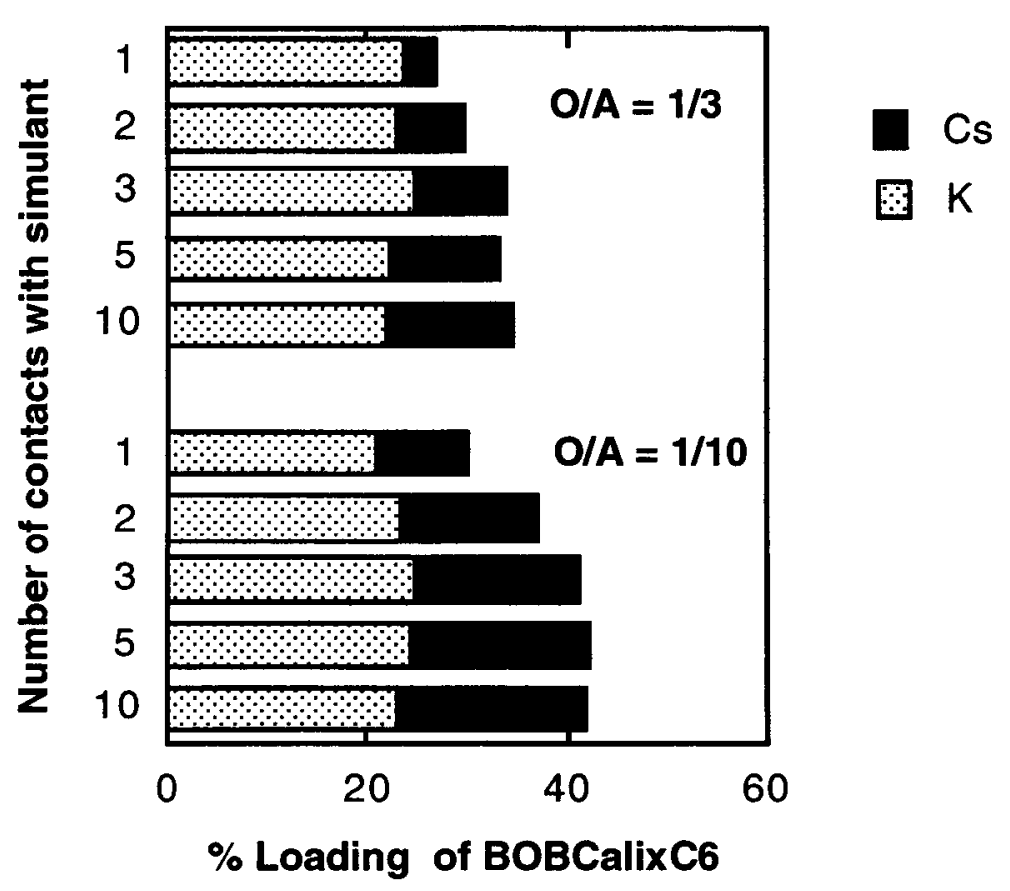

Fig. 4.13. Effect of multiple contacts of the baseline solvent with the full simulant on (A) $D_{\mathrm{Cs}}$ and (B) BOBCalixC6 loading. Organic phase: pristine washed solvent, repeatedly contacted with simulant. Aqueous phase: full simulant. $\mathrm{O} / \mathrm{A}=0.33$ or $0.1 ; T=25^{\circ} \mathrm{C}$. 


\subsubsection{Distribution of Other Metals and Selected Radionuclides}

Experiments described in this section and Section 4.3.6 were directed toward elucidating the general question of the fate of minor metal components in the simulant or waste, according to whether they distribute into the solvent and migrate through the stages in the flowsheet. One question deals with the possible buildup of other extractable metals that may not strip or wash out of the solvent, eventually leading to operational problems such as interfacial crud. Another question deals with the effect of allowing actinides and strontium to remain in the feed in the optional case that the strike with monosodium titanate is made subsequent to CSSX rather than prior to CSSX. The question of technetium was also addressed here, mainly to confirm indications from process development that pertechnetate extraction from alkaline HLW simulants is weak with the use of the family of alcohol modifiers tested. ${ }^{34}$ In Section 4.3.6, ICP-MS was used in a multi-cycle experiment to examine the fate of most of the minor metals in the simulant. In this section, actinides, strontium, and technetium are examined; these are metals not included in the full simulant.

A uniform concentration of $10^{-5} \mathrm{M}$ was desired for selected actinides, strontium, and technetium in the full simulant. Four different simulant samples were prepared, one containing only ${ }^{233} \mathrm{U}$, one only ${ }^{237} \mathrm{~Np}$, one only ${ }^{239} \mathrm{Pu}$, and one containing all three actinides plus strontium and ${ }^{99} \mathrm{Tc}$. Before performing the contacting experiments, it was important to know if all the actinides, strontium, and technetium remained in solution on addition to the full simulant. Based on the alkalinity of the simulant, it was anticipated that their concentrations after addition to the simulant would decrease relative to their initial concentrations. The concentration of $10^{-5} \mathrm{M}$ was in fact maintained for $\mathrm{Sr}$ and Tc. A labeling error on the uranium stock solution lead to a spike that was actually close to $2 \times 10^{-5}$ $M$ initially. The values reported below for the actinides were based on the alpha-counting data obtained four days after preparation of the spiked simulant solutions. They seemed more reasonable and reliable than the ICP-MS data.

$\begin{array}{llll}\text { Strontium } & 1.0 \times 10^{-5} \mathrm{M} & \text { Uranium } & 1.2 \times 10^{-5} \mathrm{M} \\ \text { Technetium } & 1.0 \times 10^{-5} \mathrm{M} & \text { Neptunium } & 5.2 \times 10^{-6} \mathrm{M} \\ \text { Plutonium } & 4.8 \times 10^{-6} \mathrm{M} & & \end{array}$

All the simulants (one element or all elements) were contacted with baseline solvent. The solvent after extraction was analyzed by alpha counting. Only uranium was barely above detection limit. The simulant after extraction was analyzed by ICP-MS, and it was found that all the actinides, strontium, and technetium remain in that phase, in agreement with the extremely low level of alpha activity in the organic phase. The solvent was then scrubbed with $50 \mathrm{mM} \mathrm{HNO}$. The two phases were then analyzed for uranium. It was deduced that the scant amount that might have been extracted was entirely scrubbed. The organic phase after scrubbing showed only 1 alpha count above background after 10 minutes. A summary of extraction results for the four metals is given below:

$$
\begin{aligned}
& D_{\mathrm{U}}=8.3 \times 10^{-4} \\
& D_{\mathrm{Np}}<1.2 \times 10^{-2} \\
& D_{\mathrm{Pu}}<1.6 \times 10^{-4} \\
& D_{\mathrm{Sr}}<6.3 \times 10^{-2} \\
& D_{\mathrm{Tc}}<0.1
\end{aligned}
$$

It was also important to check the performance of the solvent regarding cesium extraction from an aqueous simulant that contains actinides, strontium, and technetium. Organic and aqueous phases were subsampled after extraction, scrub, and two strips, then centrifuged and counted. Only the series of samples involving the simulant containing all five metals was considered, since that represented the worst and most representative case. The following cesium distribution ratios were obtained: 
Table 4.18. Cesium distribution in the presence of $\mathrm{U}, \mathrm{Np}, \mathrm{Pu}, \mathrm{Sr}$, and $\mathrm{Tc}$

\begin{tabular}{lccc}
\hline \multicolumn{1}{c}{ Stage } & $\begin{array}{c}\boldsymbol{D}_{\mathrm{Cs}} \\
\text { Measured at } 27^{\circ} \mathrm{C}\end{array}$ & $\begin{array}{c}\boldsymbol{D}_{\mathrm{Cs}} \\
\text { Corrected to } 25{ }^{\circ} \mathrm{C}^{a}\end{array}$ & $\begin{array}{c}\boldsymbol{D}_{\mathrm{Cs}} \\
\text { Average at } 25{ }^{\circ} \mathrm{C}^{b}\end{array}$ \\
\hline Extraction & 14 & 16 & 17 \\
Scrub & 1.1 & 1.3 & 1.6 \\
Strip \#1 & 0.11 & 0.14 & 0.15 \\
Strip \#2 & 0.055 & 0.069 & 0.089 \\
\hline
\end{tabular}

${ }^{a}$ Corrected using the parameters given in Table 3.13 for extraction and Table 3.11 for scrub and strips.

${ }^{b}$ Average values taken from Table 3.4 for ESS tests performed with full simulant.

The $D_{\mathrm{Cs}}$ values were slightly lower than expected, likely due to the method of contact (vortex) and the lack of temperature control in the glove box. The global temperature was estimated at $27 \pm 1{ }^{\circ} \mathrm{C}$ in the glove box, and the $D_{\mathrm{Cs}}$ values corrected to $25^{\circ} \mathrm{C}$ are in reasonable agreement with the average values obtained with full simulant (no added $\mathrm{U}, \mathrm{Np}, \mathrm{Pu}, \mathrm{Sr}$, and $\mathrm{Tc}$ ).

The results showed that the five metals $\mathrm{U}, \mathrm{Np}, \mathrm{Pu}, \mathrm{Sr}$, and $\mathrm{Tc}$ were not appreciably extracted by the CSSX baseline solvent. The results also showed that the presence of the actinides, Sr, and Tc, does not impair extraction nor stripping of cesium. Although more tests should be conducted to gather definitive data on controlled oxidation states (except $\mathrm{Sr}$ ) in varied matrices, including real waste, it appears likely that the five metals should pass through the extraction stages and exit with the waste raffinate. Tentatively, there is therefore expected to be little if any impact due to these metals on the CSSX process and the character of its solvent and strip effluent streams.

\subsubsection{Distribution of Anions by Ion Chromatography}

Baseline solvent was contacted with full simulant in an $\mathrm{ESS}_{3} \mathrm{~W}_{2}$ test having the addition of two washes with $10 \mathrm{mM} \mathrm{NaOH}$ at equal volume at the end of the protocol [labeled as solvent (A)]. Another aliquot of solvent was contacted five times with fresh simulant in an $\mathrm{E}_{5} \mathrm{SS}_{3} \mathrm{~W}_{2}$ protocol above [labeled as solvent (B)]. The aqueous phases were analyzed directly by IC for anions. A small portion of the loaded solvent was also contacted with deionized (DI) water at ten times the organic volume and then analyzed.

The nitric acid scrub solution $(50 \mathrm{mM})$ was diluted 50 times volumetrically with DI water. The analyzed concentration was $52.4 \mathrm{mM} \mathrm{HNO}_{3}$. After the large dilution, all other anions fell below detection limits (Table 4.19). The nitrate amounts scrubbed correlated well to the $\mathrm{K}^{+}$concentrations found by ICP in the scrub solution (i.e., $2.33 \mathrm{mM} \mathrm{K}^{+}$; Section 3.4 Effect of Simulant Recipe). Only slightly more nitrate is found in the scrub of the solvent that was contacted 5 times with simulant. Because of the extreme dilution, no other anions could be determined in the scrub solution, and there was no evidence of DBP.

The strip solutions were diluted 5 times with DI water and analyzed using both IC methods (see Experimental Section 4.2). The method to separate DBP from the other anions showed no evidence of DBP in the strip solutions. The nitric acid strip solution $(1 \mathrm{mM})$ was diluted 5 times volumetrically with DI water. The concentration was found to be $1.14 \mathrm{mM} \mathrm{HNO}_{3}$. Results for the anions in the strip solutions are summarized in Table 4.20. The strips of the single-contact solvent revealed no other anions other than nitrate and trace nitrite. The nitrate concentrations can be assumed to be $\mathrm{CsNO}_{3}$ and are calculated as such in Table 4.21. The strips of the multiple-contact (B) solvent had increased evidence of nitrite and trace sulfate along with slightly increased concentrations of nitrate, which are compared to the single-contact (A) strips in Table 4.20. Although nitrate stripping of the multiplecontact solvent starts out slightly high, by the third strip it has almost reached the level of the singlecontact solvent behavior. For this multiple-contact solvent (B), there is an impurity growing into the IC chromatograph that elutes before chloride. This impurity cannot be identified at this time. 
Table 4.19. Concentration of nitrate anion found in scrub solutions

\begin{tabular}{cccc}
\hline Solvent & $\begin{array}{c}{\left[\mathrm{NO}_{3}{ }^{-}\right] \text {in scrub }} \\
(\mathrm{mM})\end{array}$ & $\begin{array}{c}{\left[\mathrm{NO}_{3}{ }^{-}\right] \text {in solvent }} \\
(\mathrm{mM})\end{array}$ & $D_{\mathrm{K}}$ for extraction ${ }^{b}$ \\
\hline Single-contact (A) & 11.4 & 2.28 & 0.118 \\
Multiple-contact (B) & 13.3 & 2.66 & 0.139 \\
\hline
\end{tabular}

${ }^{a}$ Amount of nitrate anion found in the scrub solution after 50 -fold dilution with DI water and subtraction of background nitrate from nitric acid.

${ }^{b}$ Assumes the majority of the excess nitrate anion in the scrub solution is due to $\mathrm{KNO}_{3}$ extraction by the solvent. Initial $\left[\mathrm{KNO}_{3}\right]$ in the full simulant is $20 \mathrm{mM}$.

Table 4.20. Concentration of anions found in the strip solution after subtraction of $\mathrm{HNO}_{\mathbf{3}}$

\begin{tabular}{cccccccc}
\hline \multirow{2}{*}{ Anion } & \multicolumn{3}{c}{ Single contact (A) } & & \multicolumn{3}{c}{ Multiple contacts (B) } \\
\cline { 2 - 4 } \cline { 7 - 8 } & Strip 1 & Strip 2 & Strip 3 & & Strip 1 & Strip 2 & Strip 3 \\
\hline $\mathrm{Cl}^{-}$ & BDL & BDL & BDL & & BDL & BDL & BDL \\
$\mathrm{NO}_{2}^{-}$ & 0.0094 & 0.012 & 0.00525 & & 0.023 & 0.015 & 0.013 \\
$\mathrm{NO}_{3}^{-}$ & 1.65 & 0.449 & 0.143 & & 2.60 & 0.607 & 0.239 \\
$\mathrm{SO}_{4}^{2-}$ & BDL & BDL & BDL & & BDL & 0.048 & 0.0023 \\
$\mathrm{PO}_{4}{ }^{3-}$ & BDL & BDL & BDL & & BDL & BDL & BDL \\
\hline
\end{tabular}

${ }^{a}$ Concentrations are in mM. BDL refers to "below detection limits."

Table 4.21. Comparison of single- and multiple-contact strip solutions. Nitrate concentrations are expressed as $\mathrm{CsNO}_{3}$ concentrations.

\begin{tabular}{cccc}
\hline Strips & Single contact $(\mathrm{A})$ & Multiple contacts $(\mathrm{B})$ & Ratio B/A \\
\hline 1 & $5.25 \times 10^{-4} \mathrm{M}$ & $8.27 \times 10^{-4} \mathrm{M}$ & 1.58 \\
2 & $1.43 \times 10^{-4} \mathrm{M}$ & $1.93 \times 10^{-4} \mathrm{M}$ & 1.35 \\
3 & $6.58 \times 10^{-5} \mathrm{M}$ & $7.61 \times 10^{-5} \mathrm{M}$ & 1.16 \\
\hline
\end{tabular}

The washes using $10 \mathrm{mM} \mathrm{NaOH}$ were designed to remove any anionic impurities such as 4-secbutylphenol or mono- and dibutylphosphate. Monobutylphosphate was not determined. However, it was found that dibutylphosphate elutes at the same time as nitrite anion using an isocratic IC method with $\mathrm{NaOH}$ eluent. Since the chloride peak is only separated from the nitrite peak by 0.30 minutes using this method, it is possible that a large amount of DBP in the sample could cover the retention of chloride as well. A slow gradient $\mathrm{NaOH}$ eluent with $2 \% \mathrm{MeOH}$ was effective in separating the $\mathrm{Cl}^{-}$ peak from the $\mathrm{NO}_{2}^{-}$peak by an additional $0.3 \mathrm{~min}$, leaving room in the middle of these two peaks for quantitative analysis of the DBP peak, which was effectively separated from the $\mathrm{NO}_{2}^{-}$peak. The first $\mathrm{NaOH}$-wash contained excess amounts of nitrate as well as DBP. The second wash solution was practically clean, showing that all anionic impurities can be washed out in one wash at equal volume. Interestingly, the first wash of the single-contact solvent (A) had more nitrate in the wash solution than the multiple-contact solvent (B) (approximately 3.2 times as much). However, the amount of DBP found in the wash of solvent (B) had increased by a factor of 1.8 over that found in solvent (A). Likely, a conclusion can be drawn that the additional DBP in the solvent (B) tends to displace the nitrate. It will be shown below that DBP partitions to the solvent from the simulant, with $34 \mathrm{ppm}$ DBP 
found in the solvent by ${ }^{31} \mathrm{P}-\mathrm{NMR}$ after one contact with full simulant using the standard $\mathrm{O} / \mathrm{A}=0.33$ (Section 4.3.7). Furthermore, the partitioning of DBP into the solvent from $0.01 \mathrm{M} \mathrm{NaOH}$ solutions is weak, with most DBP being washed out using O/A ratios of $1\left(P=3.0 \times 10^{-3}\right)$. The presence of DBP in the aqueous $10 \mathrm{mM} \mathrm{NaOH}$ wash solutions was therefore confirmed and correlates well to the amount of DBP in the solvent after extraction with full simulant using the same O/A ratio of 0.33 as found by ${ }^{31}$ P-NMR (Table 4.22).

Table 4.22. Results of washing by $10 \mathrm{mM} \mathrm{NaOH}^{a}$

\begin{tabular}{ccccccc}
\hline \multirow{2}{*}{ Anion } & \multicolumn{2}{c}{ Single contact (A) } & & \multicolumn{2}{c}{ Multiple contacts (B) } \\
\cline { 2 - 3 } \cline { 5 - 6 } & Wash 1 & Wash 2 & & Wash 1 & Wash 2 \\
\hline $\mathrm{Cl}^{-}$ & $1.93 \times 10^{-2}$ & BDL & & BDL & BDL \\
$\mathrm{NO}_{2}^{-}$ & $\mathrm{BDL}$ & $\mathrm{BDL}$ & & BDL & BDL \\
$\mathrm{NO}_{3}{ }^{-}$ & 0.535 & 0.0211 & & 0.168 & 0.0262 \\
$\mathrm{SO}_{4}{ }^{2-}$ & $\mathrm{BDL}$ & $\mathrm{BDL}$ & & $\mathrm{BDL}$ & $\mathrm{BDL}$ \\
$\mathrm{PO}_{4}{ }^{3-}$ & $1.21 \times 10^{-3}$ & $\mathrm{BDL}$ & & $\mathrm{BDL}$ & $\mathrm{BDL}$ \\
$\mathrm{DBP}$ & 0.166 & $\mathrm{BDL}$ & & 0.298 & $\mathrm{BDL}$ \\
\hline
\end{tabular}

${ }^{a}$ Concentrations are in $\mathrm{mM}$. BDL refers to "below detection limits."

${ }^{b}$ The concentration of DBP corresponds to $35.0 \mathrm{ppm}$ after a single contact (A) and $60.4 \mathrm{ppm}$ after multiple contacts (B).

By ion chromatography, there is no evidence of DBP present in the aqueous phase of any other stage of the ESS protocol. Furthermore, water was found to be sufficient in removing the DBP from the loaded solvent. The amount removed by the water wash also correlates well to the amount removed by the first $10 \mathrm{mM} \mathrm{NaOH}$ wash, confirming the fact that the acid scrub and strip section is not capable of removing the extracted DBP from the solvent. Furthermore, the strips of the loaded solvents using DI water showed that there is, in fact, evidence of nitrite, sulfate, and nitrate extracted into the solvent, as well as DBP (Table 4.23). Nitrate results in the water wash correlate with the amount of nitrate scrubbed using $50 \mathrm{mM} \mathrm{HNO}_{3}$ for solvent (A). However, the water wash of solvent (B) did not correlate for nitrate anion as well and was somewhat lower in concentration. This

Table 4.23. Concentration of anions released in deionizedwater stripping of loaded solvents ${ }^{a}$

\begin{tabular}{lcc}
\hline Anion & Single contact (A) & Multiple contacts (B) \\
\hline $\mathrm{Cl}^{-}$ & $8.74 \times 10^{-2}$ & $7.94 \times 10^{-3}$ \\
$\mathrm{NO}_{2}^{-}$ & 0.249 & 0.239 \\
$\mathrm{NO}_{3}^{-}$ & 0.269 & 0.161 \\
$\mathrm{SO}_{4}^{2-}$ & $\mathrm{BDL}$ & $\mathrm{BDL}$ \\
$\mathrm{PO}_{4}^{3-}$ & $\mathrm{BDL}$ & $\mathrm{BDL}$ \\
$\mathrm{DBP}^{b}$ & 0.169 & 0.287 \\
\hline
\end{tabular}

${ }^{a}$ Concentrations are in $\mathrm{mM}$. BDL refers to "below detection limits."

${ }^{b}$ The concentration of DBP corresponds to $35.5 \mathrm{ppm}$ after a single contact (A) and $60.4 \mathrm{ppm}$ after multiple contacts (B). 
could be explained as incomplete stripping by water due to more complete loading of the solvent and increased DBP concentration in the loaded solvent. In addition, there appears to be a slight accumulation of nitrite ion in the strip phases of the multiple-contact solvent (B) that is not seen in the strips of the single-contact (A) solvent. This is only evident in the water washes. Further investigation to this effect may be needed.

\subsubsection{Distribution Behavior in Multi-Cycle and Multiple-Contact Batch Tests}

\subsubsection{Five-Cycle Test Results}

Additional analytical data were collected on the multi-cycle experiment presented in Chap. 3 to determine what minor components migrate from the simulant to the scrub and strip solutions. Previous work showed that the chief migrating species include $\mathrm{Cs}^{+}, \mathrm{K}^{+}$, and $\mathrm{Na}^{+}$cations. ${ }^{8,15}$ All results presented in the tables below are those of elements or species that were above the detection limits of the RMAL analytical methods (Table 4.2). For the analysis of the aqueous phases after extraction (simulant), only the results of the elements found in subsequent stages are given. It may be concluded that the elements present in the simulant, $\mathrm{Cr}, \mathrm{Cu}, \mathrm{Fe}, \mathrm{Mo}, \mathrm{Hg}, \mathrm{Ag}, \mathrm{Pb}, \mathrm{Pd}, \mathrm{Rh}, \mathrm{Ru}, \mathrm{Sn}, \mathrm{Zn}$, $\mathrm{Cl}$, and $\mathrm{F}$, do not significantly build up in the solvent on limited cycles, nor do they appear in the scrub or strip stages in significant concentrations. It might be added, however, that traces of $\mathrm{Al}$ at $\leq 10$ $\mu \mathrm{M}$ have consistently been detected in the scrub solution and first strip solution in earlier studies. 8,15 Also, traces of $\mathrm{Fe}, \mathrm{Hg}$, and $\mathrm{Pb}$ (but not $\mathrm{Cr}$ or $\mathrm{Si}$ ) were detected at $\leq 2 \mu \mathrm{M}$ in the scrub solution in the first-generation solvent system.

Table 4.24. Cesium and total phosphorus content (mol/L) of the organic phases over five cycles ${ }^{a}$

\begin{tabular}{|l|c|c|c|c|c|c|}
\hline & \multicolumn{2}{|c|}{ Cycle \#1 } & \multicolumn{2}{c|}{ Cycle \#3 } & \multicolumn{2}{c|}{ Cycle \#5 } \\
\hline & $\mathrm{Cs}^{+}$ & Total P & $\mathrm{Cs}^{+}$ & Total P & $\mathrm{Cs}^{+}$ & Total P \\
\hline Extraction & $5.07 \times 10^{-4}$ & $2.97 \times 10^{-4}$ & $5.17 \times 10^{-4}$ & $5.61 \times 10^{-4}$ & $4.76 \times 10^{-4}$ & $5.55 \times 10^{-4}$ \\
Scrub & $4.55 \times 10^{-4}$ & $3.87 \times 10^{-4}$ & $4.67 \times 10^{-4}$ & $4.39 \times 10^{-4}$ & $\left(1.94 \times 10^{-5}\right)^{b}$ & $5.77 \times 10^{-4}$ \\
Strip \#1 & & & & & & \\
Strip \#2 & & & & & & \\
Strip \#3 & & & & & & \\
Strip \#4 & $3.21 \times 10^{-6}$ & $3.21 \times 10^{-4}$ & $7.97 \times 10^{-6}$ & $4.52 \times 10^{-4}$ & $\left(5.23 \times 10^{-4}\right)^{b}$ & $3.32 \times 10^{-4}$ \\
\hline
\end{tabular}

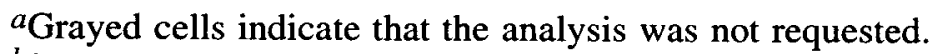

${ }^{b}$ Suspect data. The two points appear to have been switched.

It can be seen from Table 4.24 (as already observed on the $D_{\mathrm{Cs}}$ values) that the cesium concentration in the organic phase increases steadily. This also appears to be true for DBP, within some analytical uncertainty.

A few remarks can be made. From Tables 4.25 and 4.26 , the strip solution appears very clean, since only cesium and nitrate are detected. In addition, the increase in the $D_{\mathrm{Cs}}$ values observed in the scrub and strip solutions is manifested in both an increase in the organic phase and a decrease in the aqueous phase of the concentration of cesium upon cycling. The concentration of nitrate remains remarkably constant in the strip stages, leveling off at $1 \mathrm{mM}$ as expected. The only explanation for the increase of cesium concentration in the organic phase would then be an organophilic anion that is destroyed during the digestion of the organic phase prior to analyses.

Aqueous $\mathrm{pH}$ data are presented in Table 4.6 in Section 4.3.3.1. It may be concluded that little change in the $\mathrm{pH}$ profile of the aqueous scrub and strip steps occurs upon cycling. 
Table 4.25. Cation concentrations $(\mathrm{mol} / \mathrm{L})$ of the aqueous phases over five $\operatorname{cycles}^{a}$

\begin{tabular}{|l|c|c|c|c|c|c|c|c|c|}
\hline & \multicolumn{3}{|c|}{ Cycle \#1 } & \multicolumn{3}{c|}{ Cycle \#3 } & \multicolumn{3}{c|}{ Cycle \#5 } \\
\hline & $\mathrm{Na}^{+}$ & $\mathrm{K}^{+}$ & $\mathrm{Cs}^{+}$ & $\mathrm{Na}^{+}$ & $\mathrm{K}^{+}$ & $\mathrm{Cs}^{+}$ & $\mathrm{Na}^{+}$ & $\mathrm{K}^{+}$ & $\mathrm{Cs}^{+}$ \\
\hline Extraction & 5.35 & $2.50 \times 10^{-2}$ & $2.61 \times 10^{-5}$ & 6.30 & $2.47 \times 10^{-2}$ & $2.68 \times 10^{-5}$ & 6.48 & $2.51 \times 10^{-2}$ & $2.80 \times 10^{-5}$ \\
Scrub & $2.08 \times 10^{-2}$ & $1.12 \times 10^{-2}$ & $2.17 \times 10^{-4}$ & $2.26 \times 10^{-2}$ & $1.15 \times 10^{-2}$ & $1.89 \times 10^{-4}$ & $2.40 \times 10^{-2}$ & $1.16 \times 10^{-2}$ & $1.75 \times 10^{-4}$ \\
Strip \#1 & $\mathrm{BDL}$ & $4.32 \times 10^{-4}$ & $1.11 \times 10^{-3}$ & $\mathrm{BDL}$ & $2.38 \times 10^{-4}$ & $1.15 \times 10^{-3}$ & $\mathrm{BDL}$ & $5.50 \times 10^{-4}$ & $1.01 \times 10^{-3}$ \\
Strip \#2 & $\mathrm{BDL}$ & $\mathrm{BDL}$ & $6.25 \times 10^{-4}$ & $\mathrm{BDL}$ & $\mathrm{BDL}$ & $7.00 \times 10^{-4}$ & $\mathrm{BDL}$ & $\mathrm{BDL}$ & $8.05 \times 10^{-4}$ \\
Strip \#3 & & & & & \multicolumn{6}{|c|}{} & & & & & & & & \\
Strip \#4 & BDL & BDL & BDL & BDL & BDL & $7.42 \times 10^{-5}$ & $\mathrm{BDL}$ & $\mathrm{BDL}$ & $1.32 \times 10^{-4}$ \\
\hline
\end{tabular}

$a_{\mathrm{BDL}}$ denotes below detection limits. Grayed cells indicate that the analysis was not requested.

Table 4.26. Nitrate and nitrite concentrations $(\mathrm{mol} / \mathrm{L})$ of the aqueous phases over five cycles ${ }^{a}$

\begin{tabular}{|c|c|c|c|c|c|c|}
\hline & \multicolumn{2}{|c|}{ Cycle \#1 } & \multicolumn{2}{|c|}{ Cycle \#3 } & \multicolumn{2}{|c|}{ Cycle \#5 } \\
\hline & $\mathrm{NO}_{3}^{-}$ & $\mathrm{NO}_{2}^{-}$ & $\mathrm{NO}_{3}^{-}$ & $\mathrm{NO}_{2}^{-}$ & $\mathrm{NO}_{3}^{-}$ & $\mathrm{NO}_{2}^{-}$ \\
\hline Extraction & 2.00 & $5.48 \times 10^{-1}$ & 1.98 & $5.50 \times 10^{-1}$ & 2.02 & $5.39 \times 10^{-1}$ \\
\hline Scrub & 2. & $\mathrm{BD}^{a}$ & 10 & $7.65 \times 10^{-5}$ & $2.44 \times 10^{-4} c$ & $\mathrm{BDL}$ \\
\hline Strip \#1 & $3.48 \times 10^{-3}$ & BDL & $\left(1.24 \times 10^{-3}\right)^{b}$ & BDL & $3.73 \times 10^{-3}$ & BDL \\
\hline Strip \#2 & $1.87 \times 10^{-3}$ & $\mathrm{BDL}$ & & $\mathrm{BDL}$ & $1.84 \times 10^{-3}$ & $\mathrm{BDL}$ \\
\hline Strip \#3 & & & 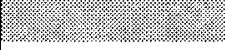 & $1:$ & 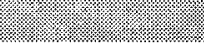 & 2 \\
\hline Strip \#4 & & & $\left(3.74 \times 10^{-3}\right)^{b}$ & BDL & $1.17 \times 10^{-3}$ & BDL \\
\hline
\end{tabular}

${ }^{a}$ BDL denotes below detection limits. Grayed cells indicate that the analysis was not requested.

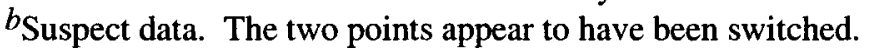

${ }^{c}$ Suspect data. The nitrate concentration should be at least $50 \mathrm{mM}$ (scrub solution).

\subsubsection{Electrospray Mass-Spectrometry Results on Multiple Contacts}

It was of interest to examine solvent samples in an ESS batch test by electrospray mass spectrometry (ES-MS) to gain qualitative information on the cationic and anionic species in the solvent. Since the solvent samples from the multiple-cycle experiment described above contained radioactive ${ }^{137} \mathrm{Cs}$ tracer, a simpler $\mathrm{E}_{5} \mathrm{SS}$ experiment was conducted to avoid contamination of the ESMS instrument. The following baseline solvent samples were analyzed by electro-spray mass spectrometry:

Pristine solvent

Solvent contacted five times with the full simulant at $\mathrm{O} / \mathrm{A}=0.33$

Solvent scrubbed with $50 \mathrm{mM} \mathrm{HNO}_{3}$ after extraction $\mathrm{O} / \mathrm{A}=5$

Solvent stripped with $1 \mathrm{mM} \mathrm{HNO}_{3}$ after extraction and scrub O/A = 5

The diluent for the solvent samples in the analysis was a $50 / 50$ mixture of acetonitrile/dichloromethane. The following results were obtained $(\mathrm{m} / z$ values in parentheses): 


\section{Pristine solvent}

Cation mode

Anion mode

predominantly protonated TOA (354.3)

nitrate (62.0), major

unknown (113.1)

sec-butylphenolate (148.8), very small

adduct of Cs-7SB with chloride or with $\mathrm{H}_{2} \mathrm{O}-\mathrm{OH}^{-}$(373.1)

adduct of Cs-7SB with anion of mass 44 (382.8)

adduct of Cs-7SB with nitrate (400.2)

\section{Solvent contacted five times with the full simulant}

Cation mode

cesium (132.8)

protonated TOA (354.3)

calixarene-potassium complex (1187.7, isotopic pattern)

calixarene-cesium complex (1281.7, isotopic pattern)

Anion mode nitrate (62.0)

dibutyl phosphate (209.0)

unknown triplet $(221,235,249)$

Cs-7SB (337.1)

adduct of Cs-7SB with nitrate (400.1)

adduct of Cs-7SB with dibutyl phosphate (547.2)

adduct of Cs-7SB with unknown triplet $(559.2,573.1,587.2)$

dimer Cs-7SB-Cs-7SB(675.2)

dimer adduct of Cs-7SB-Cs-7SB-nitrate (738.4)

dimer adduct Cs-7SB-Cs-7SB-dibutylphosphate (885.5)

trimer Cs-7SB-Cs-7SB-Cs-7SB (1013.6)

\section{Scrubbed solvent}

Cation mode

Anion mode

\section{Stripped solvent}

Cation mode

Anion mode protonated TOA (354.3)

adduct TOA-dibutylphosphoric acid (564.4)

adduct TOA-TOA-HNO ${ }_{3}-\mathrm{H}^{+}(770.8)$

series of unknowns $(917.8,929.6,943.8,957.8,973.0)$

calixarene-potassium complex (1187.7, isotopic pattern)

calixarene-cesium complex (1281.7, isotopic pattern)

nitrate (62.0)

dibutyl phosphate (209.0)

unknown quintuplet $(221.1,235.1,249.0,263.1,274.8)$

adduct of Cs-7SB with nitrate (400.1)

adduct of Cs-7SB with dibutyl phosphate (547.2)

adduct of Cs-7SB with unknown quintuplet $(559.2,573.2,587.2,601.2$, 613.2)

dimer adduct Cs-7SB-Cs-7SB-nitrate (738.3)

dimer adduct Cs-7SB-CS-7SB-dibutylphosphate (885.5)

protonated TOA (354.3)

adduct TOA-dibutylphosphoric acid (564.4)

adduct TOA-TOA-HNO $-\mathrm{H}^{+}(770.8)$

series of unknowns $(917.8,929.6,943.8,957.8,973.0,985.7)$

calixarene-cesium complex (1281.7, isotopic pattern)

nitrate (62.0)

dibutyl phosphate (209.0) 
unknown quartet $(220.9,235.1,249.2,263.1)$

adduct of Cs-7SB with nitrate (400.1)

adduct of Cs-7SB with dibutyl phosphate (547.2)

adduct of Cs-7SB with unknown quartet $(559.3,573.2,587.2,601.4)$

dimer adduct Cs-7SB-Cs-7SB-nitrate (738.3)

dimer adduct Cs-7SB-CS-7SB-dibutylphosphate (885.5)

The major conclusions that can be drawn are as follows. In the cation mode, the expected cesium and potassium complexes are present together with protonated TOA. The potassium complex drops out after scrubbing and stripping. In the anion mode, it is clear that dibutylphosphate gets extracted and remains in the organic phase through stripping. The modifier partially deprotonates in contact with the simulant, in agreement with hypothesized equilibria discussed above. The Cs-7SB modifier forms adducts with the nitrate, dibutylphosphate, and self anions, and these adducts can accept a second Cs-7SB molecule. Unknown species are observed in the cation and anion modes, and their role is not understood, except that the modifier is able to form adducts with these species. Similarities with earlier results obtained with the first-generation solvent and the simpler SRS\#2 simulant are noted, ${ }^{14}$ except for the presence of dibutylphosphate and unknowns in the present system. Further investigation is recommended.

\subsubsection{Dibutylphosphate Partitioning as Measured by NMR}

\subsubsection{Three-Contact NMR Experiment}

Baseline solvent was contacted thrice successively with the full simulant using an O/A ratio of 0.33 at $25^{\circ} \mathrm{C}$. ${ }^{31} \mathrm{P}$ NMR revealed a DBP concentration in the solvent of $0.41 \mathrm{mM}$. No other phosphorus peaks were observed. In a previous separate experiment, the solvent from a single contact with simulant was analyzed by ${ }^{31} \mathrm{P}$ NMR and found to contain only one phosphorus species; that material had the same chemical shift as DBP. No sign of TBP or MBP was observed in that sample.

An aliquot of solvent from the third contact was contacted with an equal volume of $0.5 \mathrm{M} \mathrm{NaOH}$. The ${ }^{31} \mathrm{P}$ spectrum of the solvent showed no observable DBP. A sample of the equilibrated $0.5 \mathrm{M}$ $\mathrm{NaOH}$ aqueous phase clearly showed the presence of a peak with the correct chemical shift for DBP.

\subsubsection{Partitioning of DBP Between the Solvent and the Simulant by NMR}

The full simulant contains $25 \mathrm{ppm}$ of DBP that is expected to partition into the solvent. Knowing exactly what the partition ratio is will allow calculation of how much DBP build-up should be expected in the organic phase.

Two experiments were conducted to determine the partition ratio of DBP. The first one involved one contact between pristine solvent and full simulant $(\mathrm{O} / \mathrm{A}=0.33)$. The source of DBP was solely from the simulant. A spectrum of the organic phase was obtained after contact, and the distribution was determined based on the fact that the simulant is supposed to contain $25 \mathrm{ppm}$ of DBP.

However, being unable to measure with precision the amount of DBP left in the simulant after contact because of the presence of paramagnetic species, we had to design another experiment where the initial amount of DBP in the solvent was known precisely. Therefore, some pristine solvent was spiked with $75 \mathrm{ppm}$ of DBP. A spectrum of that solution was obtained. This spiked organic phase was contacted with the simulant containing only salts and metals (no DBP). Results are shown in Table 4.27.

\subsubsection{Partitioning of DBP Between the Solvent and NaOH Solutions}

These experiments were designed to show the possibility of washing DBP from the solvent with sodium hydroxide. All sodium hydroxide solutions were freshly prepared. A spectrum of each 
spiked solvent solution was obtained before any contact. Because of the low partition values expected, the amount of DBP spiked in the solvent was increased and changed per experiment as shown in Table 4.27.

Table 4.27. Partitioning of DBP between baseline solvent and alkaline phases

\begin{tabular}{lcccc}
\hline & O/A & $\begin{array}{c}\text { Initial } \\
\text { concentration }\end{array}$ & $\begin{array}{c}\text { Concentration in the } \\
\text { organic phase after } \\
\text { contact }\end{array}$ & $\begin{array}{c}\text { Partition ratios } \\
P_{\text {DBP }}\end{array}$ \\
\hline Simulant & 0.33 & $25 \mathrm{ppm}^{a}$ & $32 \mathrm{ppm}$ & 2.23 \\
Simulant & 0.33 & $75 \mathrm{ppm}^{b}$ & $33 \mathrm{ppm}$ & 2.36 \\
$\mathrm{NaOH} \mathrm{0.01} \mathrm{M}$ & 1 & $500 \mathrm{ppm}$ & $1.5 \mathrm{ppm}$ & $3.0 \times 10^{-3}$ \\
$\mathrm{NaOH} \mathrm{0.03} \mathrm{M}$ & 1 & $500 \mathrm{ppm}$ & $1.1 \mathrm{ppm}$ & $2.1 \times 10^{-3}$ \\
$\mathrm{NaOH} \mathrm{0.1} \mathrm{M}$ & 1 & $3750 \mathrm{ppm}$ & $6.0 \mathrm{ppm}$ & $1.6 \times 10^{-3}$ \\
$\mathrm{NaOH} \mathrm{1} \mathrm{M}$ & 1 & $4600 \mathrm{ppm}$ & $46 \mathrm{ppm}$ & $1.0 \times 10^{-2}$ \\
\hline
\end{tabular}

${ }^{a}$ DBP is originally in the simulant. Use of pristine simulant.

${ }^{b}$ The solvent was spiked. The simulant used in this case was the salts + metals simulant.

From the reported results, it can be determined that the maximum amount of DBP present in the organic phase after multiple contacts with full simulant cannot exceed $57.5 \mathrm{ppm}(0.27 \mathrm{mM})$. It is remarkable that the two experiments involving the simulant gave such similar results. This is a good confirmation of the validity of the chosen method. In addition, the experiments involving an alkaline wash show the efficiency of such a wash for any concentrations of sodium concentrations from 10 $\mathrm{mM}$ to $1 \mathrm{M}$.

The results show that DBP will not build up to high levels in the solvent, as the waste raffinate will wash out $57 \%$ of the DBP in the solvent per solvent cycle. Any $\mathrm{NaOH}$ concentration in the range 0.01-1 $M$ would be efficient in washing SBP from the solvent. A single contact at $O / A=5$ with the least efficient solution, $1 \mathrm{M} \mathrm{NaOH}$, would remove $95 \%$, while $10 \mathrm{mM} \mathrm{NaOH}$ at $\mathrm{O} / \mathrm{A}=5$ would remove $98.5 \%$.

\subsubsection{Partitioning of 4-sec-Butylphenol}

The solvent breakdown component, 4-sec-butylphenol (SBP), an impurity in the modifier and a radiolytic degradation product, has been shown in Chap. 3 to have a deleterious effect on stripping performance. The SRTC team showed that this breakdown product grows steadily to approximately $3 \mathrm{mM}$ in the solvent when exposed to $16 \mathrm{Mrad}$ of external dosing. ${ }^{55}$ They also showed that it can be washed out of the solvent to the detection limit (ca. $0.04 \mathrm{mM}$ ) with three 1:1 washes with $0.5 \mathrm{M}$ $\mathrm{NaOH}$. The partitioning of SBP to several concentrations of $\mathrm{NaOH}$ and to the process solutions, including full simulant, $50 \mathrm{mM} \mathrm{HNO}$, and $1 \mathrm{mM} \mathrm{HNO}_{3}$, was examined. From the results reported in Table 4.28, it can be concluded that SBP does not partition efficiently to the simulant; salting-out apparently dominates. It was not possible to analyze the aqueous phase in the case of the simulant, and hence an approximate value of 50 is taken from the organic-phase analysis for the $\mathrm{O} / \mathrm{A}=0.1$ case as the partition ratio in the extraction step. There is also minimal transfer of SBP into $50 \mathrm{mM}$ $\mathrm{HNO}_{3}$ scrub solution $\left(P_{\mathrm{SBP}}=338 \pm 33\right)$ or $1 \mathrm{mM} \mathrm{HNO}_{3}$ strip solution $\left(P_{\mathrm{SBP}}=298 \pm 27\right)$. Equilibration with $\mathrm{NaOH}$ solutions effects a substantial removal of SBP from the solvent. The most effective $\mathrm{NaOH}$ concentrations lie in the range $0.1-1 \mathrm{M}$, the optimum being $0.3 \mathrm{M}\left(P_{\mathrm{SBP}}=0.16\right)$.

With regards to the fate of SBP in the flowsheet, the data show that the waste raffinate will not wash out the SBP rapidly (ca. 6\% each solvent cycle), and negligible SBP will be removed $(<0.1 \%)$ 
Table 4.28. Partitioning of 4-sec-butylphenol

\begin{tabular}{|c|c|c|c|c|c|}
\hline Aqueous phase & $\begin{array}{l}\text { Volume }^{a} \\
\text { (mL) }\end{array}$ & $\begin{array}{c}\text { Aqueous } \\
\text { SBP } \\
(\mathrm{mM})\end{array}$ & $\begin{array}{c}\text { Organic } \\
\text { SBP } \\
(\mathrm{mM}) \\
\end{array}$ & $\begin{array}{c}\text { Mass } \\
\text { balance } \\
(\%) \\
\end{array}$ & $\begin{array}{c}\text { Partition }^{b} \\
\text { ratio } \\
P_{\mathrm{SBP}} \\
\end{array}$ \\
\hline \multicolumn{6}{|l|}{ Full simulant } \\
\hline $\mathrm{O} / \mathrm{A}=1$ & 0 & $\mathrm{ND}^{c}$ & 0.93 & 98 & \\
\hline $\mathrm{O} / \mathrm{A}=0.33$ & 0 & ND & 0.99 & & \\
\hline $\mathrm{O} / \mathrm{A}=0.10$ & 0 & ND & 0.83 & & $\sim 50$ \\
\hline \multicolumn{6}{|l|}{$\mathrm{HNQ}_{3}$} \\
\hline $50 \mathrm{mM}$ & 89 & 0.0027 & 1.03 & 105 & 370 \\
\hline $50 \mathrm{mM}$ & 89.5 & 0.0033 & 0.99 & 102 & 305 \\
\hline $1.0 \mathrm{mM}$ & 92 & 0.0037 & 1.04 & 107 & 271 \\
\hline $1.0 \mathrm{mM}$ & 93 & 0.0031 & 1.02 & 105 & 325 \\
\hline \multicolumn{6}{|l|}{$\mathrm{NaOH}$} \\
\hline $3.0 \mathrm{M}$ & 5 & 0.67 & 0.87 & 98 & 1.30 \\
\hline $3.0 \mathrm{M}$ & 5 & 1.85 & 0.81 & 112 & 0.43 \\
\hline $1.0 \mathrm{M}$ & 4 & 3.6 & 0.53 & 111 & 0.14 \\
\hline $1.0 \mathrm{M}$ & 4 & 3.96 & 0.57 & 120 & 0.25 \\
\hline $0.30 \mathrm{M}$ & 5 & 3.16 & 0.54 & 107 & 0.17 \\
\hline $0.30 \mathrm{M}$ & 5 & 3.17 & 0.52 & 105 & 0.16 \\
\hline $0.10 \mathrm{M}$ & 5 & 1.94 & 0.85 & 117 & 0.44 \\
\hline $0.10 \mathrm{M}$ & 5 & 2.20 & 0.83 & 120 & 0.38 \\
\hline $0.03 \mathrm{M}$ & 5 & 1.28 & 0.88 & 109 & 0.69 \\
\hline $0.03 \mathrm{M}$ & 5 & 1.30 & 0.92 & 114 & 0.71 \\
\hline $0.01 \mathrm{M}$ & 5 & 0.47 & 0.91 & 98.8 & 1.94 \\
\hline $0.01 \mathrm{M}$ & 5 & 0.48 & 0.99 & 107 & 2.06 \\
\hline $0.003 \mathrm{M}$ & 5 & 0.13 & 1.01 & 103 & 7.77 \\
\hline $0.003 \mathrm{M}$ & 5 & 0.13 & 0.93 & 95.2 & 7.15 \\
\hline $0.001 \mathrm{M}$ & 5 & 0.02 & 1.00 & 100 & 50 \\
\hline $0.001 \mathrm{M}$ & 5 & 0.02 & 0.99 & 99.3 & 58 \\
\hline $0.30 \mathrm{M}$ aqueous ${ }^{d}$ & 4.5 & 0.77 & 0.12 & 115 & 0.15 \\
\hline $0.30 \mathrm{M}$ aqueous & 5 & 1.03 & 0.13 & 133 & 0.13 \\
\hline $0.30 \mathrm{M}$ control $^{e}$ & 5 & ND & 4.9 & 98.0 & $\mathrm{ND}$ \\
\hline $0.30 \mathrm{M}$ control $^{e}$ & 5 & ND & 4.9 & 98.0 & ND \\
\hline
\end{tabular}

${ }^{a}$ Volume of aqueous phase back-extracted to methylene chloride. Organic volume was $30 \mathrm{~mL}$ for each contact except $1.0 \mathrm{M} \mathrm{NaOH}$ in which the initial volume of organic phase was $25 \mathrm{~mL}$.

${ }^{b}$ Partition ratio is calculated by the equation $P_{\mathrm{DOA}}=[\mathrm{DOA}]_{\mathrm{org}} /[\mathrm{DOA}]_{\mathrm{aq}}$.

cNot determined.

$d_{\text {Aqueous }} 0.30 \mathrm{M} \mathrm{NaOH}$ was prepared with $1.36 \mathrm{mM} 4$-sec-butylphenol directly and contacted with $30 \mathrm{~mL}$ pristine solvent. The phases were then treated and analyzed as described above. The partitioning remained consistent with the reverse partition technique.

${ }^{e}$ Aqueous $0.30 \mathrm{M} \mathrm{NaOH}$ was prepared with $1.36 \mathrm{mM}$ 4-sec-butylphenol, acidified, and back-extracted into methylene chloride. The back-extraction was efficient to $97 \pm 1 \%$. 
per cycle in stripping. Note that SBP is not expected to be an important impurity in the solvent, as both chemical and radiolytic degradation of Cs-7SB is slow (see Chaps. 5 and 6). Neglecting loss of the SBP to the aqueous effluent streams, its net annual production is conservatively not expected to exceed $0.3 \mathrm{mM}$, and effects on cesium stripping are not felt until the SBP reaches $10 \mathrm{mM}$. Taking into account the net production rate and the rate of loss to the waste raffinate, only a trace steady-state level of SBP (estimated at $0.002 \mathrm{mM}$ ) can be expected. Thus, solvent washing to remove SBP is not expected to be needed. On the basis of the fact that the lower $\mathrm{NaOH}$ concentrations are more effective at removing organic acids that are more acidic than phenols (e.g., carboxylic acids; see below), a wash solution of $10 \mathrm{mM} \mathrm{NaOH}$ was recommended for the extended contactor tests. This wash solution will remove only $9 \%$ of the SBP per cycle, but this should be sufficient over the course of 2800 solvent cycles per year, given an estimated generation rate of $0.3 \mathrm{mM}$ or less. A wash with $0.3 \mathrm{M} \mathrm{NaOH}$ at $\mathrm{O} / \mathrm{A}=5$ would remove $56 \%$ of the SBP per cycle.

\subsubsection{Partitioning of Surfactant Anions}

Three surfactant anions were chosen in order to examine the partitioning of compounds having representative carboxylate and sulfate head groups and two alkyl chain lengths. The study was facilitated by the availability of a limited selection of ${ }^{14} \mathrm{C}$-labeled surfactants, allowing the use of beta liquid scintillation counting. The three surfactant anions employed in the study were laurate (12carbon carboxylate), stearate (18-carbon carboxylate), and dodecylsulfate. Although continuity with earlier studies would have made it preferable to use dodecylsulfonate, 8,14 the labeled analog was not available. Nevertheless, dodecylsulfuric acid is moderately strong acid and is judged to be a reasonable chemical analog to dodecylsulfonic acid for purposes of this study of general partitioning behavior. The distribution of the three lipophilic anions was investigated in three batch experiments at $25^{\circ} \mathrm{C}$.

The first experiment was carried out at $\mathrm{O} / \mathrm{A}=1$ and $25{ }^{\circ} \mathrm{C}$, using the baseline solvent and the nominal scrub $\left(0.05 \mathrm{M} \mathrm{HNO}_{3}\right)$, strip $\left(0.001 \mathrm{M} \mathrm{HNO}_{3}\right)$, and aqueous phases. Additionally, a single wash solution $(0.1 \mathrm{M} \mathrm{NaOH})$ was also tested. The results of the batch tests revealed that the surfactant anions distribute primarily to the solvent phase in contact with the acid solutions (see Table 4.29). Although this experiment involved insufficient activity in some of the aqueous phases to obtain a reliable partition ratio, it can be seen that the 18-carbon surfactant possesses far greater lipophilicity than the two 12-carbon surfactants. Approximately $0.1 \%$ of the stearic acid partitioned to the aqueous phase as a consequence of contact of the solvent with $0.1 \mathrm{M} \mathrm{NaOH}$. In the case of SDS, some partitioning to the aqueous phase was evident in the scrub, strip and wash contacts, namely $2.5 \%$ to the aqueous scrub phase, $1.1 \%$ to the aqueous strip phase, and $13.7 \%$ to the $\mathrm{NaOH}$ wash. In all three cases, the alkaline wash significantly lowered the surfactant partition ratio.

Table 4.29 Partition ratios for ${ }^{14} \mathrm{C}$-labeled surfactants in batch tests ${ }^{a}$

\begin{tabular}{cccc}
\hline Aqueous phase $^{b}$ & \multicolumn{3}{c}{$P$} \\
\cline { 2 - 4 } & Lauric acid & SDS & Stearic acid \\
\hline $50 \mathrm{mM} \mathrm{HNO}_{3}$ & $>3$ & 40.0 & $>4000$ \\
$1 \mathrm{mM} \mathrm{HNO}_{3}$ & $>3$ & 93.1 & $>4000$ \\
$0.1 \mathrm{M} \mathrm{NaOH}$ & 0.74 & 7.29 & 946 \\
\hline
\end{tabular}

anitial lauric acid, sodium dodecyl sulfate (SDS), and stearic acid concentrations were $2 \times 10^{-5} \mathrm{M} ;\left[\mathrm{Cs}^{+}\right]_{\mathrm{aq}, \text { init }}=0.1 \mathrm{mM}$.

${ }^{b}$ Contacts were made at $\mathrm{O} / \mathrm{A}=1, T=25^{\circ} \mathrm{C}$. 
ESS test results showed weak partitioning of all three surfactants to the process solutions (see Table 4.30). ESS results for the baseline solvent were obtained using the nominal O/A ratios of 0.33 for extraction and 5 for the scrub and three strip operations. Surfactants were added at $2 \times 10^{-5} \mathrm{M}$ relative to the simulant phase. Following the scrub and three strips, $99.66 \%$ of the SDS contained in the solvent phase following extraction remained. In the case of stearic acid, $99.81 \%$ remained in the solvent, while $99.86 \%$ of the lauric acid was still in the organic phase. Somewhat lower partition ratios for the extraction steps suggest that a limited degree of washing will take place by the waste

Table 4.30. The partitioning of ${ }^{14} \mathrm{C}$-labeled anionic surfactants in the ESS protocol ${ }^{a}$

\begin{tabular}{lccc}
\hline Process step & & $P$ & \\
& Lauric acid & SDS & Stearic acid \\
\hline Extraction & 549 & 139 & 51 \\
Scrub & 792 & 79.2 & 207 \\
Strip \#1 & 433 & 779 & 624 \\
Strip \#2 & 520 & 690 & 821 \\
Strip \#3 & 829 & 596 & 535 \\
\hline
\end{tabular}

${ }^{a} \mathrm{O} / \mathrm{A}=0.33$ (extraction), 5 (scrub and strips); $T=25^{\circ} \mathrm{C} ; 2 \times 10^{-5} \mathrm{M}$ initial surfactant relative to simulant phase; surfactants were ${ }^{14} \mathrm{C}$-labeled.

raffinate, limiting the steady-state buildup of the surfactants. The stearic acid result suggests that this surfactant could build up to $0.5 \mathrm{mM}$ in the solvent, and the other two surfactants would build up even more. Results such as these suggest the need to explore methods for removal of lipophilic anions from the solvent phase should they be present in the waste. The use of such methods as a caustic wash of the solvent or anion exchange was explored below.

Results for the effectiveness of a range of $\mathrm{NaOH}$ wash solutions for the removal of surfactants from the solvent are reported in Table 4.31 and are plotted in Fig. 4.14. The data indicate that a considerable benefit can be realized by washing the solvent with 1-10 $\mathrm{mM} \mathrm{NaOH}$ for the removal of SDS or lauric acid. The data show that these 12-carbon carboxylic acids are optimally washed out at about $3 \mathrm{mM} \mathrm{NaOH}$. The phenomenon that an optimum $\mathrm{NaOH}$ concentration is observed is explainable by opposing equilibrium processes. Increasing alkalinity results in increasing conversion of the carboxylic acids to the carboxylate sodium salts in the organic phase. A fairly alkaline $\mathrm{pH}$ $(>11)$ is needed to deprotonate an appreciable fraction of the carboxylic acid surfactant. On the other hand, increasing sodium concentration opposes the transfer of the sodium carboxylate salt to the aqueous phase by mass action. Hence, an optimum $\mathrm{NaOH}$ concentration is observed. Both carboxylic acids exhibit the minimum partition ratio at $3 \mathrm{mM} \mathrm{NaOH}$, but that for the larger 18-carbon stearic acid is too high for effective removal. By comparison, SDS exhibits no minimum, as it is presumably completely in the sodium salt form in the $\mathrm{NaOH}$ concentration range tested. Hence, its response is governed solely by the mass-action effect of aqueous sodium concentration. The use of a dilute $\mathrm{NaOH}$ wash will have a considerable utility for solvent cleanup in light of the fact that it has also been shown to be effective in the removal of 4-sec-butylphenol and dibutylphosphate. 
Table 4.31. Surfactant anion partitioning between baseline solvent and $\mathrm{NaOH}$ solutions. ${ }^{a}$

\begin{tabular}{cccc}
\hline & \multicolumn{3}{c}{$P$} \\
\cline { 2 - 4 }$[\mathrm{NaOH}], \mathrm{M}$ & Lauric acid & SDS & Stearic acid \\
\hline 1 & 8.68 & 10.2 & 4563 \\
0.3 & 1.59 & 7.12 & 2667 \\
0.1 & 0.593 & 5.41 & 1669 \\
0.03 & 0.243 & 3.24 & 763 \\
0.01 & 0.119 & 1.72 & 151 \\
0.003 & 0.072 & 0.687 & 96 \\
0.001 & 0.135 & 0.388 & 268 \\
\hline
\end{tabular}

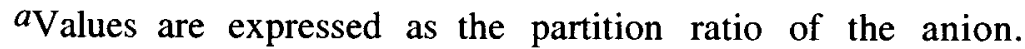
Surfactants were ${ }^{14} \mathrm{C}$-labeled; initial surfactant concentration was $1 \times 10^{-4} \mathrm{M} ; \mathrm{O} / \mathrm{A}=1, T=25^{\circ} \mathrm{C}$.

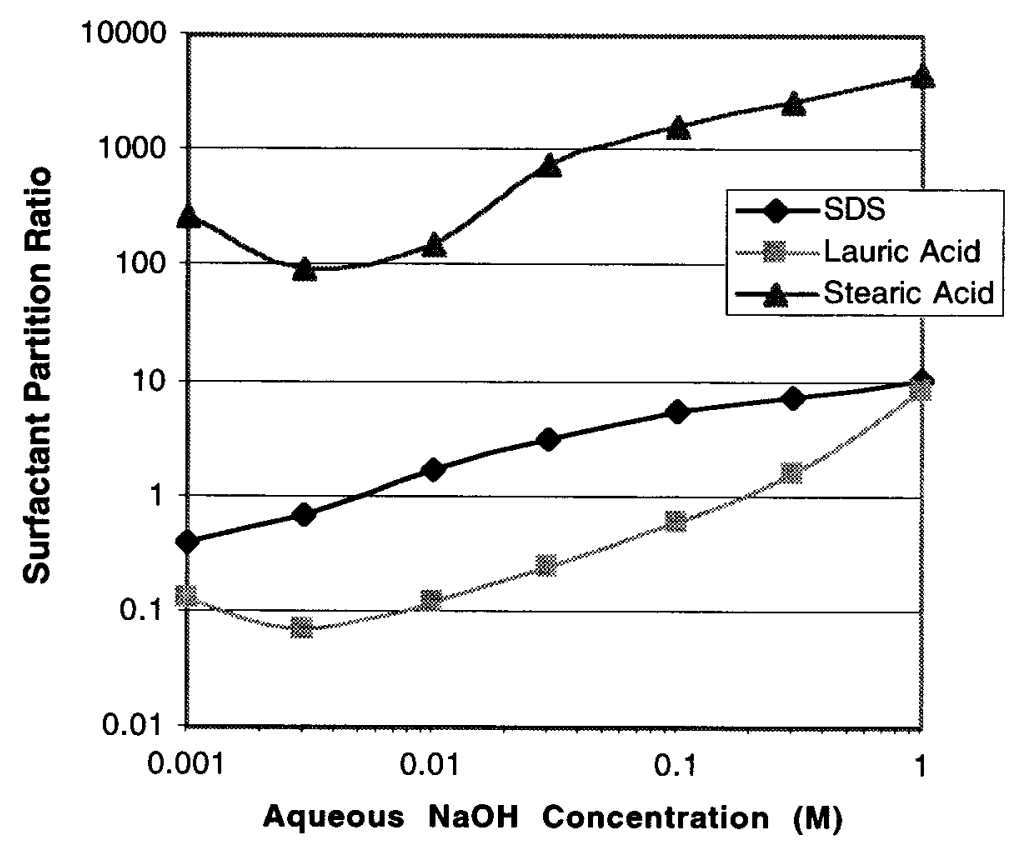

Fig. 4.14. Surfactant anion partitioning between baseline solvent and $\mathrm{NaOH}$ solutions at $25{ }^{\circ} \mathrm{C}$. 


\subsubsection{Partitioning of Amines}

The partitioning of dioctylamine (DOA), the major degradation product of trioctylamine, was examined. Questions deal with whether this fragment of TOA will report to the strip effluent or remain in the solvent. If the latter, then DOA can presumably perform the same function as TOA in promoting stripping and suppressing impurity and ion-pair dissociation. However, it is more reactive than TOA and could possibly react with nitrous acid or undergo other chemical or radiolytic processes that later need investigation. 66

As shown in Table 4.32, DOA does not partition to either the simulant or to $0.01 \mathrm{M} \mathrm{NaOH}$ wash solution. However, the acidic conditions significantly decrease the effective lipophilicity. Loss to the acid phases is greatest with $1 \mathrm{mM} \mathrm{HNO}_{3}$ strip solution, where $P_{\mathrm{DOA}}=4.7$. At $\mathrm{O} / \mathrm{A}=5$, loss of DOA to the strip effluent would be expected to be $4 \%$ per solvent cycle. Despite this relatively low loss rate, the cumulative effect of, say, one week of operation (62 solvent cycles), would be the loss of $92 \%$ of the DOA. On the other hand, the rate of production due to thermal or radiolytic degradation is at most $0.1 \mathrm{mM}$ DOA per year. Thus, the primary fate of the DOA in the process is expected to be simple washing-out by the strip solution.

As discussed in Chap. 2, trimethylamine (TMA) is a component of the waste, presumed to be a degradation product of anion-exchange resins. An ESS test was performed at $25{ }^{\circ} \mathrm{C}$ using ${ }^{14} \mathrm{C}$-labeled TMA tracer to facilitate analysis. The baseline solvent was employed together with the salts + metals simulant with ${ }^{133} \mathrm{Cs}$ added at its normal $0.14-\mathrm{mM}$ level and with TMA added at 0.169 $\mathrm{mM}$. The typical $\mathrm{O} / \mathrm{A}$ of 0.33 for extraction and 5 for the scrub and three strips was used. A second, full ESS protocol was carried out, in duplicate, for trimethylamine, but with di- $n$-butylphosphate (DBP) added at its typical simulant concentration of $0.119 \mathrm{mM}$. This second ESS series was suggested by the possibility that the DBP might enhance the TMA partitioning under the acidic conditions of scrubbing and stripping.

It may be seen in Table 4.33 that trimethylamine mildly partitions to the solvent during extraction. With an extraction factor $(3.96 \times 0.333)$ of 1.32 , the TMA will move with the solvent into the scrub stages in the flowsheet. There it will be protonated, partition into the aqueous phase, and return to the feed stage, since the scrub solution is added to the waste feed stage. Currently, the fate of the TMA is uncertain and must be investigated further. As it is very volatile, it will probably leak

Table 4.32. Partitioning of dioctylamine

\begin{tabular}{llcccc}
\hline \multicolumn{1}{c}{ Aqueous phase } & $\begin{array}{c}\text { Volume }^{a} \\
(\mathrm{~mL})\end{array}$ & $\begin{array}{c}\text { Aqueous } \\
{[\mathrm{DOA}]} \\
(\mathrm{mM})\end{array}$ & $\begin{array}{c}\text { Organic } \\
{[\mathrm{DOA}]} \\
(\mathrm{mM})\end{array}$ & $\begin{array}{c}\text { Mass } \\
\text { balance } \\
(\%)\end{array}$ & $\begin{array}{c}\text { Partition }^{b} \\
\text { ratio } \\
P_{\text {DOA }}\end{array}$ \\
\hline $\begin{array}{l}\text { Full simulant } \\
\text { Full simulant }\end{array}$ & 14.5 & 0.0093 & 52.5 & 105 & 5645 \\
$\quad \mathrm{HNO}_{3}$ & 15.0 & $<0.0008$ & 53.2 & 106 & 66500 \\
$100 \mathrm{mM}$ & & & & & \\
$100 \mathrm{mM}$ & 45 & 0.33 & 46.8 & 100 & 142 \\
$50 \mathrm{mM}$ & 44 & 0.36 & 46.4 & 99 & 128 \\
$50 \mathrm{mM}$ & 46 & 0.66 & 46.8 & 106 & 71 \\
$10 \mathrm{mM}$ & 48 & 0.79 & 49.9 & 115 & 63 \\
$10 \mathrm{mM}$ & 36 & 1.70 & 39.1 & 103 & 23 \\
$1.0 \mathrm{mM}$ & 37.5 & 1.70 & 42.4 & 110 & 25 \\
$1.0 \mathrm{mM}$ & 35 & 5.28 & 25.0 & 124 & 4.7 \\
$\mathrm{NaOH}$ & 37 & 5.44 & 25.4 & 131 & 4.7 \\
$10 \mathrm{mM}$ & & & & & 9000 \\
$10 \mathrm{mM}$ & 24 & 0.006 & 54 & 108 & 8600 \\
\hline
\end{tabular}

${ }^{a}$ Volume of aqueous phase back-extracted to methylene chloride. Organic volume was $5 \mathrm{~mL}$ for each contact.

${ }^{b}$ Partition ratio is calculated by the equation $P_{\mathrm{DOA}}=[\mathrm{DOA}]_{\mathrm{org}} /[\mathrm{DOA}]_{\mathrm{aq}}$. 
out of the system primarily via the purge gas used in the contactors. Its buildup in the solvent would be undesirable, as it may ultimately interact with the modifier, impairing its function, or it could lead to third-phase formation. As the extraction factor is rather low, a simple fix could be to reduce the solvent flow rate so that the O/A ratio in the extraction section is $<(1 / 3.96)$ or $<0.252$.

\subsubsection{Ion Exchange of Organic Anions}

The purpose of this study was to investigate the feasibility of removing lipophilic organic anions from the solvent using anion-exchange resins. Various lipophilic anions might enter the solvent during the CSSX process and acidic radiolysis-induced degradation products can alter the distribution behavior of the alkali metal ions, especially in the strip stages, thus necessitating solvent cleanup. As was observed above, simple washing with $\mathrm{NaOH}$ is unable to completely remove surfactant anions of very high lipophilicity and acidity from the solvent. Hence, it was deemed prudent to consider alternative cleanup methods in the unexpected event that surfactant buildup might occur.

Table 4.33. Trimethylamine partitioning in ESS contacting $a$

\begin{tabular}{lccc}
\hline ESS step & O/A & $\begin{array}{c}\text { Partition ratio } \\
\text { TMA only }\end{array}$ & $\begin{array}{c}\text { Partition ratio } \\
\text { TMA + DBP }\end{array}$ \\
\hline Extraction & 0.33 & 3.96 & 4.26 \\
Scrub & 5 & 0.000184 & 0.000189 \\
Strip 1 & 5 & 0.144 & 0.191 \\
Strip 2 & 5 & 0.870 & 0.973 \\
Strip 3 & 5 & 2.43 & 3.64 \\
\hline
\end{tabular}

${ }^{a}$ TMA concentration added to the simulant was $0.169 \mathrm{mM}$; DBP was added to $0.119 \mathrm{mM}$ when present.

Anion-exchange methods have some distinct advantages, since the affinity of the resin for a particular surfactant solute can be controlled by varying the nature of the tertiary amine functional group and the exchangeable counteranion. The acidity of the conjugate acid of the surfactant anion is a primary factor which affects the state of the lipophilic anion in the solvent and its partition behavior. Accordingly, two surfactant anions were selected for this study, representing "extreme" classes of strong and weak acidity, namely sodium dodecylsulfate (SDS) and stearic acid (SA), respectively. Two solutions of pristine washed solvent containing SDS or SA were prepared and put through one ESS cycle. In contact with highly alkaline simulant solution, both acids are effectively deprotonated and serve as cation-exchangers in taking up cesium and other alkali metal ions into the solvent. Weakly acidic $(\mathrm{pH} \geq 3)$ scrub and strip solutions are expected to fully protonate the weak stearate anion and leave the strong SDS anion essentially deprotonated. This was confirmed by performing a cesium distribution study using an ESS cycle employing solvent solutions containing SDS and SA (Section 3.3.11). The $\mathrm{Cs}^{+}$ion was effectively stripped into the aqueous phase from the solvent containing SA, indicating the protonation of the SA by the lack of its capability to act as a lipophilic counterion and retain $\mathrm{Cs}^{+}$in the solvent. In contrast, corresponding $D_{\mathrm{Cs}}$ values were high for the solvent containing SDS, demonstrating that this anion likely remains deprotonated after contacts with scrub and strip solutions. Hypothetically, if the chosen resin possesses trialkylammonium groups compatible with SDS, the SDS anion could exchange with any resin anion; the efficiency of this process for the particular resin is determined by the affinity of the resin anion to the solvent phase. Thus, the task is thought to be two-fold, representing a search for both the most efficient trialkylammonium functional group and the optimal counteranion associated with it. Cs $7 \mathrm{SB}$ modifier, the major component of the solvent, provides the solvation environment available for the anionic species in the solvent phase. The weakly acidic modifier possessing an -OH group is an effective hydrogen-bond donor and is expected to effectively solvate small charge-dense anions 
which can readily participate in hydrogen bonding. For the SA anion to be available for the exchange process, its conjugate acid must be deprotonated first. The hydroxide anion is the most efficient driver in this respect, and resins in the hydroxide form are thought to be the best choice. Accordingly, the hydroxide and chloride forms of the resins were selected for this study. The choice of resins applied in this experiment represents an attempt to investigate an effect of variation of the polymeric backbone and anion exchange functional group, namely the tertiary amine group, of the resin on its affinity to the surfactant anions (Table 4.34). Moreover, commercial and laboratorymade resins differ widely in the mesh size and nature and degree of cross-linking. Both are important factors participating in establishing of the selectivity of the resin. Anion-exchange resins employed in this study are characterized in Table 4.34 . The $K_{\mathrm{d}}$ values quantitatively characterize the ability of $1 \mathrm{~g}$ of dry resin to sorb organic anions from the solvent and thus can serve for the comparison of the affinity of the different resins to the particular solute.

Obtained distribution ratios $\left(K_{\mathrm{d}}\right)$ are listed in Tables 4.35 and 4.36. The most general conclusion is that performance of the resins in the hydroxide form is superior with respect to that of resins in the chloride form. As expected for SA, all resins in the chloride form exhibit similarly low $K_{\mathrm{d}}$ values that are greatly increased when corresponding resins in the hydroxide form were used. Neutralization of the protons released by SA and water formation drive the anion-exchange process. For SDS, resins in the hydroxide form are also more efficient than those in the chloride form, albeit less markedly so than with SA, which is taken as the reflection of the better compatibility of the solvation environment in the organic phase with the hydroxide than with the chloride anion. The former anion forms stronger hydrogen bonds with the modifier in the organic phase and thus favors the anion exchange reaction.

The obtained results demonstrate excellent performance of D3696 $\left[-\mathrm{N}(\mathrm{Et})_{3} /-\mathrm{N}(\mathrm{Hex})_{3}\right]$, SR-6 $\left[-\mathrm{N}(\mathrm{Bu})_{3}\right]$, UTK $\left[-\mathrm{N}(\mathrm{Bu})_{3}\right]$, and UTK $\left[-\mathrm{N}(\mathrm{Me})_{3}\right]$ resins in the hydroxide form, which showed high $K_{\mathrm{d}}$ values and respectively remove $99.6,99.6,99.4$, and $99.3 \%$ of SDS and 99.3, 99.1, 94.9, and $99.2 \%$ of SA after equilibration for 22 hours (Table 4.35). The same resins in the chloride form were also the most efficient toward SDS (Table 4.36). D3696 and SR-6 resins showed very fast kinetics, removing over $99.3 \%$ of SDS in an hour. Bulky lipophilic organic anions such as SDS and SA are thought to preferentially associate with the most lipophilic cations, and the resins with large tributylammonium groups demonstrated high affinity toward SDS and SA. The fact that the even more lipophilic trihexylammonium resin was observed to be inferior in comparison with the tributylammonium one is presumably attributable to the slow kinetics due to the large steric

Table 4.34. Description of resins used in anion-exchange experiments

\begin{tabular}{|c|c|c|c|c|}
\hline Resin & $\begin{array}{l}\text { Anion-exchange } \\
\text { group }\end{array}$ & Backbone & $\begin{array}{l}\text { Total anion-exchange } \\
\text { capacity, meq/g }\end{array}$ & $\begin{array}{l}\text { Mesh size } \\
\text { (US standard) }\end{array}$ \\
\hline $\begin{array}{c}\text { Sybron Ionac SR-6 } \\
\text { Batch no. } 6475\end{array}$ & $-\mathrm{N}(\text { Butyl })_{3}$ & Polystyrene & $1.8^{a}$ & $16-50^{a}$ \\
\hline $\begin{array}{c}\text { Purolite D3696-3 } \\
\text { SP: } 23 / 10 / \mathrm{A}\end{array}$ & $\begin{array}{l}-\mathrm{N}(\text { Ethyl })_{3} \\
-\mathrm{N}(\text { Hexyl })_{3}\end{array}$ & Polystyrene & $1.6^{b}$ & $25-40^{b}$ \\
\hline Amberlite IRA-900 & $-\mathrm{N}(\text { Methyl })_{3}$ & Polystyrene & $3.6^{a}$ & $16-50^{a}$ \\
\hline $\begin{array}{l}\text { Purolite A-850 } \\
\text { Lot no. } 139 / 98\end{array}$ & $-\mathrm{N}(\text { Methyl })_{3}$ & Acrylic & $3.90^{a}$ & $16-50^{a}$ \\
\hline UTK CS $_{2}-04-162$ & $-\mathrm{N}(\text { Methyl })_{3}$ & Polystyrene & $3.79^{c}$ & \\
\hline UTK $\mathrm{CS}_{2}-04-187$ & $-\mathrm{N}(\mathrm{Hexyl})_{3}$ & Polystyrene & $1.14^{c}$ & \\
\hline UTK CS ${ }_{2}-04-188$ & $\mathrm{~N}\left(\mathrm{CH}_{2} \mathrm{CH}_{2} \mathrm{OH}\right)_{3}$ & Polystyrene & $3.09^{c}$ & \\
\hline UTK CS $2-04-198$ & $-\mathrm{N}(\text { Butyl })_{3}$ & Polystyrene & $1.96^{c}$ & \\
\hline UTK $\mathrm{CS}_{3}-03-220$ & $-\mathrm{N}(\text { Butyl })_{3}$ & Acrylic & & \\
\hline
\end{tabular}

aTaken from ref. 66.

${ }^{b}$ Taken from company-provided technical literature.

'Taken from UTK-provided technical literature. 
hindrance of the cationic site and thus large spatial separation of the positive and negative charges. It is unlikely that an equilibrium state was reached for this particular resin in the experimental timeframe. The same effect has been observed previously with the pertechnetate anion; it has been determined that for trihexylammonium resin 24 hours represents only $10 \%$ of the time required to reach equilibrium. 65 Excellent performance of the bifunctional triethyl/trihexylammonium resin is attributed to the high affinity of the large organic anions for the lipophilic trihexylammonium groups and to the fast kinetics associated with the small triethylammonium groups. Another important aspect is that the total anion-exchange capacity of the resin, and thus the number of active sites per one gram of dry resin, decreases as the size of the trialkylammonium group increases (Table 4.35). Accordingly the obtained $K_{\mathrm{d}}$ values need to be normalized for resin capacity in order to characterize the selectivity of the particular anion-exchange group for the specific solute. This consideration explains the high $K_{\mathrm{d}}$ values observed for the UTK $\left[-\mathrm{N}(\mathrm{Me})_{3}\right]$ resin in the hydroxide form, which possesses a large number of active sites per one gram of the dry resin.

UTK $\mathrm{CS}_{2}$-04-188 resin containing $-\mathrm{N}\left(\mathrm{CH}_{2} \mathrm{CH}_{2} \mathrm{OH}\right)_{3}$ functional groups possessed poor ability to remove SDS and SA from the solvent. It is considered that the presence of hydrogen-bond donating $-\mathrm{CH}_{2} \mathrm{CH}_{2} \mathrm{OH}$ groups in the resin provides a favorable solvation environment for $\mathrm{Cl}^{-}$or $\mathrm{OH}^{-}$. As a result, more energy is needed to partition these counteranions from the resin into the solvent phase, and the efficiency of the overall anion exchange process is reduced.

Comparison of the $K_{\mathrm{d}}$ values obtained using SDS and (1) polystyrene trimethylammonium resin Amberlite IRA-900 in the chloride form and its acrylic analog Purolite A-850 and (2) polystyrene tributylammonium resin UTK CS2-04-198 in the hydroxide form and its acrylic analog UTK CS3-

03-220 reveals an effect of the polymeric backbone on the resin performance. In both cases, polystyrene resins exhibited superior behavior, which is taken as a reflection of the chemical nature of the polymeric support. The polystyrene backbone is chemically inert and does not interact with the active sites of the resin. As shown above, the Purolite A-850 polyacrylic backbone contains an ester functional group, and the UTK CS3-03-220 material is additionally functionalized with an -OH group attached to the propyl linkage between ester and trialkylamine sites.

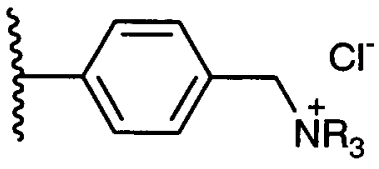

Polystyrene resin

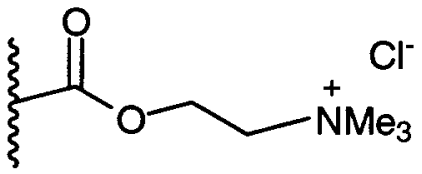

Purolite A-850<smiles></smiles>

UTK CS $2-03-220$

In the organic environment the effective positive charge of the quaternary ammonium group could be potentially reduced by its interaction with the electron-rich ester functional group. When hydroxide counteranion is used, it may be sufficiently retained in the resin by its interaction with the acidic proton of the UTK $\mathrm{CS}_{3}-03-220$ resin and thus less readily partition into the solvent phase. The other unfavorable factor is the gel-like morphology of the acrylic backbone that reduces the total active surface of the resin available for the anion-exchange reaction. Both polyacrylic resins were shown to be ineffective for the removal of the SDS and SA from the solvent. 


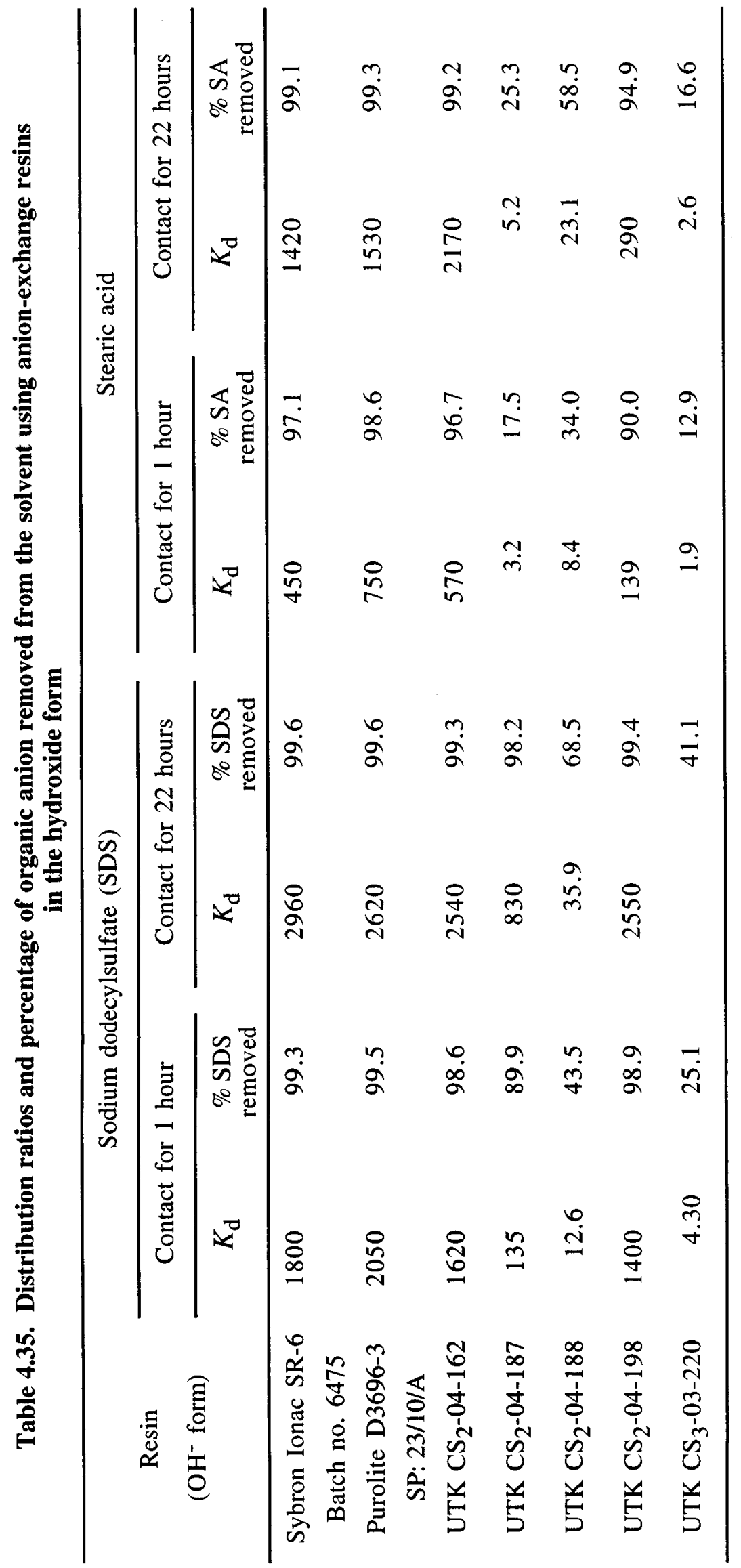




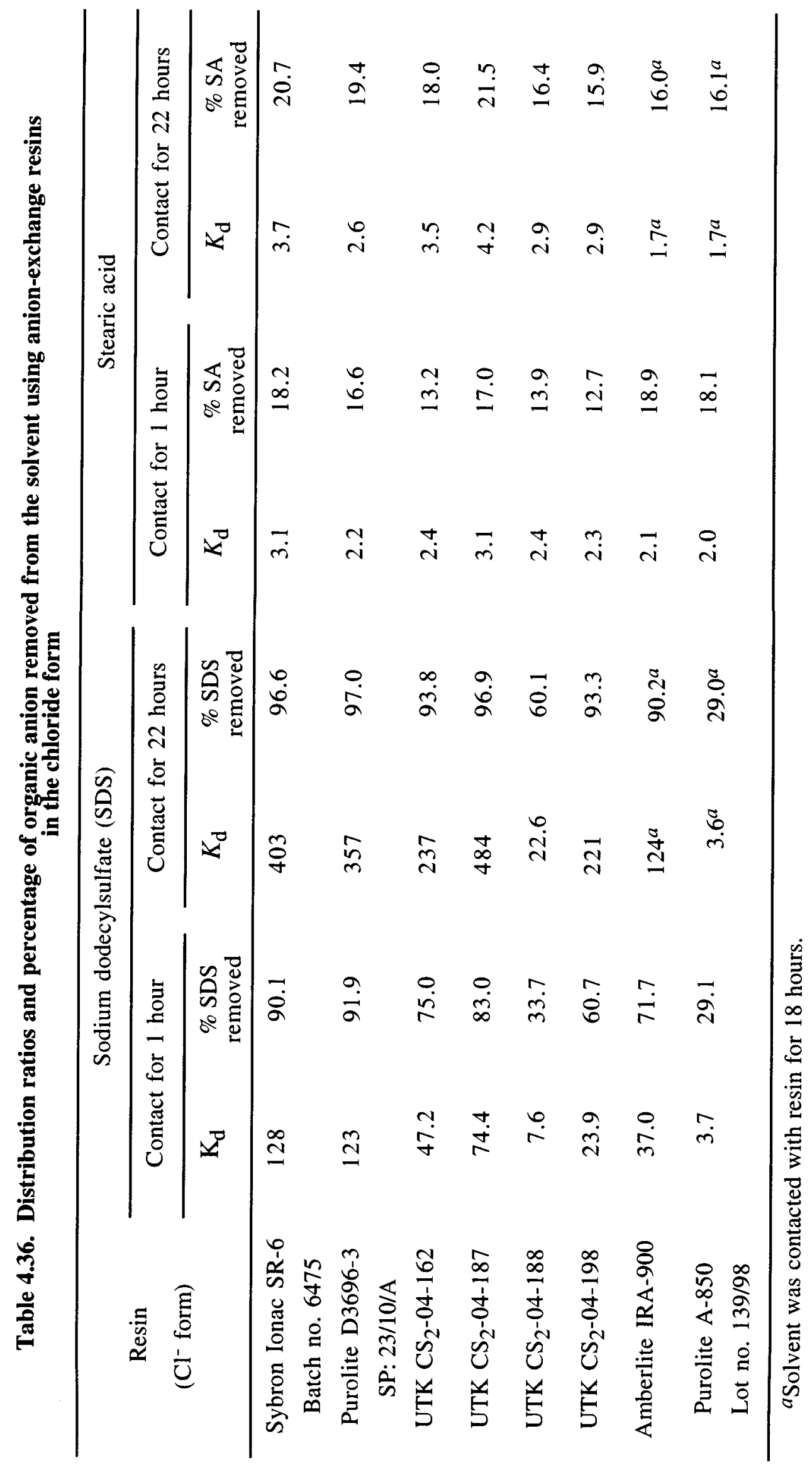




\subsubsection{Partitioning of Tributylphosphate and 1-Butanol}

Neutral organic compounds known to be present in the waste include tributylphosphate (TBP) and 1-butanol $(\mathrm{BuOH})$. Such compounds could possibly lead to problems if they do not readily wash out into any of the flowsheet aqueous phases. At sufficiently high concentrations in the solvent, TBP would be expected to interact with the Cs-7SB modifier, potentially compromising the ability of the modifier to perform its function, and lowered $D_{\mathrm{Cs}}$ values were indeed seen in Chap. 3 . Other potential problems include third-phase formation or impaired selectivity. A mitigating factor is that the more lipophilic the organic compound is, the less soluble it will likely be in the waste and the lower concentration it will be in the feed. Hence, it is important to have values of partition ratios for such compounds to predict their steady-state level in the solvent.

Straightforward batch experiments at $25{ }^{\circ} \mathrm{C}$ were performed using ${ }^{14} \mathrm{C}$ tracers to follow the TBP and $\mathrm{BuOH}$ partitioning. The baseline solvent was employed together with the salts + metals simulant with ${ }^{133} \mathrm{Cs}$ added to its normal $0.14 \mathrm{mM}$. The organic compounds were studied individually. Rather than employ an ESS series, the experiment entailed contacting the solvent directly with fresh waste simulant, scrub solution, or strip solution.

Table 4.37. Tributylphosphate partitioning in batch systems ${ }^{a}$

\begin{tabular}{lcc}
\hline \multicolumn{1}{c}{ Aqueous phase } & O/A & Partition ratio \\
\hline Simulant & 0.33 & 1880 \\
$50 \mathrm{mM} \mathrm{HNO}_{3}$ & 1 & 1180 \\
$1 \mathrm{mM} \mathrm{HNO}_{3}$ & 1 & 1660 \\
\hline
\end{tabular}

$a^{a}$ Tributylphosphate concentration added to the baseline solvent was $2.0 \times 10^{-5} \mathrm{M}$.

Table 4.38. 1-Butanol partitioning in batch systems ${ }^{a}$

\begin{tabular}{lcc}
\hline \multicolumn{1}{c}{ Aqueous phase } & O/A & Partition ratio \\
\hline Simulant & 0.33 & 7.46 \\
$50 \mathrm{mM} \mathrm{HNO}_{3}$ & 5 & 0.604 \\
$1 \mathrm{mM} \mathrm{HNO}_{3}$ & 5 & 0.600 \\
\hline
\end{tabular}

${ }^{a} 1$-Butanol concentration added to the baseline solvent was 8.1 $\times 10^{-5} \mathrm{M}$.

It may be seen in Table 4.37 that TBP partitions strongly to the solvent, but the partition ratios indicate that it could only build up in the solvent to $3-4 \mathrm{mM}$ at steady state, as the raffinate exiting stage \#1 will start to wash out the TBP when it reaches this level in the solvent. As seen in Chap. 3, this level is too low to have any effect on extraction, scrubbing, or stripping. 1-Butanol (Table 4.38) partitions mildly to the solvent, but the partition ratio is not high enough to allow it to build up to more than $0.2 \mathrm{mM}$, again too low to have any effect. 


\subsection{CONCLUSIONS}

Experiments described in this chapter probed in detail the distribution behavior of many system components, including the major solvent components, solvent degradation products, minor organic components in the waste, and major and minor inorganic components in the waste. No particular risks regarding process function were identified, as supported by the successful extended contactor tests of CSSX that have been carried out at ANL on the full simulant ${ }^{67}$ and at the SRTC on simulants and real waste. ${ }^{68}$ However, a number of questions remain for future investigation toward process improvement, better predictability, and deeper understanding.

Regarding terminology, the distinction between distribution and partitioning is arbitrary here. Both have the same operational definition of organic-phase molarity of a solute divided by its aqueous-phase molarity. Although partitioning has a strict meaning in the Nernstian sense, ${ }^{63}$ we use the term here to refer to the distribution of an organic component. It is best applied to a neutral component, where the partition ratio equates to an equilibrium constant and is likely to be constant over a reasonable range of conditions. However, the term partitioning is arbitrarily extended to all organic species, including organic anions and amines, whose partition ratios will be dependent on the particular conditions of each individual system.

The partitioning of solvent components to the aqueous phase is considered negligible. In terms of a process in which the solvent will be recycled an estimated 2800 times annually, 41,42 the high partition ratios found for the solvent components indicate that minor solvent replacement in the absence of solvent recovery will likely be required. Partition ratios $(P)$ for BOBCalixC6, Cs-7SB, and TOA in the baseline solvent in contact with the process aqueous phases at $25{ }^{\circ} \mathrm{C}$ are all very high, essentially too high for accurate measurement. The partition ratios are highest for the full simulant: $P_{\mathrm{BOBCalix} 66}>12,500, P_{\mathrm{Cs}-7 \mathrm{SB}}>50,000$, and $P_{\mathrm{TOA}}>30,000$. For scrub and strip solutions, the bounds are: $P_{\mathrm{BOBCalixC6}}>12,500, P_{\mathrm{Cs}-7 \mathrm{SB}}>8000$, and $P_{\mathrm{TOA}}>6000$. The highest aqueous flow is that of the waste. Using $P_{\mathrm{BOBCalixC} 6}=12,500$ as the most conservative basis for extractant loss, 2800 solvent cycles at $\mathrm{O} / \mathrm{A}=0.33$ implies a cumulative loss of $49 \%$ of the BOBCalixC6 annually. This replacement meets the original goal of one solvent replacement per year. However, estimates ${ }^{27}$ of the true lipophilicity of BOBCalixC6 imply a $P_{\mathrm{BOBCalix} C 6}$ value many orders of magnitude higher than the experimental lower bound of 12,500. Thus, any need for solvent recovery or replacement is expected to arise predominantly due to entrainment losses, which can be dealt with by mechanical (e.g., centrifugation, sedimentation/decantation, etc.) or chemical (e.g., washing the aqueous phase with diluent) means.

Given the use of solvent cleanup based on $\mathrm{NaOH}$ washing, the presence of lipophilic anionic species in the solvent as extracted from the waste or as formed upon degradation of the solvent components represents low risk. However, ample evidence was gathered in this chapter to show that lipophilic anions at sufficient concentration in the solvent overwhelm the tolerance provided by the TOA suppressor and impair stripping. The question then amounts to the relative rate of buildup of lipophilic anions in the solvent as governed by their concentration in the feed or their formation (in the case of degradation products) vs. how fast they will be removed from the system as governed by their partition ratios with respect to process aqueous solutions and wash solutions.

A systematic examination of the individual organic components of the full simulant revealed dibutylphosphate (DBP) to be the only organic waste component having a deleterious effect on stripping (Chap. 3). Its partition ratio $P_{\mathrm{DBP}}$ was found here to be 2.3 between the baseline solvent and full simulant at $25^{\circ} \mathrm{C}$, which at $\mathrm{O} / \mathrm{A}=0.33$ implies that only a modest buildup to $0.27 \mathrm{mM}$ in the solvent is possible, whence a steady washing by the waste raffinate will occur. Although this level of DBP is just enough to start to impair the function of the TOA, any $\mathrm{NaOH}$ concentration from $10 \mathrm{mM}$ to $1 \mathrm{M}$ efficiently washes out the $\mathrm{DBP}\left(P_{\mathrm{DBP}} \leq 0.01\right)$. A single contact at $\mathrm{O} / \mathrm{A}=5$ with the least efficient wash solution in this range, $1 \mathrm{M} \mathrm{NaOH}$, would remove $95 \%$ of the DBP, while $10 \mathrm{mM} \mathrm{NaOH}$ at $\mathrm{O} / \mathrm{A}=5$ would remove $98.5 \%$. It should be pointed out that the fact that no problem with solvent decontamination was encountered in the proof-of-concept test at ANL with four solvent cycles without a solvent wash can be attributed to the washing effect of the waste raffinate. 44

Results from Chaps. 5 and 6 show that the Cs-7SB degradation product 4-sec-butylphenol (SBP) is one of the two major solvent degradation products, the other being the TOA breakdown product, 
dioctylamine (DOA). At concentrations of greater than $3 \mathrm{mM}$, SBP in the solvent begins to interfere with stripping (Chap. 3). Since conservative estimates set the annual production of SBP in the solvent at less than $0.3 \mathrm{mM}$, though, solvent washing to remove SBP is not expected to be needed. With regards to the fate of SBP in the flowsheet, negligible SBP will be removed $(<0.1 \%)$ per cycle in stripping, but the data show that the waste raffinate washes out the SBP weakly (est. 6\% per solvent cycle, $\left.P_{\mathrm{SBP}} \sim 50\right)$. This weak washing effect would be sufficient to keep the SBP at trace levels in the solvent. For example, over the course of one week (62 solvent cycles), $97 \%$ of a given concentration of SBP would be removed from the solvent. The estimated steady-state concentration is $0.002 \mathrm{mM}$. Washing the solvent with $\mathrm{NaOH}$ solutions is more efficient. A wash with $0.3 \mathrm{M} \mathrm{NaOH}$ at $\mathrm{O} / \mathrm{A}=5$ will remove $56 \%$ of the SBP per cycle $\left(P_{\mathrm{SBP}}=0.16\right)$. At $0.01 \mathrm{M} \mathrm{NaOH}$, washing at $\mathrm{O} / \mathrm{A}=5$ would remove $9 \%$ per cycle $\left(P_{\mathrm{SBP}}=2.0\right)$.

On the basis of the fact that the lower $\mathrm{NaOH}$ concentrations are more effective at removing organic acids that are more acidic than phenols (e.g., alkyl carboxylic, sulfonic, and sulfuric acids), a wash solution of $10 \mathrm{mM} \mathrm{NaOH}$ is recommended for the extended contactor tests (Chap. 8). Although surfactant anions have not been specifically identified as waste components, tests in Chap. 3 and previous CSSX development ${ }^{8,14}$ results showed that they have significant potential to impair stripping performance. TOA negates their effect at trace levels, but it is important to understand the partitioning of typical surfactant anions so that their buildup in the solvent could be predicted and washing methods optimized should this problem be later encountered. It was found here that the 12-carbon surfactant anions dodeconoate (laurate) and dodecylsulfate and the 18-carbon surfactant octadecanoate (stearate) partition strongly to the solvent in contact with the simulant, scrub, or strip solutions. By mass-action, partitioning of the sodium salts of the surfactants is driven by the sodium concentration in the aqueous phase, making it desirable to minimize the aqueous sodium concentration. On the other hand, sufficient alkalinity is needed to deprotonate the acid forms of the surfactants. For the carboxylate surfactants, the optimum $\mathrm{NaOH}$ concentration for washing was found to be $3 \mathrm{mM}\left(P_{\text {laurate }}=0.072, P_{\text {stearate }}=96\right)$. For dodecylsulfate (and presumably related sulfonate surfactants), the optimum is less than $0.001 \mathrm{M}$. As coalescence problems increase with decreasing $\mathrm{NaOH}$ concentration, $0.01 \mathrm{M} \mathrm{NaOH}$ was accepted as the best compromise for washing. At this concentration of $\mathrm{NaOH}$ at $25^{\circ} \mathrm{C}, P_{\text {laurate }}=0.12, P_{\text {stearate }}=150$, and $P_{\text {dodecylsulfate }}=1.7$. Using a $0.01 \mathrm{M} \mathrm{NaOH}$ wash at $\mathrm{O} / \mathrm{A}=5$ and $25{ }^{\circ} \mathrm{C}$, these $P$ values correspond respectively to $62 \%, 0.13 \%$, and $10 \%$ removal of these surfactant anions. Assuming the feed has $1 \times 10^{-5} \mathrm{M}$ of any of these anions, estimated steady-state concentrations in the solvent correspond respectively to $4.8 \times 10^{-5} \mathrm{M}, 0.023 \mathrm{M}$, and $3.0 \times 10^{-4} \mathrm{M}$. Such a buildup could be tolerated in the case of the 12-carbon surfactants, but not the 18-carbon surfactant. For this reason, it was judged prudent to demonstrate an effective solventcleanup alternative, and resin anion-exchange was shown to offer the requisite capability. Resins in the hydroxide form contacted directly with solvent spiked with stearic acid and sodium dodecylsulfate were particularly effective, with $K_{\mathrm{d}}$ values greater than $1000 \mathrm{~mL} / \mathrm{g}$ for three resins, including two commercially available resins. Possibly other aqueous washing strategies could be used, but their development awaits further investigation.

The other major solvent degradation product, DOA does not partition to either the simulant or to $0.01 \mathrm{M} \mathrm{NaOH}$ wash solution, but it does partition weakly to the strip solution, where $P_{\mathrm{DOA}}=4.7$. At $\mathrm{O} / \mathrm{A}=5$, loss of DOA to the strip effluent would be expected to be $4 \%$ per solvent cycle, and the estimated steady-state concentration is $9 \times 10^{-7} \mathrm{M}$ based on $0.1 \mathrm{mM}$ DOA per year as the rate of production due to thermal or radiolytic degradation of TOA. The cumulative effect of, say, one week of operation (62 solvent cycles), would be the loss of $92 \%$ of the DOA. Thus, the primary fate of the DOA in the process is expected to be simple washing-out by the strip solution.

Three neutral organic components of the full simulant, trimethylamine (TMA), tributylphosphate (TBP), and 1-butanol $(\mathrm{BuOH})$, were examined to estimate their fate in the flowsheet. Among the three compounds, possible implications for process upset are greatest for TMA. It was found that trimethylamine mildly partitions to the solvent during extraction $\left(P_{\mathrm{TMA}}=4.3\right.$ for baseline solvent and full simulant at $25^{\circ} \mathrm{C}$ ). Accordingly, the TMA will move with the solvent into the scrub stages in the flowsheet. There it will be protonated, partition into the aqueous phase $\left(P_{\mathrm{TMA}}=1.9 \times 10^{-4}\right.$ for baseline solvent and $50 \mathrm{mM} \mathrm{HNO}_{3}$ at $25^{\circ} \mathrm{C}$ ), and return to the feed stage, since the scrub solution is added to the waste feed stage. With such a feedback loop, the TMA could in principle build up to 
very high concentrations in the solvent. As it is very volatile, the TMA would probably leak out of the system primarily via the purge gas used in the contactors. If this purge rate is insufficient, unlimited buildup of TMA would probably next lead to complete consumption of the acid in the scrub stage when the TMA reaches $10 \mathrm{mM}$ in the solvent. This would in effect limit the buildup to $10 \mathrm{mM}$ TMA, whence the TMA would start to pass into the stripping section and report to the strip effluent. At the presumed maximum $0.17 \mathrm{mM}$ level of TMA in the waste, the steady-state concentration of TMA in the solvent entering the strip section would then be $0.51 \mathrm{mM}$, equivalent to $2.5 \mathrm{mM}$ of aqueous base, more than sufficient to neutralize the acid in the strip solution. At this moment, the consequences of this scenario are not clear and are in need of further investigation. Although the chemistry of the process could change as described, whether the process would cease to function is not obvious, as acid balance is not part of the driving force in stripping. Rather, nitrate concentration is the driving force, and it should not be impacted. Options to deal with the problem include 1) sparging the aqueous feed; 2) reducing the solvent flow rate so that the O/A ratio in the extraction section gives an extraction factor less than unity, thus flushing out the TMA in the raffinate stream; 3) periodically draining the solvent from the first extraction stage (\#15) and treating it with dilute nitric acid $(<0.5 \mathrm{M})$ : or 4$)$ divert the aqueous scrub effluent to a small tank where it is neutralized and the TMA released. Currently, the fate and effect of the TMA is uncertain and must be investigated further.

TBP is present at very low concentrations in the waste $\left(\max .1 .9 \times 10^{-6} \mathrm{M}\right)$ but partitions strongly to the solvent, where $P_{\mathrm{TBP}}=1880$ for the baseline solvent equilibrated at $25^{\circ} \mathrm{C}$ with the full simulant. This partition ratio indicates that TBP could only build up in the solvent to $3.5 \mathrm{mM}$ at steady state, as the raffinate exiting stage \#1 will start to wash out the TBP when it reaches this level in the solvent. As seen in Chap. 3, this level is too low to have any effect on extraction, scrubbing, or stripping.

1-Butanol is present to at most $2.7 \times 10^{-5} \mathrm{M}$ in the waste and partitions mildly to the solvent, where $P_{\mathrm{BuOH}}=7.5$ for the baseline solvent equilibrated at $25^{\circ} \mathrm{C}$ with the full simulant. However, the partition ratio is not high enough to allow it to build up to more than $0.2 \mathrm{mM}$, again too low to have any effect on solvent performance.

A large set of data was collected for the distribution of the alkali metal cations $\mathrm{Na}^{+}, \mathrm{K}^{+}$, and $\mathrm{Cs}^{+}$ from the full simulant, simple simulants, and simple one- or two-component electrolyte solutions. Some of these data were used for computer modeling reported under separate cover. ${ }^{25}$ Experiments comparing the baseline solvent with and without BOBCalixC6, show that the calixarene has little ability to bind $\mathrm{Na}^{+}$ion, and the observed $\mathrm{Na}^{+}$extraction by the baseline solvent is largely due to ion exchange of the modifier's proton. Cesium distribution into the solvent phase is sensitive to the potassium concentration in the simulant, decreasing as the potassium loading of the calixarene increases. Potassium loading from the full simulant is $26 \%$ at $\mathrm{O} / \mathrm{A}=0.33$ and $25^{\circ} \mathrm{C}$ and increases to $47 \%$ at the bounding aqueous concentration of $50 \mathrm{mM}$. Although this effect does not pose a serious problem in sustaining desired high $D_{\mathrm{Cs}}$ values, the potassium concentration in the feed solution should be closely monitored. Potassium and sodium report almost completely to the scrub solution. Cesium loading from the full simulant at $3.6 \%$ is minor and is still not limiting even at a bounding level of $0.7 \mathrm{mM}$ in the solvent, where loading is $17.5 \%$ and $D_{\mathrm{Cs}}=15.0$. Measurements of the $\mathrm{pH}$ of the scrub and strip solutions under various conditions revealed acid-balance behavior of the system, which appears especially to be influenced by release to the strip solution of some of the acid that was extracted by TOA and modifier under scrub conditions. Ion chromatography showed that nitrate, nitrite, chloride, and dibutylphosphate are the primary anions extracted from the simulant by the baseline solvent. All the anions except for nitrate and dibutylphosphate report to the scrub solution, while the nitrate and dibutylphosphate are washed out by $\mathrm{NaOH}$ solutions.

Experiments with simple salt solutions showed that the process should be relatively tolerant to variations in the anion content among these three major anions, nitrate, nitrite, and hydroxide. Each of these anions contributes significantly to the driving force for cesium extraction, as computer modeled elsewhere, and thus they should be mutually compensating at constant sodium concentration. This desirable property was in fact observed in more complex simulant recipes tested in Chap. 3 (Section 3.3.14).

Five metals not included in the full simulant recipe, namely $\mathrm{U}, \mathrm{Np}, \mathrm{Pu}, \mathrm{Sr}$, and $\mathrm{Tc}$, were shown not to be extracted significantly by the CSSX baseline solvent. The presence of these five metals also 
does not impair extraction nor stripping of cesium. Although more tests should be conducted to gather definitive data on controlled oxidation states (except $\mathrm{Sr}$ ) in varied matrices, including real waste, it appears likely that the five metals should pass through the extraction stages and exit with the waste raffinate. As a tentative conclusion, little if any impact is therefore expected due to these metals on the CSSX process and the character of its solvent and strip effluent streams. The remaining question regarding potential placement of the monosodium titanate strike after CSSX, then, is the extent of solids removal that would be required prior to CSSX to prevent fouling the contactors with solids.

A survey of the elements present in the simulant, including $\mathrm{Cr}, \mathrm{Cu}, \mathrm{Fe}, \mathrm{Mo}, \mathrm{Hg}, \mathrm{Ag}, \mathrm{Pb}, \mathrm{Pd}, \mathrm{Rh}, \mathrm{Ru}$, $\mathrm{Sn}, \mathrm{Zn}, \mathrm{Cl}$, and $\mathrm{F}$, could not detect any buildup in the solvent on limited cycles, nor do they appear in the scrub or strip stages in significant concentrations. However, it should be noted that traces of $\mathrm{Al}$, $\mathrm{Fe}, \mathrm{Hg}$, and $\mathrm{Pb}$ have detected at $\leq 2 \mu \mathrm{M}$ in the scrub solution in the first-generation CSSX system. 


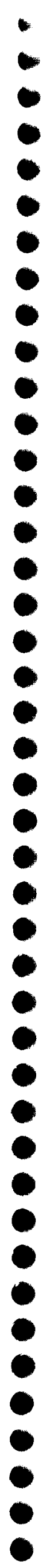




\section{THERMAL STABILITY}

\subsection{INTRODUCTION}

\subsubsection{Purpose}

In FY 1999, the immediate predecessor solvent system to the current baseline solvent was shown to have good stability to exposure to alkaline simulant and pristine scrub solution at a temperature of $52{ }^{\circ} \mathrm{C} .15$ This solvent employed the Cs-7SBT modifier, essentially the same compound as Cs-7SB except for the fact that it was synthesized with a technical grade of 4-sec-butylphenol. Otherwise, the solvent had the same composition as the current baseline solvent. After 54 days, the Cs-7SBT solvent in contact with $0.05 \mathrm{M}$ nitric acid gave no detectable nitration products by NMR spectroscopy. In a 60-day contact of the Cs-7SBT solvent with alkaline simulant, no decomposition products were detected by NMR spectroscopy, and batch extraction behavior was unchanged. The simulant used, labeled SRS\#2, had a simpler composition and lacked the noble metals and organic compounds that the more realistic simulant in present use contains.

The purpose of the thermal-stability tests being conducted in FY 2000 and FY 2001 was to increase our confidence that the solvent is sufficiently stable for process application for the duration of at least one year and to provide information on the identity and effect of any degradation products that might form. ${ }^{4}$ Although the limited test results from FY 1999 were encouraging, they were insufficient to fulfill the present purpose for several reasons. First, the full simulant used in CSSX testing since FY 2000 (Chap. 2) contains noble metals and minor components that could act as catalysts for thermal decomposition. Second, the test with $50 \mathrm{mM}$ nitric acid as the aqueous phase in FY 1999 lacked cesium and other extractable metals. Third, there was no way to extrapolate the FY 1999 results from a duration of approximately 2 months to the duration of 1 year. Fourth, no information on probable degradation products was obtained. Accordingly, the present testing was designed to address these deficiencies so as to arrive at a more reliable estimate of the operational lifetime of the solvent as limited by thermal stability only. Not only was the test much more challenging to the solvent, but much better information on degradation products was obtained from a wider array of analytical procedures. This information would then be valuable input for design of solvent-cleanup methods, and any degradation of performance would reflect on the need for solvent cleanup.

In August 2000, an interim report was issued, in which no particular thermal-stability problems could be identified after 46 days of thermal treatment. ${ }^{69}$ Results from ESS performance evaluation and NMR spectroscopy respectively showed no change in performance within statistical uncertainty and no detectable buildup of degradation products. Subsequently, the test was run to 235 days, whence it was finally possible to observe performance changes and measurable changes in system composition.

\subsubsection{Experimental Design}

The current tests involve heating the baseline solvent alone or in contact with various process aqueous phases at two different temperatures, about $35{ }^{\circ} \mathrm{C}$ and $60{ }^{\circ} \mathrm{C}$. These are the "set temperatures" for the incubators used, and accordingly these temperatures were used in the sample code extensions However, the actual average temperature ranges, as checked using a calibrated thermometer, were $34.8 \pm 0.6{ }^{\circ} \mathrm{C}$ and $61.2 \pm 0.6{ }^{\circ} \mathrm{C}$, respectively. For discussion, we will state the nominal temperatures as $35^{\circ} \mathrm{C}$ and $61{ }^{\circ} \mathrm{C}$. Although $35^{\circ} \mathrm{C}$ is considered at or near the upper end of the process operating temperature range, the higher temperature of $61{ }^{\circ} \mathrm{C}$ is needed to increase the likelihood that solvent degradation processes could be observed and characterized well enough to estimate solvent lifetime and need for cleanup. Samples of the solvent were agitated in continuous contact with the three aqueous phases specified in the basic flowsheet. Figures 5.1-5.3 define the 
order of phase contacting prior to, and subsequent to, thermal treatment. For greater fidelity to flowsheet conditions, the solvent continuously exposed to the scrub solution was first contacted with the full simulant (Fig. 5.2), and the solvent continuously exposed to the strip solution was first contacted with the full simulant and then with scrub solution (Fig. 5.3). In this manner, the two-phase systems would contain a representative mixture of extractable metal and organic species originating from the waste. To assess the effect of minor components in the full simulant that are potentially catalytically active, a fourth aqueous phase, the salts-only simulant, was also run. This simulant is identical to the full simulant but without the noble metals and organic species. Except for the temperatures, the conditions in the tests were the same as generally employed in batch contacting (Chap. 2). Following thermal treatment, samples were re-equilibrated at $25{ }^{\circ} \mathrm{C}$, whence the ESS behavior could be assessed against baseline behavior and a battery of analytical procedures applied. The latter included NMR, ES-MS, RP-HPLC, GC, and GC-MS.

In addition to the above test series, it was also planned to revisit more quantitatively the question of nitration of the extractant and modifier in solvent exposed to nitric acid at varying concentrations at elevated temperatures. Such information would allow flexibility in flowsheet optimization or in solvent washing, for example, if it were to be found that nitric acid concentrations higher than that used in scrubbing were not detrimental in limited contacting times with the solvent. In these tests, only NMR spectra were planned for examination of the treated solvents, as nitration could be readily detected and quantified upon in-growth of characteristic resonances.

\subsubsection{Work Scope}

The work described in this chapter was prescribed under WSM 4.1.419 and more elaborately outlined in SPP planning. ${ }^{4}$ The elements of this scope include thermal stability over waste simulants containing noble metals, over nitric acid as a function of concentration, over strip solution, and over other solutions (e.g., wash solutions). Although it would have been desirable to include wash solutions in the test, solvent washing was not yet defined when the thermal-stability test was initiated, and thus no actual wash solutions were included. The effect of this omission on the ability to fulfill the goals of the test is considered negligible, as exposure to actual simulant is a more severe test. It was recognized in the planning that this work is exploratory and highly dependent upon the extent of solvent degradation and performance. Analyses and tests would thus be prioritized according to the apparent severity of degradation and to the type of information needed to diagnose and remediate any identified problems. 


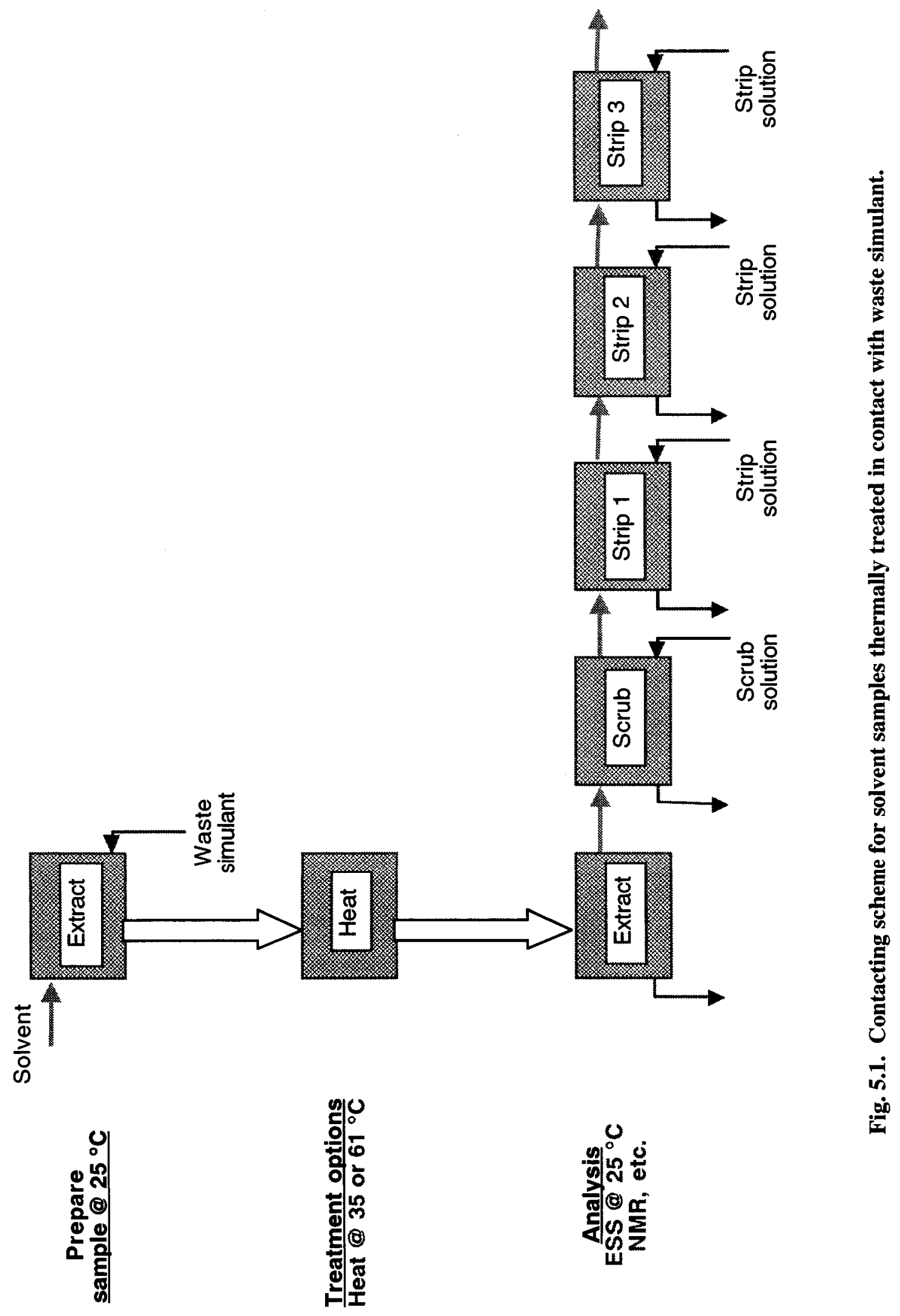




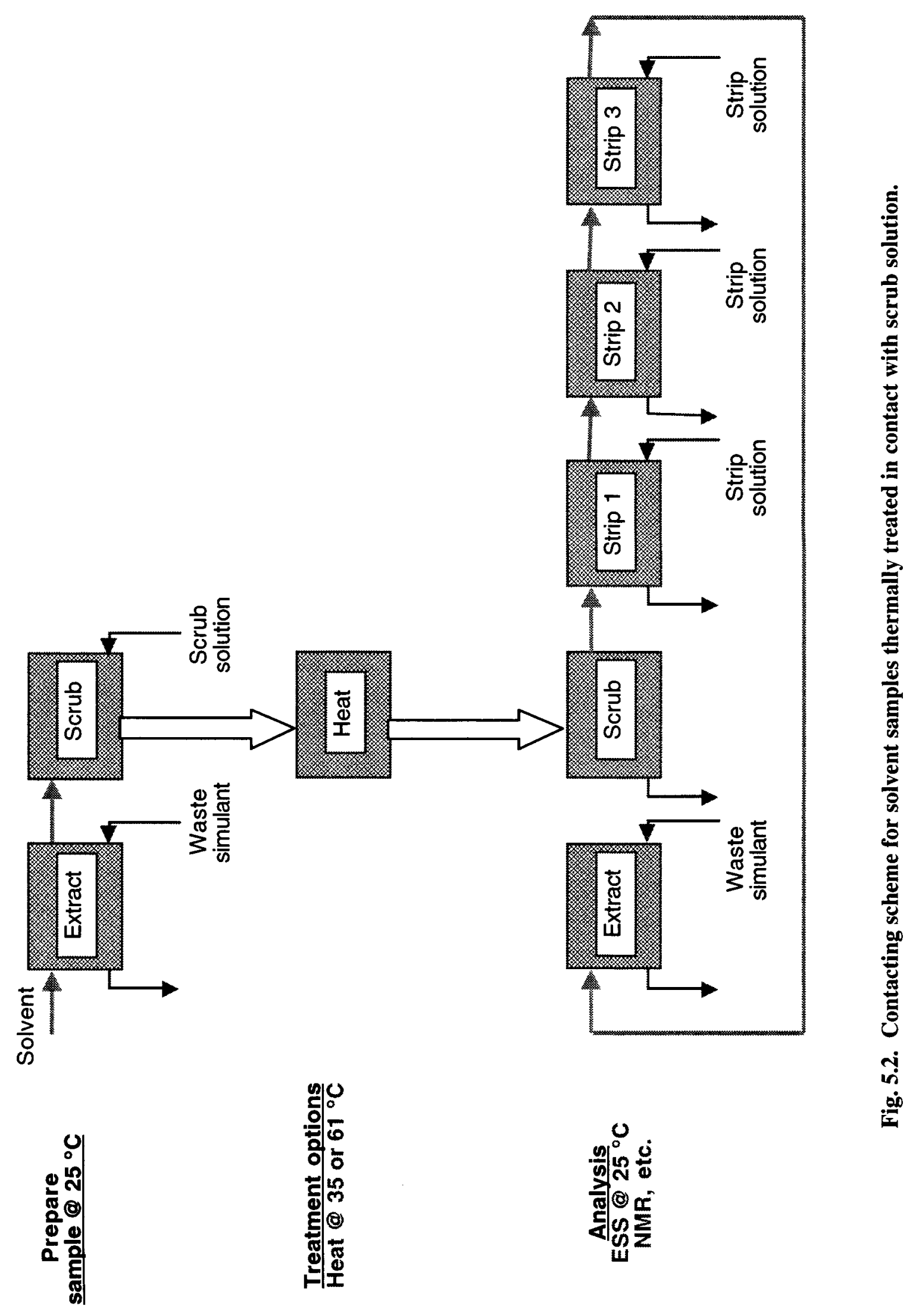




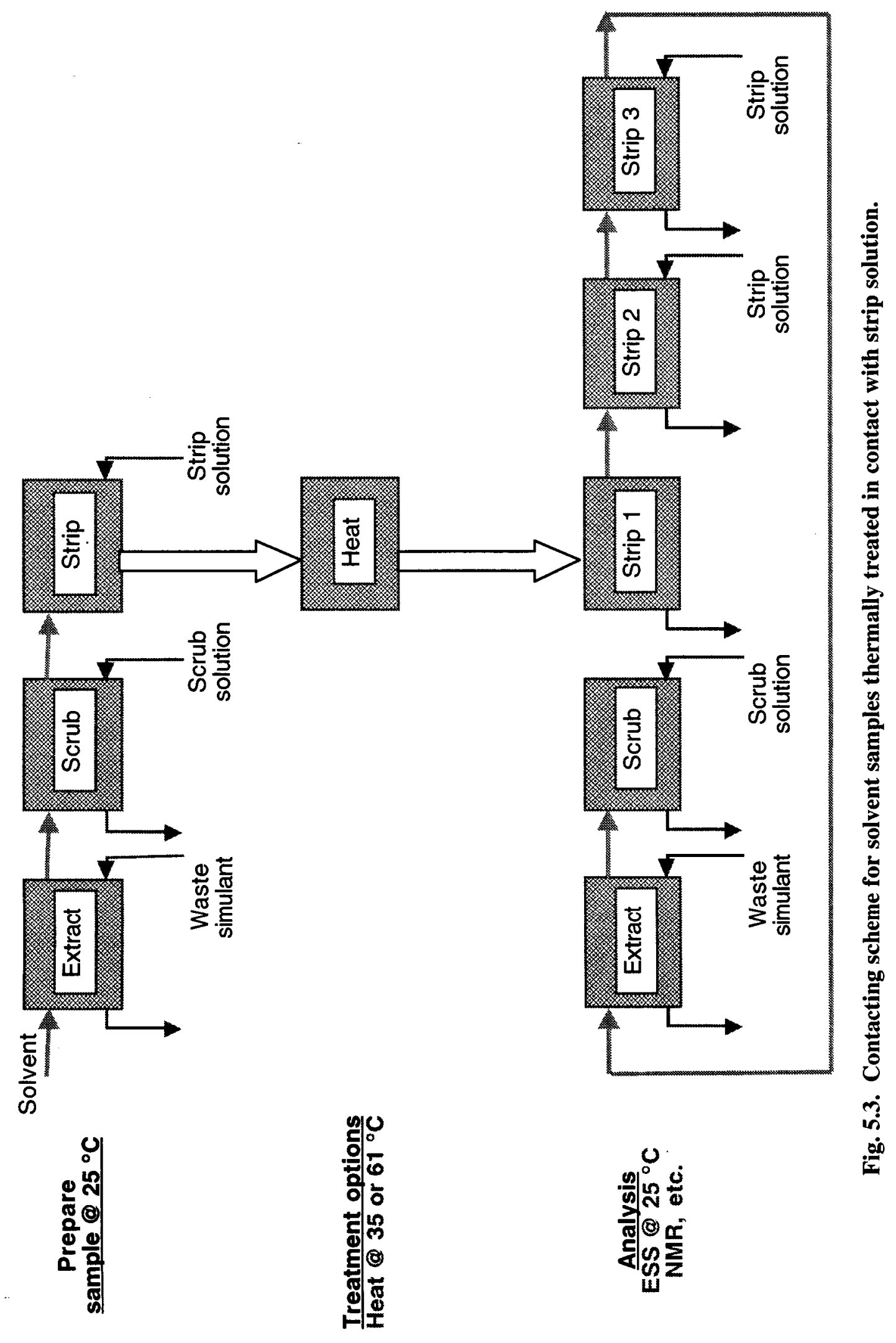

기 


\subsection{EXPERIMENTAL SECTION}

\subsubsection{Chemicals, Materials, and Equipment}

Solvent Lot B000718-132W was used for all experiments. For the simulants, the first draws of both the Full and Salts-only simulants were employed for the thermal contacts with solvent. Subsequent draws were used to supply fresh aqueous phase for the ESS tests.

Teflon FEP centrifuge tubes, cleaned as described elsewhere, ${ }^{39}$ were employed for contacting the solvents with the aqueous phases at elevated temperatures. Labline Imperial III incubators, set to $35 \pm$ $0.5^{\circ} \mathrm{C}$ and $60 \pm 1{ }^{\circ} \mathrm{C}$, were used to maintain the samples at constant temperature. Again, the actual operating temperature ranges, based on a check against a NIST traceable calibrated thermometer, were $34.8 \pm 0.5^{\circ} \mathrm{C}\left(35^{\circ} \mathrm{C}\right)$ and $61.2 \pm 0.6^{\circ} \mathrm{C}\left(61^{\circ} \mathrm{C}\right)$, respectively. The incubator model used for the $35^{\circ} \mathrm{C}$ experiments has mechanical convection, and a manufacturer stated temperature uniformity of $\pm 0.5{ }^{\circ} \mathrm{C}$. The incubator model used for the $61{ }^{\circ} \mathrm{C}$ experiments has gravity convection, and a manufacturer stated temperature uniformity of $\pm 0.9{ }^{\circ} \mathrm{C}$. The ambient temperature of the laboratory where the incubators resided varied between 16 and $22{ }^{\circ} \mathrm{C}$, and the incubator temperature ranges observed reflects both the aforementioned unit's uniformity as well as how well the incubators responded to the variance in the ambient lab temperature. The samples were agitated by end-overend rotation on Glas-Col rugged rotators placed inside the incubators. All manipulations of the solvents were performed using calibrated Eppendorf pipettes.

\subsubsection{Test Protocol}

To examine the stability of the solvent and solvent components to prolonged contact with the flowsheet solutions, aliquots of solvent were contacted with the appropriate volume of each aqueous phase as shown below in the table matrix (Table 5.1). The table sets forth the sample codes and volumes of each phase for samples placed in the $34.8 \pm 0.5{ }^{\circ} \mathrm{C}$ incubator. An identical matrix was used for samples placed in the $61.2 \pm 0.6{ }^{\circ} \mathrm{C}$ incubator, except the sample codes used " -60 -" in place of "-35-". Exposure times were set to approximately 2 weeks (12 days actual), 7 weeks (46 days actual), 16 weeks (110 days actual), and 33 weeks (235 days actual). The following aqueous phases were employed: a) full simulant, containing known organic species as well as noble metals, ${ }^{21,22}$ b) scrub solution consisting of $0.050 \mathrm{M}$ nitric acid, and c) strip solution consisting of $0.001 \mathrm{M}$ nitric acid. The salts-only simulant was also included in the test for the 2,7 , and 16-week samples. Solvent samples with no added aqueous phase were run as a control for the effect of simple storage of wet solvent.

The order of contacting prior to thermal testing and subsequent to it is defined in Figs. 5.1-5.3. Phase ratios (O/A) used in all of the tests were as prescribed for batch "ESS" tests, namely 1:3, 5:1, and 5:1 for extraction, scrubbing, and stripping, respectively. Contacting was carried out by endover-end rotation of the two-phase samples in Teflon FEP screw-cap centrifuge tubes in the $34.8 \pm$ $0.5^{\circ} \mathrm{C}$ and $61.2 \pm 0.6^{\circ} \mathrm{C}$ incubators. A set of baseline "zero-time" samples were run by preparing a set of samples as if they would be thermally treated but then proceeding directly to characterization as described below. Samples were withdrawn from the incubators at 12, 46, 110, and 235 days.

In a 4-week experiment to assess nitration of BOBCalixC6 and Cs-7SB, baseline solvent was contacted at an $\mathrm{O} / \mathrm{A}$ phase ratio of $1: 1$ with aqueous nitric acid solutions at $0,1 \mathrm{mM}, 50 \mathrm{mM}, 300 \mathrm{mM}$, $700 \mathrm{mM}$, and $1 \mathrm{M} \mathrm{HNO}_{3}$ at either $34.8 \pm 0.5^{\circ} \mathrm{C}$ or $61.2 \pm 0.6^{\circ} \mathrm{C}$. NMR spectra were obtained for all test solvent samples at $0,7,14,21$, and 28 days. 
Table 5.1. Scheme for thermal-stability samples placed in $34.8 \pm 0.5^{\circ} \mathrm{C}$ incubator

\begin{tabular}{|c|c|c|c|c|c|}
\hline $\begin{array}{c}\text { Tube } \\
\text { size } \\
(\mathrm{mL})\end{array}$ & $\begin{array}{l}\text { Sample } \\
\text { code }\end{array}$ & $\begin{array}{c}\text { Solvent } \\
\text { volume } \\
(\mathrm{mL})\end{array}$ & $\begin{array}{l}\text { Identity and volume } \\
\text { of aqueous phase }\end{array}$ & $\mathrm{O} / \mathrm{A}$ ratio & $\begin{array}{c}\text { Time in } \\
\text { incubator (days) }\end{array}$ \\
\hline 30 & C-35-2 & 10 & None & & 12 \\
\hline 30 & $C-35-7$ & 10 & None & & 46 \\
\hline 30 & $C-35-16$ & 10 & None & & 110 \\
\hline 30 & $C-35-33$ & 10 & None & & 235 \\
\hline 50 & A-35-2 & 10 & $30 \mathrm{~mL}$ Full simulant & 0.33 & 12 \\
\hline 50 & A-35-7 & 10 & $30 \mathrm{~mL}$ Full simulant & 0.33 & 46 \\
\hline 50 & A-35-16 & 10 & $30 \mathrm{~mL}$ Full simulant & 0.33 & 110 \\
\hline 50 & $A-35-33$ & 10 & $30 \mathrm{~mL}$ Full simulant & 0.33 & 235 \\
\hline 50 & B-35-2 & 9 & $27 \mathrm{~mL}$ Salts-only simulant & 0.33 & 12 \\
\hline 50 & B-35-7 & 9 & $27 \mathrm{~mL}$ Salts-only simulant & 0.33 & 46 \\
\hline 50 & $\mathrm{~B}-35-16$ & 9 & $27 \mathrm{~mL}$ Salts-only simulant & 0.33 & 110 \\
\hline 30 & $\mathrm{SC}-35-2$ & 10 & $2 \mathrm{~mL} 0.05 \mathrm{M} \mathrm{HNO}_{3}$ & 5 & 12 \\
\hline 30 & $\mathrm{SC}-35-7$ & 10 & $2 \mathrm{~mL} 0.05 \mathrm{M} \mathrm{HNO}_{3}$ & 5 & 46 \\
\hline 30 & SC-35-16 & 10 & $2 \mathrm{~mL} 0.05 \mathrm{M} \mathrm{HNO}_{3}$ & 5 & 110 \\
\hline 50 & $\mathrm{SC}-35-33$ & 20 & $4 \mathrm{~mL} 0.05 \mathrm{M} \mathrm{HNO}_{3}$ & 5 & 235 \\
\hline 30 & ST-35-2 & 10 & $2 \mathrm{~mL} 0.001 \mathrm{M} \mathrm{HNO}_{3}$ & 5 & 12 \\
\hline 30 & $\mathrm{ST}-35-7$ & 10 & $2 \mathrm{~mL} 0.001 \mathrm{M} \mathrm{HNO}_{3}$ & 5 & 46 \\
\hline 30 & $\mathrm{ST}-35-16$ & 10 & $2 \mathrm{~mL} 0.001 \mathrm{M} \mathrm{HNO}_{3}$ & 5 & 110 \\
\hline 50 & ST-35-33 & 20 & $4 \mathrm{~mL} 0.001 \mathrm{M} \mathrm{HNO}_{3}$ & 5 & 235 \\
\hline
\end{tabular}

\subsubsection{Extract/Scrub/Strip Protocol}

At the prescribed times, the sample vials were removed from the incubators, rotated for 6-14 hours at $25{ }^{\circ} \mathrm{C}$, and centrifuged. For batch ESS tests, aliquots of both the treated aqueous and organic phases were placed into clean Teflon FEP or polypropylene tubes of the appropriate size and in the appropriate phase ratios. In the case of the solvent alone, fresh full simulant was added to the tube. After equilibration at $25^{\circ} \mathrm{C}$, the sample vials were shaken by hand for a measurement of break time. The samples were then spiked with ${ }^{137} \mathrm{Cs}$ tracer and taken through a full ESS cycle by use of the end-over-end contacting technique for $30 \mathrm{~min}$ at $25 \pm 0.2{ }^{\circ} \mathrm{C}$, followed by centrifugation, subsampling, and gamma counting. For solvent treated over simulant, the ESS test yields cesium distribution ratios $\left(D_{\mathrm{Cs}}\right)$ at $25^{\circ} \mathrm{C}$ for the two phases that were thermally treated, followed by a scrub of the treated solvent with fresh scrub solution, and three strips with fresh strip solutions. For the solvent treated over scrub solution, the ESS test yields the $D_{C S}$ values for the two phases that were thermally treated, followed by 3 strips of the treated solvent with fresh strip solution, followed by an extraction with fresh simulant. For the solvent treated over strip solution, the ESS test yields the $D_{C s}$ 
values for the two phases that were thermally treated, followed by 2 strips of the treated solvent with fresh strip solution, followed by an extraction using the treated solvent with fresh simulant, followed by a scrub with fresh scrub solution. Thus, solvent treated over scrub and strip solutions is taken partly through a second ESS cycle (see Figs. 5.2 and 5.3). For the solvent thermally treated with no aqueous solution, the full ESS test was run as usual.

The size of the vial or tube employed for contacting the aqueous and organic phases is an important detail for ensuring adequate mixing. It is important to allow enough headspace in the vial or tube for both phases to be completely mixed together when rotating the samples end-over-end on the Glas-Col rotator over a 30-min period. For ESS tests starting with the solvent treated over simulant (or solvent alone), a 5-mL aliquot of the organic phase was contacted with a $15-\mathrm{mL}$ aliquot of the aqueous phase in a clean, 30-mL Teflon FEP tube. Proceeding to the scrub, $4.25 \mathrm{~mL}$ of loaded organic was transferred to a clean $15-\mathrm{mL}$ polypropylene tube and contacted with $0.85 \mathrm{~mL}$ of $50 \mathrm{mM} \mathrm{HNO} 3$ scrub solution. The strip stages were performed as follows: for strip \#1, $3.5 \mathrm{~mL}$ of organic phase from scrubbing was contacted with $0.70 \mathrm{~mL}$ of $1 \mathrm{mM} \mathrm{HNO}_{3}$ strip solution in a $15-\mathrm{mL}$ vial; for strip \#2, $2.75 \mathrm{~mL}$ of organic phase from strip \#1 was contacted with $0.55 \mathrm{~mL}$ of fresh strip solution in a 5-mL cryogenic vial; for strip \#3, $2.0 \mathrm{~mL}$ of organic phase from strip \#2 was contacted with $0.40 \mathrm{~mL}$ of fresh strip solution with added ${ }^{137} \mathrm{Cs}$ tracer in a $5-\mathrm{mL}$ cryogenic vial.

For the tests beginning with solvent treated over scrub solution, a $5.0-\mathrm{mL}$ aliquot of the organic phase and a $1.0-\mathrm{mL}$ aliquot of the aqueous phase with added ${ }^{137} \mathrm{Cs}$ tracer were equilibrated in a 15 $\mathrm{mL}$ polypropylene tube. Proceeding to the first strip, $4.25 \mathrm{~mL}$ of the organic phase from this scrub step was contacted with $0.85 \mathrm{~mL}$ of fresh strip solution in a clean $15-\mathrm{mL}$ polypropylene vial. Strip \#2 was done next by contacting $3.50 \mathrm{~mL}$ of organic phase from strip \#1 with $0.70 \mathrm{~mL}$ of fresh strip solution again in a clean $15-\mathrm{mL}$ polypropylene vial. The final strip was done by contacting $2.75 \mathrm{~mL}$ of organic phase from strip \#2 with $0.55 \mathrm{~mL}$ of fresh strip solution with added ${ }^{137} \mathrm{Cs}$ tracer in a 5 - $\mathrm{mL}$ cryogenic vial. The test looped around to the extraction by contacting $2.0 \mathrm{~mL}$ of this stripped organic phase with $6.0 \mathrm{~mL}$ of fresh simulant spiked with ${ }^{137} \mathrm{Cs}$ in a clean $15-\mathrm{mL}$ polypropylene tube.

For tests beginning with the first strip, a $5.0-\mathrm{mL}$ aliquot of organic phase was contacted with a 1.0-mL aliquot of aqueous phase spiked with ${ }^{137} \mathrm{Cs}$ in a $15-\mathrm{mL}$ polypropylene vial. The second strip was done by contacting $4.25 \mathrm{~mL}$ of this organic with $0.85 \mathrm{~mL}$ of fresh strip solution in a $15-\mathrm{mL}$ polypropylene vial. The third strip was done by contacting $3.50 \mathrm{~mL}$ of organic phase from strip \#2 with $0.70 \mathrm{~mL}$ of fresh strip solution spiked with ${ }^{137} \mathrm{Cs}$ in a $15-\mathrm{mL}$ polypropylene vial. The organic phase was taken around to the extraction again by contacting $2.75 \mathrm{~mL}$ of stripped organic phase with $8.25 \mathrm{~mL}$ of fresh simulant spiked with ${ }^{137} \mathrm{Cs}$ in a $15-\mathrm{ml}$ polypropylene vial. The scrub was done by contacting $2.0 \mathrm{~mL}$ of this organic phase with $0.40 \mathrm{~mL}$ of scrub solution in a $5-\mathrm{mL}$ cyrogenic vial.

\subsubsection{Break-Time (Coalescence) Measurements}

Break times were recorded for the contact between the phases that immediately were removed from the incubator. For the control solvent that was heated in the absence of an aqueous phase, the break time was recorded for the contact with fresh full simulant. After the samples were removed from the incubator and equilibrated to $25^{\circ} \mathrm{C}$ by rotation for 6-14 hours as stated above, the samples were hand-shaken 10 times and the time needed to reach coalescence recorded. For samples tested at $2,7,16$, and 33 weeks, Teflon vials of $25.5-\mathrm{mm}$ diameter were used for contacts between simulants and organic, while polypropylene tubes $17.0 \mathrm{~mm}$ in diameter were used for contacts between scrub or strip samples and the organic phase.

Timed observations were made for ten test samples plus a control. Each tube was inverted twice, then shaken by hand ten times. An electronic stopwatch / timer was used to record the time for the phases to coalesce. Although subjective, a common criteria was used for all observations. For test samples identified as solvent only, full simulant "A", and salts only simulant "B", coalescence was considered to be achieved when all drops disappeared at the phase boundary. Exceptions were made if a few small, persistent drops were associated with the wall of the tube. These measurements were made in $30-\mathrm{mL}$ FEP screw-capped tubes containing $5 \mathrm{~mL}$ of the test solvent and $15 \mathrm{~mL}$ of the appropriate simulant. For those test samples designated as scrub solution and strip solution, a slightly different criterion was followed in determining coalescence of the phases. Coalescence was achieved 
when all "large" drops disappeared from the region of the interface. A layer of very tiny drops, with the appearance of a concentrated haze, persisted at the interfacial region for greater than $12 \mathrm{~min}$ following shaking. This layer was approximately $4 \mathrm{~mm}$ thick and lay immediately below the phase boundary in the upper region of the aqueous phase. While the phase boundary had a convex orientation, the fine drop region in the aqueous layer was perpendicular to the vertical walls of the tube. These measurements were made in $15-\mathrm{mL}$ capacity conical bottom polypropylene tubes containing $5 \mathrm{~mL}$ of solvent and $1 \mathrm{~mL}$ of aqueous phase.

\subsubsection{NMR Analysis}

The following procedure was used for the 12- and 46-day ESS samples. Solvent samples were analyzed directly by diluting $100-\mu \mathrm{L}$ aliquots of the solvent with $900 \mu \mathrm{L}$ of deuterochloroform, which contained hexamethylbenzene (HMB, Aldrich, 99\%) at $0.0140 \mathrm{M}$ as an internal integration standard. The HMB concentration in the $1000-\mu \mathrm{L}$ prepared sample $(0.0126 \mathrm{M})$ gives a single peak for all six methyl groups (18 protons). Upon dilution, the modifier has an effective concentration in the sample of $0.050 \mathrm{M}$. Thus, in a pristine solvent sample, the ratio of the area of the HMB singlet peak (representing 18 protons) at $2.23 \mathrm{ppm}$ to the area of the triplet of triplets at $5.90 \mathrm{ppm}$ representing the $-\mathrm{CF}_{2} \mathrm{H}$ proton of the modifier should be $[0.0126 * 18] / 0.050 \sim 4.5: 1$. This ratio, as well as the ratio of the HMB singlet to other peaks of interest, was monitored to determine whether the concentration of the modifier in the solvent was changing. The ratio has a normal variance of about $\pm 10 \%$ due to normal shimming and integration reproducibility. For the 235-day samples and the variable nitric acid concentration samples, the $100-\mu \mathrm{L}$ solvent samples were diluted with $700 \mu \mathrm{L}$ of deuterochloroform containing HMB at $0.0198 \mathrm{M}$, giving an effective concentration of $0.0174 \mathrm{M}$ in the $800-\mu \mathrm{L}$ prepared sample. In these samples, the modifier has an effective concentration of 0.0625 $\mathrm{M}$, and thus the ratio of the $\mathrm{HMB}$ protons to the $-\mathrm{CF}_{2} \mathrm{H}$ proton of the modifier should be $[0.0174 * 18] / 0.0625 \sim 5: 1$. (NMR spectra were not obtained on the 110-day samples.) The amount of $\mathrm{BOBCalixC} 6$ and modifier that were nitrated relative to the amount that was unchanged was determined by comparison of the peak area integrals for selected aromatic protons for each species as described previously. ${ }^{8}$ In addition, coupling patterns and chemical shifts of all aromatic protons were inspected to determine whether other reactions (such as displacement of the tert-octyl group from the benzo-crown portion of $\mathrm{BOBCalixC} 6$ by nitrate) were occurring.

\subsubsection{Electro-Spray Mass Spectrometry (ES-MS) Analysis}

Electrospray Mass Spectrometry (ES-MS) was performed on a PE SCIEX API 165 single quadrupole mass spectrometer (Concord, Ontario, Canada) outfitted with a TurboIonSpray source, as previously described in Section 2.4.1. Samples for ES-MS were run in the order of increasing analyte concentrations to minimize contamination by retention and bleed-through of species from the more concentrated samples. Thus, for solvent samples from the thermal-stability experiments, the sample-run order was pristine solvent, followed by solvent that had been in contact with strip, scrub, salts-only simulant, and full simulant, respectively. The solvent samples exposed to all aqueous phases at $61.2 \pm 0.6{ }^{\circ} \mathrm{C}$ for 2 weeks and 16 weeks were analyzed in both anion and cation mode (2week samples analyzed first). 


\subsubsection{Chemical Analysis}

Solvent samples were prepared by dilution in isopropanol, $1 / 10$ for trioctylamine and 4-sec-butylphenol analysis, and 1/100 for analysis of the calixarene and Cs-7SB. The sample aliquot used for the analysis of TOA and 4-sec-butylphenol was made basic by the addition of $1 \%$ triethylamine. Analysis of the calixarene and modifier was carried out using reverse-phase HPLC with UV absorbance detection at $226 \mathrm{~nm}$ for quantitative analysis. Trioctylamine was analyzed by gas chromatography with flame ionization detection (samples containing no radioactivity) or by gas chromatography/mass spectrometry (samples containing radioactivity). These two methods are comparable, although the GC/MS analysis is capable of detecting and measuring 4-sec-butylphenol as well. The 4-sec-butylphenol was analyzed by gas chromatography/mass spectrometry. All methods were calibrated using the method of external standards. In all series of samples controls were used for comparison with the samples. Further details on chemical analysis procedures are given elsewhere. ${ }^{26}$

\subsection{RESULTS AND DISCUSSION}

\subsubsection{Visual Observations}

\subsubsection{Thermal-Stability Test with Flowsheet Aqueous Phases}

Perhaps the only conclusive sign of an effect of thermal treatment of the samples was the appearance of yellow color in the solvents in contact with nitric acid. The intensity of the yellow color increased with the time and temperature of the exposure, and was much more pronounced for the scrub samples. The appearance of both phases following contacts for all samples at $34.8 \pm 0.5{ }^{\circ} \mathrm{C}$ ("35 ${ }^{\circ} \mathrm{C}$ ") and $61.2 \pm 0.6^{\circ} \mathrm{C}$ ("61 ${ }^{\circ} \mathrm{C}$ ") is chronicled in Table 5.2. At 12 days, the appearance of the solvent from the $35^{\circ} \mathrm{C}$ samples was clear and colorless in all cases. However, the solvent that was in contact with the scrub solution at $61{ }^{\circ} \mathrm{C}$ possessed a pale yellow color. The solvent that was in contact with Simulant A initially possessed a faint yellow tint, which disappeared over time. Visual inspection of the 46-, 110-, and 235-day samples of solvent in contact with the alkaline simulants revealed essentially the same appearance and dissipation of color (for simulant A) as the 12-day samples. In all cases the solvent phase remained clear. Again, however, the solvent in contact with the scrub and strip solutions displayed an increasingly pronounced yellow tint with increasing length of exposure: for the 235-day samples the solvent in contact with the scrub solution at $61{ }^{\circ} \mathrm{C}$ was a deep, almost "canary" yellow when compared with the 12-day samples. The color became somewhat deeper and slightly more orange when the solvent was contacted with alkaline simulant in the ESS tests. This indicates perhaps the presence of nitrated phenolic species, which are known to exhibit a bathochromic shift of the electron-transfer band and a heightening of the color intensity upon deprotonation. It should be pointed out that even $10^{-5} \mathrm{M}$ of highly absorbing species can give rise to color but, as shall be reported below, not be detected by analysis or by effect on performance. As a fraction of bulk solvent components, the colored species thus represent negligible degradation, but they can also arise from reactions of solvent impurities, such as the trace phenols known to be present. These trace phenols could become nitrated to give rise to highly colored species. 
Table 5.2. Appearance of organic and aqueous phases following 12 (-2 series), 46 (-7 series), $110\left(-16\right.$ series), and $235(-33$ series $)$ day incubation at $34.8 \pm 0.5^{\circ} \mathrm{C}$ and $61.2 \pm 0.6^{\circ} \mathrm{C}$

\begin{tabular}{|c|c|c|c|}
\hline Sample code & Aqueous phase & $\begin{array}{l}\text { Appearance of } \\
\text { organic phase }\end{array}$ & Appearance of aqueous phase \\
\hline C-35-2 & None & Clear and colorless & \\
\hline C-35-7 & $"$ & " $\quad " \quad "$ & \\
\hline$C-35-16$ & $"$ & $"$ & \\
\hline$C-35-33$ & $"$ & $" \quad "$ & \\
\hline C-60-2 & $"$ & Clear and colorless & \\
\hline C-60-7 & $"$ & $"$ & \\
\hline C-60-16 & $"$ & $"$ & \\
\hline C-60-33 & 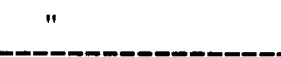 & $" \quad "$ & \\
\hline A-35-2 & Simulant A & Clear and colorless & Yellow, some white ppt \\
\hline A-35-7 & $" \quad "$ & $\begin{array}{l}\text { V. Faint yellow tint, } \\
\text { disappears with time }\end{array}$ & " " " " \\
\hline A-35-16 & $"$ & " " & " $\quad$ " \\
\hline A-35-33 & $"$ & $" \quad "$ & $" \quad " \quad "$ \\
\hline A- $60-2$ & $"$ & Same as A-35-7 & Yellow, white ppt \\
\hline A- $60-7$ & $"$ & $" \quad " \quad "$ & " " " \\
\hline$A-60-16$ & $"$ & $"$ & $" \quad "$ \\
\hline A-60-33 & $"$ & $"{ }_{n}$ & " \\
\hline B-35-2 & Simulant B & Clear and colorless & Faint blue tint, some white and tan ppt \\
\hline B-35-7 & $" \quad$ " & " $\quad " \quad "$ & " " " " " " " " " " \\
\hline B-35-16 & $"$ & $"$ & 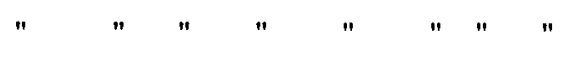 \\
\hline B-60-2 & $"$ & Clear and colorless & Faint blue tint, some white and tan ppt \\
\hline B-60-7 & $"$ & $" \quad " \quad "$ & " $\quad$ " $\quad$ " \\
\hline B-60-16 & $"$ & $n$ & " \\
\hline SC-35-2 & Scrub solution & Clear and colorless & Clear and colorless \\
\hline SC-35-7 & $"$ & Clear w/ v. pale yellow tint & $"$ \\
\hline$S C-35-16$ & $"$ & " & $"$ \\
\hline SC-35-33 & $"$ & $" \quad " \quad " \quad "$ & $" \quad " \quad "$ \\
\hline SC-60-2 & $"$ & Same as $\mathrm{SC}-35-7$ & Clear and colorless \\
\hline SC-60-7 & $"$ & Clear yellow tint & " " " " \\
\hline SC-60-16 & $"$ & " $\quad " \quad$ & Clear with pale yellow tint \\
\hline SC-60-33 & 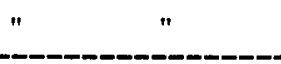 & Clear deeper yellow tint & " \\
\hline ST-35-2 & Strip solution & Clear and colorless & Clear and colorless \\
\hline ST-35-7 & $"$ & $"$ & $" \quad "$ \\
\hline ST-35-16 & $"$ & $"$ & $"$ \\
\hline ST-35-33 & $"$ & Clear w/v. pale yellow tint & $"$ \\
\hline ST-60-2 & $"$ & Clear and colorless & Clear and colorless \\
\hline ST-60-7 & $"$ & " " " & " " " \\
\hline ST-60-16 & $"$ & $"$ & $"$ \\
\hline ST-60-33 & $"$ & Clear w/pale yellow tint & $"$ \\
\hline
\end{tabular}




\subsubsection{Nitric Acid Thermal-Stability Test}

The aim of this experiment was to examine the effect of elevated concentrations of nitric acid on the solvent components, particularly with regard to nitration reactions, at temperatures of 35 and 61 ${ }^{\circ} \mathrm{C}$. The results of these experiments will serve to identify nitration products and will support options for scrubbing, stripping, and washing (e.g., how high a concentration of nitric acid can be used without excessive damage to the solvent). The test involved rotating $10-\mathrm{mL}$ aliquots of solvent Cs$7 \mathrm{SB} /$ Isopar L, lot B000718-156W, with equal volume amounts of water, and nitric acid (at 1, 50, 300, 700 and $1000 \mathrm{mM}$ ) at $35^{\circ} \mathrm{C}$ and $61{ }^{\circ} \mathrm{C}$, and sampling for analysis by NMR before incubation (time = 0 ), and at times of $7,14,21$, and 28 days.

In general, and not surprisingly, the solvents became increasingly yellow as a function of exposure time to the nitric acid, and the color intensity increased as a function of nitric acid concentration and temperature. The strong yellow coloration seen with some solvent samples is indicative of nitration of aromatic rings, presumably either on the BOBCalixC6, the modifier, or both. Another noted visual phenomenon has been the cloudiness seen in the sample contacted with 1000 $\mathrm{mM}(1 \mathrm{M}) \mathrm{HNO}_{3}$ at $61^{\circ} \mathrm{C}$ at both the 21 - and 28 -day test points. These samples indicated a waterclear strong yellow color upon removal from the centrifuge at the phase isolation step. But within 3-4 min after sitting on the bench at room temperature, the samples became increasingly cloudy, suggesting a decrease in the solubility of the nitrated species (presumably the calix) with decreasing temperature. As noted with the 28-day sample, as the solvent phase becomes increasingly cloudy, a strong yellow color builds into the aqueous phase. This suggests that some colored complex is moving from the organic phase into the aqueous phase as the temperature decreases from $61{ }^{\circ} \mathrm{C}$ to room temperature $\left(\sim 22^{\circ} \mathrm{C}\right)$. This strong yellow colored aqueous phase has not been seen, as yet, in any other samples.

The appearance of the phases following four weeks exposure is described in Table 5.3. For the solvent phases, the appearance is noted following separation from the aqueous phases, and standing in Teflon FEP tubes for several weeks in the dark. The analysis of aliquots of the solvent removed at the given sampling times will be described below in the NMR section. It was noted that in sample 60F ( 1 $\mathrm{M}$ nitric at $61^{\circ} \mathrm{C}$; the sample that showed the cloudiness above) phase separation had occurred, with a bottom orange oily phase (about $5 \%$ of the total volume), and a yellow phase on top (less yellow than sample $60 \mathrm{E}$, which was still one continuous phase). These separated phases were also analyzed by proton NMR as will be described below.

\subsubsection{Break-Time Results and Observations for Thermal-Stability Samples}

From the contacting results (Table 5.4), there were no conclusive trends seen in the break times for given solvent/aqueous phases as a function of exposure time and temperature. More importantly, there was no evidence of deterioration of break times or coalescence behavior in general for scrubbing and especially stripping. The break times and coalescence behavior on stripping would presumably be more sensitive to the presence of surface-active materials, and so if such species were being formed, an increase in the break times would be expected. However, since this was not the case, it can be concluded that surface-active materials that negatively impacted solvent coalescence behavior were not being generated. Very favorable comparisons were observed between the 110-day and 235-day test results, where the observation criteria were very strictly held. In the tests performed with the simulants for the 12-day samples, there was accidentally some solid precipitate from the simulants still present in the system, which contributed to a lengthening of the break time. When the precipitates were not present in the system, break times for the simulants are satisfactory, and there is no indication of a trend of lengthening break times. Thus, break times and coalescence behavior remained relatively stable with no indication of erosion over the course of the 235-day thermalstability experiment. 
Table 5.3. Appearance of organic and aqueous phases following 4 weeks incubation at $34.8 \pm 0.5$

${ }^{\circ} \mathrm{C}$ and $61.2 \pm 0.6^{\circ} \mathrm{C}$ with aqueous phases of varying nitric acid concentration (organic phases after standing for two weeks separated from aqueous)

\begin{tabular}{|c|c|c|c|}
\hline Sample code & Aqueous phase & Appearance of organic phase & Appearance of aqueous phase \\
\hline $36 \mathrm{~A}$ & Water & Clear and colorless & Clear and colorless \\
\hline $36 \mathrm{~B}$ & $1 \mathrm{mM}$ Nitric acid & $"$ & $"$ \\
\hline $36 \mathrm{C}$ & $50 \mathrm{mM}$ Nitric acid & Very pale yellow & $"$ \\
\hline $36 \mathrm{D}$ & $300 \mathrm{mM}$ Nitric acid & Pale yellow & $"$ \\
\hline $36 \mathrm{E}$ & $700 \mathrm{mM}$ Nitric acid & Yellow & $"$ \\
\hline $36 \mathrm{~F}$ & 1.0 M Nitric acid & Deeper yellow (like SC-60-33) & $"$ \\
\hline $60 \mathrm{~A}$ & Water & Clear and colorless & Clear and colorless \\
\hline $60 \mathrm{~B}$ & $1 \mathrm{mM}$ Nitric acid & " $\quad "$ & $"$ \\
\hline $60 \mathrm{C}$ & $50 \mathrm{mM}$ Nitric acid & Pale yellow (like 36D) & $"$ \\
\hline 60D & $300 \mathrm{mM}$ Nitric acid & Yellow (like 36E) & $"$ \\
\hline $60 \mathrm{E}$ & $700 \mathrm{mM}$ Nitric acid & Deeper yellow (like 36F) & $"$ \\
\hline $60 \mathrm{~F}$ & 1.0 M Nitric acid & $\begin{array}{l}\text { Phase separation on standing for } \\
\text { several weeks. Top } 95 \% \text { phase is } \\
\text { pale yellow; bottom } 5 \% \text { phase is } \\
\text { orange. }\end{array}$ & Yellow (see text) \\
\hline
\end{tabular}

Table 5.4. Phase disengagement break times ${ }^{a}$ at $0,12,46,110$, and 235 days

\begin{tabular}{|c|c|c|c|c|c|c|c|c|c|c|c|c|}
\hline \multirow[t]{3}{*}{ Aqueous phase } & \multirow{2}{*}{\multicolumn{2}{|c|}{$\begin{array}{l}\text { Volume } \\
(\mathrm{mL})\end{array}$}} & \multirow{3}{*}{$\begin{array}{c}\text { Vial } \\
\text { diameter } \\
(\mathrm{mm})\end{array}$} & \multicolumn{9}{|c|}{ Break times } \\
\hline & & & & \multirow{2}{*}{$\frac{0 \text { day }}{25^{\circ} \mathrm{C}}$} & \multicolumn{2}{|c|}{12 days $b$} & \multicolumn{2}{|c|}{46 days } & \multicolumn{2}{|c|}{110 days } & \multicolumn{2}{|c|}{235 days } \\
\hline & Org & $\mathrm{Aq}$ & & & $35^{\circ} \mathrm{C}$ & $61^{\circ} \mathrm{C}$ & $35^{\circ} \mathrm{C}$ & $61{ }^{\circ} \mathrm{C}$ & $35^{\circ} \mathrm{C}$ & $61{ }^{\circ} \mathrm{C}$ & $35^{\circ} \mathrm{C}$ & $61{ }^{\circ} \mathrm{C}$ \\
\hline $\begin{array}{l}\text { Full simulant } \\
\text { control }\end{array}$ & 5 & 15 & 25.5 & $\mathrm{ND}$ & 3,30 & 3,30 & $1,52^{c}$ & $1,52^{c}$ & $3,57^{c}$ & $4,29^{c}$ & $2,53^{c}$ & $2,55^{c}$ \\
\hline Full simulant & 5 & 15 & 25.5 & 3,30 & 6,00 & 6,00 & 3,10 & 3,10 & 2,58 & 3,27 & 2,00 & 3,48 \\
\hline Salts-only simulant & 5 & 15 & 25.5 & 3,30 & 5,00 & 7,30 & 1,31 & 2,00 & 1,49 & 1,51 & $\mathrm{ND}$ & $\mathrm{ND}$ \\
\hline Scrub & 5 & 1 & 17.0 & 3,10 & 1,30 & 1,30 & 2,00 & 2,00 & 2,00 & 1,56 & 1,59 & 2,06 \\
\hline Strip & 5 & 1 & 17.0 & ND & 1,30 & 1,30 & 2,00 & 2,00 & 2,01 & 2,04 & 1,58 & 2,16 \\
\hline
\end{tabular}

aTimes are given in minutes,seconds. Note: ND designates "not determined."

$b$ Precipitate was observed in the aqueous phases of the 12-day samples. These undissolved salt precipitates had been mixed into the aqueous phases that were transferred over for the ESS and break time tests, likely contributing to erroneously high break times.

cFull simulant Draw \#3 was used for the 46-day control sample while full simulant Draw \#5 was used for 110- and 235-day control samples. 


\subsubsection{Extract/Scrub/Strip Performance of Thermal-Stability Samples over the Course of 235 Days}

A component of addressing the chemical risk factors for caustic-side solvent extraction involves testing the CSSX solvent for thermal stability under the various process conditions. This exercise has served as a test of the thermal stability and robustness of the CSSX solvent to prolonged contacting periods with simulant, scrub and strip solutions, while also introducing the element of thermal stress. As previously discussed, to address this issue baseline solvent (lot B000718-132W) was tested in five tandem contacting experiments conducted at two different temperatures $\left(35^{\circ} \mathrm{C}\right.$ and $\left.61{ }^{\circ} \mathrm{C}\right)$ for a total time period of 33 weeks ( 235 days). The samples in this experiment included 1) solvent only, 2) solvent contacting full simulant $(\mathrm{O} / \mathrm{A}=0.33), 3)$ solvent contacting salts only simulant $(\mathrm{O} / \mathrm{A}=0.33)$, 4) solvent contacting scrub solution $(\mathrm{O} / \mathrm{A}=5)$, and 5) solvent contacting strip solution $(\mathrm{O} / \mathrm{A}=5)$. At stated time intervals solvent was withdrawn from the test system and the ESS performance was evaluated.

The results of the ESS evaluation of the thermal stability samples showed the solvent to be remarkably stable. Stripping behavior in particular held up well, with the only indication of significant degradation in performance being observed for solvent that had been exposed to the 50 $\mathrm{mM}$ nitric acid scrub solution at $61{ }^{\circ} \mathrm{C}$ for 235 days. As will discussed in detail in Sections 5.3 .5 and 5.3.6, the slight erosion of stripping performance observed in the SC-60-33 sample is a direct consequence of loss of trioctylamine. It should be noted that both the temperature and length of continuous contacting time for that sample are extreme cases that would not be encountered in routine operation of the CSSX process flowsheet.

Cesium distribution values for all contacts for all samples are provided in Table 5.5 and Figures 5.4 through 5.8. The figures show the extraction, scrubbing, and three stripping distribution ratios for each thermal sample (C, A, B, SC, or ST series) for each temperature as a function of time. These data points are plotted within the statistical bounds previously established for the ESS procedure for pristine washed solvent (see Table 3.4). If the data remain within bounds, then the performance is within the $95 \%$ confidence interval established for the ESS protocol. However, if the data fall outside the bounds, this is taken to indicate an effect of degradation. Although such change could indicate worsened solvent performance, flowsheet requirements would still be met as long as $D_{\mathrm{Cs}}$ values remained within specified limits. ${ }^{20}$ Mainly of interest is whether there are statistically significant deviations that reveal a trend towards degradation in solvent performance in the ESS protocol.

As can be seen from Figures 5.4 to 5.6 for the solvent alone, and in contact with the two simulants, there are no indications of a statistically significant trend indicating a fall-off in performance. Though there exist a few outliers that fall slightly above or below the bounds, the succeeding data point was observed to be back within bounds (e.g., samples A-35-16 at 110 days for extraction and scrubbing, where the data was out of bounds, but the corresponding A-35-33 data was back within bounds.) After 110 days, the experiment in which solvent was contacted with the saltsonly (B) simulant at $35^{\circ} \mathrm{C}$ and $61{ }^{\circ} \mathrm{C}$ was terminated due to the lack of adverse effects on the solvent. Up to that time, the lack of discernible differences in behavior between the full simulant and saltsonly simulant indicated that the added potential catalysts (noble metals) that are present in the full simulant have essentially no adverse effect on cesium distribution.

However, as can be seen in Figure 5.7 for the (SC) series, there are indications of statistically significant deviation trends from the boundaries, especially for the stripping operations for the SC-60 series. The stripping values for the SC-60-33 (235-day) sample was significantly elevated for all three strips, (as well as the scrub operation), indicating a genuine fall-off in stripping performance. Again, as will be discussed in more detail below, the reason for this is that the trioctylamine (TOA) in this solvent sample had been significantly degraded. A certain minimum concentration of TOA is needed to ensure the low $(<0.2)$ cesium distribution ratios can be achieved upon stripping.

In Figure 5.8 for the strip (ST) series, some statistically significant deviation is observed for the first stripping operation in both the ST-35 and ST-60 series, and a correlation was found with a somewhat diminished TOA content as will be further described in Section 5.3.6. However, the 
Table 5.5. Extraction, scrub, and strip protocol results for thermal stability test samples ${ }^{a}$

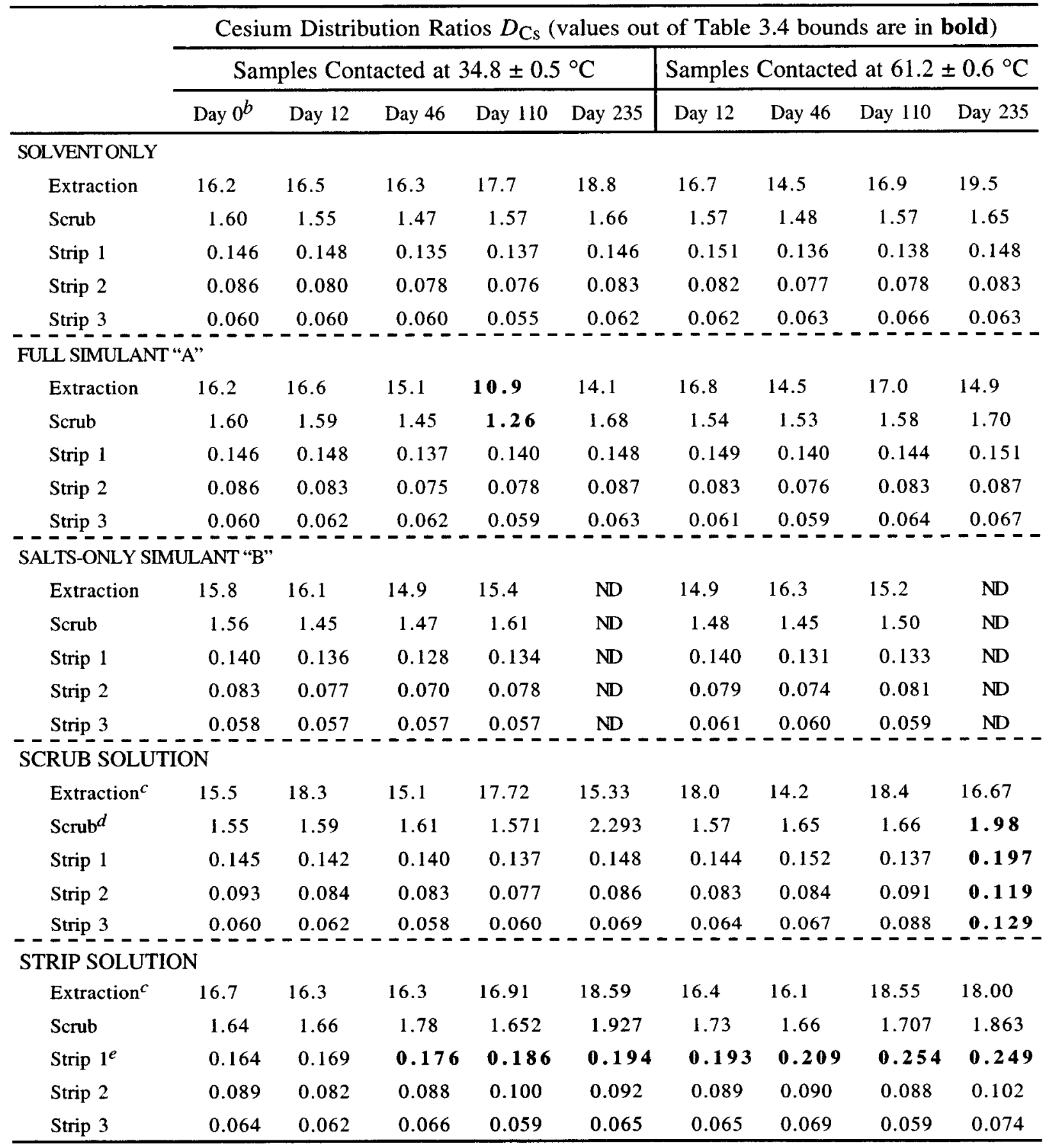

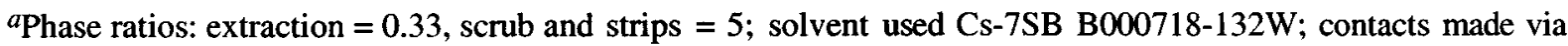
end-over-end rotation using a Glas Col rugged rotator for 35 minutes; experimental series begun on 6/24/00 and completed $2 / 15 / 01$.

bay zero values used full simulant ("A") for solvent only, full simulant "A", scrub solution, and strip solution tests; salts only simulant used for simulant "B" test.

${ }^{c}$ A second cycle started at this step.

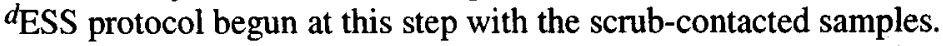

${ }^{e}$ ESS protocol begun at this step with the strip-contacted samples. Note: ND indicates that this test was not performed. 

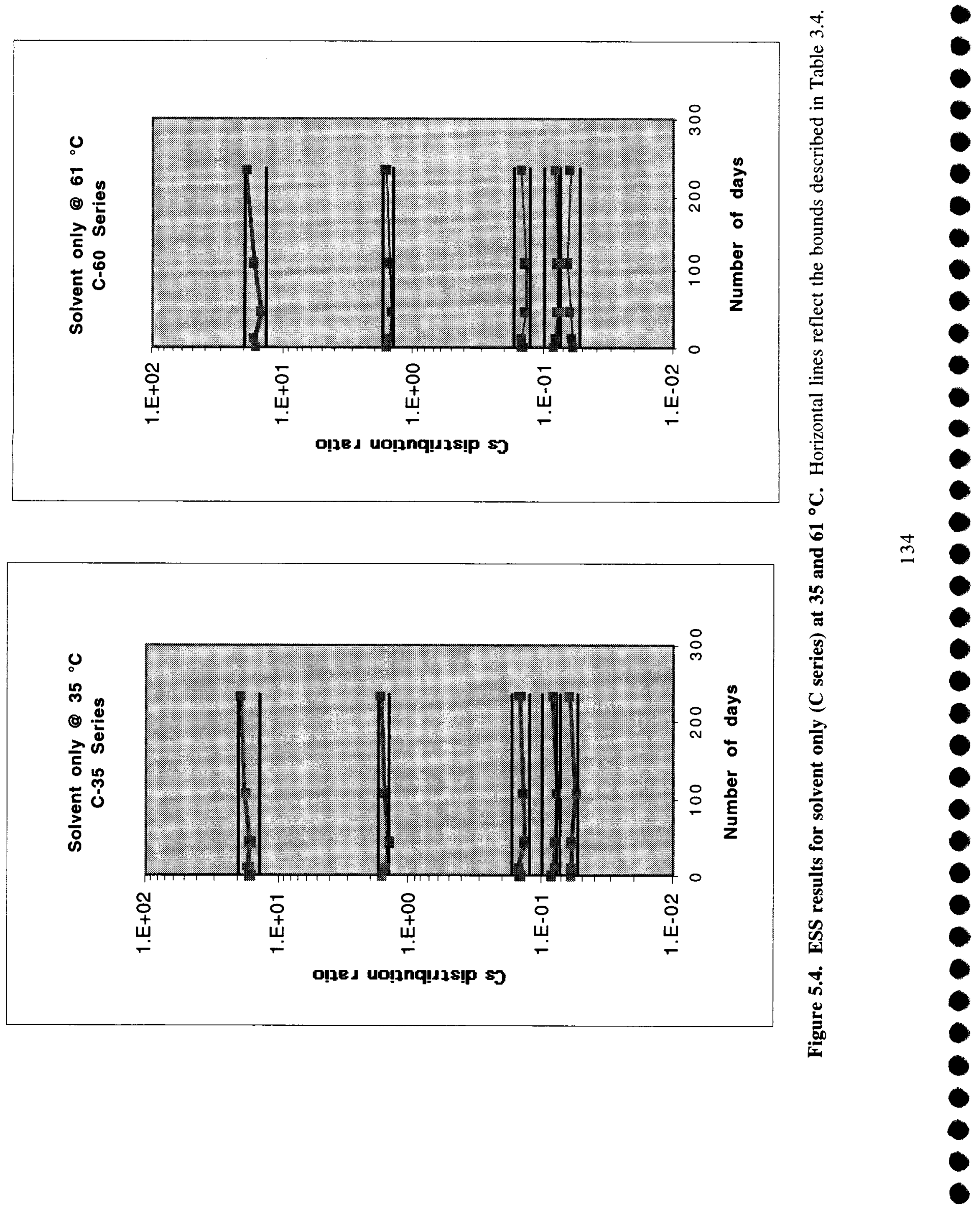

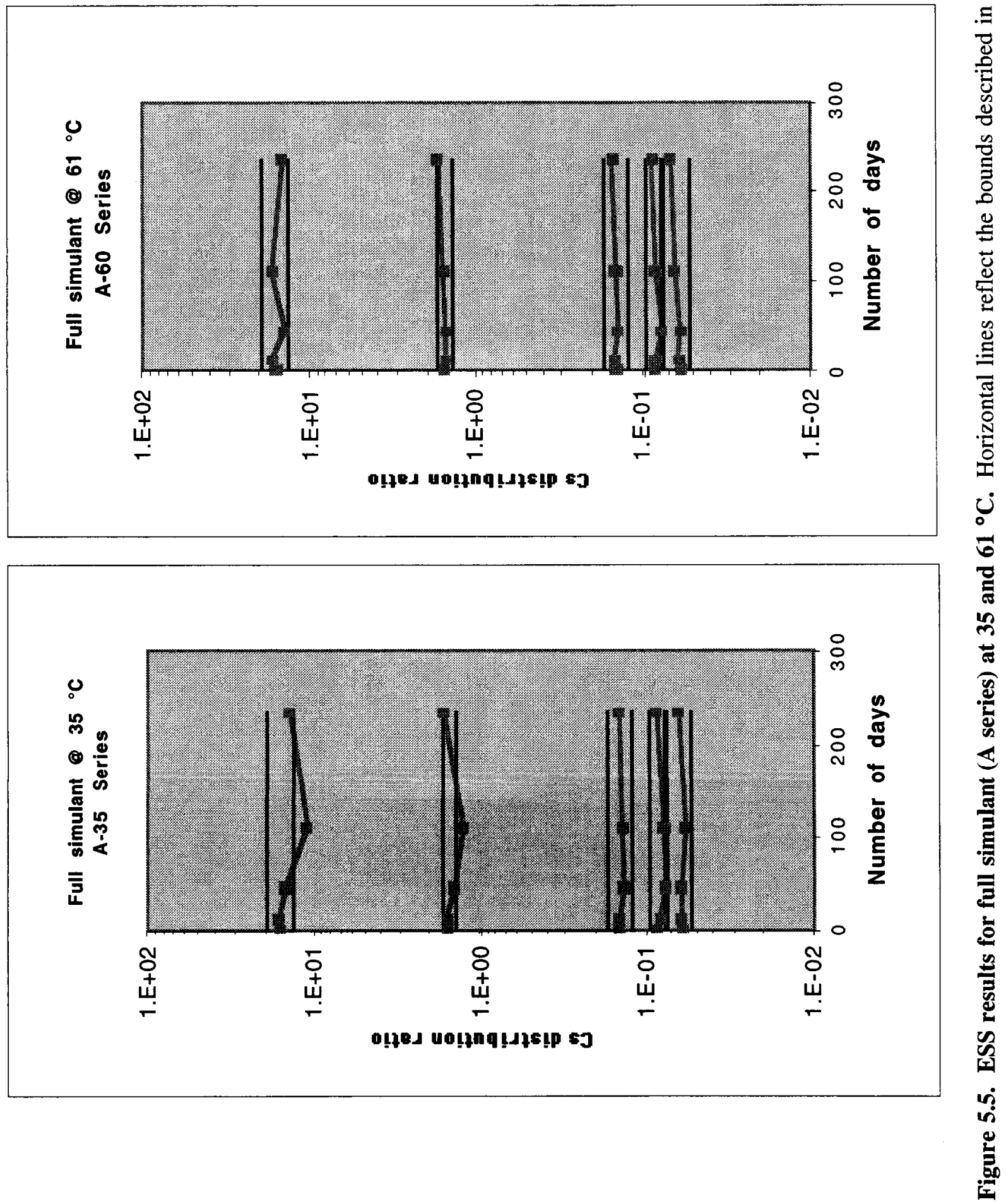


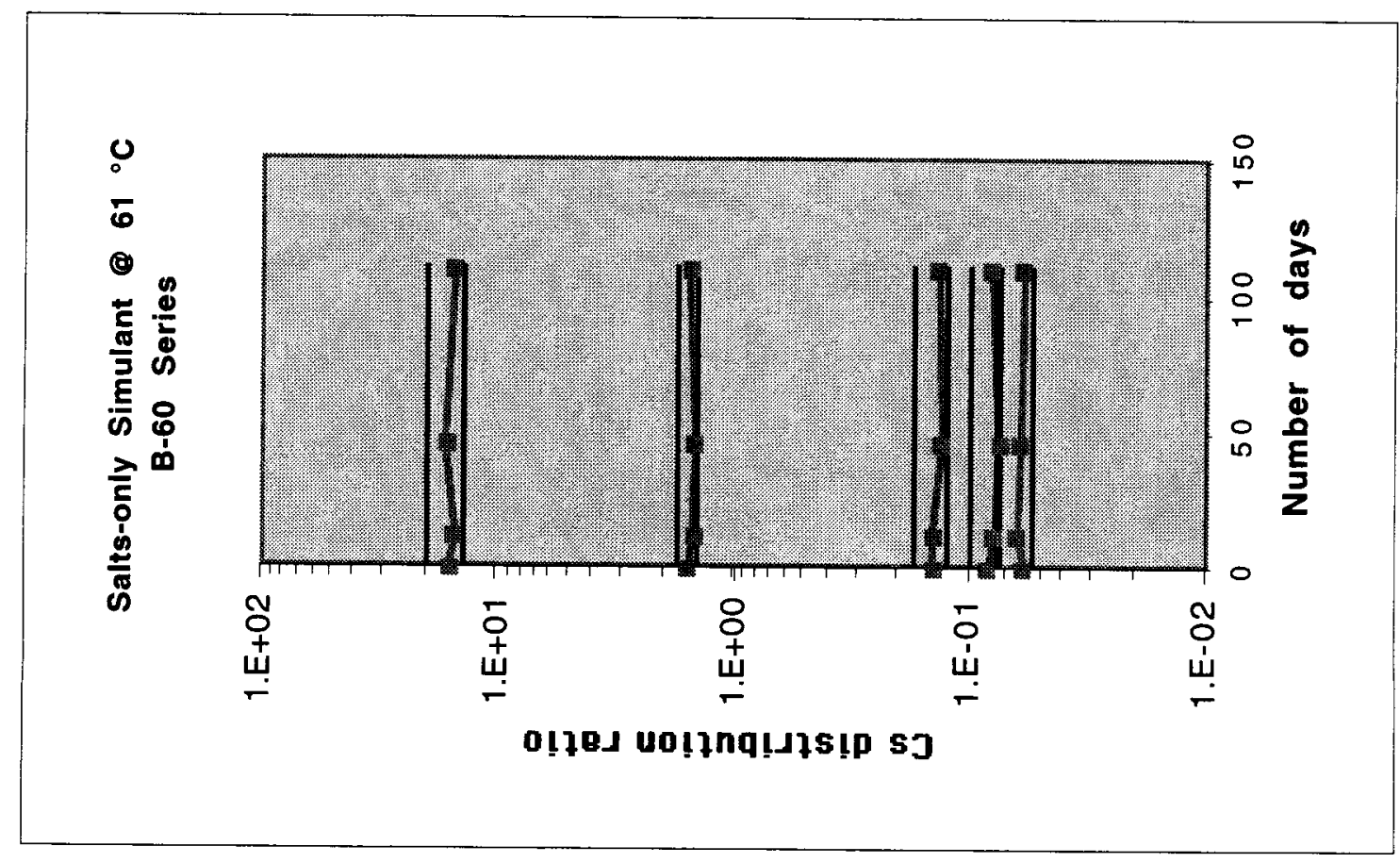

列

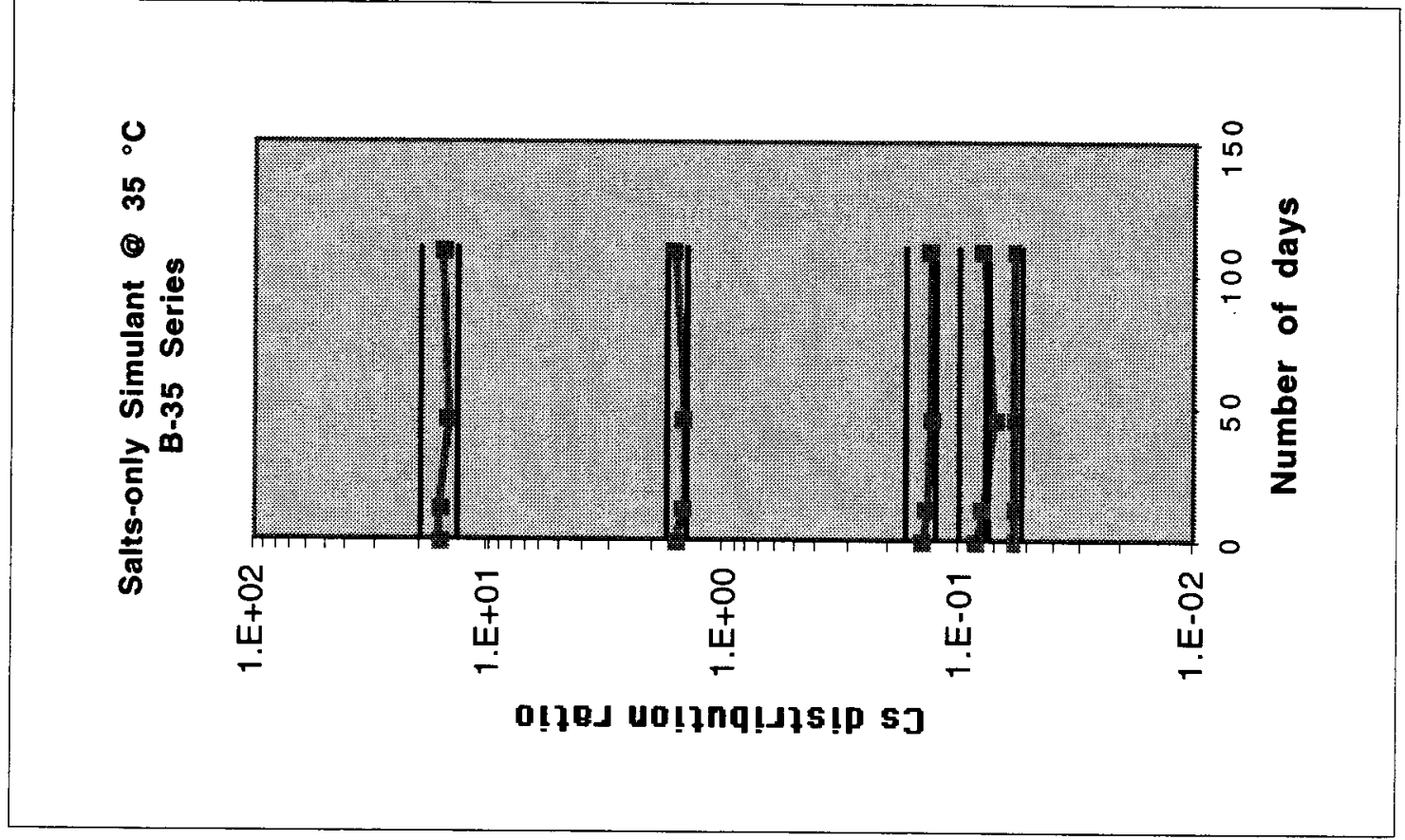



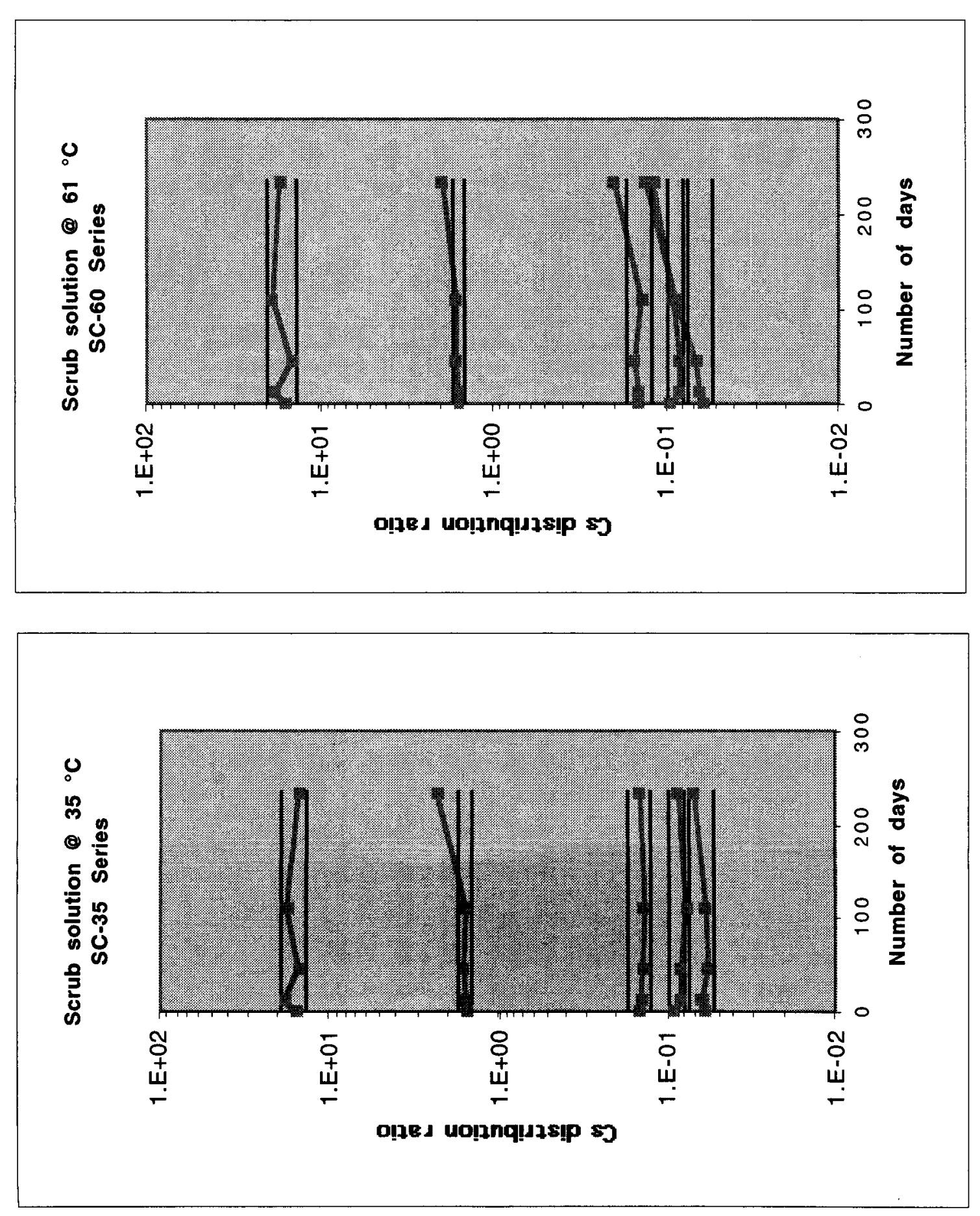

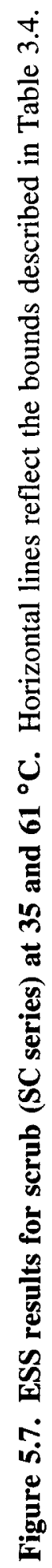



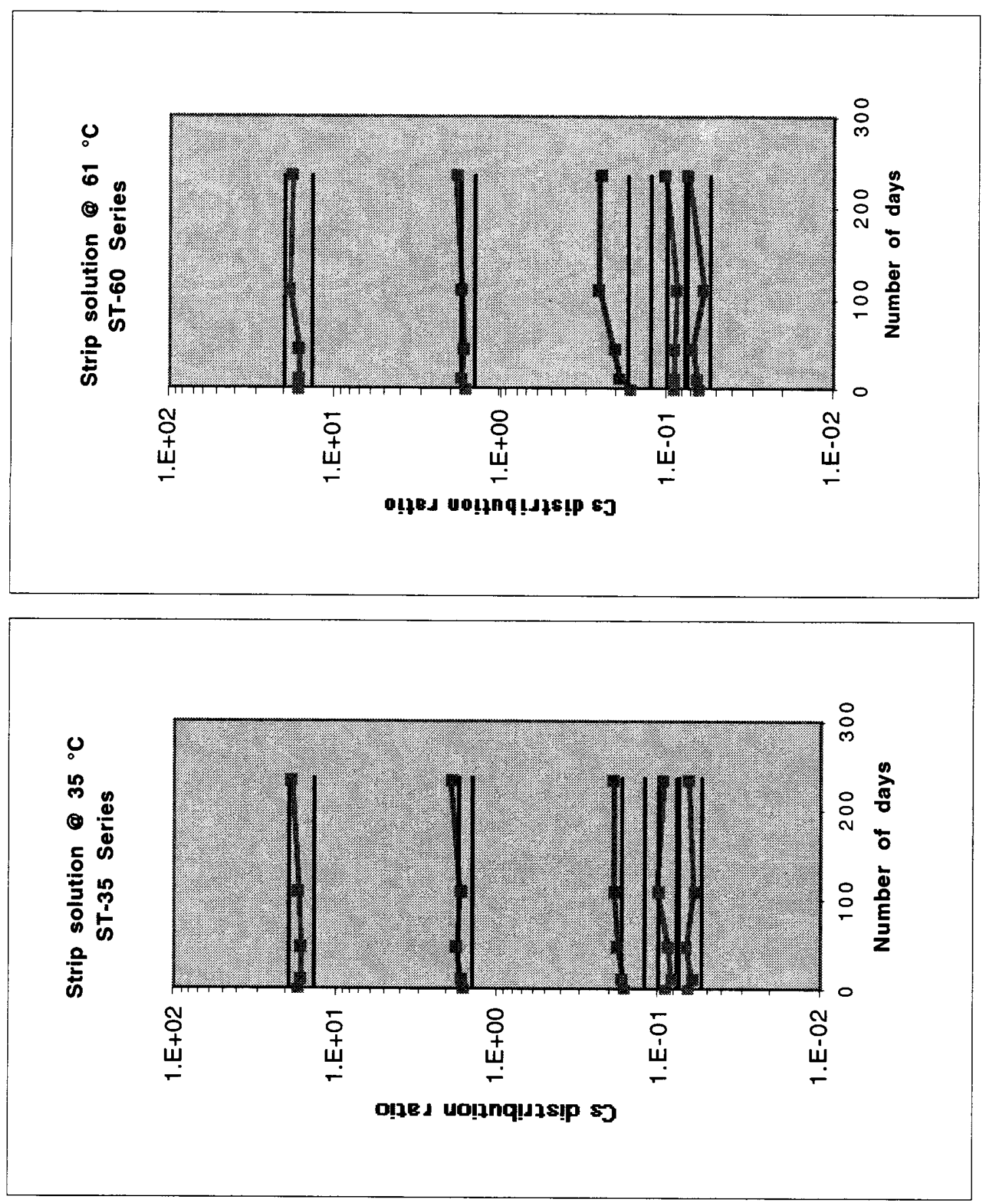

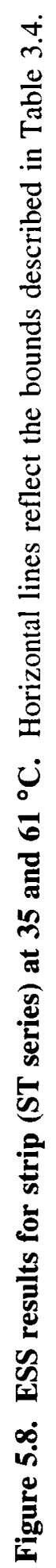


stripping $D_{\mathrm{Cs}}$ values were found to return to values that were within bounds for the second and third stripping operations (see also Table 5.5).

\subsubsection{NMR Analysis}

\subsubsection{Thermal-Stability Test with Flowsheet Aqueous Phases}

Analyses of aliquots of the solvents from the thermal stability test by proton NMR did not reveal any observable signs of solvent degradation. Although the solvent in contact with the scrub solution takes on an increasingly yellow hue with increasing exposure time and temperature, the amount of probable nitrated material corresponding to that color is very small, almost undetectable from baseline noise by NMR. Comparing pristine solvent with that of the control solvent exposed to $61{ }^{\circ} \mathrm{C}$ shows no difference, and they in turn show essentially no difference (above baseline noise) in the key aromatic region with either the strip or scrub samples exposed at either $35{ }^{\circ} \mathrm{C}$ or $61{ }^{\circ} \mathrm{C}$. Degradation of the TOA cannot be observed by proton or carbon NMR due to interference from the Isopar L diluent (the resonances from the octyl groups are obscured by the resonances from the aliphatic Isopar).

\subsubsection{Nitric Acid Thermal-Stability Test}

Proton NMR analyses of aliquots of the solvents from this test of exposure of the solvent to higher concentrations of nitric acid revealed that small amounts of nitrated modifier do form, but only under the most severe conditions ( $\geq 300 \mathrm{mM}$ nitric acid, $61^{\circ} \mathrm{C}, 28$ days exposure). No evidence of nitration of the calix was noted. In this 4-week duration experiment, solvent was contacted at an $\mathrm{O} / \mathrm{A}$ ratio of 1 with various nitric acid-containing aqueous phases $(0,1 \mathrm{mM}, 50 \mathrm{mM}, 300 \mathrm{mM}, 700 \mathrm{mM}$, and $1 \mathrm{M}$ $\mathrm{HNO}_{3}$ ) at either $35^{\circ} \mathrm{C}$ or $61{ }^{\circ} \mathrm{C}$. The exercise was designed to look into the possible nitration of either calixarene or solvent modifier under these prolonged conditions of acid concentration and temperature. Aliquots $(100 \mu \mathrm{L})$ of solvent were drawn at times of $0,1,2,3$, and 4 weeks and placed in NMR tubes. These samples were each diluted with $700 \mu \mathrm{L}$ of $\mathrm{CDCl}_{3}$ and the proton NMR spectra recorded. The critical region in the proton NMR spectrum where changes would be noted is the aromatic region spanning 9 to $6.5 \mathrm{ppm}$. Upon nitration of the aromatic rings, shifts in existing resonances with concomitant changes in coupling patterns associated with the replacement of an aromatic proton with a nitro group would be observed relative to the resonances of the parent calix and modifier. Nitrated modifier and calix were independently prepared by reaction of solutions of either calix or modifier in deuterochloroform with $5 \mathrm{M}$ nitric acid at $35^{\circ} \mathrm{C}$. The spectra for these nitrated materials were then used to assist in making peak assignments for the solvent samples.

Examination of the region between 9 and $6.5 \mathrm{ppm}$ for the samples exposed for 4 weeks at $61{ }^{\circ} \mathrm{C}$ (the most severe case) showed no signs of nitration or degradation of either the calix or the modifier at nitric acid concentrations up to and including $50 \mathrm{mM}$. However at $300 \mathrm{mM}$ nitric acid, small resonances were observed to grow in at 7.5 and $7.95 \mathrm{ppm}$ (these resonances are a few percent at most of the intensity of the peaks due to the parent calix). With increasing nitric acid concentration these resonances increase in intensity, particularly the resonance at $7.95 \mathrm{ppm}$, and a correspondingly intense resonance at $6.97 \mathrm{ppm}$. In addition, small resonances were also observed to grow in for the $300 \mathrm{mM}$ to $1 \mathrm{M}$ nitric acid samples in the olefinic region of 4.8 to $4.4 \mathrm{ppm}$. However, it is important to note that the resonances due to the calix are still present at about the intensity as would be found in a pristine sample, within the limits of integration $( \pm 10 \%)$.

Modifier alone at $0.50 \mathrm{M}$ in deuterochloroform was contacted with $5 \mathrm{M}$ nitric acid for 1 month at $35^{\circ} \mathrm{C}$, then analyzed using proton NMR. These experimental results coupled with information found in the literature, reveal the above new resonances and their coupling patterns are due to the degradation of the modifier. There appears to be no NMR evidence that the calix is being nitrated. Most interestingly, the resonances observed for the degradation product are not consistent with simple nitration of the aromatic carbon ortho to the ether group (meta to the 4-sec-butyl group), but are consistent with replacement of the 4-sec-butyl alkyl group with the nitro group at the para position. By setting the integration value of the modifier aromatic ring protons to $100 \%$, a rough quantitation 
of the degree of de-alkylation of the modifier was obtained. Over the course of the experiment the amount of modifier being so converted was quite small, amounting to about $3.6 \%$ after 28 days at 61 ${ }^{\circ} \mathrm{C}$ while contacting with $1 \mathrm{M}$ nitric acid (see Table 5.6). There is literature precedent for displacement of alkyl groups on aromatic rings by nitric acid. 70 The fate of the displaced alkyl group will be the formation of olefinic products (most likely various butenes). The olefinic protons would be observed between 5.5 and $4.4 \mathrm{ppm}$, depending on the chemical environment. The fact that resonances (albeit very small) are seen at $4.8-4.4 \mathrm{ppm}$ in the samples showing the para-nitrated modifier is consistent with displacement of the 4-sec-butyl group to liberate butene products. Although butenes are gases at room temperature, very small amounts may stay dissolved in the solvent.

As mentioned above, the 60F sample showed phase separation upon standing for several weeks. Aliquots $(100 \mu \mathrm{L})$ were removed from the bottom phase, the middle of the upper phase, and the very top of the upper phase, and each aliquot examined by proton NMR. The analyses indicated that the modifier, calix, and nitrated material were settling away from the Isopar L. In the bottom phase, the modifier and calix had moved together (their concentration ratio remained essentially the same) with an effective 3.8 -fold increase in concentration in the solvent. The ratio of the HMB peak to the $-\mathrm{CF}_{2} \mathrm{H}$ modifier peak was now 1.32 to one, instead of 5 to one as is observed for both pristine solvent and for (the NMR spectrum of) sample 60F taken right after removal from the incubator. Thus, the modifier concentration in the bottom layer is approximately $1.9 \mathrm{M}$ (pure modifier is about $3.3 \mathrm{M}$ ), and the calix is $0.038 \mathrm{M}$. The nitrated material appears to be enriched in the bottom layer, as it now comprised about $6 \%$ relative to the modifier (up from $3.6 \%$ in the original sample prior to phase separation). The sample taken at the top of the upper layer was mostly Isopar $\mathrm{L}$, with an approximately two-fold decrease in the concentration of both the modifier and the calix. The nitrated material comprised only $1 \%$ relative to the modifier, which is consistent with the observed lightening of the yellow color of this phase. Interestingly, the sample taken at the middle of the upper phase analyzed as more concentrated in the calix and modifier (about two-fold increase in modifier and calix concentration).

Overall, it is evident by proton NMR that the solvent is remarkably stable to fairly high concentrations of nitric acid for up to 1 month continuous contact, with less than $4 \%$ of the modifier being degraded. Over time, that $4 \%$ of nitrated material appears to bring about an oiling-out of the modifier and calix from the diluent.

Table 5.6. Amount of product formed by replacement of the modifier sec-butyl group with a nitro group as a function of time, temperature, and nitric acid concentration

\begin{tabular}{cccc}
\hline Time (days) & $\begin{array}{c}\text { Nitric acid } \\
\text { concentration }\end{array}$ & $\begin{array}{c}\text { Percent de-alkylated } \\
\text { modifier at } 34.8 \pm 0.5{ }^{\circ} \mathrm{C}\end{array}$ & $\begin{array}{c}\text { Percent de-alkylated } \\
\text { modifier at } 61.2 \pm 0.6^{\circ} \mathrm{C}\end{array}$ \\
\hline 7 & $300 \mathrm{mM}$ & Negligible & Negligible \\
14 & $300 \mathrm{mM}$ & $n$ & $"$ \\
21 & $300 \mathrm{mM}$ & $"$ & $"$ \\
28 & $300 \mathrm{mM}$ & Negligible & Trace \\
7 & $700 \mathrm{mM}$ & $"$ & 0.56 \\
14 & $700 \mathrm{mM}$ & Trace & 0.88 \\
21 & $700 \mathrm{mM}$ & Trace & 1.3 \\
28 & $700 \mathrm{mM}$ & Negligible & 0.4 \\
7 & $1.0 \mathrm{M}$ & Negligible & 1.4 \\
14 & $1.0 \mathrm{M}$ & Trace & 2.4 \\
21 & $1.0 \mathrm{M}$ & Trace & 3.6 \\
28 & $1.0 \mathrm{M}$ & &
\end{tabular}




\subsubsection{Electro-Spray Mass Spectrometry}

\subsubsection{Experimental Approach and General Observations}

The ES-MS analyses confirm that TOA is increasingly degraded to dioctylamine (DOA) with increasing nitric acid concentration, temperature, and duration of exposure. The ES-MS analyses also confirm that dibutylphosphate readily partitions to the solvent. Samples corresponding to 2 weeks ( 12 days actual), and 16 weeks ( 110 days actual) at $61{ }^{\circ} \mathrm{C}$ were analyzed by ES-MS to detect the presence of ionic species, both expected (e.g., BOBCalixC6-potassium adduct), and due to potential degradation of the solvent. Tables 5.7 and 5.8 below chronicle the major expected species for anion and cation mode, respectively, with calculated $\mathrm{m} / \mathrm{z}$, and whether these species were observed in the 10 samples submitted for analysis. The ten samples were C-60-2, C-60-16, ST-60-2, ST-60-16, SC-60-2, SC-60-16, B-60-2, B-60-16, A-60-2, and A-60-16. The samples were run in that order, generally first in cation mode, then anion. There were a number of peaks that could not be readily attributed to any species. These peaks are labeled as "unknown". Some of these may arise from material that was already present on the column from analyses of materials from other projects (not CSSX). For each solvent sample, the main peak in the spectrum as well as the second main peak (if the peak is at least within $50 \%$ of the main) are noted. If there was a single dominant peak, with no other peaks being anywhere close in intensity, then no "Main-2" designation was given. The relative intensities of the other peaks are given as " $T$ " for trace, "S" for small, and "L" for large. A question mark after an intensity indicates that there is some question whether the $\mathrm{m} / \mathrm{z}$ value is correct, since there was no number assigned to that peak in the spectrum.

\subsubsection{Anion-Mode Analysis}

Analyses of the solvent samples in anion mode revealed that essentially negligible solvent degradation occurred over the course of 16 weeks ( 110 days) exposure to the various aqueous phases at $61{ }^{\circ} \mathrm{C}$, in agreement with NMR results. There were somewhat higher concentrations of 4-secbutylphenolate present in both the A-60-2 and A-60-16 samples relative to that present in the other samples, but no indication that the phenolate concentration had increased between the A-60-2 and A60-16 samples. As can be seen from Table 5.7, expected anions like nitrate and the nitrate-Cs-7SB adduct were observed in every solvent sample, and these species were often the most abundant ions in the spectrum. There was an unknown peak at $m / z=113$ that appeared to be present in all the samples which cannot be readily identified and is likely due to an impurity in the system which bleedsthrough over time; its intensity, at a maximum in the first-run solvent samples (pristine washed solvent C-60 samples) essentially decreased over the course of the sample analysis. There was also another unknown peak at $\mathrm{m} / \mathrm{z}$ of 370 that cannot be as readily explained, since it persisted throughout all the analyses. Traces of a series of anions of $\mathrm{m} / z 221,235$, and 249, reminiscent of C10-C12 sulfonate surfactant acid species, appeared in the solvent samples that had been contacted with the strip, scrub, and simulant aqueous phases. Since these species were not observed in the washed solvent samples C60-2 and C-60-16, it is likely that these ions were introduced from the chemicals used to prepare the full (A) and salts-only (B) simulants. No nitrated organic compounds (e.g., 2-nitro-4-secbutylphenolate at $m / z=194.2$, or nitrated Cs-7SB at $m / z=382.3$, or para-nitrated de-alkylated Cs$7 \mathrm{SB}$ at $m / z=326.2$ ) were observed. Traces of dibutylphosphate were observed in all samples except the pristine washed solvent; however the presence of DBP in the solvent that was in contact with the salts-only simulant must be due to bleed through from the strip and scrub samples that were analyzed first, since the salts-only simulant does not contain any added phosphates. Both mono- and bisadducts of the Cs-7SB modifier with DBP (at $\mathrm{m} / z$ of 547.3 and 885.7 , respectively) were also observed whenever DBP was observed. The presence of these adducts indicates that the modifier may assist in extracting the DBP into the solvent phase. 


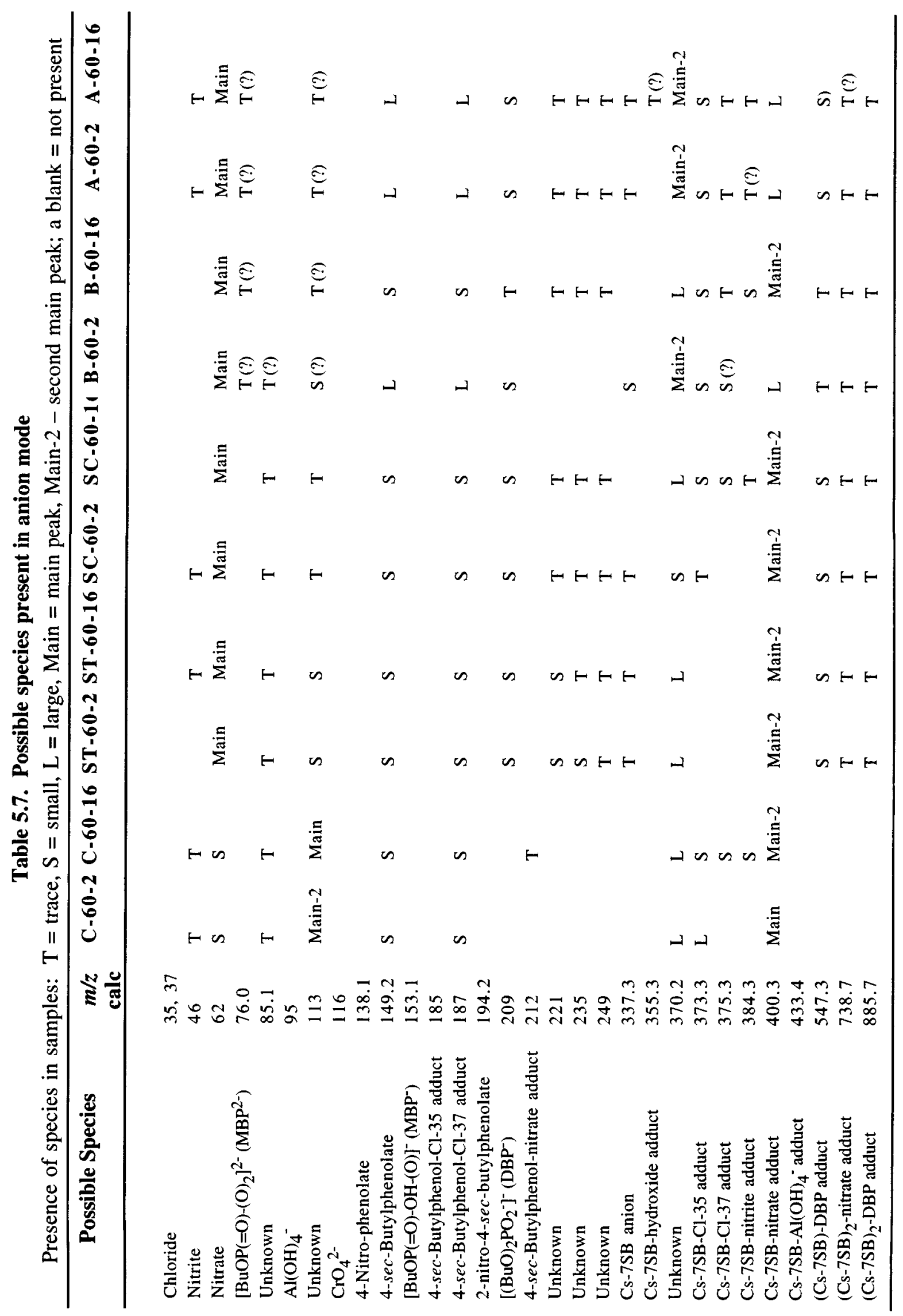




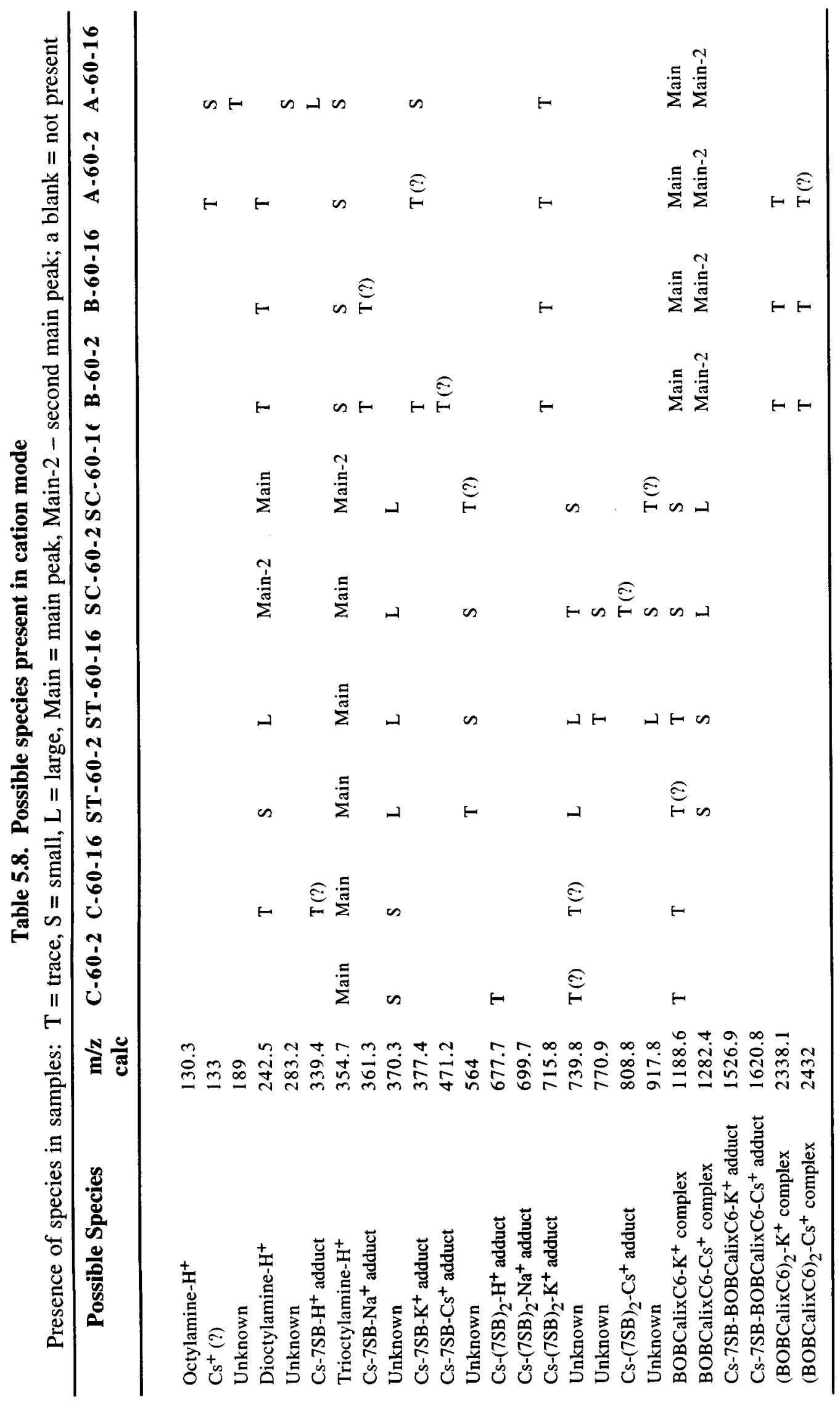




\subsubsection{Cation-Mode Analysis}

Analyses of the solvent samples in cation mode revealed that trioctylamine breaks down to form dioctylamine upon exposure to nitric acid, with the breakdown increasing with increasing nitric acid concentration and duration of exposure. (See Table 5.8.) This was the main finding, with dioctylamine being observed to increase in concentration progressively through the ST-60-2, ST-6016 , SC-60-2, and SC-60-16 samples until it was the main peak in the spectrum for the SC-60-16 sample. As will be seen below, this observation was confirmed by analyses of the solvent by reversephase HPLC (RP-HPLC).

There is some precedent for degradation of long-chain aliphatic tertiary amines by nitric acid, both with and without radiation. ${ }^{71}$ The work of Baroncelli et al. showed that tertiary amines (in benzene solution) will break down when exposed to $2 \mathrm{M}$ nitric acid for 10 hours at $110{ }^{\circ} \mathrm{C} .{ }^{71}$ The tertiary amine sequentially loses alkyl groups, giving the secondary and then primary amine. Essentially no degradation was observed at $50^{\circ} \mathrm{C}$ or $70^{\circ} \mathrm{C}$ after 10 hours. However, no studies were performed with exposure times exceeding 10 hours. Thus, it is not unreasonable that tertiary amines could degrade at $61{ }^{\circ} \mathrm{C}$ when exposed to many weeks of continuous contact with lower concentrations of nitric acid. Furthermore, the amount of nitric acid that gets into the solvent phase will be higher for the solvent (due to the calix and the modifier) than a pure benzene solution of tertiary amine. The study also found that the tertiary amines exposed to high doses of UV radiation in the presence of 2 $\mathrm{M}$ nitric acid at $25{ }^{\circ} \mathrm{C}$ were remarkably stable. At the low concentrations of nitric acid used in the scrub and strip solutions, only prolonged continuous contact (weeks) at high temperatures will produce significant degradation of TOA, as is observed.

Also observed from the ES-MS analysis in cation mode is that various adducts of the BOBCalixC6 with both potassium and cesium, including formation of trace amount of "sandwich" complexes where there are two molecules of BOBCalixC6 per one atom of potassium or cesium. Not surprisingly, the main peaks observed for solvent that was contacted with either simulant were the potassium and cesium complexes of the BOBCalixC6. The potassium complex was the dominant peak since there are two orders of magnitude more potassium than cesium present in the simulant.

There are a few unknown peaks (e.g., at $m / z=370.3,564,739.8$, and 770.8) that appear mostly during stripping and scrubbing (though some are present in the pristine washed solvent), but there is no discernable pattern. Again, it is quite likely that material on the column from prior analyses may be leaching into these samples. The only conclusive evidence of solvent degradation is the breakdown of the trioctylamine, as is evidenced by the in-growth and increase of the DOA peak relative to the TOA peak.

\subsubsection{Chemical Analysis of Thermal-Stability Samples by GC and RP-HPLC}

Detailed analyses of the solvent samples by GC and RP-HPLC revealed conclusively that the TOA content of solvents exposed to the scrub and strip solutions erodes with increasing nitric acid concentration, temperature, and duration of exposure. The concentration of dioctylamine (DOA) is observed to grow in concurrently with the disappearance of TOA. DOA is also observed to degrade in the most severe cases (SC-60-16 and -33 samples). The concentration of 4-sec-butylphenol (SBP) was not observed to increase over the course of the thermal degradation experiment, indicating that the modifier is stable over the course of the experiment.

All the thermal stability samples were analyzed by GC and reverse-phase HPLC (RP-HPLC) for the presence of TOA, DOA, and SBP. The results are shown in Table 5.9. The analyses confirmed that the only observable degradation of the solvent is the breakdown of the TOA, with the SBP concentration being below $0.02 \mathrm{mM}$ in all cases. There is very good qualitative agreement between the TOA and DOA levels observed in the ES-MS study, and the RP-HPLC study, for the ST-60-2, ST$60-16$, SC-60-2, and SC-60-16 samples. Of great interest is the fact that the DOA is found to first increase in concentration as the TOA concentration decreases (samples SC-60-2 and -7), but that the DOA concentration then decreases such that it is only present at $0.22 \mathrm{mM}$ in the SC-60-33 sample (see Figure 5.9). DOA will also function like TOA to provide low stripping distribution values; however, as the total amine content in the solvent decreases, so will the stripping efficiency decrease. 
It is reasonable to assume based on the literature ${ }^{71}$ that TOA is stepwise dealkylated when in the presence of nitric acid under forcing conditions to give first DOA, and then octylamine. The octylamine will partition rather readily to the aqueous phase, and thus will be removed from the solvent. Thus, once the DOA is broken down to octylamine, amine is lost from the solvent, and the stripping performance erodes. This is exactly what was observed (see Figure 5.7). As can be seen from Figure 5.9, after reaching a maximum concentration in sample SC-60-7, the DOA is observed to decrease in concentration in the subsequent scrub samples with increasing exposure time.

One curious observation is that the TOA concentration in all the strip samples is somewhat lower than normal but shows no clear sign of progressive degradation. Thus, for the ST-35 series, the concentrations hover around $0.78 \mathrm{mM}$ except for the ST-35-33 sample, which decreases slightly to $0.74 \mathrm{mM}$. Similarly, for the ST-60 series, the concentrations hover around $0.66 \mathrm{mM}$ except for the ST-60-33 sample, which decreases slightly to $0.64 \mathrm{mM}$. For all the ST samples, the DOA concentration remains low, about the same as is observed in pristine solvent sample $\mathrm{S}-132 \mathrm{~W}$. It is unclear why the TOA concentrations are lower in the ST-35 series than the SC-35 series. However, the only set of samples that show a clear decrease in the TOA concentration with a concomitant increase (followed by a decrease) in the DOA concentrations is the SC-60 series. 
Table 5.9. GC and RP-HPLC analyses of thermal-stability test samples for TOA, DOA, SBP

\begin{tabular}{|c|c|c|c|c|c|c|c|c|c|c|c|}
\hline Sample ID & \multicolumn{6}{|c|}{ Description } & Temp ${ }^{\circ} \mathrm{C}$ & Time (weeks) & TOA $(\mathrm{mM})$ & DOA $(\mathrm{mM})$ & $\mathrm{SBP}(\mathrm{mM})$ \\
\hline A-35-2 & \multicolumn{6}{|c|}{ Solvent incubated with full simulant } & 35 & 2 & 0.932 & 0.12 & $<0.02$ \\
\hline A-35-7 & $"$ & $"$ & " & $"$ & & 35 & 7 & 0.922 & 0.12 & $<0.02$ \\
\hline A-35-16 & $"$ & $"$ & " & $"$ & \multicolumn{2}{|l|}{$"$} & 35 & 16 & 0.95 & 0.1 & $<0.02$ \\
\hline A-35-33 & $"$ & $"$ & $"$ & $"$ & \multicolumn{2}{|l|}{$"$} & 35 & 33 & 0.975 & 0.1 & $<0.02$ \\
\hline A- $60-2$ & $"$ & $"$ & " & $"$ & \multicolumn{2}{|l|}{$"$} & 60 & 2 & 1 & 0.1 & $<0.02$ \\
\hline A- $60-7$ & $"$ & $"$ & " & $"$ & \multicolumn{2}{|l|}{$"$} & 60 & 7 & 0.9 & 0.12 & $<0.02$ \\
\hline A- $60-16$ & $"$ & $"$ & $"$ & $"$ & \multicolumn{2}{|l|}{$"$} & 60 & 16 & 0.84 & 0.15 & $<0.02$ \\
\hline A- $60-33$ & $"$ & $"$ & " & $"$ & \multicolumn{2}{|l|}{$"$} & 60 & 33 & 0.88 & 0.19 & $<0.02$ \\
\hline B-35-2 & \multicolumn{6}{|c|}{ Solvent incubated with salts-only simulant } & 35 & 2 & 0.923 & 0.12 & $<0.02$ \\
\hline B-35-7 & $"$ & $"$ & $"$ & $"$ & $"$ & $"$ & 35 & 7 & 0.971 & 0.12 & $<0.02$ \\
\hline B-35-16 & $"$ & $"$ & " & $"$ & $"$ & $"$ & 35 & 16 & 1.08 & 0.16 & $<0.02$ \\
\hline B-60-2 & $"$ & $"$ & " & $"$ & $"$ & $"$ & 60 & 2 & 0.933 & 0.13 & $<0.02$ \\
\hline B-60-7 & $"$ & $"$ & $"$ & $"$ & $"$ & $"$ & 60 & 7 & 0.894 & 0.14 & $<0.02$ \\
\hline B-60-16 & $"$ & $"$ & $"$ & $"$ & $"$ & $"$ & 60 & 16 & 0.904 & 0.13 & $<0.02$ \\
\hline$C-35-2$ & \multicolumn{6}{|c|}{ Solvent only incubated (no aqueous phase) } & 35 & 2 & 0.928 & 0.07 & $<0.02$ \\
\hline C-35-7 & $"$ & $"$ & $"$ & $"$ & $"$ & $"$ & 35 & 7 & 0.916 & 0.07 & $<0.02$ \\
\hline C. $35-16$ & $"$ & $"$ & $"$ & $"$ & $"$ & $"$ & 35 & 16 & 0.933 & 0.05 & $<0.02$ \\
\hline C-35-33 & $"$ & $"$ & $"$ & $"$ & $"$ & $"$ & 35 & 33 & 0.975 & 0.02 & $<0.02$ \\
\hline$C-60-2$ & $"$ & $"$ & $"$ & $"$ & $"$ & $"$ & 60 & 2 & 0.954 & 0.08 & $<0.02$ \\
\hline C- $60-7$ & $"$ & $"$ & $"$ & $"$ & $"$ & $"$ & 60 & 7 & 0.97 & 0.07 & $<0.02$ \\
\hline C- $60-16$ & $"$ & $"$ & $"$ & $"$ & $"$ & $"$ & 60 & 16 & 0.876 & 0.09 & $<0.02$ \\
\hline C-60-33 & $"$ & $"$ & $"$ & $"$ & $"$ & $"$ & 60 & 33 & 0.960 & 0.13 & $<0.02$ \\
\hline SC-35-2 & \multicolumn{6}{|c|}{ Solvent incubated with scrub solution } & 35 & 2 & 0.931 & 0.07 & $<0.02$ \\
\hline SC-35-7 & $"$ & $"$ & $"$ & $"$ & \multicolumn{2}{|c|}{$"$} & 35 & 7 & 0.889 & 0.05 & $<0.02$ \\
\hline SC-35-16 & $"$ & $"$ & $"$ & $"$ & \multicolumn{2}{|l|}{$"$} & 35 & 16 & 0.884 & 0.07 & $<0.02$ \\
\hline SC-35-33 & $"$ & $"$ & $"$ & $"$ & \multicolumn{2}{|l|}{$"$} & 35 & 33 & 0.930 & 0.10 & $<0.02$ \\
\hline SC-60-2 & $"$ & $"$ & $"$ & $"$ & \multicolumn{2}{|l|}{$"$} & 60 & 2 & 0.432 & 0.33 & $<0.02$ \\
\hline SC-60-7 & $"$ & $"$ & $"$ & $"$ & \multicolumn{2}{|l|}{$"$} & 60 & 7 & 0.199 & 0.68 & $<0.02$ \\
\hline SC-60-16 & $"$ & $"$ & $"$ & $"$ & \multicolumn{2}{|l|}{$"$} & 60 & 16 & 0.066 & 0.56 & $<0.02$ \\
\hline SC-60-33 & $"$ & $"$ & $"$ & $"$ & \multicolumn{2}{|l|}{$"$} & 60 & 33 & 0.047 & 0.22 & $<0.02$ \\
\hline ST-35-2 & \multicolumn{6}{|c|}{ Solvent incubated with strip solution } & 35 & 2 & 0.779 & 0.06 & $<0.02$ \\
\hline ST-35-7 & $"$ & $"$ & $"$ & $"$ & \multicolumn{2}{|l|}{$"$} & 35 & 7 & 0.786 & 0.07 & $<0.02$ \\
\hline ST-35-16 & $"$ & $"$ & $"$ & $"$ & \multicolumn{2}{|l|}{$"$} & 35 & 16 & 0.781 & 0.07 & $<0.02$ \\
\hline ST-35-33 & $"$ & $"$ & $"$ & $"$ & $"$ & & 35 & 33 & 0.740 & 0.05 & $<0.02$ \\
\hline ST-60-2 & $"$ & $"$ & $"$ & $"$ & $"$ & & 60 & 2 & 0.667 & 0.1 & $<0.02$ \\
\hline ST-60-7 & $"$ & $"$ & $"$ & $"$ & $"$ & & 60 & 7 & 0.66 & 0.1 & $<0.02$ \\
\hline ST-60-16 & $"$ & $"$ & $"$ & $"$ & $"$ & & 60 & 16 & 0.66 & 0.1 & $<0.02$ \\
\hline ST-60-33 & $"$ & $"$ & $"$ & $"$ & $"$ & & 60 & 33 & 0.64 & 0.1 & $<0.02$ \\
\hline S- $132 W$ & Pristin & vashe & solve & (no & ncuba & & NA & 0 & 1 & 0.06 & $<0.02$ \\
\hline$A-25-0$ & $"$ & $"$ & $"$ & $"$ & $"$ & & 25 & 0 & 1.01 & 0.08 & $<0.02$ \\
\hline SC-25-0 & $"$ & $"$ & $"$ & $"$ & $"$ & & 25 & 0 & 1.01 & 0.08 & $<0.02$ \\
\hline
\end{tabular}




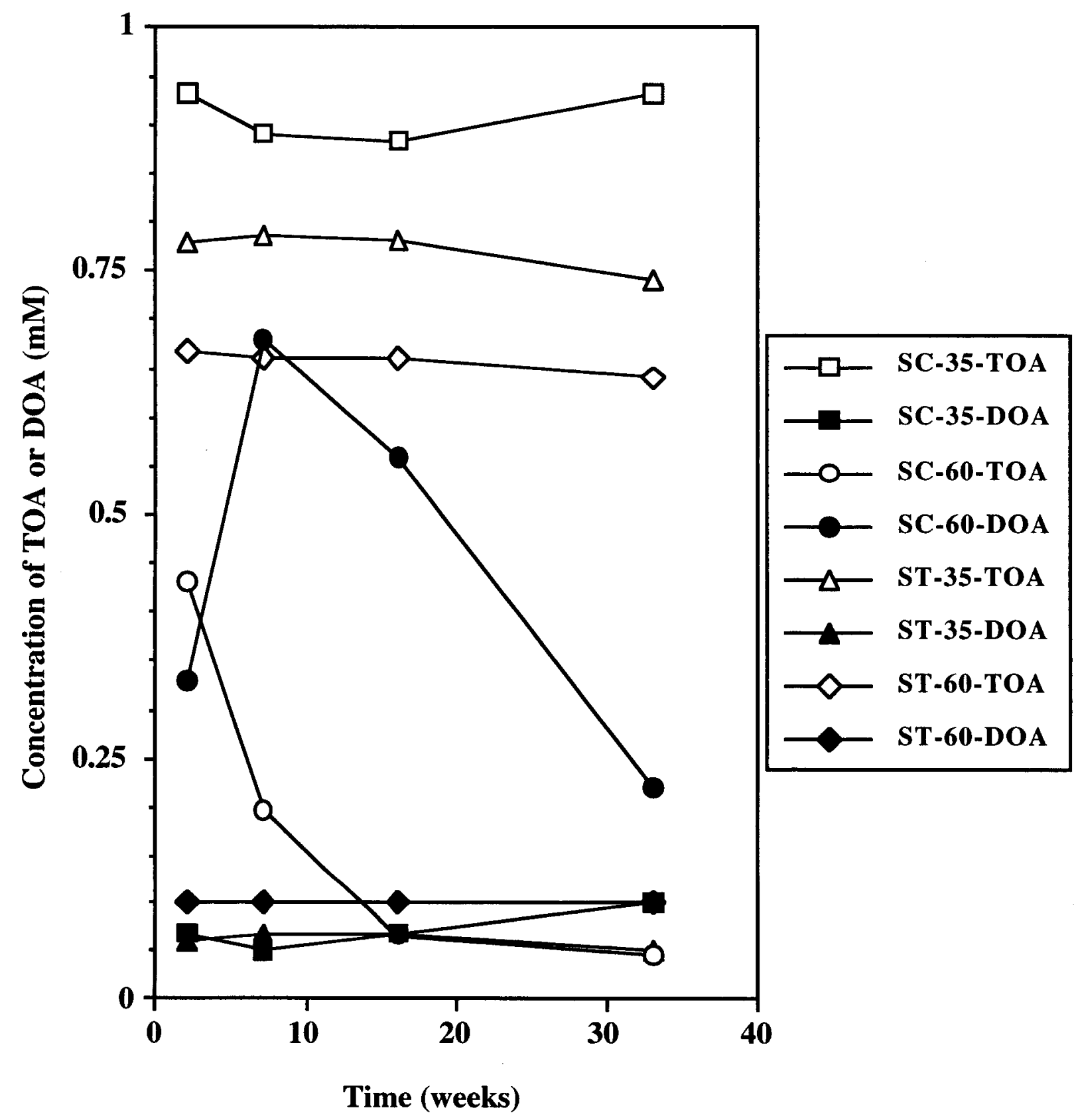

Figure 5.9. TOA and DOA concentrations in scrub (SC) and strip (ST) samples as a function of exposure time at 35 and $61^{\circ} \mathrm{C}$. 


\subsection{CONCLUSIONS}

In summary, the results present a case for high solvent thermal stability. The only solvent component that displays any sign of chemical instability to prolonged contact to aqueous solutions of the flowsheet is the trioctylamine, and the degradation manifests itself only under the most severe conditions of high temperature and very long continuous contact with the scrub solution. The decrease in the TOA concentration resulted in a slight increase in the scrub and stripping cesium distribution ratios in the ESS tests for the affected solvent samples. Though the TOA concentration in the strip series of samples analyzed a bit on the low side, there was no clear indication that the TOA concentration was actively degrading when in contact with the strip solution at either $35{ }^{\circ} \mathrm{C}$ or $61{ }^{\circ} \mathrm{C}$. Also, the TOA concentration remained constant at high concentration in the SC-35 series, indicating that the TOA possesses good long-term stability to the scrub solution at $35{ }^{\circ} \mathrm{C}$, and that it is the high $61{ }^{\circ} \mathrm{C}$ temperature that is necessary for the degradation. Since the maximum flowsheet operating temperature is not expected to exceed $35^{\circ} \mathrm{C}$, the TOA should be stable for long periods of time. For purposes of estimating the rate of replacement of solvent components, we take the TOA loss to be less than $0.1 \mathrm{mM}$ per year under continuous exposure to process solutions at $35^{\circ} \mathrm{C}$.

It is worth calculating the operating lifetime of the solvent due to thermal stability alone in the absence of solvent washing. The data show acceptable performance for the solvent for 235 days of constant exposure to the various conditions of the flowsheet at $35^{\circ} \mathrm{C}$. As the solvent is stable even at $60{ }^{\circ} \mathrm{C}$ in the absence of an aqueous phase, it is assumed that the portion of the solvent outside of the contactors is not actively degrading. Based on earlier estimates, ${ }^{41}$ it is also assumed that the solvent is cycled 3500 times per year, the cycle time is $2.5 \mathrm{~h}$, and $8.35 \%$ of the solvent inventory resides in the contactor. From the data, degradation is promoted primarily by the acid phases, and thus, at any time only $4.4 \%$ of the solvent inventory is actively degrading. In each cycle, a given volume of solvent then spends $0.105 \mathrm{~h}$ degrading, and over 3500 cycles, the annual equivalent degradation time would thus be $368 \mathrm{~h}$ or 15.3 days or 2.2 weeks. Looking only at the ESS data, then, 235 days of sustained acceptable performance corresponds to a solvent life of $15 \mathrm{y}$. In other words, thermal degradation is a negligible contributor to overall solvent loss.

Both the BOBCalixC6 and the Cs-7SB modifier possess excellent thermal stability not only to exposure to the aqueous phases of the process flowsheet (simulants and scrub and strip solution), but also to exposure to higher concentrations of nitric acid. Indeed, only about $4 \%$ degradation of the modifier (to form a nitrated product) was observed under the very severe conditions of 28 days contact at $61{ }^{\circ} \mathrm{C}$ with $1 \mathrm{M}$ nitric acid. Also, no discernible change in the concentration of 4-secbutylphenol, a likely decomposition product of the modifier, was observed in any of the samples over the full course of the 33-week thermal stability experiment.

Finally, the solvent was found to be chemically stable to both the full simulant containing various metal species (such as for example $\mathrm{Pd}$ and $\mathrm{Hg}$ which have been shown to catalytically degrade tetraphenylborate) and the salts-only simulant that does not contain those metals. No stability differences between these two cases were observed, indicating that the solvent and its components are stable in the presence of these metal species. 


\section{RADIATION STABILITY}

\subsection{INTRODUCTION}

\subsubsection{Purpose}

The external-irradiation tests performed at the SRTC were intended to provide an indication of the radiolytic stability of the solvent, from the points of view of disappearance of solvent components, appearance of degradation products, severity of the effects on flowsheet performance, and necessity of cleanup measures. ${ }^{4,55}$ Although the first-generation solvent exhibited very promising stability to external irradiation, ${ }^{72}$ the second-generation solvent composition had advanced sufficiently that further testing was desirable, especially in light of the more aggressive chemical conditions associated with the more complex full simulant planned. As in the thermal-stability tests, the presence of catalytic concentrations of noble metals and organic species provides for possibly significantly enhanced solvent breakdown. Overall, a goal of a 1-year solvent lifetime is sought, and from this experiment, an estimate of actual solvent lifetime and necessity for cleanup due to radiolytic damage is an expected outcome.

Since external irradiation may be conducted with a gamma source, experiments may be conducted quickly and without the need for hot-cell facilities. Accordingly, high-activity samples do not have to be handled, a much greater variety of analytical tools may be used, and the experiments are safer and cheaper. Internal-irradiation tests were also conducted as part of the overall program, as carried out at ORNL ${ }^{47}$ with simulated waste and at SRTC ${ }^{55}$ with real waste. Together, the overall testing program provides a realistic assessment of the radiolytic stability of the CSSX solvent and flowsheet. Comparison of the results of the internal- and external-irradiation experiments then may provide a kind of "calibration" of the easier external-irradiation experiment for subsequent use. Thus, it is implicitly recognized that the external-irradiation experiment by itself does not provide the most realistic picture of radiolytic stability, and the reader should use the conclusions made in this chapter accordingly.

\subsubsection{Experimental Design}

Four solvent systems were to be irradiated at the SRTC, 4,55 all having $0.01 \mathrm{M}$ BOBCalixC6 and $0.001 \mathrm{M}$ trioctylamine, but differing in use of either Cs-7SB, Cs-7SBT, or Cs-6 modifier (all at 0.5 M) and either Isopar L or Norpar 12 diluent. The Cs-7SBT modifier has the same structure as Cs-7SB, and since it was being considered as a more economical alternative to Cs-7SB, it was included in the test. Prior work performed at SRS in 1998 revealed that normal paraffin-based solvents (specifically Norpar 12) possessed somewhat greater radiolytic stability than isoparaffinic-based solvent (specifically Isopar L). ${ }^{72}$ The only modifier that performed well in Norpar 12 was the Cs-6 modifier, as the others tended to form third phases. Hence a Cs-6/Norpar 12 solvent was included in the radiation-stability tests, along with a Cs-6/Isopar L solvent for comparison. The four solvents that were sent to the SRTC are defined in Table 6.1.

Schematic representations of the contacting and irradiation protocol are given in Figs. 6.1-6.3. Except for having an extra extraction step and a gamma irradiation instead of a thermal treatment, the test was set up essentially the same as the thermal-stability test (see Chap. 5). The sample preparation aims for reasonable fidelity to flowsheet conditions in that the solvent irradiated over the scrub solution is first contacted with simulant. Likewise, the solvent irradiated over the strip solution is first contacted with simulant and then with scrub solution. The added extraction contact gives higher loading of cesium and organic species such as dibutylphosphate. Hence, stripping is expected to proceed with elevated $D_{\mathrm{Cs}}$ values. 
Table 6.1. Solvent systems planned for external-irradiation experiments

\begin{tabular}{ccccc}
\hline Solvent lot no. & Extractant & Modifier & Suppressor & Diluent \\
\hline PVB B000718-110W $a$ & 0.01 M BOBCalixC6 & $0.5 \mathrm{M} \mathrm{Cs-7SB}$ & $0.001 \mathrm{M}$ TOA & Isopar L \\
PVB B000718-107W & 0.01 M BOBCalixC6 & $0.5 \mathrm{M} \mathrm{Cs-7SBT}$ & $0.001 \mathrm{M}$ TOA & Isopar L \\
PVB B000718-108W & 0.01 M BOBCalixC6 & $0.5 \mathrm{M} \mathrm{Cs-6}$ & $0.001 \mathrm{M}$ TOA & Isopar L \\
PVB B000718-109W & 0.01 M BOBCalixC6 & $0.5 \mathrm{M} \mathrm{Cs-6}$ & $0.001 \mathrm{M}$ TOA & Norpar 12 \\
\hline
\end{tabular}

${ }^{a}$ Baseline solvent. Note that certain repeats were run on lot no. PVB B000718-132W that was sent to the SRTC late in June, 2000.

Dose considerations have been discussed in the SRTC report on the external irradiations, 55 and accurate calculations were performed to provide a reliable estimate for the radiation dose absorbed by the solvent under plant operating conditions and test conditions. ${ }^{41}$ Those calculations assumed the use of the present baseline flowsheet, ${ }^{20}$ which employs $25-\mathrm{cm}$ contactors in an array of 32 stages. It was assumed that at any given time only $8.35 \%$ of the solvent inventory resides inside the contactors, where the effective dose is received, with the solvent in external tankage receiving negligible dose. Based on a maximum of 3500 cycles per year and a calculated absorbed dose of 26.2 rad per cycle, it was estimated that the dose absorbed by the solvent per year is $91.7 \mathrm{krad}$. More realistically, if the plant operates $80 \%$ of the time, the absorbed dose would be $73.4 \mathrm{krad}$. It may be remarked that these absorbed dose estimates are relatively modest, which may be attributable in part to the short contacting time in each stage and the geometry of centrifugal contactors.

\subsubsection{Work Scope}

Planning called for samples to be received from irradiation experiments (WSM item 4.1.1) and subjected to analytical procedures and performance assessment. 19 The work was expected to be exploratory in nature and highly dependent upon the extent of solvent degradation and performance. Analyses were prioritized according to the apparent severity of degradation and to the type of information needed to diagnose and remediate any identified problems. Samples were submitted for organic analysis and subjected to other diagnostic experiments such as ES-MS and NMR spectrometries (WSM item 4.1.1.1). Performance assessment employed the standard batch ESS protocol, checks for third-phase formation, break-time measurement, interfacial-tension measurement, and tests of selectivity (WSM item 4.1.1.3). Most of the efforts at ORNL were intended to complement the work that was performed at the SRTC. Some intended redundancy allowed for cross-checking of results and conclusions.

Related work scope is covered elsewhere. Partitioning of degradation products is covered mostly in Chap. 4 (WSM item 4.1.1.4), including anion exchange studies (WSM item 4.1.1.6), and solvent washing is covered in Chap. 8 (WSM item 4.1.1.5). Analysis and performance assessment of samples received from the batch internal-irradiation experiments 19,47 were conducted as part of the present work (WSM item 4.1.2), but these results will be reported in detail in the corresponding report ${ }^{47}$ on the internal-irradiation test; however, Chap. 8 gives some results on cleanup of these solvent samples. 


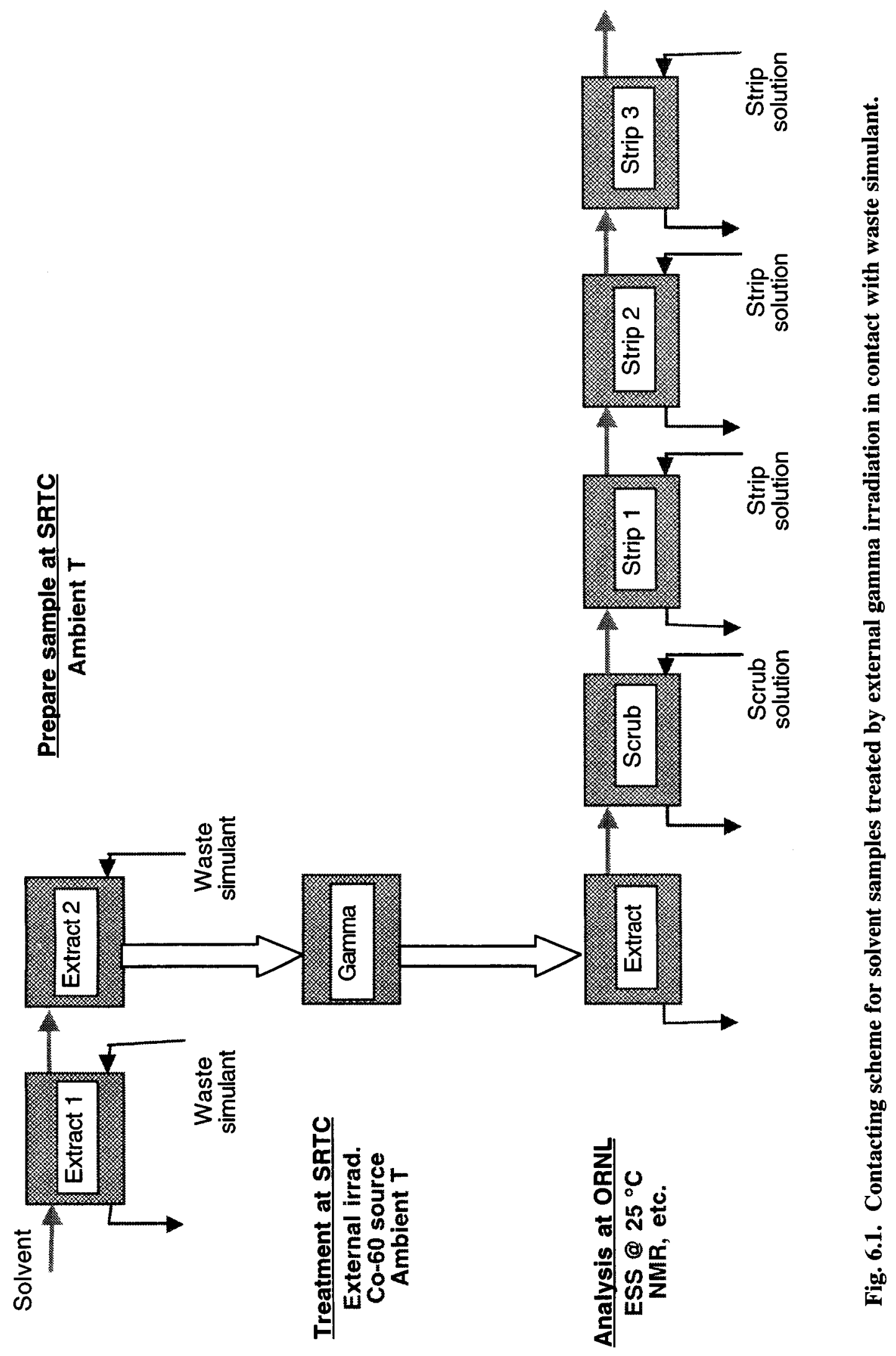




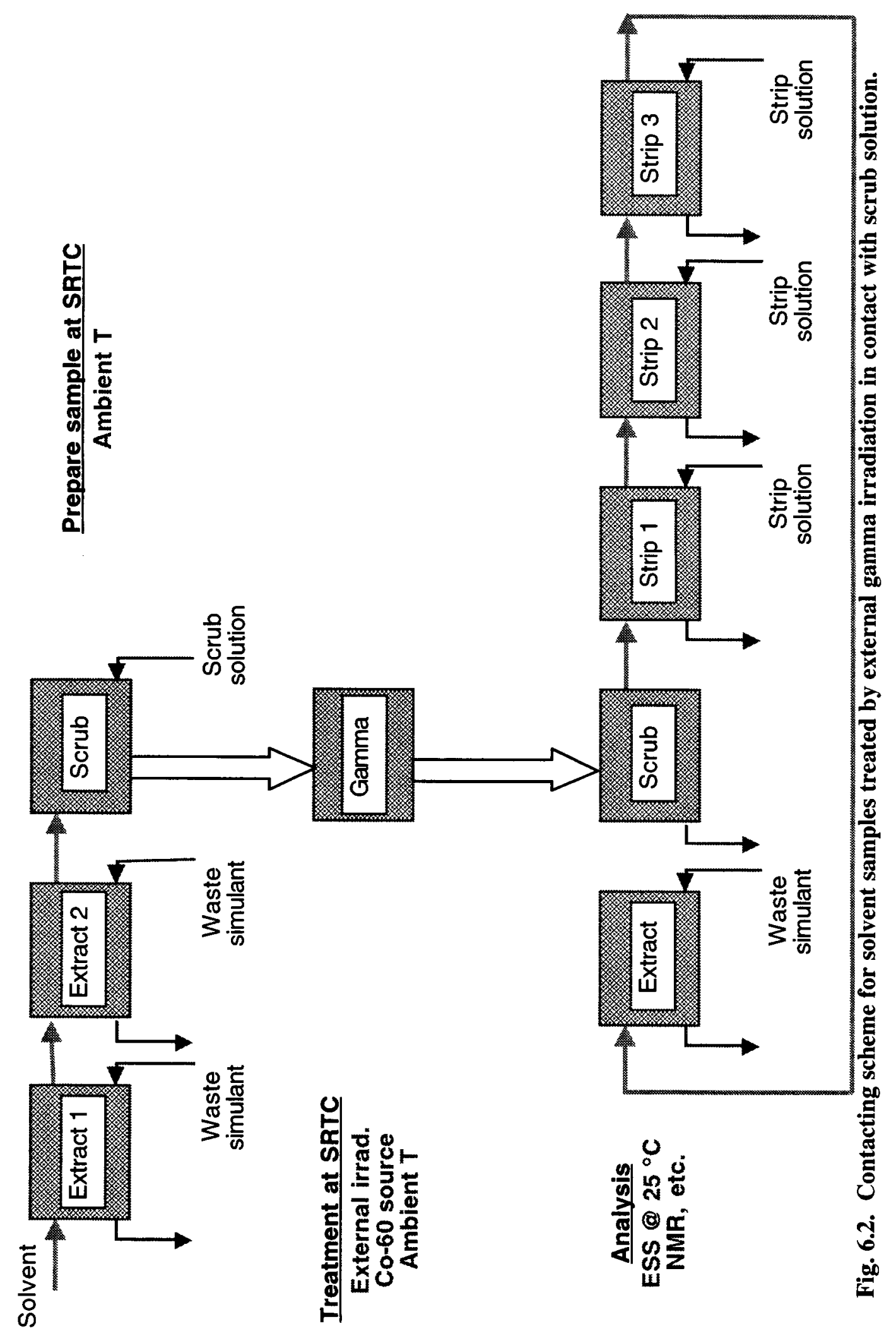




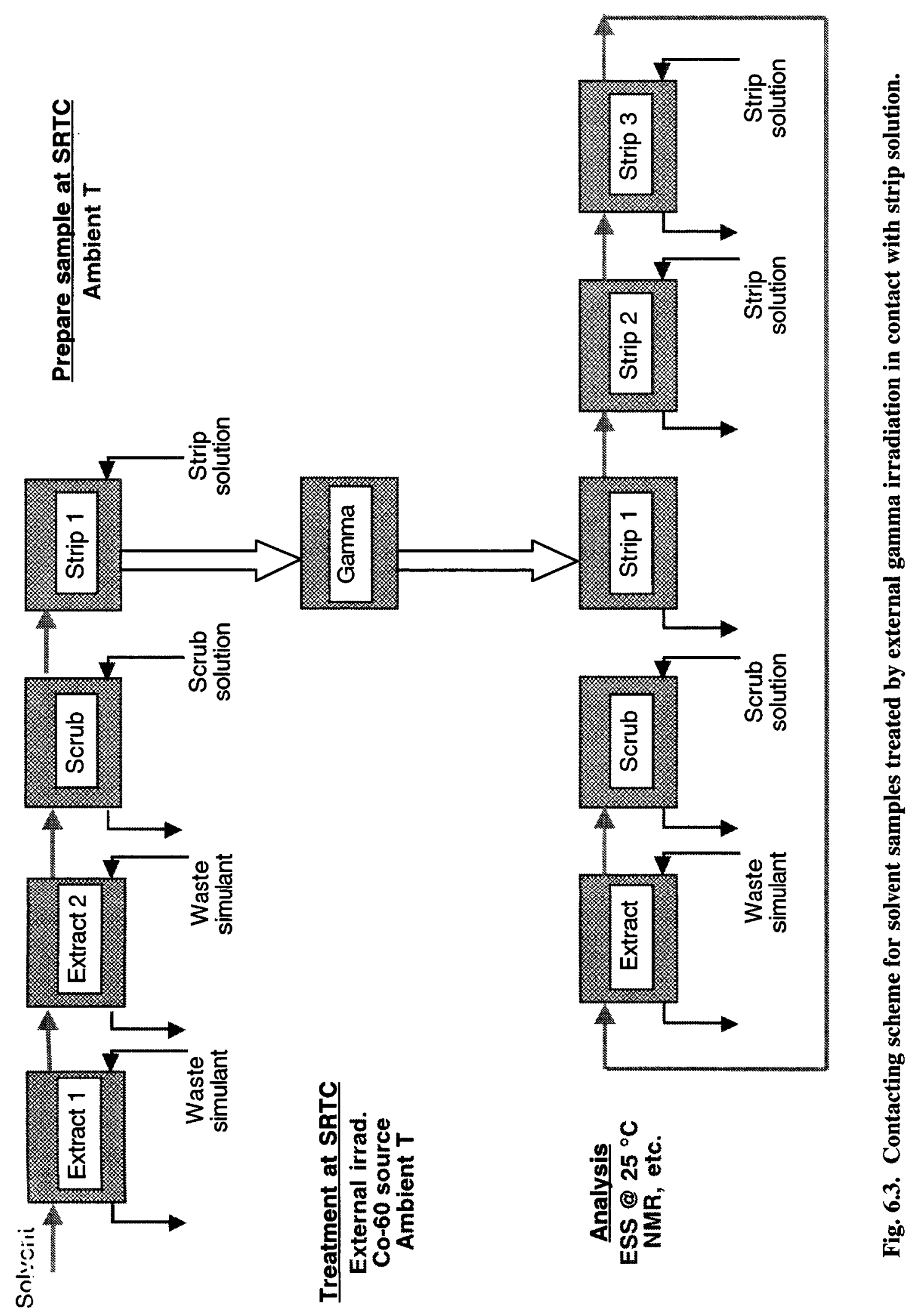




\subsection{EXPERIMENTAL SECTION}

\subsubsection{Irradiation Samples}

Solvents were prepared at ORNL (Table 6.1) and shipped to the SRTC in May, 2000. Samples of the solvent were prepared according to the schemes shown in Figs. 6.1-6.3 and were irradiated ${ }^{55}$ while being agitated in contact with the three aqueous phases specified in the baseline flowsheet: ${ }^{20}$

a) full simulant prepared at the SRTC, containing known organic species as well as noble metals, 21,22

b) scrub solution consisting of $0.050 \mathrm{M}$ nitric acid, and

c) strip solution consisting of $0.001 \mathrm{M}$ nitric acid.

Phase ratios $(\mathrm{O} / \mathrm{A})$ were those of the baseline flowsheet, namely $0.33,5$, and 5 for extraction, scrubbing, and stripping, respectively.

Samples of irradiated solvent received from Reid A. Peterson, SRTC, are summarized in Table 6.2. They were divided into three categories:

EXT: The solvent was irradiated while in contact with simulant $(\mathrm{O} / \mathrm{A}=0.33)$. The doses received were $0.5 \mathrm{Mrad}, 1 \mathrm{Mrad}, 2 \mathrm{Mrad}$, and $4 \mathrm{Mrad}$.

SC: The solvent was first contacted with the simulant $(\mathrm{O} / \mathrm{A}=0.33)$ then irradiated while in contact with the scrub solution $\left(\mathrm{HNO}_{3} 50 \mathrm{mM}, \mathrm{O} / \mathrm{A}=5\right)$. The doses received were $1.5 \mathrm{Mrad}$, $3 \mathrm{Mrad}, 6 \mathrm{Mrad}$, and $12 \mathrm{Mrad}$.

ST: The solvent was first contacted with the simulant $(\mathrm{O} / \mathrm{A}=0.33)$, then with the scrub solution $\left(50 \mathrm{mM} \mathrm{HNO}_{3}, \mathrm{O} / \mathrm{A}=5\right)$, then irradiated while in contact with the strip solution $\left(1 \mathrm{mM} \mathrm{HNO}{ }_{3}, \mathrm{O} / \mathrm{A}=5\right)$. The doses received were $2 \mathrm{Mrad}, 4 \mathrm{Mrad}, 8 \mathrm{Mrad}$, and $16 \mathrm{Mrad}$.

Only two solvents were actually irradiated, namely those with Cs-7SB and Cs-7SBT modifier. The two Cs-6 solvents solidified upon standing, as discussed in detail in Chap. 7. Note that Table 6.2 converts the listed nominal dose in Mrad to annual dose, using the conversion factor $92 \mathrm{krad} / \mathrm{year} .{ }^{41}$ As such, the external-irradiation test represents a severe dosing relative to expected dose under plant operating conditions.

\subsubsection{Analytical Procedures}

Detailed descriptions of analytical procedures are given elsewhere. ${ }^{26}$ Solvent samples were prepared by dilution in isopropanol, 1/10 for TOA and 4-sec-butylphenol analysis, and 1/100 for analysis of the calixarene and the Cs-7SB. Aqueous samples were prepared using Oasis (Waters Assoc.) solid phase extraction cartridges. The aqueous phase was passed through the cartridges and the analytes eluted with isopropanol. Analysis of the calixarene and modifier was carried out using two independent methods: reverse-phase HPLC with UV absorbance detection for quantitative analysis (also used for 4-sec-butylphenol) and size-exclusion chromatography with both absorbance and evaporative light-scattering detectors in series for qualitative purposes. Cs-7SB and Cs-7SBT. BOBCalixC6, and 4-sec-butylphenol in the solvent samples were quantitatively determined by reversed-phase HPLC. Most of the results for 4-sec-butylphenol were near the quantitation limit, 0.5 $\mathrm{mM}$. Trioctylamine was determined by gas chromatography using a flame ionization detector as described elsewhere. Aqueous fluoride was determined by ion chromatography. TOC uas determined using a Shimadzu TOC analyzer.

\subsubsection{NMR Spectrometry}

${ }^{1} \mathrm{H}$ and ${ }^{19} \mathrm{~F}$ NMR spectra were obtained on $100-\mu \mathrm{L}$ aliquots of solvent by diluting with $900 \mu_{\llcorner}$of deuterochloroform containing hexamethylbenzene (HMB, Aldrich, $99 \%$ ) at $0.0140 \mathrm{M}$ as an internal integration standard, as described in Chap. 5. 
Table 6.2. Externally-irradiated samples received from the SRTC

\begin{tabular}{|c|c|c|c|c|}
\hline Sample ID ${ }^{a}$ & $\begin{array}{l}\text { Dose } \\
\text { (Mrad) }\end{array}$ & $\begin{array}{l}\text { Annual dose } \\
\text { (years) }\end{array}$ & Modifier & Aqueous phase \\
\hline SB BE & 0 & 0 & Cs-7SB & None \\
\hline EXTO-0-SB & 0 & 0 & Cs-7SB & Full simulant \\
\hline EXTO-0-SB-R & 0 & 0 & Cs-7SB & Full simulant \\
\hline EXTO-0.5-SB & 0.5 & 5.4 & Cs-7SB & Full simulant \\
\hline EXTO-1-SB & 1 & 11 & Cs-7SB & Full simulant \\
\hline EXTO-2-SB & 2 & 22 & Cs-7SB & Full simulant \\
\hline EXTO-4-SB & 4 & 43 & Cs-7SB & Full simulant \\
\hline SCO-0-SB & 0 & 0 & Cs-7SB & $50 \mathrm{mM} \mathrm{HNO}_{3}$ \\
\hline SCO-0-SB-R & 0 & 0 & Cs-7SB & $50 \mathrm{mM} \mathrm{HNO}_{3}$ \\
\hline SCO-1.5-SB & 1.5 & 16 & Cs-7SB & $50 \mathrm{mM} \mathrm{HNO}_{3}$ \\
\hline SCO-1.5-SB-R & 1.5 & 16 & Cs-7SB & $50 \mathrm{mM} \mathrm{HNO}_{3}$ \\
\hline SCO-3-SB & 3 & 33 & Cs-7SB & $50 \mathrm{mM} \mathrm{HNO}_{3}$ \\
\hline SCO-6-SB & 6 & 65 & Cs-7SB & $50 \mathrm{mM} \mathrm{HNO}_{3}$ \\
\hline SCO-6-SB-R & 6 & 65 & Cs-7SB & $50 \mathrm{mM} \mathrm{HNO}_{3}$ \\
\hline SCO-12-SB & 12 & 130 & Cs-7SB & $50 \mathrm{mM} \mathrm{HNO}_{3}$ \\
\hline STO-0-SB & 0 & 0 & Cs-7SB & $1 \mathrm{mM} \mathrm{HNO}_{3}$ \\
\hline STO-0-SB-R & 0 & 0 & Cs-7SB & $1 \mathrm{mM} \mathrm{HNO}_{3}$ \\
\hline STO-2-SB & 2 & 22 & Cs-7SB & $1 \mathrm{mM} \mathrm{HNO}_{3}$ \\
\hline STO-2-SB-R & 2 & 22 & Cs-7SB & $1 \mathrm{mM} \mathrm{HNO} 3$ \\
\hline STO-4-SB & 4 & 43 & Cs-7SB & $1 \mathrm{mM} \mathrm{HNO}_{3}$ \\
\hline STO-8-SB & 8 & 87 & Cs-7SB & $1 \mathrm{mM} \mathrm{HNO}_{3}$ \\
\hline STO-8-SB-R & 8 & 87 & Cs-7SB & $1 \mathrm{mM} \mathrm{HNO}_{3}$ \\
\hline STO-16-SB & 16 & 174 & Cs-7SB & $1 \mathrm{mM} \mathrm{HNO} 3$ \\
\hline EXTO-0-SBT & 0 & 0 & Cs-7SBT & Full simulant \\
\hline EXTO-2-SBT & 2 & 22 & Cs-7SBT & Full simulant \\
\hline SCO-6-SBT & 6 & 65 & Cs-7SBT & $50 \mathrm{mM} \mathrm{HNO}_{3}$ \\
\hline STO-8-SBT & 8 & 87 & Cs-7SBT & $1 \mathrm{mM} \mathrm{HNO}_{3}$ \\
\hline PRISTINE 50 & 50 & 540 & Cs-7SB & None \\
\hline
\end{tabular}

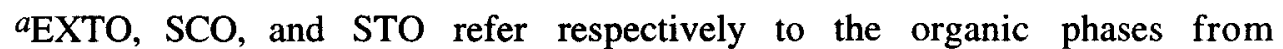
irradiated extraction, scrub, and strip samples. SB and SBT refer respectively to the modifiers Cs-7SB and Cs-7SBT. Numbers refer to the dose in Mrad. The designation $\mathrm{R}$ refers to a repeated irradiation sample received at ORNL in Sept., 2000. The sample SB BE was a sample of washed pristine baseline solvent that was simply returned to ORNL without any treatment. The sample PRISTINE 50 was washed pristine baseline solvent that received a 50-Mrad dose with no aqueous phase present. 


\subsubsection{Electro-Spray Mass Spectrometry}

Electrospray experiments were conducted as described in Section 2.4.1. A 100- $\mu \mathrm{L}$ aliquot of each irradiated solvent was diluted in $900 \mu \mathrm{L}$ of a $50 \%-50 \%$ mixture of acetonitrile-dichloromethane and analyzed.

\subsubsection{Interfacial Tension}

Interfacial tension was measured by the drop-weight (or drop-volume) method. ${ }^{73,74}$ The interfacial tension of the liquid determines the weight of a liquid drop that detaches itself from the tip of a vertically held tube, where a drop of the heavier phase is formed within the body of the lighter phase. Drops detach when the gravitational pull just exceeds the restraining force of surface tension. The mass of the drop times the acceleration due to gravity is balanced by the surface tension. The latter quantity is proportional to the diameter of the tip on which it forms. The relationship is inexact in that a correction for the fraction of the liquid forming the drop that remains on the tip is required. For interfacial measurements, the difference in density between the two phases is the important parameter:

$$
\gamma=V\left(\rho_{1}-\rho_{2}\right) g /(2 \pi r f)
$$

where $\gamma$ is the surface tension in dyne $/ \mathrm{cm}(\mathrm{mN} / \mathrm{m}), V$ is the volume of the drop in $\mathrm{cm}^{3}, \rho_{1}$ and $\rho_{2}$ are the densities of the two phases, $g$ is the acceleration due to gravity, $r$ is the radius of the drop tube, and $f$ is a correction factor proportional to $r / V^{3}$. The latter quantity was taken from published tables. ${ }^{75}$

The apparatus utilized a micrometer syringe burette to dispense drops of the aqueous phase into the organic phase. The glass vessel minimized the volume of the phases required, and the vessel was constructed so that it could be partially submerged in a water bath maintained at $25.0^{\circ} \mathrm{C}$. The burette tip was a critical feature of the apparatus and was ground to specification by the machine shop at ORNL. The measurements were carried out as described by Alexander and Hayter. ${ }^{74}$

As a test of the drop-weight apparatus, the surface tension of an air-water interface at $25{ }^{\circ} \mathrm{C}$ was measured to be 73.2 dyne $/ \mathrm{cm}$ in comparison to the known value, 72.99 dyne $/ \mathrm{cm} .{ }^{75}$ The interfacial tension of 1-octanol-water and $n$-octane-water interfaces were measured as tests with a liquid-liquid interface. The measured values of interfacial tension were in reasonable agreement with literature values. For 1-octanol-water, an interfacial tension of $7.7 \mathrm{dyne} / \mathrm{cm}$ was observed in comparison to the literature value of 8.5 dyne/cm. ${ }^{76}$ For $n$-octane-water the measured value was $42 \mathrm{dyne} / \mathrm{cm}$, to be compared to the literature value of 50.8 dyne $/ \mathrm{cm} .{ }^{76}$

\subsubsection{Sodium Extraction Capacity}

A ${ }^{22} \mathrm{Na}$ tracer experiment was conducted on selected organic-solvent and aqueous-simulant samples that had been externally irradiated. The organic solvents were specifically those containing Cs-7SB modifier (lot PVB-B000718-110W) and Cs7SBT modifier (lot PVB-B000718-107W). Only EXT series samples were run. Aliquots of the two phases from the irradiated samples were recontacted using an $\mathrm{O} / \mathrm{A}$ ratio of 2 with a total organic volume of $0.6 \mathrm{~mL}$. The samples were spiked with ${ }^{22} \mathrm{Na}$ tracer and equilibrated in $2-\mathrm{mL}$ polypropylene vials for 1 hour at $25^{\circ} \mathrm{C}$ by end-over-end rotation. After centrifugation, each phase was subsampled for gamma-counting to determine the distribution of sodium.

A ${ }^{22} \mathrm{Na}$ experiment was also conducted on the organic-solvent and aqueous-simulant samples of solvent containing Cs-6 modifier (lot PVB-B000718-107W) that had been externally irradiated and had not gelled. The samples were re-contacted in the same manner as for Cs7SB and Cs7SBT.

\subsubsection{Extract/Scrub/Strip Protocol}

ESS tests were performed on the samples received from the external irradiation tests at SRTC according to the scheme outlined in Figs. 6.1-6.3. Two cycles were involved, since to evaluate the 
performance of the samples irradiated over scrub and strip solutions requires that at least part of the second cycle is performed. Two controls were run; in both cases, the solvent used was the pristine solvent that was sent to Savannah River and returned untreated (sample SB BE in Table 6.2). The first control involved the simulant prepared at ORNL, and the second involved the simulant prepared at Savannah River (non-irradiated). The sample PRISTINE 50 was not tested by ESS contacting, as the volume was insufficient. All other samples were tested by the same protocol.

\section{First Cycle}

The first cycle involved acquiring the value of $D_{\mathrm{Cs}}$ using the actual samples of solvent and aqueous phase that were irradiated, and then completing the ESS test through the third strip.

EXT series. A 5-mL aliquot of irradiated solvent was put in contact with $15 \mathrm{~mL}$ of simulant that was in contact with the solvent during irradiation, spiked with ${ }^{137} \mathrm{Cs}$ (ca. $0.2 \mu \mathrm{Ci} / \mathrm{mL}$ ), equilibrated at $25{ }^{\circ} \mathrm{C}$ for $30 \mathrm{~min}$, and centrifuged for $3 \mathrm{~min}$ at $4000 \mathrm{rpm}$. A $0.350-\mathrm{mL}$ aliquot of each phase was then subsampled to be counted. Because of the small amount of solvent available (and the necessity to do 2 cycles with it), the counted aliquot of organic phase was recombined with the remaining solvent sample before going to the next step.

A $4.8-\mathrm{mL}$ aliquot of solvent was then transferred and put in contact with $0.960 \mathrm{~mL}$ of 50 $\mathrm{mM}$ nitric acid (scrub stage). The same protocol was used again, and $4.7 \mathrm{~mL}$ of solvent was transferred and put in contact with $0.940 \mathrm{~mL}$ of $1 \mathrm{mM}$ nitric acid (strip stage). This was repeated three times (three strips). For the second and third strips, a spike of ${ }^{137} \mathrm{Cs}$ was added to improve counting statistics and give more accurate $D_{\mathrm{Cs}}$ values.

After the third strip, $4.4 \mathrm{~mL}$ of solvent was carefully removed and stored overnight in the absence of any aqueous phase before being used for the second cycle.

Two controls were run at the same time as the EXT series using the exact same protocol.

SC series. A problem encountered with this series entailed obtaining the cesium distribution ratios using the solvent and the scrub solution that were irradiated, because of the very small amount of aqueous phase available; more than half had been submitted for analyses, and the rest was needed for surface-tension measurements. It was decided to obtain the $D_{\mathrm{Cs}}$ values on those solutions by placing $1 \mathrm{~mL}$ of the irradiated solvent in contact with $0.200 \mathrm{~mL}$ of irradiated scrub solution. Contacting and centrifuging were performed as described above. An aliquot of $0.100 \mathrm{~mL}$ of each phase was counted. The organic-phase aliquot was recovered along with the $0.900 \mathrm{~mL}$ remaining in the vial. They were added to samples containing $3.9 \mathrm{~mL}$ of solvent irradiated with the scrub solution and $0.980 \mathrm{~mL}$ of fresh $1 \mathrm{mM}$ nitric acid. From that point forward, three strips were performed using the same procedure as the one described in the EXT-series section.

ST series. The problem involving the small volume of aqueous phase available occurred with this series too and was solved identically as described for the SC series. Only two more strips were performed after obtaining the $D_{\mathrm{Cs}}$ values for the irradiated sample.

\section{Second Cycle}

The second cycle involved simply taking the first-cycle solvent samples through a second ESS test. The contacting and centrifuging protocols were the same as described for the first cycle. However, a full recovery of the solvent at each step was not performed. Volumes at each step are given in Table 6.3. As before, a spike of ${ }^{137} \mathrm{Cs}$ was added for the second and third strips. Aliquots of $0.350 \mathrm{~mL}$ of each phase were counted except for the second strip $(0.300 \mathrm{~mL})$ and third strip $(0.150 \mathrm{~mL})$. 
Table 6.3. Volumes contacted in the second ESS cycle

\begin{tabular}{lcc}
\hline \multicolumn{1}{c}{ Step } & $\begin{array}{c}\text { Organic volume } \\
(\mathrm{mL})\end{array}$ & $\begin{array}{c}\text { Aqueous volume } \\
(\mathrm{mL})\end{array}$ \\
\hline Extraction & 4.4 & 13.2 \\
Scrub & 3.5 & 0.70 \\
Strip \#1 & 2.9 & 0.58 \\
Strip \#2 & 2.1 & 0.42 \\
Strip \#3 & 1.3 & 0.26 \\
\hline
\end{tabular}

\subsection{RESULTS AND DISCUSSION}

\subsubsection{Visual Observations}

All irradiated liquid-liquid Cs-7SB and Cs-7SBT samples were inspected for possible formation of a third phase. None was detected. Moreover, no third phase formed at any time during the subsequent ESS tests (Section 6.3.8). Regarding the sample irradiated without an aqueous phase (i.e., PRISTINE 50), no other liquid or solid phase was observed. As mentioned in Section 6.2.1, the two Cs-6 solvents had solidified upon standing. These samples were not considered further for stability testing but will be treated separately in Chap. 7 on solubility phenomena.

Within a series (EXT, SC, or ST), the color of the irradiated solvent samples exhibited a dose dependence, the greater doses being associated with greater development of yellow color. This dose response was observed regardless of the aqueous phase present during irradiation. On ESS testing (Section 6.3.8), color development intensified upon contact of the solvent with the full simulant and then faded to pale yellow when in contact with the scrub and strip solutions.

Coalescence behavior during ESS testing (Section 6.3.8) was good. All of the liquid-liquid samples received from the SRTC separated in $2 \mathrm{~min}$ or less after the normal procedure of two vial inversions and ten hand-shakes.

\subsubsection{Aqueous pH}

The $\mathrm{pH}$ of the aqueous phases in the irradiated samples received from the SRTC was checked with a glass microelectrode (Table 6.4). Duplicate determinations were performed, and agreement averaged $\pm 0.02 \mathrm{pH}$ units. Control samples entailed performing a routine ESS test, except with two extractions to match the procedure used at the SRTC. Only the first strip is reported here. The ESS test was performed at ORNL with baseline solvent, baseline O/A ratios, and standard contacting protocol. All scrub samples received from the SRTC were significantly higher in pH than expected from the controls, and the two zero-dose samples were alkaline. This result indicates cross-phase contamination in the contacting procedures used to prepare the samples at the SRTC, as noted in the SRTC report. ${ }^{41}$ Most of the strip samples, except for one of the zero-dose duplicates, were not significantly higher in $\mathrm{pH}$ than the control samples. The 16-Mrad strip sample, however, gave a neutral $\mathrm{pH}$, suggesting that the irradiation-induced chemistry consumed acid.

\subsubsection{Analytical Results}

According to the SRTC data, 55 the rate of breakdown of the calixarene and modifier is minimal relative to the goal of one annual solvent replacement. The SRTC group presented data showing that the modifier concentration remains unchanged within $\pm 10 \%$ up to a $16 \mathrm{Mrad}$ dose. Over the same interval, the calixarene decreased approximately $10 \%$ in a clear trend. A significant loss of 
Table 6.4. $\mathrm{pH}$ values for aqueous phases in irradiated samples

\begin{tabular}{lcc}
\hline \multicolumn{1}{c}{ Sample ID } & \multicolumn{1}{c}{$\begin{array}{c}\text { Dose } \\
\text { (Mrad) }\end{array}$} & Aqueous pH \\
\hline SCA-0-SB & 0 & 9.60 \\
SCA-0-SB-R & 0 & 11.29 \\
SCA-1.5-SB & 1.5 & 8.78 \\
SCA-1.5-SB-R & 1.5 & 2.50 \\
SCA-3-SB & 3 & 4.12 \\
SCA-6-SB & 6 & 2.61 \\
SCA-6-SB-R & 6 & 2.41 \\
SCA-12-SB & 12 & 3.22 \\
STA-0-SB & 0 & 3.11 \\
STA-0-SB-R & 0 & 2.76 \\
STA-2-SB & 2 & 2.65 \\
STA-2-SB-R & 2 & 2.70 \\
STA-4-SB & 4 & 3.00 \\
STA-8-SB & 8 & 2.82 \\
STA-8-SB-R & 8 & 3.07 \\
STA-16-SB & 16 & 7.12 \\
SCA-6-SBT & 6 & 1.81 \\
STA-8-SBT & 8 & 2.91 \\
Scrub control $b$ & 0 & 1.82 \\
Strip control ${ }^{b}$ & 0 & 2.84 \\
\hline
\end{tabular}

${ }^{a}$ Sample IDs were defined in Section 6.2.1. The " A" following EXT, SC, or ST indicates these are aqueous samples.

${ }^{b}$ Control samples run at ORNL.

approximately half of the TOA was observed at the 16-Mrad dose. On dosing a solvent sample to 50 Mrad (no aqueous phase present), the SRTC group found that calixarene breakdown products become detectable, and the calixarene concentration drops $33 \%$. At the same time, a $10 \%$ decrease in the modifier concentration was noted. The chief breakdown product was identified as 4-sec-butylphenol (SBP), whose buildup was clearly dose related, appearing at a level of ca. $2.5 \mathrm{mM}$ at $16 \mathrm{Mrad}$. The SBP was readily washed out of the solvent with $1 \mathrm{M} \mathrm{NaOH}$.

A very similar picture emerged from the ORNL analytical work. Table 6.5 shows that loss of BOBCalixC6 and modifier is not detectable with confidence up to $16 \mathrm{Mrad}$ dose. At most, the loss of BOBCalixC6 is on the order of $10 \%$ at $16 \mathrm{Mrad}$. Loss of modifier was approximately $16 \%$ at 50 Mrad. TOA suffers the greatest fractional loss among the three solvent components. As much as $79 \%$ loss was observed. Fig. 6.4 shows the trends more clearly amidst the considerable scatter. Under stripping conditions, the loss rate appears to be steady, at most being $0.78 \mathrm{mM} / \mathrm{Mrad}$. However, under extraction and scrubbing conditions, the data suggest that the loss rate is more rapid at low doses than at higher doses. 
Table 6.5. Effect of external gamma dose on baseline solvent composition

\begin{tabular}{|c|c|c|c|c|c|}
\hline Sample ID $a$ & $\begin{array}{c}\text { Dose } \\
\text { (Mrad) }\end{array}$ & $\begin{array}{c}\text { BOBCalixC }^{b} \\
(\mathrm{~mol} / \mathrm{L})\end{array}$ & $\begin{array}{c}\text { Modifier }^{b} \\
(\mathrm{~mol} / \mathrm{L})\end{array}$ & $\begin{array}{l}\mathrm{TOA}^{c} \\
(\mathrm{mM})\end{array}$ & $\begin{array}{l}\mathrm{SBP}^{b} \\
(\mathrm{mM})\end{array}$ \\
\hline SB BE & 0 & 0.0097 & 0.44 & 0.92 & ND \\
\hline EXTO-0-SB & 0 & ND & 0.46 & 0.94 & ND \\
\hline EXTO-0-SB-R & 0 & 0.0118 & 0.53 & 1.12 & ND \\
\hline EXTO-0.5-SB & 0.5 & 0.0114 & 0.51 & 0.56 & 0.4 \\
\hline EXTO-1-SB & 1 & 0.0112 & 0.51 & 0.57 & 0.6 \\
\hline EXTO-2-SB & 2 & 0.0111 & 0.51 & 0.47 & 0.8 \\
\hline EXTO-4-SB & 4 & 0.0110 & 0.51 & 0.40 & 1.6 \\
\hline SCO-0-SB & 0 & ND & 0.45 & 0.96 & ND \\
\hline SCO-0-SB-R & 0 & 0.0108 & 0.48 & 1.03 & ND \\
\hline SCO-1.5-SB & 1.5 & 0.0112 & 0.50 & 0.50 & 0.3 \\
\hline SCO-1.5-SB-R & 1.5 & 0.0109 & 0.50 & 0.75 & 0.2 \\
\hline SCO-3-SB & 3 & 0.0113 & 0.50 & 0.62 & 0.5 \\
\hline SCO-6-SB & 6 & 0.0108 & 0.51 & 0.54 & 1.1 \\
\hline SCO-6-SB-R & 6 & 0.0104 & 0.50 & 0.24 & 0.9 \\
\hline SCO-12-SB & 12 & 0.0099 & 0.51 & 0.42 & 2.2 \\
\hline STO-0-SB & 0 & ND & 0.45 & 0.90 & ND \\
\hline STO-0-SB-R & 0 & 0.0112 & 0.50 & 0.97 & ND \\
\hline STO-2-SB & 2 & 0.0109 & 0.51 & 0.68 & 0.8 \\
\hline STO-2-SB-R & 2 & 0.0105 & 0.49 & 0.95 & 0.3 \\
\hline STO-4-SB & 4 & 0.0108 & 0.51 & 0.60 & 1.2 \\
\hline STO-8-SB & 8 & 0.0108 & 0.52 & 0.63 & 1.9 \\
\hline STO-8-SB-R & 8 & 0.0112 & 0.54 & 0.24 & 1.6 \\
\hline STO-16-SB & 16 & 0.0101 & 0.52 & 0.21 & 3.2 \\
\hline EXTO-0-SBT & 0 & 0.0114 & 0.51 & 0.97 & ND \\
\hline EXTO-2-SBT & 2 & 0.0112 & 0.52 & 0.59 & 0.9 \\
\hline SCO-6-SBT & 6 & 0.0106 & 0.51 & 0.79 & 0.8 \\
\hline STO-8-SBT & 8 & 0.0100 & 0.49 & 0.72 & 1.1 \\
\hline PRISTINE 50 & 50 & ND & 0.42 & 0.21 & 5.0 \\
\hline
\end{tabular}

${ }^{a}$ Sample IDs were defined in Section 6.2.1.

${ }^{b}$ Modifiers (Cs-7SB and Cs-7SBT), BOBCalixC6, and 4-sec-butylphenol were determined by reversed-phase HPLC. Concentrations of 4-sec-butylphenol below $0.5 \mathrm{mM}$ are below the quantitation limit and should considered estimates with considerable uncertainty.

cTrioctylamine was determined by gas chromatography. 


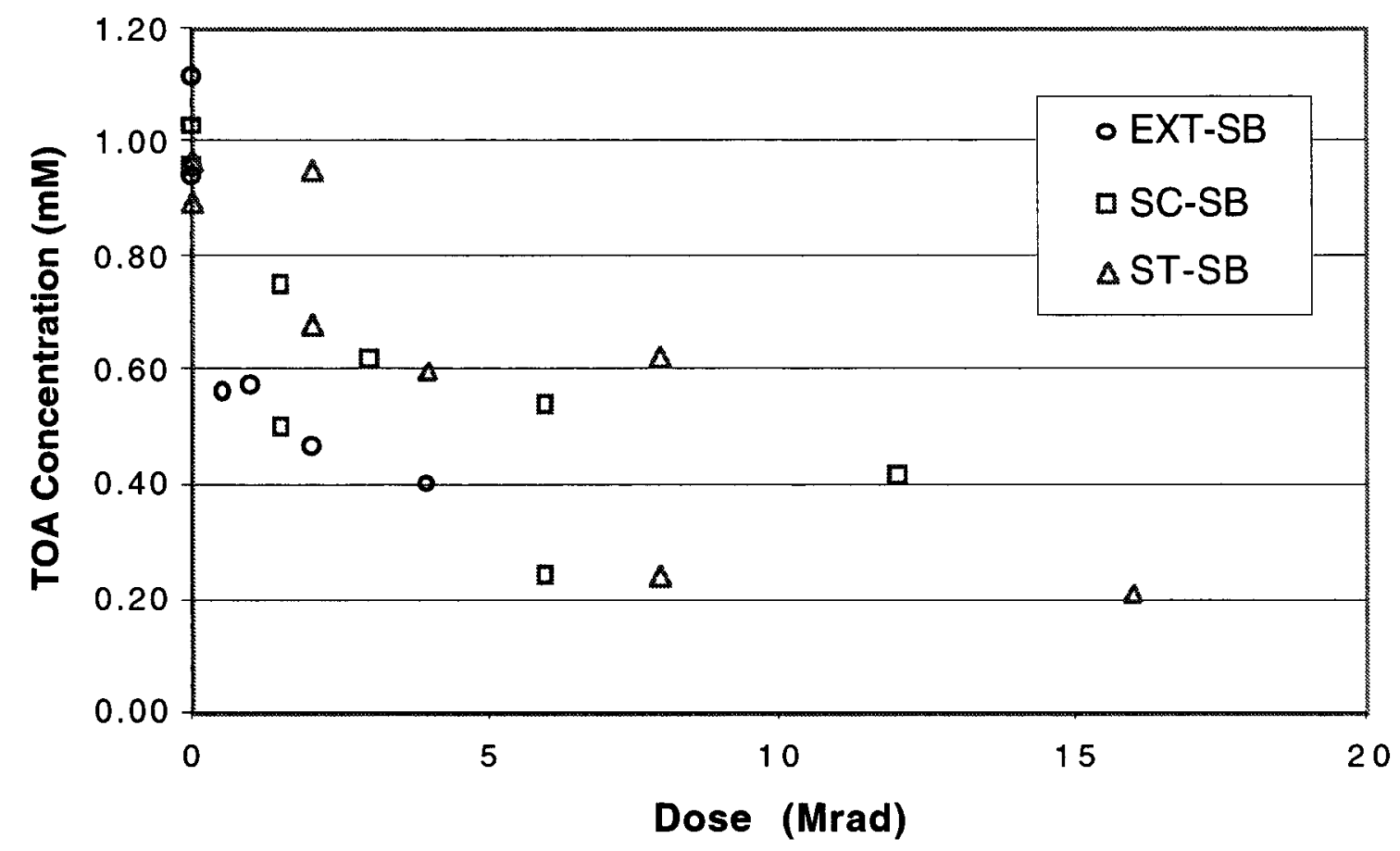

Fig. 6.4. Concentration of TOA in the baseline solvent irradiated over flowsheet solutions.

Table 6.6 shows conservative estimates of the loss rate for BOBCalixC6, Cs-7SB, and TOA in the baseline solvent irradiated in contact with full simulant, scrub solution, or strip solution. In this table, the loss rate was taken from the point exhibiting the maximum loss, or if no loss was detected, the loss rate was assumed to be $10 \%$ over the maximum dose in the series. For BOBCalixC6 and Cs-7SB, loss due to radiolytic breakdown is negligible, less than $1 \%$ being the required annual makeup. For TOA, the annual loss is conservatively expected to be $10 \%$.

The ORNL analyses confirm that the major solvent breakdown product in the organic phase is SBP, an obvious daughter of the modifier. SBP grows in steadily with dose up to $2.5 \mathrm{mM}$ in the solvent at $16 \mathrm{Mrad}$, and as such represents negligible breakdown of the parent Cs-7SB. This result

Table 6.6. Estimates of solvent loss and makeup rates

\begin{tabular}{ccccc}
\hline Component & Series & $\begin{array}{c}\text { Max. loss rate } \\
(\mathrm{mM} / \mathrm{Mrad})\end{array}$ & $\begin{array}{c}\text { Max. makeup } \\
(\mathrm{mM} / \mathrm{y})\end{array}$ & $\begin{array}{c}\text { Max makeup } \\
\text { annual }\end{array}$ \\
\hline BOBCalixC6 & EXT & 0.8 & 0.07 & $0.7 \%$ \\
& SC & 0.1 & 0.01 & $0.1 \%$ \\
& ST & 0.06 & 0.01 & $0.1 \%$ \\
Cs-7SB & EXT & 12 & 1.10 & $0.2 \%$ \\
& SC & 4.2 & 0.39 & $0.1 \%$ \\
& ST & 3.1 & 0.29 & $0.1 \%$ \\
TOA & EXT & 1.1 & 0.10 & $10.1 \%$ \\
& SC & 0.3 & 0.03 & $2.8 \%$ \\
& ST & 0.1 & 0.01 & $0.9 \%$ \\
\hline
\end{tabular}


correlates with the increase in sodium extraction capacity of the irradiated solvent (see below). NMR studies also detected the SBP product growing in to ca. $5 \pm 2 \mathrm{mM}$ at the 16-Mrad dose. The SRTC group showed that the SBP is washed out to the detection limit (ca. $0.04 \mathrm{mM}$ ) with three 1:1 washes with $0.5 \mathrm{M} \mathrm{NaOH} .{ }^{41}$ This observation validates the modifier design, in which the 4-carbon sec-butyl chain is not so large that the sodium salt of SBP cannot partition to alkaline aqueous solutions. Hence, it is expected that SBP will be washed out by the raffinate in the extraction section and in alkaline wash stages and never have an appreciable concentration in the solvent. Moreover, no effect of SBP on stripping occurs in ESS testing up to $10 \mathrm{mM}$ SBP in the solvent.

Results for the aqueous phases irradiated in the presence of baseline solvent with Cs-7SB modifier are given in Table 6.7. No calixarene or TOA were found in any of the samples. Values for the aqueous-phase modifier concentration as determined by the two methods were found to be in reasonable agreement. Since there appeared to be no dose response involved in the concentration of modifier in the aqueous phase, it is concluded that the values obtained represent the normal partitioning of the modifier. The aqueous fluoride concentration evidently has a dose response, but the concentrations are very low relative to the actual number of annual doses received and the expected large dilution by the aqueous streams in the flowsheet. TOC values for the scrub and strip solutions roughly doubled in the irradiation experiments, representing a minor loss of organic matter.

Table 6.7. Analysis of aqueous phase in external irradiation experiments

\begin{tabular}{lccccc}
\hline Sample ID & Treatment $^{b}$ & $\begin{array}{c}\left.\text { C }^{2}-7 \mathrm{SB}\right]^{c} \\
(\mathrm{~mol} / \mathrm{L})\end{array}$ & $\begin{array}{c}\left.\mathrm{FF}^{-}\right] \\
(\mathrm{mM})\end{array}$ & $\begin{array}{c}\text { TOC } \\
(\mu \mathrm{g} / \mathrm{mL})\end{array}$ & $\begin{array}{c}\mathrm{TOC}^{d} \\
(\mathrm{mM})\end{array}$ \\
\hline EXTA-0.5-SB & None & BDL & 17.8 & 410 & 34 \\
EXTA-1-SB & None & BDL & 15.3 & 120 & 10 \\
EXTA-2-SB & None & BDL & 18.6 & 390 & 32 \\
EXTA-4-SB & None & BDL & 15.8 & 380 & 32 \\
SCA-1.5-SB & None & $5.8 \times 10^{-5}$ & 0.68 & 759 & 63 \\
SCA-1.5-SB & SPE & $7.3 \times 10^{-5}$ & & & \\
SCA-3-SB & None & $6.1 \times 10^{-5}$ & 0.38 & 868 & 72 \\
SCA-3-SB & SPE & $7.2 \times 10^{-5}$ & & & \\
SCA-6-SB & None & $6.5 \times 10^{-5}$ & 0.96 & 962 & 80 \\
SCA-6-SB & SPE & $7.6 \times 10^{-5}$ & & & \\
SCA-12-SB & None & $7.1 \times 10^{-5}$ & 2.9 & 1480 & 123 \\
SCA-12-SB & SPE & $7.4 \times 10^{-5}$ & & & \\
STA-2-SB & None & $7.4 \times 10^{-5}$ & 0.73 & 299 & 25 \\
STA-2-SB & SPE & $8.1 \times 10^{-5}$ & & & \\
STA-4-SB & None & $5.2 \times 10^{-5}$ & 0.65 & 101 & 8 \\
STA-4-SB & SPE & $6.6 \times 10^{-5}$ & & & \\
STA-8-SB & None & $6.8 \times 10^{-5}$ & 2.7 & 692 & 58 \\
STA-8-SB & SPE & $7.8 \times 10^{-5}$ & & & \\
STA-16-SB & None & $6.1 \times 10^{-5}$ & 3.3 & 670 & 56 \\
STA-16-SB & SPE & $7.4 \times 10^{-5}$ & & & \\
\hline
\end{tabular}

aSample IDs were defined in Section 6.2.1. The "A" following EXT, SC, or ST indicates these are aqueous samples.

${ }^{b}$ SPE refers to Solid Phase Extraction.

${ }^{c} \mathrm{BDL}$ indicates below detection limit.

${ }^{d}$ Same TOC data converted to units of $\mathrm{mM}$ carbon. 


\subsubsection{NMR Spectroscopy}

The first set of irradiated solvent samples received from the SRTC involved the baseline solvent B000718-110W, containing the Cs-7SB modifier. These samples were received during the month of June 2000. The proton NMR results on the solvent phases for these irradiated solvents are shown in Table 6.8. In some cases, only the low- and high-dose samples were analyzed. The general observation obtained from proton NMR analysis was that the solvent is stable to the moderate doses received. The only observable degradation product was 4-sec-butylphenol, and it was present in trace quantities (the peaks were usually too small to integrate). The only observable resonances due to this phenol were the two aromatic hydrogens located ortho to the hydroxyl group at $6.75 \mathrm{ppm}$. These appeared as small bumps in the aromatic region in between aromatic protons due to the modifier $(6.84 \mathrm{ppm})$ and resonances due to the BOBCalixC6 $(6.70 \mathrm{ppm})$. Only for the sample subjected to the highest radiation dose in the presence of an aqueous phase, sample ST-16-SB in contact with the strip phase, was the signal due to the phenol large enough to be effectively integrated. The concentration was approximately $1.5-2 \%$ of the concentration of the modifier, which puts the phenol concentration on the order of $7.5-10 \mathrm{mM}$. The solvent sample that was subjected to the highest overall radiation dose was a sample of solvent only (no aqueous phase) that was irradiated to 50 Mrad. The amount of 4-sec-butylphenol in this sample was less than the amount in the ST-16-SB.

Upon nitration of the aromatic ring, which could in principle occur following extended radiolysis in the presence of nitric acid (especially the scrub solution), selected aromatic protons on the BOBCalixC6 shift positions and coupling patterns. Comparing these regions (between 7.05 and 6.95 $\mathrm{ppm}$ ) of the most irradiated samples in contact with the scrub solution (such as SC-12-SB) with corresponding regions of the pristine unirradiated solvent did not reveal any noticeable changes (none indicating that nitration was taking place). For the modifier (and also for 4-sec-butylphenol), nitration would occur at the 2-position on the aromatic ring, and according two of the aromatic protons would be expected to shift down to the region between 8.5 and $7.5 \mathrm{ppm}$. This region of the proton NMR spectrum was examined for signs of nitrated aromatic species, but hardly anything was observable beyond baseline noise. Hence, no nitrated products were observed by proton NMR.

Also, since the modifier is present at 50 times the concentration of the BOBCalixC6, it is difficult to determine within experimental error whether the BOBCalixC6 is decreasing in concentration in the solvent. The only resonance that is separated sufficiently from the aromatic peaks of the modifier to integrate adequately is the triplet that occurs at $6.7 \mathrm{ppm}$, corresponding to the four protons situated on the calix[4]arene portion of the BOBCalixC6 that are para to the oxygens connecting the crown ether. However, they still reside off the shoulder of the modifier aromatic protons, and the uncertainty in the integration due to the sloping baseline is much greater than the amount the BOBCalixC6 concentration is decreased by radiolytic degradation.

Fluorine-19 NMR spectra were obtained on selected irradiated solvent samples (EXT-4-SB, SC-12-SB, ST-16-SB, and PRIST-50-SB). The only resonances observed in the 19F NMR spectra of the first three solvent samples were those due to the modifier and its minor structural isomer ( 1.3-1.5\% of the Cs-7SB modifier). However, for the PRIST-50-SB sample, an additional set of resonances attributable to a 2,2,3,3-tetrafluoropropyl group were observed at a concentration equivalent to ca. $0.5 \%$ of the modifier. This could be the fluorinated fragment of the modifier that cleaves off during radiolysis to generate the 4-sec-butylphenol. This fragment, a 2,2,3,3-tetrafluoropropyl substituted glycerol, should be sufficiently hydrophilic to wash into the aqueous phase (note that the PRIST-50-SB sample that was not in contact with an aqueous phase).

Since the aqueous phases are likely to contain the hydrophilic fluorinated radiolytic degradation fragments of the Cs-7SB modifier, for selected samples the aqueous phases were examined by ${ }^{19} \mathrm{~F}$ NMR (these samples were generally too dilute in the degradation species to analyze by ${ }^{13} \mathrm{C}$ NMR and obtain meaningful results). Aqueous phases from the following four samples were analyzed: EXT-4-SB, SC-6-SB, ST-8-SB, and ST-16-SB. The findings are summarized in Table 6.9. The last sample was found to be alkaline rather than acidic. In alkaline media the free fluoride peak occurs generally between -118 and $-123 \mathrm{ppm}$ (relative to $\mathrm{CCl}_{3} \mathrm{~F}$, set to $0.0 \mathrm{ppm}$ ); in acidic media the free fluoride ion appears closer to $-120 \mathrm{ppm}$. The fluoride ion that is present in the simulant was the 
Table 6.8. NMR analyses of irradiated solvent sample organic phases received from SRS in June ${ }^{a}$

\begin{tabular}{|c|c|c|c|}
\hline Sample code & $\begin{array}{l}\text { Aqueous } \\
\text { phase }\end{array}$ & $\begin{array}{l}\text { Dose } \\
\text { (Mrad) }\end{array}$ & Appearance and comment \\
\hline EXT-0.5-SB & $\begin{array}{c}\text { Full } \\
\text { simulant }\end{array}$ & 0.5 & $\begin{array}{l}\text { Solvent pale yellow; barely detectable } \\
\text { 4-sec-butylphenol. }\end{array}$ \\
\hline EXT-1-SB & $\begin{array}{c}\text { Full } \\
\text { simulant }\end{array}$ & 1 & Solvent pale yellow; not analyzed. \\
\hline EXT-2-SB & $\begin{array}{c}\text { Full } \\
\text { simulant }\end{array}$ & 2 & Solvent yellow; not analyzed. \\
\hline EXT-4-SB & $\begin{array}{c}\text { Full } \\
\text { simulant }\end{array}$ & 4 & $\begin{array}{l}\text { Solvent yellow (darker than above). }{ }^{1 \mathrm{H}} \text { : trace } \\
\text { 4-sec-butylphenol. 19F: no detectable decomposition. }\end{array}$ \\
\hline $\mathrm{SC}-1.5-\mathrm{SB}$ & Scrub sol'n & 1.5 & Solvent very pale yellow; no detectable decomposition. \\
\hline $\mathrm{SC}-3-\mathrm{SB}$ & Scrub sol'n & 3 & Solvent pale yellow; not analyzed. \\
\hline SC-6-SB & Scrub sol'n & 6 & Solvent pale yellow; trace 4-sec-butylphenol. \\
\hline SC-12-SB & Scrub sol'n & 12 & $\begin{array}{l}\text { Solvent yellow. }{ }^{1} \mathrm{H} \text { : trace } 4-s e c \text {-butylphenol. }{ }^{19} \mathrm{~F} \text { : no } \\
\text { detectable decomposition. }\end{array}$ \\
\hline ST-2-SB & Strip sol'n & 2 & Solvent very pale yellow; no detectable decomposition. \\
\hline ST-4-SB & Strip sol'n & 4 & Solvent very pale yellow; not analyzed. \\
\hline ST-8-SB & Strip sol'n & 8 & Solvent pale yellow; trace 4-sec-butylphenol. \\
\hline ST-16-SB & $\begin{array}{l}\text { Strip } \\
\text { solution }\end{array}$ & 16 & $\begin{array}{l}\text { Solvent pale yellow. } 1 \mathrm{H} \text { : about } 1.5-2 \% \\
\text { 4-sec-butylphenol (most of any sample). }{ }^{19} \mathrm{~F} \text { : no } \\
\text { detectable decomposition. }\end{array}$ \\
\hline PRIST-50-SB & $\begin{array}{l}\text { None, } \\
\text { (pristine } \\
\text { solvent) }\end{array}$ & 50 & $\begin{array}{l}\text { Solvent pale yellow. }{ }^{1} \mathrm{H} \text { : trace } 4-\sec \text {-butylphenol. }{ }^{19} \mathrm{~F} \text { : ca. } \\
0.5 \% \text { (modifier }=100 \% \text { ) of a decomposition product } \\
\text { containing a } 2,2,3,3 \text {-tetrafluoropropyl group. }\end{array}$ \\
\hline
\end{tabular}

${ }^{a}$ All for solvent B000718-110W; Cs-7SB modifier; analyses by ${ }^{1} \mathrm{H}$ NMR unless otherwise noted.

major peak in the $19 \mathrm{~F}$ spectrum of the EXT-4-SB sample, with traces of resonances attributable to the modifier and an unknown degradation product (possibly the 2,2,3,3-tetrafluoropropyl substituted glycerol species) also being present. The modifier is observed to partition to the simulant very slightly $\left(\sim 2 \times 10^{-5} \mathrm{M}\right)$, and the peak assignments were made by comparison of the spectrum with one obtained on cold simulant contacted with solvent (non-irradiated). The fragment was present at about $2 \%$ of the fluoride ion peak, and assuming a fluoride concentration in the simulant of $0.023 \mathrm{M}$, the concentration of the fragment is about $5 \times 10^{-5} \mathrm{M}$, which would correspond to about $0.1 \%$ degradation of the modifier. This agrees with the trace amount of phenol seen in the simulant; trace refers to $<0.5 \%$, which is estimated to be the highest amount that can be quantified (integrated) in the solvent by proton NMR.

The solvent samples in contact with the scrub and strip phases (and also subjected to more radiolysis) displayed substantially more peaks in the $19 \mathrm{~F}$ NMR spectra indicating breakdown of the 2,2,3,3-tetrafluoropropyl substituted glycerol fragment. The modifier is still the main species in the spectrum, but resonances attributable to 2,2,3,3-tetrafluoroethanol are observed, as well as resonances that could be due to 2,2,3,3-tetrafluoropropyl substituted glycerol (likely the identity of the "2,2,3,3-tetrafluoropropyl ether" fragment listed in Table 6.9.) What is believed to be fluoride ion is only observed for the two solvent phases subjected to the highest doses (ST-8-SB, and ST-16-SB). 
The chemical shift of the fluoride ion for the ST-16-SB sample is closer to where one would expect it under alkaline conditions, and there is evidence that this sample is in fact alkaline. Increasing radiation dose should lead to increased radiolytic breakdown, and this does appear to be the case, since the smaller fragments appear to be more abundant in the ST-16-SB sample. For example, 2,2,3,3-tetrafluoropropanol and what is believed to be 1-(2,2,3,3-tetrafluoropropoxy) glycerol are the second and third most abundant species in the ST-16-SB sample, but the order is reversed in the -pathway that includes cleavage of the 2,2,3,3-tetrafluoropropoxy moiety from 1-(2,2,3,3-tetrafluoropropoxy) glycerol.

Table 6.9. Fluorine-19 NMR analyses of irradiated solvent sample aqueous phases received from SRS in June ${ }^{a}$

\begin{tabular}{|c|c|c|c|}
\hline Sample code & $\begin{array}{l}\text { Aqueous } \\
\text { phase }\end{array}$ & $\begin{array}{c}\text { Dose } \\
\text { (Mrad) }\end{array}$ & Appearance and comment \\
\hline EXT-4-SB & $\begin{array}{c}\text { Fullsimu } \\
\text { lant }\end{array}$ & 4 & $\begin{array}{l}\text { Mostly free fluoride contained in the simulant (at }-122.66 \\
\text { ppm); traces of modifier which partitioned ( } 1 \% \text { relative to } \\
\text { fluoride ion); some unidentified degradation products } \\
\text { containing the } 2,2,3,3 \text {-tetrafluoropropyl fragment }(\sim 2 \% \\
\text { relative to fluoride ion). }\end{array}$ \\
\hline SC-6-SB & $\begin{array}{c}\text { Scrubsol } \\
\text { ution }\end{array}$ & 6 & $\begin{array}{l}\text { Many peaks corresponding to Cs-7SB modifier (major } \\
\text { component); a } 2,2,3,3 \text {-tetrafluoro-propylether (second major); } \\
\text { 2,2,3,3-tetrafluoro-propanol (third major); a small amount of a } \\
\text { fourth species containing the } 2,2,3,3 \text {-tetrafluoropropyl } \\
\text { fragment; several other peaks which may be from } \\
\text { fragmentation of the tetrafluoropropyl group; free fluoride ion } \\
\text { not apparent. }\end{array}$ \\
\hline ST-8-SB & $\begin{array}{c}\text { Stripsolu } \\
\text { tion }\end{array}$ & 8 & $\begin{array}{l}\text { Many peaks corresponding to Cs-7SB modifier (major } \\
\text { component); 2,2,3,3-tetrafluoropropanol (second major); a } \\
2,2,3,3 \text {-tetrafluoropropyl-ether (third major); a small amount } \\
\text { of a fourth species containing the } 2,2,3,3 \text {-tetrafluoro-propyl } \\
\text { fragment; several other peaks which may be from } \\
\text { fragmentation of the tetrafluoropropyl group; free fluoride at } \\
-128.73 \mathrm{ppm} \text { (acidic conditions). }\end{array}$ \\
\hline ST-16-SB & $\begin{array}{c}\text { Stripsolu } \\
\text { tion }\end{array}$ & 16 & $\begin{array}{l}\text { Many peaks corresponding to Cs-7SB modifier (major } \\
\text { component); 2,2,3,3-tetrafluoropropanol (second major); a } \\
\text { 2,2,3,3-tetrafluoropropyl-ether (third major); more of the } \\
\text { fourth species containing the } 2,2,3,3 \text {-tetrafluoro-propyl } \\
\text { fragment; several other peaks which may be from } \\
\text { fragmentation of the tetrafluoropropyl group; free fluoride at } \\
-118.19 \mathrm{ppm} \text { [alkaline conditions(?)]. }\end{array}$ \\
\hline
\end{tabular}

${ }^{a}$ All for solvent B000718-110W; Cs-7SB modifier. 
Another set of irradiated samples was received from the SRS in July and August, corresponding to another set of Cs-7SB irradiated solvent, Cs-6/Isopar irradiated solvent, and Cs-7SBT irradiated solvent. The Cs- 6 modifier forms a hydrate that lowers its solubility dramatically in the solvent; this phenomenon will be discussed further in Chap. 7. Hence, very few samples remained as solutions; however, the Cs-6 solvent irradiated in the presence of the simulant at 2-Mrad dose has remained as a solution, and a proton NMR spectrum was obtained on a sample of that solvent. As shown in Table 6.10 (along with the results of proton NMR analyses on the other solvents), no detectable degradation was observed (the 4-sec-butylphenol peaks were too small to see). The results were essentially in line for what was observed previously: 4-sec-butylphenol grows in to become observable with increasing

Table 6.10. Proton NMR analyses of irradiated solvent sample organic phases received from SRS in July and August

\begin{tabular}{|c|c|c|c|c|}
\hline Sample code & Solvent phase & $\begin{array}{l}\text { Aqueous } \\
\text { phase }\end{array}$ & $\begin{array}{l}\text { Dose } \\
\text { (Mrad) }\end{array}$ & Comment \\
\hline FE-1-E-0.5-2 & $\begin{array}{l}\text { Cs 6/Isopar L } \\
\text { B000718-108W }\end{array}$ & $\begin{array}{l}\text { Full } \\
\text { simulant }\end{array}$ & 0.5 & $\begin{array}{l}\text { Solvent straw-colored; not analyzed; } \\
\text { gelled. }\end{array}$ \\
\hline FE-E-2-2 & $\begin{array}{l}\text { Cs 6/Isopar L } \\
\text { B000718 108W }\end{array}$ & $\underset{\text { simulant }}{\text { Full }}$ & 2 & $\begin{array}{l}\text { Solvent straw-colored; no detectable } \\
\text { signs of degradation; did NOT gel! }\end{array}$ \\
\hline $\begin{array}{l}\text { FD-18-SC } \\
\text { Blank }\end{array}$ & $\begin{array}{c}\text { Cs 7SB } \\
\text { B000718 132W }\end{array}$ & $\begin{array}{l}\text { Scrub } \\
\text { sol'n }\end{array}$ & 0 & Solvent colorless; clean control. \\
\hline FD-17-SC-1.5 & $\begin{array}{c}\text { Cs 7SB } \\
\text { B000718 132W }\end{array}$ & $\begin{array}{l}\text { Scrub } \\
\text { sol'n }\end{array}$ & 1.5 & $\begin{array}{l}\text { Solvent pale yellow; no detectable signs } \\
\text { of degradation. }\end{array}$ \\
\hline FD-17-SC-6 & $\begin{array}{c}\text { Cs 7SB } \\
\text { B000718 132W }\end{array}$ & $\begin{array}{l}\text { Scrub } \\
\text { sol'n }\end{array}$ & 6 & $\begin{array}{l}\text { Solvent pale yellow; trace } 4-s e c- \\
\text { butylphenol. }\end{array}$ \\
\hline $\begin{array}{l}\text { FD-18-ST } \\
\text { Blank }\end{array}$ & $\begin{array}{c}\text { Cs 7SB } \\
\text { B000718 132W }\end{array}$ & $\begin{array}{l}\text { Strip } \\
\text { sol'n }\end{array}$ & 0 & Identical to FD-18-SC Blank. \\
\hline FD-16-ST-2 & $\begin{array}{c}\text { Cs 7SB } \\
\text { B000718 132W }\end{array}$ & $\begin{array}{l}\text { Strip } \\
\text { sol'n }\end{array}$ & 2 & $\begin{array}{l}\text { Solvent almost colorless; no detectable } \\
\text { signs of degradation. }\end{array}$ \\
\hline FD-16-ST-8 & $\begin{array}{c}\text { Cs-7SB } \\
\text { B000718 132W }\end{array}$ & $\begin{array}{l}\text { Strip } \\
\text { sol'n }\end{array}$ & 8 & $\begin{array}{l}\text { Solvent pale yellow; trace 4-sec- } \\
\text { butylphenol. }\end{array}$ \\
\hline FH-E-Blank-2 & $\begin{array}{c}\text { Cs-7SBT } \\
\text { B000718 107W }\end{array}$ & $\begin{array}{l}\text { Full } \\
\text { simulant }\end{array}$ & $\mathbf{0}$ & $\begin{array}{l}\text { Solvent almost colorless; aromatic } \\
\text { region shows "SBT" impurities. }\end{array}$ \\
\hline FH-1-E-2-2 & $\begin{array}{c}\text { Cs 7SBT } \\
\text { B000718 107W }\end{array}$ & $\begin{array}{l}\text { Full } \\
\text { simulant }\end{array}$ & 2 & $\begin{array}{l}\text { Solvent straw-colored; NMR nearly } \\
\text { identical to FH E Blank } 2 \text {. }\end{array}$ \\
\hline FH-SC-6 & $\begin{array}{c}\text { Cs 7SBT } \\
\text { B000718 107W }\end{array}$ & $\begin{array}{l}\text { Scrub } \\
\text { sol'n }\end{array}$ & 6 & $\begin{array}{l}\text { Solvent yellow; trace } \\
\text { 4-sec-butylphenol. }\end{array}$ \\
\hline FH-3-ST-8 & $\begin{array}{c}\text { Cs 7SBT } \\
\text { B000718 107W }\end{array}$ & Strip & 8 & $\begin{array}{l}\text { Solvent pale yellow; trace } \\
\text { 4-sec-butylphenol. }\end{array}$ \\
\hline
\end{tabular}


radiation dose. Only at the 6-8 Mrad dose levels was 4-sec-butylphenol observable (meaning it was greater than $0.5 \%$ of the modifier concentration, or $2-3 \mathrm{mM}$ ). The Cs-7SBT solvent has more impurities in it than the Cs-7SB solvent, owing to the lower purity of the starting 4-sec-butylphenol, but it does not appear that the Cs-7SBT solvent degrades any faster than the Cs-7SB solvent (at least by proton NMR).

The ${ }^{19} \mathrm{~F}$ NMR spectra on selected aqueous phases from these samples (see Table 6.11) gave essentially the same results seen previously, except that a higher percentage of 2,2,3,3-tetrafluoropropanol was observed in the Cs-7SBT sample (FH-3-ST-8) than the Cs-7SB sample (FD-16-ST-8) that received the same dose of $8 \mathrm{Mrad}$. An unidentified singlet was found in the blank strip sample. The dependence of the chemical shift of fluoride ion with concentration and $\mathrm{pH}$ will need to be examined more closely to make more definitive assignments.

In general, the radiation stability of the solvent is excellent. Only under high ( $>6 \mathrm{Mrad}$ ) doses is any detectable sign (by NMR) of degradation observed, and even then the amount is small.

Table 6.11. Fluorine-19 NMR analyses of selected aqueous phases of irradiated samples received from SRS in August

\begin{tabular}{|c|c|c|c|c|}
\hline Sample code & Solvent phase & $\begin{array}{l}\text { Aqueous } \\
\text { phase }\end{array}$ & $\begin{array}{c}\text { Dose } \\
\text { (Mrad) }\end{array}$ & Comment \\
\hline $\begin{array}{l}\text { FD-18-ST } \\
\text { Blank }\end{array}$ & Cs 7SB & Strip & 0 & $\begin{array}{l}\text { Singlet at }-119.23 \mathrm{ppm} \\
\text { (fluoride? shift isn't correct), } \\
\text { and Cs-7SB modifier } \\
\text { (multiplet at }-124.23 \mathrm{ppm} ; \\
\text { doublet of triplets at }-138.25 \\
\text { ppm). }\end{array}$ \\
\hline FD 16 ST 8 & Cs 7SB & Strip & 8 & $\begin{array}{l}\text { Many peaks corresponding } \\
\text { to Cs-7SB modifier (major } \\
\text { component); a } \\
2,2,3,3 \text { tetrafluoropropylether } \\
\text { (second major); } \\
2,2,3,3 \text { tetrafluoropropanol } \\
\text { (third major); several other } \\
\text { peaks which may be from } \\
\text { fragmentation of the } \\
\text { tetrafluoropropyl group; no } \\
\text { obvious sign of free fluoride. }\end{array}$ \\
\hline FH-3-ST-8 & Cs-7SBT & Strip & 8 & $\begin{array}{l}\text { Like FD-16-ST- } 8 \text { with the } \\
\text { following differences: the } \\
\text { relative percentage of } \\
2,2,3,3 \text { tetrafluoro-propanol } \\
\text { is higher; a fourth species } \\
\text { containing the } \\
2,2,3,3 \text { tetrafluoro-propyl } \\
\text { fragment; an additional } \\
\text { broad singlet at }-141 \mathrm{ppm} \text {. }\end{array}$ \\
\hline
\end{tabular}




\subsubsection{Electro-Spray Mass Spectrometry}

The irradiated solvent samples were analyzed by electrospray mass spectrometry (ES-MS). The stability of the solvent is remarkable, as no changes between the irradiated samples and pristine solvent contacted with the full simulant, scrub, and strip solutions were observed. No major degradation products were found, except for the strip sample irradiated at $16 \mathrm{Mrad}$ (160-year dose). Unassigned peaks in the cation mode at 370 and $740 \mathrm{~m} / \mathrm{z}$ amu appear and seem to become major impurities in the system. A probably related species at $370 \mathrm{~m} / \mathrm{z}$ amu appears also in the anion mode. Signs of these products can also be found in the other samples, but to a lesser extent. These three presently unidentified peaks were also observed in the ES-MS analysis of the solvent samples from the thermal stability experiment described in Section 5.3.5.

\subsubsection{Interfacial Tension}

Interfacial tension was measured for the baseline solvent in contact with simulant, scrub, and strip solutions as a function of radiation dose. For the samples irradiated at the SRTC, the aqueous and organic phases were irradiated together and the interfacial tension of this equilibrated mixture determined. Unirradiated samples were used as controls.

The results shown in Table 6.12 indicate a very clear dose response for each of the extract, scrub, and strip sets. The extraction set had the largest response, going from $19.7 \mathrm{dyne} / \mathrm{cm}$ at zero dose to $7.9 \mathrm{dyne} / \mathrm{cm}$ at $4 \mathrm{Mrad}$. This would be understandable in terms of the buildup of the presumably interfacially active sodium salt of 4-sec-butylphenol. The scrub set, interestingly, has the opposite effect, wherein the interfacial tension increases from $8.4 \mathrm{dyne} / \mathrm{cm}$ at $1.5 \mathrm{Mrad}$ to $14.0 \mathrm{dyne} / \mathrm{cm}$. Stripping interfacial tensions decrease from $16.2 \mathrm{dyne} / \mathrm{cm}$ at $2 \mathrm{Mrad}$ to $13.5 \mathrm{dyne} / \mathrm{cm}$ at $16 \mathrm{Mrad}$.

Table 6.12. Interfacial tension of control and irradiated samples

\begin{tabular}{cccc}
\hline Sample identification & Aqueous phase & Dose, Mrad & Tension, dyne/cm \\
\hline Washed solvent/scrub control & Scrub & 0 & 8.79 \\
Washed solvent/strip control & Strip & 0 & 9.12 \\
EXT-0-SB & Simulant & 0 & 19.7 \\
EXT-0.5-SB & Simulant & 0.5 & 13.1 \\
EXT-1-SB & Simulant & 1 & 12.8 \\
EXT-2-SB & Simulant & 2 & 9.4 \\
EXT-4-SB & Simulant & 4 & 7.8 \\
SC-1.5-SB & Scrub & 1.5 & 8.4 \\
SC-3-SB & Scrub & 3 & 8.9 \\
SC-6-SB & Scrub & 6 & 10.8 \\
SC-12-SB & Scrub & 12 & 14.0 \\
ST-2-SB & Strip & 2 & 16.2 \\
ST-4-SB & Strip & 4 & 16.1 \\
ST-8-SB & Strip & 8 & 15.9 \\
ST-16-SB & Strip & 16 & 13.5 \\
Scrub control & Scrub & 0 & 15.4 \\
Strip control & Strip & 0 & 14.7 \\
Washed solvent/Cs control & $1 \mathrm{mM} \mathrm{HNO}+0.1 \mathrm{mM} \mathrm{Cs}$ & 0 & 14.8 \\
Washed solvent/Cs control & $1 \mathrm{mM} \mathrm{HNO}+1.0 \mathrm{mM} \mathrm{Cs}$ & 0 & 14.4 \\
\hline
\end{tabular}




\subsubsection{Sodium Extraction Capacity}

A change in the capacity of the solvent for sodium extraction under alkaline conditions was expected to be an indicator of solvent degeneration. Data for a ${ }^{22} \mathrm{Na}$ tracer experiment on irradiated solvents validate this expectation as shown in Table 6.13. Assuming the SRTC simulant is $5.6 \mathrm{M} \mathrm{Na}$, the sodium content of the pristine solvent containing modifier Cs-7SB equilibrated with simulant was found to be $3.6 \mathrm{mM}$. This increases $36 \%$ to $4.9 \mathrm{mM}$ after a dose of $4 \mathrm{Mrad}$. The difference, 1.3 $\mathrm{mM}$, serves as an indicator of the added cation-exchange capacity (probably mostly phenols) of the solvent on irradiation. The sodium content of the pristine solvent containing Cs-7SBT equilibrated with simulant is $4.2 \mathrm{mM}$. This also increases $36 \%$ to $5.73 \mathrm{mM}$ sodium extracted after 2 Mrad dosing. It may be noted that the increased sodium extraction capacity of the two solvents is on the order of the concentration of 4-sec-butylphenol formed upon irradiation (Table 6.5).

$\mathrm{A}^{22} \mathrm{Na}$ experiment was also conducted on the organic-solvent and aqueous-simulant samples of solvent containing Cs-6 modifier that had been externally irradiated and had not gelled. The samples were re-contacted in the same way as mentioned for Cs-7SB and Cs-7SBT. The 0.5-Mrad sample solvent was thick and opaque as received, but once re-contacted formed a white gel. The 2.0-Mrad sample did not gel and was still much the same consistency as when received. A pristine solvent was not tested. The sodium content of the irradiated solvent at both doses was $8.6 \mathrm{mM}$.

Table 6.13. Sodium extraction capacity of EXT series as a function of dose

\begin{tabular}{lccc}
\hline Sample ID & Dose (Mrad) & $\begin{array}{r}\text { Sodium } \\
\text { Cs-7SBT }\end{array}$ & $\begin{array}{r}\text { extracted (mM) } \\
\text { Cs-7SB }\end{array}$ \\
\hline & Pristine & 4.20 & 3.60 \\
EXT-0 & 0 & 4.41 & 4.60 \\
EXT-0.5 & 0.5 & & 4.10 \\
EXT-1 & 1 & & 4.54 \\
EXT-2 & 2 & 5.73 & 4.62 \\
EXT-4 & 4 & & 4.86 \\
\hline
\end{tabular}

\subsubsection{Extract/Scrub/Strip Performance}

Experiments on a series of 12 samples and two controls were run to determine the influence of external gamma irradiation on the solvent-extraction properties. The solvent, whatever the stage it was irradiated at, was taken through two full cycles of extraction, scrub, and three strips. It is remarkable that the first cycle shows very little change between the cesium distribution ratios obtained with the irradiated solvent and the controls. Differences start appearing for the highest doses in the scrub and strip series (see Table 6.14). This is not surprising since those doses are higher than 7 times the annual doses, and it is reasonable to anticipate some degradation and alteration of the solvent properties. For all the other samples, performance is stable.

The main increase in the distribution ratio values appear during the second cycle. Based on the previous experiments involving repeated contacts and multiple cycling of the solvent, an increase was expected. Once again, the values obtained with the irradiated solvent are marginally higher than those obtained with the controls. It appears that below twice the annual dose, the solvent shows no effect of degradation of the cesium extraction properties. The trend exhibited by the two highest doses (scrub and strip) is definitely confirmed. Similar results were obtained with samples of solvent with Cs7SB (repeated irradiation experiment) and Cs7SBT, confirming all the observations made in June (samples received in September 2000). 
Table 6.14. ESS data on externally irradiated samples

\begin{tabular}{|c|c|c|c|c|c|c|c|c|c|c|c|}
\hline \multirow[t]{3}{*}{ Sample ID } & \multirow{3}{*}{$\begin{array}{l}\text { Dose } \\
\text { (Mrad) } \\
\end{array}$} & \multicolumn{10}{|c|}{ Cesium distribution ratio $\left(D_{\mathrm{Cs}}\right)$} \\
\hline & & \multicolumn{5}{|c|}{ First cycle } & \multicolumn{5}{|c|}{ Second cycle } \\
\hline & & Extract & Scrub & Strip \#1 & Strip \#2 & Strip \#3 & Extract & Scrub & Strip \#1 & Strip \#2 & Strip \#3 \\
\hline Control $\mathrm{O} / \mathrm{O}^{a}$ & 0 & 13.2 & 1.61 & 0.149 & 0.085 & 0.065 & 15.5 & 1.84 & 0.269 & 0.123 & 0.107 \\
\hline Control $\mathrm{O} / \mathrm{S}^{b}$ & 0 & 15.8 & 1.48 & 0.119 & 0.077 & 0.054 & 17.4 & 1.67 & 0.268 & 0.102 & 0.086 \\
\hline Avg. $\mathrm{E}_{2} \mathrm{SS}^{c}$ & 0 & 17.1 & 1.57 & 0.229 & 0.136 & 0.081 & & & & & \\
\hline ORNL $E_{2} \mathrm{SS}^{d}$ & 0 & 17.0 & 1.56 & 0.188 & 0.115 & 0.086 & & & & & \\
\hline EXTO-0-SB & 0 & & & & & & & & & & \\
\hline EXTO-0-SB-R & 0 & 20.7 & 1.96 & 0.189 & 0.106 & 0.076 & 21.4 & 2.00 & 0.198 & 0.147 & 0.153 \\
\hline EXTO-0.5-SB & 0.5 & 16.8 & 1.53 & 0.171 & 0.100 & 0.072 & 18.5 & 1.74 & 0.369 & 0.127 & 0.109 \\
\hline EXTO-1-SB & 1 & 15.8 & 1.50 & 0.167 & 0.098 & 0.070 & 16.6 & 1.76 & 0.358 & 0.135 & 0.105 \\
\hline EXTO-2-SB & 2. & 17.4 & 1.54 & 0.180 & 0.102 & 0.076 & 17.6 & 1.80 & 0.440 & 0.153 & 0.118 \\
\hline EXTO-4-SB & 4 & 16.2 & 1.54 & 0.189 & 0.113 & 0.083 & 19.0 & 1.82 & 0.349 & 0.164 & 0.136 \\
\hline SCO-0-SB & 0 & & & & & & & & & & \\
\hline SCO-0-SB-R & 0 & & 1.53 & 0.121 & 0.047 & 0.028 & 20.9 & 1.82 & 0.178 & 0.121 & 0.111 \\
\hline SCO-1.5-SB & 1.5 & & 2.02 & 0.185 & 0.076 & 0.042 & 18.8 & 1.83 & 0.423 & 0.154 & 0.113 \\
\hline SCO-1.5-SB-R & 1.5 & & 1.69 & 0.191 & 0.117 & 0.082 & 21.4 & 1.85 & 0.202 & 0.225 & 0.128 \\
\hline SCO-3-SB & 3 & & 1.46 & 0.170 & 0.106 & 0.077 & 19.4 & 1.80 & 0.395 & 0.157 & 0.123 \\
\hline SCO-6-SB & 6 & & 1.30 & 0.187 & 0.116 & 0.083 & 19.2 & 1.75 & 0.462 & 0.154 & 0.129 \\
\hline SCO-6-SB-R & 6 & & 1.56 & 0.209 & 0.123 & 0.087 & 21.6 & 1.89 & 0.220 & 0.255 & 0.167 \\
\hline SCO-12-SB & 12 & & 1.14 & 0.175 & 0.112 & 0.082 & 18.5 & 1.88 & 0.480 & 0.184 & 0.174 \\
\hline STO-0-SB & 0 & & & & & & & & & & \\
\hline STO-0-SB-R & 0 & & & 0.292 & 0.121 & 0.083 & 20.9 & 1.80 & 0.183 & 0.183 & 0.103 \\
\hline STO-2-SB & 2 & & & 0.310 & 0.116 & 0.073 & 19.1 & 1.76 & 0.344 & 0.134 & 0.112 \\
\hline STO-2-SB-R & 2 & & & 0.407 & 0.130 & 0.086 & 20.4 & 1.83 & 0.204 & 0.191 & 0.111 \\
\hline STO-4-SB & 4 & & & 0.283 & 0.102 & 0.073 & 18.9 & 1.72 & 0.345 & 0.144 & 0.116 \\
\hline STO-8-SB & 8 & & & 0.127 & 0.082 & 0.066 & 8.8 & 1.68 & 0.430 & 0.157 & 0.125 \\
\hline STO-8-SB-R & 8 & & & 0.290 & 0.121 & 0.087 & 24.1 & 3.38 & 0.700 & 0.252 & 0.205 \\
\hline STO-16-SB & 16 & & & 2.74 & 0.365 & 0.181 & 18.1 & 1.84 & 0.547 & 0.224 & 0.235 \\
\hline EXTO-0-SBT & 0 & 20.1 & 1.83 & 0.210 & 0.123 & 0.086 & 21.3 & 1.99 & 0.199 & 0.139 & 0.127 \\
\hline EXTO-2-SBT & 2 & 20.1 & 1.82 & 0.222 & 0.128 & 0.090 & 21.8 & 1.92 & 0.232 & 0.176 & 0.172 \\
\hline SCO-6-SBT & 6 & & 1.39 & 0.194 & 0.121 & 0.086 & 21.6 & 1.80 & 0.208 & 0.139 & 0.154 \\
\hline STO-8-SBT & 8 & & & 0.133 & 0.098 & 0.078 & 22.3 & 2.06 & 0.212 & 0.199 & 0.181 \\
\hline
\end{tabular}

${ }^{a}$ Baseline solvent was run using ORNL full simulant.

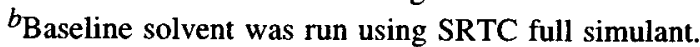

${ }^{c}$ Average ANL, SRTC, and ORNL values for $\mathrm{E}_{2} \mathrm{SS}$ test, which includes two extractions (see Table 3.6).

${ }^{d} \mathrm{ORNL}$ values for $\mathrm{E}_{2} \mathrm{SS}$ test, which includes two extractions (see Table 3.6). 


\subsection{CONCLUSIONS}

Based on the data presented above, the stability of the solvent to external irradiation, with regard to both absolute breakdown rate and performance, is very good. The major components of the solvent had the following behavior under irradiating conditions: for BOBCalixC6 and Cs-7SB, loss due to radiolytic breakdown is negligible, less than $1 \%$ being the required annual makeup. Even for TOA, the annual loss is conservatively expected to be $10 \%$.

The primary degradation product observed was 4-sec-butylphenol. Its rate of buildup, however, is low, less than $0.1 \mathrm{mM}$ per annual dose. Moreover, as mentioned in chapter 4 , this material would easily wash out during the process based on its partition ratios. Data from total organic carbon, solidphase extraction, and NMR spectrometry indicate that some organic products, especially fluorinated products from the modifier, report to the aqueous phases.

In general, the radiation stability of the solvent as measured by NMR and ES-MS is excellent. Only under high ( $>6 \mathrm{Mrad}$ ) doses is any detectable sign (by NMR) of degradation observed, and even then the amount is small. Further, no differences between the irradiated samples and corresponding pristine solvent samples contacted with the full simulant, scrub, and strip solutions were observed by ES-MS.

Interfacial behavior was acceptable in the irradiated samples. Essentially no change in break time was observed. Interfacial tensions were all above $5 \mathrm{dyne} / \mathrm{cm}$, and the response was either increasing with dose (scrub conditions) or decreasing with dose (extraction or stripping conditions).

In batch tests, extraction and scrubbing behavior were not much affected by external irradiation and remained acceptable. Stripping was also acceptable for all but the highest dose (16 Mrad under stripping conditions), where it was clear that poor performance was related to the loss of $79 \%$ of the TOA. It should be noted that not all results were straightforward, as evidence of some alkaline carryover from extraction into scrubbing and stripping was obtained from $\mathrm{pH}$ measurements. This carryover was likely responsible for some of the $D_{\mathrm{Cs}}$ values being high on the first strip, but it was seen that by the third strip, normal values were obtained in nearly all cases, except as noted above. risk.

It may also be noted that results from internal-irradiation tests ${ }^{47}$ also indicate low technology 


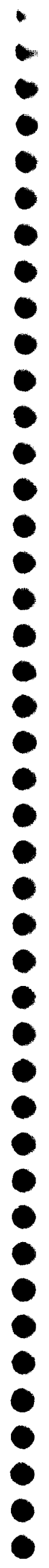




\section{SOLUBILITY PHENOMENA}

\subsection{INTRODUCTION}

\subsubsection{Purpose}

The integrity of a functional solvent requires that all solute species remain soluble under expected operating conditions. For present purposes, solubility phenomena are divided into the solubilities of the solvent components in the solvent matrix and the solubilities of extraction complexes in the solvent upon contact with aqueous flowsheet solutions. Insoluble phases encountered in solvent extraction are typically pure solid compounds or heavy liquid organic phases rich in extractants and extracted solutes. 23,77 The heavy liquid phases are typically referred to as third phases. Liquidliquid contacting equipment is normally not designed to accommodate formation of solid precipitates or third-phases. Although minor amounts of such phases might be tolerable, continued buildup eventually causes poor hydraulic performance or deterioration of extraction, stripping, or selectivity.

The purpose of the work reported in this chapter was to determine the relevant solubility limits of solute species in the solvent. Three individual studies were carried out. The first entailed determining the solubilities of the modifier and extractant in the solvent matrix. The second entailed observing the solvent for possible solids formation over a long period of time under various conditions relevant to the CSSX flowsheet. The third entailed identifying the variables controlling third-phase formation and determining the window of operating conditions under which no third-phase formation would be expected.

\subsubsection{Background and Experimental Design}

Table 6.1 defines the solvent compositions that were of interest initially. Namely, four solvent systems were to be considered, all containing $0.01 \mathrm{M}$ BOBCalixC6 and $0.001 \mathrm{M}$ trioctylamine, but differing in use of either Cs-7SB, Cs-7SBT, or Cs-6 modifier (all at $0.5 \mathrm{M}$ ) and either Isopar L or Norpar 12 diluent. As discussed below, the planned work scope included solubility studies of the major solvent components. ${ }^{4,19}$ In the planning phase, it was recognized that little concrete data existed upon which to build confidence that the solubilities of solvent components were in fact adequate for process operation. During execution of the plan in FY 2000, it was found that solvents containing the Cs-6 modifier were prone to solidify under moist conditions, and the issue of solubility was thus raised as a matter of greater concern. Modifier Cs- 6 consequently ceased to stand as a potential modifier for CSSX, but it still remained to understand the factors associated with its insolubility and to determine whether there might be an analogous limitation with the other modifier candidates Cs-7SB and Cs-7SBT. As Cs-7SB and Cs-7SBT are completely miscible with the Isopar L diluent, the logical experimental design entailed observing selected solvent compositions in contact with various aqueous phases at ambient and cool (ca. $4{ }^{\circ} \mathrm{C}$ ) conditions. The solid phase associated with Cs-6 was also studied to determine its composition and solubility behavior.

In CSSX development studies, ${ }^{34}$ BOBCalixC6 had routinely been used at concentrations of 20 $\mathrm{mM}$ or more without difficulty, and solutions stored for up to a year exhibited no signs of phase instability. Nevertheless, its solubility behavior was an important property to quantify. Experiments were therefore designed to examine the solubility of BOBCalixC6 in the solvent matrix under relevant conditions, such as varied modifier concentration. It was also planned to approach solubility equilibrium from undersaturation as well as supersaturation.

As neat TOA is miscible with the solvent matrix, its solubility per se is not an issue. However, considerable literature shows that on conversion to their ammonium salt forms, TOA and other alkylamines tend to form third phases, both solid and liquid, in aliphatic diluents. ${ }^{77-80}$ This phenomenon is simply related to the fact that the highly polar ion pairs are incompatible with the essentially nonpolar, nonpolarizable aliphatic diluent. ${ }^{77}$ The usual practice is simply to add a fatty 
alcohol modifier to the diluent to solvate the alkylammonium salt, ${ }^{23,77}$ and the question therefore becomes one of ensuring that an adequate concentration of modifier is used to prevent third-phase formation under expected operating conditions. In the baseline flowsheet, TOA is converted to the salt form under the acidic conditions of scrubbing and stripping, and a lipophilic alcohol is in fact employed as a diluent modifier.

By analogy with the tendency of alkylammonium ion pairs to form third phases, it was expected that the ion pairs formed upon extraction of $\mathrm{Cs}^{+}$and $\mathrm{K}^{+}$salts by BOBCalixC6 might also form third phases. In fact, early CSSX process development ${ }^{34}$ identified third-phase formation as a limitation that could be controlled according to the choice of modifier structure. In this respect, Cs-6 was superior, but the Cs-7SB modifier exhibited adequate performance such that the solvent could be used at high loading down to $20^{\circ} \mathrm{C} .15$ In the present work, it was intended to survey the particular loading behavior leading to third-phase formation and to determine the lower operating temperature at the bounding process conditions. In this respect, the third-phase formation observed is the total effect of the solubility behavior of TOA salts and $\mathrm{Cs}^{+}$and $\mathrm{K}^{+}$salts extracted by BOBCalixC6.

\subsubsection{Work Scope}

WSM item 5.1.1 specified the determination of the solubilities of major solvent components, 19 and this need was addressed in task planning. ${ }^{4}$ The solubility of BOBCalixC6 was to be measured as a function of modifier and amine concentration, neat and in the presence of flowsheet and other aqueous solutions. Induced third-phase formation was to be taken as an effective solubility limit for extraction complexes upon loading.

\subsection{EXPERIMENTAL SECTION}

\subsubsection{Chemicals and Materials}

BOBCalixC6 (IBC Advanced Technologies, Inc., Lot 000714HMKC-0004) was recrystallized for the solubility experiments. A $10.5-\mathrm{g}$ quantity was dissolved in about $25 \mathrm{~mL}$ of hot dichloromethane (EM Science HPLC grade) in a $250-\mathrm{mL}$ Erlenmeyer flask. The flask was covered and allowed to cool to room temperature, then placed in a refrigerator for several hours. As no solids had crystallized, $25 \mathrm{~mL}$ of $n$-pentane (J. T. Baker) was layered onto to the dichloromethane layer and the flask placed back in the refrigerator overnight. This procedure was repeated until a total of $100 \mathrm{~mL}$ of $n$-pentane had been added (final solution 4:1 v:v $n$-pentane/dichloromethane), whereupon a yellow oil finally came out of the cold solution. The nearly colorless supernatant liquid was decanted into a clean 250-mL Erlenmeyer flask, and that solution was allowed to concentrate down by slow evaporation over the course of several days. When the volume had been reduced to about $50 \mathrm{~mL}$, semi-transparent crystals were found to have formed on the walls of the flask. The semi-transparent crystals were collected by filtration and washed with about $10 \mathrm{~mL}$ of cold $n$-pentane. About $7.8 \mathrm{~g}$ of white solid was recovered. Approximately $6.2 \mathrm{~g}$ of this was ground to a fine powder using an agate mortar and pestle and then dried under vacuum for several days at $60-65{ }^{\circ} \mathrm{C}$ to remove residual solvent. Additional BOBCalixC6 of slightly lesser purity can be recovered from the mother liquor.

The purity of the $7.8 \mathrm{~g}$ of white crystalline powder was estimated to be $99+\%$ BOBCalixC6 as assayed by NMR and HPLC. From the NMR spectra, it was seen that the recrystallization almost entirely removed impurities that contained unfunctionalized phenolic groups on the belt of the calixarene, which arise as side-products of the synthesis. Among three large resonances in the region 9-10 ppm where calixarene phenolic groups are located, only a trace of one with $\mathrm{S} / \mathrm{N}$ ratio 2 to 3 remained after crystallization. There were only traces left of impurities in the aromatic and ether regions. The HPLC analysis on the BOBCalixC6 before and after recrystallization indicated the removal of one impurity peak that had been observed as a shoulder on the BOBCalixC6 peak corresponding to the material before recrystallization. The HPLC traces showed minor tailing of the BOBCalixC6 peak (3.75\% of the total response), which in light of the NMR results was interpreted as the compound sticking to the column rather than the presence of an impurity. 
The yellow oil that was isolated first from the 4:1 v:v $n$-pentane/dichloromethane solution was found by proton NMR to contain both BOBCalixC6 and what appeared to be a polymeric material. In HPLC analysis the material eluted before the BOBCalixC 6 on a GPC column, suggesting that it possessed a higher molecular weight than the BOBCalixC6 $\left(\mathrm{C}_{72} \mathrm{H}_{92} \mathrm{O}_{12}\right.$, MW 1149.51). The yellow material was separated from the BOBCalixC6 and isolated by HPLC. A proton NMR analysis on this isolated material revealed broad peaks in the aromatic, ether, and aliphatic regions, suggesting that the material is indeed polymeric in nature. The relative integration of the peaks in the aromatic, ether, and aliphatic regions suggests that the material could have only one calix[4]arene group, with a bis1,2-[2'(2"-hydroxyethoxy)ethoxy]-4-tert-octylbenzene moiety connected by one arm to each phenolic group of the calixarene belt (in other words, four bis-1,2-[2'(2"-hydroxyethoxy)ethoxy]4-tert-octylbenzene groups per one calix[4]arene group). Such material would have a molecular formula of $\mathrm{C}_{116} \mathrm{H}_{168} \mathrm{O}_{24}$ and a MW of $1946.59 \mathrm{~g} / \mathrm{mol}$.

\subsubsection{Solubility Measurements}

\subsubsection{Preparation of the Solubility Samples}

Seven $10-\mathrm{mL}$ samples of Cs-7SB modifier in Isopar $\mathrm{L}$ at various concentrations $(0,0.01,0.03$, $0.1,0.25,0.5$, and $0.75 \mathrm{M}$ ) were prepared in duplicate and placed into Teflon FEP vials. The weighed amounts of BOBCalixC6 added to each sample were adjusted so that after solubility was reached, some solid BOBCalixC6 would still remain in the sample. The experimental conditions are summarized in Table 7.1 (Samples \#1-7). The finely ground BOBCalixC6 formed a fine dispersion in each sample.

It was of interest to examine whether the solubility of BOBCalixC6 in the $0.5 \mathrm{M} \mathrm{Cs-7SB}$ modifier solution in Isopar $\mathrm{L}$ would depend on the sample preparation. Additional samples containing $5 \mathrm{~mL}$ of $0.5 \mathrm{M} \mathrm{Cs-7SB}$ and $0.001 \mathrm{M}$ TOA in Isopar L, either dry, or washed using the standard solvent washing protocol (namely two times with $0.1 \mathrm{M} \mathrm{NaOH}$, two times with $0.05 \mathrm{M} \mathrm{HNO}_{3}$, and three times with DI water), and an amount of BOBCalixC6 corresponding to $0.05 \mathrm{M}$ solution (Table 7.1, Samples \#8 and \#9) were prepared and placed for equilibration in the air box at $25{ }^{\circ} \mathrm{C}$. Also, a weighed amount of BOBCalixC6 corresponding to a $0.01 \mathrm{M}$ solution was dissolved in a solution containing $0.5 \mathrm{M} \mathrm{Cs}-7 \mathrm{SB}$ and $0.001 \mathrm{M}$ TOA in Isopar L by applying a combination of heating to $60{ }^{\circ} \mathrm{C}$ and continuous sonication in an ultrasonic bath (Table 7.1, Sample \#10). After cooling to room temperature, the BOBCalixC6 remained in solution. This sample was seeded with a few $\mathrm{mg}$ of recrystallized $\mathrm{BOBCalixC6}$ and placed for equilibration in a constant-temperature air box at $25^{\circ} \mathrm{C}$.

To determine the effect of impurities contained in the commercial BOBCalixC6, two samples were prepared in duplicate using BOBCalixC6 as received from IBC (Lot 000714 HM KC-0004, 97\%). These samples consisted of $10-\mathrm{mL}$ solutions of 0.5 or $0.75 \mathrm{M}$ Cs-7SB in Isopar L. Initially, an amount of BOBCalixC6 was added corresponding to approximately 0.05 and $0.075 \mathrm{M}$ solutions, respectively (Table 7.1, Samples \#11 and \#12). After overnight equilibration at $25{ }^{\circ} \mathrm{C}$, the BOBCalixC6 was completely dissolved in the $0.5 \mathrm{M}$ modifier solution. The $0.75 \mathrm{M}$ Cs-7SB sample was hazy and contained a very small amount of fine solids. An additional portion of BOBCalixC6 was added to each sample. Sample \#13 was prepared as a $0.048 \mathrm{M}$ solution of BOBCalixC6 in a prewashed (using standard solvent washing protocol) solution containing $0.5 \mathrm{M} \mathrm{Cs}-7 \mathrm{SB}$ and $0.001 \mathrm{M}$ TOA in Isopar $\mathrm{L}$, the total volume of the sample being $10 \mathrm{~mL}$. The sample was placed on the rotating wheel in the air box at $25^{\circ} \mathrm{C}$; in an hour it was found that the entire amount of BOBCalixC6 was dissolved, giving a homogeneous solution of pale yellow color. After 3 days, light precipitation of BOBCalixC6 was observed. The amount of precipitate gradually increased with time.

For equilibration, all samples were rotated in the air box at $25{ }^{\circ} \mathrm{C}$. The concentration of the BOBCalixC6 in the liquid phase was periodically checked by reverse-phase HPLC analysis over the period of 52 days. 
Table 7.1. Preparation of samples for determination of BOBCalixC6 solubility

\begin{tabular}{|c|c|c|c|c|c|c|c|}
\hline \multirow[t]{2}{*}{ Sample ${ }^{a}$} & \multicolumn{2}{|c|}{ BOBCalixC6 } & \multicolumn{5}{|c|}{ Solution composition } \\
\hline & $\begin{array}{c}\text { Weight } b \\
\text { (g) }\end{array}$ & Condition & $\begin{array}{c}\text { [Cs-7SB] } \\
\text { (M) }\end{array}$ & $\begin{array}{c}{[\mathrm{TOA}]} \\
(\mathrm{M})\end{array}$ & $\begin{array}{c}\text { Volume } \\
\text { (mL) }\end{array}$ & $\begin{array}{c}\text { Weight }^{b} \\
(\mathrm{~g})\end{array}$ & Condition \\
\hline $1-A$ & 0.173 & Recryst. & 0 & 0 & 10 & 7.55 & Dry \\
\hline $1-B$ & 0.173 & & & & & 7.56 & \\
\hline 2-A & 0.173 & Recryst. & 0.01 & 0 & 10 & 7.57 & Dry \\
\hline $2-B$ & 0.173 & & & & & 7.57 & \\
\hline $3-A$ & 0.173 & Recryst. & 0.03 & 0 & 10 & 7.61 & Dry \\
\hline $3-B$ & 0.173 & & & & & 7.61 & \\
\hline $4-\mathrm{A}$ & 0.346 & Recryst. & 0.1 & 0 & 10 & 7.67 & Dry \\
\hline $4-B$ & 0.346 & & & & & 7.67 & \\
\hline $5-A$ & 0.576 & Recryst. & 0.25 & 0 & 10 & 7.84 & Dry \\
\hline $5-B$ & 0.576 & & & & & 7.84 & \\
\hline $6-A$ & 0.575 & Recryst. & 0.5 & 0 & 10 & 8.11 & Dry \\
\hline $6-B$ & 0.575 & & & & & 8.13 & \\
\hline $7-\mathrm{A}$ & 0.863 & Recryst. & 0.75 & 0 & 10 & 8.40 & Dry \\
\hline $7-B$ & 0.863 & & & & & 8.42 & \\
\hline 8-A & 0.145 & Recryst. & 0.5 & 0.001 & 5 & 4.07 & Dry \\
\hline $8-B$ & 0.155 & & & & & 4.04 & \\
\hline $9-\mathrm{A}$ & 0.149 & Recryst. & 0.5 & 0.001 & 5 & 4.05 & Washed \\
\hline $\begin{array}{l}9-\mathrm{B} \\
10-\mathrm{A}^{c}\end{array}$ & 0.152 & Recrvst & 0.5 & 0001 & 3 & 4.04 & Dry \\
\hline $10-\mathrm{B}^{c}$ & & Necryst. & 0.3 & 0.001 & 3 & & Diy \\
\hline $11-\mathrm{A}$ & $0.579+0.394$ & As rec'd & 0.5 & 0 & 10 & 8.20 & Dry \\
\hline $11-B$ & $0.581+0.414$ & & & & & 8.19 & \\
\hline $12-A$ & $0.885+0.400$ & As rec'd & 0.75 & 0 & 10 & 8.41 & Dry \\
\hline $12-B$ & $0.890+0.413$ & & & & & 8.41 & \\
\hline $13-\mathrm{A}$ & 0.552 & As rec'd & 0.5 & 0.001 & $10^{d}$ & & Washed \\
\hline
\end{tabular}

aThe designations $\mathrm{A}$ and $\mathrm{B}$ denote duplicate samples.

$b$ Not all weight values were obtained, as indicated by blanks.

${ }^{c} 0.01 \mathrm{M}$ BOBCalixC6 solution prepared applying sonication and heating at $60{ }^{\circ} \mathrm{C}$ to dissolve the BOBCalixC6, cooling the sample to room temperature, and seeding with an additional small amount of pure BOBCalixC6.

${ }^{d}$ This volume corresponds to the volume of the whole sample including BOBCalixC6.

\subsubsection{Preparation of Solubility Samples for HPLC Analysis}

The Teflon FEP vials containing the solubility samples were taken out of the air box and centrifuged for an hour at $25^{\circ} \mathrm{C}$. Even after centrifuging, the liquid phase in all samples contained a fine dispersion of solid. A $0.5-\mathrm{mL}$ volume of each sample was withdrawn and filtered through Whatman No. 40 filter paper. A $0.1-\mathrm{mL}$ subsample of filtrate was collected, diluted with chloroform by a factor of 10 , and analyzed by HPLC.

\subsubsection{Preparation of Standard Samples for HPLC Analysis}

Initially, BOBCalixC6 calibration standards were prepared in isooctane. It was found that these standards could not be used because of precipitation of the calixarene and clogging of the injection port. A second set of BOBCalixC6 calibration standards was prepared in chloroform. It was 
inconvenient to handle these samples, because the fast evaporation of the chloroform made it possible to use only freshly prepared standards. Moreover, the matrices of the actual sample and calibration standards described above are different due to the high content of the Cs-7SB modifier in the solubility samples. To more accurately match the sample matrices, the full, non-washed baseline solvent [0.5 M Cs-7SB, 0.01 M BOBCalixC6 (IBC, Lot 00714HMKC-0004), and 0.001 M TOA in Isopar L] was used to prepare the standards, as diluted 1:10 v:v with chloroform. The concentration of the BOBCalixC6 in this standard is $1,150 \mathrm{ppm}$. A series of HPLC calibration standards was prepared by the dilution of this 1,150-ppm standard solution with isopropanol. The observed linear dynamic range using reversed-phase HPLC analysis with UV detection at $226 \mathrm{nM}$ is $1.3-115 \mathrm{ppm}$, with a detection limit of $0.6 \mathrm{ppm}$ and a QA limit of $1.3 \mathrm{ppm}$. The linear-regression was given by the relation: Response $=12.1+51.7[\mathrm{BOBCalixC6}], r^{2}=0.99995$. To test the reliability of this calibration, the standard samples containing $0.008,0.01$, and $0.02 \mathrm{M}$ BOBCalixC6 in a solution matrix containing $0.5 \mathrm{M}$ Cs-7SB and $0.001 \mathrm{M}$ TOA solution in Isopar L were prepared. The 0.008and 0.02-M samples were prewashed using the standard washing protocol for the solvent. For HPLC analysis, the samples were diluted with chloroform by the factor of 10 .

\subsubsection{Solids-Formation Tests}

For the solids-formation tests, Cs-7SB modifier (Lot PVB B000718-24DM) was dried under vacuum at $50{ }^{\circ} \mathrm{C}$ for 3 days. A weight loss of $5 \%$ presumably due to the water evaporation was detected. Isopar L (ExxonMobil Chemical Co.) and BOBCalixC6 (IBC, Lots 000711HMKC-0004 and 000714HMKC-0004) were used as received. Compositions of the prepared samples are described in Table 7.2. Solutions of $0.5 \mathrm{M}$ and $0.75 \mathrm{M}$ Cs-7SB in Isopar L were prepared under dry conditions or washed using the standard solvent-washing protocol. Solvent solutions containing 0.01 M BOBCalixC6 were prepared containing Cs-7SB modifier at the baseline concentration of $0.5 \mathrm{M}$ or $0.75 \mathrm{M}$ and kept dry or washed using the standard solvent-washing protocol. Washed samples were put into contact with aqueous solutions of different compositions (Table 7.2). The volume of the solvent phase was $5 \mathrm{~mL}$, and the $\mathrm{O} / \mathrm{A}$ ratio for the samples containing the aqueous phase was 0.33 . Two identical sets of samples were prepared, placed in 50-mL Nalgene Teflon FEP screw-capped bottles, subjected to agitation by rotating on a wheel in an air box set at $25{ }^{\circ} \mathrm{C}$ for $30 \mathrm{~min}$, then kept for 7.5 months in the dark at $4{ }^{\circ} \mathrm{C}$ and at room temperature. Each sample was periodically checked for solids formation and then agitated by rotation (Glas-Col rotator) in an air box set at $25{ }^{\circ} \mathrm{C}$ for 30 $\min$.

\subsubsection{Third-Phase Tests}

Loading and third-phase tests were carried out using solvent containing modifier Cs-7SB (Lots PVB B000718-110W, B000718-132W, and B000718-156W). Extraction experiments were performed to determine BOBCalixC6 loading and its effect on third-phase formation at different metal ion concentrations in the aqueous phase. Solvent was equilibrated with an aqueous phase in a temperature-controlled air box at $25{ }^{\circ} \mathrm{C}$ for $30 \mathrm{~min}$ at various O/A ratios. Subsequently, the samples were centrifuged at $4000 \mathrm{rpm}$ for $3 \mathrm{~min}$. Each sample was visually observed for third-phase formation. To determine cesium distribution ratios, each phase was subsampled and analyzed by ${ }^{137} \mathrm{Cs}$ radiometric techniques. To determine $\mathrm{K}^{+}$and $\mathrm{Na}^{+}$distribution ratios, subsamples of the solvent phase were mixed with 1,2-diisopropylbenzene in a 1:1 v:v ratio, stripped with deionized water, and the stripping phases analyzed by ICAP. Distribution ratios of $\mathrm{K}^{+}$and $\mathrm{Na}^{+}$were calculated assuming that a mass balance of $100 \%$ is sustained for each sample. In the loading calculations, it was assumed based on previous distribution experiments (Chap. 4) that extracted $\mathrm{Na}^{+}$does not contribute to the BOBCalixC6 loading. 


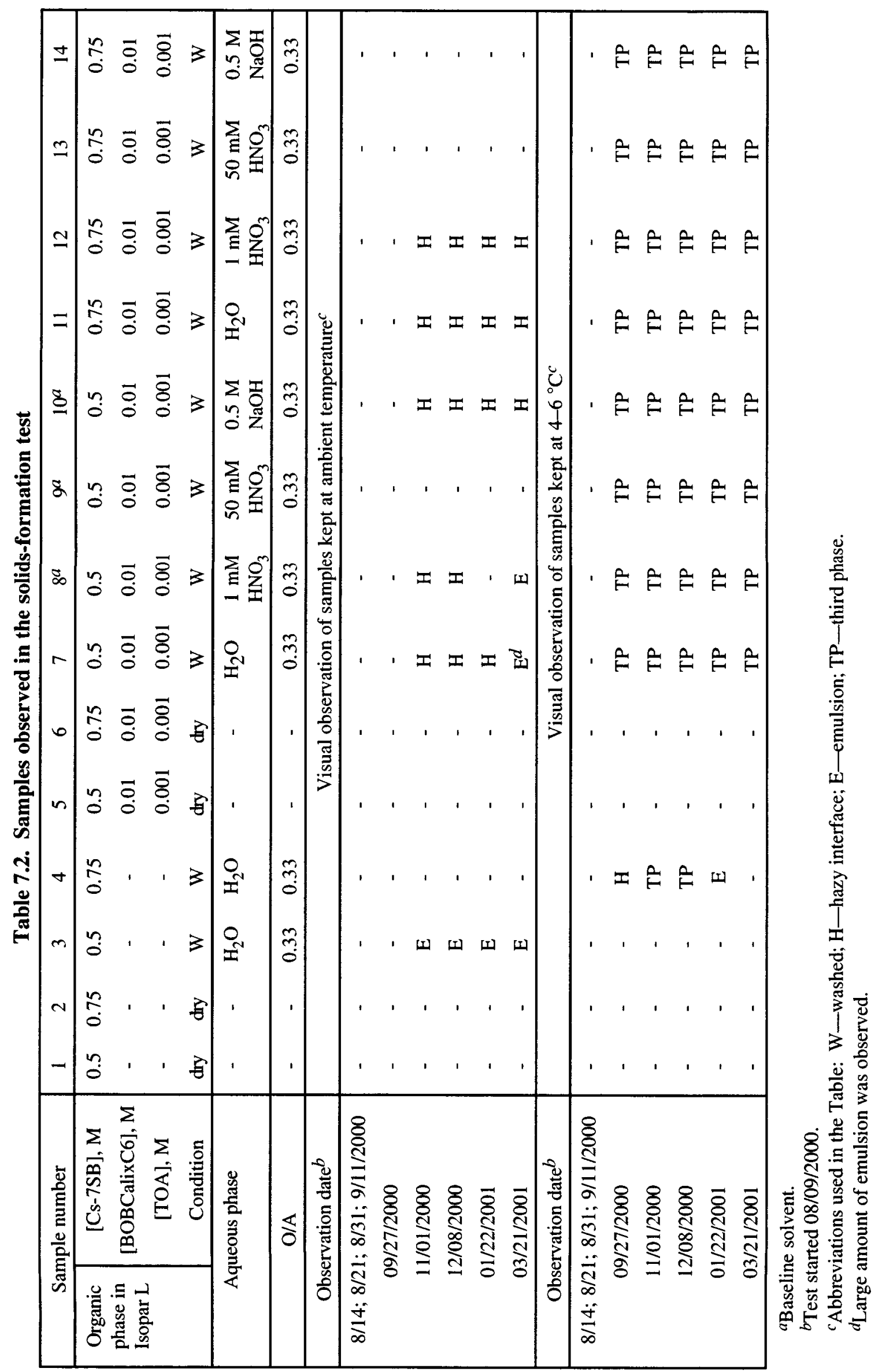




\subsection{RESULTS AND DISCUSSION}

\subsubsection{Solubility of BOBCalixC6}

The key finding is that, with respect to BOBCalixC6 solubility, the baseline solvent is somewhat supersaturated at $25{ }^{\circ} \mathrm{C}$. When highly supersaturated solutions employing the modifier at $0.50 \mathrm{M}$ and as-received BOBCalixC6 at $0.05 \mathrm{M}$ are prepared by warming and sonication of the solution, the BOBCalixC6 is observed to precipitate out after standing at $25{ }^{\circ} \mathrm{C}$, giving a final concentration in the solvent of only $0.0079 \mathrm{M}$, below the nominal $0.01-\mathrm{M}$ baseline-solvent concentration. However, when the modifier is used at $0.75 \mathrm{M}$, the equilibrium concentration of $\mathrm{BOBCalixC6}$ following precipitation from a $0.05 \mathrm{M}$ solution is $0.0127 \mathrm{M}$. It is therefore recommended that solvent-optimization in possible follow-on tasks consider raising the modifier concentration.

Data collected for the BOBCalixC6 solubility tests are summarized in Table 7.3. The purpose of this experiment was to determine the solubility of BOBCalixC6 in Isopar L under different conditions. First, HPLC measurements of the recrystallized BOBCalixC6 concentration in Isopar L (Samples \#1-7) performed after 12 days of the equilibration at $25^{\circ} \mathrm{C}$ afforded much lower values of the BOBCalixC6 molar concentration than measurements performed after more that 20 days of equilibration. Moreover, the molar concentration values of BOBCalixC6 obtained using isooctane as the calibration solvent are expected to be somewhat higher that the actual values, since precipitation of the BOBCalixC6 was observed in the isooctane BOBCalixC6 calibration standards. (Thus isooctane cannot be considered a suitable solvent for the preparation BOBCalixC6 calibration standards.) The values of the BOBCalixC6 molar concentration obtained after 20,27, 35, and 52 days of equilibration at $25{ }^{\circ} \mathrm{C}$ (Table 7.3, Samples \#1-7) reached a plateau, although some samples continued to exhibit slowly increasing values. Checks of the 8- and 10-mM BOBCalixC6 standard samples using Isopar L calibration solvent resulted in reasonably close values of 8.23 and $9.49 \mathrm{mM}$, respectively (duplicate average, Table 7.3). When chloroform calibration solvents were used, analysis of the 8- and 20-mM BOBCalixC6 standard samples gave 7.18 and $15.85 \mathrm{mM}$, respectively (duplicate average, Table 7.3). The larger disagreement observed using 20-mM standard sample could be explained by the fact that $20-\mathrm{mM}$ concentration of the BOBCalixC6 in the $0.5 \mathrm{M} \mathrm{Cs}-7 \mathrm{SB}$ / $0.001 \mathrm{M}$ TOA / Isopar L system significantly exceeds its solubility and constitutes a supersaturated solution. Thus, chloroform is a preferable solvent for the preparation of the BOBCalixC6 calibration samples.

The most important factor affecting the calixarene solubility is the concentration of the Cs-7SB modifier in Isopar L. As shown in Fig. 7.1, it was determined that the solubility of the pure recrystallized BOBCalixC6 depends linearly on the concentration of Cs-7SB in Isopar L. The solubility of BOBCalixC6 in Isopar L containing $0.5 \mathrm{M}$ Cs-7SB modifier was found to be $4.18 \mathrm{mM}$ (Table 7.3, Sample \#6, averaged). Addition of TOA at 0.001-M concentration resulted in only a slight increase of solubility, which reached the level of $5.0 \mathrm{mM}$ (Table 7.3, Sample \#8, averaged). Prewashing of the $0.5 \mathrm{M}$ Cs-7SB and $0.001 \mathrm{M}$ TOA solution in Isopar L (using standard solvent washing protocol) further increased the solubility of BOBCalixC6 to $5.5 \mathrm{mM}$ (Table 7.3, Sample \#6, averaged); presumably, this effect is due to the water content of the solvent. Thus, it may be concluded that the solubility of pure BOBCalixC6 in the baseline solvent obtained in the forward direction (dissolution vs. precipitation) is less than the prescribed concentration of $0.010 \mathrm{M}$. This observation was made using recrystallized BOBCalixC6.

It was found that the solubility obtained in the reverse direction (precipitation) gave a higher solubility value than the forward direction (dissolution). Sample \#10 (Table 7.1) was prepared following the procedure similar to the one used for the baseline-solvent preparation, namely completely dissolving BOBCalixC6 in the $0.5 \mathrm{M} \mathrm{Cs-7SB} \mathrm{/} 0.001 \mathrm{M}$ TOA solution in Isopar L applying sonication. The sample was then seeded with a small amount of pure BOBCalixC6, showed a much higher BOBCalixC6 equilibrium concentration of 0.00944 (Table 7.3, Sample \#10, averaged) which is very close to the $0.01 \mathrm{M}$ concentration of BOBCalixC6 in the baseline solvent. 

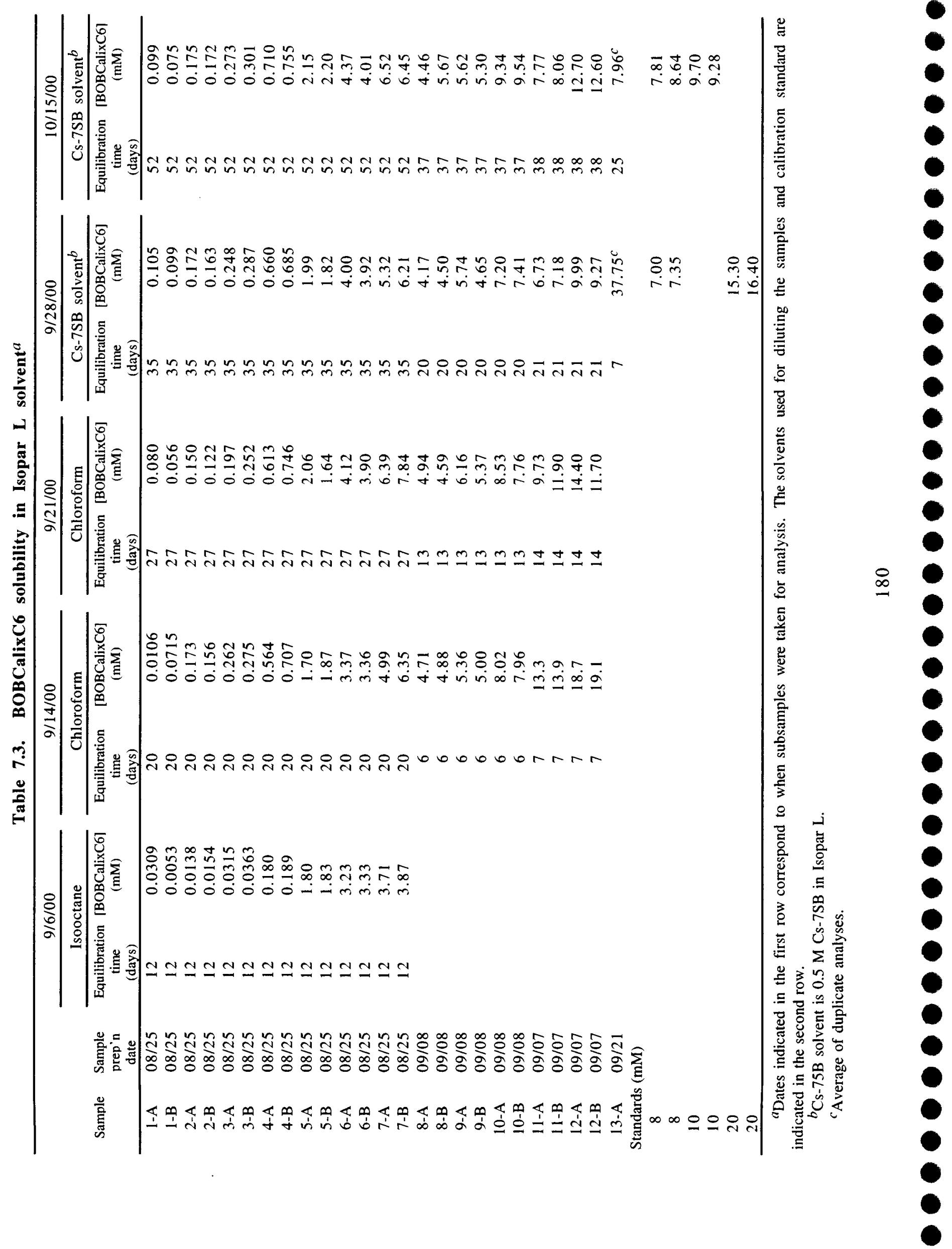
As discussed at the end of this chapter, the disagreement between the forward and reverse solubility values for BOBCalixC6 calls into question whether equilibrium had been reached in any of the experiments. It would appear likely that the dissolution process is very slow, and the appearance of a plateau may imply a slow approach to equilibrium. Further work will be needed to answer this question. However, the most practical values for use in discussing the supersaturation of the solvent would be the data from the reverse-direction experiments, as these values would be more likely to predict the concentration of $\mathrm{BOBCalixC} 6$ in case of possible precipitation from the baseline solvent.

To investigate the effect of impurities in the commercial BOBCalixC6 on its solubility in Isopar L, Samples \#11-13 were prepared using BOBCalixC6 as received from the vendor. It was observed that its dissolution behavior differs from that of recrystallized material. Significant energy input is needed (sonication and heating) to dissolve the latter to $0.01 \mathrm{M}$ in the $0.5 \mathrm{M}$ Cs-7SB solution in Isopar L. Unlike the recrystallized BOBCalixC6, the as-received BOBCalixC6 could be easily dissolved at $\sim 0.05 \mathrm{M}$ in $0.5 \mathrm{M}$ Cs-7SB solution in Isopar $\mathrm{L}$, forming a pale yellow, homogeneous, supersaturated solution. Seeding or extended agitation caused precipitation of the BOBCalixC6. It is noteworthy that equal equilibrium concentrations of BOBCalixC6 $(0.0079 \mathrm{M})$ were determined in both Samples \#11 and \#13 (Table 7.3). This result appeared to be independent of the total amount of the BOBCalixC6 in the sample and thus on the total amount of impurities introduced into the samples along with BOBCalixC6. It could be seen from the Table 7.1 that the amount of BOBCalixC6 used in Sample \#11 was $176 \%$ of that of used in Sample \#13 and presumably contains a greater level of impurities by the same percentage. The observed solubility of $0.0079 \mathrm{M}$ is slightly less than the corresponding value of $0.00944 \mathrm{M}$ (Table 7.3, Sample \#10, averaged) obtained using purified $\mathrm{BOBCalixC6}$ in the reversed direction (precipitation). This observation suggests that impurities in the macrocycle material do not improve its solubility in the solvent. In this experiment, the properties of the Isopar L are greatly modified by the presence of Cs-7SB at high concentration, so that introduction of minor quantities of the impurities along with BOBCalixC6 has no effect on its solubility. Reverse-direction solubility of the as-received BOBCalixC6 in the $0.75 \mathrm{M}$ Cs-7SB solution in Isopar L was determined to be $0.0127 \mathrm{M}$. These results show that the effect of impurities on solubility of BOBCalixC6 is mainly on the rate of dissolution, rather than on the solubility itself.

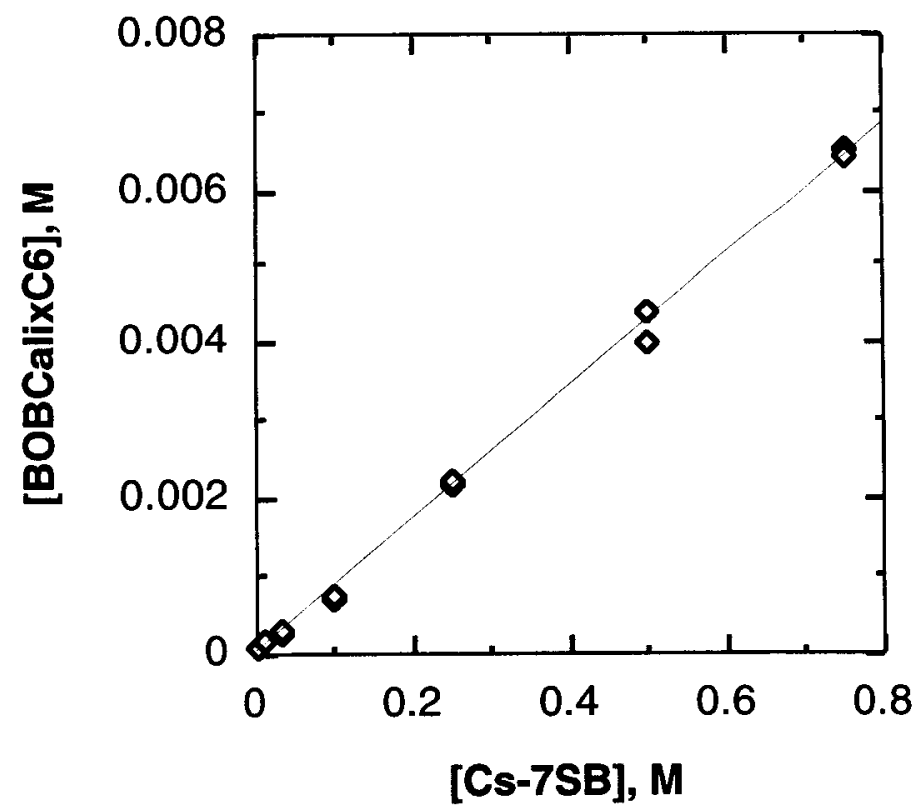

Fig. 7.1. Forward solubility of BOBCalixC6 in Isopar $L$ as a function of Cs-7SB modifier concentration. Symbols represent the observed values, and the line represents the linear-regression. 


\subsubsection{Solubility of Modifiers}

The key finding is that the baseline Cs-7SB modifier, which possesses an alkyl group with an asymmetric center and exists as a mixture of four stereoisomers in equal amounts, has excellent solubility properties and cannot be induced to precipitate from the solvent even at temperatures as low as $-18{ }^{\circ} \mathrm{C}$. However, certain related modifiers that possess alkyl groups with no asymmetric center and exist only as enantiomeric pairs were observed to precipitate from the solvent as a solid hydrate.

The Cs-6 modifier [1-(2,2,3,3-tetrafluoropropoxy)-3-(4-tert-octylphenoxy)-2-propanol] was the first of the second-generation modifiers to be prepared following the discovery that the Cs- 3 modifier was chemically unstable to prolonged contact with warm alkali. The second-generation modifiers are shown in Fig. 7.2, and differ from one another only in the type of alkyl group connected to the aromatic ring. The Cs- 6 modifier possesses a tert-octyl group, whereas the Cs-7SB modifier possesses a sec-butyl group, the Cs-7TB modifier possesses a tert-butyl group, and the Cs-7TA modifier possesses a tert-amyl group. Note that the sec-butyl group in the Cs-7SB modifier possesses a chiral carbon, whereas for the other modifiers the alkyl groups are all achiral. All the modifiers possess a chiral carbon on the alcohol carbon (carbon the hydroxyl group is attached to.) Hence, there are two stereoisomers (enantiomeric pair) for modifiers Cs-6, Cs-7TA, and Cs-7TB, but due to the two chiral centers present, there are four stereoisomers for the Cs-7SB modifier.

For the solvents to be evaluated in FY 2000 for radiolytic stability, in addition to the baseline Cs-7SB solvent, two solvents containing the Cs-6 modifier were included. Prior work performed in FY 1998 at SRS revealed that normal paraffin-based solvents (specifically Norpar 12) possessed somewhat greater radiolytic stability than isoparaffinic-based solvent (specifically Isopar L). The only modifier that performed well in Norpar 12 was the Cs-6 modifier, ${ }^{34}$ hence a Cs-6/Norpar 12 solvent was included in the radiation stability tests, along with a Cs-6/Isopar L solvent for comparison. (The other modifiers tended to form third phases in Norpar 12.)

All solvents in FY 2000 were washed according to the standard washing procedure, which resulted in the solvents being essentially saturated with water as a result of the final water-washing step. During manipulation of both Cs-6 based solvents (Cs-6/Isopar L and Cs 6 / Norpar 12), it was observed at the SRTC that the solvents "gelled" to a solid mass. The gellation occurred first when the solvent was contacted with aqueous scrub and strip solutions, and later when a pipette tip was inserted into the bottle of the washed solvent. Crystallization appeared to be induced by the pipette tip, and the entire solvent bottle eventually formed a gel-like mass. The identical phenomenon was observed at Oak Ridge with the same two lots of Cs-6 solvents. The solvents, which had been stored undisturbed on the laboratory bench for several months, solidified upon manipulation (i.e., specifically, after aliquots had been withdrawn from the bulk solvent using an Eppendorf pipette). The mass so formed was opaque in appearance and possessed a somewhat waxy consistency, though shaking the gel resulted in a suspension of white microcrystalline material in a thick oil-like medium.

Another observation made at the SRTC was that, when water was added directly to the pure Cs- 6 modifier, a white solid formed. This observation was replicated at ORNL. The conditions to form the solid hydrate are facilitated by seeding and also by cooling. Isolation of this white solid material revealed it to be a hydrate of Cs-6. Filtration of the gelled solvent and washing of the gel with small amounts of pentane led to the isolation of white microcrystalline material, which is sparingly soluble in Isopar $\mathrm{L}$ and which by proton NMR analysis was revealed to also be a hydrate of Cs-6. It appears that 1-2 molecules of water are closely associated with the Cs-6 solid hydrate, though more waters may be associated in the solution phase. Strong intermolecular hydrogen-bonding forces between water and the modifier alcohol group could be involved in the hydrate formation.

Some water was also added to samples of pure Cs-7SB and Cs-7TA modifiers. As no solids were observed to form at room temperature, the vials were placed in the refrigerator. No solid was ever observed to form with Cs-7SB; however a white solid did form after cooling in the refrigerator $\left(4{ }^{\circ} \mathrm{C}\right)$ for the Cs-7TA sample. That solid persisted after warming back to room temperature. Cooling the 
Cs-7SB sample to $-18{ }^{\circ} \mathrm{C}$ in a freezer caused the water in the sample to freeze (and pure Cs-7SB forms a very viscous colorless material at $-18{ }^{\circ} \mathrm{C}$ ), but warming the sample back to room temperature returned the samples to normal (no white solid formation).

Modifier Name

\section{Modifier Structure}

1-(2,2,3,3-tetrafluoropropoxy)-3-

(4-tert-octylphenoxy)-2-propanol

Code Name: Cs-6

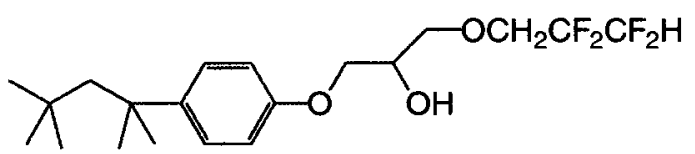

1-(2,2,3,3-tetrafluoropropoxy)-3-

(4-tert-butylphenoxy)-2-propanol

Code Name: Cs-7TB

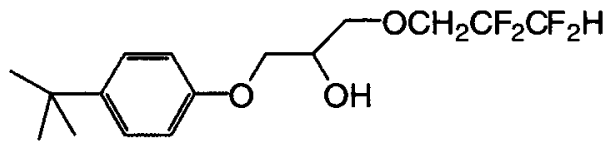

1-(2,2,3,3-tetrafluoropropoxy)-3-

(4-sec-butylphenoxy)-2-propanol

Code Name: Cs-7SBT and Cs-7SB

1-(2,2,3,3-tetrafluoropropoxy)-3-

(4-tert-amylphenoxy)-2-propanol

Code Name: Cs-7TA

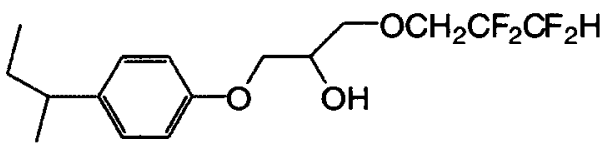

Fig. 7.2. Modifiers having different alkyl structures.

It appears that Cs-7SB resists solidification to a solid hydrate material. This could be due to the greater number of stereoisomers (four) for the Cs-7SB, due to the two chiral centers that the molecule possesses. (Thus, in addition to enantiomers there are also diastereomers for Cs-7SB.) Both Cs-6 and Cs-7TA exist only as enantiomers. All things being equal, it is reasonable to suggest that the more stereoisomers there are (especially diastereomers), the more difficult it is for the molecules to become arranged with sufficient for crystallization to take place. Thus it is reasonable to suggest that the Cs -SB modifier should resist forming organized crystalline solids, and to date, no solid formation has been observed with Cs-7SB.

\subsubsection{Solids-Formation Tests}

The purpose of this study was to determine the long-term stability of the solvent to precipitation of solids under various conditions. Two different solvent solutions containing 0.5 and $0.75 \mathrm{M}$ Cs 7SB modifier were examined, the first being the baseline solvent. Two controls consisting of 0.5 and $0.75 \mathrm{M}$ solutions of $\mathrm{Cs}-7 \mathrm{SB}$ modifier in Isopar $\mathrm{L}$ in the absence of BOBCalixC6 and TOA were tested. The four organic phases (two solvent solutions and two controls) were pre-washed using the standard solvent-washing protocol used for the baseline solvent, except that one dry set was not exposed to aqueous solutions at any experimental stage. To determine whether solids precipitate on extended exposure of the solvent to aqueous solutions, solvent samples were placed in contact with water, $0.001 \mathrm{M} \mathrm{HNO}_{3}, 0.05 \mathrm{M} \mathrm{HNO}_{3}$, or $0.5 \mathrm{M} \mathrm{NaOH}$ solutions at an O/A phase ratio of 0.33 . Each 
sample was prepared in duplicate, one being stored in the refrigerator and one being stored at room temperature in the dark. Periodically, the samples were removed for examination. Results of this test are reported in Table 7.2.

Over the entire test, no solids were observed in any sample. The final visual examination was conducted at 224 days. More specific observations are described below.

For the room-temperature samples, neither third-phase formation nor presence of solids was detected. No changes of any kind were observed in any sample for one month. After 83 days, minor changes related to coalescence were observed in certain samples. Some fine uncoalesced droplets were observed as a cloudy zone on the interface of the baseline solvent sample contacted with 0.001 $\mathrm{M} \mathrm{HNO}_{3}$ and of solvent samples containing $0.75 \mathrm{M} \mathrm{Cs-7SB}$ contacted with water and $0.001 \mathrm{M}$ $\mathrm{HNO}_{3}$. Baseline solvent contacted with $0.05 \mathrm{M} \mathrm{HNO}_{3}$ and solvent samples containing $0.75 \mathrm{M}$ Cs-7SB contacted with $0.05 \mathrm{M} \mathrm{HNO}_{3}$ and $0.5 \mathrm{M} \mathrm{NaOH}$ remained unchanged. Among the control samples (containing neither BOBCalixC6 nor TOA), the $0.5 \mathrm{M}$ Cs-7SB sample contacted with water formed an emulsion at the interface, though the $0.75 \mathrm{M} \mathrm{Cs-7SB}$ sample contacted with water remained unchanged. All dry samples remained unchanged. At 165 and 224 days, the baseline solvent sample contacted with $1 \mathrm{mM} \mathrm{HNO}_{3}$ exhibited a clear interface, though cloudy interfacial spots were observed after 83 and 120 days. The baseline solvent sample contacted with water formed a large amount of emulsion after 224 days. Other samples examined at 120, 165, and 224 days were found to be in the same state as after 83 days.

For the refrigerated samples, no presence of solids was detected in any sample, though third phases were observed in some cases. No changes were observed in any sample for one month. After 1.5 months, the presence of a third phase was detected in solvent samples exposed to the aqueous solutions. The third phase may be described as a transparent liquid located at the interface. The amount of the third phase was found to be dependent on the concentration of the Cs-7SB modifier in the sample and seemed to decrease with time thereafter. In the baseline solvent samples, only a small quantity of the third phase was observed, while in the solvent samples containing $0.75 \mathrm{M} \mathrm{Cs}-7 \mathrm{SB}$, the volume of the third phase was estimated to be $20 \%-30 \%$ of the total volume of the organic phase. At 120 days, the third phase was present but in smaller quantity. At 165 and 224 days, the interface was cloudy, and very little amount of third phase remained in the baseline solvent samples. Solvent samples containing $0.75 \mathrm{M} \mathrm{Cs}-7 \mathrm{SB}$ contacted with (1) water and $1 \mathrm{mM} \mathrm{HNO}_{3}$ exhibited a cloudy interface and a small amount of third phase, (2) $50 \mathrm{mM} \mathrm{HNO}$ exhibited a clear interface and no third phase, and (3) $0.5 \mathrm{M} \mathrm{NaOH}$ exhibited a moderate amount of third phase, ca. $20 \%$ of the total volume of organic phase. In control samples, $0.75 \mathrm{M}$ Cs-7SB sample contacted with water exhibited (1) a cloudy interface after 1.5 month, (2) a third phase after 83 and 120 days, and (3) and no third phase but cloudy interface after 165 days. The $0.5 \mathrm{M} \mathrm{Cs-7SB}$ control contacted with water and all dry samples remained unchanged.

It may be remarked that hydration appears to be associated with reduced modifier solubility. Unlike the case of Cs-6, however, hydration of Cs-7SB leads to only liquid third phases that form only at much reduced temperature. Thus, the alkyl structure of Cs-7SB successfully prevents solids formation.

\subsubsection{Third-Phase Formation}

\subsubsection{Visual Observations}

Physical properties of the third phase depend on the chemical nature of insoluble extraction species. Three different kinds of third phase have been observed. 1) Normally, it is a viscous liquid with density intermediate between that of the solvent and aqueous phase and thus located at their interface. 2) At $0.25 \mathrm{M} \mathrm{Cs}-7 \mathrm{SB}$ and $0.01 \mathrm{M}$ BOBCalixC6 in the solvent, the third phase often is a dense gel heavier than the aqueous phase and precipitates at the bottom of the extraction vial. Dissolution of this gel-like third phase upon raising the temperature, addition of solvent in the extraction system, or dilution of the aqueous phase with water requires long and intensive agitation. 3 ) Often, especially when alkalinity of the aqueous phase is high, the densities of the third phase and the solvent are very similar, and they form a fine emulsion or dispersion. In this case, it is hard to 
detect formation of the third phase, since centrifuging does not separate these two organic phases, and at least overnight settling time is needed. A good indication of the formation of such finely dispersed third phases is that the solvent phase loses its transparency and becomes hazy.

\subsubsection{Effect of BOBCalixC6 Loading}

In a study of distribution behavior of alkali metal ions between different aqueous phases and full solvent (Section 4.3.3), formation of the third phase was observed and correlated with the loading of BOBCalixC6 extractant. Figs. 4.2-4.4, and Tables 4.9-4.11 and 7.4 summarize data collected in the distribution experiments.

Formation of the third phase was found to be highly dependent on the composition of the aqueous phase. As seen from Figs. 4.2 and 4.3, a third phase was observed in the solvent contacted with aqueous solutions containing high concentrations of $\mathrm{Cs}^{+}$or $\mathrm{K}^{+}$ion and when BOBCalixC6 loading exceeded $74 \%$. Comparison of the BOBCalixC6 loading values collected using aqueous phases containing only $\mathrm{Cs}^{+}$or $\mathrm{K}^{+}$salts under neutral or acidic conditions (Experiments \#1, \#2, \#4, and \#5 in Table 7.4) suggests that the solvent is capable of tolerating twice the amount of extracted $\mathrm{Cs}^{+}$species than $\mathrm{K}^{+}$without formation of the third phase. For $\mathrm{Cs}^{+}$ion at $\mathrm{pH} \leq 7$, loading exceeds $100 \%$, indicating formation of both $1: 1$ and $2: 1$ metal-calixarene species. An important observation is that under alkaline conditions (Experiment \#3 in Table 7.4) the solvent can tolerate less $\mathrm{Cs}^{+}$, and a third phase was observed at $80 \%$ loading. This speculatively could be explained by the consideration that Cs-7SB modifier becomes partially deprotonated under alkaline conditions, which changes not only the extraction mechanism but also the solvation environment in the organic phase.

In a study of the effect of $\mathrm{O} / \mathrm{A}$ ratio and number of contacts with full simulant (Sections 4.3.3.6 and 4.3.3.7), no formation of a third phase was observed. It was determined that the number of solvent contacts with simulant and $\mathrm{O} / \mathrm{A}$ ratio have negligible effect on the calix loading and hence third-phase formation. In the extreme case, the maximum total BOBCalixC6 loading upon 10 contacts of the full solvent with full simulant at an O/A of 0.1 was determined to be $36 \%$. This is half of the loading level at which third phase was observed in the alkali metal ion distribution experiments.

In conclusion, formation of a third phase should be anticipated when loading exceeds $70 \%$, and $\mathrm{K}^{+}$is expected to be the primary source of the third-phase formation.

\subsubsection{Effect of $\mathrm{Cs}^{+}$and $\mathrm{K}^{+}$in Full Simulant and Cs-7SB Modifier in Solvent}

In the extraction experiments performed at different Cs-7SB modifier concentrations, $\mathrm{Cs}^{+}$or $\mathrm{K}^{+}$ in the solvent and different ion concentrations in the full simulant (described in Section 4.3.3), each sample was observed for third-phase formation at $25^{\circ} \mathrm{C}$ (Tables 4.12 and 4.13 and Figs. 4.3.5 and 4.3.7). It was found that the tendency to a form third phase greatly depends on the concentration of the modifier in the solvent. The higher the modifier concentration in the solvent is, the less is the risk of third-phase formation. Observed results are summarized as follows.

At $0.25 \mathrm{M} \mathrm{Cs}-7 \mathrm{SB}$, a third phase formed when $\mathrm{Cs}^{+}$or $\mathrm{K}^{+}$concentrations in the simulant reached 0.003 or $0.05 \mathrm{M}$ respectively, and corresponding total loading values were 45 and $25 \%$. At $0.5 \mathrm{M}$ Cs-7SB (baseline solvent), a third phase was found at $0.12 \mathrm{M} \mathrm{K}^{+}$concentration in the simulant at $74 \%$ total loading and not found for the entire tested $\mathrm{Cs}^{+}$concentration range up to $0.01 \mathrm{M}$. Solvent containing $0.75 \mathrm{M}$ Cs-7SB did not form a third phase for the entire tested concentration ranges up to $0.01 \mathrm{M}$ for $\mathrm{Cs}^{+}$and $0.86 \mathrm{M}$ for $\mathrm{K}^{+}$ion in the simulant. These observations are consistent with the data collected in a study of the effect of BOBCalixC6 loading on third-phase formation performed using simple aqueous phases (Section 7.3.4.2). Formation of a third organic phase is governed by the solubility of the extraction complexes in the solvent, presumably $\left(\mathrm{M}^{+} \cdot \mathrm{BOBCalixC}\right) \mathrm{X}^{-}$(where $\mathrm{X}^{-}$ is inorganic anion) ion-paired complexes. The ability of the solvent to solvate and thus solubilize such extraction complexes is greatly enhanced at elevated modifier concentrations. The solubility of the $\mathrm{K}^{+}$extraction complexes is much less than those of the corresponding $\mathrm{Cs}^{+}$species. As shown in Figs. 4.5 and 4.7 , at $0.25 \mathrm{M}$ Cs-7SB in the solvent, a third phase is formed when the total 


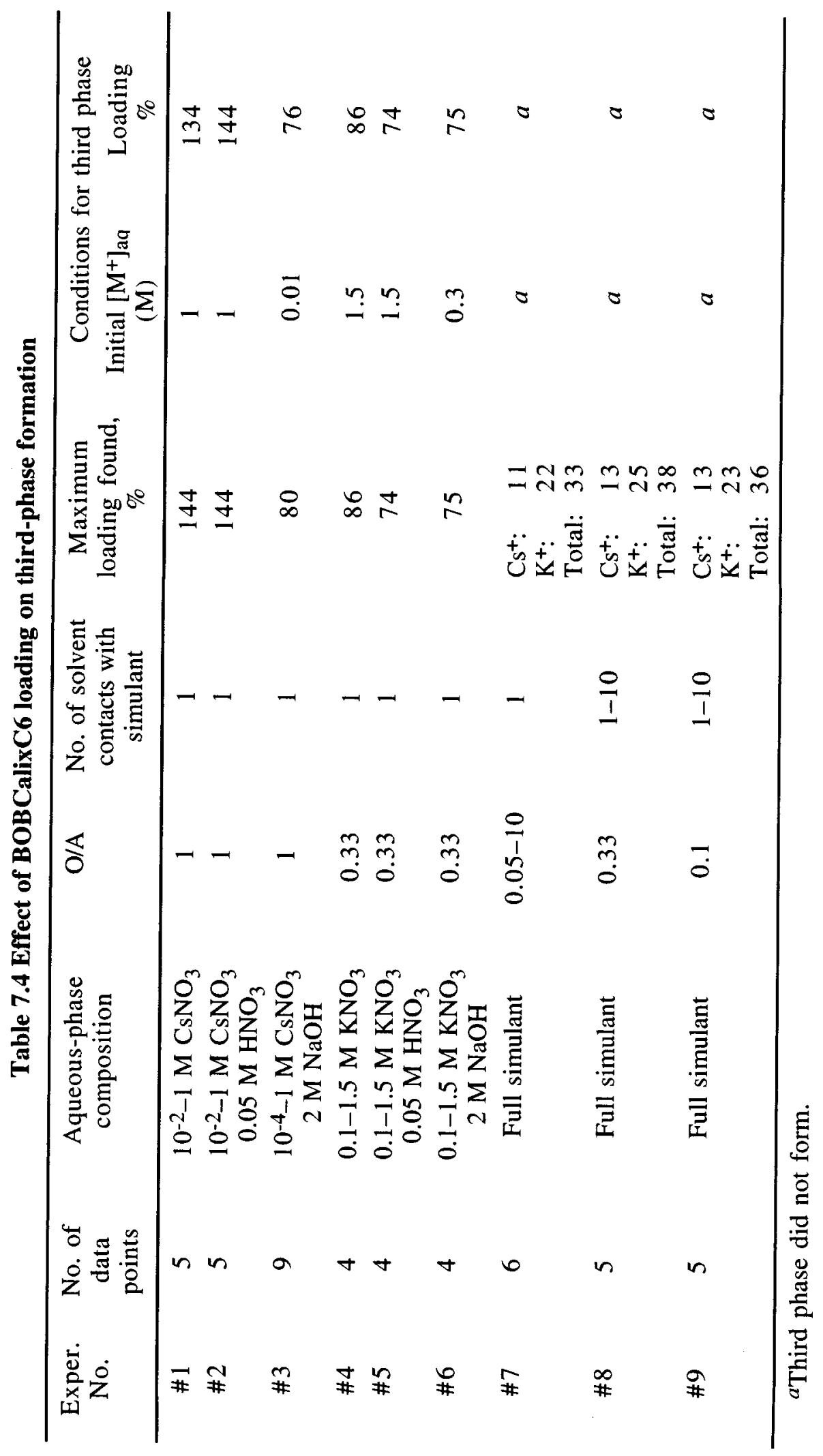


concentration of $\mathrm{K}^{+}$in the solvent is $0.0025 \mathrm{M}(25 \%$ loading), while the same solvent can tolerate

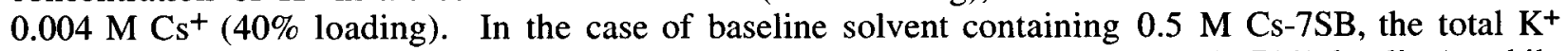
concentration in the organic phase at which a third phase formed was $0.0071 \mathrm{M}$ ( $71 \%$ loading), while a third phase was not observed up to $0.0093 \mathrm{M} \mathrm{Cs}^{+}$(93\% loading).

Additional third-phase formation tests were carried out at $25^{\circ} \mathrm{C}$ using solvent containing $0.02 \mathrm{M}$ BOBCalixC6 and $0.25,0.5$, or $0.75 \mathrm{M} \mathrm{Cs-7SB}$ modifier contacted with aqueous phases of different compositions. Collected data are summarized in Table 7.5. Overall conclusion is that increased BOBCalixC6 concentration increases the tendency to form a third phase, which is formed at much smaller initial $\mathrm{Cs}^{+}$or $\mathrm{K}^{+}$concentrations compared with the baseline solvent containing $0.01 \mathrm{M}$ BOBCalixC6. When the concentration of the BOBCalixC6 is increased from 0.01 to $0.02 \mathrm{M}$ in the

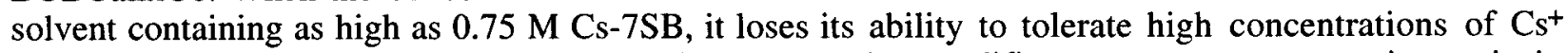
or $\mathrm{K}^{+}$and readily forms a third phase. This suggests that modifier:extractant concentration ratio in the solvent is an important factor which affects the solvation properties of the solvent.

Table 7.5. $\mathrm{CsNO}_{3}$ or $\mathrm{KNO}_{3}$ concentration in the aqueous phase and $\mathrm{O} / \mathrm{A}$ ratios corresponding to third-phase formation in extraction experiments using solvent containing $0.02 \mathrm{M}$ BOBCalixC6

\begin{tabular}{|c|c|c|c|}
\hline \multirow{2}{*}{$\begin{array}{l}\text { Aqueous } \\
\text { phase }\end{array}$} & \multicolumn{3}{|c|}{$\begin{array}{c}{\left[\mathrm{Cs}^{+}\right]_{\mathrm{aq}} \text { or }\left[\mathrm{K}^{+}\right]_{\mathrm{aq}} \text { and corresponding O/A ratio at which a third }} \\
\text { phase was observed }\end{array}$} \\
\hline & $\begin{array}{c}0.25 \mathrm{M} \mathrm{Cs}-7 \mathrm{SB} \\
0.02 \mathrm{M} \\
\text { BOBCalixC6 } \\
0.001 \mathrm{M} \text { TOA }\end{array}$ & $\begin{array}{c}0.5 \mathrm{M} \mathrm{Cs}-7 \mathrm{SB} \\
0.02 \mathrm{M} \\
\text { BOBCalixC6 } \\
0.001 \mathrm{M} \text { TOA }\end{array}$ & $\begin{array}{c}0.75 \mathrm{M} \text { Cs-7SB } \\
0.02 \mathrm{M} \\
\text { BOBCalixC6 } \\
0.001 \mathrm{M} \text { TOA }\end{array}$ \\
\hline $\mathrm{CsNO}_{3}$ & $0.015-0.02$ & $0.03-0.04$ & 0.4 \\
\hline $\mathrm{O} / \mathrm{A}$ & 0.25 & 0.5 & 0.83 \\
\hline $\begin{array}{c}\mathrm{CsNO}_{3} \\
2 \mathrm{M} \mathrm{NaOH}\end{array}$ & 0.003 & $0.007-0.0085$ & 0.01 \\
\hline $\mathrm{O} / \mathrm{A}$ & 1 & 1 & 1 \\
\hline $\mathrm{KNO}_{3}$ & $0.1-0.2$ & $0.2-0.3$ & 1.2 \\
\hline $\mathrm{O} / \mathrm{A}$ & 1 & 1 & 0.5 \\
\hline $\begin{array}{c}\mathrm{KNO}_{3} \\
2 \mathrm{M} \mathrm{NaOH}\end{array}$ & 0.079 & $0.053-0.065$ & 0.09 \\
\hline $\mathrm{O} / \mathrm{A}$ & 0.33 & 0.59 & 1 \\
\hline $\begin{array}{c}\mathrm{KNO}_{3} \\
\text { Simulant }\end{array}$ & 0.015 & 0.02 & 0.12 \\
\hline $\mathrm{O} / \mathrm{A}$ & 1 & 0.19 & 0.67 \\
\hline
\end{tabular}

It was concluded that three most important compositional factors regulate formation of the third phase in the solvent / simulant extraction system, namely concentration of the potassium ion in the simulant, concentration of the modifier in the solvent, and the modifier:BOBCalixC6 concentration ratio in the solvent. 


\subsubsection{Effect of Temperature}

The temperature at which distribution experiments are performed is a main factor that affects the thermodynamics of the extraction process, reflecting distribution and loading values and therefore conditions of third-phase formation. In the discussion above, the higher Cs-7SB concentration in the solvent and the higher modifier:extractant ratio resulted in the reduction of third-phase formation. In order to find optimum values for the modifier concentration and modifier:extractant concentration ratio in the solvent, two series of solvent samples were tested containing 0.008 or $0.01 \mathrm{M}$ BOBCalixC6 concentration and $0.5,0.55,0.6,0.65,0.7$, or $0.75 \mathrm{M}$ Cs-7SB modifier concentration. Also, three different simulant solutions were examined: 1) a "worst-case" simulant containing $0.05 \mathrm{M} \mathrm{KNO}_{3}$ and $0.00044 \mathrm{M} \mathrm{CsNO}_{3} ; 2$ ) a "worst-case" $\mathrm{K}^{+}$simulant containing the highest expected $\mathrm{K}^{+}$ concentration of $0.05 \mathrm{M}$ while maintaining the baseline $\mathrm{Cs}^{+}$concentration of $0.00014 \mathrm{M}$; and 3) a baseline simulant containing the average $\mathrm{Cs}^{+}$and $\mathrm{K}^{+}$ion concentrations of 0.00014 and $0.02 \mathrm{M}$, respectively. Solvent samples were contacted with simulant solutions twice at $25{ }^{\circ} \mathrm{C}$ and an $\mathrm{O} / \mathrm{A}$ ratio of 0.33 and allowed to remain in contact with simulant after the second equilibration. The temperature was lowered by $0.5{ }^{\circ} \mathrm{C}$ increments. At each temperature, samples were agitated for at least $30 \mathrm{~min}$, then left to stand at least $30 \mathrm{~min}$ (hazy samples were kept for several hours with subsequent centrifuging at the same temperature). Samples were then observed for the presence of a third phase. Each data point was collected in duplicate, and temperature-dependent observations were performed twice. The obtained results are summarized in Table 7.6. It is shown that the temperature of third-phase formation was gradually decreased by decrements of $0.5{ }^{\circ} \mathrm{C}$ on average as the Cs-7SB concentration in the solvent was increased by $0.05 \mathrm{M}$ increments from 0.5 to $0.7 \mathrm{M}$ for all three simulant solutions. When the modifier concentration was further increased from 0.7 to $0.75 \mathrm{M}$, an abrupt drop from 2.5 to $5{ }^{\circ} \mathrm{C}$ of the third-phase formation depending on the simulant composition was observed. This observation suggests that the solvation properties of the solvent phase containing $0.75 \mathrm{M}$ modifier change significantly from those of the solvent containing modifier at $0.7 \mathrm{M}$ or lower concentration. Solvent solutions with enhanced modifier:extractant concentration ratio

Table 7.6. Formation of the third phase as a function of temperature, solvent, and simulant compositions

\begin{tabular}{|c|c|c|c|c|}
\hline \multicolumn{2}{|c|}{ Solvent component concentrations } & \multicolumn{3}{|c|}{$\begin{array}{l}\text { Temperature of third-phase appearance }\left({ }^{\circ} \mathrm{C}\right) \text { upon two } \\
\text { contacts with simulant }(\mathrm{O} / \mathrm{A}=0.33)\end{array}$} \\
\hline $\begin{array}{c}\text { [BOBCalixC6] } \\
\mathrm{M} \\
\end{array}$ & $\begin{array}{c}{[\mathrm{Cs}-7 \mathrm{SB}]} \\
\mathrm{M}\end{array}$ & $\begin{array}{c}0.049 \mathrm{M} \mathrm{K}^{+} \\
0.44 \mathrm{mM} \mathrm{Cs}^{+} \\
\end{array}$ & $\begin{array}{r}0.049 \mathrm{M} \mathrm{K}^{+} \\
0.14 \mathrm{mM} \mathrm{Cs}^{+} \\
\end{array}$ & $\begin{array}{c}0.02 \mathrm{M} \mathrm{K}^{+a} \\
0.14 \mathrm{mM} \mathrm{Cs}^{+}\end{array}$ \\
\hline 0.01 & $\begin{array}{c}0.50^{a} \\
0.55 \\
0.60 \\
0.65 \\
0.70 \\
0.75\end{array}$ & $\begin{array}{l}19.0 \\
18.5 \\
18.0 \\
17.5 \\
17.0 \\
13.2\end{array}$ & $\begin{array}{l}18.0 \\
18.0 \\
17.5 \\
17.0 \\
16.5 \\
12.7\end{array}$ & $\begin{array}{l}15.5 \\
15.5 \\
15.0 \\
14.5 \\
13.7 \\
11.2\end{array}$ \\
\hline 0.008 & $\begin{array}{l}0.50 \\
0.55 \\
0.60 \\
0.65 \\
0.70 \\
0.75\end{array}$ & $\begin{array}{l}17.0 \\
16.5 \\
16.0 \\
15.5 \\
15.0 \\
11.2\end{array}$ & $\begin{array}{l}16.5 \\
16.0 \\
15.5 \\
15.0 \\
14.7 \\
10.7\end{array}$ & $\begin{array}{c}13.7 \\
13.2 \\
13.2 \\
13.2 \\
12.7 \\
9.7\end{array}$ \\
\hline
\end{tabular}

${ }^{a}$ Baseline solvent or simulant. 
containing $0.008 \mathrm{M}$ BOBCalixC6 form a third phase at $2{ }^{\circ} \mathrm{C}$ lower than do the solvent solutions with baseline 0.01 M extractant concentration. Comparison of the performance of different simulant solutions indicates that an increase of the $\mathrm{K}^{+}$concentration in the simulant increases the temperature of the third-phase formation more rapidly than when it is caused by increase of $\mathrm{Cs}^{+}$concentration.

An important finding is that the baseline solvent in contact with full simulant forms a third phase at $15.5{ }^{\circ} \mathrm{C}$, setting a lower process limit for the extraction temperature. Addition of $\mathrm{K}^{+}$in the simulant to the bounding concentration in the waste increases this temperature to $18{ }^{\circ} \mathrm{C}$. In the worstcase simulant (maximum $\left[\mathrm{Cs}^{+}\right]$and $\left[\mathrm{K}^{+}\right]$), a third phase forms at $19{ }^{\circ} \mathrm{C}$. Not only the process temperature, but also the $\mathrm{K}^{+}$content in the feed solution, should be closely monitored.

\subsubsection{Effect of Scrub}

Solvents containing 0.01 or $0.008 \mathrm{M}$ BOBCalixC6 and 0.5 or $0.75 \mathrm{M}$ Cs-7SB were contacted twice with the full simulant $\left(0.02 \mathrm{M} \mathrm{K}^{+}\right.$and $\left.0.00014 \mathrm{M} \mathrm{Cs}^{+}\right)$, "worst-case simulant" $\left(0.05 \mathrm{M} \mathrm{K}^{+}\right.$and $0.00044 \mathrm{M} \mathrm{Cs}^{+}$), and simulant containing $0.05 \mathrm{M} \mathrm{K}^{+}$and $0.00014 \mathrm{M} \mathrm{Cs}^{+}$at $25{ }^{\circ} \mathrm{C}$ and $\mathrm{O} / \mathrm{A}=0.33$. Solvent subsamples were then withdrawn, contacted with scrub solution at $\mathrm{O} / \mathrm{A}=5$, and cooled with agitation to $10^{\circ} \mathrm{C}$. No third phase was observed in these experiments, since the majority of the organic-phase $\mathrm{K}^{+}$, but not $\mathrm{Cs}^{+}$, is released into the aqueous scrub solution. This observation supports the conclusion that $\mathrm{K}^{+}$loading is the primary cause of third-phase formation.

\subsection{CONCLUSIONS}

Long-term observation of the baseline solvent under various conditions revealed no tendency to form solid phases at $25^{\circ} \mathrm{C}$ or at $4-6{ }^{\circ} \mathrm{C}$. Thus, the supersaturation of BOBCalixC6 represents a metastable condition that over the course of a year's time has not posed any experimental difficulty in any of the CSSX tasks. Furthermore, the solubility phenomena associated with crystallization of Cs-6 hydrate do not apply to the case of Cs-7SB. Although Cs-7SB is undoubtedly hydrated in the solvent, no solid hydrated material was ever observed to precipitate. Modifier Cs-6 exists as a pair of enantiomers due to the presence of one chiral carbon atom, whereas modifier Cs-7SB exists as four stereoisomers due to the presence of two chiral carbon atoms; the greater number of isomers present for Cs-7SB is believed to play a key role in its resistance to solids formation. Hence, no issues are identified for the modifier solubility.

In contrast to the observations on modifier solubility, close inspection of BOBCalixC6 solubility data revealed that the baseline solvent is somewhat supersaturated at $25^{\circ} \mathrm{C}$. Highly supersaturated solutions employing the modifier at $0.50 \mathrm{M}$ can be prepared by warming and sonication of the solution. However, after standing at $25{ }^{\circ} \mathrm{C}$ with the aid of seeding, BOBCalixC6 is observed to crystallize out. After crystallization at $25{ }^{\circ} \mathrm{C}$, the resultant solutions contained approximately $8 \mathrm{mM}$ of the extractant. When the modifier concentration is raised to $0.75 \mathrm{M}$, however, the extractant solubility so obtained is $12.7 \mathrm{mM}$. Thus, there is motivation to test higher modifier concentrations in future solvent-optimization efforts. This opportunity also offers advantages in raising $D_{\mathrm{Cs}}$ values, allowing the BOBCalixC6 concentration to be decreased (affords cost savings), as well in decreasing the operating temperature of the solvent to $15^{\circ} \mathrm{C}$ (see below). Further investigation of solubility issues is warranted in that solubilities for BOBCalixC6 in the forward direction (i.e., approaching equilibrium from undersaturation) were significantly less than the values obtained from the reverse direction. Careful experiments were conducted approaching equilibrium in the forward direction at $25^{\circ} \mathrm{C}$ using recrystallized BOBCalixC6. The solubility of BOBCalixC6 in Isopar L containing only $0.5 \mathrm{M}$ Cs-7SB modifier was thus found to be $4.18 \mathrm{mM}$. Addition of TOA at $0.001 \mathrm{M}$ concentration resulted in only a slight increase of solubility, which reached the level of $5.0 \mathrm{mM}$. Prewashing the 0.5 $\mathrm{M}$ Cs-7SB and $0.001 \mathrm{M}$ TOA solution in Isopar L (using standard solvent washing protocol) further increased the solubility of BOBCalixC6 to $5.5 \mathrm{mM}$; presumably, this effect is due to the water content of the solvent. Although the data suggested that the concentrations of BOBCalixC6 had reached a plateau, inconsistencies raise the likelihood that equilibrium is reached very slowly and that the values obtained in the forward direction may under-represent the true solubility. At present, the solubility 
data taken from the reverse direction are taken to be the most applicable values, as equilibrium is approached in the same direction that it would be approached in the event that the calixarene precipitates from the baseline solvent. Impurities in as-received BOBCalixC6 were found to enhance the dissolution rate of the material but not to affect the solubility itself. Obviously, further data are needed, both to resolve the question of the true equilibrium solubility of BOBCalixC6 and to optimize the solvent so that a supersaturated state cannot be reached.

Third-phase formation was found to occur as a result of either $\mathrm{Cs}^{+}$or $\mathrm{K}^{+}$ion loading and is associated with the generally recognized limited solubility of ion-pair complexes in nonpolar organic solvents. It was found that high $\mathrm{Cs}^{+}$ion loading can be tolerated, however, and conditions leading to high loading will not be found in the flowsheet. On the other hand, $\mathrm{K}^{+}$ion loading is significant, and the solubility limit of its complexes can be exceeded at the bounding $\mathrm{K}^{+}$aqueous concentration if the temperature falls below $20{ }^{\circ} \mathrm{C}$. Hence, this property sets the operating limit of the process at $20{ }^{\circ} \mathrm{C}$. If it is desirable to operate at a lower temperature for seasonal reasons, then feed blending or other control of the potassium concentration is needed. Alternatively, a higher modifier concentration can be employed, in which case the process could be operated down to $15^{\circ} \mathrm{C}$ with $0.75 \mathrm{M} \mathrm{Cs}-7 \mathrm{SB}$. 


\section{SOLVENT CLEANUP}

\subsection{INTRODUCTION}

\subsubsection{Purpose}

Solvent-extraction technology normally includes some form of solvent cleanup. Over many solvent cycles, minor components from the waste stream, solvent degradation products, and other matter build up in the solvent and ultimately impair performance. Problems can arise from poor extraction and stripping, lower selectivity, crud formation, third-phase formation, slow phase disengagement, etc. In the present system, evidence obtained in earlier chapters pointed to a need for solvent cleanup mainly to remove extractable minor waste components, such as dibutylphosphate (DBP), that remain in the solvent throughout the solvent cycle. Solvent degradation does not appear to be as great of a concern with regard to necessitating solvent cleanup. The chief breakdown products identified, namely 4-sec-butylphenol and dioctylamine, will wash out into respectively the waste raffinate and strip effluent streams and will therefore not be expected to build up to concentrations in the solvent that could have an effect on solvent performance. Nevertheless, since phenols and secondary amines are much more reactive than the baseline solvent components, it would be prudent to ensure that their concentrations in the solvent remain at trace levels.

The purpose of the tests reported in this chapter has been to demonstrate solvent washing strategies using mainly $\mathrm{NaOH}$ solutions. Data are provided on the performance of used or treated baseline solvent samples before and after washing. Another purpose was to recommend, based on systematic test data, a solvent wash that could be incorporated into extended contactor testing of CSSX.

\subsubsection{Background}

It has been the intention in this report to provide a basis for rational choices of solvent-cleanup methodology through knowledge of the species that are likely to build up in the solvent and interfere with performance. Toward this end, experiments described in earlier chapters were designed to provide as much information as possible about the identity, effect, and partitioning of a) interfering minor components in the waste feed and b) interfering solvent-degradation products. In so doing, much has already been learned about effective approaches to solvent rejuvenation. The most harmful species remain lipophilic anions, as demonstrated during CSSX development. ${ }^{8,14}$ Built into the solvent already is a degree of tolerance to lipophilic anions to approximately $0.3 \mathrm{mM}$, owing to the beneficial effect of the TOA suppressor component. Since DBP at its bounding concentration $(0.12$ $\mathrm{mM}$ ) in the waste can build up in the solvent to concentrations comparable to the $0.3 \mathrm{mM}$ solvent tolerance, it is clear that some form of solvent cleanup is needed as an integral part of each solvent cycle.

A recommendation for solvent washing may be made according to the following general chemical logic. Lipophilic anions represent the primary poison for the solvent, impairing stripping of cesium. To most efficiently wash out organic anions requires that the aqueous wash solution possess sufficient alkalinity to maintain expected lipophilic anions in their deprotonated state, since they will be most hydrophilic in their salt form. Phenols as a class are the least acidic ionizable species expected in the solvent, and high alkalinity $([\mathrm{NaOH}] \geq 0.1 \mathrm{M})$ is needed to convert an appreciable fraction to the salt form. ${ }^{51,52}$ High alkalinity or ionic strength also benefits phase disengagement. To maximize alkalinity for removal of phenol degradation products, hydroxide vs. carbonate is the preferred aqueous anion for the wash solution. Suitable cations for washing include $\mathrm{Li}^{+}$and $\mathrm{Na}^{+}$. Larger alkali cations such as $\mathrm{K}^{+}$are complexed by the calixarene, and their salts would therefore be less easily washed out. Being most economical, $\mathrm{NaOH}$ was therefore employed here. Although the preceding arguments favor higher concentrations of $\mathrm{NaOH}$, chemical mass-action 
favors lower $\mathrm{NaOH}$ concentrations, because removal of sodium salts from the organic solvent becomes increasingly efficient as the sodium concentration in the aqueous phase decreases. Economics also places value on minimizing the $\mathrm{NaOH}$ concentration.

As a compromise, $10 \mathrm{mM} \mathrm{NaOH}$ was chosen as a suitable wash solution for the extended contactor tests and for further flowsheet evaluation. Dispersion-number tests at ANL ${ }^{67}$ showed that this concentration is acceptable, though tendency to form emulsions tended to be greater in batch tests than obtained with higher $\mathrm{NaOH}$ concentrations. Since 4-sec-butylphenol will likely be washed from the solvent sufficiently by the waste raffinate, it was deemed best to base the recommended $\mathrm{NaOH}$ wash solution on the partitioning behavior of other interfering lipophilic anions. DBP washes out with excellent efficacy over a wide range of $\mathrm{NaOH}$ concentrations $(P \leq 0.01$ for 0.01 to $1 \mathrm{M}$ $\mathrm{NaOH}$ ) and thus sets no constraints. Carboxylates require $0.003 \mathrm{M} \mathrm{NaOH}$ optimally, but such a low concentration of $\mathrm{NaOH}$ would be both challenging to maintain and troublesome with regards to phase disengagement. Hence, $0.01 \mathrm{M} \mathrm{NaOH}$ was chosen as a good compromise. It is expected that lipophilic organic phenolate, carboxylate, phosphate, sufonate, and sulfate compounds will all be washed out by this solution. Although waste-characterization data do not indicate their presence as significant waste components, surfactant anions having more than 12 aliphatic carbon atoms may not wash out effectively at any alkalinity, and for this as-yet hypothetical possibility, we have provided an effective option involving resin anion exchange, as discussed in Chap. 4.

\subsubsection{Experimental Design}

Unless otherwise noted, most of the tests described herein employed only the standard ESS protocol or simple batch contacting procedures used throughout this report.

\subsubsection{Work Scope}

In connection with examination of lipophilic anion partitioning (WSM task 5.1.5), experiments to demonstrate solvent rejuvenation were carried out (WSM task 5.2.3) as specified by the Work Scope Matrix. ${ }^{19}$ Solvent samples received from several other tasks carried out at ORNL, SRTC, and ANL were received and subjected to analytical procedures and performance assessment. Exploratory solvent-cleanup tests were dependent upon the extent of solvent degradation and performance, and analyses and tests were prioritized according to the apparent severity of degradation and to the type of information needed to diagnose and remediate any identified problems. Specifically, on receipt of a sample of spent solvent from the 4-cycle flowsheet test at ANL, analysis, ESS protocol, and diagnostics were performed (WSM task 3.1.4.2); a similar set of tests will be conducted on a solvent sample from a "5-day" test, 67 though the results are not yet available to include in the present report (WSM task 3.2.4.3). In cooperation with the SRTC, solvent washing with selected aqueous phases was carried out upon receipt of degraded solvent samples from ${ }^{60}$ Co external-irradiation tests (WSM task 4.1.1.5.). Solvent samples received from the ORNL-CTD batch internal-radiolysis tests were also subjected to selected performance tests, diagnostic experiments, and cleanup procedures (WSM task 4.1.2.1); these solvent samples were considered to be representative of the closed-loop batch internalradiolysis tests (WSM item 4.1.3.1-4.1.3.5).

\subsection{EXPERIMENTAL SECTION}

\subsubsection{Solvent Washing After Multiple ESS Cycles}

The potential buildup of lipophilic acidic impurities in the solvent may cause unwanted effects on cesium distribution behavior. In order to regain solvent performance, it should be periodically cleaned up. A proposed solvent cleanup procedure involving consecutive washings with equal volumes of $0.1,0.01$, and $0.001 \mathrm{M} \mathrm{NaOH}$ solutions was undertaken using two solvent lots. The first had been subjected to five ESS cycles (see Section 3.3.16), and the second had been spiked with $0.00144 \mathrm{M}$ dibutylphosphate (DBP) and then subjected to a single ESS (see Section 3.3.12). 
Following the washing procedure, the two solvent samples were then subjected to a typical ESS analysis. Baseline data, for comparative purposes, were drawn from Tables 3.4, 3.19, and 3.26.

\subsubsection{Solvent Washing After Multiple Contacts With Full Simulant}

A given volume of pristine solvent was contacted five times with fresh simulant, then scrubbed, then stripped four times while utilizing the normal volume ratios of 0.33 on extraction and 5 on scrubbing and stripping. It was then split into five different vials. One was set aside, the four others were respectively contacted at $\mathrm{O} / \mathrm{A}=1$ for $30 \mathrm{~min}$ at $25^{\circ} \mathrm{C}$ with $\mathrm{NaOH}$ at $3 \mathrm{mM}, 10 \mathrm{mM}, 30 \mathrm{mM}$, and $100 \mathrm{mM}$. Following this step, a full ESS test was conducted on those four washed samples; the unwashed sample and one control test were run in parallel. To determine an appropriate O/A ratio for washing with $10 \mathrm{mM} \mathrm{NaOH}$, further tests were conducted. Solvent previously contacted five times with the full simulant was used. Separate washing contacts were performed as follows: $\mathrm{O} / \mathrm{A}=2,30$ $\min$ (wheel); $\mathrm{O} / \mathrm{A}=5,30 \mathrm{~min}$ (wheel); $\mathrm{O} / \mathrm{A}=5,10$ s (vortexing). Immediately after the wash, the samples were centrifuged and the solvent separated. An ESS (extraction, scrub, and four strips) test was run.

\subsubsection{Washing Internally Irradiated Solvent Samples}

A series of internally irradiated samples (see Table 8.1 ), ${ }^{47}$ provided by ORNL-CTD, were subjected to a series of wash steps for the removal anionic species. The aim of this work was to establish a wash-based clean-up protocol for the removal of anionic species produced in the solvent as a product of internal irradiation. The samples selected were from two test regimens, one involving a 20-day contacting period (T1) with simulant, scrub, or strip solution, and the second from an 80-day contact period (T3) with solutions comparable to the T1 series. All solvent samples had been extensively stripped prior to washing. Chemical analyses revealed some consumption of TOA (Table 8.1).

Table 8.1. Internally irradiated solvents selected for establishing a wash-based cleanup process

\begin{tabular}{|c|c|c|c|c|}
\hline Test process & T1 samples ID ID $^{a}$ & {$\left[\mathrm{TOA}^{b}, \mathrm{mM}\right.$} & T3 samples ID ${ }^{c}$ & [TOA], mM \\
\hline \multirow[t]{3}{*}{ Extraction } & T1-4-0 & 0.697 & T3-16-0-S & 0.503 \\
\hline & & & T3-44-0-S & 0.417 \\
\hline & & & T3-46-0-S & 0.457 \\
\hline Scrub & $\mathrm{T} 1-22-0$ & 0.917 & T3-34-0-S & 0.877 \\
\hline Strip & $\mathrm{T} 1-12-0$ & 1.020 & T3-40-0-S & 0.823 \\
\hline
\end{tabular}

${ }^{a} \mathrm{~T} 1$ solvent samples were contacted for 20 days with simulant (extraction test), scrub, or strip solution.

${ }^{b}$ Trioctylamine concentrations [TOA] are given as measured following internal irradiation.

cT3 solvent samples were contacted for 80 days with their indicated aqueous phases.

The planned procedure for solvent washing and subsequent performance testing included washing each sample $(4.3 \mathrm{~mL})$ at $\mathrm{O} / \mathrm{A}=1$ with $10 \mathrm{mM} \mathrm{NaOH}$ for $45 \mathrm{~min}$ using the normal end-overend contacting protocol in a $25{ }^{\circ} \mathrm{C}$ air box. The phases were separated by centrifugation at 3600 rpm $(2221 \times \mathrm{g})$ for $3 \mathrm{~min}$ using a Mistral 2000R refrigerated tabletop centrifuge. The solvent phase was isolated and an appropriate amount of TOA added (to T3 samples only) in order to replenish the TOA levels to $1 \mathrm{mM}$. Performance of the solvent was then gauged by performing the standard ESS procedure using full simulant (Draw \#5) spiked with ${ }^{137} \mathrm{Cs}$ at $0.2 \mu \mathrm{Ci} / \mathrm{mL}$. A minimal amount of available starting solvent required that organic phase that was withdrawn for counting at each ESS step be returned to the solvent pool prior to performing the subsequent ESS operation. 


\subsubsection{ESS Procedure for Washed Internally Irradiated Samples}

Solvent phases were isolated from all samples following the final centrifugation, and solvent performance was judged via the ESS protocol. For each sample, $3.0 \mathrm{~mL}$ of solvent was available for ESS. The ESS protocol followed the normal procedure of O/A phase ratios of 0.33 for extraction and 5 for the scrub and three strip steps. The simulant used was SRS Full Simulant, Draw \#5, with Cs added to $1.4 \times 10^{-4} \mathrm{M}$, then spiked with ${ }^{137} \mathrm{CsNO}_{3}$ at $0.2 \mu \mathrm{Ci} / \mathrm{mL}$. Trioctylamine $(0.2 \mathrm{M}$ in Isopar L) was added to the T3-extraction solvents (T3-16-0-S, T3-44-0-S, and T3-40-0-S) to replenish TOA levels to $1 \mathrm{mM}$. Contacts involved shaking 10 times followed by end-over-end tumbling on a rotating wheel in a $25^{\circ} \mathrm{C}$ air box for $50 \mathrm{~min}$. Samples were centrifuged for $3 \mathrm{~min}$ at $3600 \mathrm{rpm}$ in a Mistral 2000R tabletop centrifuge. The small volumes $(3.0 \mathrm{~mL})$ of starting solvent required that organic samples that were withdrawn for counting at each ESS step be returned to the solvent pool for the subsequent contacting and sampling operation with the extraction, scrub, and first two steps. Phases were separated and subsampled for gamma counting. A Packard Cobra II AutoGamma

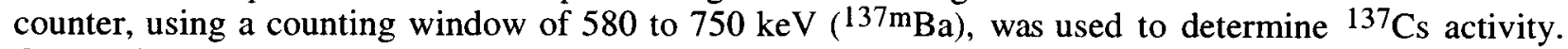
Count data were collected and entered into a spreadsheet to determine solvent performance as indicated by the cesium distribution values obtained for the various ESS steps.

\subsubsection{Washing Externally Irradiated or Thermally Treated Samples}

Solvent samples from two stress tests were subjected to the nitric acid/sodium hydroxide washing procedure for the removal of anionic surface/interfacially active species. The effectiveness of this washing process was then measured by observing cesium distribution behavior in the ESS Protocol. Solvents selected for testing included four samples from the 235-day thermal-stability study: solvent contacted with scrub (SC-35-33, SC-60-33) or strip (ST-35-33, ST-60-33) solution at $35{ }^{\circ} \mathrm{C}$ and $61{ }^{\circ} \mathrm{C}$. Also tested were two solvents from the SRTC external irradiation tests, ${ }^{55}$ one (ST-16-SB) having received a 16-Mrad dose while in contact with strip solution, and a second (FD-17-SC-6) receiving $6 \mathrm{Mrad}$ while in contact with scrub solution.

The washing protocol involved two contacts, at an O/A of 1 , with $50 \mathrm{mM} \mathrm{HNO}$, then twice with $0.5 \mathrm{M} \mathrm{NaOH}$, once with $0.1 \mathrm{M} \mathrm{NaOH}$, and finally once with $10 \mathrm{mM} \mathrm{NaOH}$. Following the final wash, the organic layer was isolated, and defined volumes were subjected to the ESS protocol. Previously performed chemical analyses indicated that all of the candidate solvent samples had varying amounts of their trioctylamine (TOA) content consumed in the tests in which they had been previously involved. Trioctylamine $(0.2 \mathrm{M}$ in Isopar $\mathrm{L})$ was added to the tested solvents (see Table 8.2 below) to replenish TOA levels to $1 \mathrm{mM}$.

The ESS protocol followed the normal procedure using O/A phase ratios of 0.33 for extraction and 5 for the scrub and three strip steps. The simulant used was the full simulant, Draw \#5, with Cs added to $1.4 \times 10^{-4} \mathrm{M}$, then spiked with ${ }^{137} \mathrm{CsNO}_{3}$ at $0.2 \mu \mathrm{Ci} / \mathrm{mL}$.. Contacts involved shaking 10 times followed by end-over-end tumbling on a rotating wheel in a $25{ }^{\circ} \mathrm{C}$ air box for $50 \mathrm{~min}$. Samples were centrifuged for $3 \mathrm{~min}$ at $3600 \mathrm{rpm}$ in a Mistral 2000R tabletop centrifuge. Phases were separated and subsampled for gamma counting in a Packard Cobra II AutoGamma counter using a counting window of 580 to $750 \mathrm{keV}\left({ }^{137} \mathrm{mBa}\right)$ to determine ${ }^{137} \mathrm{Cs}$ activity. Count data were collected and entered into a spreadsheet to determine solvent performance as indicated by the cesium distribution values obtained from the various ESS steps.

\subsubsection{ESS of Washed Solvents Used in Contactor Tests}

An ESS Protocol (extraction, scrub, three strips) was undertaken with several solvent samples that had been repeatedly contacted with simulant in various testing regimens. The purpose of this ESS experiment was to see, indirectly, if any contaminants had accumulated in the solvents (which had seen considerable use), that would affect the distribution of Cesium-137 between the organic and aqueous phases. Some of the solvents had also been subjected to various post-test washing procedures to investigate their effectiveness in removing accumulated contaminants. The solvents 
Table 8.2. Trioctylamine (TOA) content of test samples and amount of TOA added to replenish that which was consumed in earlier testing (thermal-stability test or external-irradiation test) prior to performing the ESS protocol ${ }^{a}$

\begin{tabular}{|c|c|c|c|c|}
\hline Sample $\mathrm{ID}^{b}$ & & $\begin{array}{l}\text { Original }[\mathrm{TOA}] \text { via } \\
\text { chemical analysis } \\
(\mathrm{mM})\end{array}$ & $\begin{array}{c}\text { Volume of TOA } \\
\text { added to } \\
\text { solvent to make } 1 \mathrm{mM} \\
(\mu \mathrm{L})\end{array}$ & Moles of added $\mathrm{TOA}^{d}$ \\
\hline $\mathrm{SC}-35-33$ & & 0.93 & 1.47 & $2.94 \times 10^{-7}$ \\
\hline SC-60-33 & & 0.047 & 20.0 & $4.00 \times 10^{-6}$ \\
\hline ST $-35-33$ & & 0.74 & 5.46 & $1.09 \times 10^{-6}$ \\
\hline ST-60-33 & & 0.64 & 7.56 & $1.51 \times 10^{-6}$ \\
\hline ST-16-SB & - & 0.24 & 16.6 & $3.32 \times 10^{-6}$ \\
\hline FD-17-SC-6 & - & 0.21 & 16.0 & $3.19 \times 10^{-6}$ \\
\hline
\end{tabular}

$a$ Volume of solvent tested by ESS was $4.2 \mathrm{~mL}$.

${ }^{b} \mathrm{SC}$ series and ST series are 33-week contacts at $35{ }^{\circ} \mathrm{C}$ or $61{ }^{\circ} \mathrm{C}$ from thermal-stability tests; ST-16-SB (strip contacted while receiving $16 \mathrm{Mrad}$ ) and FD-17-SC-6 (scrub contacted while receiving $6 \mathrm{Mrad})$ are both from the SRTC externally-irradiated samples.

cTrioctylamine used was $0.20 \mathrm{M}$ in Isopar diluent.

${ }^{d}$ A $4.2-\mathrm{mL}$ solvent sample containing $1 \mathrm{mM}$ TOA will contain $4.2 \times 10^{-6}$ moles of TOA.

tested included a pristine solvent control, a sample provided by ANL, and four samples from internalirradiation tests.

The ANL solvent had been subjected to four full-cycle tests using a centrifugal contactor and full simulant that contained ${ }^{137} \mathrm{Cs}$. Following the contactor testing protocol, the solvent was repeatedly stripped with $\mathrm{HNO}_{3}$ resulting in solvent gamma activity levels consistent with background. The ORNL solvent had been subjected to some number of consecutive hydrodynamic studies followed by a variety of post-contact treatments designed to remove contaminants that may have accumulated in the solvent during the testing phase. These samples included an untreated sample, a sample washed with $\mathrm{NaOH}$, a sample contacted with $\mathrm{HNO}_{3}$, and one solvent sample contacted with $\mathrm{H}_{2} \mathrm{O}$.

The ESS experiments were performed using the standard procedure with full simulant, Draw \#5, containing cesium at $1.4 \times 10^{-4} \mathrm{M}$ and spiked with ${ }^{137} \mathrm{Cs}$ at $0.2 \mu \mathrm{Ci} / \mathrm{mL}$. An $\mathrm{O} / \mathrm{A}$ ratio of 0.33 was used for extraction, and an $\mathrm{O} / \mathrm{A}$ ratio of 5 for the scrub and strip operations. The volumes used in the contacting steps were extraction $-7 \mathrm{~mL}$ solvent, $21 \mathrm{~mL}$ simulant; scrub $-6 \mathrm{~mL}$ solvent, $1.2 \mathrm{~mL}$ scrub solution; strip \#1 $-5 \mathrm{~mL}$ solvent, $1 \mathrm{~mL}$ strip solution; strip \#2 $-4 \mathrm{~mL}$ solvent, $0.8 \mathrm{~mL}$ strip solution; and strip \#3-3 mL solvent, $0.6 \mathrm{~mL}$ strip solution. A fourth strip was also performed using $2.5 \mathrm{~mL}$ solvent and $0.5 \mathrm{~mL} 1 \mathrm{mM} \mathrm{HNO}_{3}$ strip solution spiked with ${ }^{137} \mathrm{Cs}$ at approximately 0.15 $\mu \mathrm{Ci} / \mathrm{mL}$. For sampling and gamma counting, 300- $\mu \mathrm{L}$ subsamples were withdrawn from each phase following contacting, centrifugation, and phase separation.

\subsection{RESULTS AND DISCUSSION}

\subsubsection{Solvent Washing after Multiple ESS Cycles}

Figure 8.1 shows the effect of washing the solvent following five ESS cycles. The data shown are in part taken from the five-cycle experiment in Section 3.3.16 and the washing data from Table 8.3. In the table, Set \#1 is the average ESS performance given in Table 3.4. Sets \#2 and \#3 represent the ESS data from the first and fifth cycles in Section 3.3.16 (Table 3.26). Set \#4 shows the 
improvement upon washing with the sequence of the following three $\mathrm{NaOH}$ solutions at $\mathrm{O} / \mathrm{A}=1$ and $25^{\circ} \mathrm{C}$ : $0.1 \mathrm{M}, 0.01 \mathrm{M}$, and $0.001 \mathrm{M}$.

In the experiment corresponding to Set \#5 (taken from Table 3.19), $1.44 \mathrm{mM}$ DBP was added to the solvent before the test to determine whether DBP alone could account for the worsened stripping seen on the fifth cycle (Set \#3). The amount $1.44 \mathrm{mM}$ represents the concentration of DBP that would be in the solvent after four cycles if all of the DBP is extracted and remains in the solvent through scrubbing and stripping. By reference to the data in Table 3.19, it may be concluded that DBP causes elevated $D_{\mathrm{Cs}}$ values in stripping, and especially in the first strip. However, its effect on subsequent stripping is not enough to account entirely for the elevated $D_{\mathrm{Cs}}$ values in those stages. It may be seen from the data corresponding to Set \#6 that washing readily restores performance, as it efficiently removes the DBP (Chap. 4).

Table 8.3. ESS performance after washing ${ }^{a}$

\begin{tabular}{ccccccc}
\hline Set & Test description & Extraction & Scrub & Strip \#1 & Strip \#2 & Strip \#3 \\
\hline 1 & Average ESS & 16.9 & 1.59 & 0.148 & 0.089 & 0.067 \\
2 & ESS cycle \#1 & 18.6 & 1.63 & 0.154 & 0.093 & 0.071 \\
3 & ESS cycle \#5 & 20.0 & 2.24 & 0.272 & 0.166 & 0.150 \\
4 & After washing & 17.4 & 1.42 & 0.188 & 0.110 & 0.083 \\
5 & ESS with 1.44 mM DBP & 17.9 & 1.22 & 0.289 & 0.116 & 0.098 \\
6 & After washing & 17.8 & 1.07 & 0.255 & 0.085 & 0.061 \\
\hline
\end{tabular}

${ }^{a}$ Baseline solvent and full simulant were used for all ESS tests. Tests were carried out by the normal protocol at $25^{\circ} \mathrm{C}, \mathrm{O} / \mathrm{A}$ (extraction) $=0.33$, and $\mathrm{O} / \mathrm{A}$ (scrub and strip) $=5$. Washing in Sets \#4 and \#6 was carried out by successively contacting the solvent at $\mathrm{O} / \mathrm{A}=1$ with $0.1 \mathrm{M}, 0.01 \mathrm{M}$, and $0.001 \mathrm{M} \mathrm{NaOH}$ solutions at $25^{\circ} \mathrm{C}$.

\subsubsection{Solvent Washing after Multiple Contacts with the Full Simulant}

Based on the partition results of dibutyl phosphate and other organophilic anions, solutions of $\mathrm{NaOH}$ at low concentrations were tested. Solvent that had already been through 5 contacts with the simulant, 1 scrub, and 4 strips was then washed with different solutions of $\mathrm{NaOH}$. This test showed (see Table 8.4) that the solvent is rejuvenated with all the $\mathrm{NaOH}$ washes (the best one being $10 \mathrm{mM}$ ), while the unwashed solvent gives poor stripping values. Results were obtained with an O/A ratio of 1 and a gentle contact of the two phases. The influence of the volume ratios and of the nature of the contact was also investigated to answer some of the questions that pertain to the solvent wash when in real conditions, using centrifugal contactors. It was found that whatever the $\mathrm{O} / \mathrm{A}$ ratios, the results are identical (within the experimental error). The vortex method with a 10 -second contact allowed the same level of solvent rejuvenation as the gentle contacts on the wheel.

\subsubsection{Solvent Washing of Internally Irradiated Solvent Samples}

\subsubsection{Genesis of Solvent Washing Procedure Due to Emulsion Formation}

The planned procedure for solvent washing was not adhered to due to the unanticipated formation of emulsion layers as a result of washing these solvents with $10 \mathrm{mM} \mathrm{NaOH}$. A $4.3-\mathrm{mL}$ volume of each organic phase was isolated and placed in $15-\mathrm{mL}$ conical-bottomed polypropylene tubes. An equal volume of $10 \mathrm{mM} \mathrm{NaOH}$ was added to each tube, and the samples were rotated endover-end at $25{ }^{\circ} \mathrm{C}$ for $45 \mathrm{~min}$. 


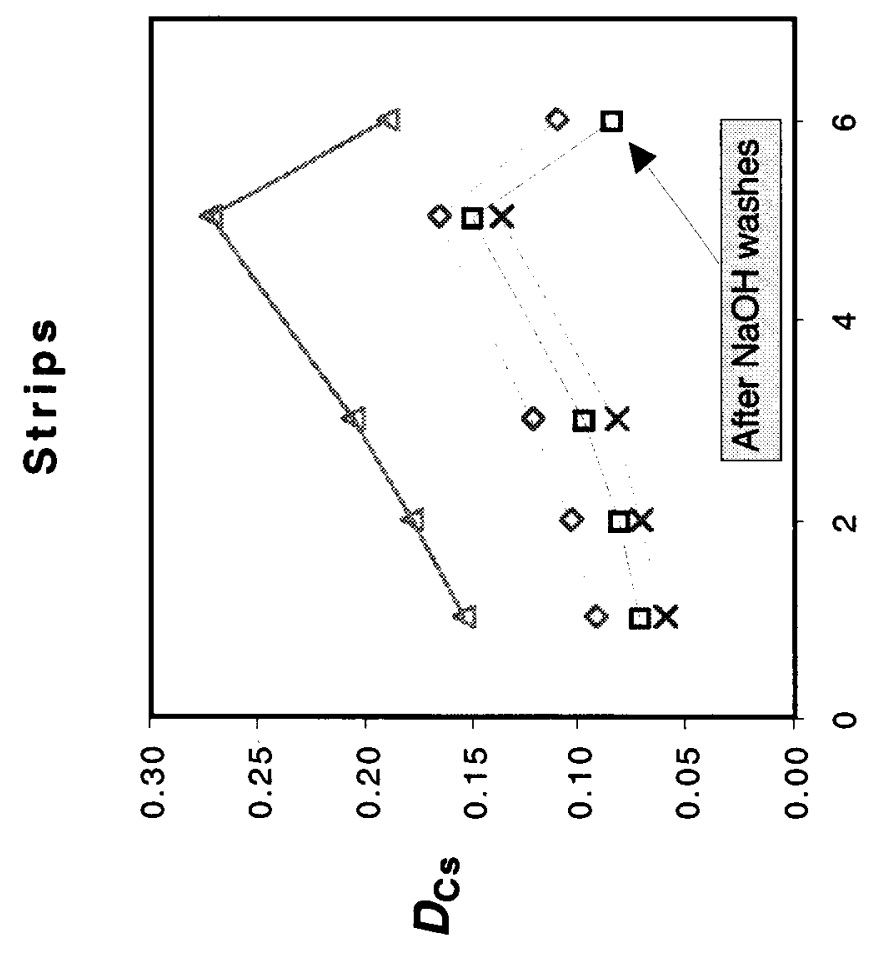

家

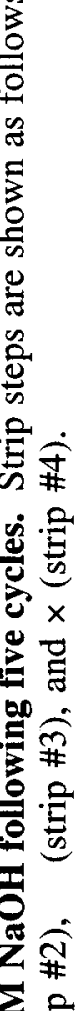

是景

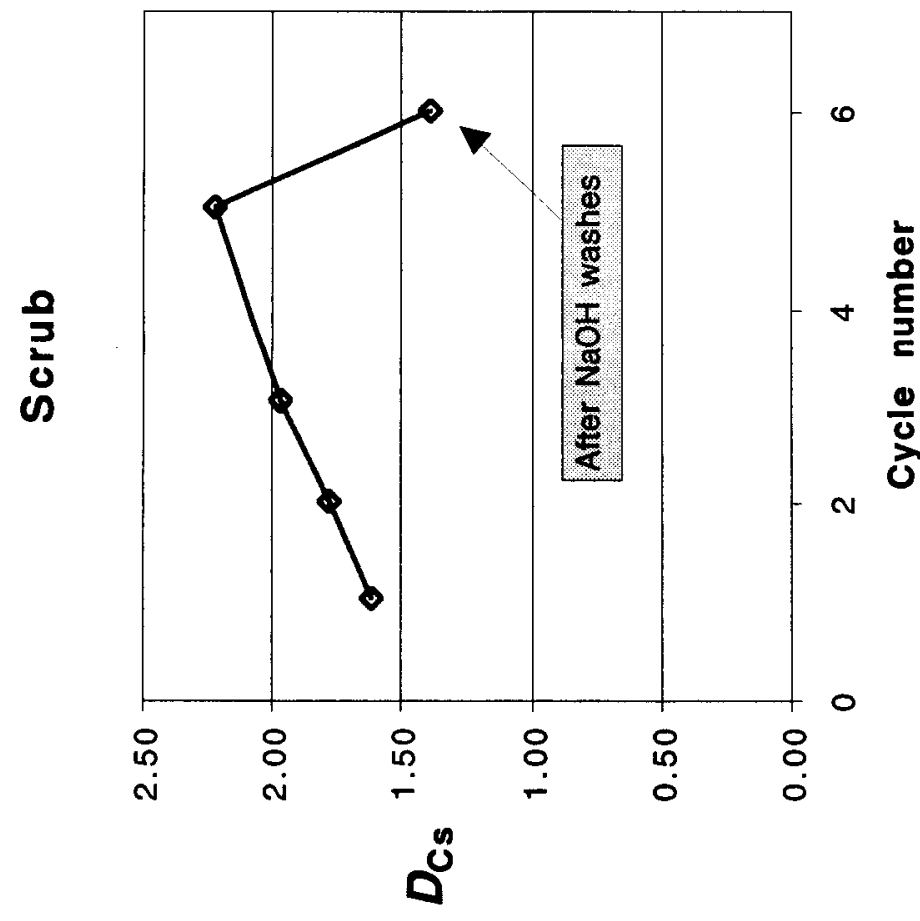

吾

最㬝

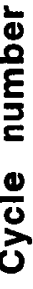

를

$\dot{\infty}$

禹 
Table 8.4. Performance of multiply contacted solvent following $\mathrm{NaOH}$ washing

\begin{tabular}{|c|c|c|c|c|c|c|}
\hline & \multicolumn{6}{|c|}{$D_{\mathrm{Cs}}$} \\
\hline & Ext'n & Scrub & Strip \#1 & Strip \#2 & Strip \#3 & Strip $\# 4^{a}$ \\
\hline \multicolumn{7}{|l|}{ After 5 contacts, scrub, 4 strips } \\
\hline \multicolumn{7}{|l|}{ Wash: $\mathrm{O} / \mathrm{A}=1$} \\
\hline No wash & 18.08 & 2.13 & 0.304 & 0.223 & 0.209 & 0.211 \\
\hline Wash with $3 \mathrm{mM} \mathrm{NaOH}$ & 17.13 & 1.56 & 0.156 & 0.098 & 0.075 & 0.067 \\
\hline Wash with $10 \mathrm{mM} \mathrm{NaOH}$ & 17.45 & 1.60 & 0.154 & 0.096 & 0.073 & 0.059 \\
\hline Wash with $30 \mathrm{mM} \mathrm{NaOH} b$ & 17.51 & 1.60 & 0.156 & 0.083 & 0.114 & 0.052 \\
\hline Wash with $100 \mathrm{mM} \mathrm{NaOH}$ & 17.65 & 1.66 & 0.160 & 0.102 & 0.073 & 0.062 \\
\hline 1 contact (as a comparison) & 16.79 & 1.50 & 0.141 & 0.084 & 0.063 & 0.055 \\
\hline \multicolumn{7}{|l|}{ After 5 contacts, scrub, 4 strips } \\
\hline \multicolumn{7}{|l|}{ Wash with $10 \mathrm{mM} \mathrm{NaOH}$} \\
\hline $\mathrm{O} / \mathrm{A}=2$, gentle rocking, $30 \mathrm{~min}$ & 17.73 & 1.66 & 0.166 & 0.103 & 0.078 & 0.064 \\
\hline $\mathrm{O} / \mathrm{A}=5$, gentle rocking, $30 \mathrm{~min}$ & 19.16 & 1.66 & 0.169 & 0.102 & 0.076 & 0.066 \\
\hline $\mathrm{O} / \mathrm{A}=5$, vortex $10 \mathrm{~s}$ & 18.51 & 1.70 & 0.171 & 0.108 & 0.081 & 0.073 \\
\hline
\end{tabular}

${ }^{a} A$ spike of ${ }^{137} \mathrm{Cs} 0.1 \mu \mathrm{Ci} / \mathrm{mL}$ was added for Strip \#4.

${ }^{b}$ Mistake for Strip \#2: in that case, $\mathrm{O} / \mathrm{A}=2.5$ (and not 5).

Following the $\mathrm{NaOH}$ wash, the tubes were centrifuged $(2200 \mathrm{x}$ g) at 3600 RPM. Emulsion layers were noted with six of the eight samples (T1-4-0, T1-22-0, T3-16-0-S, T3-44-0-S, T3-46-0-S, and T3-34-0-S). All samples were then recentrifuged for $3 \mathrm{~min}$. Emulsion layers persisted in all six tubes that had exhibited them previously, though with a reduction in layer thickness (Table 8.5).

Table 8.5. Volume of persistent emulsion layers in internally irradiated samples following various centrifugations ${ }^{a}$

\begin{tabular}{lcccc}
\hline & \multicolumn{4}{c}{ Approximate emulsion layer volumes (mL) following centrifugation } \\
\cline { 2 - 5 } Sample ID & $\begin{array}{c}\text { After 1st } \\
\text { centrifugation }\end{array}$ & $\begin{array}{c}\text { After 2nd } \\
\text { centrifugation }\end{array}$ & $\begin{array}{c}\text { Following 3rd } \\
\text { centrifugation }\end{array}$ & $\begin{array}{c}\text { Following 4th } \\
\text { centrifugation }\end{array}$ \\
\hline T1-4-0 & 1.25 & 1.0 & 0.9 & 0.75 \\
T1-22-0 & 0.75 & 0.4 & 0.4 & 0.4 \\
T1-12-0 & None & None & None & None \\
T3-16-0-S & 0.25 & 0.25 & 0.25 & 0.25 \\
T3-44-0-S & 4.0 & 3.75 & 3.0 & 2.4 \\
T3-46-0-S & 3.75 & 3.0 & 2.4 & 1.8 \\
T3-34-0-S & 2.0 & 1.15 & 0.8 & 0.6 \\
T3-40-0-S & None & None & None & None \\
\hline
\end{tabular}

${ }^{a}$ Total volume of each sample is approximately $8.6 \mathrm{~mL}$, equally divided between organic and aqueous layers. 
The samples were re-centrifuged a third time for a duration of $6 \mathrm{~min}$. The emulsion layers were still present. The six samples with the persistent emulsion layer were recentrifuged (for 7 min at 2800 RPM, $1791 \times \mathrm{g}$; followed immediately for $7 \mathrm{~min}$ at $3200 \mathrm{RPM}, 2340 \times \mathrm{g}$ ). The emulsion layers, while generally decreasing in size with subsequent centrifugations, still persisted. Solvent that could be isolated from each contact was removed to separate tubes. After two days, no further breakdown of the emulsion layers was noted.

High concentrations of surface-active anionic compounds are thought to be responsible for the persistent emulsion layers. Accordingly, it was decided to wash the solvents with higher ionic-strength $\mathrm{NaOH}$ to remove any bulk surfactant present, then to wash again with $10 \mathrm{mM} \mathrm{NaOH}$.

Sample T3-44-0-S had the largest emulsion layer, and thus it served as the test subject for this expanded test procedure. The solvent that had been removed from the wash sample was recombined with the aqueous $(10 \mathrm{mM} \mathrm{NaOH})$ layer and the emulsion layer. A 384- $\mu \mathrm{L}$ aliquot of $5.6 \mathrm{M} \mathrm{NaOH}$ was then added directly to the aqueous layer effectively increasing the $\mathrm{NaOH}$ concentration from 10 $\mathrm{mM}$ to $0.5 \mathrm{M}$. The tube was shaken ten times, then placed on the wheel at $25{ }^{\circ} \mathrm{C}$ for $50 \mathrm{~min}$. A very clean phase separation with no color or cloudiness at the interface resulted. The phases were segregated, and the organic phase was transferred to a clean tube. To the organic phase was added an equal volume of $10 \mathrm{mM} \mathrm{NaOH}$. The phases were contacted by shaking the tube ten times, then placing it on the wheel at $25^{\circ} \mathrm{C}$ for $50 \mathrm{~min}$. The phases were separated by centrifugation at 3600 RPM $(2200 \times \mathrm{g})$ for $3 \mathrm{~min}$. An emulsion layer was present $(\sim 1.3 \mathrm{~mL})$, but much diminished from the original emulsion layer of $4.0 \mathrm{~mL}$ that was seen after the original washing with $10 \mathrm{mM} \mathrm{NaOH}$. The solvent/emulsion layer was again isolated and washed at an O/A ratio of 1 with $100 \mathrm{mM} \mathrm{NaOH}$. Following centrifugation and phase separation, a slight cloudiness was noted at the interface. Following isolation, the solvent layer was washed with $10 \mathrm{mM} \mathrm{NaOH}$. After centrifugation, the phases separated well with only a slight milkiness noted at the interface. The solvent phase, amounting to $\sim 3.5-3.8 \mathrm{~mL}$, was isolated and recovered.

Suspecting that the principal interfacially active species in these samples is 4-sec-butylphenol (SBP), it was decided to subject the remaining samples to a washing protocol favorable to the removal of SBP. This procedure consists of two contacts with $0.5 \mathrm{M} \mathrm{NaOH}$, one with $0.1 \mathrm{M} \mathrm{NaOH}$, and one with $10 \mathrm{mM} \mathrm{NaOH}$, all at an $\mathrm{O} / \mathrm{A}$ ratio of 1 . This was done uniformly with all samples. Following this procedure, emulsion layers were still noted with three samples, T1-4-0, T3-16-0-S, and T3-46-0-S (see Table 8.6).

Since the emulsion phenomenon seemed to improve with washing, all eight samples were subjected to one final round of washes, but this time to include both acidic and basic washes. All samples were washed twice with $50 \mathrm{mM}$ nitric acid, twice with $0.5 \mathrm{M} \mathrm{NaOH}$, then once with $0.1 \mathrm{M}$ $\mathrm{NaOH}$, and finally once with $10 \mathrm{mM} \mathrm{NaOH}$. Sample T3-44-0-S was subjected to one extra wash with $0.5 \mathrm{M} \mathrm{NaOH}$ so that all samples would have the same number of contacts with concentrated base. Following this process, emulsion layers were found in four samples (see column 2 of Table 8.7). The emulsion layers were further reduced by a very aggressive centrifugation at $2657 \times \mathrm{g}$ for $30 \mathrm{~min}$. The final emulsion layer volumes are shown in column 3 of Table 8.7 below.

\subsubsection{ESS Performance of Washed Internally Irradiated Samples}

Washing and restoring the TOA concentration was successful at nearly equalizing the performance of the $\mathrm{T} 1$ and $\mathrm{T} 3$ samples (Table 8.8). The $\mathrm{T} 1$ samples before washing had not changed much relative to $\mathrm{T} 0$, and thus the potential for improvement by washing was understandably limited. As a consequence, not much improvement was seen, except for perhaps $10 \%$ better stripping for the T1-extract sample (T1-4-0). The T3 samples, and especially the T3-extract sample (T3-16-0-S), gave improved stripping performance upon washing. The most severely degraded sample (T3-16-0-S) behaved like the others after washing, showing that performance can be restored. 
Table 8.6. Observations of internally irradiated samples following wash sequence Twice with $0.5 \mathrm{M} \mathrm{NaOH}$, once with $0.1 \mathrm{M} \mathrm{NaOH}$, and once with $10 \mathrm{mM} \mathrm{NaOH}$

\begin{tabular}{llc}
\hline Sample ID & \multicolumn{1}{c}{$\begin{array}{c}\text { Aqueous-phase color } \\
\text { observations after initial } \\
0.5 \mathrm{M} \mathrm{NaOH} \text { wash }\end{array}$} & $\begin{array}{c}\text { Emulsion-layer volume } \\
(\mathrm{mL}), \text { if present, } \\
\text { following final (10 mM } \\
\mathrm{NaOH}) \text { washing }\end{array}$ \\
\hline T1-4-0 & No color & $\sim 0.5 \mathrm{~mL}$ \\
T1-22-0 & Orange color & $\mathrm{CI}^{a}$ \\
T1-12-0 & Tan color magnitude $3^{b}$ & $\mathrm{SCI}^{c}$ \\
T3-16-0-S & Tan color magnitude 2 & $\sim 1.5 \mathrm{~mL}$ \\
T3-44-0-S & No data & $\mathrm{CI}$ \\
T3-46-0-S & Tan color magnitude 1 & $\sim 1.0 \mathrm{~mL}$ \\
T3-34-0-S & Intense orange color & $\mathrm{CI}$ \\
T3-40-0-S & Tan color magnitude 4 & $\mathrm{SCI}$ \\
\hline
\end{tabular}

${ }^{a} \mathrm{CI}=$ cloudy interface.

$b$ Increasing coloration judged on an arbitrary scale from 1 to 4 . ${ }^{c} \mathrm{SCI}=$ slightly cloudy interface.

${ }^{d}$ T3-44-0-S was used in a previous clean-up trial.

Table 8.7. Emulsion layer characteristics of internally irradiated solvent samples following final wash and centrifugation procedure

\begin{tabular}{|c|c|c|}
\hline \multirow[b]{2}{*}{ Sample ID } & \multicolumn{2}{|c|}{ Selected internally irradiated samples } \\
\hline & $\begin{array}{l}\text { Emulsion layer volume } \\
(\mathrm{mL}) \text { following final } \\
\text { wash procedure }\end{array}$ & $\begin{array}{l}\text { Emulsion layer volume } \\
\text { (mL) following final } \\
\text { aggressive } \\
\text { centrifugation }\end{array}$ \\
\hline $\mathrm{T} 1-4-0$ & $\sim 0.4 \mathrm{~mL}$ & $\sim 0.1 \mathrm{~mL}$ \\
\hline $\mathrm{T} 1-22-0$ & $\mathrm{CI}^{a}$ & None \\
\hline $\mathrm{T} 1-12-0$ & CI & $\mathrm{Cl}$ \\
\hline T3-16-0-S & $\sim 2.6 \mathrm{~mL}$ & $\sim 0.7 \mathrm{~mL}$ \\
\hline T3-44-0-S & $\sim 0.5 \mathrm{~mL}$ & $\sim 0.1 \mathrm{~mL}$ \\
\hline T3-46-0-S & $\sim 1.9 \mathrm{~mL}$ & $\sim 0.4 \mathrm{~mL}$ \\
\hline T3-34-0-S & $\mathrm{SCI}^{b}$ & $\mathrm{SCI}$ \\
\hline T3-40-0-S & $\mathrm{CI}$ & SCI \\
\hline
\end{tabular}




\subsubsection{Solvent Washing of Externally Irradiated Samples}

The two externally irradiated samples from the SRTC tests appear to have degraded over time. Once loaded with cesium in the extraction cycle, solvent ST-16-SB, dating from June 2000, failed to subsequently release its cesium load to either the scrub or strip solutions (Table 8.9). Solvent FD-17SC-6, dating from September 2000, also showed signs of degradation and a diminished capacity to release cesium, as indicated by the high $D_{\mathrm{Cs}}$ values at all process steps. Original ESS data collected on June 26, 2000, for ST-16-SB and October 20, 2000, for FD-17-SC-6 are included in Table 8.9 for comparison purposes.

Table 8.8. Summary of the effectiveness of washing on cesium distribution ratios of selected solvent samples from ORNL internal-irradiation tests

\begin{tabular}{lcccccccc}
\hline & \multicolumn{7}{c}{$D_{\mathrm{Cs}}$ by identifying sample number } \\
\cline { 2 - 9 } Process step & T1-4-0 & T1-12-0 & T1-22-0 & T3-16-0-S & T3-34-0-S & T3-40-0-S & T3-44-0-S & T3-46-0-S \\
\hline Extraction & 22.07 & 20.89 & 20.75 & 21.98 & 22 & 20.95 & 20.26 & 20.74 \\
Scrub & 2.064 & 2.057 & 1.999 & 2.016 & 2.247 & 2.033 & 1.855 & 1.795 \\
Strip 1 & $0.22 C$ & 0.219 & 0.218 & 0.222 & 0.231 & 0.228 & 0.192 & 0.193 \\
Strip 2 & 0.132 & 0.126 & 0.131 & 0.125 & 0.135 & 0.131 & 0.114 & 0.109 \\
Strip 3 & 0.111 & 0.096 & 0.099 & 0.098 & 0.105 & 0.104 & 0.094 & 0.085 \\
\hline
\end{tabular}

Table 8.9. Extraction, scrub, and strip results for externally irradiated samples ${ }^{a}$

\begin{tabular}{|c|c|c|c|c|}
\hline \multirow[b]{2}{*}{ Process step } & \multicolumn{2}{|c|}{$\begin{array}{l}\text { Cesium distribution values obtained } \\
\text { post-wash }\end{array}$} & \multicolumn{2}{|c|}{ Original $D_{\mathrm{Cs}}$ values } \\
\hline & $\begin{array}{l}\text { ST-16-SB } \\
(3 / 22 / 01)\end{array}$ & $\begin{array}{c}\text { FD-17-SC-6 } \\
(3 / 22 / 01)\end{array}$ & $\begin{array}{l}\text { ST-16-SB } \\
(6 / 26 / 00)\end{array}$ & $\begin{array}{l}\text { FD-17-SC-6 } \\
(10 / 20 / 00)\end{array}$ \\
\hline Extraction & 21.86 & 28.49 & & \\
\hline Scrub & 16.42 & 5.78 & & 1.56 \\
\hline Strip 1 & 12.06 & 2.73 & 2.74 & 0.209 \\
\hline Strip 2 & 23.73 & 1.17 & 0.365 & 0.123 \\
\hline 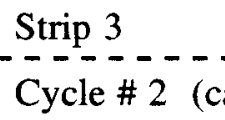 & out with $6 / 26 /$ & $\begin{array}{c}0.57 \\
-0 / 20 / 00 \text { samples }\end{array}$ & 0.181 & $\begin{array}{r}0.087 \\
-----\end{array}$ \\
\hline Extraction & & & 18.1 & 21.58 \\
\hline Scrub & & & 1.838 & 1.89 \\
\hline Strip 1 & & & 0.547 & 0.22 \\
\hline Strip 2 & & & 0.224 & 0.25 \\
\hline Strip 3 & & & 0.235 & 0.17 \\
\hline
\end{tabular}

${ }^{a}$ Original values are compared with those obtained after standing at room temperature for 6 months (FD-17-SC-6) and 9 months (ST-16-SB). 


\subsubsection{Solvent Washing of 235-Day Thermal-Stability Samples}

ESS performance data collected for the washed 235-day thermal-stability scrub and strip samples (designated post-wash) were compared with the data set collected prior to washing at the end of the 235-day experiment (2-20-01). Samples treated at $35{ }^{\circ} \mathrm{C}$ had not originally shown severe degradation, and thus only slight improvement in performance upon washing could be expected, as was observed (Strip \#1). More significant improvement in stripping $D_{\mathrm{Cs}}$ values was seen upon washing of the solvents treated at $61{ }^{\circ} \mathrm{C}$.

Table 8.10. ESS performance of 235-day thermal-stability test samples before and after washing protocol

\begin{tabular}{|c|c|c|c|c|}
\hline \multirow[b]{3}{*}{ Process step } & \multicolumn{4}{|c|}{ Scrub and strip samples at $35^{\circ} \mathrm{C}$} \\
\hline & \multicolumn{2}{|c|}{ ST-35-33 } & \multicolumn{2}{|c|}{ SC-35-33 } \\
\hline & Pre-wash $D_{\mathrm{Cs}}$ & Post-wash $D_{\mathrm{Cs}}$ & Pre-wash $D_{\mathrm{Cs}}$ & Post-wash $D_{\mathrm{Cs}}$ \\
\hline Extraction & 18.6 & 18.7 & 15.3 & 18.99 \\
\hline Scrub & 1.93 & 1.58 & 2.29 & 2.20 \\
\hline Strip \#1 & 0.194 & 0.158 & 0.148 & 0.122 \\
\hline Strip \#2 & 0.092 & 0.092 & 0.086 & 0.069 \\
\hline \multirow{3}{*}{ Strip \#3 } & 0.065 & 0.073 & 0.069 & 0.069 \\
\hline & \multicolumn{4}{|c|}{ Scrub and strip samples at $60^{\circ} \mathrm{C}$} \\
\hline & \multicolumn{2}{|c|}{ ST-60-33 } & \multicolumn{2}{|c|}{ SC-60-33 } \\
\hline Process step & Pre-Wash $D_{\mathrm{Cs}}$ & Post-Wash $D_{\mathrm{Cs}}$ & Pre-Wash $D_{\mathrm{Cs}}$ & Post-Wash $D_{\mathrm{Cs}}$ \\
\hline Extraction & 18.0 & 18.71 & 16.7 & 18.57 \\
\hline Scrub & 1.86 & 1.58 & 1.98 & 1.51 \\
\hline Strip \#1 & 0.249 & 0.177 & 0.197 & 0.163 \\
\hline Strip \#2 & 0.102 & 0.093 & 0.119 & 0.103 \\
\hline Strip \#3 & 0.074 & 0.074 & 0.129 & 0.086 \\
\hline
\end{tabular}

\subsubsection{ESS Performance of Solvents Used in Contactor Tests}

Five solvent samples were received from collaborators at ANL and ORNL. The ANL solvent was used in the CS25 32-stage proof-of-concept test using full simulant. ${ }^{44}$ The ORNL solvent had been used in contactor-efficiency tests. ${ }^{45}$ The results of this ESS series (Table 8.11) show no significant difference in ${ }^{137} \mathrm{Cs}$ distribution ratios for any of the solvents. All five treated solvents (ANL sample; ORNL pretreatment; ORNL post $\mathrm{NaOH}$ wash; ORNL post $\mathrm{HNO}_{3}$ wash; and ORNL $\mathrm{H}_{2} \mathrm{O}$ washed) did show slightly elevated $D_{\mathrm{Cs}}$ values for all stages of the ESS protocol when compared to the pristine solvent. See below for discussion of Strip \#3. 
Table 8.11. Summary of effects of solvent washing upon $D_{\mathrm{Cs}}$ values in solvents used in contactor tests

\begin{tabular}{lcccccc}
\hline & \multicolumn{6}{c}{ Cesium distribution $D_{\mathrm{Cs}}$ in the ESS Protocol } \\
\cline { 2 - 7 } & $\begin{array}{c}\text { Pristine } \\
\text { solvent }\end{array}$ & Solvent & $\begin{array}{c}\text { ORNL } \\
\text { solvent } \\
\text { pretreatment }\end{array}$ & $\begin{array}{c}\text { ORNL } \\
\text { solvent } \\
\text { post-NaOH }\end{array}$ & $\begin{array}{c}\text { ORNL } \\
\text { solvent } \\
\text { post HNO }\end{array}$ & $\begin{array}{c}\text { ORNL } \\
\text { solvent } \\
\text { post } \mathrm{H}_{2} \text { O wash }\end{array}$ \\
\hline Extraction & 16.8 & 17.6 & 17.1 & 19.0 & 19.2 & 19.7 \\
Scrub & 1.47 & 1.58 & 1.65 & 1.63 & 1.78 & 1.81 \\
Strip \#1 & 0.130 & 0.1479 & 0.1699 & 0.1608 & 0.162 & 0.158 \\
Strip \#2 & 0.078 & 0.086 & 0.103 & 0.097 & 0.094 & 0.092 \\
Strip \#3 & 0.172 & 0.222 & 0.281 & 0.278 & 0.186 & 0.227 \\
Strip \#4 & 0.057 & 0.069 & 0.078 & 0.075 & 0.066 & 0.067 \\
\hline
\end{tabular}

Upon examination of the data collected from the ESS experiments (Table 8.11), it was very apparent that something was wrong with the data collected from Strip \#3. For all solvents tested, including the pristine solvent, the distribution coefficients were seen to increase for Strip \#3. A fourth strip was undertaken using $0.15 \mu \mathrm{Ci}{ }^{137} \mathrm{Cs} / \mathrm{mL}$ in $1 \mathrm{mM} \mathrm{HNO}_{3}$. Values of $D_{\mathrm{Cs}}$ resulting from Strip \#4 were much lower and in the range expected. An obvious conclusion drawn from the results from Strip \#3 would be that equilibrium was not reached in the 1-h contact time on the rotating wheel. Strip \#3 was carried out in a $5-\mathrm{mL}$ polypropylene tube, whereas Strips \#1 and \#2 were performed in $15-\mathrm{mL}$ tubes. While the headspace in the $5-\mathrm{mL}$ tube was $28 \%$ of its capacity, equilibrium was not achieved during the 1-h end-over-end contacting period. It would seem prudent that, if the smaller capacity, smaller dimensioned tubes are to be used, they should be shaken vigorously prior to placement on the wheel.

\subsection{CONCLUSIONS}

As demonstrated in this chapter, the ability of solvent-cleanup methods to restore solvent performance after solvent degradation further reduces technical risk associated with solvent thermal and radiolytic stability. Data from Chapters 5 and 6 show that thermal and radiolytic degradation processes are minor relative to the goal of a 1-year solvent lifetime. However, with the equivalent of many years of thermal and radiolytic degradation, solvent performance eventually becomes problematic, as correlated with loss of TOA and buildup of identified products such as 4-secbutylphenol. In the case of the most severely degraded samples from the thermal-stability and internal-irradiation tests, it was possible to demonstrate recovery of solvent extraction, scrubbing, and stripping performance after $\mathrm{NaOH}$ washing and replenishment of the TOA concentration. Results were not all completely satisfactory. Emulsion formation during washing with $10 \mathrm{mM} \mathrm{NaOH}$ was encountered with some of the washed internally irradiated samples, a reflection of the low ionic strength of the wash solution combined with buildup of interfacially-active impurities. Although the emulsion tendency was reduced, it was not completely eliminated upon washing, but it was not a difficulty at higher $\mathrm{NaOH}$ concentrations. The most severely degraded externally irradiated samples could not be cleaned up, and in fact, these samples exhibited signs of further deterioration upon storage.

The baseline flowsheet specifies both an alkaline and an acid wash. Data to date show the sufficiency of an alkaline wash, leaving little benefit to be demonstrated for an acid wash. Although an acid wash could later be shown to be beneficial, it is not clear on empirical or technical grounds what solvent impurities would be removed by aqueous acid nor how to choose the appropriate acid and concentration. On the other hand, batch tests clearly show the restoration of stripping performance on washing the solvent with $0.003-0.1 \mathrm{M} \mathrm{NaOH}$ solutions following contact of the 
solvent multiple times with the full simulant. Analytical experiments have identified dibutylphosphate (DBP) in the solvent after contact with the full simulant; performance tests show that DBP causes impaired performance; and partitioning experiments show that DBP is efficiently removed from the solvent by washing with $0.01-1 \mathrm{M} \mathrm{NaOH}$ solutions. Similarly, 4-sec-butylphenol, an impurity in the modifier and also a degradation product, has a deleterious effect on stripping performance and is washed from the solvent by $\mathrm{NaOH}$ solutions. Surfactant impurities having 12 aliphatic carbons or less are also efficiently removed by $\mathrm{NaOH}$ washing, and the most effective $\mathrm{NaOH}$ concentrations lie in the range $0.001-0.01 \mathrm{M}$. Although such surfactants are not reported to be waste constituents, their presence in trace amounts could still lead to their concentration in the solvent and subsequent stripping impairment, as they would not be washed out into the raffinate. Fortunately, the ANL proof-of-concept experiment $(\mathrm{CS} 25)^{44}$ did not show signs of degraded stripping impairment, implying the lack of significant minor-component buildup. Although this result suggests that a wash step might not be needed (at least for a few cycles), it is not sufficient for complete confidence that minor components would not build up in the solvent over many more cycles with real waste, or even with simulant. Hence, an alkaline wash was judged a prudent measure of insurance that anionic minor components would not put the critical real-waste contactor test at risk. With implementation of a wash stage using $10 \mathrm{mM} \mathrm{NaOH}$ at $\mathrm{O} / \mathrm{A}=5$, the multi-day contactor tests at $\mathrm{ANL}^{67}$ with simulant and the SRTC with real waste 68 in fact encountered no phenomena that indicated possible buildup and interference by minor components. 


\section{CONCLUSIONS AND PATH FORWARD}

\subsection{TECHNOLOGY RISKS ADDRESSED}

The overall conclusion of these studies is that the CSSX solvent meets all of the performance requirements within the bounding conditions defined by the Savannah River Site. High technology risks that have been addressed relating to successful implementation of the CSSX process include: (1) chemical stability of the solvent matrix, (2) radiolytic stability of the solvent matrix, (3) proof-ofconcept performance of the proposed process flowsheet with simulated waste, and (4) performance of the CSSX flowsheet with actual SRS high-level waste.

The risk associated with chemical and thermal stability was the primary subject matter of this report. These studies show that chemical and thermal degradation under the operating conditions of the process is slow relative to the goal of one annual solvent replacement. In fact, extrapolation of the data implies that adequate performance can continue for 15 years without solvent cleanup. The chemical consequences of degradation were understood mainly in terms of loss of the TOA solvent component. With restoration of the TOA and solvent washing, even severely degraded solvent was restored to good performance. Moreover, the trace products that were detected are not harmful to the process and will wash out of the solvent, into either the process effluents or the added wash stages.

Solvent integrity with regard to component solubility loss to the aqueous phase, resistance to precipitation of solids or third-phases, and susceptibility to impurity effects is good with implementation of temperature management and solvent washing. The solvent is, in fact, robust to a wide variation in feed composition and performs satisfactorily at the bounding concentrations of organic compounds and competing potassium ions in the waste. Solubility phenomena will dictate solvent optimization to move the solvent out of the condition of supersaturation with regard to BOBCalixC6. This will be readily accomplished by raising the modifier concentration, which also provides other benefits, such as lowering the operating temperature and reducing reagent costs. Thus, the findings on solubility do not effectively pose unexpected risk factors. Impurity effects, particularly with regard to lipophilic anions, were recognized during CSSX development and are successfully dealt with by the TOA suppressor component and use of a $\mathrm{NaOH}$ wash, both of which were already implemented in the baseline flowsheet. Accordingly, the data presented here serve to quantify these effects and to provide the basis for rational solvent cleanup. As a backup solventcleanup technique, resin anion exchange was shown to remove the most difficult lipophilic anions, thus providing further reduction in risk.

This work also supported other tasks in addressing the radiation-stability risk. Specifically, analytical and performance data showed that the solvent can withstand the dose equivalent of several years without the need for solvent washing. Moreover, the most severely degraded solvent samples in the internal-irradiation test at ORNL were successfully cleaned up and performance restored. However, the same could not be said for 16-Mrad (170-year dose) externally-irradiated solvent samples from the SRTC, which were not successfully cleaned up.

Finally, this task provided batch distribution data and solvent-washing data that were critical in designing the proof-of-concept and real-waste tests. These data supported implementation of a caustic wash stage using $10 \mathrm{mM} \mathrm{NaOH}$ at an O/A ratio of 5 .

Overall, the data imply that the chemical- and thermal-stability risk for implementation of the CSSX process is low. Given future solvent optimization, no major issues were identified that cannot be dealt with outside normal engineering practice with regards to flow-rate adjustment, temperature management, and possibly feed blending. In fact, some opportunities to gain major savings in footprint are within the realm of possibility if actinide and strontium removal is moved from an upstream to a downstream operation, where it can be accomplished without heavy shielding. Distribution data suggest that actinides and strontium are not significantly extracted and do not interfere with CSSX performance. 


\subsection{QUESTIONS REMAINING AND SUGGESTIONS FOR FUTURE WORK}

\subsubsection{Summary Remarks}

The results of this extensive report provide a good understanding of the factors that influence cesium distribution behavior in CSSX, leading to a basis for predicting and controlling flowsheet behavior under varying conditions. The manner in which key system components, including inorganic and organic species, distribute between the solvent and the process aqueous phases has been described. As a result, the nature of the aqueous effluent streams is reasonably well characterized.

Not all chemical phenomena are completely understood, and further work is suggested to improve the foundation for both better predictability of flowsheet behavior and improving the process. Discussed below are some areas for future tests and development as an outgrowth of this report specifically. The reader is also referred to Chap. 9 of the R\&D Plan, ${ }^{4}$ which lays out a broad scope of future work related to CSSX.

\subsubsection{Solvent Optimization}

No thermal or radiolytic stability issue provides incentive to question the choice of extractant, modifier, suppressor, or diluent components in the baseline solvent. Stability is excellent, and the trace breakdown products identified will leave the system, with or without solvent washing. However, the supersaturation of the solvent with respect to BOBCalixC6 makes it necessary to consider adjustment of component concentrations. The most direct approach is to raise the modifier concentration, shown in Chap. 7 to raise the BOBCalixC6 solubility. At the same time, the BOBCalixC6 concentration can be decreased, and the large cost of the extractant relative to all other solvent components therefore implies direct cost savings. In addition, the operating temperature of the process can likely be decreased from $20^{\circ} \mathrm{C}$ to $15^{\circ} \mathrm{C}$, owing to increased resistance to third-phase formation. BOBCalixC6 solubility studies should be extended to a range of temperatures and a range of concentrations of the other components, primarily the modifier. The question of the true equilibrium solubility as attained in the forward and reverse directions needs to be resolved. Increasing the TOA concentration would also be beneficial, as the bounding concentration of dibutylphosphate in the waste saturates the ability of the TOA to neutralize its effect. Hence, stripping could be improved (lower $D_{\mathrm{Cs}}$ values). Alternatives to raising the modifier concentration are more substantial but are still within the realm of standard solvent-extraction practice. These alternatives would include testing other diluents, especially aliphatic ones, whose structures might provide the needed slight boost in solvation. In addition, the structure of the aliphatic substituents on the benzo groups of the BOBCalixC6 could be modified with likely no effect on any system property other than the extractant solubility.

\subsubsection{Actinide and Strontium Extraction}

As pointed out in the R\&D plan, a significant opportunity to reduce the overall shielded footprint exists in moving actinide and strontium removal downstream of CSSX. A more detailed actinide study is warranted, in which the oxidation state of the actinides is better controlled. Tolerance of the CSSX flowsheet to solids in the feed is also a major issue, as addressed in the R\&D plan. Another opportunity for major savings should be examined in that perhaps CSSX could be modified so as to extract the actinides and strontium itself. This challenging chemical problem would entail addition of other extractants to the solvent matrix, with likely consequences related to the needed cesium extraction, scrubbing, and stripping performance.

\subsubsection{Radiation Stability}

Although the solvent exhibits excellent radiation stability, some questions have arisen from the current studies. For example, it would be prudent to confirm and understand the observation in Chap. 8 that the performance of externally irradiated solvent degrades further upon storage of the 
solvent for several months. The ultimate fate of dioctylamine and 4-sec-butylphenol in a static system is also of interest, though mostly for chemical understanding, as these degradation products will leave the system in aqueous effluent streams.

\subsubsection{Thermal Stability}

The current study has provided detailed knowledge of thermal stability, revealing that the solvent possesses high stability, but when subjected to high temperatures under acidic conditions the solvent begins to suffer loss of TOA. The main degradation product is dioctylamine. It would be worthwhile to understand the chemical mechanism of breakdown of TOA and the ultimate fate of the more reactive dioctylamine.

\subsubsection{Solvent Cleanup}

Investigation of alternative cleanup approaches to augment the $\mathrm{NaOH}$ washing step would be prudent, as the possibility remains that lipophilic anions that do not wash out well in the alkaline wash could build up over time and overwhelm the solvent tolerance for them. Anion exchange is an effective current backup, and it is recommended to develop this technology further. In addition, perhaps alternative certain aqueous washes might be effective.

\subsubsection{Minor Species}

Solvents from the multi-day contactor tests at ANL and the SRTC should be carefully analyzed to determine what minor species have built up in the solvent. Electrospray mass spectrometry revealed traces of unknown cationic and anionic species in the solvent after multiple contacts with the full simulant. It would be prudent to identify them and determine if they continue to build up over time.

\subsubsection{Fate of Trimethylamine}

The feedback loop between extraction and scrubbing is predicted to trap trimethylamine in flowsheet stages \#15 and \#16. It will likely be lost to volatilization and has not been observed to cause any performance issues. However, its effect at high concentrations needs to be better understood and its fate precisely determined.

\subsubsection{Role of Nitrite}

This anion is abundant in the waste and contributes significantly to the driving force of extraction after nitrate and hydroxide. It is thus one of the counteranions for cesium extraction and is carried over into scrubbing. Under the acidic conditions of scrubbing, it is converted to nitrous acid, a reactive species. Evidence in Chap. 3 shows that very high nitrite concentration in the feed starts to be felt in terms of hindered stripping, and this question needs to be understood. The particular form of nitrite in the solvent is not clear, as deliberate addition of nitrite to the scrubbing stage has no effect on either scrubbing or stripping. Thus, a study directed specifically at the role of nitrite is needed.

\subsubsection{Modeling}

The model developed elsewhere ${ }^{25}$ needs to be expanded to include explicit incorporation of temperature effects, Pitzer mixing parameters, and effects of minor species. The model also needs to be incorporated into flowsheet calculations. 


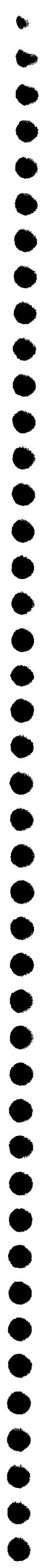




\section{REFERENCES}

1. National Research Council, Long-Term Research Needs for High-Level Waste at Department of Energy Sites: Interim Report, Washington, DC, 2000, http://www.nap.edu/catalog/9992.html.

2. USDOE Office of Environmental Management, Program Announcement To DOE National Laboratories, LAB 01-16, Environmental Management Science Program: Basic Science Research Related to High Level Radioactive Waste, Washington, DC, 2001, http://emsp.em.doe.gov/announcements.htm; see also http://www.pnl.gov/tfa/emsp/needs.stm.

3. D. D. Walker, M. J. Barnes, C. L. Crawford, R. A. Peterson, R. F. Swingle, and S. D. Fink, "InTank Precipitation with Tetraphenylborate: Recent Progress and Research Results," pp. 219-230 in Science and Technology for Disposal of Radioactive Tank Wastes, eds. W. W. Schultz and N. J. Lombardo, Plenum Press, New York, 1998.

4. H. Harmon, S. Schlahta, J. Walker, D. Wester, K. Rueter, and S. Fink, Tanks Focus Area, Savannah River Site Salt Processing Project, Research and Development Program Plan, PNNL13253, Rev. 1, Pacific Northwest National Laboratory, Richland, WA, November 2000.

5. U.S. Department of Energy, Independent Assessment of the Savannah River Site High-Level Waste Salt Disposition Alternatives Evaluation, DOE/ID-10672, Idaho Falls, ID, December 1998.

6. B. A. Moyer, P. V. Bonnesen, R. A. Sachleben, and D. J. Presley, "Solvent and Process for Extracting Cesium from Alkaline Waste Solutions," U.S. Patent 6,174,503, January 16, 2001.

7. Z. Asfari, S. Wenger, and J. Vicens, "Calixcrowns and Related Molecules." J. Inclusion Phenom. Mol. Recognit. Chem. 19, 137-148 (1994).

8. P. V. Bonnesen, L. H. Delmau, T. J. Haverlock, and B. A. Moyer, Alkaline-Side Extraction of Cesium from Savannah River Tank Waste Using Calixarene-Crown Ether Extractant, ORNL/TM13704, Oak Ridge National Laboratory, Oak Ridge, TN, 1998.

9. B. A. Moyer, P. V. Bonnesen. L. H. Delmau, T. J. Haverlock, R. A. Sachleben, R. A. Leonard, C. Conner, and G. J. Lumetta, "Solvent Extraction of Tc and Cs from Alkaline Nitrate Wastes," Proceedings of the International Solvent Extraction Conference (ISEC '99), Barcelona, Spain, July 11-16, 1999, vol. 2, pp. 1365-1369.

10. R. A. Leonard, C. Conner, M. W. Liberatore, J. Sedlet, S. B. Aase, and G. F. Vandegrift, Evaluation of an Alkaline-Side Solvent Extraction Process for Cesium Removal from SRS Tank Waste Using Laboratory-Scale Centrifugal Contactors, ANL-99/14, Argonne National Laboratory, Argonne, IL, August, 1999.

11. R. A. Leonard, C. Conner, M. W. Liberatore, J. Sedlet, S. B. Aase, G. F. Vandegrift, L. H. Delmau, P. V. Bonnesen, and B. A. Moyer, "Development of a Solvent Extraction Process for Cesium Removal from SRS Tank Waste," Sep. Sci. Technol. 35(5\&6), 743-766 (2001).

12. S. F. Piccolo, F. R. Fowler, R. A. Jacobs, P. L. Rutland, and R. A. Peterson, Bases, Assumptions, and Results of the Flowsheet Calculations for the Short List Disposition Alternatives, WSRC-RP-98-00168, Westinghouse Savannah River Company, Aiken, SC, October 29, 1998.

13. J. Barnes, Decision Phase Final Report, WSRC-RP-99-00007, Rev. 0, Westinghouse Savannah 
River Company, Aiken, SC, November 1, 1999.

14. L. H. Delmau, G. J. Van Berkel, P. V. Bonnesen, and B. A. Moyer, Improved Performance of the Alkaline-Side CSEX Process for Cesium Extraction from Alkaline High-Level Waste Obtained by Characterization of the Effect of Surfactant Impurities, ORNL/TM-1999/209, Oak Ridge National Laboratory, Oak Ridge, TN, October 1999.

15. P. V. Bonnesen, L. H. Delmau, B. A. Moyer, and R. A. Leonard, "A Robust Alkaline-Side CSEX Solvent Suitable for Removing Cesium from Savannah River High Level Waste," Solvent Extr. Ion Exch. 18(6), 1079-1107 (2000).

16. U.S. Department of Energy, Independent Assessment of the Savannah River Site High-Level Waste Salt Disposition Alternatives Evaluation-Phase IV, DOE/ID-10716, Idaho Falls, ID, November, 1999.

17. National Research Council, Interim Report-Committee on Cesium Processing Alternatives for High-Level Waste Salt Processing at the Savannah River Site, letter report to E. J. Moniz, U.S. Department of Energy, Washington, DC, October 14, 1999.

18. National Research Council, Committee on Cesium Processing Alternatives for High-Level Waste Salt Processing at the Savannah River Site, Alternatives for High-Level Waste Salt Processing at the Savannah River Site, National Academy Press, Washington, D.C., 2000.

19. Westinghouse Savannah River Company, Science and Technology Work Scope Matrix for Caustic-Side Solvent Extraction (Demonstration Phase), HLW-SDT-2000-00051, Rev. 4, Westinghouse Savannah River Company, Aiken, SC, November 9, 2000.

20. R. A. Dimenna, H. H. Elder, J. R. Fowler, R. C. Fowler, M. V. Gregory, T. Hang, R. A. Jacobs, P. K. Paul, J. A. Pike, P. L. Rutland, F. G. Smith III, S. G. Subosits, G. A. Taylor and S. G. Campbell, Bases, Assumptions, and Results of the Flowsheet Calculations for the Decision Phase Salt Disposition Alternatives, WSRC-RP-99-00006, Rev. 1, Westinghouse Savannah River Company, Aiken, SC, October 2000.

21. R. A. Peterson, Preparation of Simulated Waste Solutions for Solvent Extraction Testing, WSRC-RP-2000-00361, Westinghouse Savannah River Company, Aiken, SC, May 1, 2000.

22. P. L. Rutland, Savannah River Site High Level Waste Salt Disposition Process Engineering Position Paper on the Simulant for the Caustic Side Solvent Extraction Research and Development, HLW-SDT-2000-00134, Westinghouse Savannah River Company, Aiken, SC, May 2, 2000.

23. G. M. Ritcey and A. W. Ashbrook, Solvent Extraction, Principles and Applications to Process Metallurgy, part I, Elsevier, New York, 1984; part II, 1980.

24. J. Rydberg, C. Musikas, and G. R. Choppin, eds., Principles and Practices of Solvent Extraction, Marcel Dekker, New York, 1992.

25. L. H. Delmau, T. J. Haverlock, T. G. Levitskaia, F. V. Sloop, Jr., and B. A. Moyer, CausticSide Solvent Extraction Chemical and Physical Properties: Equilibrium Modeling of Distribution Behavior, ORNL/TM-2001/267, Oak Ridge National Laboratory, Oak Ridge, TN, December 2001; interim report issued April 16, 2001 as CERS/SR/SX/018, Rev. 0.

26. M. P. Maskarinec, J. E. Caton, Jr., and T. L. White, Analytical Methods Development in Support of the Caustic-Side Solvent Extraction System, ORNL/TM-2001/130, Oak Ridge National Laboratory, Oak Ridge, TN, April 2001. 
27. T. J. Haverlock, P. V. Bonnesen, R. A. Sachleben, and B. A. Moyer, "Analysis of Equilibria in the Extraction of Cesium Nitrate by Calix[4]arene-bis(t-octylbenzo-crown-6) in 1,2Dichloroethane," J. Incl. Phenom. Mol. Recognit. Chem. 36, 21-37 (2000).

28. R. Ungaro, A. Casnati, F. Ugozzoli, A. Pochini, J.-F. Dozol, C. Hill, and H. Rouquette, "1,3Dialkoxycalix [4]arenecrowns-6 in 1,3-Alternate Conformation: Cesium-Selective Ligands that Exploit Cation-Arene Interactions," Angew. Chem., Int. Ed. Engl. 33, 1506-1509 (1994).

29. J.-F. Dozol, J. Casas, and A. M. Sastre, "Transport of Cesium from Reprocessing Concentrate Solutions Through Flat-Sheet-Supported Liquid Membranes: Influence of the Extractant," Sep. Sci. Technol. 30, 435-48 (1995).

30. J.-F. Dozol, N. Simon, V. Lamare, H. Rouquette, S. Eymard, B. Tournois, D. De Marc, and R. M. Macias, "A Solution for Cesium Removal from High Salinity Acidic or Alkaline Liquid Waste: The Crown Calix[4]arenes," Sep. Sci. Technol. 34, 877-909 (1999).

31. R. A. Sachleben, P. V. Bonnesen, T. Descazeaud, T. J. Haverlock, A. Urvoas, and B. A. Moyer, "Surveying the Extraction of Cesium Nitrate by 1,3-Alternate Calix[4]arene Crown-6 Ethers in 1,2-Dichloroethane," Solvent Extr. Ion Exch. 17, 1445-1459 (1999).

32. J. C. Bryan and P. V. Bonnesen. unpublished results. August 21, 2000.

33. P. Thuéry, M. Nierlich, J. C. Bryan, V. Lamare, J.-F. Dozol, Z. Asfari, and J. Vicens, "Crown Ether Conformations in 1,3-Calix[4]arene Bis(Crown Ethers): Crystal Structures of a Caesium Complex and Solvent Adducts and Molecular Dynamics Simulations," J. Chem. Soc., Dalton Trans., 4191-4202 (1997).

34. P. V. Bonnesen, L. H. Delmau, B. A. Moyer, and G. J. Lumetta, "Development of Effective Solvent Modifiers for the Solvent Extraction of Cesium from Alkaline High-Level Tank Waste," Solvent Extr. Ion Exch., in preparation.

35. C. R. Duchemin, N. L. Engle, P. V. Bonnesen, T. J. Haverlock, L. H. Delmau, and B. A. Moyer, "Solvatochromic Solvent Polarity Measurements of Alcohol Solvent Modifiers and Correlation with Cesium Extraction Strength," Solvent Extr. Ion Exch. 19, 1037-1058 (2001).

36. L. H. Delmau, P. V. Bonnesen, G. J. Van Berkel, and B. A. Moyer, "A Solution to Stripping Problems Caused by Organophilic Anion Impurities in Crown-Ether-Based Solvent Extraction Systems: A Case Study of Cesium Removal from Radioactive Waste," Hydrometallurgy, submitted for publication (2002).

37. T. J. Keever and P. V. Bonnesen, Method for Evaluating CSSX Solvent Quality, Oak Ridge National Laboratory, CERS/SR/SX/005, Rev. 0, Oak Ridge, TN, June 23, 2000.

38. D. R. Lide, Jr., and H. P. R. Frederikse, CRC Handbook of Chemistry and Physics, 75th ed., CRC Press, Boca Raton, FL, 1994, pp. 15-21.

39. J. A. Dean, Lange's Handbook of Chemistry, 15th ed., McGraw Hill, New York, 1999, p. 11.102 .

40. R. A. Leonard, "Solvent Characterization Using the Dispersion Number," Sep. Sci. Technol. 30(7-9), 1103-1122 (1995).

41. G. D. Kerr, K. F. Eckerman, L. N. Klatt, and B. A. Moyer, Radiation Dosimetry for the CSSX 
Process, Oak Ridge National Laboratory, Oak Ridge, TN, in preparation. G. D. Kerr and K. F. Eckerman, Radiation Dosimetry for the CSSX Process, ORNL letter report to K. J. Rueter, Westinghouse Savannah River Company, October 12, 2000.

42. R. Blackmon, G. Lumetta, M. Hodges, R. Peterson, P. Rutland, R. Leonard, J. D. Law, L. Klatt, B. A. Moyer, T. Todd, A. Delley and M. A. Nadeau, Real Waste Feasibility Study for Caustic Side Solvent Extraction Alternative, HLW-SDT-2000-00251, Rev. 0, Savannah River Site High Level Waste Salt Disposition Program, Westinghouse Savannah River Company, Aiken, SC, July 17, 2000.

43. R. A. Leonard, D. B. Chamberlain, and C. Conner, "Centrifugal Contactors for LaboratoryScale Solvent Extraction Tests," Sep. Sci. Technol. 32, 193-210 (1997).

44. R. A. Leonard, S. B. Aase, H. A. Arafat, C. Conner, J. R. Falkenberg, and G. F. Vandegrift, Proof-of-Concept Flowsheet Tests for Caustic-Side Solvent Extraction of Cesium from Tank Waste, ANL-00/30, Argonne National Laboratory, Argonne, IL, November 2000.

45. J. F. Birdwell, Jr., and K. K. Anderson, Evaluation of 5-cm Centrifugal Contactor Hydraulic and Mass Transfer Performance for Caustic-Side Solvent Extraction of Cesium, Report ORNL/TM2001/137, Oak Ridge National Laboratory, Oak Ridge, TN, August 2001.

46. R. A. Leonard, G. J. Bernstein, A. A. Ziegler, and R. H. Pelto, "Annular Centrifugal Contactors for Solvent Extraction," Sep. Sci. Technol. 15, 925-943 (1980).

47. R. D. Spence, L. N. Klatt, L. H. Delmau, F. V. Sloop, Jr., P. V. Bonnesen, and B. A. Moyer, Batch-Equilibrium Hot-Cell Tests of Caustic-Side Solvent Extraction (CSSX) with SRS Simulant Waste and Internal ${ }^{137}$ Cs Irradiation, ORNL/TM-2001/49, Oak Ridge National Laboratory, Oak Ridge, TN, September 2001.

48. W. J. McDowell, "Crown Ethers as Solvent Extraction Reagents: Where Do We Stand?" Sep. Sci. Technol. 23, 1251-1268 (1988).

49. B. A. Moyer, "Complexation and Transport," pp. 377-416 in Molecular Recognition: Receptors for Cationic Guests, vol. 1, ed. G. W. Gokel, Comprehensive Supramolecular Chemistry, eds. J. L. Atwood, J. E. D. Davies, D. D. MacNicol, F. Vögtle, and J.-M. Lehn, Pergamon, Elsevier, Oxford, 1996, chap. 10.

50. L. H. Delmau, T. J. P. LeFranc, P. V. Bonnesen, J. C. Bryan, and B. A. Moyer, "Modeling of the Extraction of Alkali Metals by a Calix(4)arene Biscrown-6 Ether and Various Modifiers," Solvent Extr. Ion Exch., in preparation.

51. B. A. Moyer, P. V. Bonnesen, C. K. Chambliss, T. J. Haverlock, A. P. Marchand, H.-S. Chong, A. S. McKim, K. Krishnudu, K. S. Ravikumar, V. S. Kumar, and M. Takhi, "Use of CageFunctionalized Macrocycles and Fluorinated Alcohols in the Liquid-Liquid Extraction of $\mathrm{NaOH}$ and Other Sodium Salts. Strategies Toward Waste-Volume Reduction," pp. 114-132 in Nuclear Site Remediation: First Accomplishments of the Environmental Science Program, ACS Symposium Series 778, eds. P. G. Eller and W. R. Heineman, American Chemical Society, Washington, DC, 2001.

52. C. K. Chambliss, T. J. Haverlock, P. V. Bonnesen, N. L. Engle, and B. A. Moyer, "Recovery of Hydroxide from Alkaline Media by Liquid-Liquid Extraction with Weakly Acidic Alcohols," Environ. Sci. Technol., in press (2002).

53. T. G. Levitskaia, B. A. Moyer, P. V. Bonnesen, and A. P. Marchand K. Krishnudu, Z. Chen, Z. Huang, H. G. Kruger, and A. S. McKim, "Novel Approach to Sodium Hydroxide Separation: Synergistic Pseudo-Hydroxide Extraction by a Fluorinated Alcohol and Cage-Functionalized Crown 
Ethers," J. Amer. Chem. Soc. 123, 12099-12100 (2001).

54. P. L. Rutland. Westinghouse Savannah River Company, Aiken, SC, personal communication to B. A. Moyer, Oak Ridge National Laboratory, Oak Ridge, TN, August 2, 2000.

55. R. A. Peterson, T. L. White, S. Crump, and L. H. Delmau, Solvent Extraction External Radiation Stability Testing, WSRC-TR-2000-0413, Rev. 0, Westinghouse Savannah River Company, Aiken, SC, October 30, 2000. R. A. Peterson, T. L. White, and N. E. Gregory, Solvent Extraction External Irradiation Update, SRT-LWP-2000-00101, Westinghouse Savannah River Company, Aiken, SC, June 16, 2000.

56. B. A. Moyer and Y. Sun, "Principles of Solvent Extraction of Alkali Metal Ions. Understanding Factors Leading to Cesium Selectivity in Extraction by Solvation," pp. 295-391 in Ion Exchange and Solvent Extraction, vol. 13, eds. Y. Marcus and J. A. Marinsky, Marcel Dekker, New York, 1997.

57. W. D. Kumler and J. J. Eiler, "The Acid Strength of Mono and Diesters of Phosphonic Acid. The $n$-Alkyl Esters from Methyl to Butyl, the Esters of Biological Importance, and the Natural Guanidine Phosphoric Acids," J. Am. Chem. Soc. 65, 2355-2361 (1943).

58. R. A. Peterson. Westinghouse Savannah River Company, Aiken, SC, personal communication to B. A. Moyer, Oak Ridge National Laboratory, Oak Ridge, TN, November 2000.

59. Y. Marcus and L. E. Asher, "Extraction of Alkali Halides from Their Aqueous Solutions by Crown Ethers," J. Phys. Chem. 82, 1246-1254 (1978).

60. G. Ritcey, "Development of Industrial Solvent Extraction Processes," pp. 449-510 in Principles and Practices of Solvent Extraction, eds. J. Rydberg, C. Musikas, and G. R. Choppin, Marcel Dekker, New York, 1992.

61. R. K. Winge, V. J. Peterson, and V. A. Fassel, "Inductively Coupled Plasma-Atomic Emission Spectroscopy: Prominent Lines," Appl. Spectroscopy 33(3), 206-208 (1979).

62. K. R. Ashley and J. R. Ball, "Sorption Behavior of Pertechnetate on Reillex ${ }^{\mathrm{TM}}-\mathrm{HPQ}$ Anion Exchange Resin from Nitric Acid Solution," Solvent Extr. Ion Exch. 12(2), 239-259 (1994).

63. H. Irving and R. J. P. Williams, "Liquid-Liquid Extraction," chap. 31 in Treatise on Analytical Chemistry, part I, vol. 3, eds. I. M. Kolthoff and P. J. Elving, Interscience, New York, 1961.

64. A. F. D. de Namor, R. M. Cleverley, and M. L. Zapata-Ormachea, "Thermodynamics of Calixarene Chemistry," Chem. Rev. 98, 2495-2526 (1998).

65. P. V. Bonnesen, G. M. Brown, S. D. Alexandratos, L. B. Bavoux, D. J. Presley, V. Patel, R. Ober, and B. A. Moyer, "Development of Bifunctional Anion Exchange Resins with Improved Selectivity and Sorptive Kinetics for Pertechnetate. Batch-Equilibrium Experiments," Environ. Sci. Tcchnol. 34, 3761-3766 (2000).

66. B. C. Challis and A. R. Butler, "Substitution at an Amino Nitrogen," chap. 6 in The Chemistry of the Amino Group, ed. S. Patai, Interscience, New York, 1968.

67. R. A. Leonard, S. B. Aase, H. A. Arafat D. B. Chamberlain, C. Conner, M. C. Regalbuto, and G. F. Vandegrift, Interim Report on a Multi-day Test of the Caustic-Side Solvent Extraction Flowsheet for Cesium Removal from a Simulated SRS Tank Waste, ANL-01/10, Argonne National Laboratory, Argonne, IL, April 2001. 
68. S. G. Campbell, M. W. Geeting, C. W. Kennell, J. D. Law, R. A. Leonard, M. A. Norato, R. A. Pierce, T. A. Todd, D. D. Walker, W. R. Wilmarth, Demonstration of Caustic-Side Solvent Extraction with Savannah River Site-High-Level Waste, WSRC-TR-2001-00223, Rev. 0, Westinghouse Savannah River Company, Aiken, SC, April 19, 2001.

69. B. A. Moyer, Interim Report on Solvent Stability to Thermal Degradation, letter report to K. J. Rueter, Westinghouse Savannah River Company, CERS/SR/SX/004, Rev. 0, Oak Ridge National Laboratory, Oak Ridge, TN, August 15, 2000.

70. P. B. D. De La Mare and J. H. Ridd, Aromatic Substitution: Nitration and Halogenation, Academic Press, New York, 1959.

71. F. Baroncelli, G. Grossi, and A. Novegno, "Degradation des Amines Tertiaires Aliphatiques a Longue Chaine par l'Acide Nitrique et sous l'Influence des Radiations. I. Irradiation par les Rayons U.V.," Radiochim. Acta. 7(2/3), 135-141 (1967).

72. R. A. Peterson, C. L. Crawford, F. F. Fondeur, and T. L. White, "Radiation Stability of Calixarene-Based Solvent System," pp. 45-55 in Calixarenes for Separations, eds. G. J. Lumetta, R. D. Rogers, and A. S. Gopalan, ACS Symposium Series 757, American Chemical Society, Washington, DC, 1999.

73. A. W. Adamson, Physical Chemistry of Surfaces, Wiley-Interscience, New York, 1990, p. 21.

74. A. E. Alexander and J. B. Hayter, Determination of Surface and Interfacial Tension, p. 501 in Physical Methods of Chemistry, vol I, part V, eds., A. Weissberger and B. W. Rossiter, WileyInterscience, New York, 1971.

75. N. B. Vargaftik, B. N. Volkov, and L. D. Voljak, "International Tables of the Surface Tension of Water," J. Phys. Chem. Ref. Data 12, 817 (1983).

76. J. T. Davies and E. K. Rideal, Interfacial Phenomena, Academic Press, New York, 1961, p. 17.

77. Y. Marcus and A. S. Kertes, Ion Exchange and Solvent Extraction of Metal Complexes, Wiley Interscience, New York, 1969.

78. B. A. Moyer and W. J. McDowell, "Characterization of Molybdenum Interfacial Crud in a Uranium Mill that Employs Tertiary Amine Solvent Extraction," pp. 503-516 in Hydrometallurgy: Research, Development, and Plant Practice, eds. K. Osseo-Asare and J. D. Miller, The Metallurgical Soc. of AIME, New York (1983).

79. B. A. Moyer, C. O. Price, and W. J. McDowell, "Alkane-Insoluble Trialkylammonium Double Salts Involving the Dodecamolybdophosphate Anion. I. Model Studies Using Trioctylamine in Dodecanol-Modified Nonane," Hydrometallurgy 16, 177-195 (1986).

80. B. A. Moyer and W. J. McDowell, "Alkane-Insoluble Trialkylammonium Double Salts Involving the Dodecamolybdophosphate Anion. II. Effect of Amine Structure on Third Phase Formation," Sep. Sci. Technol. 22, 417-445 (1987). 


\section{INTERNAL DISTRIBUTION}

1. J. F. Birdwell, Jr., 7601, MS-6305; birdwelljfjr@ornl.gov

2. P. V. Bonnesen, 4500S, MS-6119; bonnesenpvornl.gov

3. L. H. Delmau, 4500S, MS-6119; delmaulh@ornl.gov

4. R. T. Jubin, 4501, MS-6223; letourneauva@ornl.gov

5. T. J. Keever, 4500S, MS-6119; keevertj@ornl.gov

6. T. G. Levitskaia, 4500S, MS-6119; levitskaiatg@ornl.gov

7-16. B. A. Moyer, 4500S, MS-6119; moyerba@ornl.gov

17. F. V. Sloop, Jr., 4500S, MS-6119; sloopfvjr@ornl.gov

18. ORNL Central Research Library

19. Laboratory Records, RC

20. DOE Office of Scientific and Technical Information (OSTI)

\section{EXTERNAL DISTRIBUTION}

21. S. D. Alexandratos, Office of Academic Affairs, City University of New York, 535 East 80th Street, New York, NY 10021; sdabh@cunyvm.cuny.edu

22. J. T. Carter, Westinghouse Savannah River Company, P.O. Box 616, Buidling 766-H, Aiken, SC 29808; joe.carter@srs.gov

23. S. D. Fink, Westinghouse Savannah River Company, P.O. Box 616, Building 773-A, Aiken, SC 29808; samuel.fink@srs.gov

24. H. D. Harmon, Tank Focus Area Salt Processing Program, P.O. Box 616, Building 704-3N, Aiken, SC 29808; harry.harmon@srs.gov

25. R. T. Jones, Westinghouse Savannah River Company, P.O. Box 616, Building 704-3N, Aiken, SC 29808; robertted.Jones@srs.gov

26. R. A. Leonard, Argonne National Laboratory, Building 205, 9700 South Cass Avenue, Argonne, IL 60439; leonard@cmt.anl.gov

27. R. Leugemors, Pacific Northwest National Laboratory, P.O. Box 999, Richland, WA 99352; robert.leugemors@pnl.gov

28. M. A. Norato, Westinghouse Savannah River Company, P.O. Box 616, Building 786-5A, Aiken, SC 29808; michael.norato@srs.gov

29. R. A. Pierce, Westinghouse Savannah River Company, P.O. Box 616, Building 773-A, Aiken, SC 29808; robert.pierce@srs.gov

30. M. C. Regalbuto, Argonne National Laboratory, Building 205, 9700 South Cass Avenue, Argonne, IL 60439; regalbuto@cmt.anl.gov

31. P. C. Suggs, U.S. Department of Energy, Savannah River Operations Office, P.O. Box A, Building 704-3N, Aiken, SC 29808; patricia.suggs@ srs.gov

32. M. C. Thompson, Westinghouse Savannah River Company, P.O. Box 616, Building 773-A, Aiken, SC 29808; major.thompson@srs.gov

33. T. A. Todd, Idaho National Engineering \& Environmental Laboratory, Building 637, MS-5218, Idaho Falls, ID 834415-5218; ttodd@inel.gov 
34. G. F. Vandegrift, Argonne National Laboratory, Building 205, 9700 South Cass Avenue, Argonne, IL 60439; vandegrift@cmt.anl.gov

35. D. D. Walker, Westinghouse Savannah River Company, P.O. Box 616, Building 773-A, Aiken, SC 29808; darrel.walker@ srs.gov

36. W. R. Wilmarth, Westinghouse Savannah River Company, P.O. Box 616, Building 773-42A, Aiken, SC 29808; bill.wilmarth@srs.gov

37. Tanks Focus Area Technical Team, c/o B. J. Williams, Pacific Northwest National Laboratory, P.O. Box 999, MSIN K9-69, Richland, WA 99352

38. Tanks Focus Area Field Lead, c/o T. P. Pietrok, U.S. Department of Energy, Richland Operations Office, P.O. Box 550, K8-50, Richland, WA 99352

39. Tanks Focus Area Headquarters Program Manager, c/o K. D. Gerdes, DOE Office of Science and Technology, 19901 Germantown Rd., 1154 Cloverleaf Building, Germantown, MD 20874-1290

\section{ELECTRONIC DISTRIBUTION ONLY}

\section{S. B. Aase, ANL, aase@cmt.anl.gov}

H. A. Arafat, ANL, arafat@cmt.anl.gov

M. J. Barnes, WSRC, mark.barnes@srs.gov

G. M. Brown, ORNL, browngm1@ornl.gov

D. Camaioni, PNNL, donald.camaioni@pnl.gov

J. Campbell, PNNL, james.campbell@pnl.gov

S. G. Campbell, WSRC, seth.campbell@srs.gov

J. E. Caton, ORNL ret., catonjejr@msn.com

D. B. Chamberlain, ANL, chamberlain@cmt.anl.gov

W. D. Clark, DOE-SR, bill.clark@srs.gov

S. L. Clifford, PNNL, shari.clifford@srs.gov

J. L. Collins, ORNL, collinsjl@ornl.gov

C. Conner, ANL, conner@cmt.anl.gov

C. R. Duchemin, CEA, duchem $1 @$ aol.com

M. C. Duff, WSRC, martine.duff@srs.gov

R. E. Edwards, DOE-SR, richardedwards (asrs.gov

H. H. Elder, WSRC, herbert.elder@srs.gov

N. L. Engle, ORNL, englenl@ornl.gov

J. R. Falkenberg, ANL, falkenberg@ cmt.anl.gov

F. F. Fondeur, WSRC, fermando. fondeur (Q) srs.gov

L. Greenwood, WSRC, larry.greenwood@pnl.gov

R. N. Hinds, WSRC, robert.hinds@srs.gov

D. T. Hobbs, WSRC, david.hobbs@srs.gov

R. D. Hunt, ORNL, huntrd@ornl.gov

V. G. Kay, DOE-SR, virginia.kay@srs.gov

L. N. Klatt, ORNL Ret., Inklatı@ esper.com

D. P. Lambert, WSRC, dan.lambert@srs.gov

J. D. Law, INEEL, jdlaw@inel.gov

D. D. Lee, ORNL, leedd@ornl.gov

B. L. Lewis, WSRC, brenda.lewis@sss, gov

T. J. Lex, WSRC, thomas.lex $@$ srs.goy

G. J. Lumetta, PNNL, gregg.lumetta@pnl.gov
J. C. Marra, WSRC, james.marra@srs.gov

M. P. Maskarinec, ORNL, maskarinecmp@ornl.gov

A. J. Mattus, ORNL, mattusaj@ornl.gov

J. W. McCullough, Jr., DOE-SR, james.mccullough@srs.gov

C. P. McGinnis, ORNL, mcginniscp@ornl.gov

J. P. Morin, WSRC, jerome.morin@srs.gov

J. R. Noble-Dial, DOE-OR, nobledialjr@oro.doe.gov

L. M. Papouchado, WSRC, lucien.papouchado@srs.gov

F. M. Pennebaker, WSRC, frank.pennebaker@srs.gov

T. B. Peters, WSRC, thomas.peters@ srs.gov

J. A. Pike, WSRC, jeff.pike(a) srs.gov

M. R. Poirier, WSRC, michael.poirier@srs.gov

R. A. Sachleben, sachleben2001@aol.com

S. N. Schlahta, PNNL, steve.schlahta@pnl.gov

R. D. Spence, ORNL, spencerd@ornl.gov

R. H. Spires, WSRC, renee spires@srs.gov

M. E. Stallings, WSRC, mary stallings $(\hat{\theta}$ srs.gov

W. E. Stevens, WSRC, ed.stevens@srs.gov

C. L. Stine, UTK, stine@ novell.chem.utk.edu

S. G. Subosits, WSRC, steve.subosits@ srs.gov

W. L. Tamosaitis, WSRC, walter.tamosaitis@srs.gov

G. A. Taylor, WSRC, glemn.tavlor@srs.gov

W. B. Van Pelt, WSRC, bill.van-pelt@ srs.gov

J. F. Walker, ORNL, walkerjfjr@ornl.gov

F. A. Washburn, WSRC, fatina.washburn@srs.gov

J. S. Watson, ORNL, watsonjs@ornl.gov

D. W. Wester, PNNL, dennis.wester@pnl.gov

T. L. White, WSRC, thomas.white@srs.gov 